\title{
THE INFLUENCE OF SIMULATED PURGE FLOW ON THE SECONDARY FLOW OF A LOW-SPEED TURBINE CASCADE
}

\author{
by \\ Gordon MacIsaac B. Eng. (Aerospace) \\ A thesis submitted to the Faculty of Graduate and Postdoctoral Affairs \\ in partial fulfilment of the degree requirements of

\section{Doctor of Philosophy} \\ in Aerospace Engineering \\ Ottawa-Carleton Institute for Mechanical and Aerospace Engineering \\ Department of Mechanical and Aerospace Engineering \\ Carleton University \\ Ottawa, Ontario, Canada
}

Copyright $(\mathrm{C}$ Gordon MacIsaac

December 2015 


\section{Abstract}

This thesis documents experimental measurements of a low-speed linear turbine cascade. Experiments were carried out in the Low-Speed Turbomachinery Laboratory of Carleton University. The overall objective of the thesis was to enhance the understanding of turbine near endwall flows, termed secondary flows. Of particular interest was the loss generation and energy dissipation mechanisms present within the turbine blade row. Point measurements of the downstream pressure fields for different cascade configurations were made using a seven-hole pneumatic pressure probe. The generation of total pressure losses were examined in relation to the inferred secondary flow structures. Also, the corresponding turbulence field was measured for one of the cascade configurations using a hot-wire probe thereby providing insights into the role of the turbulence field in the generation of total pressure losses. The interpretations of the flow fields were aided with experimental oil film flow visualizations and corresponding CFD (Computational Fluid Dynamics) simulations.

In a real turbine engine there is an axial gap between the stationary and rotating bladerows. The term rim-seal refers to the geometry of the axial gap and the cooling flow, termed purge flow, which is typically injected into the mainstream from within the gap to prevent ingress of the hot mainstream gases. The present work examines the effects of the rim-seal geometry on the secondary flow and associated losses for different levels of purge flow. It was found that the presence of the rim-seal geometry significantly alters the secondary flow compared to that of a traditional flat-endwalled turbine cascade. The addition of purge flow further enhances the secondary flow. The bladerow losses are shown to increase with increasing purge flow. It was therefore recommended that modern turbine designs consider both the rim-seal geometry and the purge flow during the design process. 
Previous studies have shown that a secondary loss mitigation technology, termed nonaxisymmetric endwall contouring, significantly reduces the endwall losses generated through a turbine bladerow. The present work investigated a computationally optimized endwall design for the present turbine cascade for varying levels of purge injection. The experimental measurements found that the endwall contouring, with the upstream rim-seal geometry, was effective at significantly reducing the bladerow losses for the range of positive net purge flows investigated. 


\section{Acknowledgements}

The completion of the present thesis was a significant challenge and could not have been completed without the support and encouragement from several individuals and organizations.

I hold the utmost regard for my thesis supervisor Steen A. Sjolander. He has navigated me through difficult technical and personal challenges and for this I am very grateful. He was influential in developing many skills that I hold in high regard. Thank you.

I also acknowledge the financial and technical support of our research partner, Pratt and Whitney Aircraft (PWA). The technical guidance from an industry leader has given purpose to the present research. I give special mention to the technical guidance and support of Tom Praisner and Renee Jurek of PWA.

I gratefully acknowledge the financial support from the National Sciences and Engineering Research Council (NSERC) of Canada through NSERC Postgraduate Scholarships, the Government of Ontario through Ontario Graduate Scholarships (OGS) and the Department of Mechanical and Aerospace Engineering of Carleton University.

As a graduate student I have formed many relationships with my peers and professors. Many obstacles have been overcome with conversations and interactions with these friends. I therefore acknowledge the many individuals that I have worked with and specifically acknowledge Farzad Taremi, Daniel Knezevici and Professor Metin Yaras.

Lastly, I acknowledge the support and continued sacrifice of my wife Justyne. Your unwavering support has fueled me through the most difficult times. Your selflessness has made it possible for me to reach my goals and for that I am forever grateful. 


\section{Table of Contents}

$\begin{array}{ll}\text { Abstract } & \text { i }\end{array}$

Acknowledgements $\quad$ iii

List of Figures $\quad$ xi

$\begin{array}{lc}\text { List of Tables } & \text { xix }\end{array}$

List of Symbols $\quad$ xxi

Chapter 1: Introduction 1

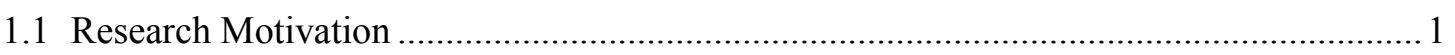

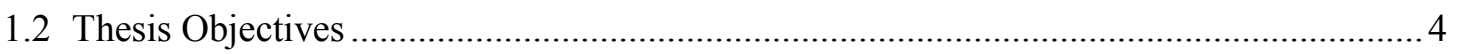

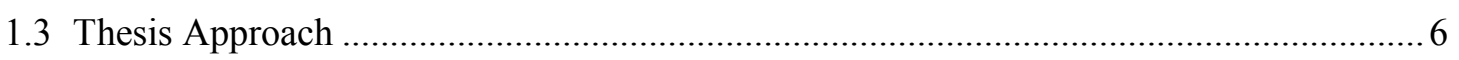

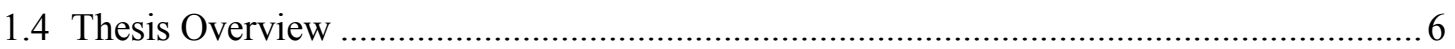

Chapter 2: Background and Literature Review $\quad 8$

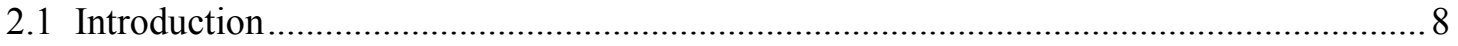

2.2 The Flow Field and Losses in Axial Turbine Blade Passages ......................................... 9

2.3 The Role of Low-speed Linear Turbine Cascade Research ............................................. 10

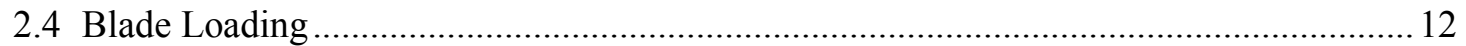

2.5 Profile and Secondary Losses in Linear Turbine Cascades ............................................ 15

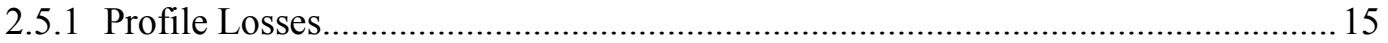

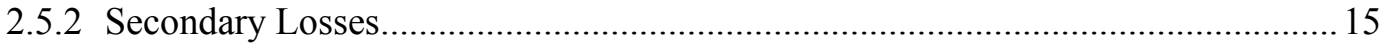

2.6 Research on Secondary Losses in Linear Turbine Cascades .......................................... 18

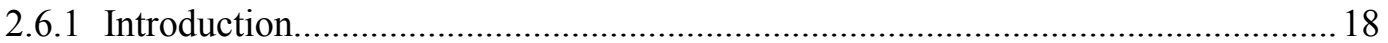

2.6.2 Effects of Inlet Endwall Boundary Layer and Free-stream Turbulence Intensity .. 19

2.6.3 Effects of Blade Loading and Blade Loading Distributions ..................................21

2.6.4 Turbulence and Losses within the Secondary Flow ..............................................2. 23 


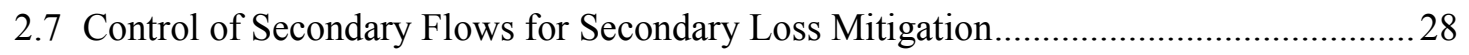

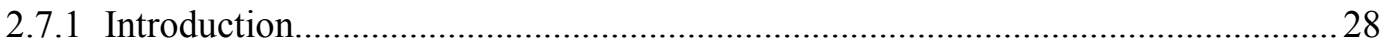

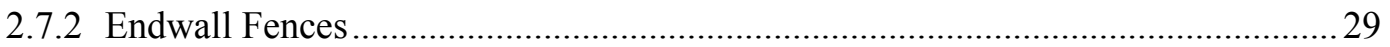

2.7.3 Leading Edge and Near Endwall Blade Profile Modifications............................... 30

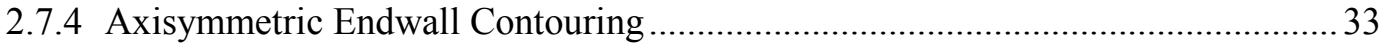

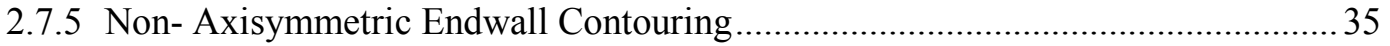

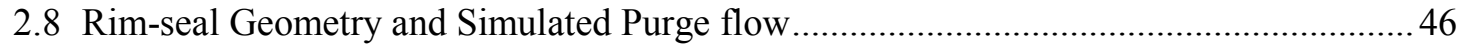

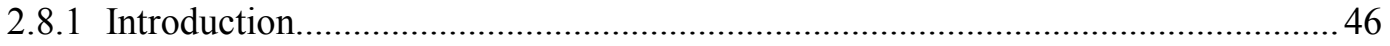

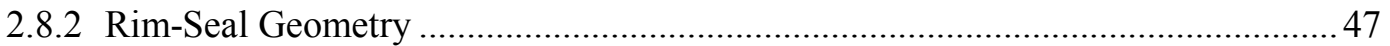

2.8.3 The Interaction between Purge and Mainstream Flows......................................... 49

Chapter 3: $\quad$ Experimental Methods and Data Reduction Procedures 57

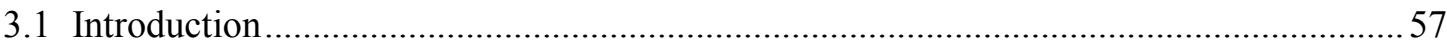

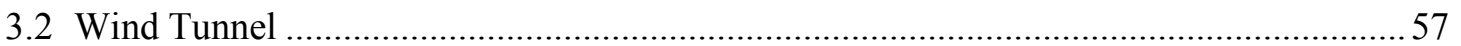

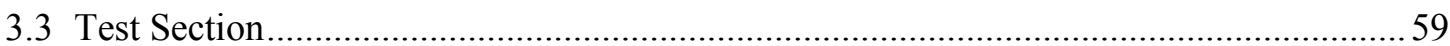

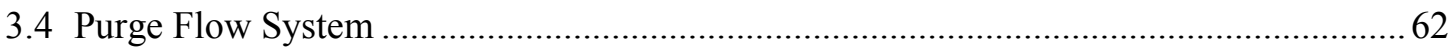

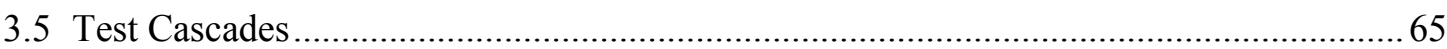

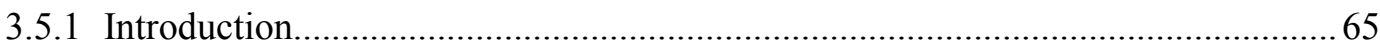

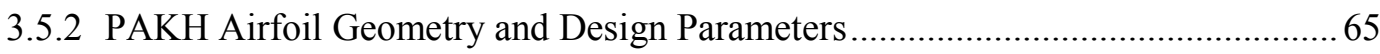

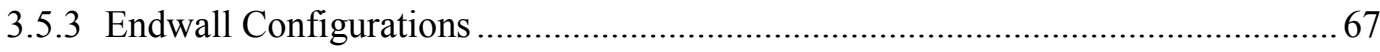

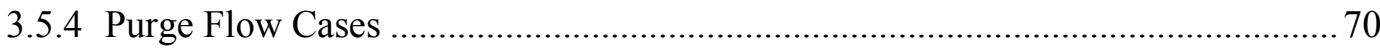

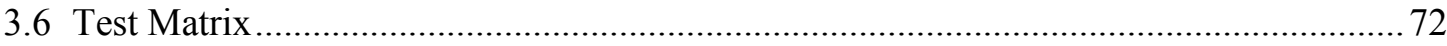

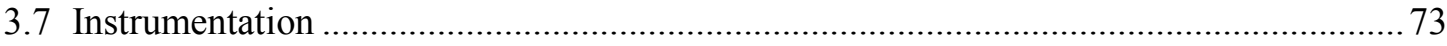

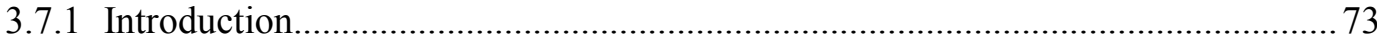

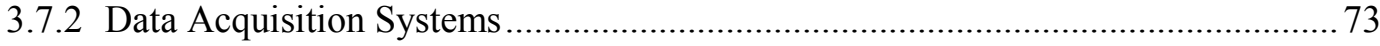

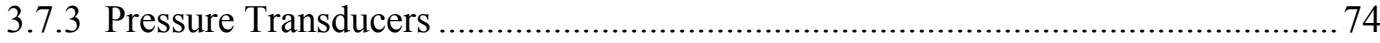

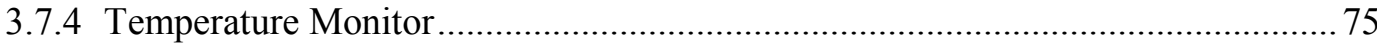

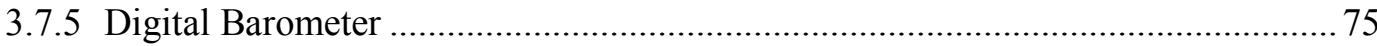

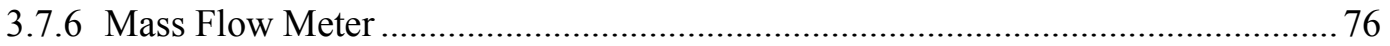

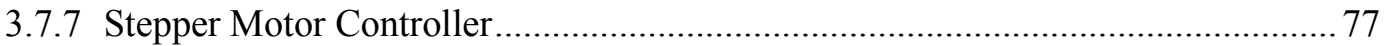

3.8 Measurement Probes, Measurement Procedures, Calibration Procedures, and

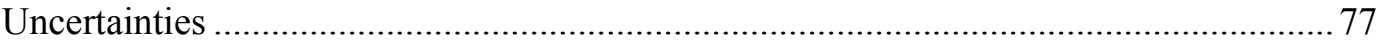

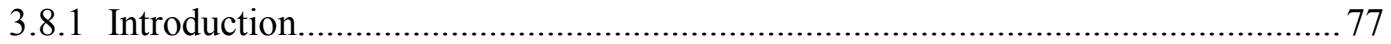


3.8.2 Pressure Measurement Sampling Rate and Time ............................................. 78

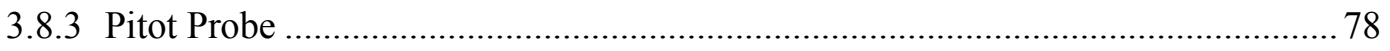

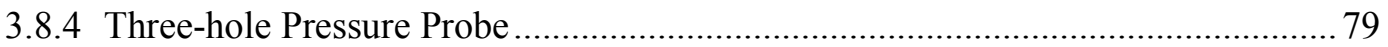

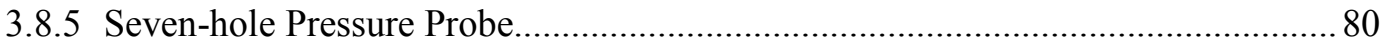

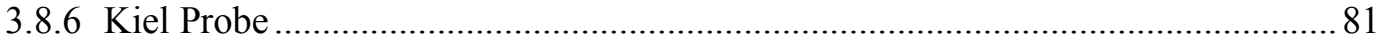

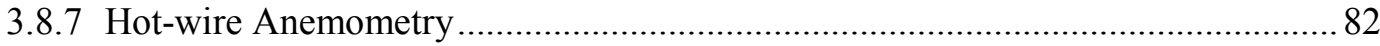

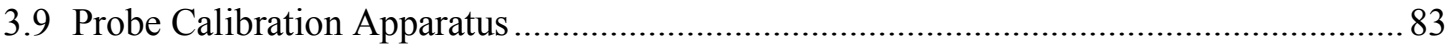

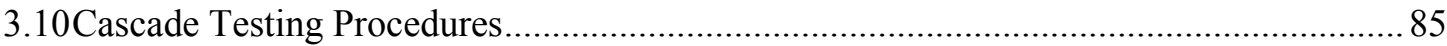

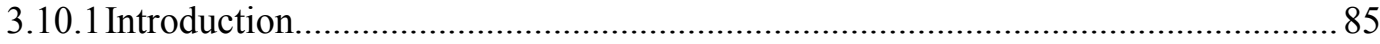

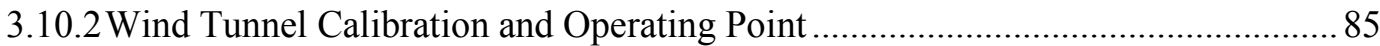

3.10.3 Downstream Traverse Locations and Measurement Grid ...................................... 86

3.10.4Blade Surface Static Pressure Measurements ........................................................... 88

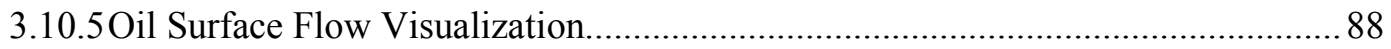

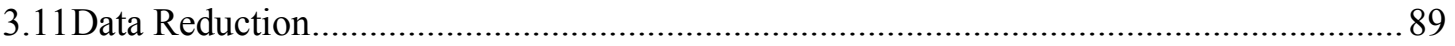

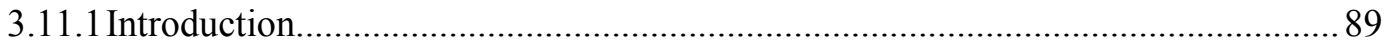

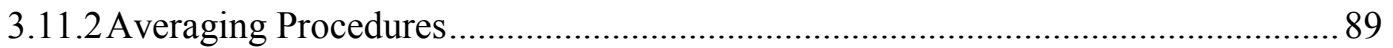

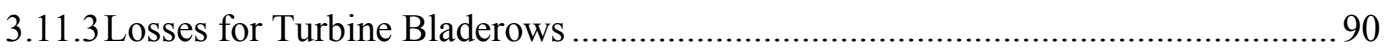

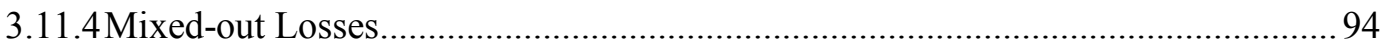

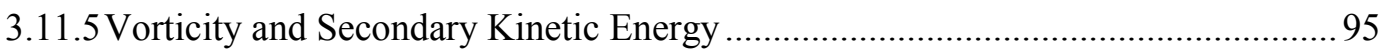

$\begin{array}{lll}\text { Chapter 4: } & \text { Computational Methods } & 97\end{array}$

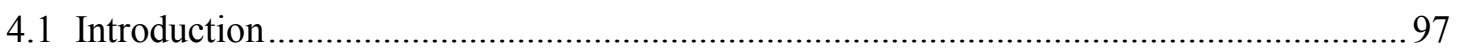

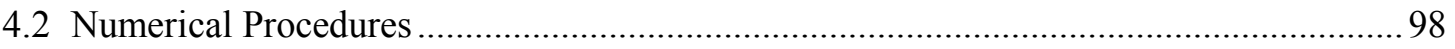

4.3 Domain Geometry, Blocking and Meshing Strategies.................................................... 99

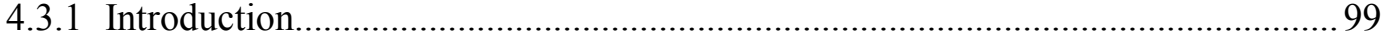

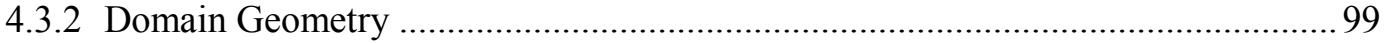

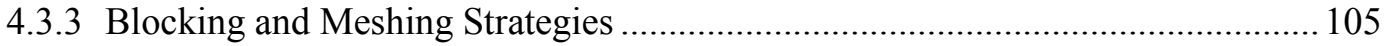

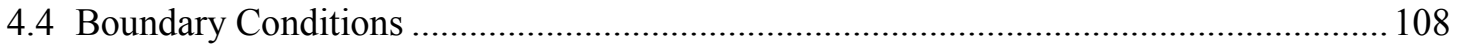

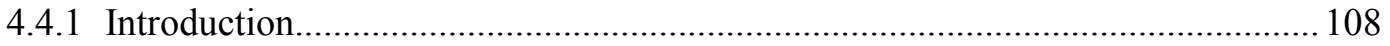

4.4.2 Definition of the Mainstream Inlet Boundary Conditions .................................... 109

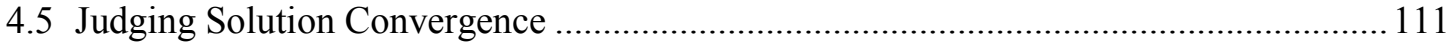

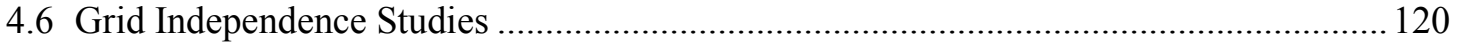

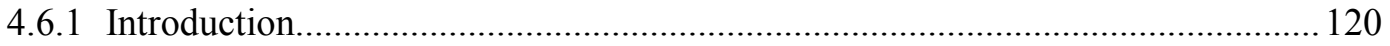




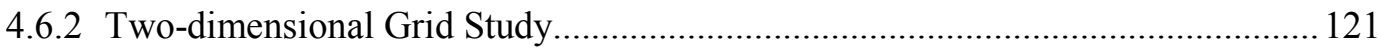

4.6.3 Three-dimensional Grid Sensitivity Study for the Flat Endwall Case.................. 129

4.6.4 Three-dimensional Grid Sensitivity Study for the Endwall Cavity Case ............. 133

\section{Chapter 5: Reynolds Stresses and Loss Production within the Secondary Flow Downstream of a Turbine Cascade 139}

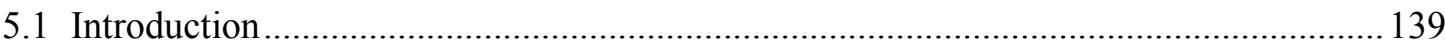

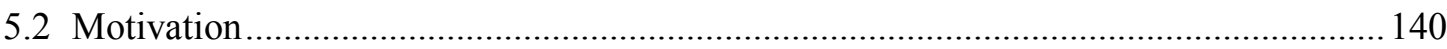

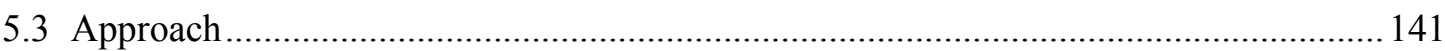

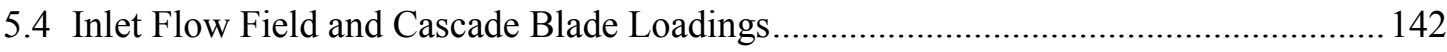

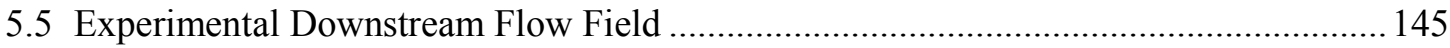

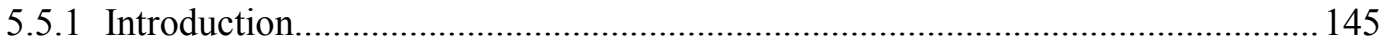

5.5.2 Development of the Secondary Flow with Downstream Distance ....................... 145

5.5.3 Integrated Losses and Other Flow Quantities ..................................................... 149

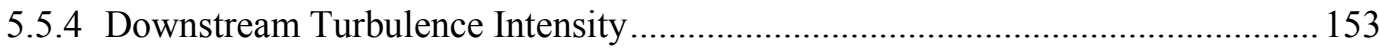

5.5.5 Turbulence and Loss Production from the Mean Flow ........................................ 156

5.6 Computational Results for the Downstream Flow Field ............................................... 168

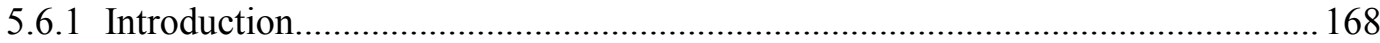

5.6.2 Development of the Secondary Flow with Downstream Distance ....................... 168

5.6.3 Integrated Losses and Other Flow Quantities .................................................... 171

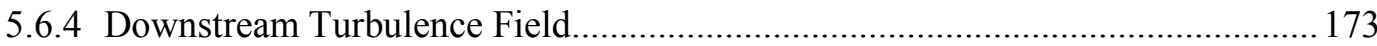

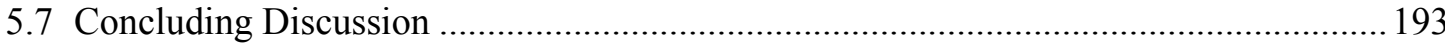

\section{Chapter 6: Secondary Flows of Turbine Cascades with Simplified Upstream Platform Overlaps and Endwall Cavities $\quad 196$}

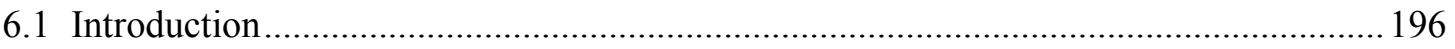

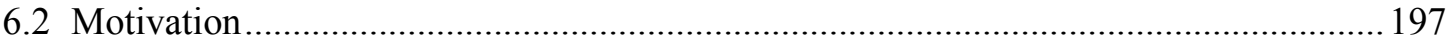

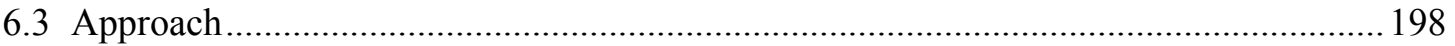

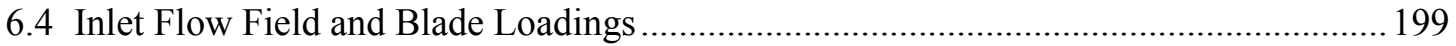

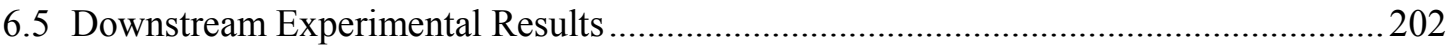

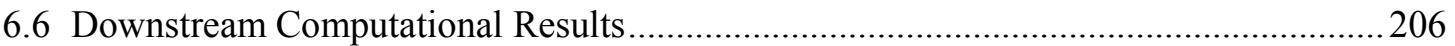

6.7 Experimental and Computational Integrated Results...................................................209

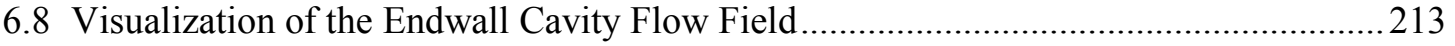

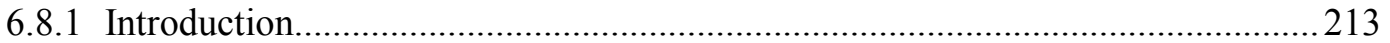


6.8.2 Interpretation of the Flow Fields for the Flat and Cavity Endwall

Configurations 213

6.8.3 The Effect of Restricting the Bladerow Potential Field with a Simplified Step ... 221

6.9 Concluding Discussion 226

Chapter 7: Secondary Flow of a Turbine Cascade with Simulated Purge Flow

7.1 Introduction .228

7.2 Motivation 229

7.3 Approach 230

7.4 Inlet Flow Field and Purge Flow Velocity Triangles. 230

7.5 Blade Loadings with Purge Flow Injection... 232

7.6 Downstream Experimental Results .236

7.6.1 Introduction. 236

7.6.2 Periodicity of the Secondary Flow with Purge Flow Injection. 236

7.6.3 The Effects of Purge Flow on the Downstream Flow field. 239

7.7 Downstream Computational Results. 247

7.8 Experimental and Computational Integrated Losses and Other Flow Quantities . 253

7.9 Flow Visualization of the Endwall Flow for Different Levels of Purge Flow Injection. 259

7.10Interpretation of the Loss Growth through the Blade Passage ....................................... 268

7.11Concluding Discussion 271

\section{Chapter 8: Secondary Loss Mitigation Technology: Non-Axisymmetric Endwall Contouring and an Elliptically Shaped Platform Overlap}

8.1 Introduction 273

8.2 Motivation .275

8.3 Approach.... .276

8.4 Inlet Flow Field. .276

8.5 The Effects of Non-axisymmetric Endwall Contouring on the Secondary Flow of the Linear Turbine Cascade 277

8.5.1 Introduction 277

8.5.2 Downstream Flow Field with Non-axisymmetric Endwall Contouring. 277

8.5.3 Integrated Flow Quantities for the Endwall Contouring Configuration 284

8.5.4 Surface flow visualization .292 
8.6 The Effects of an Elliptical Platform Overlap on the Secondary Flow of the Linear Turbine Cascade with Flat Intra-passage Endwalls ....................................................... 300

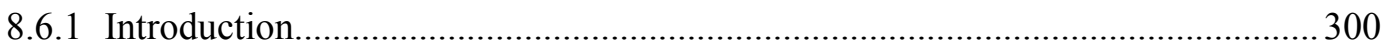

8.6.2 The Downstream Flow Field with an Elliptical Platform Overlap .......................300

8.6.3 Integrated Flow Quantities for the Elliptical Platform Overlap Configuration .... 306

8.6.4 Flow Visualization for the Elliptical Overlap Configuration................................. 310

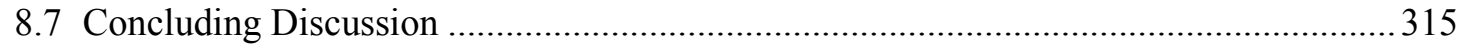

Chapter 9: Summary, Conclusions and Recommendations 318

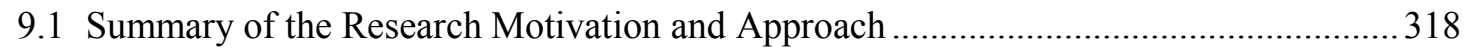

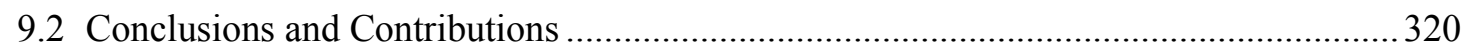

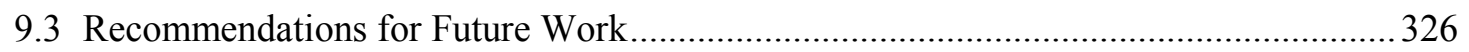

$\begin{array}{ll}\text { References } & 328\end{array}$

Appendix A: $\quad$ Pressure Transducer Calibration $\quad$ A-1

Appendix B: Procedure for Determining Experimental Uncertainties B-1

Appendix C: Calibration Procedures and Data Reduction Methods for the Three-hole Pressure Probe C-1

Appendix D: Calibration Procedures and Data Reduction Methods for the Seven-hole Pressure Probe D-1

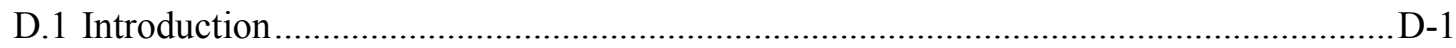

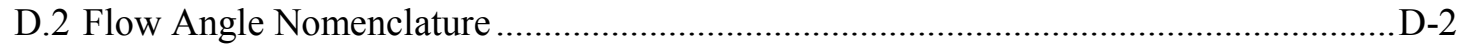

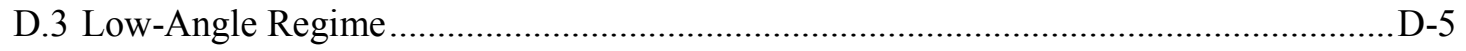

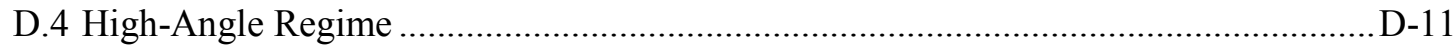

D.5 Challenges in Measuring Total Pressure within Turbine Cascade Secondary Flows

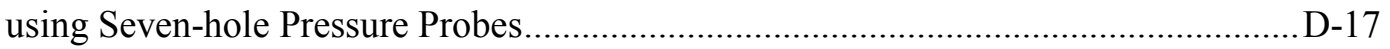

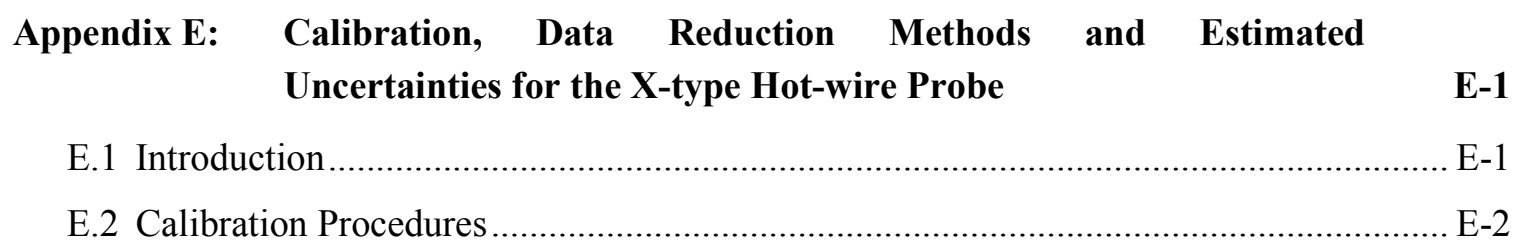




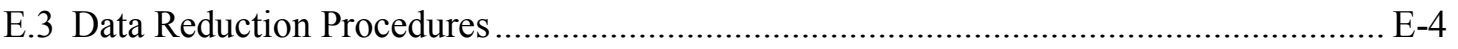

E.4 Uncertainties of the X-type Hotwire Probe .................................................................... E-5

E.4.1 Uncertainty of the Effective Cooling Velocity ...................................................... E-6

E.4.2 Uncertainty of the Mean Velocity Vector............................................................... E-7

E.4.3 Uncertainty of the Reynolds Stresses ............................................................. E-8

Appendix F: Additional Experimental Results for the Cascade Configuration with a Rectangular Platform Overlap and Flat Intra-passage Endwalls (RF) $\quad$ F-1

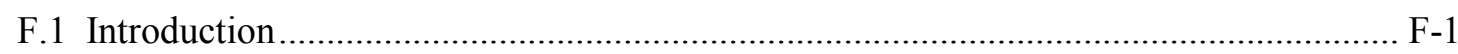

F.2 Downstream Measurements for the Rectangular Flat (RF) configuration at the $1.20 C_{x}$

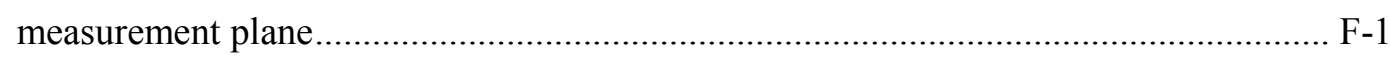

Appendix G: Experimental Results for the Cascade Configuration with an (EC) G-1

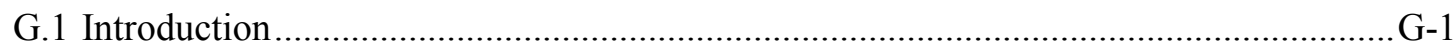

G.2 Downstream Measurements and Overall Integrated Flow Quantities for the EF configuration at the $1.40 \mathrm{Cx}$ measurement plane.

G-1 


\section{List of Figures}

Figure 1.1: PW1000G gas turbine engine cut-away [Source: www.pw.utc.com] ......................2

Figure 1.2: Schematic of stationary and rotating engine blades with platform overlap

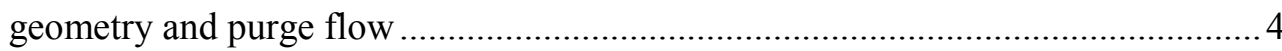

Figure 2.1: $\quad$ Main features of a turbine flow field, from Sjolander (2007)............................... 9

Figure 2.2: Turbine pressure distribution (a) and control volume definition (b), from

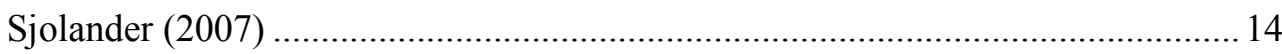

Figure 2.3: Interpretation of endwall flow structures, from Wang et al. (1997) ....................... 17

Figure 2.4: $\quad$ Example of leading edge bulbs, from Sauer et al. (2001) ....................................... 31

Figure 2.5: Contoured endwall heights ( $\mathrm{mm})$, reproduced from Hartland et al. (2000) ........... 36

Figure 2.6: Recessed and raised leading edge (LE) platform shapes, adapted from

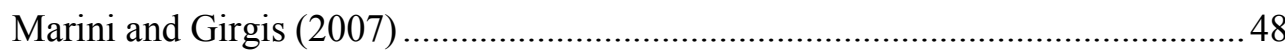

Figure 2.7: Schematic of the mainstream and purge flow relative inlet velocity triangles .......52

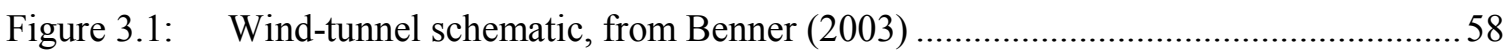

Figure 3.2: Linear cascade test-section, from Benner (2003) …............................................59

Figure 3.3: Upstream (a) and downstream (b) midspan flow quality measurements................ 61

Figure 3.4: A photograph of the flat intra-passage platform overlap cascade hardware (a) and a schematic drawing of cascade cross-section (b) .......................................... 62

Figure 3.5: A photograph (a) and schematic drawing (b) of the purge flow delivery system

Figure 3.6: A typical pitchwise distribution of the endwall cavity static pressure coefficient $\left(C_{P S}\right)$ with purge flow.....

Figure 3.7: Cascade nomenclature showing the measurement coordinate systems ................. 67

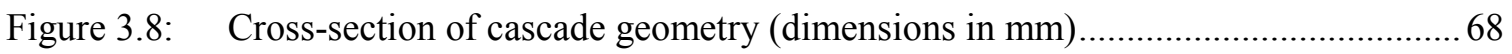

Figure 3.9: Endwall contouring geometry with a colour flood of the perpendicular distance $\left(\% C_{x}\right)$ from to the flat intra-passage endwall (RF)............................... 70

Figure 3.10: Geometric simplifications of the platform overlap (dimensions in $\mathrm{mm}$ )............... 71

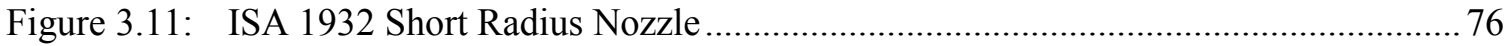




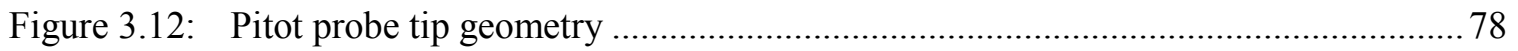

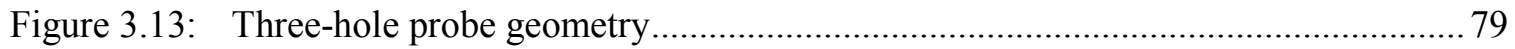

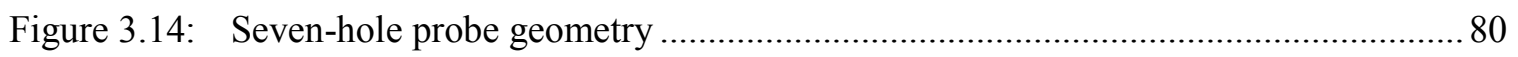

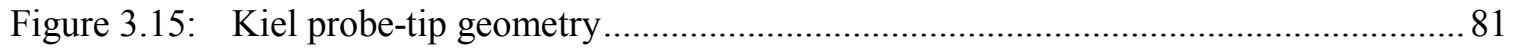

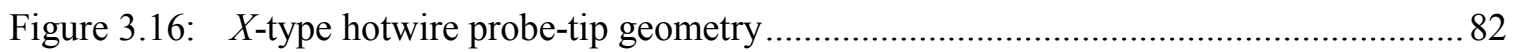

Figure 3.17: Schematic drawing of the low-speed calibration facility (Benner, 2003)............... 84

Figure 3.18: Colour flood of the total pressure coefficient $\left(C_{P 0}\right)$ with the measurement

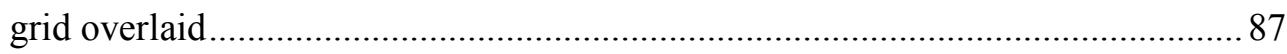

Figure 3.19: Schematic diagram showing the mass flow balance for a bladerow with purge flow.

Figure 3.20: Sensitivity of the total loss coefficient $(Y)$ with respect to the purge exit flow angle.

Figure 4.1: CFD domain and boundary conditions (a). CFD mesh (b).

Figure 4.2: Total pressure coefficient $\left(C_{P 0}\right)$ along the periodic boundaries at midspan $(\mathrm{z} / \mathrm{h}=0.5)$ for (a) Mesh 1 and (b) Mesh 2

Figure 4.3: (a) CFD domain with one-to-one periodic boundaries and (b) CFD mesh........... 102

Figure 4.4: (a) CFD domain with the platform overlap geometry (b) CFD mesh................... 105

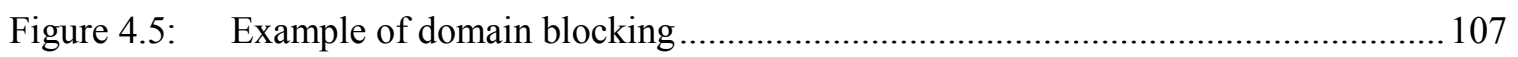

Figure 4.6: Boundary conditions applied to domain surfaces .............................................. 109

Figure 4.7: Inlet turbulence boundary condition profiles..................................................... 111

Figure 4.8: Example of the RMS (a) and MAX (b) residuals for the RF 125 case ................. 113

Figure 4.9: Total pressure coefficient $\left(C_{P 0}\right)$ at the $1.40 C_{x}$ plane output at $250^{\text {th }}$ (a) and

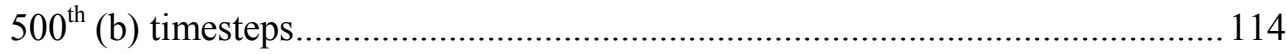

Figure 4.10: Example of RMS residuals for a case with periodic unsteady oscillations .......... 115

Figure 4.11: Example of RMS residuals for a case with periodic unsteady oscillations .......... 116

Figure 4.12: Point-by-point differences of the total pressure coefficients $\left(C_{P 0}\right)$ from the $810^{\text {th }}$ and $840^{\text {th }}$ timesteps at the $1.40 C_{x}$ plane

Figure 4.13: Pitchwise integrated loss coefficient for the $810^{\text {th }}$ and $840^{\text {th }}$ timesteps at the $1.40 C_{x}$ plane 118

Figure 4.14: RMS residuals for the modified near endwall grid 119

Figure 4.15: Pitchwise integrated loss coefficient for the converged steady-state simulation at the $1.40 C_{x}$ plane 120 
Figure 4.16: Two-dimensional distributions of $C_{P 0}, C_{q}$ and $\beta$ at $1.20 C_{x}$ (a-c) and $1.40 C_{x}$ (d-f)

Figure 4.17: Total pressure coefficient $\left(C_{P 0}\right)$ at the blade trailing edge.

Figure 4.18: (a) Profile loss coefficient and (b) pitchwise area averaged flow angle (b) at $1.40 C_{x}$

Figure 4.19: Blade surface static pressure coefficient.

Figure 4.20: (a) Blade suction surface skin friction coefficient $\left(C_{f}\right)$ and (b) the suction surface pressure gradient acceleration parameter (AC)

Figure 4.21: Colour floods of the streamwise vorticity coefficient $\left(C_{\omega s}\right)$ overlaid with line contours of the total pressure coefficient $\left(C_{P 0}\right)$ at $1.40 C_{x}$

Figure 4.22: Pitchwise averaged loss coefficient (a), outlet pitchwise flow angle (b) and the secondary kinetic energy coefficient at $1.40 C_{x}$

Figure 4.23: (a) Overall loss coefficient and (b) Secondary loss coefficient at $1.40 C_{x}$

Figure 4.24: Colour floods of the streamwise vorticity coefficient $\left(C_{\omega s}\right)$ overlaid with line contours of the total pressure coefficient $\left(C_{P 0}\right)$ at $1.40 C_{x}$ 136

Figure 4.25: Pitchwise averaged loss coefficient (a), outlet pitchwise flow angle (b) and the secondary kinetic energy coefficient at $1.40 C_{x}$

Figure 4.26: (a) Overall loss coefficient and (b) Secondary loss coefficient at $1.40 C_{x} \ldots \ldots \ldots \ldots . . . .138$

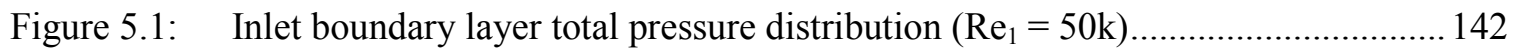

Figure 5.2: Midspan turbulence intensity (left) and Reynolds stresses (right)....................... 143

Figure 5.3: $\quad$ Blade surface static pressure distribution measured at midspan $(\mathrm{z} / \mathrm{h}=0.5) \ldots \ldots \ldots 144$

Figure 5.4: Total pressure coefficient $\left(C_{P 0}\right)$ floods at (a) $1.00 C_{x}$, (b) $1.20 C_{x}$ and (c) $1.40 C_{x}$

Figure 5.5: Streamwise vorticity coefficient $\left(C \omega_{s}\right)$ floods with contour lines of total pressure coefficient $\left(C_{P 0}\right)$ and secondary velocity vectors at (a) $1.00 C_{x}$, (b) $1.20 C_{x}$ and (c) $1.40 C_{x}$ 146

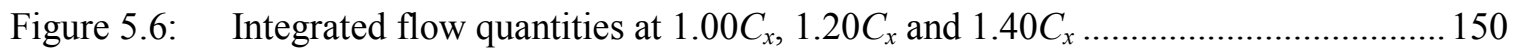

Figure 5.7: Mixing loss breakdown at $1.20 C_{x}$ and $1.40 C_{x}$

Figure 5.8: Floods of local turbulence intensity $\left(T u_{l o c}\right)$ with contour lines of total pressure coefficient $\left(C_{P 0}\right)$ at (a) $1.00 C_{x}$, (b) $1.20 C_{x}$ and (c) $1.40 C_{x}$.

Figure 5.9: Floods of the deformation work term, $\Psi_{\mathrm{i}, \mathrm{j}}$, with contour lines of total pressure coefficient and the secondary velocity vectors at (a) $1.20 C_{x}$ and (b) $1.40 C_{x}$ 160 
Figure 5.10: Pitchwise distributions of the deformation work components at $\mathrm{z} / \mathrm{h}=0.5$, 0.18 and 0.08 from the $1.20 C_{x}$ plane.

Figure 5.11: Pitchwise distributions of the deformation work components at $\mathrm{z} / \mathrm{h}=0.5$, 0.18 and 0.08 from the $1.40 C_{x}$ plane 166

Figure 5.12: Measured and computed total pressure coefficient $\left(C_{P 0}\right)$ floods at $1.20 C_{x}$ and $1.40 C_{x}$

Figure 5.13: Measured and computed streamwise vorticity coefficient $\left(C_{\omega s}\right)$ floods and secondary velocity vectors at $1.20 C_{x}$ and $1.40 C_{x}$

Figure 5.14: Comparison between the measured and CFD integrated flow parameters 172

Figure 5.15: Predicted non-dimensional eddy viscosity at $1.20 \mathrm{Cx}$. 176

Figure 5.16: Floods of the measured non-dimensional shear eddy viscosity components at $1.20 C_{x}$

Figure 5.17: Pitchwise distributions of the non-dimensional eddy viscosity $\left(v_{t 1,2} / v_{L}\right)$, mean strain rate $\left(S_{1,2}\right)$ and Reynolds stress $\left(\overline{u^{\prime} v^{\prime}}\right)$ at $\mathrm{z} / \mathrm{h}=0.10$ of the $1.20 C_{x}$ plane.

Figure 5.18: Pitchwise distribution of the non-dimensional eddy viscosity $\left(v_{t 1,3} / v_{L}\right)$, mean strain rate $\left(S_{1,3}\right)$ and Reynolds stress $\left(\overline{u^{\prime} w^{\prime}}\right)$ at $\mathrm{z} / \mathrm{h}=0.14$ of the $1.20 C_{x}$ plane.

Figure 5.19: Pitchwise distributions of the non-dimensional eddy viscosity $\left(v_{T 2,3} / v_{L}\right)$, mean strain rate $\left(S_{2,3}\right)$ and Reynolds stress $\left(\overline{v^{\prime} w^{\prime}}\right)$ at $\mathrm{z} / \mathrm{h}=0.08(\mathrm{a}-\mathrm{b})$ and $\mathrm{z} / \mathrm{h}$ $=0.40(\mathrm{c}-\mathrm{d})$ of the $1.20 C_{x}$ plane.

Figure 5.20: Pitchwise distributions of (a) the non-dimensional secondary velocity components and (b) the velocity gradients at $\mathrm{z} / \mathrm{h}=0.40$

Figure 5.21: Floods of the measured and predicted Reynolds normal stresses with line contours of the streamwise vorticity coefficient at $1.20 C_{x}$

Figure 5.22: Floods of the measured and predicted Reynolds shear stresses with line contours of the streamwise vorticity coefficient at $1.20 C_{x}$

Figure 5.23: Floods of the measured and predicted deformation work, $\Psi$, with line contours of the streamwise vorticity coefficient at $1.20 C_{x}$ and $1.40 C_{x}$

Figure 5.24: Streamlines through the counter vortex core coloured with the local turbulence kinetic energy coefficient, $C_{T K E}$

Figure 6.1: Geometric simplifications of the platform overlap (dimensions in $\mathrm{mm}$ ).......... 198 
Figure 6.2: Inlet boundary layer total pressure distribution $\left(\operatorname{Re}_{1}=75 \mathrm{k}\right)$

Figure 6.3: Measured blade surface static pressure distribution at $\mathrm{z} / \mathrm{h}=0.5$ and $\mathrm{z} / \mathrm{h}=0.05 \ldots .200$

Figure 6.4: Predicted blade surface static pressure distribution at $\mathrm{z} / \mathrm{h}=0.5$ and $\mathrm{z} / \mathrm{h}=0.05 \ldots .201$

Figure 6.5. Floods of the experimentally measured $C \omega_{S}$ overlaid with the line contours of $C_{P 0}$ for the (a) Baseline, (b) Cavity, (c) Step and (d) Ramp configurations .....203

Figure 6.6. Floods of the experimentally measured $C_{S K E}$ overlaid with the secondary velocity vectors for the (a) Baseline, (b) Cavity, (c) Step and (d) Ramp configurations ..... 205

Figure 6.7: Floods of the predicted (CFD) $C_{\omega S}$ overlaid with the line contours of $C_{P 0}$ for the (a) Baseline, (b) Cavity, (c) Step and (d) Ramp configurations. 207

Figure 6.8: Floods of the predicted $C_{S K E}$ overlaid with the secondary velocity vectors for the (a) Baseline, (b) Cavity, (c) Step and (d) Ramp configurations......................208

Figure 6.9: Experimental and computational pitchwise-averaged results

Figure 6.10: ISO-surface of constant TKE and surface streamlines showing the secondary flow of the (a) baseline and (b) cavity case 215

Figure 6.11: Endwall surface visualization and interpretation. 218

Figure 6.12: Cut-planes oriented with the design inlet flow angle showing the endwall cavity streamlines for the (a) Step and (b) cavity case .....

Figure 6.13: Cavity static pressure at $\mathrm{z} / \mathrm{h}=-0.048$ (one half the step height) ........................ 223

Figure 6.14: Purge slot axial velocity. .225

Figure 7.1: $\quad$ Purge flow velocity triangles ( $5 \mathrm{x}$ the mainstream scale) with a fixed injection angle for (a) low and (b) high purge mass flow ratios. 231

Figure 7.2: Measured blade surface static pressure distribution at $\mathrm{z} / \mathrm{h}=0.5$ and $\mathrm{z} / \mathrm{h}=0.05$ with varying levels of purge flow 233

Figure 7.3: Predicted blade surface static pressure distribution at $\mathrm{z} / \mathrm{h}=0.5$ and $\mathrm{z} / \mathrm{h}=0.05$ with varying levels of purge flow

Figure 7.4: Measured and predicted axial velocity ratio (AVR) with increasing purge mass flow ratio $(\phi)$

Figure 7.5: Secondary flow periodicity measurements at different spanwise locations for $\phi=0.90$. .238

Figure 7.6: Total pressure coefficient measured over two adjacent passages for $\phi=0.9$ at $1.40 C_{x}$. .239

Figure 7.7: Schematic of the cascade numbering and purge slot termination points 239 
Figure 7.8: Floods of the total pressure coefficient $\left(C_{P 0}\right)$ for varying levels of purge flow measured at $1.40 C_{x}$.

Figure 7.9: Floods of the streamwise vorticity coefficient $\left(C_{\omega S}\right)$ with contour lines of the total pressure coefficient $\left(C_{P 0}\right)$ for varying levels of purge flow measured at $1.40 C_{x}$

Figure 7.10: Measured passage vortex flow quantities with increasing flow purge flow.....

Figure 7.11: Floods of the secondary kinetic energy coefficient $\left(C_{S K E}\right)$ with the secondary velocity vectors overlaid for varying levels of purge flow measured at $1.40 C_{x} \ldots 246$

Figure 7.12: Floods of the predicted $C_{\omega S}$ overlaid with the line contours of $C_{P 0}$ for $\phi=$ (a) 0.00 , (b) 0.75 , (c) 1.00 , and (d) 1.25

Figure 7.13: Floods of the predicted $C_{S K E}$ overlaid with the secondary velocity vectors for $\phi=$ (a) 0.00 , (b) 0.75 , (c) 1.00, and (d) 1.25 .250

Figure 7.14: Predicted and measured passage vortex flow quantities with increasing flow purge flow

Figure 7.15: Pitchwise integrated flow quantities for different purge flow levels at the $1.40 \mathrm{C}_{\mathrm{x}}$ axial plane.

Figure 7.16: Overall in-plane $(Y)$ and mixed-out $\left(Y_{\text {mixed }}\right)$ loss coefficients for different levels of purge flow at $1.40 \mathrm{C}_{\mathrm{x}}$

Figure 7.17: Endwall surface visualization and interpretation. 261

Figure 7.18: Velocity streamlines within the endwall cavity for the (b) RF 000 and (c) RF 125 cases. 263

Figure 7.19: Visualization of the intra-passage flow field 265

Figure 7.20: Predicted relative loss growth through the passage for varying levels of purge flow

Figure 8.1: Floods of the total pressure coefficient $\left(C_{P 0}\right)$ for varying levels of purge flow measured at $1.40 C_{x}$ for the $\mathrm{RF}$ and $\mathrm{RC}$ configurations

Figure 8.2: Floods of the streamwise vorticity coefficient $\left(C_{\omega S}\right)$ with contour lines of the total pressure coefficient $\left(C_{P 0}\right)$ for varying levels of purge flow measured at $1.40 C_{x}$ for the RF and RC configurations.....

Figure 8.3: Floods of the secondary kinetic energy coefficient $\left(C_{S K E}\right)$ with the secondary velocity vectors overlaid for varying levels of purge flow measured at $1.40 C_{x}$ for the RF and RC configurations. .282

Figure 8.4: Pitchwise-averaged results measured at the $1.40 \mathrm{C}_{\mathrm{x}}$ plane for the contoured endwall configuration (RC) 286 
Figure 8.5: Overall $(Y)$ and Mixed-out $\left(Y_{\text {mixed }}\right)$ loss coefficients for different levels of purge flow at $1.40 \mathrm{C}_{\mathrm{x}}$ for the $\mathrm{RF}$ and $\mathrm{EF}$ configurations

Figure 8.6: Endwall surface visualization and interpretation for the RC configuration..........293

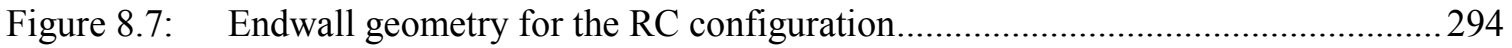

Figure 8.8: Flow field endwall cavity interpretation .295

Figure 8.9: Floods of the total pressure coefficient $\left(C_{P 0}\right)$ for varying levels of purge flow measured at $1.40 C_{x}$ for the RF and EF platform overlap configurations

Figure 8.10: Floods of the streamwise vorticity coefficient $\left(C_{\omega S}\right)$ with contour lines of the total pressure coefficient $\left(C_{P 0}\right)$ for varying levels of purge flow measured at $1.40 C_{x}$ for the RF and EF platform overlap configurations

Figure 8.11: Floods of the secondary kinetic energy coefficient $\left(C_{S K E}\right)$ with the secondary velocity vectors overlaid for varying levels of purge flow measured at $1.40 C_{x}$ for the RF and EF platform overlap configurations 305

Figure 8.12: Overall $(Y)$ and Mixed-out $\left(Y_{\text {mixed }}\right)$ loss coefficients for different levels of purge flow at $1.40 \mathrm{C}_{\mathrm{x}}$ for the $\mathrm{RF}$ and $\mathrm{EF}$ configuations.

Figure 8.13: Floods of the total pressure coefficient $\left(C_{P 0}\right)$ and the streamwise vorticity coefficient $\left(C_{\omega S}\right)$ for $\phi=0.90$ measured at $1.40 C_{x}$ 309

Figure 8.14: Endwall surface visualization and interpretation for the EF configuration 313

Figure A.1: Water micromanometer, from Benner (2003) A-2

Figure A.2: Calibration data and curve fits for the ASG DRAL501DN $\left( \pm 1\right.$ inch $\left.\mathrm{H}_{2} \mathrm{O}\right)$ transducers

Figure A.3: Calibration data and curve fits for the ASG DRAL505DN $\left( \pm 5\right.$ inch $\left.\mathrm{H}_{2} \mathrm{O}\right)$ transducers A-4

Figure C.1: Sample calibration data for the three-hole probe at two Reynolds numbers ........ C-3

Figure D.1: Probe tip numbering and Cartesian coordinate system.

Figure D.2: Tangential probe coordinate system, reproduced from Scribner (2011) and originally adapted from Gerner et al. (1984) D-3

Figure D.3: Polar probe coordinate system, reproduced from Scribner (2011) and originally adapted from Gerner et al. (1984) ..................................................... D-3

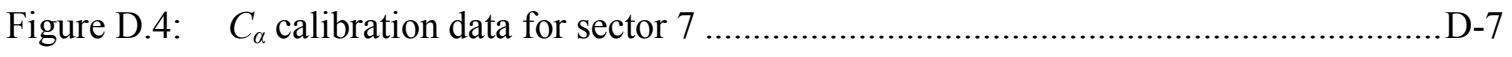

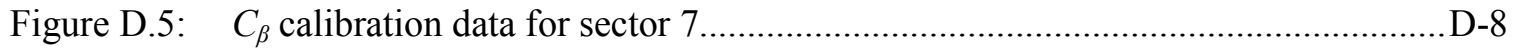

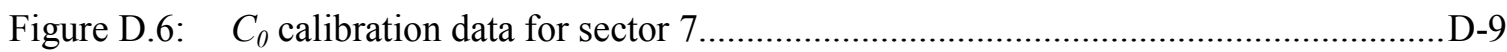

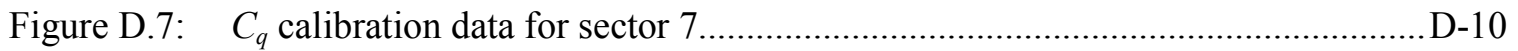


Figure D.8: (a) Division of probe tip angular space, reproduced from Gerner et al. (1984) and (b) the experimental probe tip angular space ............................................

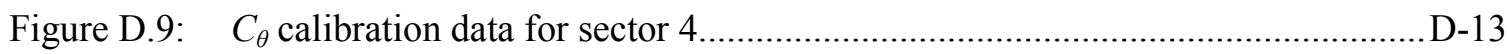

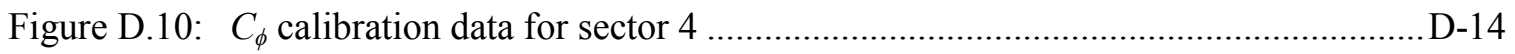

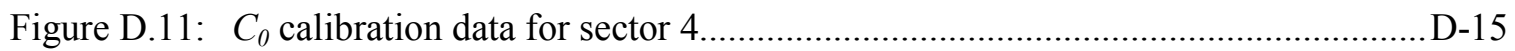

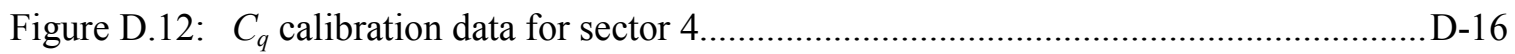

FigureD.13: The effect of shear flow on the inferred flow angle of a seven-hole probe ........D-18

Figure D.14: $C_{P 0}$ measured using the Kiel and seven-hole probe (a) and point by point total pressure difference, $\% q_{1, C L}$, with line contours of $T u_{l o c}$ (b) ...................... D-19

Figure E.1: Thermal Anemometry Circuit................................................................................. E-2

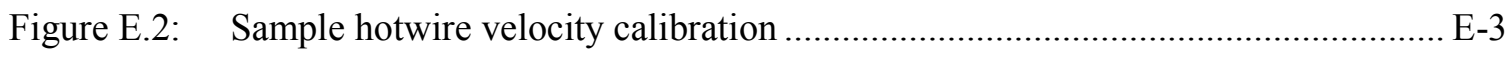

Figure E.3: Directional sensitivity functions for the $x$-type hotwire probe ............................ E-4

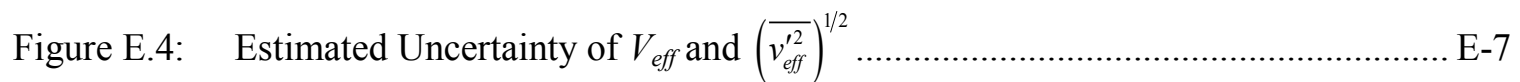

Figure E.5: Overall uncertainty of the measured mean velocity ......................................... E-8

Figure F.1: Floods of the total pressure coefficient $\left(C_{P 0}\right)$ for varying levels of purge flow measured at $1.20 C_{x}$

Figure F.2: Floods of the streamwise vorticity coefficient $\left(C_{\omega S}\right)$ with contour lines of the total pressure coefficient $\left(C_{P 0}\right)$ for varying levels of purge flow measured at $1.20 C_{x}$

Figure F.3: Floods of the secondary kinetic energy coefficient $\left(C_{S K E}\right)$ with the secondary velocity vectors overlaid for varying levels of purge flow measured at $1.20 C_{x} \ldots \mathrm{F}-4$

Figure G.1: Floods of the total pressure coefficient $\left(C_{P 0}\right)$ for varying levels of purge flow measured at $1.40 C_{x}$

Figure G.2: Floods of the streamwise vorticity coefficient $\left(C_{\omega S}\right)$ with contour lines of the total pressure coefficient $\left(C_{P 0}\right)$ for varying levels of purge flow measured at $1.40 C_{x}$

Figure G.3: Floods of the secondary kinetic energy coefficient $\left(C_{S K E}\right)$ with the secondary velocity vectors overlaid for varying levels of purge flow measured at $1.40 C_{x} \ldots \mathrm{G}-4$

Figure G.4: Overall $(Y)$ and Mixed-out ( $\left.Y_{\text {mixed }}\right)$ loss coefficients for different levels of purge flow at $1.40 \mathrm{C}_{\mathrm{x}}$ for the $\mathrm{RC}$ and $\mathrm{EC}$ configurations G-5 


\section{List of Tables}

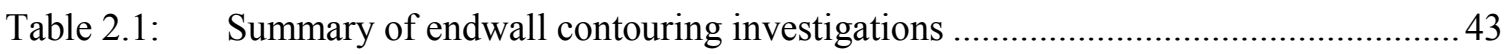

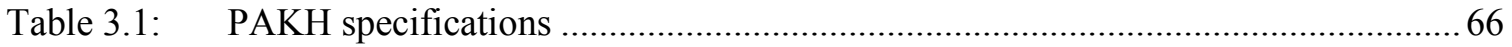

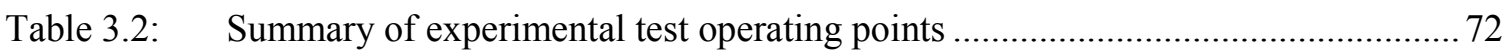

Table 3.3: $\quad$ Seven-hole probe measurement uncertainties (Scribner, 2011)............................. 81

Table 4.1: $\quad$ Summary of integrated flow parameters from the $1.40 C_{x}$ plane for two different domain inlet positions

Table 4.2: $\quad$ Summary of integrated flow parameters from the $1.40 C_{x}$ plane for three different domain outlet positions

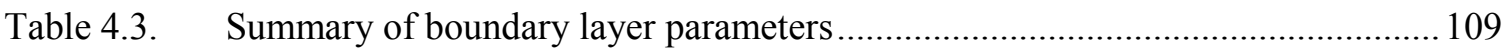

Table 4.4: $\quad$ Summary of the 2D grid sensitivity parameters ................................................ 121

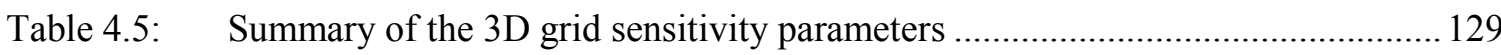

Table 4.6: $\quad$ Summary of the 3D grid sensitivity parameters for the endwall cavity

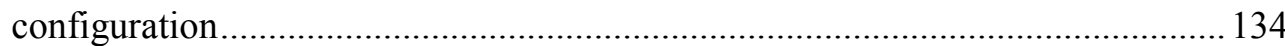

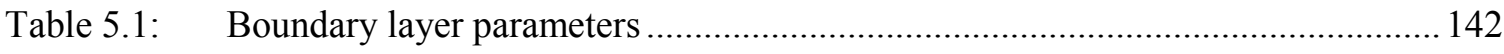

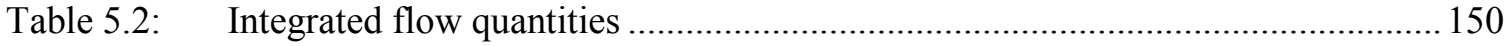

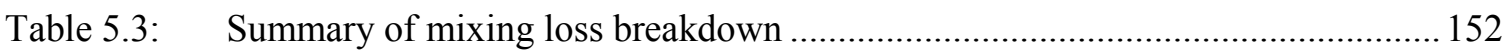

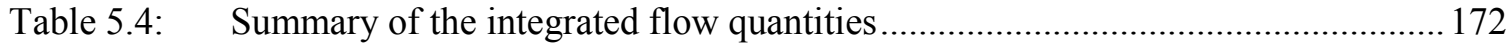

Table 6.1: $\quad$ Summary of peak losses and streamwise vorticity within the passage vortex $(\mathrm{PV})$ and the relative in-plane area of the inviscid core.

Table 6.2: $\quad$ Summary of the measured and predicted overall integrated flow quantities........211

Table 6.3: Summary of the measured and predicted overall integrated flow quantities relative to the baseline measurements

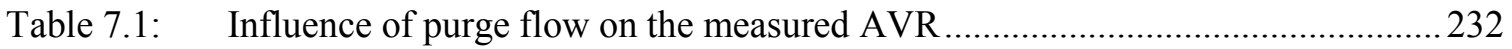

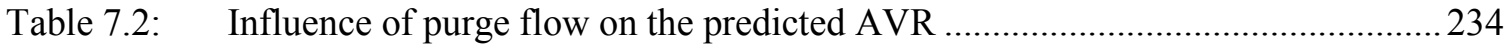

Table 7.3: $\quad$ Summary of peak losses and streamwise vorticity within the passage vortex $(\mathrm{PV})$, the passage vortex location and the relative in-plane area of the inviscid core 
Table 7.4: Summary of the measured and predicted overall integrated flow quantities........258

Table 7.5: $\quad$ Summary of the measured and predicted overall integrated flow quantities relative to the zero net purge flow case (RF 000) ..............................................258

Table 8.1: $\quad$ Summary of the measured overall integrated flow quantities..............................289

Table 8.2: $\quad$ Summary of the measured and predicted overall integrated flow quantities relative to the zero net purge flow case (RF 000) .............................................290

Table 8.3: $\quad$ Summary of the measured overall integrated flow quantities................................308

Table 8.4: Summary of the measured overall integrated flow quantities relative to the zero net purge flow case (RF 000)

Table A.1: Calibration coefficients for the ASG DRAL501DN $\left( \pm 1\right.$ inch $\left.\mathrm{H}_{2} \mathrm{O}\right)$

transducers

Table A.2: $\quad$ Calibration coefficients for the ASG DRAL505DN $\left( \pm 5\right.$ inch $\left.\mathrm{H}_{2} \mathrm{O}\right)$

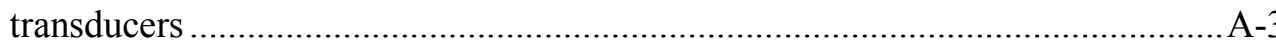

Table E.1: $\quad$ Summary of velocity cases for each level of turbulence intensity........................ E-9

Table E.2: Overall uncertainty of the measured Reynolds stresses and the turbulence

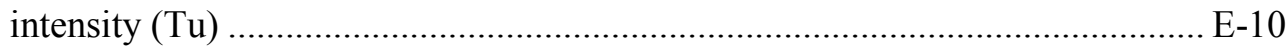

Table G.1: $\quad$ Summary of the measured overall integrated flow quantities................................ G-5

Table G.2: $\quad$ Summary of the measured and predicted overall integrated flow quantities relative to the zero net purge flow case (RF 000) ............................................... 


\section{List of Symbols}

\begin{tabular}{ll}
$C$ & True chord \\
$C_{x}$ & Axial chord \\
$C_{a}$ & Axial velocity \\
$\mathrm{CR}$ & Convergence ratio, $\cos \beta_{1} / \cos \beta_{2}$ \\
$C_{P 0}$ & Total pressure coefficient \\
$C_{\omega_{S}}$ & Streamwise vorticity coefficient \\
$C_{S K E}$ & Secondary kinetic energy coefficient \\
$\mathrm{d}$ & Probe tip diameter \\
$F$ & Force \\
$H_{S F}$ & Boundary Layer Shape factor \\
$h$ & Span \\
$T_{l}$ & Turbulence intensity, $\sqrt{\frac{2}{3} k} / V_{l o c} \times 100$ \\
$P_{S}$ & Turbulence kinetic energy, $\frac{1}{2}\left(\overline{u^{\prime 2}}+\overline{v^{\prime 2}}+\overline{w^{\prime 2}}\right)$ \\
$P_{0}$ & Total pressure \\
$\mathrm{Ma}$ & Mach number \\
& Mass flow rate \\
\hline &
\end{tabular}




\begin{tabular}{|c|c|}
\hline$U$ & Blade speed \\
\hline$u_{i}$ & Instantaneous velocity vector, $(u, v, w)=\left(\bar{u}+u^{\prime}, \bar{v}+v^{\prime}, \bar{w}+w^{\prime}\right)$ \\
\hline $\bar{u}_{i}$ & Time-averaged velocity vector, $(\bar{u}, \bar{v}, \bar{w})$ \\
\hline$u, v, w$ & Instantaneous velocity components \\
\hline $\bar{u}, \bar{v}, \bar{w}$ & $\begin{array}{l}\text { Time-averaged velocity components (the overbar notation may be dropped if time-averaged } \\
\text { velocity is contextually implied) }\end{array}$ \\
\hline$u^{\prime}, v^{\prime}, w^{\prime}$ & Turbulent fluctuations \\
\hline$V$ & Resultant time-averaged velocity vector, $\sqrt{\bar{u}^{2}+\bar{v}^{2}+\bar{w}^{2}}$ \\
\hline$x, y, z$ & Axial, pitchwise and spanwise directions \\
\hline Y & Loss coefficient \\
\hline$Y_{\text {mid }}$ & Midspan loss coefficient \\
\hline$Y_{p}$ & Profile loss coefficient \\
\hline$Y_{\text {sec }}$ & Secondary loss coefficient \\
\hline $\mathrm{y}^{+}$ & Non-dimensional distance from the wall, $\Delta y u_{\tau} / v$ \\
\hline $\mathrm{Z}_{\mathrm{TE}}$ & Spanwise penetration height of the secondary flow \\
\hline $\mathrm{Zw}$ & Zweifel coefficient, $\frac{2 s}{C_{x}} \cos ^{2} \beta_{2}\left(\tan \beta_{2}-\frac{C_{a 1}}{C_{a 2}} \tan \beta_{1}\right)$ \\
\hline$\Lambda$ & Turbulence integral length scale \\
\hline$\Psi_{i, j}$ & Total deformation work, $\frac{\overline{u_{i}^{\prime} u_{j}^{\prime}}}{V_{C L, 1}^{2}} \cdot \frac{\partial\left(\bar{u}_{i} / V_{C L, 1}\right)}{\partial\left(x_{j} / C_{x}\right)}$ \\
\hline$\alpha$ & Yaw or spanwise flow angle (measured from tangential direction) \\
\hline$\beta$ & Pitch or pitchwise flow angle (measured from axial direction) \\
\hline$\gamma$ & $\begin{array}{l}\text { Turbulence intermittency or the angle of the pressure side tangent measured from } \\
\text { the axial direction }\end{array}$ \\
\hline$\delta$ & Boundary-layer thickness \\
\hline
\end{tabular}



$\delta^{*}$
Boundary-layer displacement thickness
$\varepsilon$
Turbulence dissipation rate
$\theta$
Boundary-layer momentum thickness
$\mu$
Dynamic viscosity
$v \quad$ Kinematic viscosity
$\rho \quad$ Density
$\theta \quad$ Boundary-layer momentum thickness
$\phi \quad$ Purge flow ratio, $\frac{\dot{m}_{\text {purge }}}{\dot{m}_{W T}} \times 100$
$\omega$
Vorticity or specific turbulence dissipation rate

\section{Superscripts and Overbars}

\author{
Pitchwise mass-averaged flow quantity or fluctuating velocity component \\ Two-dimensional Mass-averaged flow quantity \\ $\bar{\Omega} \quad$ Pitchwise area-averaged or time-averaged flow quantity
}
$\overline{\bar{\Omega}} \quad$ Two-dimensional Area-averaged flow quantity

\section{Subscripts}

1,2 Upstream and downstream

$C L \quad$ Centre line of blade span, $z / h=0.5$

$i \quad$ Wire number

IS Isentropic 


$\begin{array}{ll}\text { loc } & \text { Local } \\ L & \text { Laminar } \\ \text { prim } & \text { Primary flow direction } \\ \text { purge } & \text { Purge flow } \\ \text { sec } & \text { Secondary flow direction (perpendicular to the primary flow direction) } \\ \text { WT } & \text { Wind tunnel coordinate system }\end{array}$

\section{Abbreviations}

$\begin{array}{ll}\text { CFD } & \text { Computational Fluid Dynamics } \\ \text { EC } & \begin{array}{l}\text { Elliptical platform overlap with non-axisymmetric intra-passage endwall } \\ \text { contouring }\end{array} \\ \text { EF } & \text { Elliptical platform overlap with flat intra-passage endwalls } \\ \text { H } & \text { Hump } \\ \text { HP } & \text { High pressure } \\ \text { ID } & \text { Inner diameter } \\ \text { IR } & \text { Injection rate } \\ \text { LEF } & \text { Leading Edge fillet } \\ \text { LF } & \text { Leakage fraction } \\ \text { LP } & \text { Low pressure } \\ \text { LSTL } & \text { Low-Speed Turbomachinery Laboratory } \\ \text { NEC } & \text { Non-axisymmetric endwall contouring } \\ \text { NGV } & \text { Nozzle guide vane } \\ \text { OD } & \text { Outer diameter }\end{array}$




$\begin{array}{ll}\text { OHR } & \text { Over-heat ratio } \\ \text { PKE } & \text { Primary kinetic energy } \\ \text { PS } & \text { Pressure side } \\ \text { PV } & \text { Passage vortex } \\ \text { PWA } & \text { Pratt \& Whitney Aircraft, East Hartford, CT } \\ \text { R } & \text { Ridge } \\ \text { RANS } & \text { Reynolds-Averaged Navier Stokes } \\ \text { RC } & \text { Rectangular platform overlap with non-axisymmetric intra-passage endwall } \\ \text { contouring } & \text { Rectangular platform overlap with flat intra-passage endwalls } \\ \text { RF } & \text { Secondary kinetic energy } \\ \text { SKE } & \text { Dot product of the secondary kinetic energy and helicity } \\ \text { SKEH } & \text { Stereolithography } \\ \text { SLA } & \text { Suction side } \\ \text { SS } & \text { Shear stress transport } \\ \text { TKT } & \text { Trough } \\ \text { T } & \text { Turbulence kinetic energy } \\ & \end{array}$




\section{Chapter 1}

\section{Introduction}

\subsection{Research Motivation}

The growth of the air-travel industry coupled with volatile fuel prices and more stringent environmental restrictions has forced aircraft manufacturers to consider new designs that improve upon current operational costs and emission levels. A reduction in aircraft fuel consumption has the direct benefit of reducing the overall life cycle (operational) cost as well as the carbon footprint of the aircraft. Of course, the aircraft engine is an important factor in the improvement

of the overall aircraft performance. Thus, new state-of-the-art aircraft engine designs must be fuel efficient and light weight while also having low capital, manufacturing and operating costs. Engine weight reductions and efficiency improvements can be obtained through the optimization of the individual engine components. Figure 1.1 shows the typical components of a gas turbine engine including the fan, low-pressure (LP) and high-pressure (HP) compressor, combustor and the LP and HP turbines. The current thesis investigates aerodynamic design improvements to the HP turbine stages, with particular focus on the complex, three-dimensional flow on the inner endwalls. 


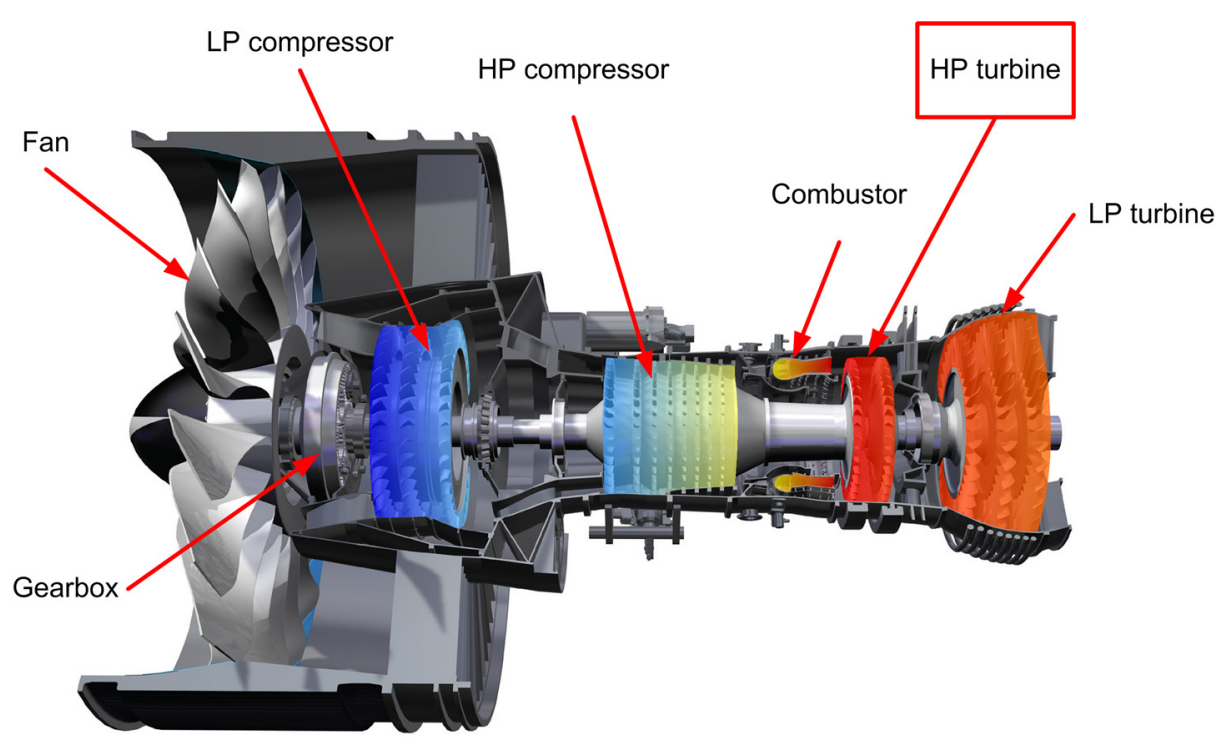

Figure 1.1: PW1000G gas turbine engine cut-away [Source: www.pw.utc.com]

The next emerging market for aircraft engines is the short- to medium-haul single-aisle aircraft, such as the Airbus A320 and Boeing 737 replacements (Szodruch et al., 2011). Reductions in fuel consumption, noise and emission levels can be achieved through new engine technologies. One engine option for these aircraft is the Pratt \& Whitney geared turbofan (PW1000G) engine, shown in Figure 1.1. The larger fan diameter and the addition of a gearbox, although necessary for the performance benefits, tend to increase the overall engine weight relative to equivalent-duty turbofan engines. Therefore, to be a viable solution, additional weight savings must be gained through the optimization of other engine components.

The current research project has been completed under contract with the Turbine Aerodynamics group at Pratt \& Whitney Aircraft, East Hartford, CT (PWA) and is part of an ongoing research collaboration (Popovic, 2005; Zoric, 2006; Knezevici, 2011). An objective of the research group has been to achieve weight and cost reductions by reducing the number of blades in a given turbine stage. To obtain the same power output with a reduced blade count, the aerodynamic loading per blade must be increased. Generally, more highly loaded blades result in 
higher pressure losses through the bladerow and thus lower stage efficiencies. Wisler (1998) estimates that a $1 \%$ change in HP turbine efficiency leads to about a $1 \%$ change in the specific fuel consumption (SFC) for a typical gas turbine engine. Small improvements to the efficiency of the individual engine components are therefore significant to the aircraft operational economics.

Specialized endwall profiles, developed using computational fluid dynamics (CFD), have been shown to reduce some of the losses associated with higher blade loadings (Praisner et al., 2007; Knezevici, 2011). As a relatively new technology, the effectiveness of these endwall designs under more engine representative conditions is still largely unknown. For example, within an actual HP turbine there is a relatively large endwall step, termed the platform overlap, between the stationary and moving bladerow, as shown in Figure 1.2. In addition to the step, the flow is complicated by the injection of a small amount of air, known as purge flow, which is used to cool the blade disk and prevent ingress of the hot mainstream gas in the actual engine. The step and purge flow significantly alter the near wall flow physics relative to traditional cascade studies. Thus, by better understanding how the platform overlap and purge flow affect the main gas-path flow, improvements can be made to the current and future loss-mitigation technologies (eg. endwall profiling).

The current research is partly motivated by the development of these loss-mitigation technologies. Previous studies involving turbine purge flows have generally focused on minimizing the purge mass flow required to prevent hot-gas ingestion into the turbine wheelspace. According to Bohn et al. (2006) the disk cooling and sealing flows (including purge flows) increase the engine specific fuel consumption by about $6 \%$. These flows must be bled from the compressor and bypass the combustor, therefore contributing little to the expansion work in the turbine. Thus, any reductions have obvious benefits for the overall engine performance. However, as noted by McLean et al. (2001), the rim seal geometry and purge flow characteristics also affect the endwall boundary layer, mainstream pressure field and the vortex development in the main gas-path. Therefore, the rim-seal (platform overlap, endwall cavity and purge flow level) design must also aim to limit any adverse effects on the mainstream flow. The 
present research will investigate the effects of different platform overlap geometries as well as varying levels of purge mass flow.

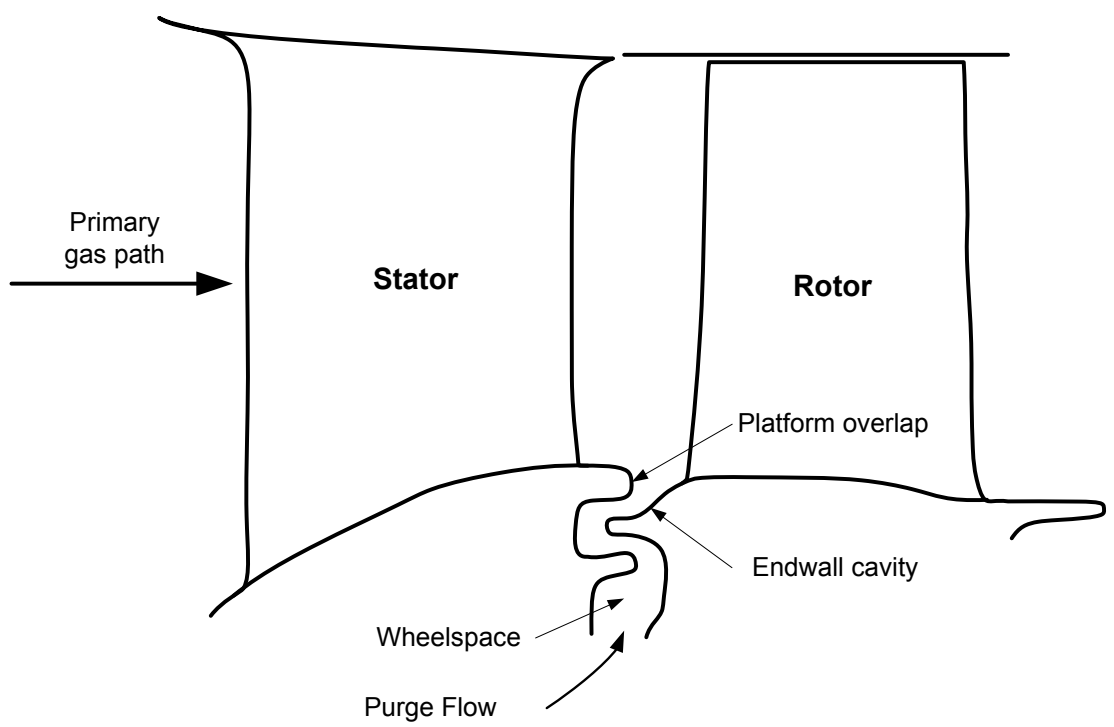

Figure 1.2: Schematic of stationary and rotating engine blades with platform overlap geometry and purge flow

\subsection{Thesis Objectives}

The overall objective of the research project is to improve the physical understanding of the near-endwall flow, also termed the secondary flow, of turbine bladerows. The current thesis will focus on the subtle effects of the platform overlap, endwall cavity and purge flow (see Figure 1.2) on the secondary flow physics and the associated losses, termed secondary losses. The purpose of studying these at relatively engine representative conditions in a controlled cascade facility is to individually understand how these features affect the behaviour of the secondary flows. This understanding will ultimately provide guidance for new turbine designs and thus will aid the development of new state-of-the-art engine technology. 
As mentioned in Section 1.1, the thesis will explore the effectiveness of endwall profiling, also known as endwall contouring, with the inclusion of the upstream platform overlap, the endwall cavity and purge flow. Previous authors (Hartland et al., 2000; Knezevici, 2011) have provided detailed explanations of how endwall contouring alters the secondary flows of turbine cascades to reduce the secondary losses in the absence of a platform overlap and simulated purge flow. The present work will expand upon the current understanding by addressing the influence of the upstream rim-seal. Geometric changes to the shape of the platform overlap as well as different levels of purge mass flow are considered.

The research is primarily experimental and is supplemented with computational simulations (i.e. CFD). A particular contribution of this thesis is the experimentally measured turbulence quantities of the turbine secondary flow. These measurements, although only collected for the traditional flat-endwall cascade, provide insights into how irreversibilities are generated from the mean flow and thus improve the general understanding of secondary loss production. The observed levels of elevated turbulence within the secondary flow have also prompted the development of improvements to the experimental measurement techniques within highly turbulent flows (Scribner, 2011).

The computational simulations presented herein are performed with two intentions: to gain physical insights within regions of the flow field that are difficult to measure experimentally and to evaluate the prediction capabilities of a commercially available CFD code. CFD predictions are an important tool for the development of new engine technologies. However, the complexity of the near endwall flow makes obtaining accurate CFD predictions difficult. In particular, the prediction of absolute pressure losses using a steady RANS (Reynolds-Averaged Navier Stokes) simulation is generally unsatisfactory as a result of limitations of the two-equation turbulence models. Thus, an integral part of new technology development is the experimental investigation and validation of the computational models. 


\subsection{Thesis Approach}

Experimental investigations were conducted in the linear-cascade wind tunnel located in the Low-Speed Turbomachinery Laboratory (LSTL) at Carleton University. Downstream point measurements focused on the near-wall vortical structures and the associated total pressure losses, termed secondary losses. Surface flow visualizations were used in combination with CFD predictions to interpret the inner-passage flow physics.

Tests were performed for one airfoil geometry, designated PAKH, with three different endwall configurations and two different platform overlap features, totaling five cascade configurations. All of the hardware were designed and supplied by PWA. The first configuration is the baseline planar endwall. The second configuration includes an upstream non-axisymmetric platform overlap step and a corresponding endwall cavity feature. The third configuration attempts to reduce the secondary losses by applying a specially designed endwall contour within the bladerow passage. The fourth and fifth configurations replace the rectangular platform overlap shape with a more gradual elliptical shape. The influence of the purge flow, for varying velocity levels, is investigated for all the step configurations.

\subsection{Thesis Overview}

Chapter 2 introduces the background information relevant to the study of turbine secondary flows. A description of the traditional turbine flow field and loss-breakdown scheme is provided. The literature review includes brief overviews of the seminal works on secondary flows generally and then summarizes the more recent studies that are particularly pertinent to the current work. Passive loss-mitigation techniques that involve geometric modifications are also discussed. The chapter concludes with a summary of literature on the interaction between the mainstream flow and the rim-seal. 
As mentioned, the experimental investigations were conducted in the Low-speed Turbomachinery Laboratory at Carleton University. Chapter 3 provides details of the experimental apparatus, procedures and estimates of the experimental uncertainties. The experimental work of this thesis involved commissioning a purge flow delivery system which is also described in this chapter.

Computational simulations are used throughout the thesis to support the discussion of the secondary flow physics. A common approach to CFD is used throughout and the CFD methods are discussed in Chapter 4. The domain shape, mesh sensitivity, inlet boundary conditions as well as general guidelines for solving turbine cascade flows are discussed.

The experimental and computational results are presented in Chapters 5 through 8 . Chapter 5 discusses the turbulent flow field of the planar endwall cascade. The rate of turbulence production from the mean flow is examined from multiple downstream measurement planes. The chapter also addresses the short-comings of CFD predictions when using two-equation turbulence models to predict turbine secondary flows.

Chapter 6 examines the influence on the cascade secondary flow of the upstream platform overlap cavity without the presence of purge flow. A description of how the secondary flow is changed relative to the traditional planar case is provided. This chapter also investigates geometric approximations of the platform overlap that may be viable simplifications for the preliminary design of turbine stages.

Chapter 7 examines the influence of varying levels of purge flow on the secondary flows for the cascades with the rectangular platform overlap and flat intra-passage endwalls. Chapter 8 examines the secondary loss-mitigation technology. Endwall contouring and the elliptical platform overlap shape are discussed separately.

Lastly, a summary of the main conclusions and recommendations for future work are provided in Chapter 9. 


\section{Chapter 2}

\section{Background and Literature Review}

\subsection{Introduction}

This chapter examines the background and literature relevant to the present thesis. It begins with a general introduction to the flow field in axial turbine blade passages followed by a discussion regarding the role of linear turbine cascade research. The discussion includes the effects of the inlet conditions, blade loadings and turbulence on the secondary loss production. In the later sections of this chapter, significant attention is given to secondary loss mitigation through geometric modifications. Such modifications include endwall fences, leading edge modifications and axisymmetric and non-axisymmetric endwall contouring. Lastly, the chapter concludes with the literature that has focused on the effects of the rim-seal geometry and purge flow on the secondary flows of turbine bladerows. 


\subsection{The Flow Field and Losses in Axial Turbine Blade Passages}

The main features of the turbine passage flow field are shown schematically in Figure 2.1, and will be discussed in terms of their associated total pressure losses.

In general, estimations of the pressure losses generated within new bladerow designs are obtained from empirical correlations. Examples of such correlations are given by Kacker and Okapuu (1982), Sharma and Butler (1987) and Benner et al. (2006a; 2006b). The losses are usually divided into three components, which are assumed to be independent of each other: the profile losses, tip-leakage losses and secondary losses.

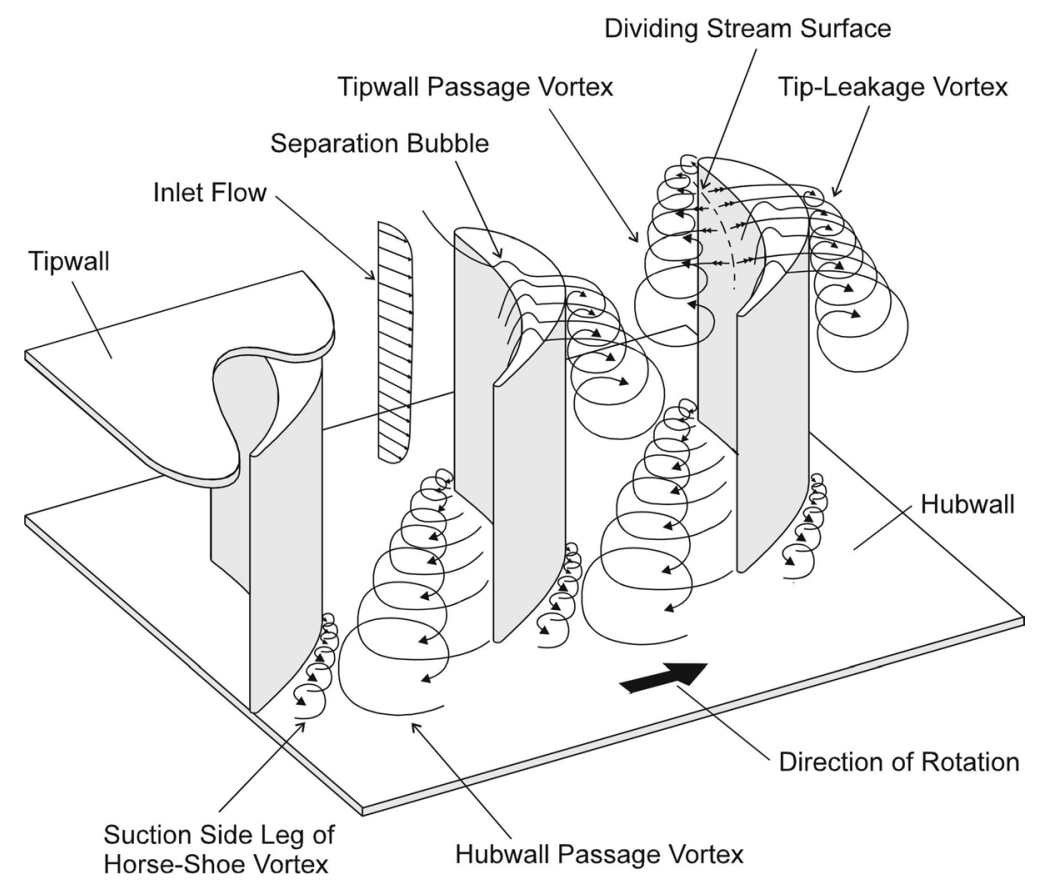

Figure 2.1: Main features of a turbine flow field, from Sjolander (2007)

Profile losses are generated away from the endwalls within the blade surface boundary layers. The profile losses are typically measured at midspan where the flow is two-dimensional. Additional losses from the trailing edge sudden expansion and downstream mixing are generally included in the profile losses. 
Secondary losses are generated within the endwall surface boundary layers and through the downstream mixing of the endwall vortical structures. The vortical structures, shown in Figure 2.1, are a result of interactions between the inlet boundary layer and the blade surface

pressure distribution. The difference in pressure between the suction and pressure sides of the blade gives rise to a blade-to-blade (cross-passage) pressure gradient. The cross-passage gradient causes the low momentum fluid of the endwall boundary layer to be turned more strongly than the higher momentum fluid of the midspan region. This results in a sweeping of the fluid across the endwall surface towards the suction surface of the blade. When the swept fluid, or secondary flow, encounters the blade suction surface it is forced up and into the blade passage forming the passage vortex as shown in Figure 2.1. The formation of the vortical structures is largely an inviscid process and the secondary losses are primarily generated though viscous mixing of the vortices and interactions with blade surface boundary layers.

Tip-leakage losses are generated primarily from the downstream mixing of the vortex that forms due to the leakage through the gap between the stationary tip-wall and moving rotor blade, as shown in Figure 2.1. The tip-leakage losses are not investigated in this study.

Secondary flows and the associated losses have been studied for many years and a general understanding of the flow behaviour exists. The current study will investigate the differences that arise in the secondary flow when an upstream platform overlap feature is present and when purge flow is activated.

\subsection{The Role of Low-speed Linear Turbine Cascade Research}

The research proposed here was conducted under contract with PWA and the results of this investigation will likely find uses in new engine developments. The research is concerned with the secondary flows of a low-speed linear turbine cascade. The periodic conditions of an actual annular bladerow are simulated in cascade tests using a finite number of equally spaced blades 
arranged on a planar endwall. In this investigation, tests are performed at low-speed, incompressible conditions, using large scale geometry.

Cascade testing provides an idealized but highly controlled environment in which the flow fields can be observed in great detail; many complex features of an actual engine flow can be decoupled and investigated individually. For example, Mahallati and Sjolander (2007) investigated the effects of flow unsteadiness on boundary layer transition using a linear cascade with a wake generator installed upstream. Investigations of this level of detail would be costly and extremely difficult to implement in a real engine environment. Another example is the tipleakage flows simulated by Yaras and Sjolander (1992) and Yaras et al. (1992). A belt-driven moving endwall, installed on a linear cascade test-section, was used to simulate the relative motion between the rotor blade tip and the annulus of a real engine. The measurements of the tipgap flow are representative of the real engine flow physics, and therefore facilitate engine technology improvements.

In low-speed testing the parameter of similarity is the Reynolds number. Typically, this is based on the axial chord and outlet velocity. In many instances engine representative Reynolds numbers are not matched exactly but are sufficiently high to achieve Reynolds number independent conditions. The limitations of low-speed cascade testing are related to the thermal conditions within the engine. Compressibility effects are not considered in the present work and therefore Mach numbers cannot be matched to engine levels. The effects of compressibility are generally studied separately in a high-speed cascade facility. For low-speed testing, blade shapes are inverse-designed with non-dimensional surface pressure distributions equal to an equivalent high speed design. Some of the typical features of engine flows that are not considered in lowspeed cascade testing include:

1. Elevated temperatures

2. Compressibility

3. Radial pressure gradients

4. Inlet boundary skew 
5. Flow unsteadiness (wakes from upstream bladerow)

6. Blade surface and endwall cooling flows

7. Tip-leakage flows

8. Rim-seal purge flow

9. Upstream and downstream platform overlaps (rim-seal geometry)

In low-speed cascade testing items 1 through 3 are difficult, if not impossible, to simulate. However, items 4 through 9 can be introduced by modifying the test-setup. As mentioned, Mahallati and Sjolander (2007) studied the effects of unsteadiness caused by the upstream blade wake using a wake-generating apparatus. Yaras and Sjolander (1992) applied a modified belt driven endwall to simulate the tip-gap flow. In this thesis, the effects of items 8 and 9 are studied. Details of the test-section modifications are provided in Section 3.5.

Typically, low-speed testing is focused on understanding the flow physics, rather than the evaluation of engine ready technology. Lessons learned can therefore be applied to the engine component design philosophy. The current investigation will attempt to address the effects of the upstream platform overlap geometry and the simulated purge flow on the secondary losses of a turbine cascade.

\subsection{Blade Loading}

Although blade loading is not a parameter investigated in this study, the loading and loading distribution are known to affect the overall bladerow losses. Thus, a brief background is provided here.

The turbine blade loading is defined as the net tangential force, $F_{\text {actual }}$, acting on an airfoil cross-section. It is determined by integrating, over the axial chord distance, the difference in pressure between the pressure side (PS) and suction side (SS) of the airfoil. An example pressure distribution is shown in Figure 2.2 (a). A bladerow is shown schematically in Figure 2.2 (b) with the corresponding nomenclature. 
In 1945, Zweifel introduced a non-dimensional loading parameter, defined as,

$$
Z_{w}=\frac{F_{\text {actual }}}{F_{\text {ideal }}}=2\left(\frac{s}{C_{x}}\right) \cos ^{2} \beta_{2}\left(\tan \beta_{2}-\frac{C_{a 1}}{C_{a 2}} \tan \beta_{1}\right)
$$

where $s$ is the blade spacing (pitch), $C_{x}$ is the axial chord length, $C_{a 1}$ and $C_{a 2}$ are the inlet and outlet axial velocities and $\beta_{1}$ and $\beta_{2}$ are the inlet and outlet flow angles, respectively. It represents the ratio of the actual tangential force, $F_{\text {actual }}$, to an ideal reference value, $F_{\text {ideal }}$. The ideal force is defined as the maximum loading that could be achieved for the same inlet and outlet conditions, without the presence of adverse pressure gradients on the suction surface of the blade and with the maximum available pressure applied over the full length of the pressure surface. Such a pressure distribution is not physically realizable since it would have uniform pressures, equal to the inlet stagnation pressure $\left(P_{0}\right)$ on the pressure side and the outlet static pressure $\left(P_{S 2}\right)$ on suction side of the airfoil. Also, at the leading and trailing edge there would be instantaneous changes of pressure over zero surface length. The ideal pressure distribution is shown in Figure 2.2 (a) by the dashed lines.

Cascade test data from the 1930's and 1940's was used by Zweifel to determine the optimum value for minimum profile losses. He concluded that a value of 0.8 represents the best compromise between losses incurred from boundary layer separation and frictional boundary layer losses (Sjolander, 2007). Advancements in turbine designs have led to higher Zweifel coefficients for both HP and LP turbines. The cascade presented in this study has a Zweifel coefficient of approximately 0.97 , which is considered moderately high for HP turbine blades.

Several studies have investigated the effects of higher loading on the losses of turbine blade rows. Section 2.6.3 discusses the relevant literature. In general, increases in the aerodynamic loading result in higher total pressure losses. 
(a)

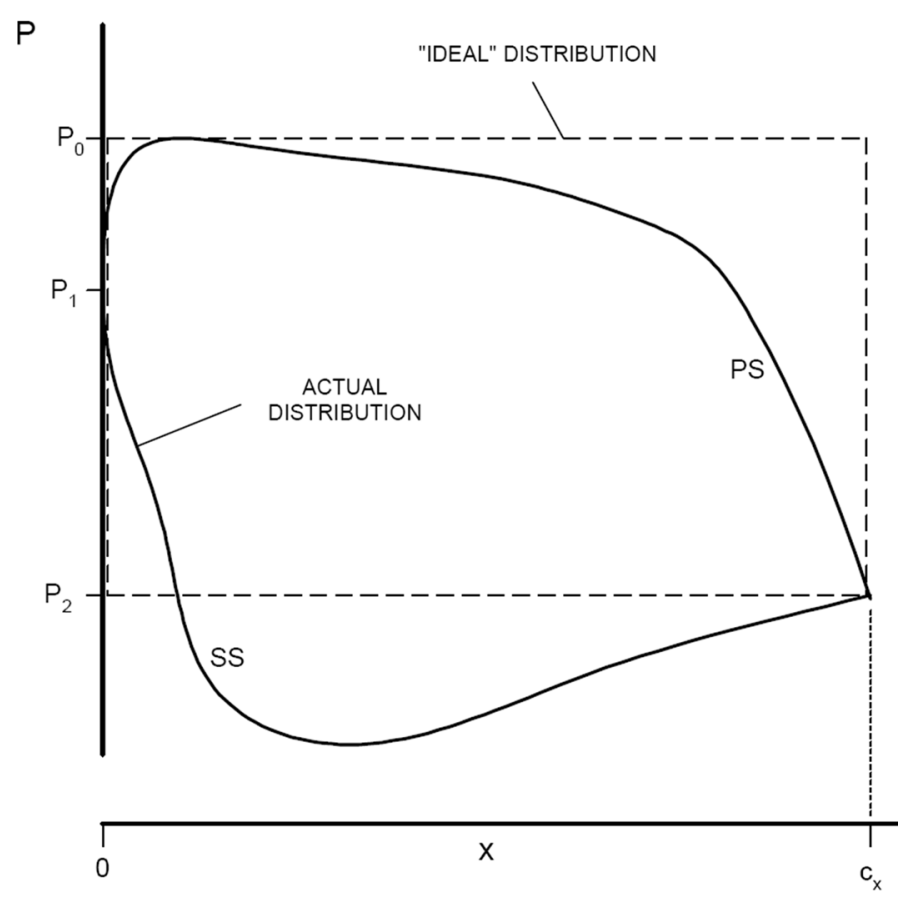

(b)

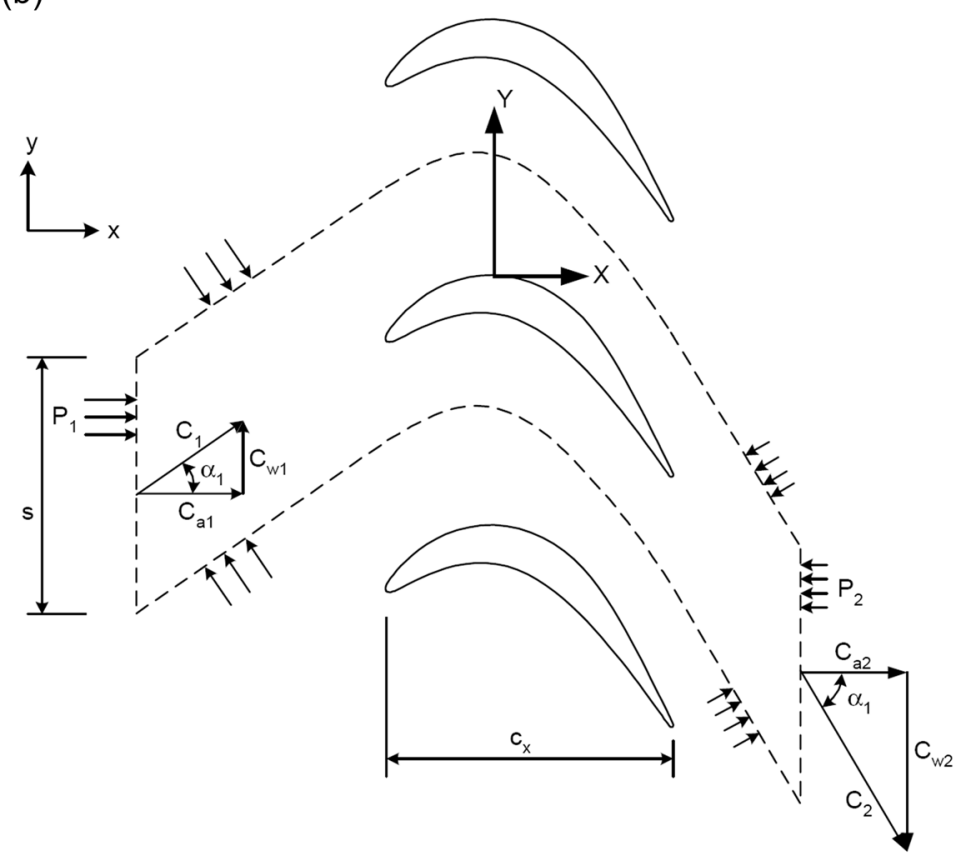

Figure 2.2: Turbine pressure distribution (a) and control volume definition (b), from Sjolander (2007) 


\subsection{Profile and Secondary Losses in Linear Turbine Cascades}

\subsubsection{Profile Losses}

The profile pressure losses described in Section 2.2 are generated within the twodimensional blade surface boundary layers at locations away from the endwalls. The magnitude of the profile losses is dependent on the state of the boundary layer, the streamwise pressure gradient and the Reynolds number.

In low Reynolds number applications, a laminar boundary layer may exist for a significant portion of the blade suction surface. Depending on the severity of the adverse pressure gradient present on the suction surface of the blade, laminar separation may occur. The flow may then be fully stalled or reattached forming a separation bubble. If laminar separation is unavoidable, the latter is desirable as the increase in losses from a separation bubble is much lower than for the fully stalled conditions.

\subsubsection{Secondary Losses}

A classic definition of the total row losses of turbine bladerows is that of Kacker and Okapuu (1982). In the absence of tip-leakage losses, the secondary losses then become the difference between the total row losses and the profile losses:

$$
Y_{\text {secondary }}=Y_{\text {total }}-Y_{\text {profile }}
$$

The definition is somewhat simplified from the physical descriptions provided in Section 2.2. In this form, it is assumed that the profile losses are uniform over the blade span. Benner et al. (2004b) suggests that this conventional breakdown is unphysical. They showed that erroneously low secondary losses were obtained for high incidence cases despite that the secondary flows were clearly strengthened. Essentially, the conventional scheme implies that the losses generated near the endwall, along the blade surface, are the same as those observed at midspan. For design incidence cases this assumption may be appropriate and thus the 
conventional scheme is acceptable (Benner et al., 2004b). However, as incidence levels increase for off-design conditions the conventional profile losses increase and represent a larger fraction of the total losses. As a result, negligible or near zero secondary losses may be obtained for high incidence cases.

In a follow up paper, Benner et al. (2006a) proposed modifications to the definition of Equation 2.2. The profile losses are re-defined to represent the losses along the blade surface in the region unaffected by the secondary flow, termed the primary region. A key parameter for this breakdown is the penetration height $\left(Z_{T E}\right)$ of the secondary flow which represents the spanwise extent of the secondary flow at the blade trailing edge. In practice, the profile losses would be determined according to:

$$
Y_{\text {profile }}=Y_{\text {mid }}\left(1-\frac{Z_{T E}}{h}\right)
$$

where $Y_{\text {mid }}$ is the measured losses at midspan, $Z_{T E}$ is the penetration height measured from the endwall towards midspan at the blade trailing edge and $h$ is the blade span. In this form, the secondary loss coefficient ( $\left.Y_{\text {seconday }}\right)$ may still be obtained using Equation 2.2; however, the profile losses are now more physical in that they represent the losses associated with blade surface boundary layer in the absence of secondary flow interactions. Although Benner et al. (2006a) showed this breakdown may be a significant improvement from the conventional scheme, it has not been widely adopted because of the limited experimental data. The penetration height is not commonly documented in many cascade studies because the measurement requires blade surface visualizations which can be experimentally challenging to obtain. As mentioned previously, the conventional scheme appears to be reasonable for design point cases despite the unphysical assumptions.

To further understand the mechanisms of the secondary loss generation, a detailed interpretation of the flow field is necessary. One of the earliest depictions of the endwall flows of an annular cascade was presented by Sjolander (1975). Other influential works include that of 
Langston et al. (1977), Sharma and Butler(1987), Hodson and Dominy (1987b), Wang et al. (1997) and Benner et al. (2004).

The general interpretation of the flow field presented by Wang et al. (1997) is shown in Figure 2.3. Images were obtained by seeding the flow using smoke wires and illuminating it using laser sheets. As shown, the inlet flow near the blade leading edge rolls up and separates at the location known as the saddle point. The separation is induced by the adverse streamwise pressure gradient that is present as the endwall boundary layer approaches the stagnation point. This separation gives rise to the horseshoe vortex, which has two legs: the suction side $\left(\mathrm{V}_{\mathrm{sh}}\right)$ and pressure side $\left(\mathrm{V}_{\mathrm{ph}}\right)$ legs. As shown, the legs of the horseshoe vortex develop into a complex and highly three-dimensional flow. The secondary losses are generated by viscous shearing between vortices, between vortices and the free-stream fluid and within the endwall boundary layers. The development of the main vortical structures begins as the pressure side leg, $\mathrm{V}_{\mathrm{ph}}$, is driven across the passage by the action of the blade-to-blade pressure gradient. The vortex size increases as it

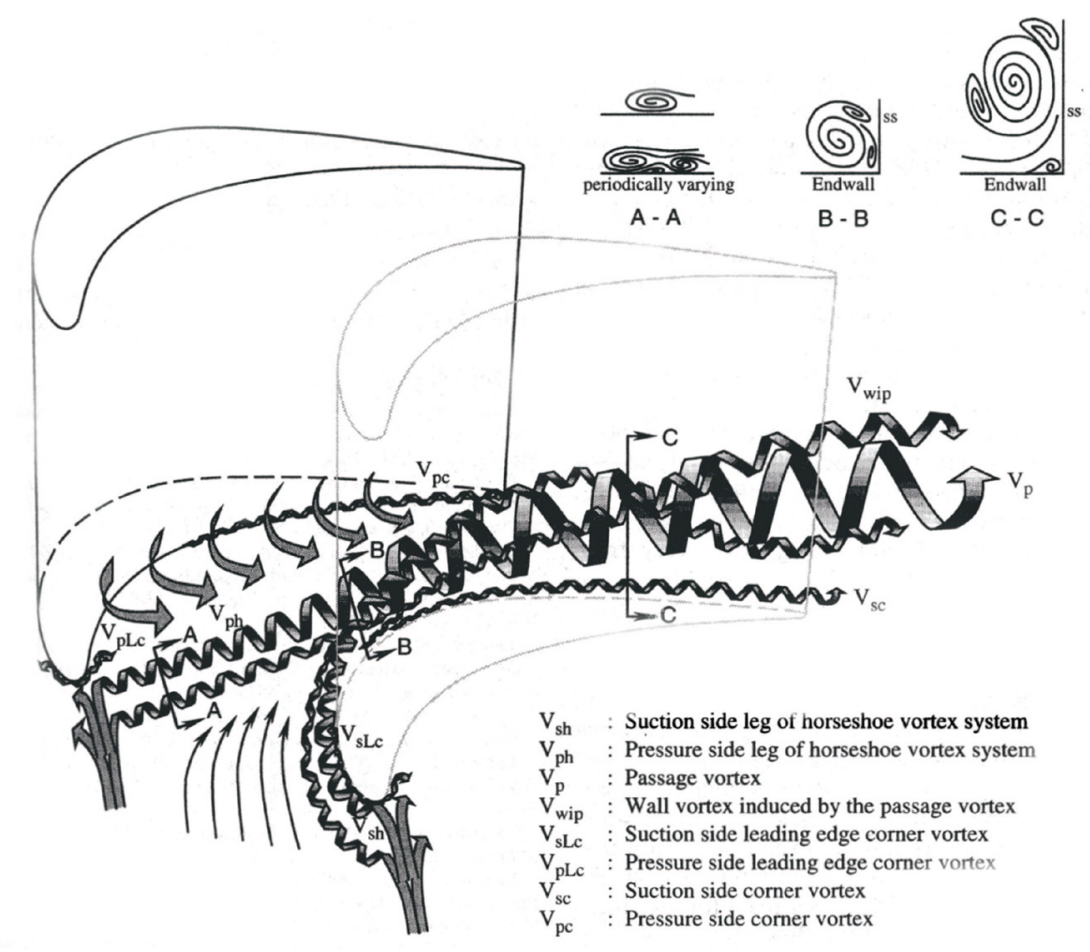

Figure 2.3: Interpretation of endwall flow structures, from Wang et al. (1997) 
migrates. It entrains low momentum endwall boundary fluid and eventually forms the passage vortex $\left(\mathrm{V}_{\mathrm{p}}\right)$. The smaller suction side leg of the horseshoe vortex, $\mathrm{V}_{\text {sh }}$, remains close to the blade suction surface up to a point where it may either wrap around the passage vortex $\left(V_{p}\right)$ as shown, or continue in close proximity to the blade and endwall corner as described by Langston et al. (1977).

The pitchwise convection of the passage vortex continues until it reaches the suction surface of the adjacent blade, at which point there is a strong interaction with the suction surface boundary layer (Benner et al., 2004a). The size, strength and proximity of the passage vortex to the blade and endwall surfaces results in the formation of two-counter rotating vortices: the wall induced vortex $\mathrm{V}_{\text {wip }}$ (also referred to as the counter vortex) and the suction surface corner vortex $\mathrm{V}_{\mathrm{c}}$.

The downstream cross-sectional view labelled C-C shows the well-developed secondary flow features. The passage vortex is largely responsible for the size and strength of other vortical structures, and therefore the generation of additional secondary losses. A number of investigations have shown that the development of the passage vortex is strongly influenced by the magnitude of the blade loadings, the loading distribution, the blade leading edge geometry and the total flow turning (Gregory-Smith et al., 1988a; Gregory-Smith and Cleak, 1992; Benner et al., 2004a; Zoric et al., 2007a).

\subsection{Research on Secondary Losses in Linear Turbine Cascades}

\subsubsection{Introduction}

The investigation and understanding of secondary losses are important for the improvement of turbine technology as they represent a significant portion of the total bladerow losses. Denton (1993) suggests that the secondary losses can represent up to $1 / 3$ of the total row losses, 
and for low-aspect ratio blades Gregory-Smith and Cleak (1992) suggest this amount can be as large as $1 / 2$ the total losses.

Secondary losses have been well studied for since the 1940's. Sieverding (1985) provided a thorough review of secondary flow experiments performed prior to 1985 while Langston (2001) has followed with a review documenting the important papers from 1985 to 2001.

As mentioned in Section 2.4.2, past research regarding the details of the secondary flow structures also include the work of Sjolander(1975), Langston et al. (1977), Sharma and Butler (1987), Hodson and Dominy (1987b), Wang et al. (1997) and Benner et al. (2004) . The loss generating mechanisms have been investigated by Moore and Adhye (1985), GregorySmith et al. (1988a) and Harrison (1990).

The following sections will discuss some of the aerodynamic variables that are known to affect the generation of the secondary losses.

\subsubsection{Effects of Inlet Endwall Boundary Layer and Free-stream Turbulence Intensity}

The inlet endwall boundary layer parameters were not varied in the present study. However, the choice of an appropriate boundary layer is necessary to have meaningful secondary flow results. In this study, the boundary layer is fully turbulent with a shape factor $\left(\mathrm{H}_{\mathrm{SF}}\right)$ of 1.32 and thickness of approximately $9 \%$ of the blade span. The free-stream turbulence intensity is $3.3 \%$ and length scale is approximately $20 \%$ of the axial chord.

Chen and Dixon (1986) investigated boundary-layer thickness ranging from $4 \%$ to $22 \%$.

Thickening the inlet boundary resulted in significantly higher total pressure losses but did not change the location of the peak losses (in terms of the pitchwise averaged values). However, when the inlet boundary layer total pressure deficit was subtracted from the downstream results (to calculate the generated losses), no significant differences were observed. 
Sharma and Butler (1987) reviewed many past studies and concluded that the boundarylayer thickness had little effect on the overall losses. Gregory-Smith et al. (1988a) investigated the inner passage flow field and downstream secondary losses for three different endwall boundary-layer thicknesses $(18 \%, 22 \%$ and $33 \%$ of the blade span). Low-speed investigations were completed at an operating Reynolds number of 500,000 (based on blade chord and outlet velocity). It was concluded that a thicker inlet boundary layer, which has lower levels of vorticity near the endwall, causes the secondary flow features to appear larger and less intense. Although differences were observed in the loss distributions, the overall losses from each case showed no significant differences.

The effects of free-stream turbulence intensity were investigated by Gregory-Smith and Cleak (1992). The baseline wind-tunnel intensity of $1.4 \%$ was increased to $4.5 \%$ using a turbulence generating grid. The grid also had the effect of thinning the endwall boundary layer. The mixed-out profile losses were increased by $7 \%$ and the secondary losses were reduced by $18 \%$ for the higher inlet turbulence level. Although these differences seem significant, the authors indicated that the differences are comparable with their experimental levels of uncertainty.

Zoric et al. (2007a) examined the effects of two free-stream turbulence levels $(1.4 \%$ and $4 \%$ ) on the secondary losses for a family of three low-pressure turbine cascades. The cascades had different blade loading magnitudes and distributions but the same velocity triangles. For the higher levels of free-stream turbulence, the mixed-out secondary losses were reduced by $20 \%$, $23 \%$ and $11 \%$ relative to the respective low-turbulence cases. The conclusions suggest that much of the observed benefit could be attributed to a reduction of secondary flow interactions with the suction surface separation bubble. The benefits observed by Gregory-Smith and Cleak (1992) were obtained in the absence of any suction surface separation bubbles.

In summary, the literature appears to indicate that the boundary layer thickness does not affect overall losses, but can affect the development of the secondary flow and thus the distribution of losses. The free-stream turbulence intensity increases can be linked to secondary 
loss reductions, but there is conflicting evidence about the origins of the reductions. Thus, in a controlled study that will not examine the effects of the inlet boundary layer parameters, it would appear that selecting a boundary layer thickness that is representative of engine conditions is most important. The current boundary layer thickness corresponds to that used by PWA for the design of the geometry used in this study.

\subsubsection{Effects of Blade Loading and Blade Loading Distributions}

As mentioned in Section 1.1, turbine weight reductions can be achieved by reducing the number of blades in a given bladerow. In doing so the spacing between adjacent blades must increase, resulting in higher Zweifel coefficients as well as increased profile and secondary losses. To mitigate the higher endwall losses, a passive flow control measure, termed endwall contouring can be applied. The effectiveness of endwall contouring is dependent not only on the magnitude of the loading but also the distribution of the loading relative to the axial chord (Knezevici et al., 2009, 2010).

The Zweifel coefficient of the current cascade is 0.97 which is considered moderately high for HP turbine designs. Endwall contouring is investigated in the present study, as this cascade would likely only find practical applications with reductions in the endwall losses. Although the effect of blade loading is not specifically investigated in this research, a brief overview of the effects of loadings and loading distribution is included.

Past investigations by Hodson and Dominy (1987a) and Perdichizzi and Dossena (1993) arrived at different conclusions when investigating the effects of higher blade loadings on secondary losses. Each study examined the effects of increasing the cascade pitch-to-chord ratios (increased Zweifel) on planar endwall cascades. Hodson and Dominy (1987a) found distinct changes to the secondary flows and that the highest losses are generated for the most highly loaded blades (largest pitch-to-chord ratio). Perdichizzi and Dossena (1993) found that only increases in loading near the blade leading edge (forward loading) could be correlated to stronger secondary flows. No distinct trends could be associated with the levels of pitch-to-chord ratio. 
More recently, Zoric et al. (2007a; b) compared the profile and secondary losses for design and off-design conditions of a family three-highly loaded LP turbine cascades, PAKB, PAKD-A and PAKD-F. The baseline airfoil, PAKB, has a Zweifel coefficient of 1.08 and is considered an aft-loaded design since the suction peak is located at an axial distance greater than $50 \%$. The more highly loaded cascades, PAKD-A and PAKD-F have the same Zweifel coefficient of 1.37, but have pressure distributions that are aft (A) and front loaded (F) respectively. The aft loaded PAKD-A airfoil had higher profile losses and similar secondary losses relative to more lightly loaded baseline case. The agreement of the secondary losses between PAKD-A and the baseline case was attributed to the relatively high baseline secondary losses. It was suggested that perhaps the secondary flow interacts with the suction surface separation bubble in the baseline case causing an increase in the endwall losses. The forward loaded PAKD-F airfoil showed the highest secondary losses with the lowest profile losses. This is not a unique result as observed by several other researchers, such as Perdichizzi and Dossena (1993), Weiss and Fottner (1995) and Benner et al. (2004). Forward loaded airfoils were generally found to have higher secondary losses than equivalent aft-loaded airfoils, but have lower profile losses. The conclusions of Zoric et al. $(2007 \mathrm{a}$; b) are consistent with this pattern in that the aft-loaded distribution is favourable for secondary loss reduction, and front loaded designs are preferred for profile losses. This suggests that an airfoil with mixed spanwise loading distributions, aft loading near the endwall and front loading towards midspan, might represent an optimum design for engine use.

Knezevici (2011) expanded upon the work Zoric et al. (2007a; b) by investigating the use of endwall contouring to reduce the increase in secondary losses associated higher blade loading. He presented data from the same family of airfoils as used by Zoric et al. (2007a; b) with and without endwall contouring. Also, another extremely highly-loaded cascade, PAKF, was investigated. This cascade has a Zweifel coefficient of 1.78 and to the present author's knowledge is the highest loading level to appear in open literature. Previous to this, Houtermans et al. (2004) presented data from an airfoil with a Zweifel coefficient of 1.473. The PAKF cascade produces similar profile losses compared to the baseline (PAKB) cascade. This is truly remarkable considering the $65 \%$ increase in loading. The forward loading distribution and 
smaller separation bubble are the factors that lead to favourable profile performance. On the other hand, the secondary flow of PAKF is the strongest of the PAK family. Downstream measurements show that the peak losses are similar to the baseline case, while the secondary flow is larger and pushed further away from endwall. Also, the secondary vortices have higher levels of secondary kinetic energy (SKE) and streamwise vorticity. Thus, the in-plane and mixed-out secondary losses are significantly higher than the other PAK airfoils. It is unlikely that an airfoil with loadings of this level would be used in a commercial engine. Instead, the PAKF airfoil represents a successful midspan design that is well beyond the typical design scope. Having been designed with state-of-the-art tools, this airfoil is an affirmation that a successful design outside of the current design guidelines, albeit at lower loading levels than PAKF, is possible.

\subsubsection{Turbulence and Losses within the Secondary Flow}

Irreversibilities within a general turbulent flow arise from the dissipation of eddies to internal energy. The production of turbulence from the mean flow is therefore considered the first step in the production of losses (Gregory-Smith et al., 1988a). Very limited research exists on the downstream turbulence field of turbine cascades. Therefore, a portion of the present research includes measurements of the turbulence field to further understand how losses are produced from the mean flow field.

Moore et al. (1987) performed measurements of the mean and turbulent flow downstream of a low-speed $(\mathrm{Ma} \approx 0.07)$ cascade using pressure and hotwire probes. They examined the rates at which turbulence, and thus ultimately total pressure losses, were produced from the mean kinetic energy. Much of the discussion surrounds analysis of the deformation work term. This parameter appears in both the mean and turbulence kinetic energy equations and represents the rates of turbulence production from the mean flow (Kundu and Cohen, 2004b). Moore et al. (1987) showed downstream planar distributions of the deformation work in an effort to reveal the loss production mechanisms. Area-averages of the deformation work components show that the highest rates of turbulence production were associated with the $\overline{v^{\prime} w^{\prime}}$ Reynolds shear 
stress acting against the mean spanwise velocity gradient $(\partial \bar{v} / \partial z)$. They assert that the secondary kinetic energy dissipation within the secondary flow, particularly in the spanwise direction, is largely influenced by the action of this Reynolds stress.

Moore et al. (1987) also found the regions with the highest rates of turbulence production, or losses, from the mean flow appear adjacent to the secondary vortices and are not coincident with the location of peak turbulence kinetic energy or peak total pressure loss. The highest turbulence intensities, of approximately $23 \%$ based on the inlet free-stream velocity, were found within the passage vortex region.

Gregory-Smith and Cleak (1992) performed a similar study to that of Moore et al. (1987). Again, high turbulence intensities of about $30 \%$ were associated with the passage vortex. Lower values of about $10 \%$ were local to the blade wakes. Unlike Moore et al. (1987) they found that the highest rates of turbulence production (deformation work) were associated with the $\overline{u^{\prime} v^{\prime}}$ Reynolds shear stress and the corresponding velocity gradient $(\partial \bar{u} / \partial y)$. In this work (GregorySmith and Cleak, 1992) in the $\overline{v^{\prime} w^{\prime}}$ component was not measured and therefore the results are not directly comparable to those of Moore et al. (1987). Gregory-Smith and Cleak (1992) also conclude that the normal stresses $\left(\overline{u^{\prime 2}}, \overline{v^{\prime 2}}, \overline{w^{\prime 2}}\right)$ can act to either extract or return energy to the mean flow, whereas the shear stresses tend to only be dissipative. Although it was not stated explicitly, the results appear to show that the shear stresses primarily dissipate secondary kinetic energy rather than primary kinetic energy along the primary flow direction.

Perdichizzi et al. (1992) present downstream turbulence results from a cascade at much higher outlet velocities $(\mathrm{Ma}=0.3)$. Peak turbulence intensities of approximately $10 \%$ were associated with the passage vortex. Area-averages of the deformation work terms were not obtained, but it appears that the Reynolds shear stresses closely follow the development of the secondary flow features. It was suggested that the observed reductions in the spanwise secondary velocities with downstream distance could be attributed to the action of the $\overline{v^{\prime} w^{\prime}}$ shear stress within the passage vortex region. Again, this is consistent with the study by Moore et al. (1987) 
and Gregory-Smith and Cleak (1992) in that the Reynolds shear stresses are largely responsible for the dissipation of secondary kinetic energy from the mean flow.

Experimental investigations of the nature discussed here form the basis for understanding the complexity of the turbulence field of turbine secondary flows, which can then be applied to numerical models. CFD is widely used for turbomachinery design and the simulations are largely performed using RANS solvers with two-equation turbulence models. An assumption of these turbulence models is the Boussinesq approximation: the Reynolds stresses are proportional to the corresponding mean velocity gradients. The eddy-viscosity is the coefficient of proportionality and is assumed isotropic.

Wilcox (1998) discusses the deficiencies of these eddy viscosity based turbulence models. He refers to Bradshaw (1973) and indicates that these models are inaccurate for flows with rapidly changing mean strain rates, suggesting that the Reynolds stresses adjust to rates unrelated to the mean flow. Turbine secondary flows have many of the characteristics that commonly lead to rapid changes of local mean strain rates, such as flow separations, three-dimensionality and swirling or rotating flows. It is therefore not surprising the validity of the Boussinesq approximation for turbine secondary flows has also been previously investigated. However, there are again limited data.

Both Gregory-Smith and Cleak (1992) and Perdichizzi et al. (1992) investigated the isotropy of the eddy viscosity downstream of turbine cascades. Gregory-Smith and Cleak (1992) found that the eddy viscosity was reasonably isotropic while Perdichizzi et al. (1992) found high levels of anisotropy at the measurement plane closest to the trailing edge. Only at a plane further downstream did Perdichizzi et al. (1992) measure more isotropic eddy viscosities. In any case, the results of both studies suggest that within at least some regions of the secondary flow the Boussinesq approximation is invalid. The level to which turbine flow predictions are affected by this anisotropy is not addressed in these studies.

Cleak and Gregory-Smith (1992) performed CFD predictions of a turbine secondary flow using three turbulence models: a mixing-length, a one-equation and a hybrid $k-\varepsilon$ mixing length 
model. They found the choice of turbulence model has a large impact on the mean flow, with the least complex mixing length model providing the best predictions. All the models produced inaccurate predictions of the turbulence stresses and the authors also suggest that transition is important to the accurate prediction of turbine secondary flows.

Today, one of the most widely used turbulence models for turbomachinery applications is the shear-stress transport (SST) model. Developed by Menter (1994), it is designed to take advantage of the strengths of both the $k-\omega$ and $k-\varepsilon$ models (Wilcox, 1998). In the laminar sublayer and logarithmic regions of the boundary layer, the $k-\omega$ model is preferred, while in the outer, or wake, region the $k-\varepsilon$ model is used to avoid the undesired sensitivity to the freestream turbulence intensity. The SST model switches between the $k-\omega$ and $k-\varepsilon$ formulations depending on the flow region. Furthermore, the eddy-viscosity formulation of the SST model accounts for the transport of the turbulence shear stresses. This ultimately improves the model for use in flows with adverse pressure gradients and pressure induced separations: both are characteristic of turbomachinery flows (Menter, 1994). The SST model can also be combined with a transition model for prediction of both laminar and transitional flow regimes. Although the SST model provides excellent results for many engineering applications, it is still based on the Boussinesq approximation. As mentioned, this assumption is likely not strictly valid for secondary flows. Thus, part of the present research will investigate experimentally the anisotropy of the turbine secondary flow and then address short-comings of the predictions using the SST turbulence model.

The $\gamma-\operatorname{Re}_{\theta}$ transition model available in ANSYS-CFX was developed Menter et al. (2006) and interfaces with the SST model. Part II of the paper, Langtry et al. (2006), provides several test cases to validate the model. Both two-dimensional and three-dimensional turbomachinery cases are included. The paper focuses on the prediction of the near surface flow: specifically the accuracy of the surface skin-friction coefficients. The secondary flow physics and turbulence quantities are not investigated. The availability of secondary flow turbulence data, as well as the complexity of the turbulence field, has previously made it difficult to assess the accuracy of the 
predicted turbulence fields. Generally, comparisons with experimental data have focused on the accuracy of the predicted mean flow. The turbulence measurements in the present work therefore provide the unique opportunity to assess the prediction accuracy of the turbulence field and the mean flow using the CFX solver with the SST and transition model.

To this point the discussion has only focused on the time-independent flows of turbine cascades. In an actual engine environment there are significant unsteady effects. For example, the relative motion of the rotor results in periodic unsteady inlet conditions that then propagate through the downstream blade passage. In traditional cascade studies these effects are generally disregarded. However, evidence exists that the turbine cascade secondary flow still exhibits some level of unsteadiness even in the absence of these real engine effects. Wang et al. (1997) visualized the secondary flow of a turbine cascade using smoke wires and laser illumination. They report that the pressure-side leg of the horse-shoe vortex is periodically varying through the passage. This is illustrated in cross-section A-A of Figure 2.3. Periodic motion of this nature should yield a distinct frequency in the temporal history of time-accurate measurements. Different investigations have yielded different results regarding the inherent unsteadiness within the passage vortex. This suggests that perhaps these effects may be cascade specific. On the other hand, the frequency of the periodic motion is likely relatively low and thus may be difficult to identify using spectral analysis.

Gregory-Smith et al. (1988b) and Gregory-Smith and Cleak (1992) investigated the frequency of the unsteadiness using frequency spectra obtained at selected points within the secondary flow. Initially, a distinct frequency of $30 \mathrm{~Hz}$ was observed and attributed to unsteadiness within the passage vortex (Gregory-Smith et al., 1988b). In the latter investigation, the cascade was slightly modified to remove a suction surface separation bubble. As a result, no distinct frequency peaks were observed and the initial dominant frequency was attributed to the unsteadiness originating from the separation bubble, not the passage vortex. The possible unsteadiness of the passage vortex is particularly relevant to the use of pressure probes in cascade flows, which generally do not have a sufficient frequency response to measure flow unsteadiness. 
Appendix D.5 discusses the challenges of using seven-hole pressure probes for turbine cascade secondary flows.

A study by Förster et al. (2011) examined the differences between steady and timeresolved pressure probe measurements of "Durham" cascade. The outlet Reynolds number was approximately $4 \times 10^{5}$ based on the blade axial chord $(181 \mathrm{~mm})$. They used traditional and fastresponse five-hole probe for comparison purposes. Their measurements showed distinct differences between the mean total pressure fields measured using the two probes. These differences are similar to those identified in the present work between the Kiel and seven-hole probes, as discussed in Appendix D.5. Förster et al. (2011) attributes the differences to the inability of the traditional probe to respond to the transient behavior of the secondary flow.

In a subsequent paper, Förster et al. (2012) investigated the apparent movement of the secondary flow structures. The fast-response five-hole probe was used to make point measurements at a downstream measurement plane. To correlate the measurements of the entire plane in time, a fixed position hot-wire probe is also logged during the data collection process. During post-processing the hot-wire serves as a trigger to indicate the required phase shift at each point in the pressure probe data set. A spectral analysis of points within the passage vortex shows that the most significant frequency associated with the passage vortex was about $2.7 \mathrm{~Hz}$. The frequency was not related to spatial movements of the structure but to periodic fluctuations of the total pressure field within the vortex. The apparent steadiness of the secondary structures perhaps helps to explain the success of engine technologies designed using steady-state computations.

\subsection{Control of Secondary Flows for Secondary Loss Mitigation}

\subsubsection{Introduction}

As discussed in Section 2.4, engine weight reductions can be achieved through blade count reductions. The higher secondary losses associated with the increased aerodynamic loading can 
be reduced by passively manipulating the development of the passage vortex. The endwall geometry is typically modified using either endwall fences or endwall contouring. Modifications to the blade leading edge, the blade profile and loading in the near endwall region have also proven successful in the reduction of the secondary losses. The following subsections discuss endwall fences, near endwall leading edge and blade profile modifications and then both axisymmetric and non-axisymmetric endwall contouring.

\subsubsection{Endwall Fences}

An endwall fence, as the name suggests, is a spanwise protrusion from the endwall that typically extends from the inlet to outlet of the blade passage. The purpose of the fence is to alter the cross-passage trajectory of the passage vortex by deflecting it away from the suction surface of the blade. By maintaining a position near the pressure surface the low momentum fluid of the vortex will mix with the free-stream in a more favourable, accelerating, pressure field. In doing so, as suggested by Denton (1993), the mixing that occurs between the passage vortex and the free-stream will produce lower mixing losses than if the mixing occurred in a decelerating or constant pressure flow region closer to the blade suction surface.

A seminal work by Prumper (1972) investigated over 400 configurations of endwall fences and blade suction surface grooves on an annular cascade of inlet guide vanes. His investigation led to an optimum design that had shallow groves on the blade suction surface, positioned a short distance from the endwall. These were found to produce lower losses when compared to cases with guide elements (fences) positioned on the endwall surface between the blades. Although the endwall fence acts as intended by deflecting the cross-passage flow away from the blade suction surface, the relatively high impingement angles with the fence lead to higher losses than the cases with the blade suction surface groves.

Chung et al. (1991) and Chung and Simon (1993) also investigated the use of endwall fences to improve the effectiveness of the suction surface film cooling in the near endwall region. They found reductions in both the size and strength of passage vortex and improvements of the 
film cooling effectiveness. In the latter study, the fences demonstrated similar effectiveness when the free-stream turbulence intensity was elevated to about $10 \%$. The disadvantage of the endwall fence is its durability in the high temperature turbine stages.

\subsubsection{Leading Edge and Near Endwall Blade Profile Modifications}

Modifications to the leading edge for secondary loss reduction are designed to alter the development of the horseshoe vortex. Sauer et al. (2001) used leading edge bulbs in the near endwall blade profile, as shown in Figure 2.4, to strengthen the suction-side (SS) leg of the horseshoe vortex. A stronger SS leg, with an opposite sense of rotation relative to the passage vortex, delays the interaction of the passage vortex and suction surface of the adjacent blade. They report a $47 \%$ reduction in the secondary losses relative to their baseline geometry. In the review paper on secondary flows by Langston (2001), he was cautiously optimistic about his study but suggests that further investigation is required because strengthening any structure within the secondary flow could in fact lead to higher mixed out losses.

Leading edge fillets, as opposed to bulbs, have also been successful in reducing the strength of the passage vortex and the associated losses. Zess and Thole (2002) tested a leading edge fillet design in a four blade cascade. A fiber optic laser Doppler velocimetry (LDV) system was used to measure the mean and turbulent flow fields in the turbine vane passage. Corresponding CFD computations are also shown. The measurements reveal that the fillet was effective in accelerating the inlet boundary layer to prevent the formation of the pressure-side leading edge horseshoe vortex. As a result the formation of the passage vortex was delayed and the downstream levels of secondary kinetic energy (SKE) and turbulence kinetic energy (TKE) were significantly reduced. Their CFD is in good agreement with the experiment and also predicts significant benefits in terms of the losses. Experimental loss measurements were not performed and therefore the predicted loss benefits were not verified. 
(a)

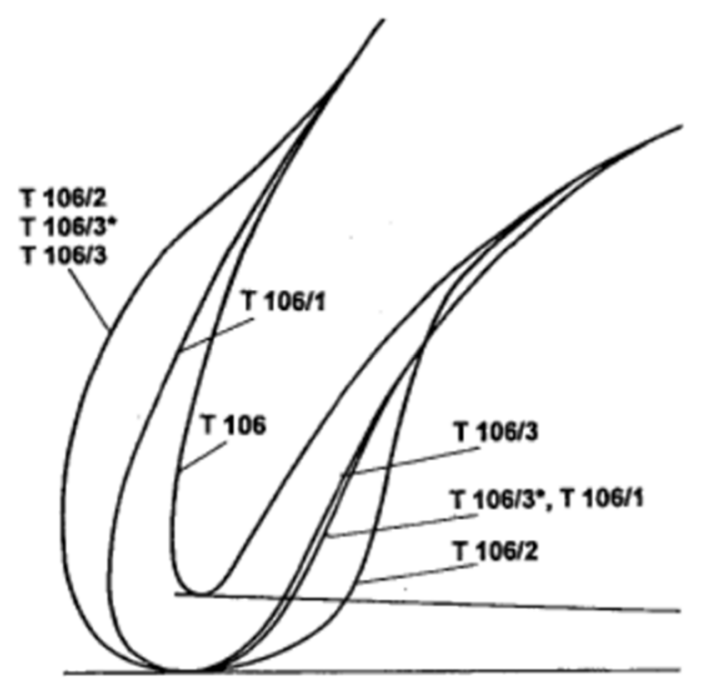

(b)

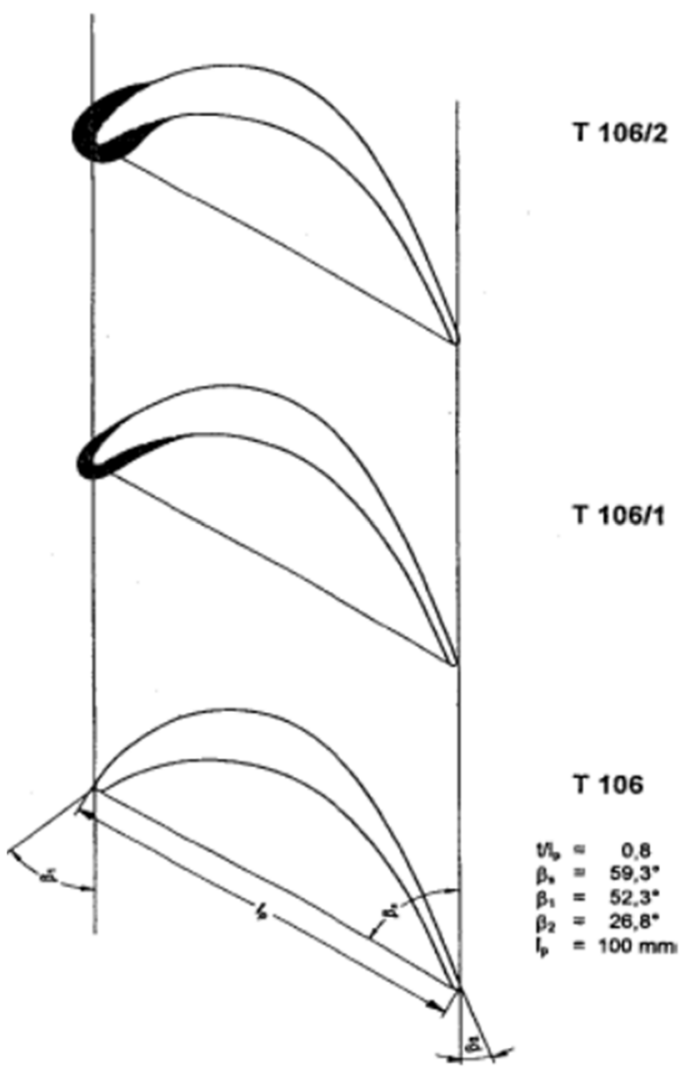

Figure 2.4: Example of leading edge bulbs, from Sauer et al. (2001)

Becz et al. (2003) and Becz et al. (2004) investigated the potential of both leading edge bulbs and fillets. They examined two bulb shapes, one large and one small, and one leading edge fillet geometry in a linear cascade facility. In the 2003 paper, loss measurements were made using a Kiel probe and therefore the overall loss coefficients were area-averaged. The fillet and small bulb showed equal loss reductions of about $8 \%$, while the large bulb provided no benefit. The 2004 study used multi-hole probes to document both the pressure and velocity fields of the same flows. As such, mass-averaging was possible. The bulb geometry showed no benefit in terms of the overall mass-averaged losses. The fillet, however, still showed a benefit of about 7.3\%. Again, the fillet appeared effective in reducing the strength of the passage vortex. 
More recently, Saha et al. (2012) investigated leading edge fillets in an annular cascade for mid-to-high sub-sonic conditions $\left(\mathrm{M}_{\mathrm{a} 2, \mathrm{IS}}=0.9,0.7\right.$ and 0.2$)$. The objective was to investigate the effectiveness of leading edge fillets in an annular cascade, as opposed to a linear cascade. Downstream measurements revealed no significant difference in the losses, blade loading, downstream SKE and streamwise vorticity from the baseline case (no fillets). They provide little explanation except that in this study the baseline case was manufactured with a small fillet around the entire blade profile. In the previous studies that report benefits for the leading edge fillets, such as Becz et al. (2004), the baseline geometry was not filleted and was flush with the linear endwalls.

Another approach to passively reducing the secondary losses is by altering the shape, and loading, of the blade pressure surface. The novelty arises from the observation that the lower the streamwise momentum of the fluid along the blade pressure surface the more strongly it is accelerated across the endwall by the blade-to-blade pressure gradient. Knezevici (2011) introduces an airfoil with a "modified-loading" distribution that is the subject of a patent filing by PWA. Effectively, the momentum of the pressure-side fluid is increased by lowering the static pressure. Of course, the suction surface had to be modified accordingly to maintain the overall loading level. This airfoil, PAKD-FP, has a Zweifiel that is $25 \%$ higher than the baseline PAKB airfoil, which is consistent with the PAKD-A and PAKD-F airfoils. The results of Knezevici (2011) show that the modified-loading approach is an effective means to reducing the secondary losses.

Brear et al. (2002a:b) present similar results to those of Knezevici (2011) in a study that focused on the effects of reducing the size of a pressure-side separation bubble. They studied a family of airfoils with modified pressure-surfaces. Downstream planar measurements revealed consistent profile losses for all cases and Airfoil (D) had the lowest overall mass-averaged losses that were $10.2 \%$ less than the baseline case (A). The authors attribute the benefit to the elimination of the pressure-side bubble, and the increase in streamwise momentum of the pressure-side fluid. In the study by Knezevici (2011) the airfoils did not have pressure-side 
separation bubbles. Thus, he demonstrated that the pressure-side modifications can be beneficial even if there is no pressure-side separation.

Most recently, a two-part paper by Lyall et al. (2013) and Sangston et al. (2013) discusses the design and validation of a near endwall fillet, respectively. These papers are mentioned now because of the relation to the modified loading approach of Knezevici (2011). The endwall fillet designs of these studies have near endwall airfoil cross-sections that are significantly thickened relative to the midspan airfoil. The most prominent changes occur along the pressure-surface, much like the thickening of the PAKD-FP of Knezevici (2011). The design approach of Lyall et al. (2013) was based on the observation that more forward-loaded airfoils, with higher stagger angles, generate higher secondary losses than their more aft-loaded and lower-stagger counterparts. The fillet design focused on creating a low-stagger (LS) profile in the near endwall region that would reduce the secondary losses associated with the forward-loaded baseline airfoil. The CFD simulations show that the fillet is effective at eliminating the near endwall separation bubble present on the pressure surface of the baseline airfoil. As a result, the overall predicted losses are reduced by $7.1 \%$. The experimental validation shows a reduction of $9.9 \%$.

The validation paper by Sangston et al. (2013) suggests that the loss benefits associated with the fillet result from a weaker inlet boundary layer separation. In their opinion, the weakened pressure-side leg of the horseshoe vortex results in a passage vortex that is smaller and also lower in strength. Their results are strikingly similar to those of Knezevici (2011). The loading distribution of the LS fillet has lower static pressures which is indicative of an increase in the pressure-side streamwise momentum. Perhaps, this study has taken another approach to arrive at a design that benefits from the same mechanisms proposed in the modified-loading approach of Knezevici (2011).

\subsubsection{Axisymmetric Endwall Contouring}

Endwall contouring is another type of passive flow control for the reduction of secondary losses. Axisymmetric endwall contouring usually refers to contraction of annulus in the axial 
direction. Generally, the contraction leads to either hub or tip endwall being "S" shaped. Deich et al. (1960) was one of the first studies to test the S-shape applied to the hub endwall. They showed efficiency improvements for the low aspect ratio stages with large contraction ratios. Generally, axisymmetric contouring is applied to nozzle guide vanes where the contraction between the combustion chamber and the turbine inlet continues through the first vane. The $\mathrm{S}$ shape permits a larger contraction over a shorter distance than a more traditional conical contraction. Morris and Hoare (1975) presented another early investigation of S-wall contouring. Tests were performed in a low speed linear cascade and they found significant loss reductions on the planar endwall side. Kopper et al. (1981) performed cascade tests at higher outlet Mach numbers $\left(\mathrm{M}_{\mathrm{a} 2}=0.85\right)$ and show loss reductions of about 17\%. Again, most of the improvements were local to the planar endwall side of the cascade.

Dossena et al. (1999) presented experimental and computational results from a linear cascade of nozzle guide vanes with an S-type endwall contour applied at the blade tip endwall. Overall and secondary loss reductions were reported as $26 \%$ and $35 \%$ relative to the baseline case with two planar endwalls. In their study the secondary flow was significantly altered on the contoured side of the passage, with lower loss levels confined to the near endwall region. On the planar side (hub), the losses were similar to the baseline case and the vortices were smaller with lower levels of streamwise vorticity. Overall, the Dossena et al. (1999) study showed benefits over the entire span, unlike the earlier studies.

Other examples of axisymmetric contouring applied to turbine vanes include the works of Boletis (1985), Moustapha and Williamson (1986), Warner and Tran (1987), Burd and Simon (2000) and Piggush and Simon (2013). In general, this type of contouring provides a benefit because the intra-passage mixing of the low momentum secondary fluid occurs in a more favourable pressure gradient. 


\subsubsection{Non- Axisymmetric Endwall Contouring}

Non-axisymmetric endwall contouring is one of the secondary loss reduction technologies investigated in this thesis. It is similar in concept to endwall fences in that the growth of the passage vortex is altered by shaping (contouring) the intra-passage endwall. However, this is geometrically a more gradual approach. Rather than applying a physical barrier to alter the trajectory of passage vortex, the blade-to-blade cross-passage pressure gradient is reduced by changing the endwall static pressure distribution. This is accomplished using endwall streamline curvature.

Rose (1994) pioneered the concept of locally accelerating and diffusing the endwall flow using concave and convex curvatures (relative to a planar datum) to raise or lower the local static pressures respectively. The objective of the investigation was not to reduce the downstream losses, but rather to reduce the purge flow requirements by altering the circumferential static pressure at the gap between stator and rotor endwalls. In his study, the radial height of the threedimensional endwall of a nozzle guide vane (NGV) was designed using distribution functions in the circumferential and axial directions. A sinusoidal wave-form, with a wave length equal to one pitch, was applied in the circumferential direction. This approximates the distribution of the endwall static pressure observed on the uncontoured hub-wall. In the axial direction, the radial distribution was prescribed using a combination of a parabola and sinusoid, resulting in a gradual curvature increase through the passage and a more abrupt curvature change at the trailing edge. Rose (1994) admits that his methodology leads to two geometric deficiencies: (1) there is curvature discontinuity between the axial parabola and sinusoid and (2) the curvature of the trailing edge sinusoid may be too large. However, as a proof of the concept his CFD study showed that the endwall contouring leads to a $70 \%$ reduction of the static pressure nonuniformities at the platform trailing edge. Although the losses were not presented, the uniformity improvements would likely lead to loss benefits as well.

Harvey et al. (2000) developed an endwall profile, shown in Figure 2.5, using threedimensional CFD simulations as part of a design study. The endwall profile was applied to the 
well researched "Durham" cascade (e.g. Gregory-Smith and Cleak, 1992) that is representative of an HP turbine rotor. The design methodology is based on a forward and reverse linear design system introduced by Shahpar et al. (1999) for the design of turbine blades. In this method, the endwall geometry is defined by a B-spline with six control points. The surface profile is altered using six perturbations per control point, generating a total of 36 different geometries. CFD simulations are then performed for each endwall configuration. Since loss predictions from CFD simulations can be somewhat unreliable, the endwall static pressure and exit flow overturning were used as the parameters for evaluating the effects of contouring.

Overturning, or underturning, is the term that describes the deviation of the pitchwiseaveraged outlet angle, relative to the midspan design angle, across the blade span. The magnitude of overturning/underturning is well correlated to the size and strength of the secondary flow. Deviations from the design angle are important since they can alter the incidence for the downstream bladerow thereby increasing losses for that subsequent passage.

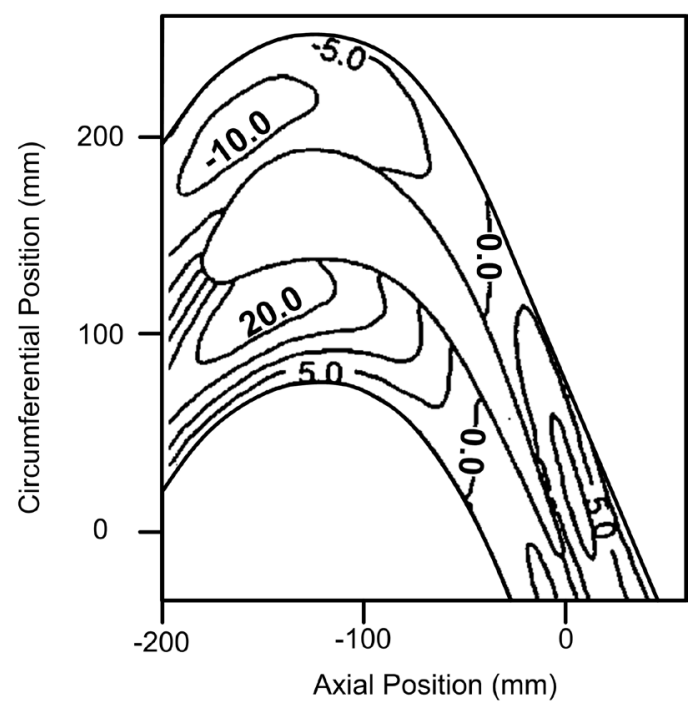

Figure 2.5: Contoured endwall heights (mm), reproduced from Hartland et al. (2000) 
In Figure 2.5, the design study results have led to an endwall profile that appropriately controls the secondary flow. In the centre of passage and towards the blade leading edge, there are convex (positive) and concave (negative) regions. The convex curvature accelerates the flow which impedes the rollup of the pressure side leg of the horseshoe vortex, while the concave regions diffuse the flow and restrict the development of the suction side of the horseshoe vortex. Towards the trailing edge another profiled region is used to reduce the exit flow overturning, with the disadvantage of increasing the strength of the corner vortex.

Hartland et al. (2000) experimentally validated the performance of the endwall designed by Harvey et al. (2000). The endwall static pressures were found to be in good agreement with the predictions. The measured secondary flow was significantly reduced in size and strength, as predicted by the CFD, with the exception of a stronger corner vortex. Near the endwall the pitchwise-averaged flow angles of the contoured case shows a reduction in underturning of about $2^{\circ}$ relative to the contoured case. Again, this is consistent with the CFD predictions. The contouring provided an overall loss improvement of $30 \%$ relative to the baseline case, which was significantly more than predicted by the CFD. It was concluded, that further loss improvements could be obtained by reducing the strength of the corner vortex but at the cost of sacrificing the reductions of outlet overturning.

As discussed, cascade studies have investigated endwall contouring designs with different design intentions. Rose (1994) focused his design on reducing the non-uniformities of the static pressure distribution downstream of the nozzle guide vane (NGV) in an effort to reduce the rimseal leakage flow entering the rotor. Harvey et al. (2000) focused on altering endwall static pressure distribution to reduce the cross-passage gradient to reduce the strength of the secondary flow. In their case, the spanwise distribution of the outlet flow angle was used as a design metric to compare the effectiveness of different endwalls. In many endwall contouring studies the predicted downstream total pressure losses $(Y)$ are not commonly used as a design metric because the values predicted by CFD are generally unreliable. The downstream secondary kinetic energy (SKE) has become an attractive parameter because (1) it is generally better predicted than $Y$ and 
(2) it represents the kinetic energy loss from the primary flow that will be realized as total pressure losses with further downstream mixing. Brennan et al. (2003) point out that solely using the SKE parameter may lead to shape optimization in undesirable endwall regions. For example: the shape optimization is not necessary near the blade leading and trailing edges where the locally elevated SKE is unrelated to secondary flow structures but is a product of the blade potential field. As an alternative, they suggest using the dot product of the SKE and the helicity (SKEH) as a design metric. The helicity is defined as the flux of streamwise vorticity. Several others have since adopted this methodology, although Praisner et al. (2013) contest this practice and suggest that perhaps the row losses $(Y)$ are still the most appropriate design metric if the objective of contouring is to reduce the overall row losses.

Since the introduction of endwall contouring, nearly 20 years ago, there have been many investigations of the design and validation of non-axisymmetric endwall contouring. The studies vary widely in the design techniques as well the test conditions. In an effort to organize some of this research Table 2.1 was created. It summarizes many of the important endwall contouring investigations of the past 20 years. The references are listed chronologically and are categorized according to: (1) the study type, (2) contouring design characteristics and (3) the flow parameters.

The study type (1) heading is divided into seven sub-headings. The first two indicate if numerical (Num.) and/or experimental (Exp.) results were presented. The following three indicate the type of bladerow used: a linear cascade (L. Cas.), an annular cascade (A. Cas.) or stage/rig (Stg./rig). The last two sub-headings indicate whether the investigation focussed on the design methodology (Des.) or the validation of a particular design (Val.).

The second main heading is the contour design (2). The sub-headings here are perhaps less descriptive than originally intended due to the wide variety of contouring designs. In many cases, the endwalls are optimized for specific blade loadings and turbine operating conditions. Therefore, the first two sub-headings of this group differentiate between non-axisymmetric endwall contouring (NEC) and leading edge fillet (LEF) designs. The latter is sometimes referred to as endwall contouring (Lyall et al., 2013; Sangston et al., 2013) and therefore some of these 
studies are included in Table 2.1. The third sub-heading is specific to the current thesis topic and indicates if upstream endwall cavity geometry and/or purge flow were investigated. The last subheading indicates the design metric(s) (DM) used to evaluate the effectiveness of the contouring. Several abbreviations are used here and were adopted from the nomenclature of each reference. Descriptions of each are given below:

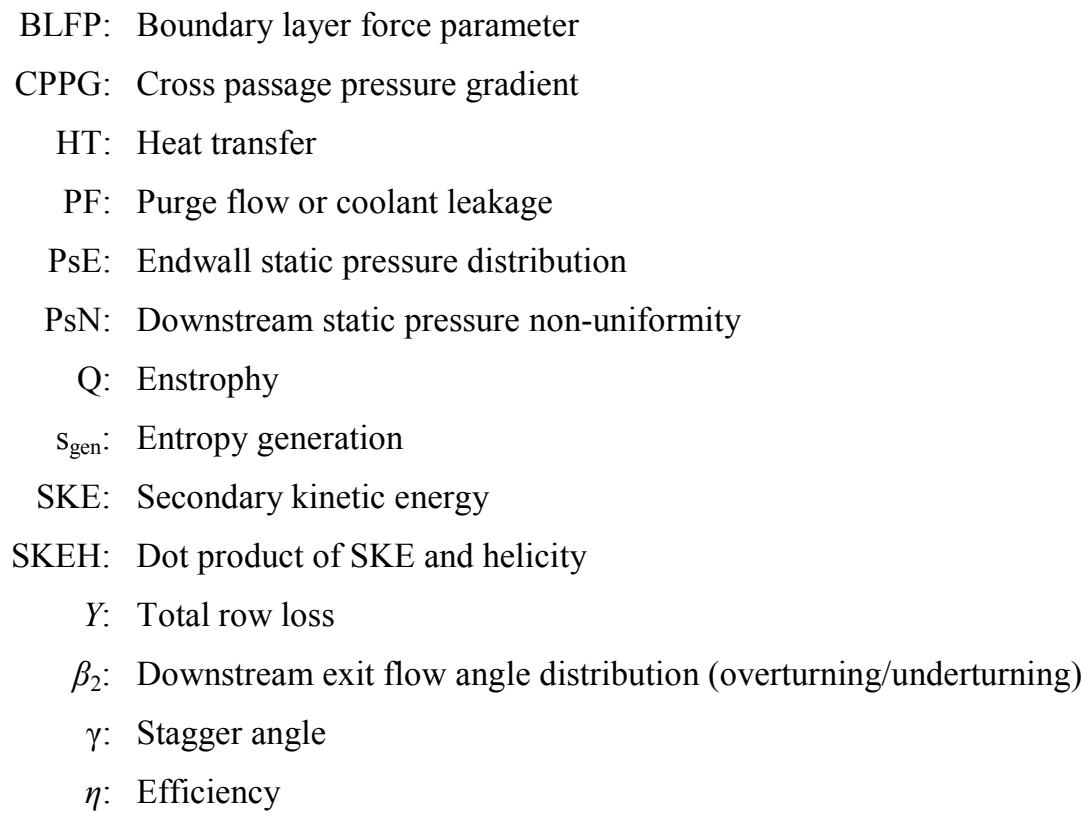

The third main heading, flow parameters (3), is divided into sub-categories for the exit Reynolds number $\left(\operatorname{Re}_{2}\right)$, the exit isentropic Mach number $\left(\mathrm{M}_{\mathrm{a} 2}\right)$, the inlet turbulence intensity $(T u)$ and the purge flow level (PF). The last main column, entitled comments, summarizes key points for each study.

As shown in Table 2.1, many of the investigations have been validation studies that demonstrate the effectiveness of endwall contouring as a secondary loss mitigation technology. As mentioned, the initial linear cascade study by Hartland et al. (2000) demonstrated that contouring could reduce the overall losses of the Durham cascade by about $30 \%$. Following this, Ingram et al. (2002) tested a second generation endwall alongside that of Hartland et al. (2000). The cascade measurements show reductions of the secondary losses by $18 \%$ and $30 \%$ for the first 
and second generation designs, respectively. This paper was also one of the first to include physical interpretations of the contoured flow field using endwall flow visualizations and 3D CFD results. In general, the profiled endwall weakened the cross-passage flow and the pressureside leg of the horseshoe vortex. The latter was indistinguishable in the experimental surface visualizations.

In the same time period, Rose et al. (2001) extended the application of endwall contouring to a cold flow singe stage HPT test rig of the Rolls-Royce Trent 500. The CFD design study by Rose et al. (2001) predicted a turbine stage efficiency improvement of $0.4 \%$. The experimental validation exceeded the predictions with a measured turbine stage efficiency increase of $0.59 \%$. Overall, the uniformity of the downstream flow was improved with contouring and lower overturning was observed in the hub wall region. Others such as Snedden et al. (2009), Germain et al. (2010), Schüpbach et al. (2010), Poehler et al. (2010) and Bergh et al. (2012) have shown similar benefits for rig or stage tests that include endwall contouring.

An aggressive endwall design was developed by Ingram et al. (2005) in an attempt to push the limits of endwall contouring. The endwall was designed using curvature values at the peaks and troughs nearly double those used in the previous study (Ingram et al., 2002). Both the predictions and experiment showed reductions in SKE for the profiled endwall. However, loss benefits were not realized in the experimental measurements. In fact, the secondary losses increased significantly by about $9 \%$ relative to the baseline case. Although this design may benefit the downstream blade rows due to the reduced SKE, this study shows the importance of experimental testing and perhaps raises questions as to the merit of using the SKE as a design parameter.

Many of the studies mentioned to this point have been focused on endwall designs for HP turbines. Generally, the HP bladerows have low aspect ratios and the secondary losses are a large fraction of the overall losses. This makes them a good candidate for endwall contouring. Recently, more highly loaded LP turbine designs have become popular for engine weight and cost savings. Endwall contouring has therefore become useful for LP designs as well to reduce the 
increase in losses associated with the more highly loaded bladerows. For several years highlyloaded LP turbine bladerows have been the focus of the research at Carleton University in both the low- and high-speed linear cascade facilities. Knezevici et al. (2010) and Knezevici et al. (2009) investigated the application of endwall contouring to the low-speed PAKB and PAKD-F linear cascades. A related transonic investigation was performed by Taremi et al. (2013). In these cases, the endwall geometry was designed by PWA using the methods outlined by Praisner et al. (2013).

The profiled endwall for the current thesis was also designed by PWA using similar procedures. The following paragraphs summarize the methods of Praisner et al. (2013).

For each design, a total of 25 control points were used to define the endwall shape. Five control points were equally distributed along five offset camber lines spaced equally spaced in the pitchwise direction. The first and last control points along each camber line are positioned at the blade leading and trailing edge. These points are fixed at the nominal endwall height to prevent the contouring from extending upstream and downstream of the bladerow.

The endwall profiles were optimized using three-dimensional steady RANS CFD simulations using the $k-\omega$ turbulence model. The control points are perturbed according to a sequential quadratic gradient-based algorithm. The primary optimization objective was to reduce the mass-averaged total pressure losses. Praisner et al. (2013) presented measured and predicted results for the PAKB, PAKD-A and PAKD-F airfoils. In general, lower losses were obtained for the contoured endwalls with the largest benefits observed for the forward-loaded PAKD-F airfoil. The inability of the CFD to predict the complex passage vortex resulted in the overall measured losses being over predicted by $12 \%$ compared with the measurements. However, it was concluded that the CFD could still reliably predict the trends in losses for the modified endwall configurations.

Many of the design concepts described in Table 2.1 have been effective at reducing endwall losses for a variety of flow conditions. However, only a small number of these studies have specifically investigated the effects of the platform overlap or endwall cavity geometry, and 
even fewer have investigated the effects of purge flow. As will be discussed in the following section, the upstream platform overlap, endwall cavity and purge flow have significant effects on the secondary flow physics. Jenny et al. (2011) suggest that the stage efficiency is strongly influenced by the ratio of the purge mass flow rate to mass flow rate in the main gas-path (known as the purge flow ratio and denoted by $\phi$, see Section 3.5.4). In their study, the most significant effect of contouring was to reduce the sensitivity of the stage efficiency to the purge flow ratio. This was achieved by reducing the interaction of the purge flow and main gas path secondary flow. In another stage test, Schuepbach et al. (2011) showed that the endwall contouring loses its benefit with increasing purge flow ratio. In fact, the baseline case (no contouring) was the most resistant to changes in the purge ratio, thereby suggesting a potential vulnerability of endwall contouring in real engine environments.

Chapter 8 of the current thesis will investigate an endwall contouring design for two different platform overlaps with the addition of varying purge flow levels. The purpose is to add to the relatively small body of data that has investigated the performance of endwall contouring under these more engine-like conditions. The focus is on the physical understanding of the flow field with axisymmetric endwall contouring. 
Table 2.1: Summary of endwall contouring investigations

\begin{tabular}{|c|c|c|c|c|c|c|c|c|c|c|c|c|c|c|c|c|}
\hline \multirow[b]{2}{*}{ References } & \multicolumn{7}{|c|}{ (1) Study Type } & \multicolumn{4}{|c|}{ (2) Contouring Design } & \multicolumn{4}{|c|}{ (3) Flow Parameters } & \multirow[b]{2}{*}{ Comments } \\
\hline & Num. & Exp. & L.Cas. & A. Cas. & $\begin{array}{c}\text { Stg./ } \\
\text { Rig }\end{array}$ & Des. & Val. & NEC & LEF & $\begin{array}{c}\text { Cav.l } \\
\mathrm{PF}\end{array}$ & DM. & $\mathrm{Re}_{2} \times 10^{-5}$ & $\mathrm{Ma}_{2, \text { Is }}$ & Tu (\%) & Pur. FI & \\
\hline Rose (1994) & $\checkmark$ & - & - & $\checkmark$ & - & $\checkmark$ & - & $\checkmark$ & - & - & PSN, PF & - & - & - & - & NGV contour shows $70 \%$ red. in static pressure non-uniformities \\
\hline Hartland et al. (1998) & $\checkmark$ & $\checkmark$ & $\checkmark$ & - & - & $\checkmark$ & $\checkmark$ & $\checkmark$ & - & - & PSN, PF & - & - & - & - & Exp. show a small increase in loss; CFD trends are similar \\
\hline Harvey et al. (2000) & $\checkmark$ & - & $\checkmark$ & - & - & $\checkmark$ & - & $\checkmark$ & - & - & PsE, $\beta_{2}$ & 4.0 & 0.1 & $\approx 5.0$ & - & $\begin{array}{l}\text { Part I-HP turbine (Durham cascade) des. study; } \\
\text { lower SKE and } \beta_{2} \text { deviations; small benefit in } Y\end{array}$ \\
\hline Brennan et al. (2003) & $\checkmark$ & - & - & - & $\checkmark$ & $\checkmark$ & - & - & - & - & SKEH, $\beta_{2}$ & $\begin{array}{r}\mathrm{NGV}=6.8 \\
\text { Rotor }=4.5\end{array}$ & 0.8 & - & - & $\begin{array}{l}\begin{array}{l}\text { Part I - design of contour for HP turbine NGV and rotor cold flow } \\
\Delta \eta=+0.4 \%\end{array}\end{array}$ \\
\hline Rose et al. (2001) & - & $\checkmark$ & - & - & $\checkmark$ & - & $\checkmark$ & $\checkmark$ & - & - & SKEH, $\beta_{2}$ & $\begin{array}{c}\mathrm{NGV}=6.8 \\
\text { Rotor }=4.5\end{array}$ & 0.8 & - & - & Part II - validation; $\Delta n=+0.59 \%$ \\
\hline Harvey et al. (2002) & - & $\checkmark$ & - & - & $\checkmark$ & $\checkmark$ & $\checkmark$ & $\checkmark$ & - & - & SKEH, $\beta_{2}$ & $\begin{array}{c}\mathrm{NGV}=6.8 \\
\text { Rotor }=4.5\end{array}$ & 0.8 & - & - & Design $\Delta \eta=+0.96 \% ; \operatorname{Exp} \Delta \eta=+0.90 \%$ \\
\hline Ingram et al. (2002) & - & $\checkmark$ & $\checkmark$ & - & - & - & $\checkmark$ & $\checkmark$ & - & - & PsE, $\beta_{2}$ & 4.0 & 0.1 & $\approx 5.0$ & - & $\begin{array}{l}\text { Durham cascade two endwalls tested (P1 and } \mathrm{P} 2) \text {. Flow vis. } \mathrm{P} 2 \text { has } \\
24 \% \text { red. in Ysec. }\end{array}$ \\
\hline Ingram et al. (2005) & $\checkmark$ & $\checkmark$ & $\checkmark$ & - & - & $\checkmark$ & $\checkmark$ & $\checkmark$ & - & - & SKE, $\beta_{2}$ & 4.0 & 0.1 & $\approx 5.0$ & - & $\begin{array}{l}\text { Durham cascade aggressive endwall design; larger peak and dips; } \\
43 \% \text { red. in SKE, } 9 \% \text { inc. in Ysec }\end{array}$ \\
\hline Gustafson et al. (2007) & - & $\checkmark$ & $\checkmark$ & - & - & - & $\checkmark$ & $\checkmark$ & - & - & r & 2.33 & $<0.3$ & 4.0 & - & $\begin{array}{l}\text { HPT intra-passage physical interpretation using flow vis, endwall surf. } \\
\text { Ps, flow measurements. } 61 \% \text { red. Y }\end{array}$ \\
\hline Corral and Gisbert (2008) & $\checkmark$ & - & $\checkmark$ & - & - & $\checkmark$ & - & $\checkmark$ & - & - & SKEH & 1.2 & 0.6 & - & - & $\begin{array}{l}\text { LPT endwall optimization using gradient-based method. } \\
\text { SKEH cost function not effective in reducing small corner separations }\end{array}$ \\
\hline Gisbert et al., 2008 (2008) & $\checkmark$ & - & - & & $\checkmark$ & $\checkmark$ & & $\checkmark$ & - & $\checkmark$ & SKEH & 1 & 0.6 & - & & $\begin{array}{l}\text { Endwall optimized (gradient-based) with and without upstream cavity. } \\
\text { Endwall contour is altered in leading edge region. HSV is strong within } \\
\text { cavity. }\end{array}$ \\
\hline Gregory-Smith et al. (2008) & - & $\checkmark$ & $\checkmark$ & & - & - & $\checkmark$ & $\checkmark$ & $\checkmark$ & - & SKEH, $\beta_{2}$ & 4.0 & 0.1 & $\approx 5.0$ & & $\begin{array}{l}\text { Endwall contouring combined with blade reverse compound lean and } \\
\text { L.E extensions. } 20 \% \text { red. in Ymix for combined case. }\end{array}$ \\
\hline Knezevici et al. (2009) & - & $\checkmark$ & $\checkmark$ & 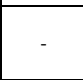 & - & - & $\checkmark$ & $\checkmark$ & - & - & $\mathrm{Y}, \beta_{2}$ & 2.1 & 0.1 & 4 & & $\begin{array}{l}\text { High lift front loaded LPT (PAKD-F). Contouring red. } \mathrm{Y}_{\text {sec, mix }} \text { by } 32 \% \text {. } \\
\text { The strong pitchwise gradient in early part of passage mitigated by } \\
\text { contouring }\end{array}$ \\
\hline Lott et al. (2009) & $\checkmark$ & - & - & & $\checkmark$ & $\checkmark$ & & $\checkmark$ & - & $\checkmark$ & $\begin{array}{l}\text { SKEH, Y, } \\
\text { PsN }\end{array}$ & 2.1 & 0.85 & - & & $\begin{array}{l}\text { Two endwall profiles were optimized using steady sim. W. different } \\
\text { objectives (SKEH, Y and Ps distribution). Unsteady results show diff. } \\
\text { trends. }\end{array}$ \\
\hline Snedden et al. (2009) & $\checkmark$ & $\checkmark$ & - & & $\checkmark$ & - & $\checkmark$ & $\checkmark$ & - & - & SKEH, $\beta_{2}$ & 1.27 & $<0.3$ & 1 & & $\begin{array}{l}\text { Inverse design of Durham cascade generic profile. Exp. } \Delta \eta=+0.4 \% \\
\text { with red. } \beta_{2} \text { non-uniformities. Increase in tip-clearance flow. }\end{array}$ \\
\hline
\end{tabular}




\begin{tabular}{|c|c|c|c|c|c|c|c|c|c|c|c|c|c|c|c|c|}
\hline \multirow[b]{2}{*}{ References } & \multicolumn{7}{|c|}{ Study Type } & \multicolumn{4}{|c|}{ Design Parameters } & \multicolumn{4}{|c|}{ Flow Parameters } & \multirow[b]{2}{*}{ Comments } \\
\hline & Num. & Exp. & L.Cas. & A. Cas. & $\begin{array}{l}\text { Stg. } / \\
\text { Rig }\end{array}$ & Des. & Val. & NEC & LEF & $\begin{array}{c}\text { Cav./ } \\
\text { PF }\end{array}$ & $\mathrm{DM}$ & $\mathrm{Re}_{2} \times 10^{-5}$ & $\mathrm{Ma}_{2, \text { Is }}$ & Tu (\%) & $\mathrm{PF}$ & \\
\hline Germain et al. (2010) & $\checkmark$ & $\checkmark$ & - & - & $\checkmark$ & $\checkmark$ & $\checkmark$ & $\checkmark$ & - & - & $\mathrm{Y}, \mathrm{SKE}$ & $\begin{array}{l}\mathrm{S} 1=7.1, \mathrm{R} 1= \\
3.8, \mathrm{R} 2=5.6\end{array}$ & 0.53 & - & - & $\begin{array}{l}\text { Part I - Design and Validation. Contouring lowers Ysec and surprisingly } \\
\text { Yp. Yp benefits are explained by unsteady results of Part II. }\end{array}$ \\
\hline Schüpbach et al. (2010) & $\checkmark$ & $\checkmark$ & - & - & $\checkmark$ & $\checkmark$ & $\checkmark$ & $\checkmark$ & - & - & $\mathrm{Y}, \mathrm{SKE}$ & $\begin{array}{l}\mathrm{S} 1=7.1, \mathrm{R} 1= \\
3.8, \mathrm{R} 2=5.7\end{array}$ & 0.53 & - & - & $\begin{array}{l}\text { Part II - Time resolved unsteady exp. measurements. Contouring results } \\
\text { in a } \Delta \eta=+1.0 \% \text {. S1 had a } 12 \% \text { reduction in Ysec. }\end{array}$ \\
\hline Knezevici et al. (2010) & - & $\checkmark$ & $\checkmark$ & - & - & - & $\checkmark$ & $\checkmark$ & - & - & $\gamma, \beta_{2}$ & 2.1 & 0.1 & 4 & - & $\begin{array}{l}\text { Baseline LPT (PAKB) airfoil. Contouring red. Ysec, mix by } 15 \% \text { and SKE } \\
\text { by } 13 \% \text {. Flow vis. and flow physics discussed }\end{array}$ \\
\hline Poehler et al. (2010) & $\checkmark$ & $\checkmark$ & - & - & $\checkmark$ & $\checkmark$ & $\checkmark$ & $\checkmark$ & - & - & SKEH & $\mathrm{S} 1=8.2$ & 0.45 & - & - & $\begin{array}{l}\text { Num. and Exp. results show benefits for } n \text { and SKE. Contouring shows } \\
\text { no effect on at the rotor tip due to the dominating tip clearance system }\end{array}$ \\
\hline Jenny et al. (2011) & $\checkmark$ & $\checkmark$ & - & - & $\checkmark$ & $\checkmark$ & $\checkmark$ & $\checkmark$ & - & $\checkmark$ & SKE, $\eta$ & $\begin{array}{l}\mathrm{S} 1=7.1, \mathrm{R} 1= \\
3.8, \mathrm{R} 2=5.1\end{array}$ & $\begin{array}{c}0.52 / 0.28 \\
10.48\end{array}$ & - & $\begin{array}{l}\mathrm{IR}=0.4 \\
0.8,1.2 \%\end{array}$ & $\begin{array}{l}\eta \text { is strongly influence by purge flow ratio. The contouring red. Purge } \\
\text { flow sensitivity by } 18 \% \text { by mitigating PF and sec. flow interaction. }\end{array}$ \\
\hline Knezevici (2011) & $\checkmark$ & $\checkmark$ & $\checkmark$ & - & - & - & $\checkmark$ & $\checkmark$ & $\checkmark$ & - & $Y, \beta_{2}$ & 2.1 & 0.1 & 4 & - & $\begin{array}{l}\text { PhD thesis including results discussed in Knezevici et al. (2009)and } \\
\text { Knezevici et al. (2010) }\end{array}$ \\
\hline Luo et al. (2011) & $\checkmark$ & - & $\checkmark$ & - & - & $\checkmark$ & - & $\checkmark$ & - & - & $s, \beta 2$ & - & - & - & - & $\begin{array}{l}\text { Adjoint gradient-based optimization. Contouring benefits are attributed } \\
\text { to red. of cross-passage pressure gradient. Ysec red. by } 17 \% \text {. }\end{array}$ \\
\hline Panchal et al. (2011) & $\checkmark$ & - & $\checkmark$ & - & - & $\checkmark$ & & $\checkmark$ & - & - & Yvs. SKE & - & - & - & - & $\begin{array}{l}\text { Y. red. for contour A (optimized based on Y) was } 3 \% \\
\text { Y. red for contour B (optimized based on SKE) was 1.7\% }\end{array}$ \\
\hline Schuepbach et al. (2011) & $\checkmark$ & $\checkmark$ & - & - & $\checkmark$ & - & $\checkmark$ & $\checkmark$ & - & $\checkmark$ & SKE, $\beta 2$ & $\begin{array}{l}\mathrm{S} 1=7.1, \mathrm{R} 1= \\
3.8, \mathrm{R} 2=5.1\end{array}$ & $\begin{array}{c}0.54 / 0.26 \\
10.46\end{array}$ & - & $\begin{array}{l}\mathrm{IR}=-0.1 \\
\quad 0.9 \%\end{array}$ & $\begin{array}{l}2 \text { contour designs were compared to the baseline with purge flow added. } \\
\text { Baseline case most resistant to efficiency deterioration with PF. }\end{array}$ \\
\hline Torre et al. (2011) & $\checkmark$ & $\checkmark$ & $\checkmark$ & - & - & - & $\checkmark$ & $\checkmark$ & - & - & - & 2.3 & 0.07 & 0.5 & - & $\begin{array}{l}\text { Endwall designed to enhance SS leg of HSV. } \\
72 \% \text { red. of SKEH and } 20 \% \text { red. Ymix. }\end{array}$ \\
\hline Abraham et al. (2012) & $\checkmark$ & $\checkmark$ & $\checkmark$ & - & - & - & $\checkmark$ & $\checkmark$ & - & - & Ysec, HT & low & low & - & - & $\begin{array}{l}\text { Aero and heat transfer optimized endwall were compared. Aero } \\
\text { optimized showed } 17 \% \text { red. in Ymix. }\end{array}$ \\
\hline Bergh et al. (2012) & $\checkmark$ & - & - & - & $\checkmark$ & $\checkmark$ & $\checkmark$ & $\checkmark$ & - & - & SKE, $\beta_{2}$ & 1.27 & $<0.3$ & 1 & - & $\begin{array}{l}\text { Design of Sneddenet al. (2009) was generic. This study optimized } \\
\text { endwall contour design. Total-to-total } \Delta \eta=+0.14 \text { for optimized endwall }\end{array}$ \\
\hline Hilfer et al. (2012) & $\checkmark$ & $\checkmark$ & - & . & - & - & $\checkmark$ & $\checkmark$ & - & & SKE, $\beta 2$ & 4.0 & 0.1 & $\approx 5.0$ & - & $\begin{array}{l}\text { Durham cascade fitted with tip-clearance. Most of contouring benefit is } \\
\text { realised on the planar tip side of the cascade }\end{array}$ \\
\hline Turgut and Camci (2012) & - & $\checkmark$ & - & - & $\checkmark$ & - & $\checkmark$ & $\checkmark$ & $\checkmark$ & 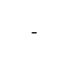 & & $S 1=9$ & - & - & - & $\begin{array}{l}\text { NGV Endwall contour, leading edge fillet and combination type designs. } \\
\text { Area avg. loss show conflicting trends with CFD. Mass-average? }\end{array}$ \\
\hline Dunn et al. (2012) & - & $\checkmark$ & - & - & $\checkmark$ & - & $\checkmark$ & $\checkmark$ & - & - & SKEH, $\beta_{2}$ & 1.27 & $<0.3$ & 1 & - & $\begin{array}{l}\text { Exp. unsteady investigation using rig of Snedden et al. (2009). } \\
\text { Contouring red. unsteady velocity variation. Yields a smoother profile. }\end{array}$ \\
\hline
\end{tabular}




\begin{tabular}{|c|c|c|c|c|c|c|c|c|c|c|c|c|c|c|c|c|}
\hline \multirow[b]{2}{*}{ References } & \multicolumn{7}{|c|}{ Study Type } & \multicolumn{4}{|c|}{ Contouring Design } & \multicolumn{4}{|c|}{ Flow Parameters } & \multirow[b]{2}{*}{ Comments } \\
\hline & Num. & Exp. & L.Cas. & A. Cas. & $\begin{array}{c}\text { Stg./ } \\
\text { Rig }\end{array}$ & Des. & Val. & NEC & LEF & $\begin{array}{c}\text { Cav./ } \\
\text { PF }\end{array}$ & DM & $\mathrm{Re}_{2} \times 10^{-5}$ & $\mathrm{Ma}_{2, \text { Is }}$ & $\mathrm{Tu}(\%)$ & $\mathrm{PF}$ & \\
\hline Lyall et al. (2012) & $\checkmark$ & - & $\checkmark$ & - & - & $\checkmark$ & - & - & $\checkmark$ & - & $\gamma$, BLFP & $0.2-1.0$ & 0.2 & - & - & \multirow{2}{*}{$\begin{array}{l}\text { Part I - High lift front loaded LPT. PAKB derivative. High stagger cause } \\
\text { for high secondary loss, not front loading. Endwall fillet contour design } \\
\text { w. low stagger at endwall. Predicted Ysec red by 17- 24\%; exp. Ysec } \\
\text { red. by } 20 \%\end{array}$} \\
\hline Sangston et al. (2013) & - & $\checkmark$ & $\checkmark$ & - & - & - & $\checkmark$ & - & $\checkmark$ & - & - & $0.2-1.0$ & 0.2 & - & - & \\
\hline Lynch et al. (2013) & - & $\checkmark$ & $\checkmark$ & - & - & - & $\checkmark$ & $\checkmark$ & - & $\checkmark$ & $Y, \beta_{2}$ & 2 & 0.06 & 6 & $\phi=0.75 \%$ & $\begin{array}{l}\text { Endwall contour no sig. effect. The purge flow intensifies the PV and the } \\
\text { low loaded airfoil yields the contouring ineffective. }\end{array}$ \\
\hline Praisner et al. (2013) & $\checkmark$ & $\checkmark$ & $\checkmark$ & - & - & $\checkmark$ & $\checkmark$ & $\checkmark$ & - & - & $Y, \beta_{2}$ & 1.26 & 0.1 & 4 & - & $\begin{array}{l}\text { Endwall contour design applied to PAKD-F, a front highly loaded } \\
\text { derivative of PAKB. Ytotal red. } 12 \text { and } 13 \% \text { for Num. and Exp. }\end{array}$ \\
\hline Taremi et al. (2013) & - & $\checkmark$ & $\checkmark$ & - & & - & $\checkmark$ & $\checkmark$ & - & - & $\gamma, \beta_{2}$ & 6 & 0.78 & 4 & - & $\begin{array}{l}\text { Flow vis, surf. Ps and flow measurements. SKE and vorticity weakened } \\
\text { by contour but design loss benefits not measured exp. }\end{array}$ \\
\hline Turgut and Camci (2013) & $\checkmark$ & $\checkmark$ & - & - & $\checkmark$ & $\checkmark$ & $\checkmark$ & $\checkmark$ & $\checkmark$ & $\checkmark$ & - & $\mathrm{S} 1=9$ & - & - & $\phi=0.75 \%$ & Turgut and Camci (2012) study is expanded to include purge flow \\
\hline Schobeiri and Lu (2014) & $\checkmark$ & $\checkmark$ & - & - & $\checkmark$ & $\checkmark$ & $\checkmark$ & $\checkmark$ & $\checkmark$ & - & - & - & - & - & - & $\begin{array}{l}\text { New method using "continuous diffusion" to red. CPPG was tested. } \Delta \eta \\
=+0.51 \% \text {. Endwall design not optimized. }\end{array}$ \\
\hline Lipfert et al. (2014) & $\checkmark$ & $\checkmark$ & - & - & $\checkmark$ & - & $\checkmark$ & $\checkmark$ & - & - & - & $330-810 \mathrm{k}$ & $\sim 0.4$ & $0.4 \%$ & - & $\begin{array}{l}1.5 \text { rig stage test. Evaluate } 3 \mathrm{D} \text { airfoil and endwall design. Improvement } \\
\text { in mechanical efficiency for new designs. Agreement with predictions. }\end{array}$ \\
\hline Poehler et al. (2015) & $\checkmark$ & - & - & - & $\checkmark$ & $\checkmark$ & & $\checkmark$ & - & - & nstage & $330-810 \mathrm{k}$ & $\sim 0.4$ & $0.4 \%$ & - & 1.5 rig test design for above validation paper. \\
\hline Niewoehner et al. (2015) & $\checkmark$ & $\checkmark$ & - & - & $\checkmark$ & - & $\checkmark$ & $\checkmark$ & - & $\checkmark$ & Ysec & $40-180 \mathrm{k}$ & 0.6 & - & - & $\begin{array}{l}\text { Off design Re conditions test for } 2 \text { stage rig test. Change of incidence in } \\
\text { the second vane leads to higher secondary losses. }\end{array}$ \\
\hline
\end{tabular}




\subsection{Rim-seal Geometry and Simulated Purge flow}

\subsubsection{Introduction}

A typical example of a rim seal is shown in Figure 1.2. As shown, it consists of the platform overlap and endwall cavity features. The purge mass flow creates the aerodynamic seal to prevent hot-gas ingress into the disk cavity region. The primary objective of the rim-seal design is first to protect the disk wheel-space and second to limit the amount of purge mass flow injected into the mainstream. According to Bohn et al. (2006) the disk cooling and sealing flows of a typical gas turbine engine account for an increase in the engine specific fuel consumption of about $6 \%$. The careful design of the rim seal is therefore important to the overall engine performance. As suggested by Bohn et al. (1999), rim seal design improvements can lead to a $30 \%$ reduction in the required purge mass flow. It is not a surprise that much of literature on rimseal geometry has focused on reducing the purge mass flow. However, as noted by McLean et al. (2001), the rim seal geometry and purge flow characteristics also affect the endwall boundary layer, mainstream pressure field and the vortex development. Therefore, the rim-seal design must also aim to limit any adverse effects on the secondary flow.

The literature presented in the following sub-sections will focus on the aerodynamic effects of the purge flow on the main gas path flow. Of course the near endwall flows are the focus. For brevity, investigations of endwall heat transfer, film cooling and sealing effectiveness (in terms of heat transfer) are not considered in this section. However, early studies of cavity flows investigating the rim-seal effectiveness include those by Bunker et al. (1992a; 1992b). Also, the Owen and Wilson (2001) paper provides a summary of earlier research in this area.

The following subsections will first discuss literature investigating the rim-seal geometry followed a section focussing on the interaction between purge flow and mainstream flow. 


\subsubsection{Rim-Seal Geometry}

In the present thesis the effects of purge flow are investigated for two rim-seals designs with different platform overlap shapes. Others have also investigated the effects of varying the rim-seal geometry on the interaction of purge mainstream flow. This section summarizes some of these studies.

De la Rosa Blanco et al. (2009) examined the effects of purge flow and upstream geometry on a low-speed LP linear turbine cascade. The effects of a backward and forward facing step as well as two levels of purge flow $(0.3 \%$ and $0.7 \%)$ with four different injection directions were investigated. Comparisons between the cases without purge flow found that an interaction of the endwall flow and a pressure surface separation bubble increased the losses for the forward step case. When the leakage flow was introduced a skewed shear layer between the leakage and mainstream flow developed. In cases where the purge flow tangential velocities were beyond $44 \%$ of the main flow axial velocity, a number of vortices were formed within the skewed shear layer. The vortices were entrained into the passage vortex resulting in a significant increase in the secondary losses. For purge flows with tangential velocities below $44 \%$ this phenomenon was not observed, suggesting a practical limit for turbine and purge flow designs.

Numerical simulations of a high pressure turbine stage were performed by Marini and Girgis (2007) for two rim seal designs, a recessed leading edge and a raised leading edge platform. As shown in Figure 2.6, the recessed platform (a) is a gradual ramp that extends from beneath the nozzle platform into the main gas-path, while the raised platform (b) is an abrupt step (the rim-seal channel is perpendicular to the main gas-path). Although the recessed shape (a) generated secondary flows with lower penetration heights, the enhanced mixing (relative to the raised platform case) lead to higher entropy generation in the hub wall region. Consequently, only a small efficiency benefit was found for the raised platform (b) case.

A similar numerical study by Schuler et al. (2010) compared the secondary flows of a turbine rotor for a simple axial gap rim-seal and for a more complex compound seal geometry; similar to the raised platform of Marini and Girgis (2007). The compound seal showed lower 
losses than the axial gap, which was explained by the gradual injection path created by the compound seal geometry. This result is somewhat contradictory to the results of Marini and Girgis (2007), as they found that higher losses were generated by the gradual ramp configuration (recessed platform).

(a)

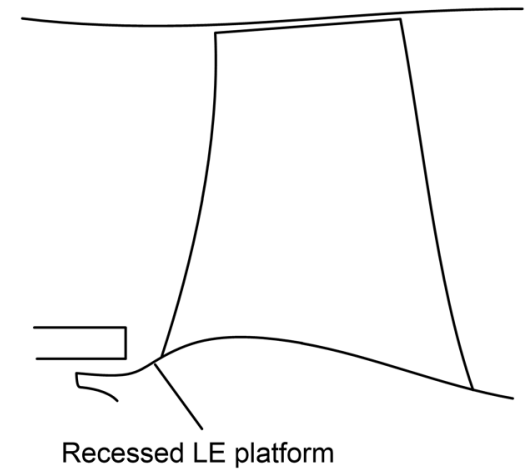

(b)

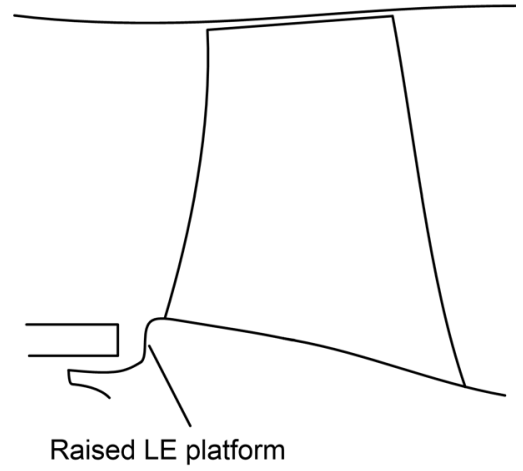

Figure 2.6: Recessed and raised leading edge (LE) platform shapes, adapted from Marini and Girgis (2007)

Perhaps two of the most comprehensive investigations regarding the rim-seal geometry are those by Popovic and Hodson (2013b) and Popovic and Hodson (2013c). They present numerical and experimental results from a linear cascade with purge injection. The first paper documents a numerical parametric study of several rim-seal designs. The effects of varying purge levels on the leakage losses and sealing effectiveness are shown for all cases. The study parameters include the axial and radial seal clearance between the stator platform and rotor fin as well as the length of the overlap between the stator and rotor. Also, forward and backward facing rotor hub-walls of different heights were considered. The authors draw several conclusions regarding the specific geometries considered. In general, it was found that to improve the sealing effectiveness the rim-seal clearance should be reduced in both the axial and radial directions. Therefore, the authors suggest that rotor disc designs should favour upstream and outward disc motions which would act to reduce the rim-seal clearance in the axial and radial directions 
respectively. Also, the backward facing rotor step was found to be detrimental to the losses and the sealing effectiveness in all cases.

The second study by Popovic and Hodson (2013c) investigates several engine representative rim-seals, ranging from simple axial gaps to a variety of overlapping configurations. This investigation includes experimental measurements with supplemental CFD results to improve the physical interpretations. The investigation suggests that size and location of the recirculation zones within the rim-seal are the key flow features that affect the rim-seal effectiveness and the downstream leakage losses. Unfortunately, optimizing for high sealing effectiveness tends to increase the leakage losses causing the designer to trade-off between sealing effectiveness and losses. The best compromise was a design with the smoothest trajectory from the purge cavity into the main gas-path. This reduces what the authors refer to as "spoiling losses". The design representing the best compromise showed a leakage loss reduction of $33 \%$ relative to their datum seal design. In full stage CFD simulations this was translated to efficiency benefits of nearly $0.2 \%$.

It is apparent from the studies mentioned here that the rim-seal geometry is an important consideration for not only the seal effectiveness but also the mainstream losses. This suggests that rim-seal representative geometry should be included early in the design process. The following section will discuss the importance of also considering the purge flow.

\subsubsection{The Interaction between Purge and Mainstream Flows}

McLean et al. (2001a; 2001b) presented one of the first investigations of the aerodynamic effects of the rim-seal purge flow on the main gas-path flow. Measurements were performed in a cold-flow single-stage turbine rig at the ATRF (Axial Turbine Research Facility) of Pennsylvania University. In the first paper, the experimental measurements were made downstream of a rotor in the stationary frame for cases with three types of purge injection: root injection, radial cooling, and impingement cooling. The radial injection is most similar to the purge flow applied in the current thesis. In all cases it was found that small amounts of purge flow (less than $1 \%$ of the 
main gas path mass flow) had significant effects on the downstream flow. The authors report both increases and decreases in the total-to-total efficiency of for the radial injection case. They suggest that for different flow rates the mixing interaction produces different levels of turbulence kinetic energy (TKE), dissipation and thus the losses. Higher flow rates likely cause a separation of the hub-wall flow leading to higher downstream losses and lower efficiencies.

Measurements in the rotational frame are presented in the second paper (McLean et al., 2001b). The objective was to physically understand how the secondary vortices are affected. They concluded that the injection of purge flow energizes the rotor inlet boundary layer, thereby altering the secondary flow development and deflecting the secondary flow towards midspan. As a result, the downstream flow is significantly changed. It was therefore concluded that the effects of purge flow were not only important for the current bladerow design but also for the design of the subsequent bladerows.

Gier et al. (2003) numerically investigated the interaction of hub-leakage flow with the mainstream flow of a three-stage turbine. Their predictions were also compared to experimental measurements and flow visualizations. Similar to McLean et al. (2001a; 2001b), the focus was on understanding the physical interaction between the purge and mainstream. They suggest that different loss mechanisms are present in the interaction of the purge flow the mainstream flow. They found that mixing losses, as described by Denton (1993), constitute about $50 \%$ of the entropy generation caused by purge injection. Other mechanisms described as the "by-pass" and step losses were approximately $20 \%$ of the cavity losses. The other $30 \%$ was a combined effect of the "windage" and "subsequent row" losses. The latter represents the detriment to the downstream flow as a result of increased SKE and flow angle deviation.

Paniagua et al. (2004) investigated the effects of a hub-endwall purge flow on the downstream flow field of an HP turbine stage. The tests were performed in the von Karman Institute (VKI) compression tube facility that provides fully simulated engine conditions. Three purge mass flow ratios (relative to the main gas path primary flow rate) of $-0.5 \%, 0.6 \%$ and $1.5 \%$ were tested. The results included static pressure measurements on the endwall, in the endwall 
cavity and on the blade surfaces. In general, it was found that with increasing purge flow levels, the secondary flows were stronger and migrated increasingly towards the blade tip. The injection of the purge flow also led to a large blockage of the transonic vane exit increasing the static pressure upstream of the cavity. It was concluded that the purge flow should be considered during the turbine design phase to prevent changes to the stage parameters, such as the degree of reaction.

Reid et al. (2006) offer similar conclusions regarding the inclusion of the purge flow as part of the design process. They present numerical and experimental results from a full-scale single stage low-speed axial flow turbine. A number of purge mass flows with varying tangential velocities were tested. Downstream pitchwise mass-averaged and overall efficiencies constitute the majority of the results. They introduce two categories of loss production to describe loss of efficiency with increased purge mass flow: mixing losses; and the losses incurred due to the alteration of the secondary flow physics. The former is similar to that described by Gier et al. (2003), but the latter is somewhat more general. Perhaps the most important conclusion of the paper is that increasing the relative tangential velocity of the purge flow increases the losses associated with purge injection. Similar conclusions have also been found in experimental cascade studies by de la Rosa Blanco et al. (2009), Lynch et al. (2013) and Popovic and Hodson (2013a).

To further explain how increased tangential purge velocities lead to higher purge injection losses, a schematic of the relative inlet velocity triangles for a rotor blade passage are shown in Figure 2.7. The purge flow velocity is shown in red and the mainstream velocity is shown in blue. Dashed lines indicate the axial and tangential components of both vectors. The blade speed is labelled $U$. As shown, sub-figure (a) and (b) represent cases of low and high tangential purge velocity respectively. Popovic and Hodson (2013a) explain that cases of low tangential purge flow will have lower mixing losses because the mismatch between the mainstream and purge flow tangential velocities is reduced. Theoretically, the lowest injection losses would then occur when the tangential purge velocity is zero. Lynch et al. (2013) termed this case "unswirled" 
(a) Low tangential purge velocity

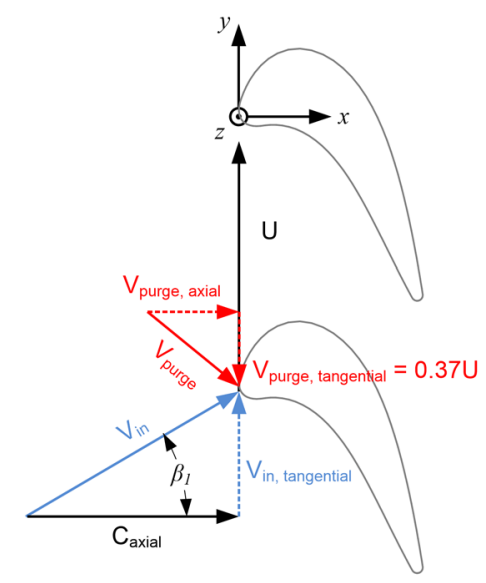

(b) High tangential purge velocity

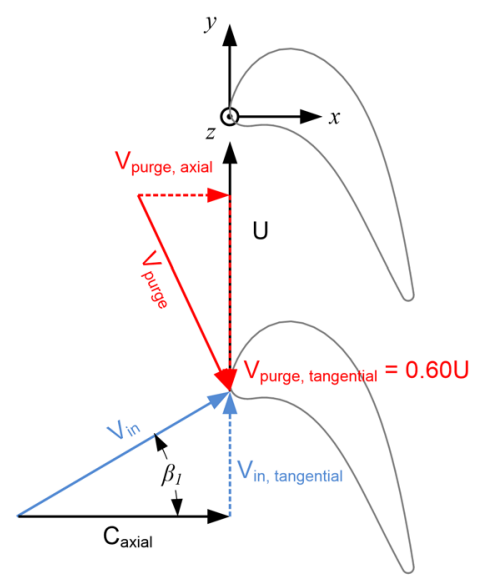

Figure 2.7: Schematic of the mainstream and purge flow relative inlet velocity triangles

because in the stationary frame the purge flow tangential velocity is equal the blade speed $(U)$. As noted by Lynch et al. (2013), the unswirled case is not representative of engine conditions; however, their measurements downstream of a turbine cascade with unswirled purge flow did produce lower losses compared to a swirled case (high tangential velocity).

An experimental investigation by Schuepbach et al. (2010) examined the effects of purge flow injection and suction on the secondary flow of a high work turbine stage. Steady and unsteady measurements were performed upstream and downstream of the rotor. A detailed analysis of the flow physics was provided. The results included pitchwise and overall massaveraged losses as well as downstream contours of the time-averaged and rms values of the fluctuating relative total pressure and outlet flow angles. Vorticity contours were used to identify the strength of the secondary vortices. The results showed that increasing the purge flow from suction $(I R=-0.14 \%)$ to positive injection $(I R=0.9 \%)$ resulted in a total-to-total efficiency drop of $\Delta \eta_{t t}=0.6 \%$. For the injection case, the peak vorticity of the hub passage vortex was reduced indicating a reduction in rotation about the streamwise axis. However, they also reported that the integrated circulation of the passage vortex increased by $10 \%$ with injection. To aid the physical interpretations and visualize the regions of high viscous dissipation, the authors introduced a non- 
dimensional dissipation function. The downstream integrated value of this function increases by nearly $40 \%$ with injection which indicates increased loss generation. The results show that although the passage vortex rotation is attenuated with purge injection, the entropy generation through mixing is still significantly enhanced.

In a follow up paper by Schuepbach et al. (2011), full-stage turbine tests were performed with and without the application of endwall contouring. Two contouring designs were tested. The first was designed with the objective of reducing the secondary kinetic energy coefficient, while the second was based on an improved methodology that also included optimization criteria to yield more homogenous radial distributions of the outlet swirl angle. The results included contour plots of loss and vorticity as well as the non-dimensional efficiency reductions. In each case, the addition of positive purge flow resulted in the reduction of the stage efficiency relative to a low-suction case. Interestingly, the first contouring design showed the largest deterioration in efficiency even when compared to the axisymmetric endwall. It was concluded that large variations in the circumferential pressure at the purge exit resulted in higher localized purge flows and therefore increased the mixing losses. The second contouring case was designed to smooth the blowing circumferentially and therefore reduce the losses. This case showed the lowest efficiency deterioration relative to corresponding low-suction case.

Other studies involving contouring have also shown mixed benefits when purge injection is introduced. Lynch et al. (2013) examined the effects of purge injection on the secondary flows of a large-scale low-speed linear cascade. The results include the relative integrated losses, downstream contours of total pressure and vorticity as well as oil-film visualizations. The purge flow for all cases had the same purge ratio $(\phi=0.75 \%)$ but was either swirled or unswirled relative to the cascade frame of reference. As mentioned previously, the flat unswirled case produced the lowest overall losses. The corresponding contouring case showed a loss increase $5 \%$, while the contoured swirled case showed a 4\% benefit. Lynch et al. (2013) suggest that the swirled leakage may reduce the blade incidence near the endwall. The unloading of the airfoil then improved the effectiveness of the contouring. 
The most recent and comprehensive study of rim-seal geometry and purge flows was performed at the Whittle Turbomachinery Laboratory at Cambridge University and is reported by Popovic and Hodson (2013a), Popovic et al. (2013), Popovic and Hodson (2013b) and Popovic and Hodson (2013c). The first two papers focus on the aerothermal interaction of the purge and mainstream flow and will be discussed next. The latter two were discussed in Section 2.8.2 and focused on the effects of the rim-seal geometry.

Popovic and Hodson (2013a) investigated the aerothermal effects of purge flow for a single rim-seal geometry in a large-scale low-speed linear cascade. Numerical results are also included. The study examines the physical effects of purge flow on the mainstream by varying the purge mass flow and the swirl. The measured and predicted leakage losses increase with increasing leakage fraction (LF) much like the previous studies mentioned here. LF is analogous to the purge flow ratio $(\phi)$ described previously. Also, with increasing swirl the leakage losses were found to increase. The rate at which the leakage losses increase with purge injection is not linear. In fact, beyond a leakage fraction of $1.1 \%$, the rate of leakage loss increase nearly doubles compared to leakage fractions below 1.1\%. The lower loss rate below the LF of $1.1 \%$ is attributed the small amount of mainstream ingestion into the cavity. This ingestion thins the mainstream boundary layer entering the blade row and attenuates the losses. At leakage fractions above $1.1 \%$, the purge flow is sufficiently high to prevent any mainstream ingestion. This prevents the thinning, and actually may increase the thickness of the mainstream inlet boundary layer. The thicker inlet boundary layer results in a stronger secondary flow with larger mixing losses. Beyond a LF value of $1.5 \%$ the leakage loss rate levels off. This is caused by the purge flow being sufficiently high to energize the inlet boundary layer thereby preventing cross-flow and suppressing the secondary flow development.

The second paper, Popovic et al. (2013), builds upon the initial work and investigates the compressibility and unsteady effects on the purge flow interaction. Flow compressibility was found to have an insignificant effect on the interactions between the purge and mainstream flow. 
In general, the interaction occurs in regions where the Mach numbers are relatively low and away from any of the downstream shocks.

Popovic et al. (2013) also found that the periodic unsteadiness caused by the relative motion of the upstream blade row resulted in significant periodic changes to the purge and secondary flows. Adjacent passages showed diametrically opposite effects. Despite the significant unsteadiness, the time-averaged losses were relatively unaffected: the unsteadiness only accounted for about a $10 \%$ increase in the leakage losses compared to steady conditions. The results from this study justify the procedures used for the present thesis. Here, both unsteadiness and compressibility are neglected but meaningful results should still be obtainable. Also, this suggests the results of the present investigation can be compared meaningfully to compressible or unsteady investigations.

For instance, the results of the present thesis can be compared to those from a complimentary study conducted in the transonic linear cascade facility at Carleton University by Abo El Ella et al. (2012). This experimental study presented detailed loss and vorticity measurements from two transonic linear cascades, one with a flat endwall and one with a simplified upstream endwall cavity. The classic backward-facing step shown in Figure 1.2 was approximated by an inclined ramp that gradually transitioned into the endwall cavity region. Data for both design and off-design Mach numbers showed that the presence of the upstream cavity resulted in stronger passage and counter vortices than the flat endwall case. Complementary oil film flow visualizations indicated the presence of cavity induced vortices which strengthen the development of the passage vortex. The flow structures (size and strength) are significantly altered relative to the flat endwall cascade. Chapter 6 further discusses the results of this study for comparison purposes.

In summary, the literature has shown that the purge flow and rim-seal geometry significantly affect the secondary flow physics and downstream losses. Generally, larger leakage fractions lead to higher losses; however, this relationship is not linear. The interpretation of the loss production mechanisms introduced with purge injection is not unified throughout the 
literature. This is likely a result of the sensitivity of these flows to the specific conditions for each study. To this end, it was stressed by several researchers that the effects of purge flow and the rim-seal geometry should both be considered early in the turbine design process. 


\section{Chapter 3}

\section{Experimental Methods and Data Reduction Procedures}

\subsection{Introduction}

The experimental measurements were performed in the Low-speed Turbomachinery Laboratory (LSTL) at Carleton University. This chapter will describe the wind tunnel facility, the test-section, the purge flow system and the research cascades. Also included is an overview of the instrumentation, probes, probe calibration, data acquisition, data reduction methods and the experimental uncertainties.

\subsection{Wind Tunnel}

The low-speed wind-tunnel facility consists of an open circuit wind-tunnel that is interfaced with a modular test section. Recently, the facility has been used mainly for the investigation of turbine and compressor cascade secondary flows and secondary losses (Benner, 2003; Zoric, 2006; Knezevici, 2011; Prevost, 2013). 
The wind-tunnel, shown schematically in Figure 3.1, was originally commissioned by Rodger (1992) and is of the open circuit type. The centrifugal fan (Daltec Industries, Model No. IE17P) is belt driven using a four-pole 40HP AC motor. The rotational speed of the motor is controlled using a Mitsubishi variable frequency drive (VFD).
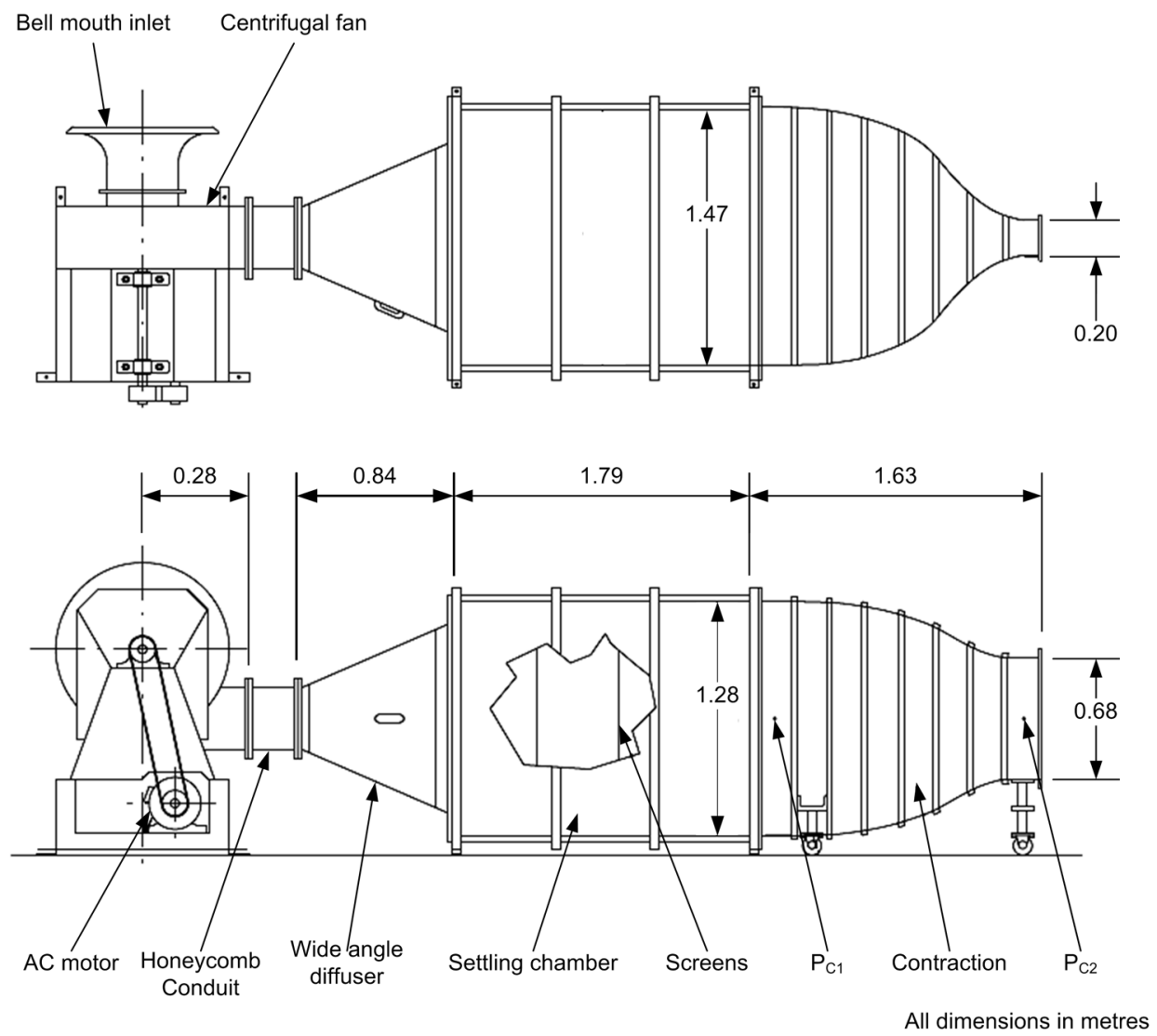

Figure 3.1: Wind-tunnel schematic, from Benner (2003)

The ambient air enters the centrifugal fan through a bell mouth inlet. The flow exits the fan and passes through a honeycomb conduit to remove the swirl. A wide angle diffuser, with screens to prevent boundary-layer separation, reduces the flow velocity prior to entering the settling chamber. Four equally spaced screens reduce the mean flow non-uniformities and dissipate large scale turbulence. The contraction, with an area reduction of $14: 1$, accelerates the flow into the test-section. The inlet and outlet of the contraction is instrumented with reference 
static pressure taps, giving pressures designated $P_{C l}$ and $P_{C 2}$, which are used to monitor the wind tunnel operating point. $P_{C 2}$ also serves are the reference pressure for the differential-type pressure transducers.

\subsection{Test Section}

The linear cascade test section, shown in Figure 3.2, was designed by Goobie (1989). The cascade is mounted to a turntable (item 1) which can rotate to vary the nominal inlet flow angle. Measurements of the flow field are made using Pitot, Kiel, and multi-hole pressure probes as well as hot-wire probes. The probes enter the test-section through slots in the turntable endwall and measurements are made on the turn-table side of the test-section. The opposite wall, made of Plexiglass, is mounted to a steel frame with casters. It can be rolled away for access to the testsection.

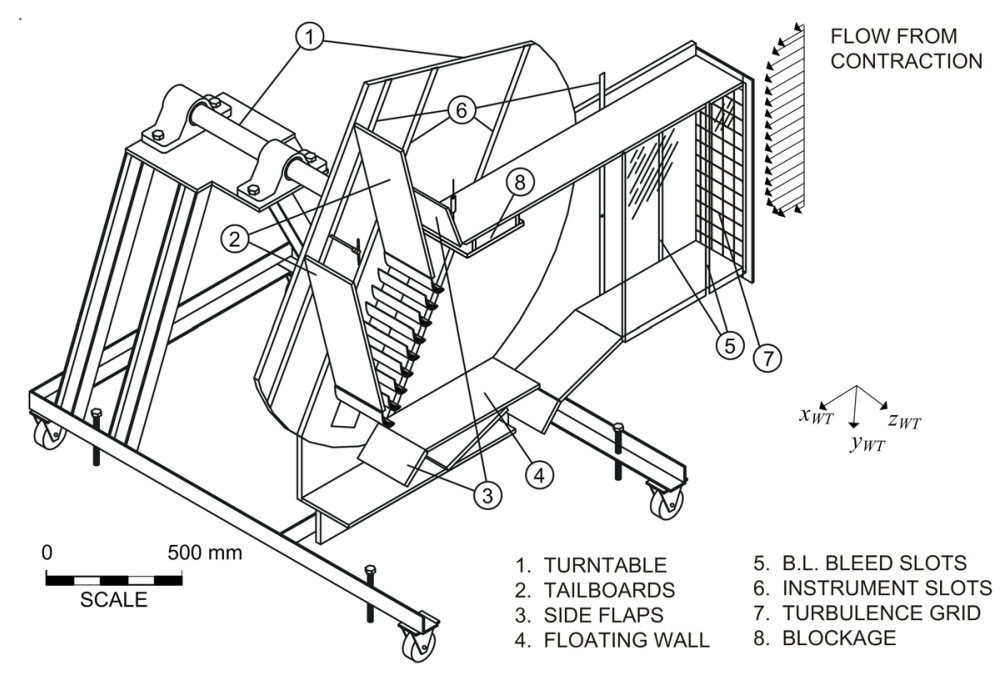

Figure 3.2: Linear cascade test-section, from Benner (2003)

To simulate an infinite bladerow the upstream flow must be pitchwise uniform and the downstream flow periodic. The upstream side flaps (items 3 and 4) are used to set the inlet flow 
uniformity, while the downstream tailboards (item 2) are positioned to obtain periodic outlet flow. A blockage mechanism, designed by Mahallati (2003), is used to improve the inlet uniformity and the effectiveness of the upper inlet side flap.

A three-hole pressure probe (see Section 3.8.4) is traversed at midspan both upstream and downstream and the measurements are used as the basis for adjusting the control surfaces. The influence of the control surfaces is coupled between the cascade inlet and outlet and therefore the process for setting the positions is iterative. Sample results of the upstream and downstream midspan flow, measured at $-1.20 C_{x}$ and $1.40 C_{x}$ relative to the blade leading edge, are shown in Figure 3.3 (a) and (b) respectively.

In Figure 3.3 (a), the upstream potential field of the blades is observed in the periodically varying inlet flow angle. The average value is equal to the design inlet flow angle $\left(\beta_{1, \text { design }}\right)$. While setting the control surfaces, the dynamic pressure is closely monitored as it provides an indication of the mass-flow that is passing through each passage. The inlet total, dynamic and static pressure distributions are uniform at the cascade inlet.

The downstream periodicity is shown in Figure 3.3 (b). The midspan outlet flow angle is periodic from passage-to-passage and the mean value is equal to the design value $\left(\beta_{2, \text { design }}\right)$. The downstream total pressure distribution shows that the peak losses within the blade wakes are equal for each passage. Similar periodic trends are observed for the dynamic and static pressure coefficients. 

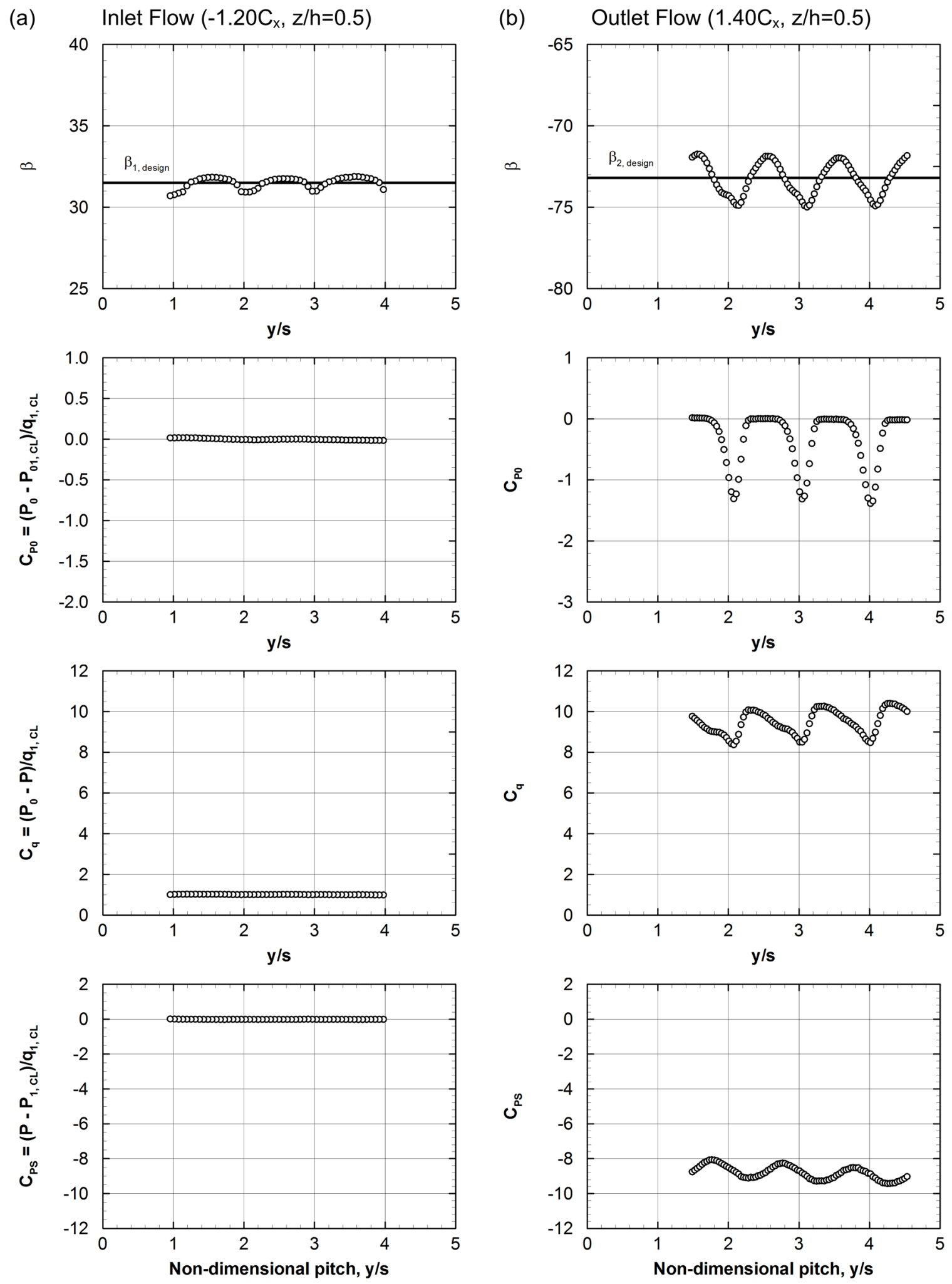

Figure 3.3: Upstream (a) and downstream (b) midspan flow quality measurements 


\subsection{Purge Flow System}

As mentioned in Chapter 1, the influence of the platform overlap and purge flow on the secondary flows were investigated. A photograph of the turbine cascade mounted on the endwall is shown in Figure 3.4 (a). The endwall and blades were manufactured by PWA using stereolithography (SLA). The cross-sectional geometry is shown in Figure 3.4 (b). The geometric details of each cascade are provided in Section 3.5.

(a)

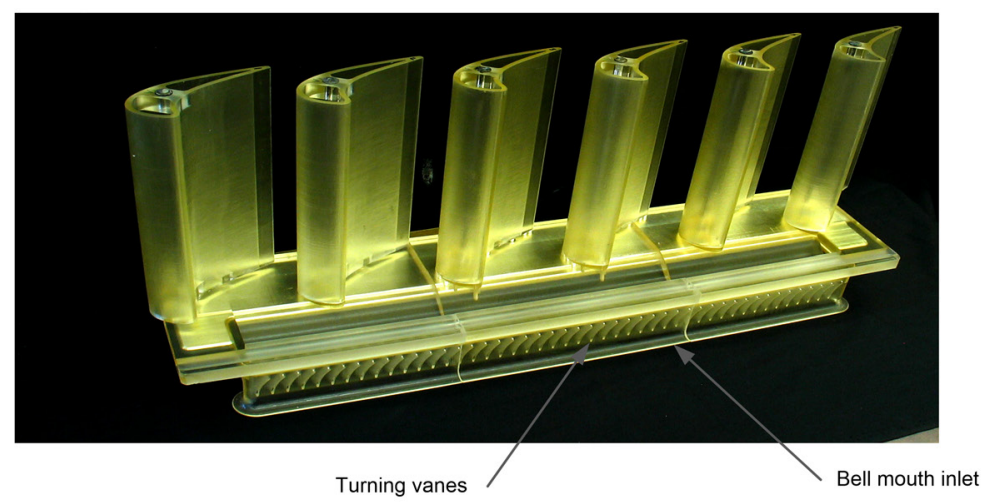

(b)
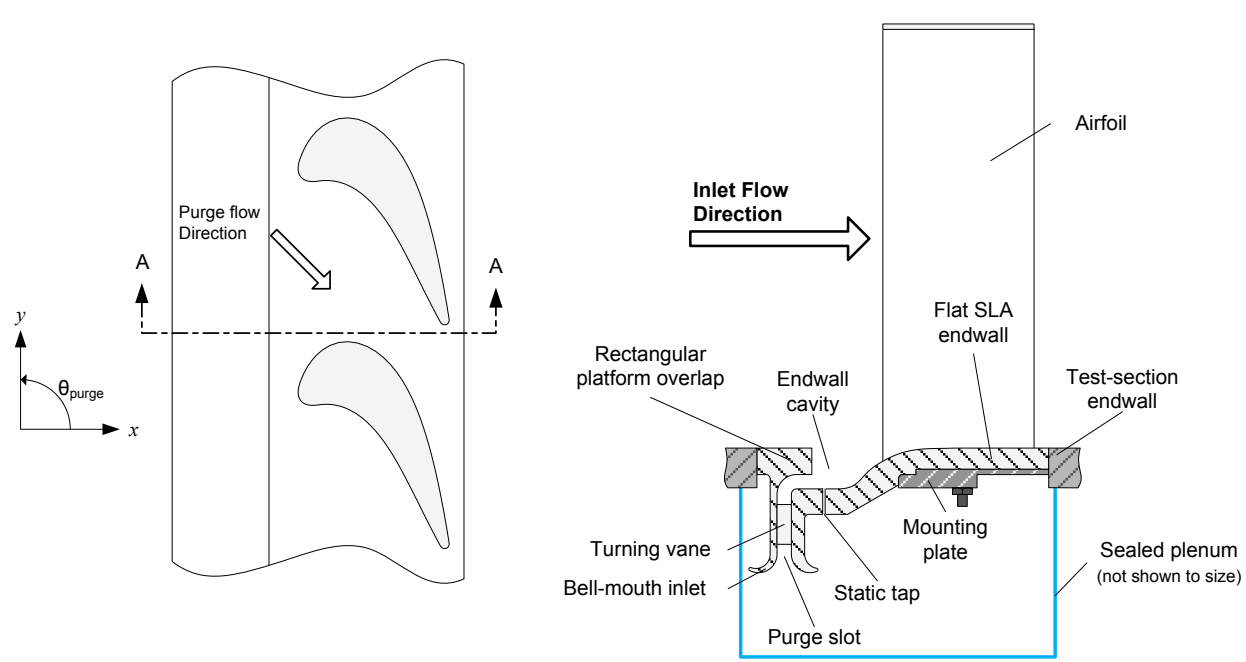

Figure 3.4: A photograph of the flat intra-passage platform overlap cascade hardware (a) and a schematic drawing of cascade cross-section (b) 
The purge flow system delivers the air to the test-section from a pressurized plenum through a bell mouth inlet, as shown in Figure 3.4 (b) and Figure 3.5 (b). In an engine, the relative motion between the stator and rotor results in the purge flow having a tangential component of velocity relative to the rotor. In this experiment, the tangential velocity was obtained using turning vanes in the purge slot, just downstream of the bell mouth. The turning vanes were designed to eject the flow at $-45^{\circ}$ angle relative to the axial direction. The ejection angle was selected with guidance from PWA and is typical of real gas turbine engine conditions (Lynch et al., 2013). The selected angle represents the median between the hypothetical maximum of $0^{\circ}$ corresponding to unswirled purge injection and a minimum of $-90^{\circ}$ corresponding to zero net purge injection. Section 7.4 describes the purge flow velocity triangles in further detail.

The purge flow air supply system is shown in Figures 3.5 (a) and (b). It consists of a radial blower, a flow meter (ISA 1932 short radius nozzle), a supply plenum, a mixing plenum and the bell-mouth inlet. The blower can be throttled using an upstream gate to adjust the mass flow rate. Six 1 inch diameter tubes are evenly spaced around the circumference of the supply plenum to evenly distribute the flow along the mixing plenum. The total purge mass flow rate, $\dot{m}_{\text {purge, total }}$, is calculated from the pressure difference across the nozzle $\left(\Delta P=P_{P 1}-P_{P 2}\right)$ as described later in Section 3.7.6.

During the commissioning of this system a number of different methods were attempted to measure the purge mass flow rate entering a single passage. Measurements within the purge slot were attempted using a single-normal hotwire and a specially designed three-hole cobra probe. These measurements were unsuccessful due to the presence of reversed flow within the endwall cavity region (see Chapter 6). Also, it was suspected that the presence of the cobra-probe tip within the slot caused a significant blockage. Thus, it was decided to assume that the purge flow was uniformly distributed along the slot and therefore the mass flow rate per passage, $\dot{m}_{\text {purge }}$, is taken as: 


$$
\dot{m}_{\text {purge }}=\dot{m}_{\text {purge, total }} \frac{s}{l_{\text {purge slot }}}
$$

where $l_{\text {purgeslot }}$ is the total length of the purge slot $(498.48 \mathrm{~mm})$ and $s$ is the cascade pitch spacing $(107.15 \mathrm{~mm})$.

The purge flow periodicity is monitored using 45 pitchwise static taps within the endwall

(a)

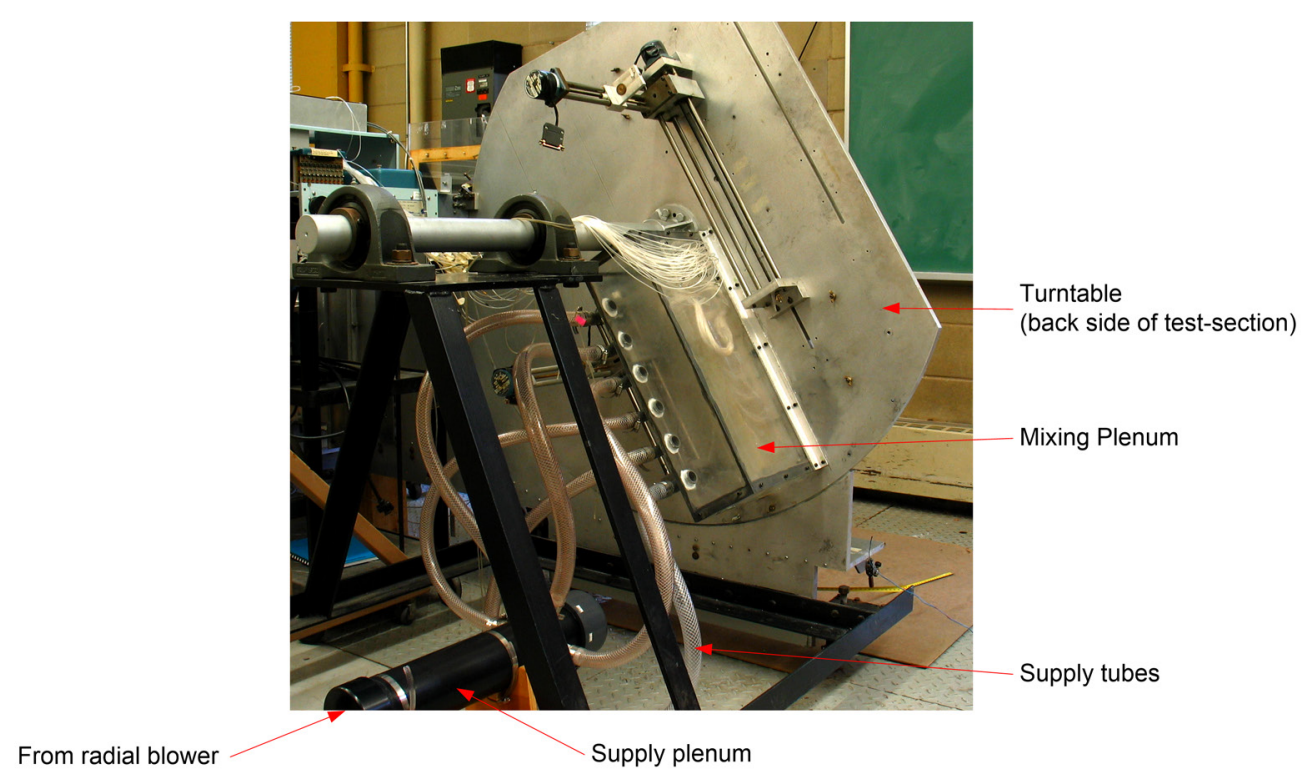

(b)

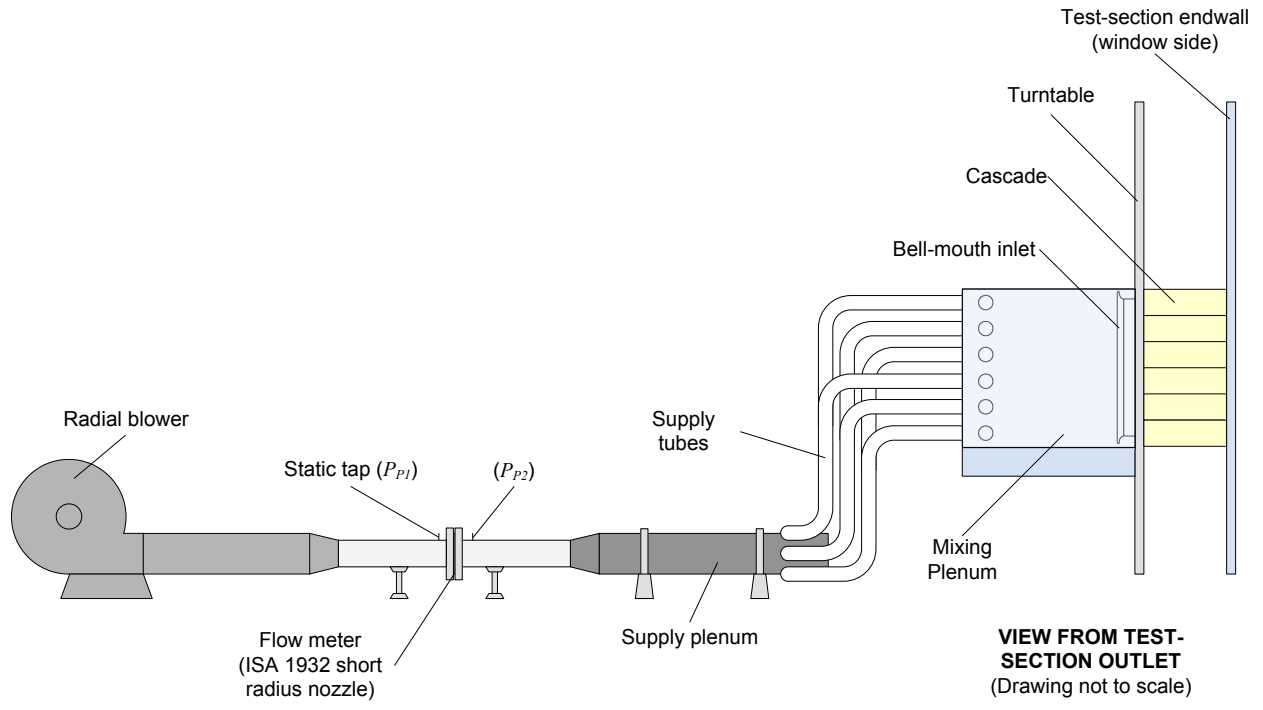

Figure 3.5: A photograph (a) and schematic drawing (b) of the purge flow delivery system 
cavity, as shown in Figure 3.4 (b). An example of the distribution of cavity static pressure is shown in Figure 3.6. The leading edge of the top blade of the cascade is located at $y / s=0$. The upstream potential effect of cascade is clearly seen from the pitchwise distribution. The degradation of the periodicity at $y / s=0.5$ and $y / s=4.5$, is likely caused by the end effects of the purge slot. As a consequence there are minor differences of the downstream secondary flow periodicity at the top and bottom of the cascade. This is discussed further in Chapter 7.

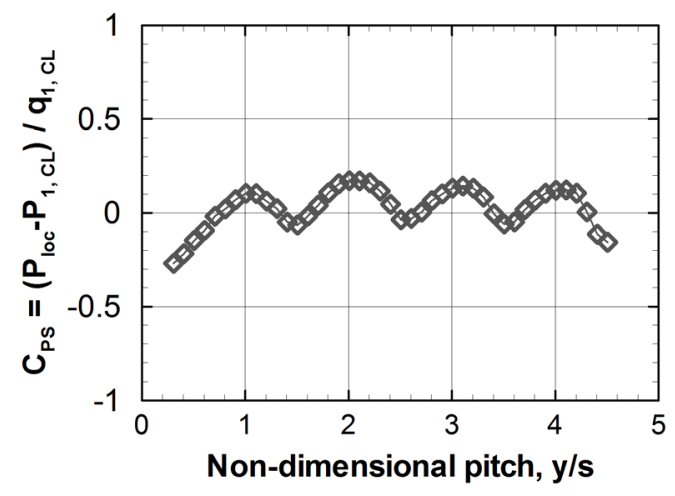

Figure 3.6: A typical pitchwise distribution of the endwall cavity static pressure coefficient $\left(C_{P S}\right)$ with purge flow

\subsection{Test Cascades}

\subsubsection{Introduction}

The experimental tests were performed for one airfoil geometry, PAKH, with three different endwall configurations and two different platform overlap features. This section describes the airfoil geometry, the endwall geometries and the platform overlap shapes.

\subsubsection{PAKH Airfoil Geometry and Design Parameters}

PAKH is a research airfoil specifically designed and manufactured by PWA for low-speed testing. It is representative of the root-section of a high-pressure turbine rotor blade. 
The geometric and design parameters of PAKH are given in Table 3.1 and two adjacent airfoils are shown to scale in Figure 3.7. The cascade consists of six blades and measurements are collected for the centre passage. The overall cascade turning at mid-span is $104.7^{\circ}$. The acceleration through the passage is relatively high as the convergence ratio $\left(C R=\cos \beta_{1} / \cos \beta_{2}\right)$ is about 2.95. The cascade has a moderate Zweifel coefficient of about 1.00. The design Reynolds number is 75, 000 based on the blade axial chord and inlet velocity (220,000 based on outlet velocity).

Figure 3.7 shows the two Cartesian coordinate systems used in this work. The origin of the cascade coordinate system is located at the blade leading edge with the $x$-axis aligned with the axial direction, the $y$-axis with the pitchwise direction and the $z$-axis with the spanwise direction. A mean flow coordinate system is used to present results from the downstream measurement plane. The $x_{p r i m}$-axis is aligned with the primary mean flow direction (streamwise) and the $y_{\text {sec }}{ }^{-}$ and $z_{s e c}$-axes define the secondary velocity directions. The primary flow direction for twodimensional downstream measurement planes is defined by

$$
\overline{\overline{\beta_{2}}}=\tan ^{-1}\left(\overline{\overline{v_{2}}} / \overline{\overline{u_{2}}}\right)
$$

where $\overline{\overline{v_{2}}}$ and $\overline{\overline{u_{2}}}$ are the area-averaged axial and pitchwise velocities.

Table 3.1: PAKH specifications

\begin{tabular}{lc}
\hline \hline True chord, $C[\mathrm{~mm}]$ & 102.1 \\
\hline Axial chord, $C_{x}[\mathrm{~mm}]$ & 73.3 \\
\hline Pitch, $\mathrm{s}[\mathrm{mm}]$ & 107.2 \\
\hline Span, $\mathrm{h}[\mathrm{mm}]$ & 203.2 \\
\hline Inlet design flow angle, $\beta_{1}\left[{ }^{\circ}\right]$ & 31.5 \\
\hline Outlet design flow angle, $\beta_{2}\left[^{\circ}\right]$ & -73.2 \\
\hline Pressure side tangent stagger angle, $\gamma\left[{ }^{\circ}\right]$ & -46.2 \\
\hline Number of blades & 6 \\
\hline Convergence ratio, $\mathrm{CR}$ & 2.95 \\
\hline Zweifel coefficient, $\mathrm{Zw}$ & 1.00 \\
\hline \hline
\end{tabular}




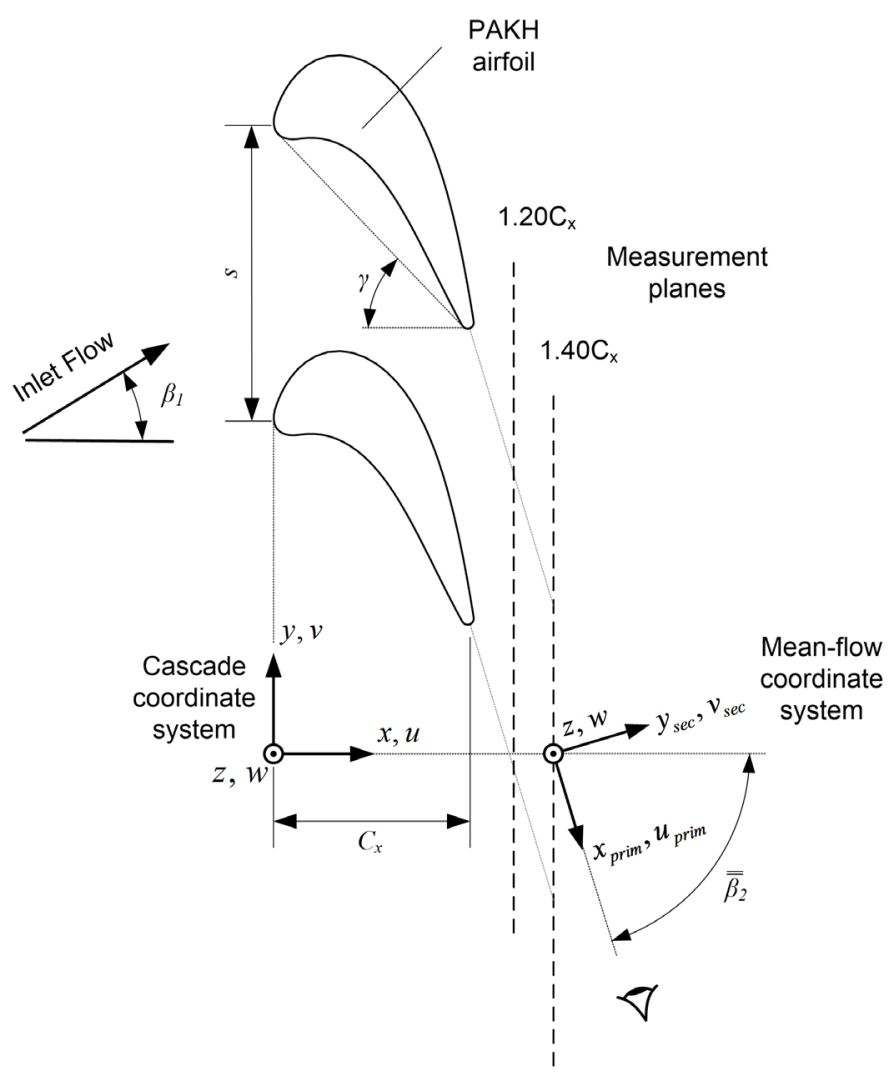

Figure 3.7: Cascade nomenclature showing the measurement coordinate systems

\subsubsection{Endwall Configurations}

The PAKH airfoil was mounted to three different endwalls (including the flat baseline) with two different platform overlap geometries, totalling five geometric cases. For each geometric case different levels of purge flow were examined as discussed in Section 3.5.4.

For the Baseline case, the blades are affixed to a flat mounting plate that is flush with the test-section endwall. For the second case, the endwall includes the step and cavity features as shown in Figure 3.8 (a). The platform overlap geometry is representative of that in an actual engine. This configuration is referred to as the Rectangular Flat (RF) case as the platform overlap shape is rectangular and the endwall is flat through the flow passage. Figure 3.8 (b) shows 
(a) Flat Intra-passage Endwall (RF)

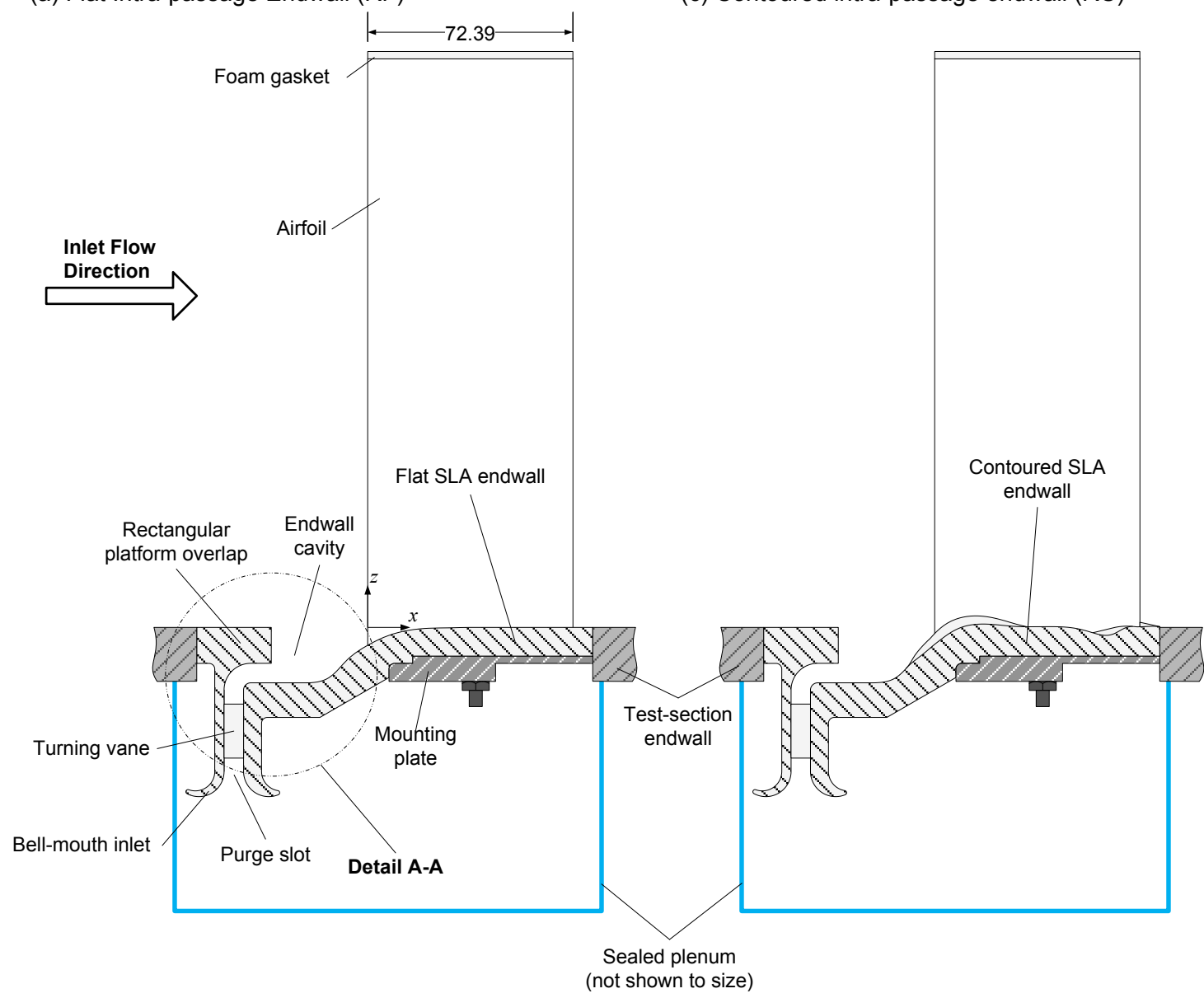

(b) Rectangular Platform Overlap

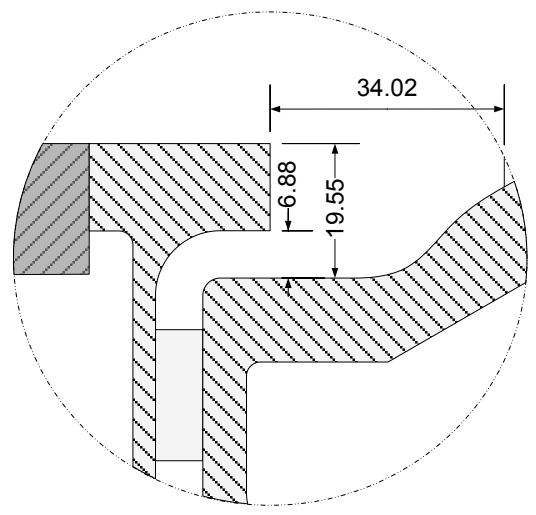

Detail A-A (d) Elliptical Platform Overlap

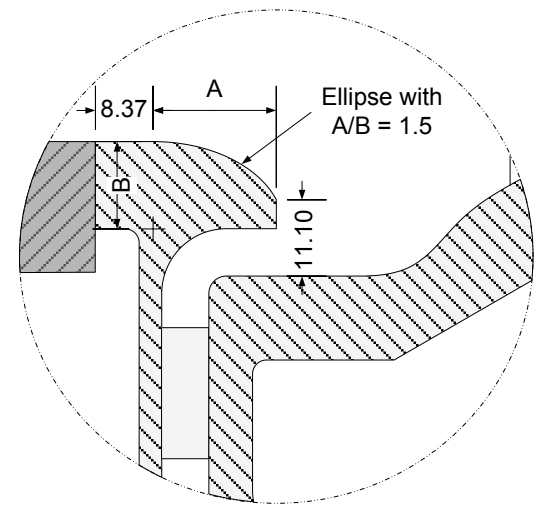

Detail A-A

Figure 3.8: Cross-section of cascade geometry (dimensions in $\mathbf{m m}$ ) 
detailed dimensions of the platform geometry. The axial gap, the distance from platform overlap to the blade leading edge, is about $47 \%$ of $C_{x}$.

The third case involved the same platform overlap geometry but with endwall contouring applied through the bladerow passage, as shown in Figure 3.8 (c). This case is referred to as the Rectangular Contoured (RC). The curvature of the contouring changes locally the endwall static pressure with the goal of reducing the development and cross-passage migration of the passage vortex. The endwall contouring was designed by PWA using the procedures described by Praisner et al. (2007). The surface profiles are optimized using steady-state RANS CFD simulations that minimize the outlet mass-averaged losses. The optimization was performed with a simplified rectangular platform overlap shape and no purge flow to reduced the mesh complexity and improve the solution convergence. This simplified geometry is discussed in the following section. Subsequent CFD simulations with purge flow were performed by PWA to evaluate the predicted performance benefits.

The contoured endwall is shown in Figure 3.9. The colour flood shows the contour amplitudes relative to the baseline $\mathrm{RF}$ endwall surface and expressed as a percentage of the axial chord $\left(\% C_{x}\right)$. At the blade leading edge there are two regions of convex curvature that are referred to as "humps" and are labelled $\mathrm{H}_{1}$ and $\mathrm{H}_{2}$. Such humps will locally produce regions of lower static pressure due to the resulting convex streamline curvature. Between the humps is a depression referred to as a "trough" (T) that extends downstream within the passage. Towards the passage exit there is a streamwise "ridge" (R) which is used to guide the trajectory of the passage vortex. The maximum and minimum amplitudes of the contouring features are $+8 \%$ and $-8 \% C_{x}$ relative to the RF surface, respectively. For this particular endwall optimization, the endwall features were not confined to the bladerow passage: they extend upstream and downstream of the blade leading and trailing edge by 12 and $10 \%$ respectively. The effect of contouring on the secondary flow and downstream losses is discussed in detail in Chapter 8 . 


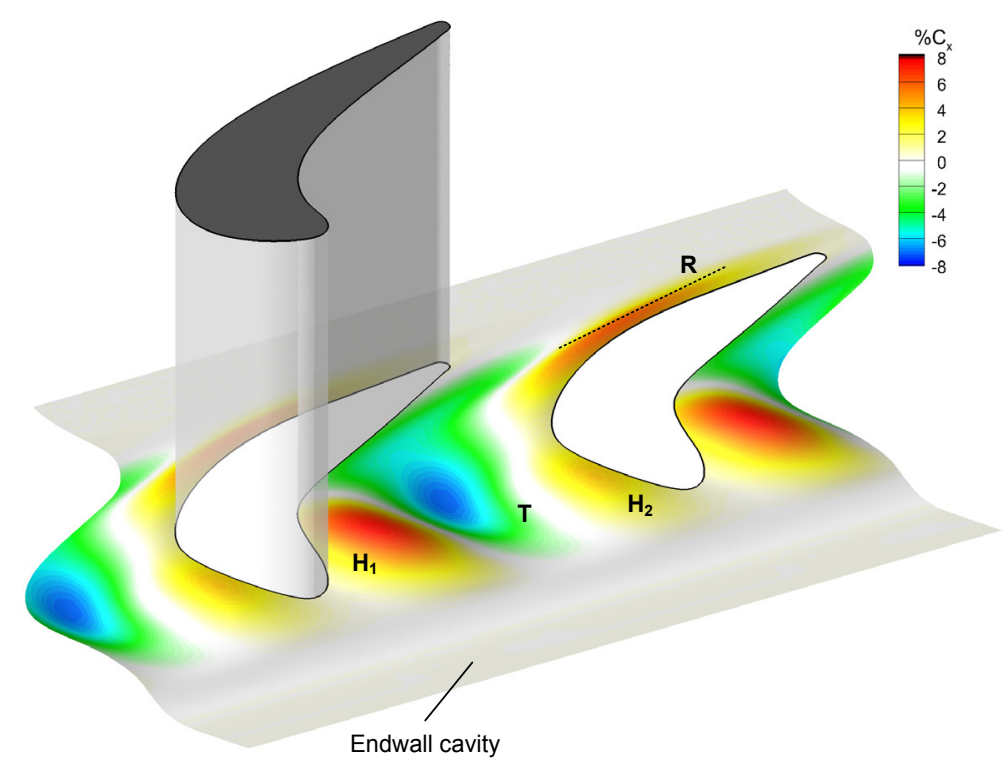

Figure 3.9: Endwall contouring geometry with a colour flood of the perpendicular distance $\left(\% C_{x}\right)$ from to the flat intra-passage endwall (RF).

In Section 2.8.2, it was mentioned that the shape of the platform overlap can affect the development of the secondary flow. In this work a novel elliptical overlap shape was developed by PWA and is shown in Figure 3.8 (d). The elliptical platform overlap was manufactured as a separate aluminum part (not shown for here for simplicity) which mates with the modified SLA endwalls of the RF and RC cascades. Thus, a variety of platform overlap shapes could be tested using these endwall geometries. The elliptical platform overlap with flat intra-passage endwalls is referred to as the Elliptical Flat (EF) and the contoured intra-passage is the Elliptical Contoured (EC). The effect of this platform overlap shape on the flow fields is also discussed in Chapter 8.

\subsubsection{Purge Flow Cases}

The effects of purge flow were investigated for three different levels and for zero net purge flow. Early during the turbine design process, it is common to assume that the aerodynamic effects of the purge flow are small and therefore to omit the purge flow and use an approximate 
endwall cavity geometry. In the current investigation, the purge slot can be plugged to investigate such approximations.

Three-different endwall cavity configurations were considered. In Figure 3.10 (a), the purge slot is blocked using a sloped plug to simulate the simplified geometry sometimes used in computational design studies. Figure 3.10 (b) shows a flush plug. The third cavity configuration, shown in Figure 3.10 (c), is an open purge slot that is sealed upstream of the supply plenum (see Figure 3.5), allowing both inflows and outflows to the purge slot but no net purge flow. This case was used to first assess the effects of the blade potential field on the slot prior to testing with the purge flow turned on.

A minimum of three levels of net purge flow were considered for each geometric configuration. The purge flow levels are presented as a percentage of the main gas path mass flow,

$$
\phi=\frac{\dot{m}_{\text {purge }}}{\dot{m}_{W T}} \times 100
$$

where $\dot{m}_{\text {purge }}$ is the purge mass flow per passage, as defined in Equation 3.1, and $\dot{m}_{W T}$ is the passage mass flow rate of the main gas path flow (full span). The latter is computed using the areaaveraged inlet velocity profile measured at $1.20 C_{x}$ upstream of the blade leading edge.

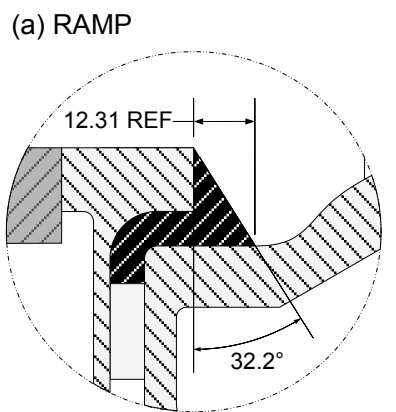

Detail A-A

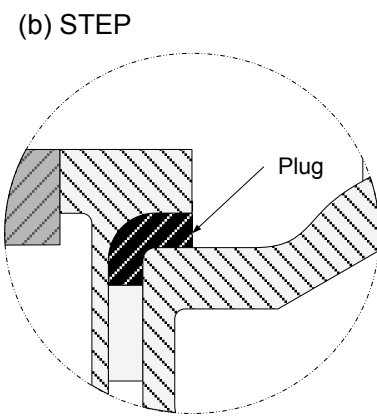

Detail A-A (c) CAVITY

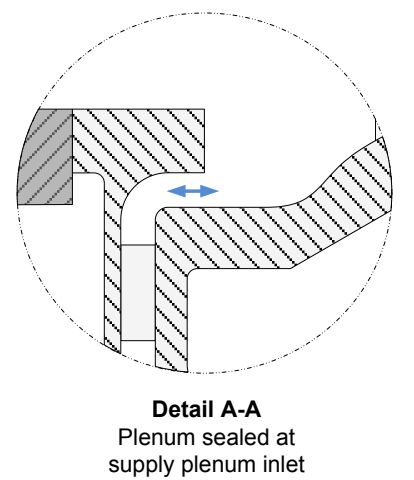

Figure 3.10: Geometric simplifications of the platform overlap (dimensions in mm) 


\subsection{Test Matrix}

For each combination of the geometry and purge flow, two downstream planes (at $1.20 C_{x}$ and $1.40 C_{x}$ from the leading edge) were measured using the seven-hole pressure probe described in Section 3.8.5. The data are supplemented with mid-span blade surface static pressure measurements and blade and endwall surface flow visualizations. A summary of the test matrix is provided in Table 3.2. Each test case will be referenced using a naming convention that uses the abbreviations given in Table 3.2. For example, RF 075 refers to the rectangular flat endwall with a purge flow level of $0.75 \%$.

Table 3.2: $\quad$ Summary of experimental test operating points

\begin{tabular}{|c|c|c|c|c|c|c|c|c|}
\hline \multirow{2}{*}{$\begin{array}{l}\text { Endwall } \\
\text { Geometry }\end{array}$} & \multicolumn{6}{|c|}{ Purge Flow Level $(\phi)$} & \multirow{2}{*}{$\begin{array}{c}\text { Inlet } \\
\text { Reynolds No. } \\
\left(\operatorname{Re}_{1}\right)\end{array}$} & \multirow{2}{*}{$\begin{array}{l}\text { Downstream } \\
\text { Measurements }\end{array}$} \\
\hline & $0.00 \%$ & $0.00 \%$ & $0.00 \%$ & $0.75 \%$ & $1.10 \%$ & $1.25 \%$ & & \\
\hline Baseline & - & - & - & - & - & - & $50 \mathrm{k}$ & $7 \mathrm{H}, \mathrm{K}, \mathrm{x}-\mathrm{HW}$ \\
\hline Baseline & - & - & - & - & - & - & $75 \mathrm{k}$ & $7 \mathrm{H}$ \\
\hline $\mathrm{RF}$ & RAMP & STEP & 000 & 075 & 110 & 125 & $75 \mathrm{k}$ & $7 \mathrm{H}$ \\
\hline $\mathrm{RC}$ & RAMP & STEP & 000 & 075 & 110 & 125 & $75 \mathrm{k}$ & $7 \mathrm{H}$ \\
\hline EF & - & STEP & 000 & 075 & 110 & 125 & $75 \mathrm{k}$ & $7 \mathrm{H}$ \\
\hline EC & - & STEP & 000 & 075 & 110 & 125 & $75 \mathrm{k}$ & $7 \mathrm{H}$ \\
\hline
\end{tabular}

*Seven-hole probe $(7 \mathrm{H})$, Kiel Probe $(\mathrm{K})$ and x-type hotwire probe $(\mathrm{x}-\mathrm{HW})$

As shown in Table 3.2, all of the test cases were measured at the design inlet Reynolds number $\left(\operatorname{Re}_{1}\right)$ of 75,000 . The Baseline data was also supplemented with measurements at $R e_{1}=50,000$ as part of a study of the turbulence quantities in the downstream flow field. The lower than design Reynolds number was necessary to keep the fluctuating velocity components within the hotwire calibration. Chapter 5 presents and discusses the turbulence results.

The cases measured at the design Reynolds number $\left(R e_{1}=75,000\right)$ are more applied in nature. They focus on effects of the platform overlap and endwall geometry as well as the influence of purge flows on the flow physics and the losses. As shown in Table 3.2, there are three zero flow cases for the $\mathrm{RF}$ and $\mathrm{RC}$ configurations and only two for the $\mathrm{EF}$ and $\mathrm{EC}$ as the Ramp geometry is not compatible with the elliptical overlap. The effects of the platform overlap 
geometric simplifications and the application to preliminary turbine designs is discussed in Chapter 6.

There are three cases with net positive purge ratios $(\phi)$ of $0.75,1.10$ and $1.25 \%$. This range is representative of purge ratios observed in actual engines and the test points where chosen with guidance from PWA. During the initial setup of the purge flow system, unsteady flow behavior was observed in the downstream measurements for purge ratios close to $1.0 \%$ and below $0.5 \%$. It is believed that the apparent instabilities originate from the simulated purge flow system and are not representative of engine conditions. Thus, the selected purge ratios were chosen to avoid these effects. Chapter 7 examines the effects of the purge flow on the secondary flow development for the RF configuration.

In Chapter 8, the effectiveness of the loss mitigation techniques (the endwall contouring and the elliptical overlap) is discussed. The data from RC, EF and EC configurations form the basis of this chapter.

\subsection{Instrumentation}

\subsubsection{Introduction}

The following section describes the wind-tunnel instrumentation. This includes the data acquisition systems, the pressure transducers, the temperature monitor, the digital barometer, the mass flow meter and the stepper motor controller.

\subsubsection{Data Acquisition Systems}

Two different data acquisition systems have been used for this work and will be referred to as System 1 and System 2. System 1 was used for the Baseline (flat endwall) test case, including the hot-wire measurements, and was previously used in the same wind-tunnel by Knezevici (2011) and Scribner (2011). System 2 was used for all the remaining cases, with and 
without purge flow. It was developed in anticipation of the need for additional analog input channels to measure the purge mass flow rate.

System 1 consists of United Electronic Industries, Inc. PowerDAQ PD2-MFS-8-800/14 analog-to-digital converter PCI card installed on Windows 2000 based computer. This simultaneous sample-and-hold card has eight single-ended analog input channels which can be sampled at a rate of $800 \mathrm{kHz}$ with 14-bit resolution over $\pm 10 \mathrm{~V}$. LabVIEW software, developed by the current and previous researchers, was used for data acquisition and control of the traverse gear.

System 2 consists of a National Instruments data acquisition card (NI PCI-6221) installed on a Windows XP based computer. This card can sequentially sample a maximum of 16 analog inputs at a rate of $250 \mathrm{kHz}$ with a 16-bit resolution over $\pm 10 \mathrm{~V}$. The present author developed data acquisition software for use with both systems using MATLAB R2010a. A graphical user interface (GUI) allows the user to configure the acquisition of individual data points or automate multi-point runs. The software is also used to operate the other instruments such as the temperature monitor, barometer and stepper motor controller. Equivalent measurements of the Baseline case completed using both System 1 and System 2 showed negligible differences.

\subsubsection{Pressure Transducers}

Pressure measurements were made using a maximum of four Data Instruments ASG DRAL501DN and eight ASG DRAL505DN differential pressure transducers with full-scale operating ranges of $\pm 250 \mathrm{~Pa}$ and $\pm 1250 \mathrm{~Pa}$ respectively. The transducers are temperature compensated from 0 to $50^{\circ} \mathrm{C}$ and share a common 14VDC power supply. The estimated uncertainty for the ASG DRAL501DN- and ASG DRAL505DN-type transducers due to calibration, non-linearity and hysteresis, is $\pm 0.5 \mathrm{~Pa}$ and $\pm 2 \mathrm{~Pa}$ respectively. Seven transducers were used for the probe pressure ports, while one was reserved for monitoring the wind-tunnel contraction pressure difference $\left(P_{C l}-P_{C 2}\right)$. The pressure difference across the mass flow meter, at two circumferential locations, was monitored using two of the low-range transducers. 
The transducers were calibrated using the micromanometer designed by Benner (2003) and Mahallati (2003). The calibration procedures and typical calibration coefficients are presented in Appendix A. The transducer calibrations were checked prior to testing new configurations and were recalibrated annually.

\subsubsection{Temperature Monitor}

A pseudo-static temperature within the wind-tunnel test-section was monitored using a type-T (Copper-Constantan) thermocouple and an Omega DPi8-C24 temperature meter. The meter processes the thermocouple voltage signal with resolution of 15-bits at a frequency of $3 \mathrm{~Hz}$. The data acquisition system communicates with the meter through an RS-232 serial port. The uncertainty of the measured temperature is $\pm 0.4^{\circ} \mathrm{C}$.

The thermocouple was place downstream of the lower-bypass duct of the test section (see Figure 3.2) and therefore senses a pseudo-static temperature. For low-speed flows with $\mathrm{Ma}<0.15$ the difference between the total and static temperatures is about $0.45 \%$ of the local static pressure. Applying the root-sum of squares (RSS) method given in Appendix B, the uncertainty of the inferred static temperature from the measured pseudo-static temperature is then $\pm 1.4^{\circ} \mathrm{C}$. The inferred static temperature is used to compute the air viscosity and density for monitoring the test-section Reynolds number (see Section 3.10.2).

\subsubsection{Digital Barometer}

An Omega DPI740 digital barometer with a pre-calibrated (absolute) pressure transducer was used to monitor the ambient pressure within the wind-tunnel facility. The barometer has an uncertainty of $\pm 0.02 \%$ full scale range and a sampling frequency of $2 \mathrm{~Hz}$. The data acquisition

system communicates with the meter through an RS-232 port. The ambient pressure and temperature were used to compute the density using the ideal gas law. 


\subsubsection{Mass Flow Meter}

An ISA 1932 Short Radius Nozzle, shown in Figure 3.11, was used to measure the total purge mass flow rate, $\dot{m}_{\text {purge, total }}$, delivered to the cascade test-section. The inlet and outlet of the nozzle is instrumented with static-pressure taps at two different circumferential positions. The differential pressures across the nozzle $(\Delta P)$ were used to compute two values, which were later averaged, of the purge mass flow:

$$
\dot{m}_{\text {purge, total }}=\rho Q_{P}=\rho C_{D} A_{t} \sqrt{\frac{2 \Delta P}{\rho\left(1-\beta^{4}\right)}}
$$

The discharge coefficient $\left(C_{D}\right)$ is given by an empirical formula:

$$
C_{D}=0.99-0.2262 \beta^{4.1}+\left(0.000215-0.001125 \beta+0.00249 \beta^{4.7}\right)\left(10^{6} / \operatorname{Re}_{D}\right)
$$

where $\beta$ the ratio of the nozzle diameter $(d)$ to the pipe inside diameter $(D)$ and $\operatorname{Re}_{\mathrm{D}}$ is the Reynolds number.

The nozzle was manufactured by K-Flo Fluid Meters, Inc. to tolerances of 0.001 inch and as such a mass flow calibration was not required. The quoted uncertainty of the inferred mass flow rate is $\pm 1.0 \%$ of the measured value (Bean, 1971).

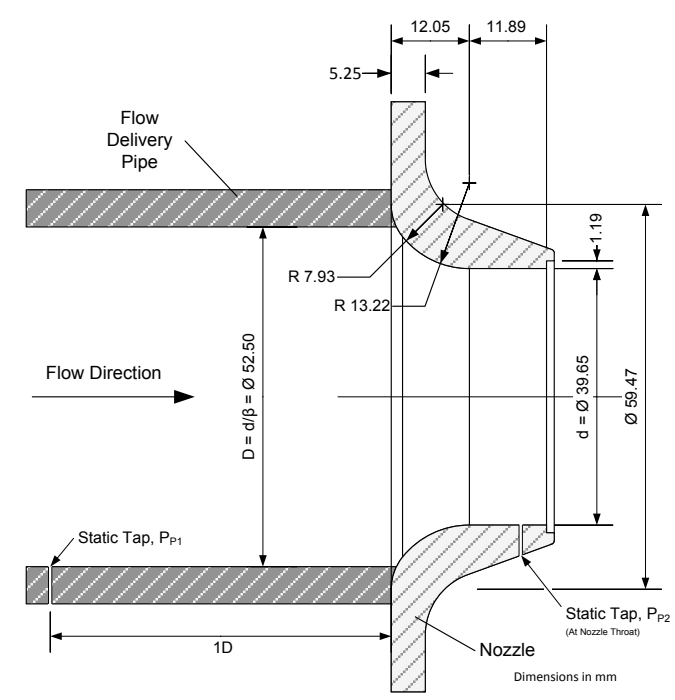

Figure 3.11: ISA 1932 Short Radius Nozzle 


\subsubsection{Stepper Motor Controller}

Two linear actuators driven by stepper motors automate the movement of the measurement probe in the pitchwise and spanwise directions. The minimum step size in either direction is $0.003175 \mathrm{~mm}$. A Velmex VXM motor controller translates ASCII directions from the user (or the data acquisition program) and moves the stepper motors a specified number of steps at a specified rate. The motor controller interfaces with the data acquisition computer via a RS-232 port.

\subsection{Measurement Probes, Measurement Procedures, Calibration Procedures, and Uncertainties}

\subsubsection{Introduction}

The following section describes the pressure and hot-wire probes and their respective measurement procedures, calibration procedures and estimated uncertainties. The choice of sampling rates and times for the pressure measurements are described first followed by descriptions of the probes. The measurement and calibration procedures as well as the estimates of the uncertainties are also given. Details of the probe calibration facility are provided in Section 3.9 and the general procedure for estimating the uncertainties is given in Appendix B.

In the present research, flow field data were obtained exclusively by using pressure and hot-wire probes. The choice to use point measurements, opposed to other non-invasive measurements such as particle image velocimetry (PIV), was largely dictated by the flow quantities of interest. For turbomachinery applications the bladerow losses are determined using measurements of the upstream and downstream total pressures (see Section 3.11.3). Pneumatic point measurements are the only reliable method for measuring the total pressure field of lowspeed cascades. Thus, for the present thesis point measurements were used exclusively. 


\subsubsection{Pressure Measurement Sampling Rate and Time}

All pressure measurements were made by collecting 10, 000 samples at $1 \mathrm{kHz}$, equalling 10 seconds of sampling. The sampling rate is sufficiently high for steady measurements and the number of samples was chosen for an adequate settling time. Running averages of the time signals showed that a constant mean was obtained within about 5 to 8 seconds. Thus, 10 seconds of sampling was chosen conservatively.

\subsubsection{Pitot Probe}

An L-shaped Pitot probe shown in Figure 3.12 was used to measure the total pressure distribution of the inlet endwall boundary. Relative to the boundary layer, the probe tip is approximately $3.5 \%$ of its thickness. Estimates of the uncertainty are based on the transducer calibration which includes errors due to non-linearity and hysteresis. Thus, the estimated uncertainty of the measured total pressure is $0.3 \%$ of the inlet midspan dynamic pressure. It is well known that a number of factors can affect the pressure sensed by Pitot probe including the Reynolds number, turbulence, wall proximity, shear flow and flow misalignment. Corrections for these conditions were not applied in the present work.

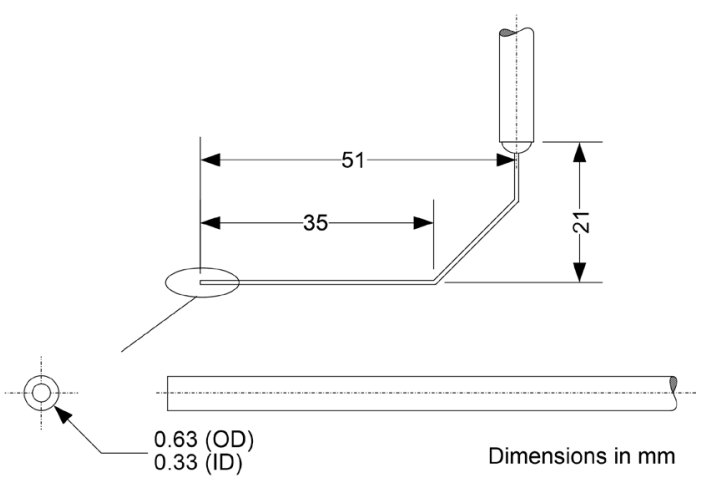

Figure 3.12: Pitot probe tip geometry 


\subsubsection{Three-hole Pressure Probe}

A three-hole pressure probe, designed by Mahallati (2003) and shown schematically in Figure 3.13, was used to make measurements of two-dimensional flows. The probe-tip has a $45^{\circ}$ wedge angle, a width of $1.89 \mathrm{~mm}$ and a thickness of $0.63 \mathrm{~mm}$. Prior to measuring an unknown flow field, the probe is calibrated in the uniform jet (see Section 3.9) by pitching it through $0.5^{\circ}$ increments over the range of $\pm 21^{\circ}$. The three-port pressures and the known jet conditions are used to formulate calibration functions using the procedure of Lewis (1966). At lower velocities, the probe shows some Reynolds number sensitivity and therefore calibrations were made at jet velocities of $10,15,30$ and $42 \mathrm{~m} / \mathrm{s}$ which span the range of velocities encountered in the experiment. Details of the calibration and the data reduction procedures are described in Appendix C.

This probe and experimental apparatus have been used by several past researchers (Mahallati, 2003; Popovic, 2005; Zoric, 2006 and Knezevici, 2011). Estimates of the uncertainty were made by repeating measurements in known flow conditions. The estimated uncertainty of the measured flow angle is $\pm 0.4^{\circ}$. The uncertainty of the total and dynamic pressure is $\pm 0.4 \%$ and $\pm 0.6 \%$ of the local dynamic pressure, respectively.

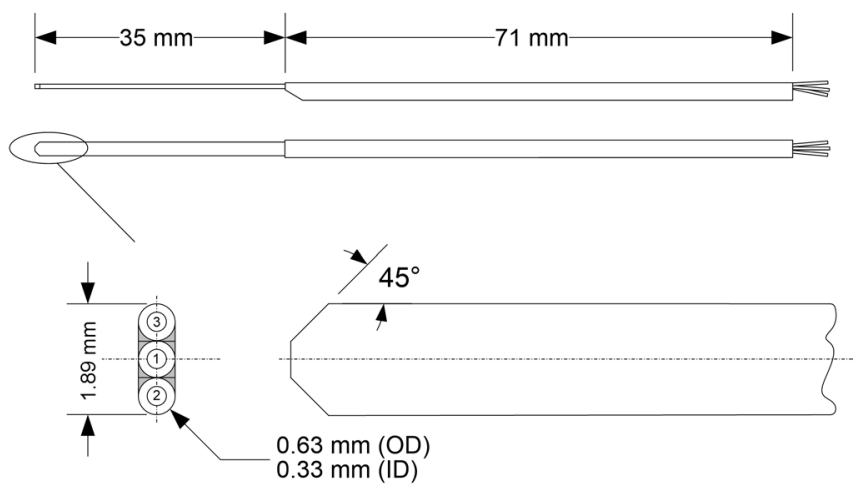

Figure 3.13: Three-hole probe geometry 


\subsubsection{Seven-hole Pressure Probe}

Downstream measurements of the three-dimensional secondary flow were made using a seven-hole pressure probe, shown schematically in Figure 3.14. The probe was designed by Knezevici (2011) and is bent in the shape of a "goose-neck" to access the near wall positions. The probe-tip has a nominal cone angle of $60^{\circ}$ and an outer diameter of $1.83 \mathrm{~mm}$. The calibration and data reduction procedures for the seven-hole probe have been adapted from Gerner et al. (1984) and are described in Appendix D. The seven-hole probe is calibrated through the pitch $(\beta)$ and yaw $(\alpha)$ angle range of $-50^{\circ}<\alpha, \beta<+50^{\circ}$ in $2^{\circ}$ increments for five separate velocities $(20,25,30,35$ and $42 \mathrm{~m} / \mathrm{s})$.

The uncertainty of seven-hole probe measurements was thoroughly investigated by Scribner (2011) using the same apparatus and calibration procedures as in the present work. The probe reading errors were tested for range of operating conditions that varied the calibration jet Reynolds number and turbulence intensity. The uncertainties are reproduced in Table 3.3 and are applicable to the measurements of this thesis.
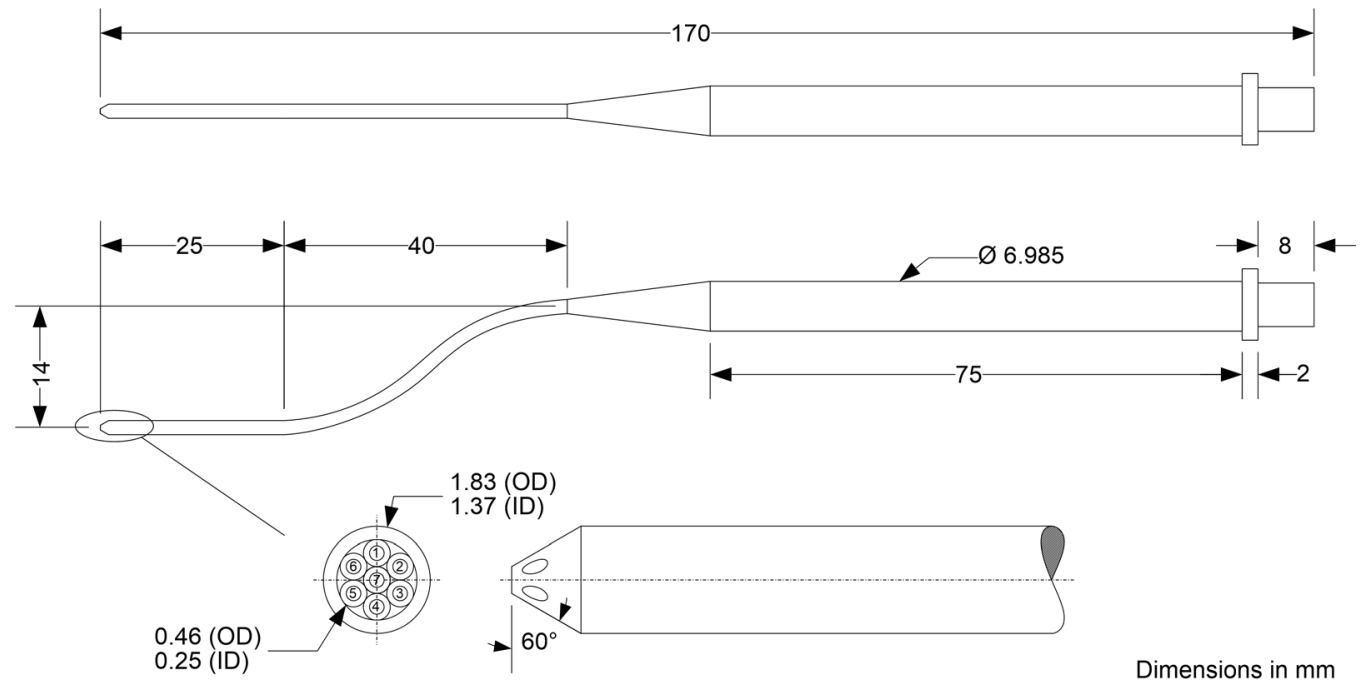

Figure 3.14: Seven-hole probe geometry 
Table 3.3: Seven-hole probe measurement uncertainties (Scribner, 2011)

\begin{tabular}{|c|c|c|c|c|c|}
\cline { 2 - 6 } \multicolumn{1}{c|}{} & \multicolumn{5}{c|}{ Uncertainty (all Reynolds numbers) } \\
\cline { 2 - 6 } \multicolumn{1}{c|}{} & $T u=1.5 \%$ & $T u=3 \%$ & $T u=5 \%$ & $T u=8 \%$ & $T u=12 \%$ \\
\hline $\begin{array}{c}\text { Total Pressure } \\
(\% \text { of local dynamic pressure) }\end{array}$ & \pm 0.5 & \pm 0.5 & \pm 0.5 & \pm 0.8 & \pm 1.0 \\
\hline $\begin{array}{c}\text { Dynamic Pressure } \\
(\% \text { of local dynamic pressure) }\end{array}$ & \pm 0.7 & \pm 0.7 & \pm 0.7 & \pm 0.9 & \pm 1.1 \\
\hline Flow Angle $\left({ }^{\circ}\right)$ & \pm 0.6 & \pm 0.6 & \pm 0.8 & \pm 0.8 & \pm 1.2 \\
\hline
\end{tabular}

\subsubsection{Kiel Probe}

The Kiel probe was designed by PWA and has a similar probe body to that of the sevenhole probe. The probe tip, shown in Figure 3.15, consists of an inner Pitot tube covered by an outer sheath. Although Pitot tubes typically read erroneous total pressures when the flow misalignment is greater than about $5^{\circ}$ (Chue, 1975), the outer sheath of the Kiel probe aligns the streamlines with the inner Pitot tube.

The Kiel probe is relatively insensitive to misalignment less than $35^{\circ}$ (Chue, 1975) and was used to compare with seven-hole probe measurements. Differences were observed in flow regions where the turbulence intensity and the velocity gradients were both high. A summary is provided in Section D.5 of Appendix D and further details are given by Scribner (2011). His project investigated the response of the seven-hole probe over a range of Reynolds numbers for varying levels of turbulence intensity. The current project continued using the seven-hole probe as the primary downstream measurement probe; however the uncertainties were updated as recommended by Scribner (2011).

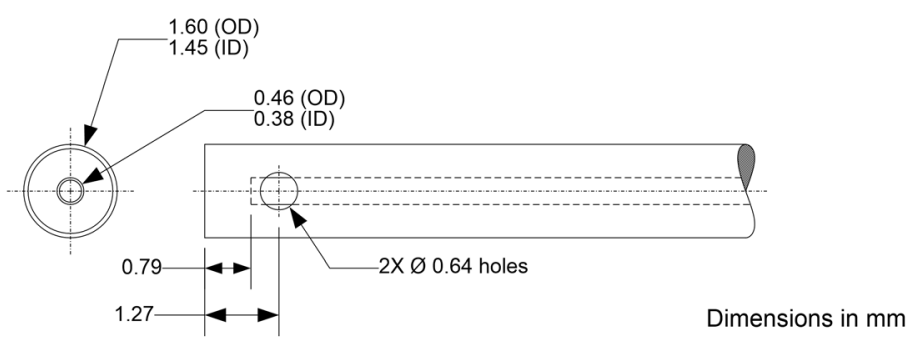

Figure 3.15: Kiel probe-tip geometry 


\subsubsection{Hot-wire Anemometry}

A rotatable $x$-type hotwire probe, shown in Figure 3.16, was used to measure the three mean velocity components and the six Reynolds stresses downstream of the cascade. The $x$-type probe has four prongs with two tungsten wires spot welded to the tips. The nominal wire diameters are $5 \mu \mathrm{m}$.

Each wire sensor of the probe is connected to one channel of an AN-1003 (A.A. Lab Systems) anemometer. The unit was originally used by Mahallati (2003) and has 10 CTA (Constant Temperature Anemometry) channels. Eight are identically configured while two have an additional tuning option for high frequency applications. Two of the basic channels were used here.

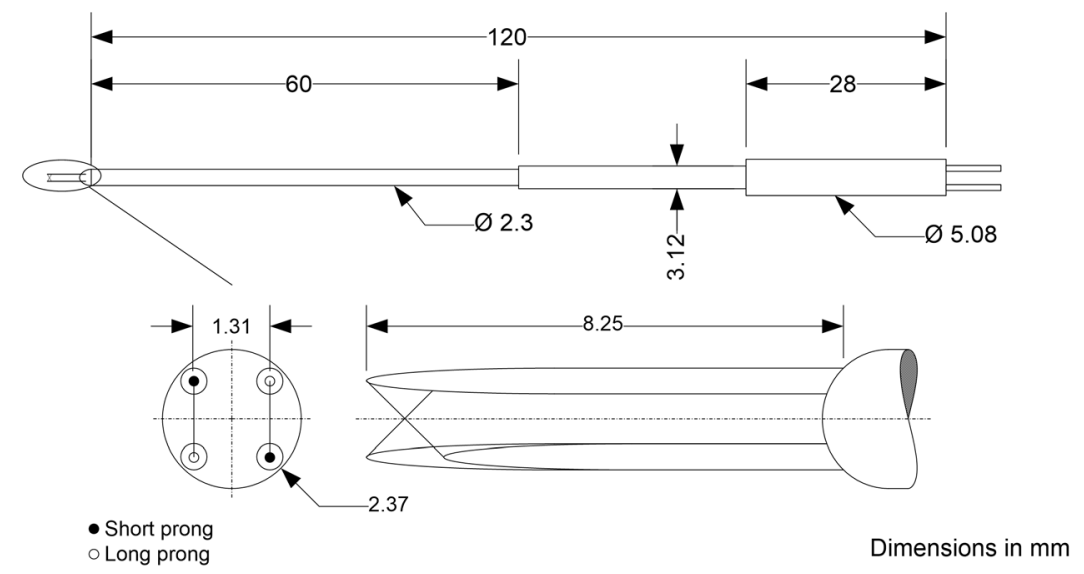

Figure 3.16: $X$-type hotwire probe-tip geometry

Prior to performing hotwire measurements, the wire temperature was set by selecting the over-heat ratio $(\mathrm{OHR})$ and balancing the Wheatstone bridge. A high overheat value improves the response of the hotwire circuit but also promotes sensor oxidation which can lead to wire burnout. A moderate value of OHR of 1.5 was chosen and the corresponding frequency response was determined to be about $150 \mathrm{kHz}$ by observing the response of a pulsed step input on an oscilloscope. 
All hotwire measurements were made by collecting 131,072 $\left(2^{17}\right)$ samples at a rate of $28 \mathrm{kHz}$ per channel, equalling about 4.7 seconds of data sampling. FFT algorithms require the number of samples $(N)$ to be a power of 2 (i.e. $N=2^{x}$ ). The sampling rate was twice the value of the low-pass filter $(14 \mathrm{kHz})$ to avoid aliasing.

The calibration of the $x$-type hotwire probe requires a velocity and a directional sensitivity (angular) calibration. The velocity calibration was performed with the probe axis aligned in the calibration jet over the range of $7<V_{j e t}<42 \mathrm{~m} / \mathrm{s}$. The directional sensitivity of the two wires was measured by rotating the probe through a range of pitch $(\beta)$ and yaw $(\alpha)$ flow angles $\left(-30^{\circ}<\alpha, \beta<+30^{\circ}\right)$ in $2^{\circ}$ increments. The directional sensitivity of the probe is independent of Reynolds number at higher flow velocities $(V>5 \mathrm{~m} / \mathrm{s})$ and was measured at a constant jet velocity of $30 \mathrm{~m} / \mathrm{s}$. Details of the calibration procedures and sample calibration data are given in Appendix E.

The data reduction procedure of Döbbeling et al. (1992), originally derived for quadruplewire probes, was adapted for the $x$-type probe. In an unknown flow, the mean velocity components are determined by performing measurements in two roll orientations, $0^{\circ}$ and $90^{\circ}$, to simulate a quadruple-wire probe. The signals are not temporally correlated and therefore Reynolds stresses were solved using the procedure similar to that of Buresti and Di Cocco (1987). In order to improve the accuracy of the Reynolds stresses, the directional calibration and flow field measurements were collected for a third roll orientation at $45^{\circ}$. Details of the data reduction procedures and uncertainty estimates are given in Appendix E.

\subsection{Probe Calibration Apparatus}

Pressure and hotwire probe calibrations are performed in the low-speed calibration facility shown in Figure 3.17. The calibration rig is of the suction-type and has a convergent nozzle to create a calibration jet in a closed test section. Air filters enclose the inlet to remove dust and dampen aerodynamic disturbances from the laboratory. Two turntables, designed by 
Yaras (1987), permit the computer-controlled rotation of the probe through pitch $(\beta)$ and yaw $(\alpha)$ angles while maintaining the probe tip at the same position within the calibration jet. Details of the flow angle nomenclature are described in Section D.2 of Appendix D. The velocity of the jet $\left(V_{j e t}\right)$ can be varied from 0 to $43 \mathrm{~m} / \mathrm{s}$ by adjusting a gate throttle at the blower inlet. The axial and radial uniformity of the jet has been documented by Benner (2003) and verified by the current author. The turbulence intensity of the jet is approximately $0.5 \%$ (Mahallati, 2003).

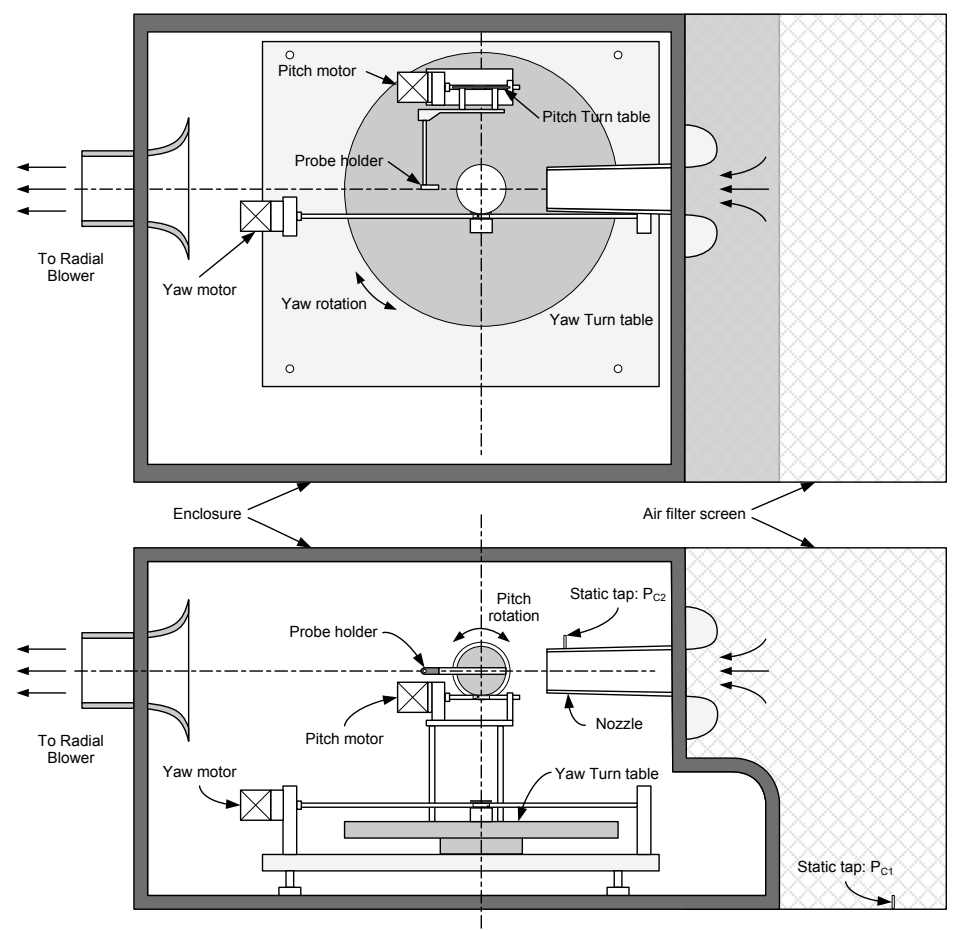

Figure 3.17: Schematic drawing of the low-speed calibration facility (Benner, 2003)

Prior to calibrating pressure or hotwire probes, the flow conditions were characterized for several velocity levels. The total $\left(P_{0}\right)$ and static pressure $\left(P_{S}\right)$ within the jet and at centre of the pitch turn table were measured using Pitot and static pressure probes, respectively. The nozzle calibration coefficients are then given by:

$$
C_{P 0 \text { Cal }}=\frac{P_{0}-P_{C 1}}{P_{C 1}-P_{C 2}}
$$




$$
\begin{gathered}
C_{P S_{C a l}}=\frac{P_{S}-P_{C 2}}{P_{C 1}-P_{C 2}} \\
C_{q_{C a l}}=\frac{P_{0}-P_{S}}{P_{C 1}-P_{C 2}}=C_{P 0}-C_{P S}+1
\end{gathered}
$$

During probe calibrations these coefficients are used to determine the total, static and dynamic pressure at the probe tip based on $P_{C l}$ and $P_{C 2}$. The jet velocity is given by:

$$
V_{j e t}=\sqrt{\frac{2 \cdot\left(P_{C 1}-P_{C 2}\right) \cdot C_{q_{C a l}}}{\rho}}
$$

where $\rho$ is the air density calculated using ideal gas law.

The uncertainties in the calibration coefficients was investigated by Mahallati (2003) and included the quoted uncertainties of Section 3.8.

\subsection{Cascade Testing Procedures}

\subsubsection{Introduction}

The following section describes the cascade testing procedures. This includes descriptions of the wind-tunnel calibration, the downstream measurement grid and the oil-film surface visualization procedures.

\subsubsection{Wind Tunnel Calibration and Operating Point}

The cascade inlet Reynolds number $(\mathrm{Re})$ is set by adjusting the rotational speed of the wind tunnel blower. The contraction pressure difference $\left(P_{C 1}-P_{C 2}\right)$ is calibrated to the midspan conditions at $1.20 C_{x}$ upstream of the blade leading edge giving the wind-tunnel total and dynamic pressure coefficients: 


$$
\begin{gathered}
C_{P 0_{W T}}=\frac{P_{0, C L}-P_{C 1}}{P_{C 1}-P_{C 2}} \\
C_{q_{W T}}=\frac{P_{0, C L}-P_{C L}}{P_{C 1}-P_{C 2}}
\end{gathered}
$$

where $P_{0, C L}$ and $P_{C L}$ are the total and static pressure at the cascade centre-line (midspan), respectively. The cascade Reynolds number based on the centre-line velocity $\left(V_{C L}\right)$ is given by:

$$
\operatorname{Re}=\frac{\rho \cdot V_{C L} \cdot C_{x}}{\mu}
$$

where $\rho$ and $\mu$ is the air density and the dynamic viscosity, calculated using the ideal gas law and Sutherland's law for viscosity. The centre-line velocity is calculated using the measured contraction pressure difference:

$$
V_{C L}=\sqrt{\frac{2 \cdot\left(P_{C 1}-P_{C 2}\right) \cdot C_{q_{W T}}}{\rho}}
$$

The test-section Reynolds number is continuously monitored during measurement runs but is not adjusted for drift. Runs are started after an initial warm-up period of about 30 minutes. From the author's experience the Reynolds number will drop over a typical 24 hour run by about 1000. The variable frequency drive of the wind tunnel fan is not programmed to automatically control the fan speed as the air temperature rises. Therefore, the operating Reynolds number is initially set above the design inlet Reynolds number by about 500. The change in Reynolds number over a run equates to about $\pm 0.1 \mathrm{~m} / \mathrm{s}$ of the inlet mid-span velocity.

\subsubsection{Downstream Traverse Locations and Measurement Grid}

The downstream measurements were made at two axial planes (at 1.20 and $1.40 C_{x}$ ) using the seven-hole pressure probe for the cases described in Section 3.6. Typically, measurements were made over the centre passage from $-0.15<y / s<1.15$ and for one half span $(0.005<z / h<0.5)$. Sample results for the Baseline case, measured at the $1.40 C_{x}$ plane, are shown in Figure 3.18. The dashed vertical lines at $y / s=0$ and $y / s=1$ represent the projection of 
the trailing edge along the blade metal angle. The measurement grid is overlaid on a colour flood of the total pressure coefficient $\left(C_{P 0}\right)$. The grid consists of 90 equally spaced pitchwise positions and 62 spanwise locations, totalling 5580 data points. The resolution was chosen such that the blade wake and peak loss regions are resolved with several data points, as shown. For this cascade the pitchwise spacing between the measurement points was $0.78 \%$ of the blade span while a spanwise resolution of $0.63 \%$ span was used for the secondary flow region, from $z / h=0$ to $z / h=0.3$. For the two-dimensional region, from $z / h=0.3$ to $z / h=0.5$, a larger spacing of $1.56 \%$ span was used. Probe reading errors occur for measurements made very close to the endwall and therefore measurements below $z / h=0.01$ are discarded, as shown. The probe movement is automated by the data acquisition program and sampling is only initiated 2 seconds after the probe movement is complete to allow the pressure transients to dissipate. The sampling rates and times specified in section 3.8.2 were used and the complete grid was measured in a continuous run lasting approximately 23 hours.

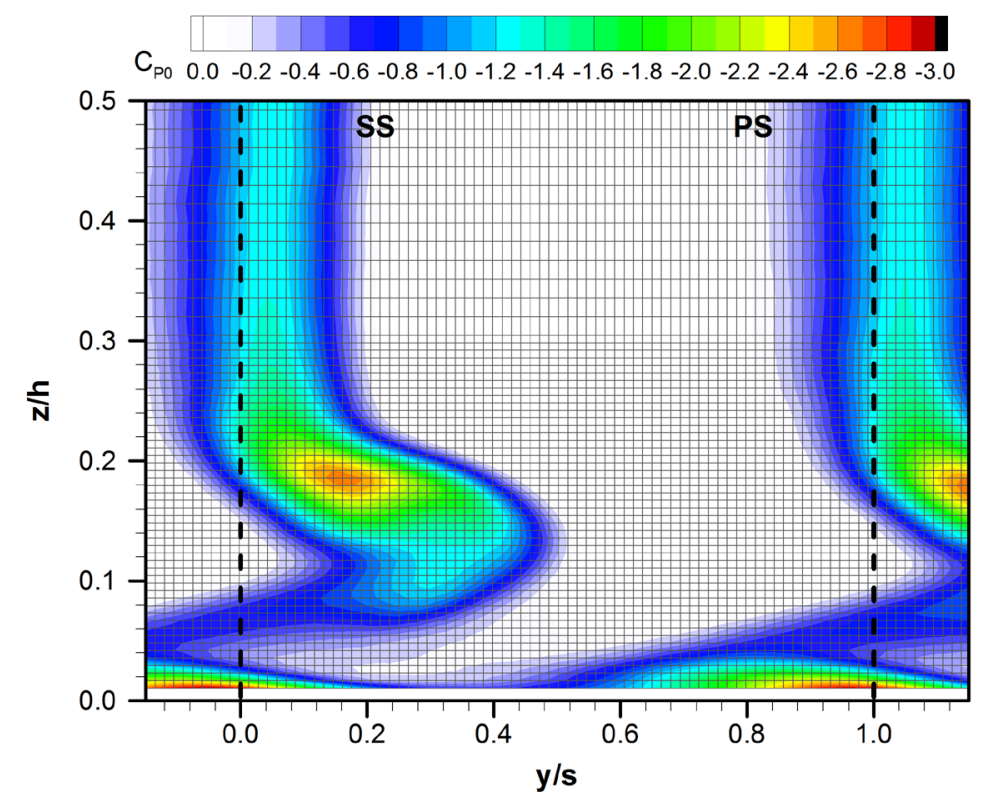

Figure 3.18: Colour flood of the total pressure coefficient $\left(C_{P 0}\right)$ with the measurement grid overlaid 


\subsubsection{Blade Surface Static Pressure Measurements}

Blade surface pressure measurements, or blade loadings, were used to aid the interpretation of the midspan and near endwall flows. The blade loadings are also used to ensure the cascade is setup as intended. Measurements were made using a 48-port Scani-valve and a specially design blade. The blade has two rows of 14 suction surface and 6 pressure surface static taps positioned at $5 \%$ and $50 \%$ span. During the cascade setup, preliminary results showed significant differences between the measured and predicted (CFD) pressures over the suction surface. Several causes were investigated; however, the root-cause was traced to subtle differences between the designed and as-built coordinates of the PAKH airfoil. Using the as-built coordinates for the CFD predictions resulted in good agreement between the predicted and measured loadings. Therefore, all of the CFD results were generated using the as-built blade coordinates, which were measured using a CMM (coordinate measuring machine) at the National Research Council.

\subsubsection{Oil Surface Flow Visualization}

Surface flow visualizations were used to aid the interpretation of the secondary flow physics. Several pigment-oil mixtures of varying viscosities were tested on to the blade suction surface and the endwall to visualize the shear stress streamlines. It was found that lighter 5W-30 motor oil was best for the endwalls while a heavier 10W-30 was better for the higher shear levels on the blade suction surface. For the cases with purge flow, the visualizations were made for the zero flow (cavity configuration) and the highest purge flow cases. The intermediate purge flow levels were not considered.

The first visualizations were made using Titanium oxide as the pigment in the oil mixtures. This was effective on the blade suction surface, however, these mixtures would tend to flocculate when used on the endwall. Additives, such as linseed oil, were not effective in reducing the flocculation. As an alternative, a fine florescent red-powder pigment that requires ultra-violet (UV) light for illumination was used. Photographs were taken in a temporary tent constructed 
using an arrangement of UV backlights and tarpaulins covering the test-section to eliminate as much visible-spectrum light as possible.

In all cases, the best visualizations were obtained using very thin layers of the oil-mixture. This prevented pooling in the low-shear stress regions and limited dripping when the wind-tunnel was shut-off. A thin-layer of oil has the added benefit of reducing any effects on the surface flow itself.

\subsection{Data Reduction}

\subsubsection{Introduction}

This section describes general averaging procedures followed by the methods for computing the losses, mixed-out losses, vorticity and secondary kinetic energy coefficients.

\subsubsection{Averaging Procedures}

It is often useful to examine the averaged flow quantities to assess the overall bladerow performance. The averaging procedures used here follow the recommendations of Cumpsty and Horlock (2006). In general, it is appropriate to mass-average extensive properties such as entropy or total pressure which are defined on a per unit mass basis. For the static pressure which is defined as the force per unit area, area-averaging is appropriate. For other quantities the choice of averaging procedure may be unclear. Cumpsty and Horlock (2006) recommend that the averaging should reflect the physical interpretation of the quantity. For example, the outlet flow angle is not a mass-based quantity and is therefore computed using area-averaged velocities. Mass-averaging would only confuse the physical interpretation. The secondary kinetic energy (SKE) however is physically interpreted as an extensive parameter and is therefore massaveraged. 
Here, both one-dimensional (pitchwise) and two-dimensional averages are used. Pitchwise area- and mass-averages are computed for a desired flow quantity, say $\Omega$, using cell-centred averages at constant spanwise positions $(j)$, defined by:

$$
\begin{gathered}
\text { Pitchwise area-averaged: } \quad \bar{\Omega}=\frac{\int_{0}^{s} \Omega \cdot d y}{\left.\int_{0}^{s} d y\right|_{j}} \\
\text { Pitchwise mass-averaged: } \quad \Omega^{\prime}=\frac{\int_{0}^{s} \rho \cdot u \cdot \Omega \cdot d y}{\left.\int_{0}^{s} \rho \cdot u \cdot d y\right|_{j}}
\end{gathered}
$$

Two-dimensional area- and mass-averages are computed at cell-centers for one full pitch $(s)$ and over one-half of the blade span $(h)$, defined by:

$$
\begin{aligned}
\text { 2D area-averaged: } \quad & \overline{\bar{\Omega}}=\frac{\int_{0}^{0.5 h} \int_{0}^{s} \Omega \cdot d y d z}{\int_{0}^{0.5 h} \int_{0}^{s} d y d z} \\
\text { 2D mass-averaged: } \quad \quad \Omega^{\prime \prime} & =\frac{\int_{0}^{0.5 h} \int_{0}^{s} \rho \cdot u \cdot \Omega \cdot d y d z}{\int_{0}^{0.5 h} \int_{0}^{s} \rho \cdot u \cdot d y d z}
\end{aligned}
$$

The density of the measured flows is essentially constant $\left(\mathrm{Mach}_{\max } \approx 0.12\right)$ and in practice is dropped from the mass-averaged integrations (Equations 3.15 and 3.17). Since the total pressure cannot physically be measured at the endwall, $z=0$, its value is approximated for the integrated results. It is assumed that the static pressure gradient normal to the wall and the wall velocity is zero. The total pressure at the endwall is then taken as the static pressure measured at a position close to the endwall (typically $z / h=0.01$ or $1 \%$ span).

\subsubsection{Losses for Turbine Bladerows}

The term losses refers fundamentally to the production of entropy in the flow. However, as discussed in Section 2.5, for incompressible flow and stationary geometries, the loss in total pressure is an alternative and convenient measure of the entropy production (Denton, 1993). 
For the present experiment, the total pressure measured at a point is first expressed as a total pressure coefficient,

$$
C_{P 0}=\frac{P_{0}-P_{01, C L}}{q_{1, C L}}
$$

where the reference total and dynamic pressures, $P_{01, C L}$ and $q_{1, C L}$, are the values on the centreline at the cascade inlet. These pressures are always known during the measurements. Values of $C_{P 0}$ that have been mass-averaged along a pitchwise line, using Equation 3.15, are designated $C_{P 0}{ }^{\prime}$. The mass-averaged value over a plane, obtained with Equation 3.17, is written as $C_{P 0}{ }^{\prime \prime}$.

The total pressure loss between two planes can be obtained by taking the difference between the respective values of $C_{P 0}{ }^{\prime \prime}$. However, the resultant total pressure loss coefficient nondimensionalizes the losses by the upstream dynamic pressure. As discussed in Chapter 2, the losses in turbine blade rows scale on the passage outlet dynamic pressure. It is therefore conventional to define the total pressure loss coefficient as

$$
Y=\frac{P_{01}^{\prime \prime}-P_{02}^{\prime \prime}}{q_{2}^{\prime \prime}}
$$

where 1 is the upstream plane and 2 the downstream one. Then in terms of $C_{P 0}$,

$$
Y=\left(C_{P 0_{1}}{ }^{\prime \prime}-C_{P 0_{2}}{ }^{\prime \prime}\right)\left(\frac{q_{1, C L}}{q_{2}}\right)^{\prime \prime}
$$

The secondary loss coefficient $\left(Y_{\text {sec }}\right)$ is then obtained by subtracting the profile losses $\left(Y_{p}\right)$ from the total loss coefficient $(Y)$. $Y_{p}$ is computed by applying Equation 3.15 to the midspan measurements. The profile and secondary loss coefficient are then given by:

$$
\begin{gathered}
Y_{P}=-C_{P 0_{2}}{ }^{\prime} \cdot\left(\frac{q_{1, C L}}{q_{2}}\right)^{\prime} \\
Y_{s e c}=Y-Y_{p}
\end{gathered}
$$


With purge flow present, both the mass flow rate and the total pressure of the purge flow must be taken into account in determining the losses. Figure 3.19 shows the mass flow exiting the bladerow $\left(\dot{m}_{2}\right)$ as the sum of the main-gas path $\left(\dot{m}_{1}\right)$ and purge mass flows $\left(\dot{m}_{\text {purge }}\right)$. Since there are two inlet flows, the total loss coefficient becomes:

$$
Y=\left(C_{P 0_{1}}^{\prime \prime}\left(\frac{\dot{m}_{1}}{\dot{m}_{2}}\right)+C_{P 0_{\text {purge }}}{ }^{\prime \prime}\left(\frac{\dot{m}_{\text {purge }}}{\dot{m}_{2}}\right)-C_{P 0_{2}}^{\prime \prime}\right) \cdot\left(\frac{q_{1, C L}}{q_{2}}\right)^{\prime \prime}
$$

Downstream measurements are made for one half span and therefore this definition represents the losses for a hypothetical bladerow with purge flow injected at both the blade root and tip, $\mathrm{z}=0$ and $\mathrm{z}=\mathrm{h}$ respectively.

Equation 3.23 is only useful if the purge flow mass flow rate $\left(\dot{m}_{\text {purge }}\right)$ and total pressure are known. The author has attempted to measure the purge flow with limited success. Instead, the purge flow total pressure was determined according to:

$$
C_{P 0_{\text {purge }}}=C_{q_{\text {purge }}}+C_{P S_{\text {purge }}}-1
$$

where $C_{P S_{\text {purge }}}$ and $C_{q_{\text {purge }}}$ are approximations of the purge flow static and dynamic pressure coefficients respectively. $\quad C_{P S_{\text {purge }}}$ was obtained by integrating the endwall cavity static pressure

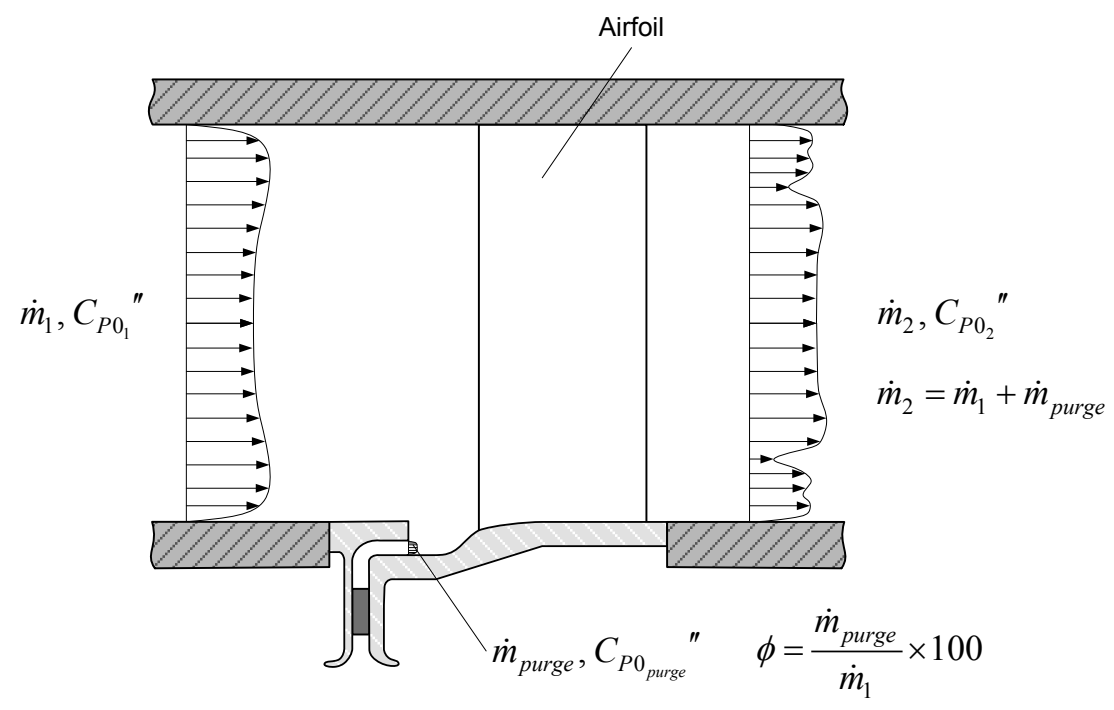

Figure 3.19: Schematic diagram showing the mass flow balance for a bladerow with purge flow. 
measurements (see Figure 3.6 of Section 3.4) for one pitch, while $C_{q_{\text {purge }}}$ was computed using the following procedure. First, the non-dimensional axial velocity $\left(u_{\text {purge }}\right)$ was determined using:

$$
u_{\text {purge }}=\left(\frac{\phi}{100}\right) \cdot\left(\frac{\dot{m}_{1}}{\rho A_{\text {purge }}}\right) \cdot\left(\frac{1}{V_{1, C L}}\right)
$$

where $\dot{m}_{1}$ is the mass-flow rate of the main gas path and $\phi$ is the purge mass flow percentage. The resultant velocity $\left(V_{\text {purge }}\right)$ is then approximated using $u_{\text {purge }}$ and an estimate of the purge flow exit angle, $\theta_{\text {purge }}$. The purge flow dynamic pressure coefficient $\left(C_{q_{\text {purge }}}\right)$ is then given by:

$$
C_{q_{\text {purge }}}=\left(\frac{q_{\text {purge }}}{q_{1, C L}}\right)=\left(\frac{V_{\text {purge }}}{V_{1, C L}}\right)^{2}=\left(\left(u_{\text {purge }}\right) \frac{1}{\cos \theta_{\text {purge }}}\right)^{2}
$$

Equations 3.23 through 3.26 show that $C_{P 0_{\text {purge }}}$, and therefore the total pressure loss coefficient $(Y)$, are functions of $\theta_{\text {purge. }}$. Since the purge flow angle was estimated and not measured, the sensitivity of the total loss coefficient $(Y)$ to this angle was examined.

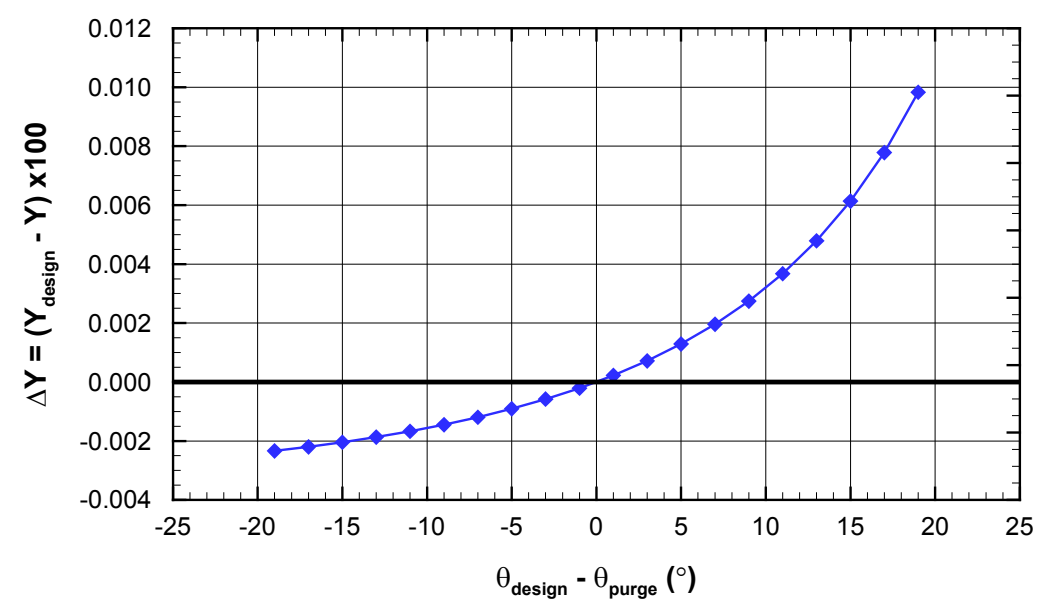

Figure 3.20: Sensitivity of the total loss coefficient $(Y)$ with respect to the purge exit flow angle

At the design purge flow angle $\left(\theta_{\text {design }}=-45^{\circ}\right)$ a typical value of the overall loss coefficient, $\left(Y_{\text {design }}\right)$ is 0.0504 . Figure 3.20 shows that if $\theta_{\text {purge }}$ deviates by $+19^{\circ}$ or $-19^{\circ}$ from $\theta_{\text {design }}$ the change in loss coefficient $\left(\Delta Y=Y_{\text {design }}-Y\right)$ is $+0.01 \%$ or $-0.002 \%$ respectively. This change is negligible 
compared to the uncertainty of the measured loss coefficient of $\pm 0.35 \%$. Also, if the loss coefficient is computed using either Equations 3.20 or 3.23 the values only differ by $0.08 \%$. Thus, including the purge flow terms may be unnecessary; however, for completeness Equation 3.23 was used for all cases with purge flow.

\subsubsection{Mixed-out Losses}

The loss coefficients, $Y$, defined in the last section are the values at the measurement plane. The measured flow is non-uniform and thus as it mixes with downstream distance additional losses are generated. The mixed-out losses are often quoted as they allow results obtained at different facilities with different measurement planes to be compared.

In this work the mixed-out losses are computed using an analysis, adapted by Benner (2003) from Harrison (1990). The procedure applies the momentum and continuity equations to a control volume that extends downstream from the measurement plane, at constant area, to a hypothetical mixed-out plane. The mixing losses are realised through the dissipation of the secondary kinetic energy, the recovery of static pressure and the mixing-out of nonuniformities in the primary kinetic energy. The analysis assumes that no additional losses are generated by the wall shear stress between the measurement and mixed-out planes. The mixedout total, profile and secondary loss coefficients are given by:

$$
\begin{gathered}
Y_{\text {mixed-out }}=\left(C_{P 0_{1}}^{\prime \prime}-C_{P 0_{\text {mixed-out }}}{ }^{\prime \prime}\right)\left(\frac{q_{1, C L}}{q_{\text {mixed-out }}}\right)^{\prime \prime} \\
Y_{P}=-C_{P 0_{\text {mixed-out }}} \cdot\left(\frac{q_{1, C L}}{q_{\text {mixed-out }}}\right)^{\prime} \\
Y_{\text {sec }}=Y_{\text {mixed-out }}=Y_{\text {mixed-out }}-Y_{P_{\text {mixed-out }}}
\end{gathered}
$$




\subsubsection{Vorticity and Secondary Kinetic Energy}

The size, intensity and location of the secondary flow structures can be identified using vorticity and secondary kinetic energy.

The vorticity is defined as the curl of the velocity vector.

$$
\omega=\nabla \times V
$$

The components of the vorticity vector (in the Cartesian coordinate system) are given by:

$$
\begin{gathered}
\omega_{x}=\frac{\partial w}{\partial y}-\frac{\partial v}{\partial z} \\
\omega_{y}=\frac{\partial u}{\partial z}-\frac{\partial w}{\partial x} \approx \frac{1}{u}\left(v \omega_{x}+\frac{1}{\rho} \frac{\partial P_{0}}{\partial z}\right) \\
\omega_{z}=\frac{\partial v}{\partial x}-\frac{\partial u}{\partial y} \approx \frac{1}{u}\left(w \omega_{x}-\frac{1}{\rho} \frac{\partial P_{0}}{\partial y}\right)
\end{gathered}
$$

Strictly speaking, the pitchwise $(y)$ and spanwise $(z)$ vorticity components require measurements from multiple closely-spaced streamwise planes to obtain the axial velocity gradients. In this work, the approximation given in Equations 3.32 and 3.33, which require data from a single plane only, were used. The expressions were derived by Gregory-Smith et al. (1988a) using the incompressible Euler equations. Yaras (1990) provides a full derivation and also tested the accuracy of the approximations. The single plane approximation showed very good agreement to the multiple plane method in all regions of the flow except where the velocity gradients change rapidly (e.g. the vortex centre). The maximum error in the local vorticity was less than $10 \%$.

The streamwise vorticity $\left(\omega_{s}\right)$ is the component of vorticity that is aligned with the primary flow direction (defined in Section 3.5.2; Equation 3.2) and is given by:

$$
\omega_{s}=\omega_{x} \cos \overline{\overline{\beta_{2}}}+\omega_{y} \sin \overline{\overline{\beta_{2}}}
$$

where $\overline{\overline{\beta_{2}}}$ is the area-averaged outlet primary flow direction. The streamwise vorticity is non-dimensionalized and presented as the streamwise vorticity coefficient. 


$$
C_{\omega_{s}}=\frac{\omega_{s} \cdot C_{x}}{V_{1, C L}}
$$

where $C_{x}$ is the blade axial chord.

The secondary kinetic energy (SKE) is the kinetic energy associated with the secondary velocity vectors: that is those that lie in the plane perpendicular to the primary flow direction (see Section 3.5.2). The secondary kinetic energy is expressed as:

$$
S K E=\frac{1}{2} \rho\left(v_{s e c}^{2}+w_{s e c}^{2}\right)
$$

where $v_{\text {sec }}=-u \sin \overline{\overline{\beta_{2}}}+v \cos \overline{\overline{\beta_{2}}}$ and $w_{\text {sec }}=w$. The non-dimensional SKE coefficient is:

$$
C_{S K E}=\frac{v_{s e c}^{2}+w_{s e c}^{2}}{V_{1, C L}^{2}}
$$

The secondary kinetic energy represents an irreversible loss from the primary flow and eventually dissipates with downstream distance through viscous mixing. At the fully mixed-out conditions all of the secondary kinetic energy is dissipated appearing as additional mixing losses. 


\section{Chapter 4}

\section{Computational Methods}

\subsection{Introduction}

The following chapter outlines the computational (CFD) procedures. Two different approaches were used for the investigations which were either fundamental or applied in nature.

In Chapter 5, CFD is used to make comparisons with the experimental measurements of the mean and turbulent flow field and assess the suitability of two-equation turbulence models to turbine secondary flows. The fundamental nature of this study required a relatively high density computational mesh. In Chapters 6 and 7, the simulations were performed using lower density grids that are similar to those used for design applications. Thus, the purpose of these appliedtype simulations was twofold: (1) to aid the interpretation of the experimental measurements and (2) to evaluate the ability of the commercial code to predict these flows for industrial applications.

The current chapter will first discuss the numerical procedures for both the fundamental and applied investigations. Descriptions of the domain geometry and meshing strategies, the boundary conditions and convergence criteria follow. The chapter concludes with sample results from the two-dimensional and three-dimensional grid sensitivity studies. 


\subsection{Numerical Procedures}

Simulations were performed using the ANSYS CFX-v-12.1 solver. The solver implements an element-based finite volume technique in which the conservation of mass and momentum equations are satisfied for each mesh volume. The CFX solver is considered a pressure-based, coupled solver that utilizes an Algebraic Multigrid technique to improve the convergence rates. The mass and momentum advection terms were discretized using a second-order central differencing scheme. (ANSYS, 2009)

The steady-state RANS equations were solved with air modelled as an incompressible fluid (constant density) at a temperature of $25^{\circ} \mathrm{C}$. The domain walls are considered adiabatic and the energy equation was not solved. The two-equation SST turbulence model was chosen for this investigation as it is a widely used model based on the Boussinesq eddy-viscosity assumption. The predicted eddy-viscosity distribution is therefore isotropic, and thus, this model provides a good test of the effects this assumption has on the prediction of flows in turbomachinery cascades. The transition model, $\gamma-\operatorname{Re}_{\theta} \mathrm{CFX}-\mathrm{v}-12.1$, was enabled to capture the laminar and transitional regions of the boundary layers on both the blade and endwall surfaces. The transport equations for the turbulence intermittency $(\gamma)$ and for the transition onset momentum thickness Reynolds number $\left(\operatorname{Re}_{\theta}\right)$ were discretized using a high resolution (bounded second-order upwind biased) scheme (ANSYS, 2009).

For turbine design purposes one would generally model the real engine conditions. Flow compressibility and heat transfer can be modeled using the total energy formulation in ANSYS CFX. In the present work only low-speed flows were modeled and the energy equation was not solved. 


\subsection{Domain Geometry, Blocking and Meshing Strategies}

\subsubsection{Introduction}

Prior to meshing the flow passage, important decisions were made regarding the domain shape that affect the mesh quality and solution accuracy. The following sub-section will first discuss these choices. The remaining sub-sections will then discuss the blocking and meshing strategies. The discussions in this section only pertain to structured-type meshes with hexahedral elements. A structured mesh, as opposed to an unstructured, allows the user more control over the grid density, wall spacing and overall mesh quality. Unstructured meshes will also typically lead to higher node counts and were not used in this work, although they tend to be faster to generate.

\subsubsection{Domain Geometry}

For the more fundamental investigations of Chapter 5, the flow through the flat endwall cascade is modelled for one flow passage and one half-span, taking advantage of the cascade pitchwise periodicity and spanwise symmetry, as shown in Figure 4.1 (a). The inlet and outlet boundaries were positioned at $1.50 C_{x}$ upstream and $3.0 C_{x}$ downstream of the blade leading edge. As shown, the flow passage at the inlet and outlet of the cascade is aligned with the cascade design inlet and outlet flow angles. For a hexahedral element mesh, this reduces the discretization errors by aligning the majority of the elements with primary flow direction (ANSYS, 2009). Since the design outlet flow angle of this cascade is relatively large at $-72.3^{\circ}$ the domain was flared at the outlet. This ensured the hexahedral elements near the outlet have internal angles that are greater than $10^{\circ}$ as recommend by the solver manual (ANSYS, 2009). 

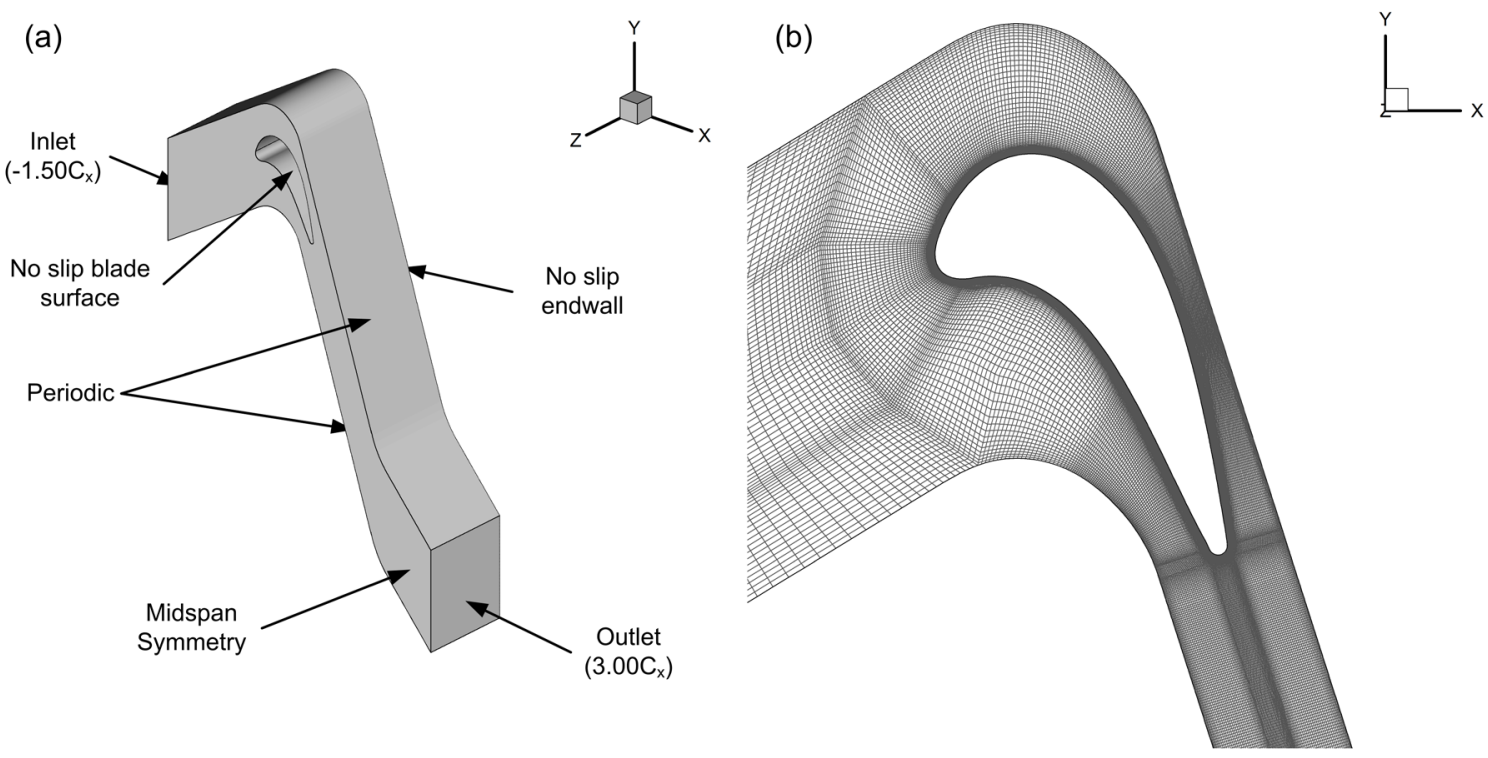

Figure 4.1: CFD domain and boundary conditions (a). CFD mesh (b)

A portion of the CFD mesh is shown in Figure 4.1 (b). As shown, the grid is orthogonal to the primary flow direction downstream of the blade trailing edge. Although this is advantageous for reducing discretization errors, this domain shape does not permit one-to-one node matching on the periodic surfaces. For this domain, if the user attempts to force node matching along these boundaries the resulting mesh quality is poor: the large outlet angle of the domain results in highly skewed elements with small internal angles. Since the nodes could not be matched, the ANSYS CFX solver then requires an interpolation scheme, referred to as a General Grid Interface (GGI) connection, to enforce the periodic boundary conditions. The drawback of this option is that the final solution may have locally unequal flow variables along the periodic boundaries. 
Figure 4.2 shows axial distributions of the total pressure coefficient $\left(C_{P 0}\right)$ extracted along the periodic boundaries at midspan $(z / h=0.5)$. For a coarse mesh, identified as Mesh 1 and shown in Figure 4.2 (a), there are significant differences between the two periodic boundaries at $x / C_{x}=2.2$. Increasing the number of nodes along the periodic boundaries improves the consistency of the flow quantities between the two surfaces. This is shown in Figure 4.2 (b) which shows results from a second mesh (Mesh 2) that has approximately three times the number of nodes. Of course, increasing the number elements comes with the penalty of increased computational time. For the CFD results of Chapter 5 this was not an important consideration as there was only one test case. However, for design applications involving multiple test cases this would have been inefficient. The domain shape was therefore altered to permit one-to-one node matching for the design level computations performed for Chapters 6 and 7.

Figures 4.3 (a) and (b) show the updated computational domain and corresponding sample mesh. The shape of the domain permits one-to-one node matching along the periodic surfaces while also maintaining high quality elements through the passage and downstream of the bladerow. The most significant advantage of this mesh is that the node count could be significantly reduced without sacrificing the computational accuracy along the periodic

(a) Mesh 1 (coarse): 38, 926 nodes on the midspan plane

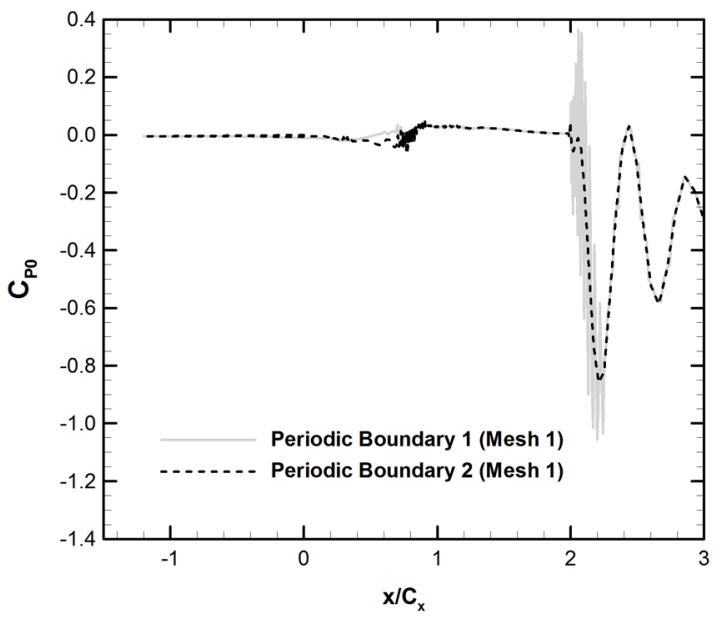

(b) Mesh 2 (fine): 111,373 nodes on the midspan plane

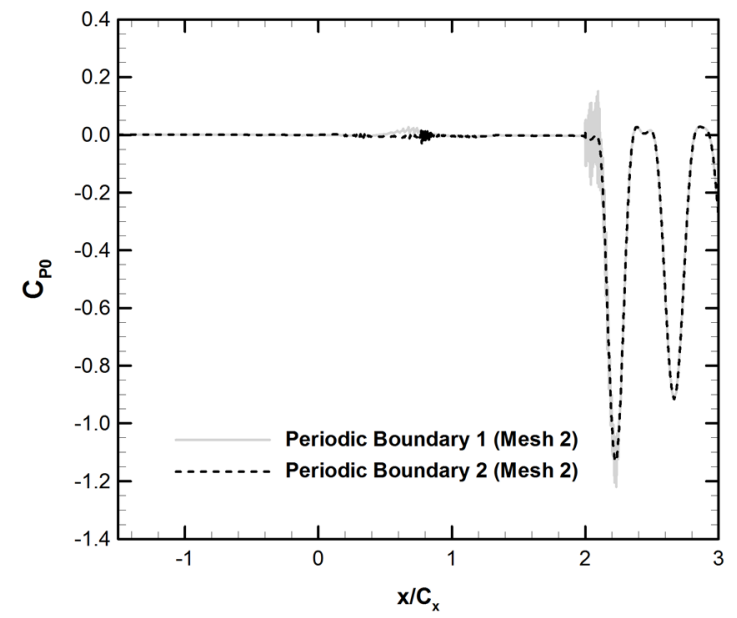

Figure 4.2: $\quad$ Total pressure coefficient $\left(C_{P \theta}\right)$ along the periodic boundaries at midspan $(\mathrm{z} / \mathrm{h}=0.5)$ for (a) Mesh 1 and (b) Mesh 2 
boundaries.

(a)

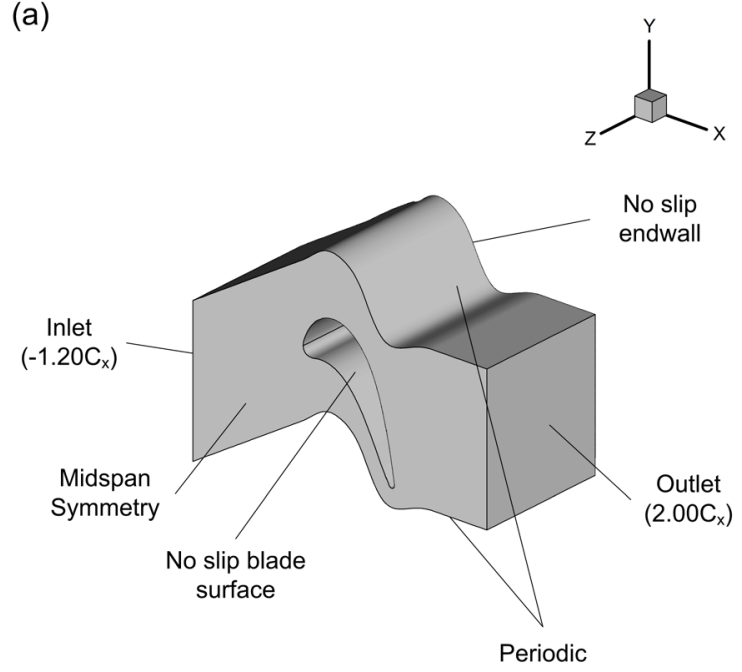

(b)
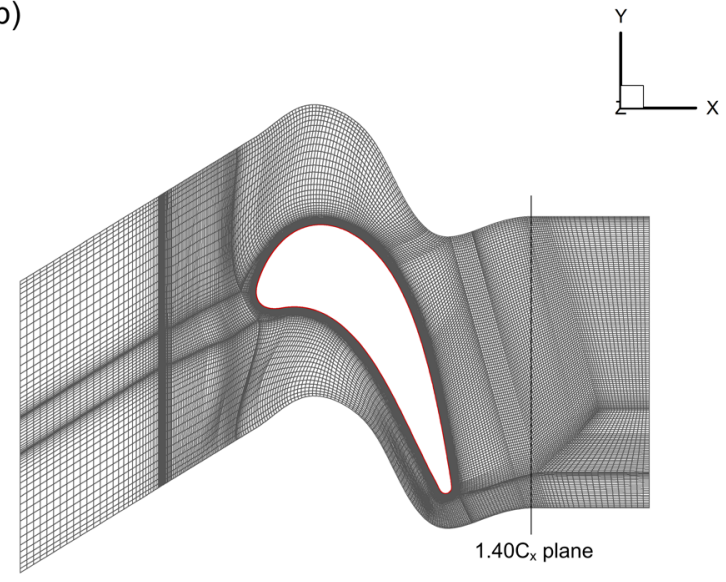

Figure 4.3: (a) CFD domain with one-to-one periodic boundaries and (b) CFD mesh

For the updated domain (Figure 4.3), the length of the domain was also reduced by altering the axial location of the inlet and outlet boundaries. Previously, as shown in Figure 4.1 (a), the inlet of the domain was located at $-1.50 C_{x}$ upstream of the blade leading edge, which is further upstream from the experimental measurement plane of $-1.20 C_{x}$. In the experiment, the inlet measurement plane shows some periodic non-uniformity upstream of the blades. Thus, the CFD inlet was originally placed further upstream than the $-1.20 \mathrm{Cx}$ location to allow this potential field to develop with the solution. As it turns out this was unnecessary and had little effect on the solution. A comparison of the results for two equal density meshes with the alternative inlet boundary locations showed negligible differences. The downstream integrated flow parameters are shown in Table 4.1. Both the in-plane and mixed-out loss coefficients and outlet flow angles show negligible differences. It should be noted that these simulations were run for fully turbulent conditions. 
Table 4.1: Summary of integrated flow parameters from the $1.40 C_{x}$ plane for two different domain inlet positions

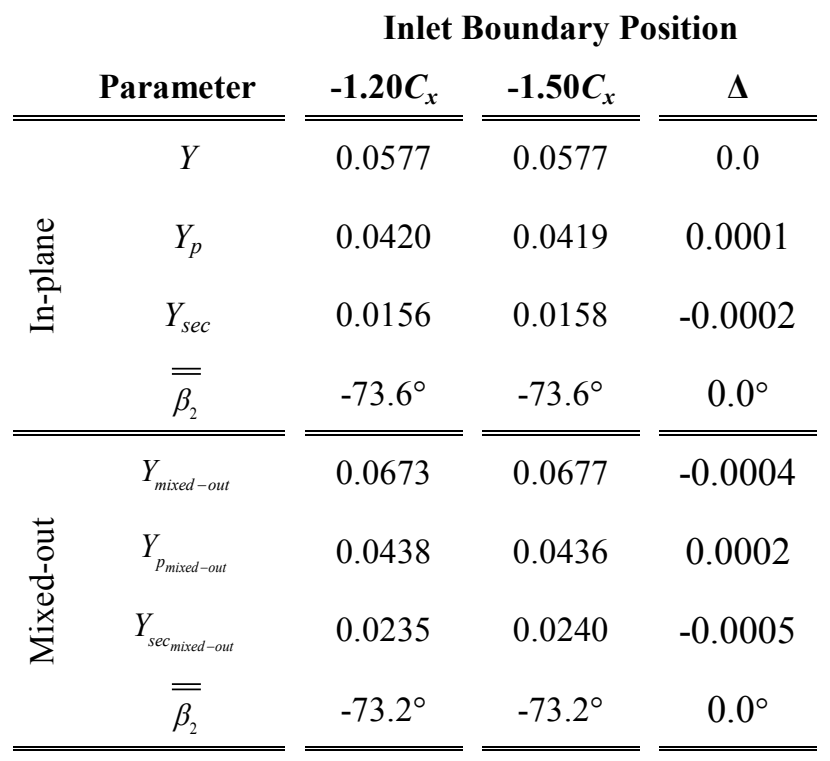

A similar test was performed for the outlet boundary location. Table 4.2 shows the integrated flow quantities for domains with the outlet boundary positioned at 1.80, 2.00, 2.50 and $3.00 C_{x}$. The respective node counts are also included. These simulations were run using the transition model, and therefore the predicted losses are different than those shown in Table 4.1. Table 4.2 shows that the position of the outlet boundary had a negligible effect on the downstream flow. The outlet boundary location was then set at $2.00 C_{x}$ for all subsequent simulations, thereby reducing the mesh size by $27 \%$.

In order to simulate the effects of the platform overlap and purge flow, the flat endwall domain of Figure 4.3 (a) was modified as shown in Figure 4.4 (a). Here, two domains were used. The first includes the main gas path, platform overlap, endwall cavity and purge slot cavity regions, as labelled. The turning vanes, previously described in Section 3.4, extend from the purge slot cavity and were modelled as a separate domain. This was done to ensure a high quality mesh through the vanes. It was not possible to match the nodes between the turning vane outlet surface and purge slot inlet surface. Therefore, the domains were connected using the GGI method described previously. Although this is an undesirable approximation, the local effects 
within the purge slot were not expected to significantly alter the physics of the purge flow and main gas-path interaction.

Table 4.2: Summary of integrated flow parameters from the $1.40 C_{x}$ plane for three different domain outlet positions

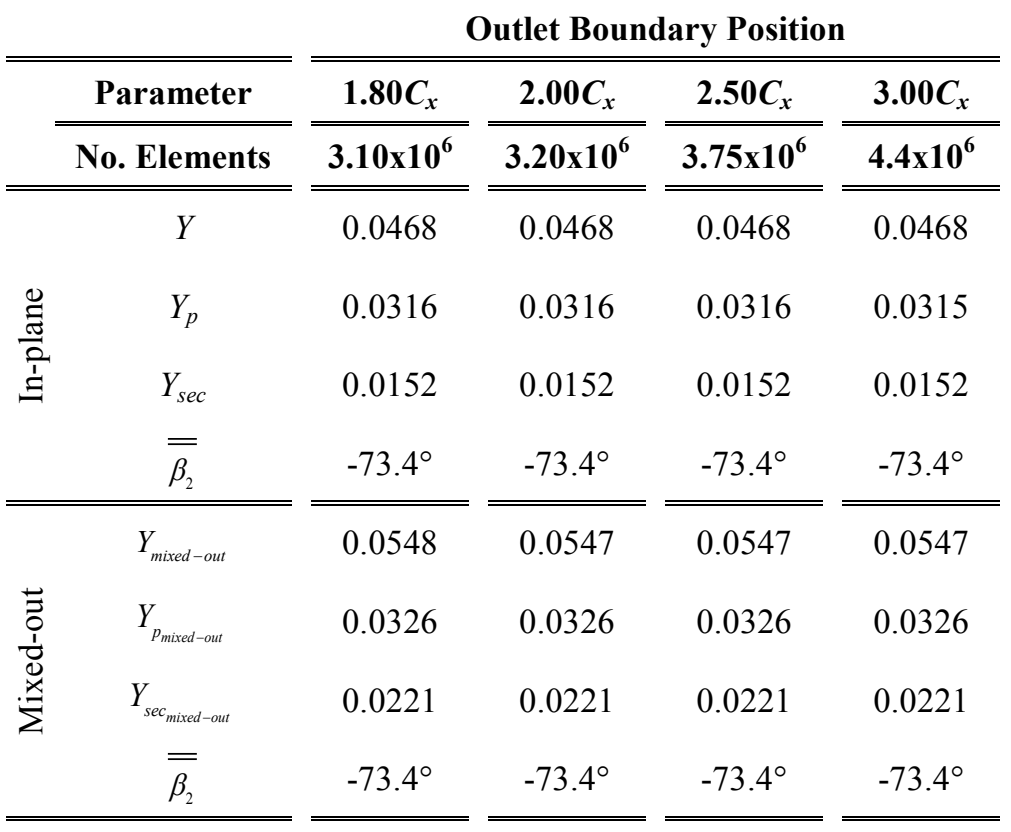

For all simulations involving purge flow the turning vane domain was required. It was not possible to prescribe a boundary condition that would accurately represent the complex flow that exits the turning vanes. More details of the flow field within the purge slot are provided in Chapter 7.

Section 3.6 describes other geometric test cases, such as the simplified platform overlaps (RAMP and STEP), the elliptical overlap and the endwall contour. For each case a new domain was created by altering the domain of Figure 4.4 (a). By using similar geometries, the initial blocking and meshing were easily adapted thereby ensuring consistency between the resulting meshes for each geometric variation. 
(a)

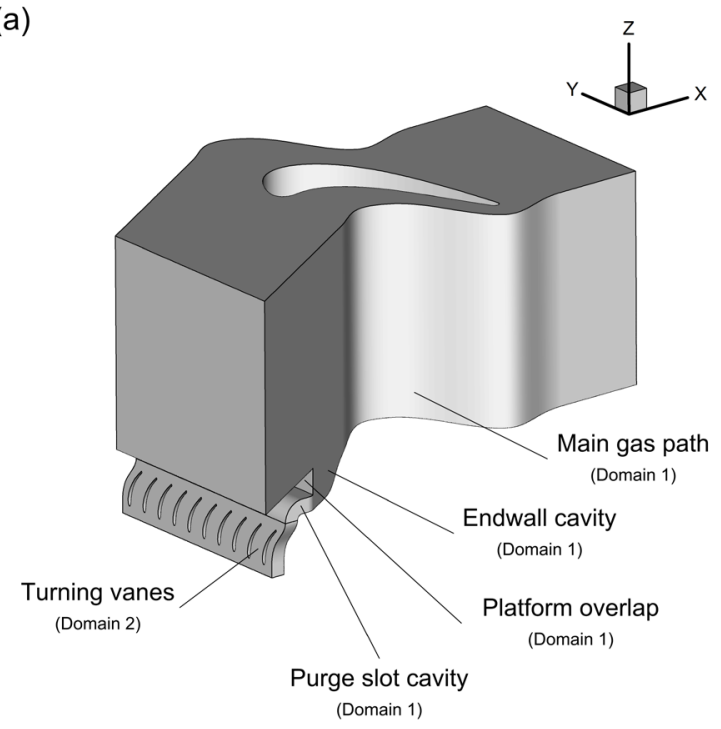

(b)

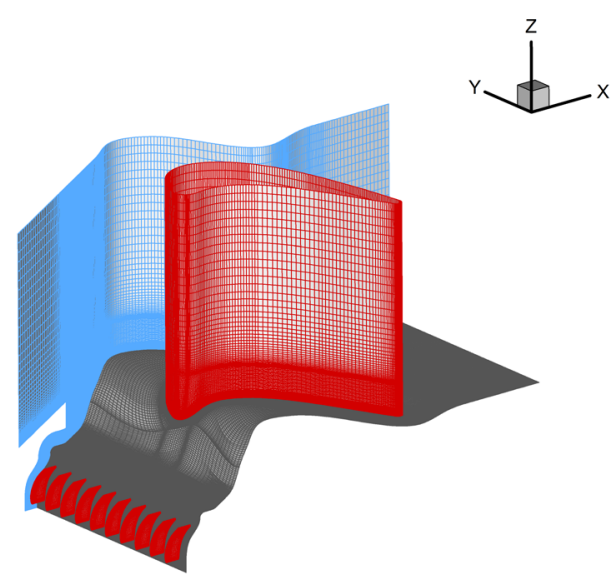

Figure 4.4: (a) CFD domain with the platform overlap geometry (b) CFD mesh

\subsubsection{Blocking and Meshing Strategies}

The structured grids of hexahedral elements were created using ANSYS ICEM CFD v12.0.1. This type of mesh was chosen in order to reduce the overall node count as well as control the wall normal expansion ratio, the grid orthogonality and the maximum/minimum element aspect ratios. Meshes were designed to conform to the requirements of the ANSYS CFX-v-12.1 solver (ANSYS, 2009) including those of the CFX transition model (Langtry et al., 2006; Menter et al., 2006). The requirements specified in the ANSYS CFX-v-12.1 manual under the section entitled "Mesh Visualization Advice" are summarized as follows for hexahedral elements:

- Edge Length Ratio: the ratio of an element's longest to shortest lengths $<100$

- Min Face Angle: the minimum angle between adjacent faces within an element $>10^{\circ}$

- Element Volume Ratio: volume ratio of neighbouring elements $<5$

- Connectivity Number: the number of elements connected to a single node $<24$

- Near wall spacing: the first node height should yield $\mathrm{y}+\approx 1$ and the expansion ratio within the boundary layer should be 1.1 (Langtry et al., 2006; Menter et al., 2006) 
Structured grids were first created by sub-dividing the domain into several "blocks". The block edges and faces are associated with the nearby curves and surfaces of the domain. During mesh creation, parameters are assigned to the edges, such as the number of nodes and node spacing, and are projected to the associated geometry. An advantage of this meshing method, is that the mesh is computed quickly allowing the user to visualize the result of small changes to the blocking parameters. Also, the same blocking may be re-associated to different domains with similar geometric dimensions. This ensured consistency between the meshes for the different cases.

An example of the blocking used for the design level meshes is shown in Figures 4.5 (a) and (b). The size and position of the blocks were specified manually. As shown, the block edges extend perpendicular to the geometric surfaces. This ensures proper node projection onto the surface and prevents skewing of the near surface elements. The edges of each block are assigned parameters such as the number of nodes and node spacing. In general, node spacing was prescribed by a geometric relation with an expansion ratio of approximately 1.1. The edge parameters were typically first assigned to edges that project from no-slip surfaces in order to control the $y+$ value. The expansion ratio $(\approx 1.1)$ and element volume ratio $(<5)$ then dictate the choice of the edge parameters for the blocks positioned away from these surfaces.

The blocking shown in Figure 4.5 (a) has two distinct regions. Near the blade surface an ogrid is used while away from surface standard three dimensional blocks are applied. The o-grid enhances the control over the near wall mesh and ensures elements through the boundary layer are largely orthogonal to the surface. Higher quality elements in the boundary layer reduce discretization errors and improve the solution accuracy. Downstream of the blade trailing edge the blocks are positioned such that the mesh elements are oriented with the design outlet flow angle. Further downstream, the blocks labelled "periodic wake region" are also aligned with the primary flow direction. 
(a)
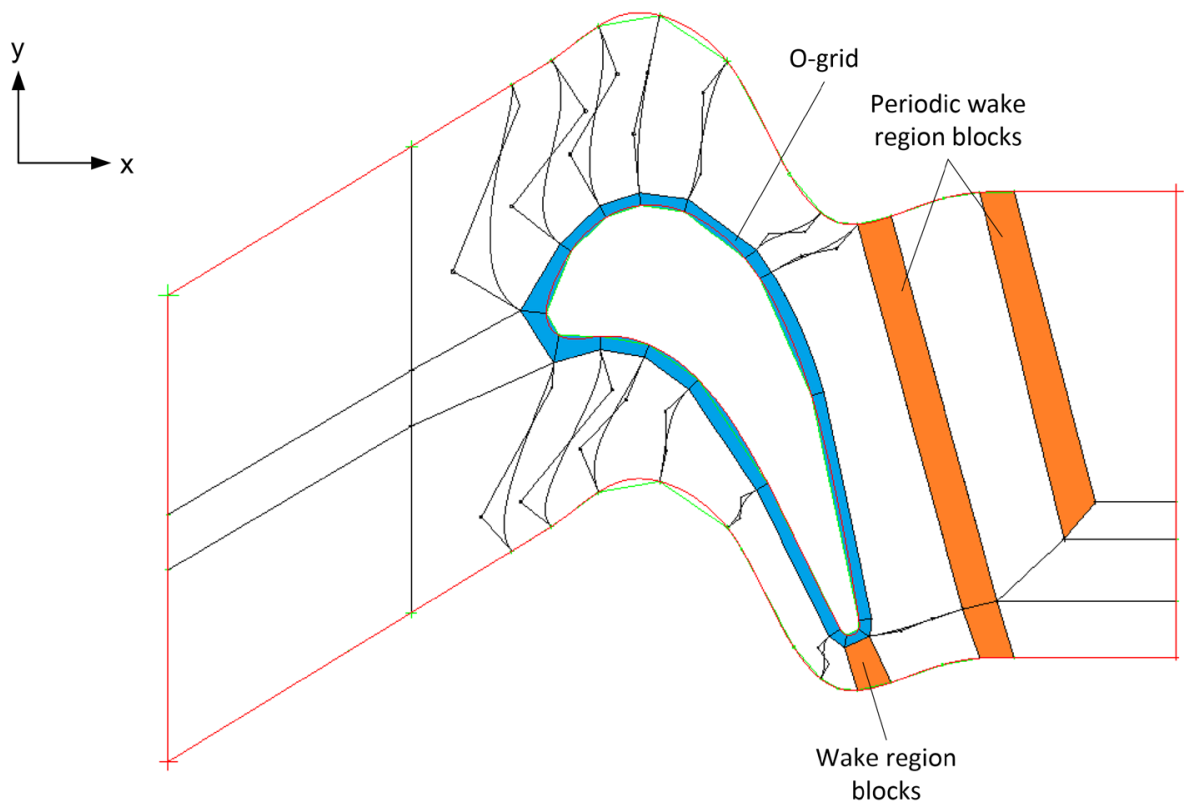

(b)
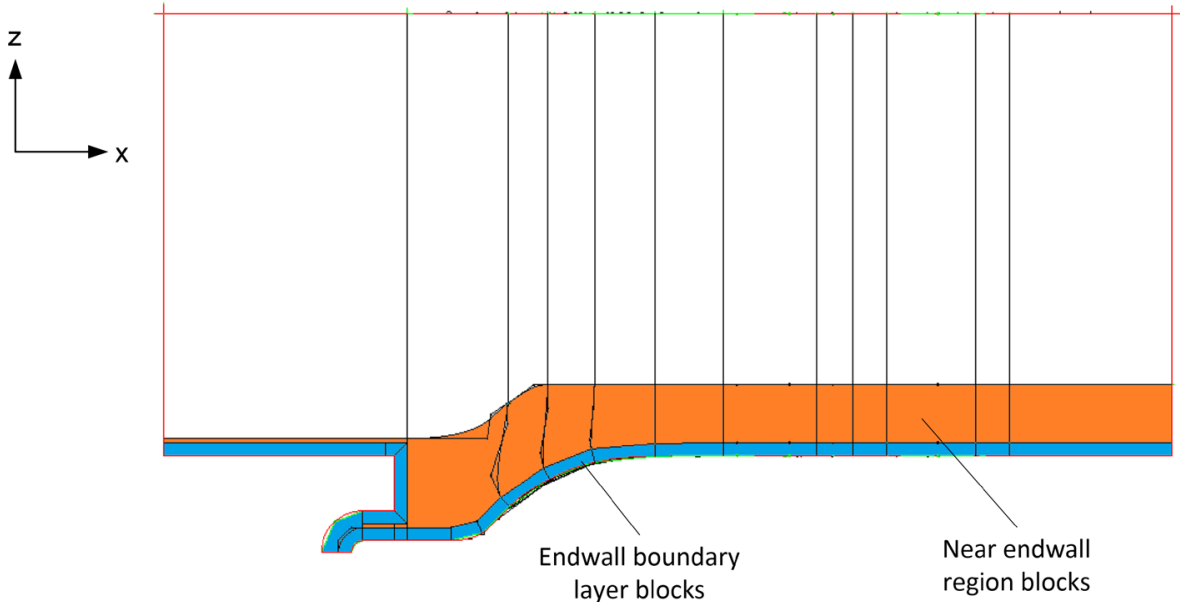

Figure 4.5: Example of domain blocking

Figure 4.5 (b) shows the blocking viewed along the pitchwise direction. Here, small blocks similar to the blade surface o-grid are applied at the endwall and platform overlap surfaces. Without these, the curvature of the endwall cavity geometry would compromise the orthogonality of the near endwall mesh. These blocks were also generated pre-emptively for the later domains with larger changes to the endwall curvature, such the cases with endwall contouring or the 
elliptical platform overlap. The added control improved node projection and therefore quality of the near endwall elements. Again, the node spacing was first set at the endwall surface to ensure a $\mathrm{y}+$ of about 1 and the geometric expansion ratio was set to about 1.1.

The details of the blocking parameters for each mesh used in the grid independence study are described in Section 4.6.

\subsection{Boundary Conditions}

\subsubsection{Introduction}

An example of the domain boundary conditions is shown in Figure 4.6 for the RF (Rectangular Flat) configuration. The solid wall surfaces have no-slip conditions. The repeating blades of the cascade are simulated with one-to-one periodic boundaries and a symmetry boundary condition is applied to the mid-span plane. As mentioned previously, the turning vane and main gas-path domain are interfaced using a GGI connection. The purge flow inlet boundary was specified as a constant mass flow condition. Depending on the test case, the actual mass flow value was computed from the desired mass flow ratio $(\phi)$. To prevent round-off errors during the computations, the solution uses relative pressures throughout. In this case the pressure reference was set to standard atmospheric pressure, $P_{r e f}=101,325 \mathrm{~Pa}$. Thus, the outlet boundary condition is specified as an average static pressure $\left(P_{\text {out }}=0 \mathrm{~Pa}\right)$ relative to the reference value.

Because of the focus on the endwall flows, the inlet boundary condition is important to the solution accuracy. Thus, the following subsection will describe the procedure for generating and applying the inlet boundary conditions that are most representative of the experimental conditions. 


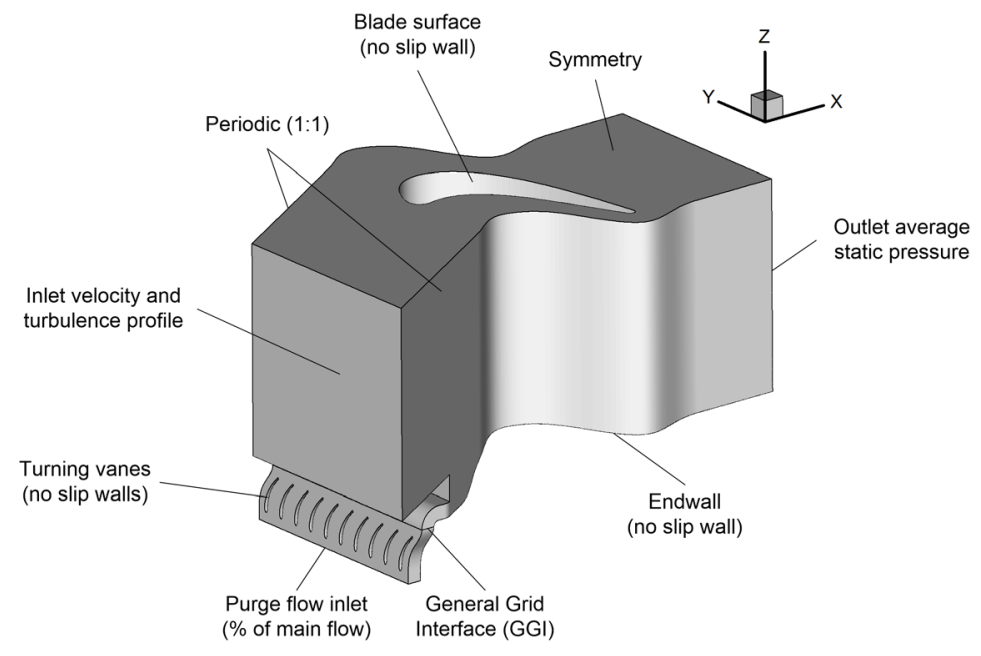

Figure 4.6: Boundary conditions applied to domain surfaces

\subsubsection{Definition of the Mainstream Inlet Boundary Conditions}

The inlet boundary conditions are specified using velocity and turbulence distributions at the inlet location. The velocity distribution was specified from the experimentally measured velocity profile, oriented at the design inlet flow angle of $-31.5^{\circ}$. An example of the inlet boundary layer distribution is shown in Figure 5.1 of Section 5.4. The experimental boundarylayer parameters are shown in Table 4.3.

Table 4.3. Summary of boundary layer parameters

\begin{tabular}{lcccc}
\hline \hline Boundary layer thickness $\left(u / u_{\mathrm{e}}=0.995\right)$ & & $\delta / h$ & & 0.092 \\
Displacement thickness $(\mathrm{mm})$ & & $\delta^{*}$ & & 2.13 \\
Momentum thickness $(\mathrm{mm})$ & & & 1.62 \\
Boundary layer shape factor & & $H_{S F}$ & & 1.32 \\
Free stream turbulence intensity & & $T u_{l o c}$ & & $3.3 \%$ \\
Free stream integral length scale & & $\Lambda / C_{x}$ & 0.25 \\
\hline \hline
\end{tabular}

The inlet turbulence conditions are defined by spanwise profiles of turbulence kinetic energy $(k)$ and specific turbulence dissipation rate $(\omega)$. In a two-equation $k$ - $\omega$ based model these two parameters also define the integral length scale $(\Lambda)$, eddy viscosity $\left(v_{T}\right)$ and turbulence 
dissipation rate $(\varepsilon)$. Since the free stream (midspan) values of the length scale $(\Lambda)$ and turbulence intensity $\left(T u_{l o c}\right)$ were measured experimentally, the turbulence profiles were specified using these parameters. The solver then determines $k$ and $\omega$ according to Equations 4.1 and 4.2 respectively (ANSYS, 2009).

$$
\begin{gathered}
T u_{l o c}=\sqrt{2 / 3 k} \\
\Lambda=\frac{k^{\frac{1}{2}}}{C_{\mu} \omega}
\end{gathered}
$$

where $C_{\mu}$ is a non-dimensional constant.

Although the midspan turbulence quantities were measured experimentally, the full spanwise profile was not. Thus, the inlet turbulence profiles were generated by simulating the growth of the boundary layer upstream of the cascade. Separate simulations were performed with a domain, referred to as the pre-domain, corresponding to the streamtube approaching a single bladerow passage. For each solution, the predicted boundary layer was compared to the experiment using the parameters and free-stream values given in Table 4.3. By iteratively adjusting the inlet conditions for the pre-domain, many different boundary layers were generated and analyzed. In practice, it was possible to generate a boundary layer that was well matched to the experimental one with the exception of the free-stream length scale. Further iterations to improve the length scale would tend to alter the other boundary layer parameters. Therefore, the $\omega$ profile corresponding to best matched boundary layer was scaled such that the free-stream length scale would match the experimentally determined value. Examples of the nondimensional profiles of turbulence intensity and length scale are shown in Figure 4.7. 


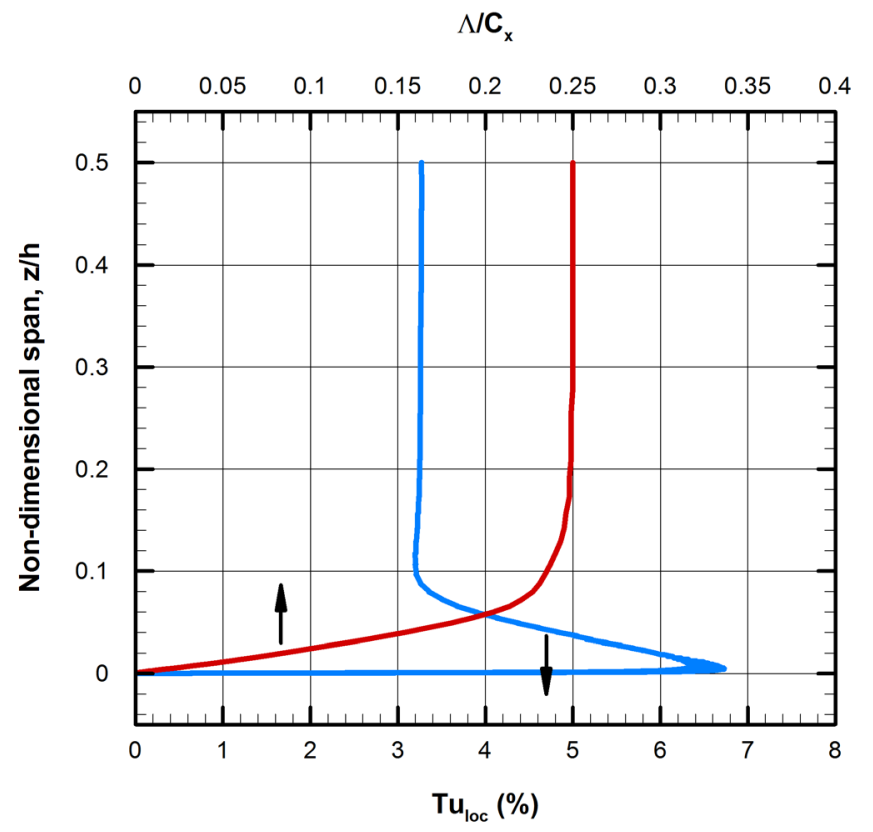

Figure 4.7: Inlet turbulence boundary condition profiles

\subsection{Judging Solution Convergence}

Solution convergence was first judged based on the normalized residuals of the solutions of the mass, momentum and turbulence equations. For a given flow variable $\phi$, the ANSYS CFX solver normalizes the raw residual $\left(r_{\phi}\right)$ of each linearized equation according to:

$$
\tilde{r}_{\phi}=\frac{r_{\phi}}{a_{p} \Delta \phi}
$$

where $a_{p}$ is a representative control volume coefficient and $\Delta \phi$ is representative of the range of the variable in the domain. The exact formulation of $a_{p}$ and $\Delta \phi$ is not given in the CFX manual.

For steady-state simulations, the most conservative approach of judging convergence is to ensure that the maximum (MAX) normalized residuals throughout the domain are below a desired threshold. In some cases, however, small unsteady disturbances are resolved in the 
solution. These can cause this approach to yield MAX residuals that do not converge to the desired threshold. This is misleading if these localized effects have a negligible effect on the overall physics of the solution.

An alternative approach is to monitor the root mean square (RMS) of the normalized residuals throughout the domain. It was found that this approach, along with observing other flow parameters, such as the integrated loss coefficient $\left(Y^{\prime \prime}\right)$, provided the best indication of solution convergence. An example of a typical convergence history is shown in Figure 4.8 for the RF 125 case. The RMS and MAX residuals are plotted separately in Figures 4.8 (a) and (b) respectively. As shown, beyond 300 timesteps the RMS residuals approach near constant values that are less than $10^{-6}$. The only exception is the intermittency residual (RMS Intermit) from the $\gamma-\operatorname{Re}_{\theta}$ transition model which reaches a near constant level of $2 \times 10^{-4}$ at about 225 timesteps. It was not possible to reduce the turbulence intermittency residuals in any of the cases examined. Adjustments were made, with limited success, to the grid density, physical timestep, the adaptive timestepping method and the initial guess. Only by observing the time history of the integrated flow parameters and as well as visualizing the measurement planes at different time steps could the solution be deemed converged.

Figure 4.8 (a) shows the time history of the integrated loss coefficient at the domain outlet plane $\left(2.00 C_{x}\right)$. It clearly converges to a value of 0.060 after approximately 250 timesteps. Figures 4.9 (a) and (b) show planar distributions of the total pressure coefficient $\left(C_{P 0}\right)$ extracted from the $1.40 C_{x}$ location for the $250^{\text {th }}$ and $500^{\text {th }}$ timestep. There are no obvious differences between the two data sets. Other parameters such as the streamwise vorticity and secondary kinetic energy coefficients also showed negligible differences. It therefore appears that the solution is converged after about 250 timesteps. 
(a)

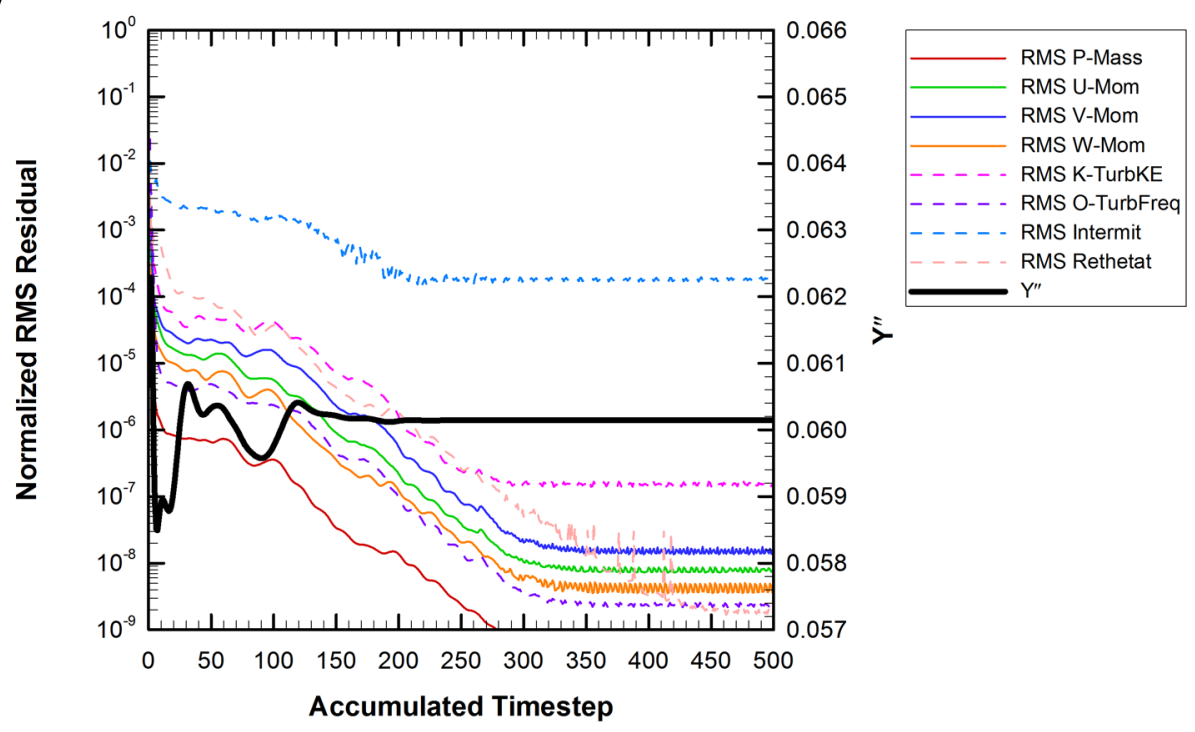

(b)
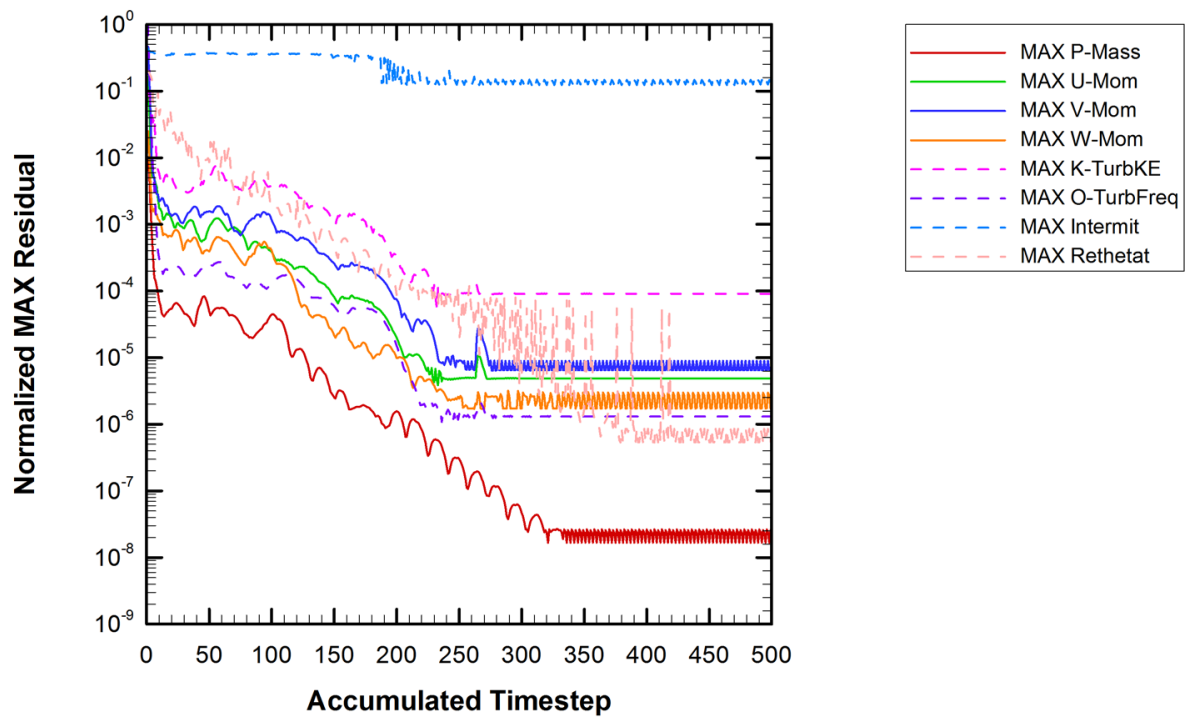

Figure 4.8: Example of the RMS (a) and MAX (b) residuals for the RF 125 case

As a final check, the individual elements with high intermittency residuals were visualized in three dimensions (not shown here). Elements with high values were localized and could not be attributed to any particular flow features. For this case, the number of nodes with intermittency residuals above $10^{-6}$ was 2733 , or $0.08 \%$ of entire domain. Therefore, further investigation into 
the convergence of the intermittency residuals did not seem necessary and the overall solution convergence was deemed to be satisfactory.

The corresponding MAX residuals for this case are shown in Figure 4.8 (b). The mass and momentum equations are sufficiently converged beyond 350 timesteps with residuals below $10^{-5}$ : one order of magnitude greater than RMS values. All of the turbulence parameters also have residuals below $10^{-5}$ with the exception of the turbulence intermittency (as described above) and the turbulence kinetic energy (k-TurbKE). Similar to the intermittency parameter, the number of elements with high k-TurbKE residuals is a small percentage of the entire domain. Only 160 nodes of the entire domain have k-TurbKE residuals above $10^{-5}$ and only 785 are above $10^{-6}$. These correspond to $0.005 \%$ and $0.02 \%$ of the domain respectively. Therefore, similar to the intermittency, these locally high residuals were not a concern relative to the overall solution convergence.

(a) Timestep 250

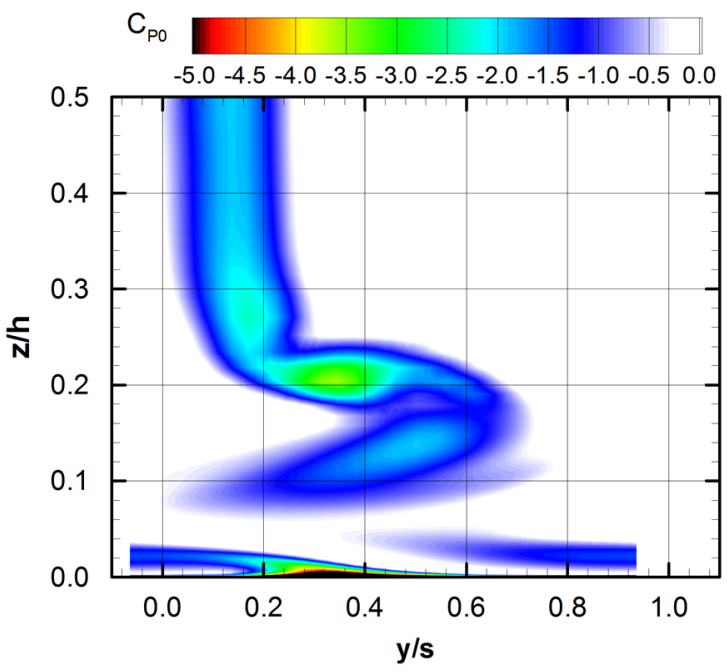

(b) Timestep 500

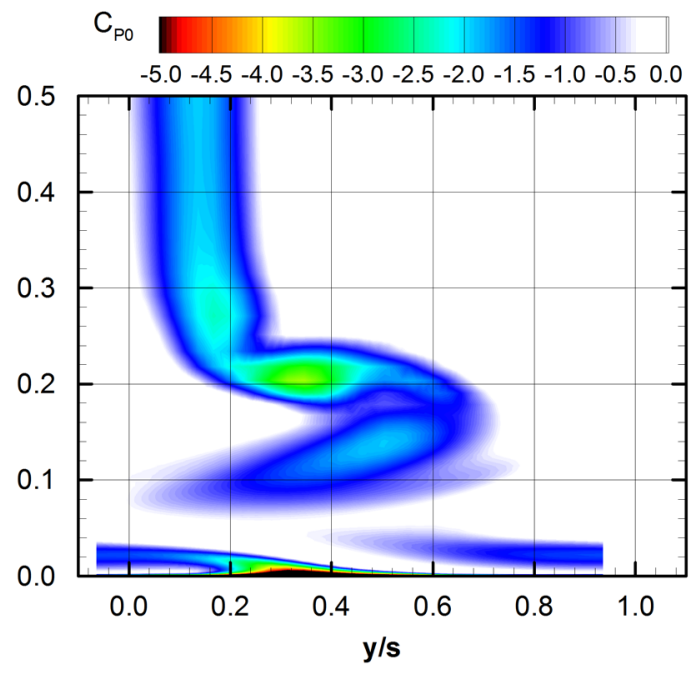

Figure 4.9: Total pressure coefficient $\left(C_{P 0}\right)$ at the $1.40 C_{x}$ plane output at $250^{\text {th }}$ (a) and $500^{\text {th }}(\mathrm{b})$ timesteps

The analysis shown here is a representative example of the convergence analyses applied to all simulations. Of course, each case is different and there were some other convergence 


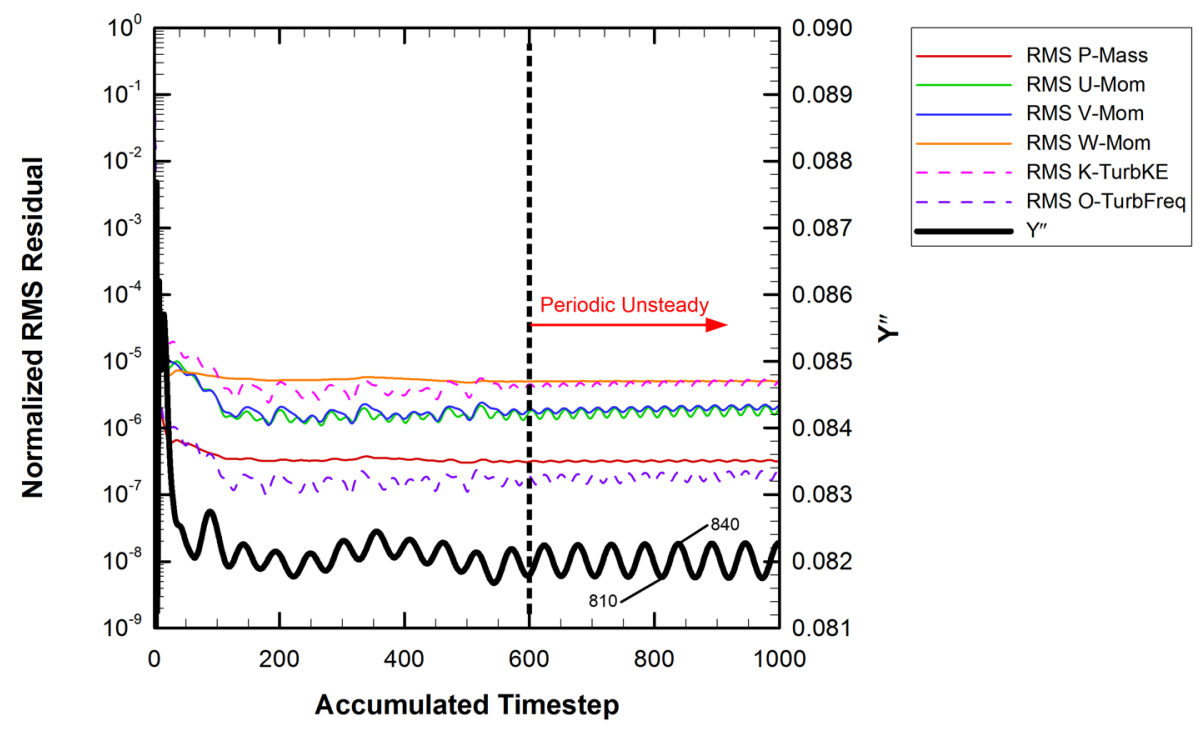

Figure 4.10: Example of RMS residuals for a case with periodic unsteady oscillations

problems not yet mentioned. One in particular is the development of periodic unsteady flow; manifested as periodic fluctuations in both the residuals and integrated flow quantities. It is the author's experience that over-refinement of the grid can lead to the development of small periodic oscillations which then prevent the solution from reaching steady-state. Figure 4.10 shows the RMS residuals of such a case. Note that this example is for a fully turbulent simulation. As shown, the residuals and the integrated loss coefficient oscillate with a constant period beyond about 600 timesteps. If the flow is genuinely unsteady then a time resolved analysis should be performed. However, transient simulations significantly increase the required computational resources and may not be necessary depending on the information required from the simulation. In cases where converged solutions could not be obtained, like that of Figure 4.10, the following questions were posed:

1) Are the oscillations a numerical effect or genuine unsteadiness in the flow?

2) If they are genuine unsteadiness, to what degree is the overall solution affected?

3) If the mesh is coarsened and the unsteadiness is reduced how are the physics affected? 
Question (1) was addressed by altering the physical timestep of the solution. If the oscillations are in fact caused by periodic unsteadiness in the flow, then the period of the oscillations should be independent of the physical timestep. For example, if the original timestep is halved, then to complete a new oscillation it should take twice the number of timesteps. For the case shown in Figure 4.10, it was found that the oscillations were caused by inherent physical unsteadiness in the flow.

To determine if the unsteadiness was localized or more widespread through the domain, the residuals were visualized in three-dimensions. Figure 4.11 shows two views of the full domain. The element volumes are coloured if the local absolute residuals are greater than $1 \times 10^{-5}$.

(a)

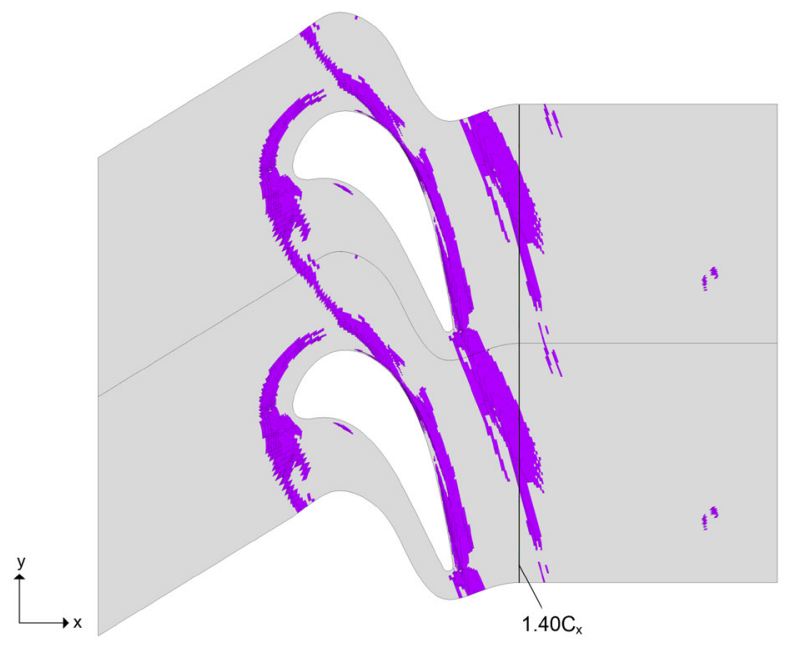

(b)

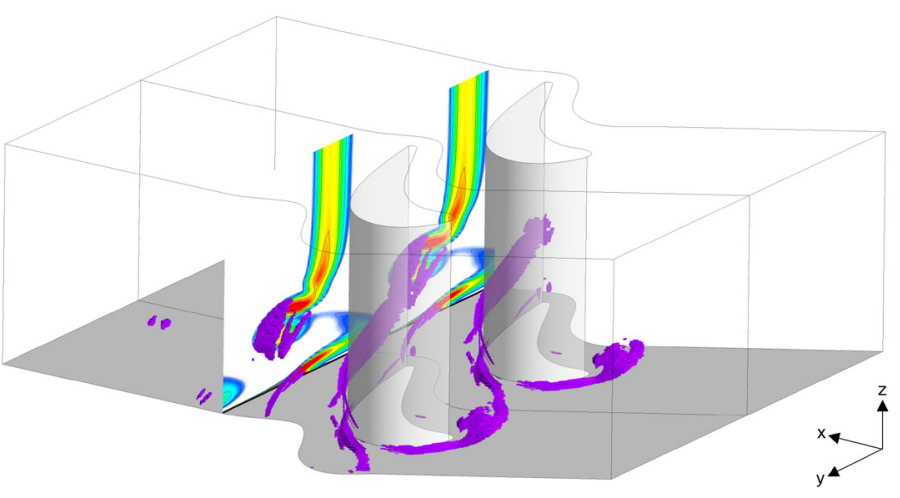

Figure 4.11: Example of RMS residuals for a case with periodic unsteady oscillations 
Distributions of the total pressure coefficient at the $1.40 C_{x}$ plane are also shown. The coloured elements appear to originate upstream of the blade, near the saddle point, where the inlet boundary layer separates from the endwall. This region then appears to propagate through the passage. First, it follows the trajectory of each leg of the horseshoe vortex and then progresses through the passage and counter vortices. The path shown suggests that the secondary flow has some tendency to periodic unsteady oscillations.

To further investigate the effect of the unsteadiness on the downstream flow field, data were extracted from the $1.40 C_{x}$ plane at two different timesteps, 810 and 840 . These timesteps corresponds to a trough or peak in the time history of the outlet loss coefficient, as indicated in Figure 4.10. In general, the differences between the downstream flows at two timesteps are subtle. Therefore, to compare the data, point-by-point differences were taken at the $1.40 C_{x}$ plane for different flow parameters. Figure 4.12 shows the differenced total pressure coefficient, defined by:

$$
\left|\Delta C_{P 0}\right|=\left|C_{P 0,840}-C_{P 0,810}\right|
$$

where the subscripts 840 and 810 indicate the different timesteps. By definition, this coefficient represents a percentage of the cascade inlet dynamic pressure $\left(q_{1, \mathrm{CL}}\right)$. As a spatial reference, Figure 4.12 also shows contour lines of the streamwise vorticity coefficient. Positive values are indicated by the solid lines and negative values by the dashed lines. The locations of the secondary vortices are also indicated.

Figure 4.12 shows that there are significant differences between the two timesteps. The largest differences, about $14 \%$ of the inlet dynamic pressure, are adjacent to the passage vortex at about $y / s=0.60$ and $z / h=0.15$. Other regions with significant differences are local to both the counter and corner vortices, as indicated. The differences between the timesteps shown here only indicate differences in the in-plane distribution of the total pressure losses. This should not be confused with differences in the predicted loss levels. For example: integrating the loss distributions from each timestep yields overall loss-coefficients that are within $\pm 0.1 \%$. Thus, the overall loss levels are equal but the in-plane distributions are not. This is further demonstrated in 
Figure 4.13 which shows that the pitchwise mass-averaged losses are nearly identical. Only minor differences are seen in the vicinity of the passage vortex at the spanwise location of $z / h=0.14$, as indicated

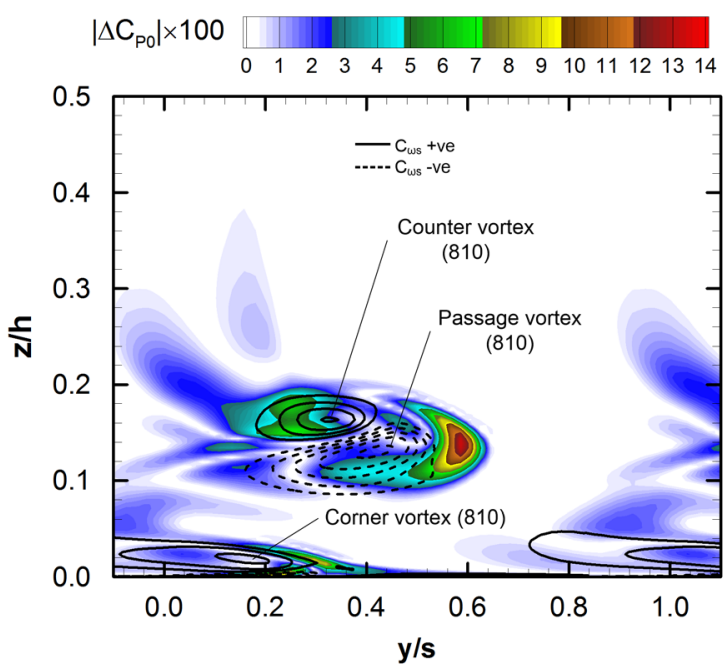

Figure 4.12: Point-by-point differences of the total pressure coefficients $\left(C_{P 0}\right)$ from the $810^{\text {th }}$ and $840^{\text {th }}$ timesteps at the $1.40 C_{x}$ plane

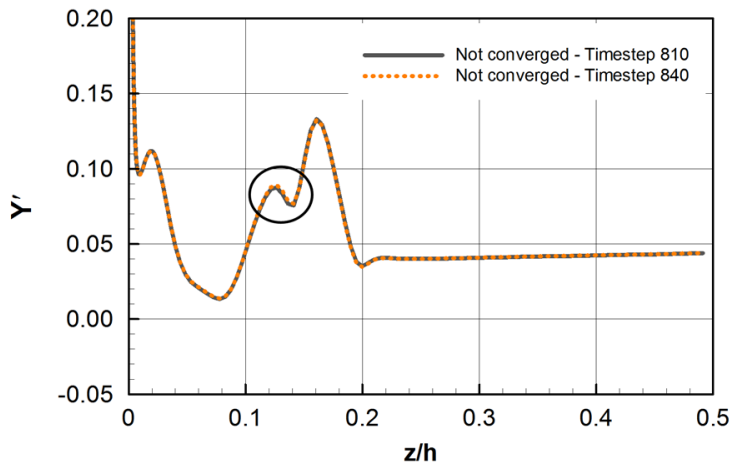

Figure 4.13: Pitchwise integrated loss coefficient for the $810^{\text {th }}$ and $840^{\text {th }}$ timesteps at the $1.40 C_{x}$ plane 
Since the differences in the integrated losses between the timesteps were so insignificant, performing transient solutions for each case was largely unnecessary. Instead, alternative approaches to arrive at a converged steady-state solution were investigated.

Figure 4.11 showed that the unsteadiness originates near the endwall, upstream of the blade leading edge, at the saddle point location. To avoid this unsteadiness, the mesh was coarsened in this near endwall region in an attempt to artificially dampen the oscillations. An identical grid with the same number of total nodes but with an increased first node height on the endwall was used to obtain a fully converged solution. The corresponding convergence history is shown in Figure 4.14. Essentially, the maximum endwall $\mathrm{y}+$ was doubled from about 1.2 to about 2.4. Although this value is beyond that recommended for the CFX solver, this appeared to have little effect on the overall solution. Figure 4.15, shows the pitchwise mass-averaged losses for the converged solution overlaid on the previously unconverged timesteps. The integrated losses of Figure 4.15 show only minor differences. It appears that for this cascade the slight increase to first node height was a necessary compromise to obtain a converged solution. Therefore, for the design level simulations of Chapters 6 and 7, an increased first node height on the endwall surface was applied.

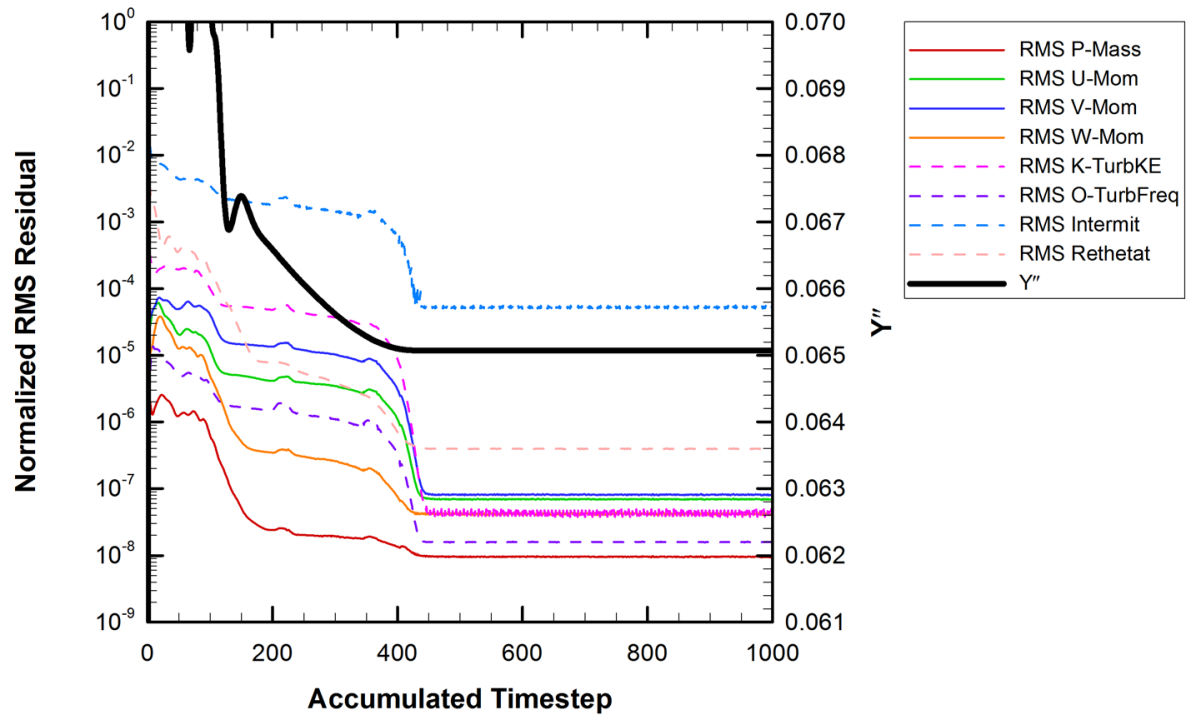

Figure 4.14: RMS residuals for the modified near endwall grid 


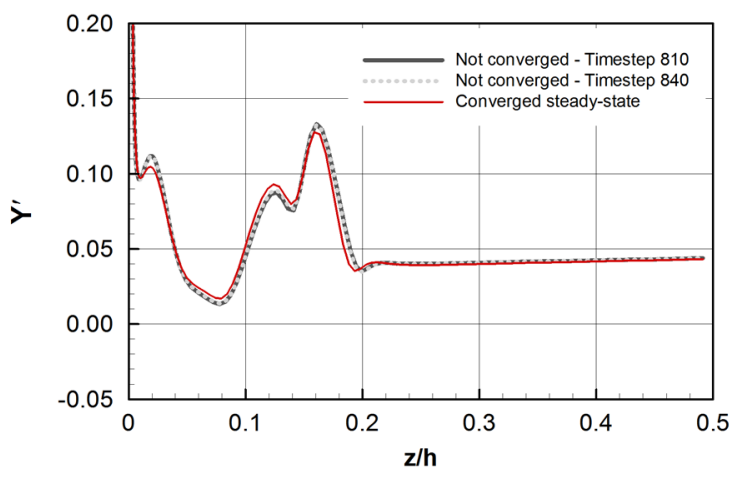

Figure 4.15: Pitchwise integrated loss coefficient for the converged steady-state simulation at the

$$
1.40 C_{x} \text { plane }
$$

\subsection{Grid Independence Studies}

\subsubsection{Introduction}

This section will show example results from grid sensitivity studies for the design level simulations used in Chapters 6 and 7. The purpose of this section is to demonstrate the grid study procedures as well the sensitivity of the simulations results. The results shown here are from simulations with a Reynolds number $\left(\operatorname{Re}_{1}\right)$ of 75,000 based on the inlet midspan velocity and axial chord. A similar grid-independence study was conducted for the more fundamental simulations presented in Chapter $5\left(\operatorname{Re}_{1}=50 \mathrm{k}\right)$, but the results are not included here for brevity.

For both cases, the grid independence study began by first investigating the flow for a thin two-dimensional mid-span slice - effectively simulating the two-dimensional profile flow. Downstream distributions of the total and dynamic pressures as well as the outlet flow angle were used to compare the simulations from the different grids. After achieving grid-independent results, the two-dimensional grid was extruded over one half-span, and full three-dimensional study was performed. Following this, a final three-dimensional grid study was performed for the case involving the platform overlap and endwall cavity geometries. 


\subsubsection{Two-dimensional Grid Study}

Table 4.4 summarizes the grid sensitivity parameters for the two-dimensional gridindependence study. The six grids are numbered G01 through G06. The relevant grid parameters are: the number of trailing edges nodes (a), the number of o-grid nodes perpendicular to the blade surface (b), the first node height away from the blade surface (c) and the corresponding wall normal expansion ratio (d). After setting these values (a through d), the remainder of the mesh body is then generated by adjusting the nodes counts of the interior blocks such that the mesh conforms to the requirements of the solver. As mentioned in Section 4.3.3 the solver requires:

- element volume ratios $<5$

- element aspect ratio $<100$

- $\quad$ minimum face angles $>10^{\circ}$

Table 4.4 also includes the total number of nodes in a two-dimensional cross-section of the grid (e) and the node density on that plane (f). The latter provides an overall average value of the mesh density that is independent of the domain length. This was necessary because the twodimensional grid study and the domain length sensitivity study were investigated concurrently. As previously mentioned in Section 4.3.2, the length of the domain had negligible effects on the simulation results. Thus, it became unnecessary to continue the grid study with excessively long domains.

Table 4.4: Summary of the 2D grid sensitivity parameters
(a)
(b)
(c)
(d)
(e)
(f)

\begin{tabular}{ccccccc} 
Grid & $\begin{array}{c}\text { Trailing } \\
\text { Edge Nodes }\end{array}$ & $\begin{array}{c}\text { O-grid } \\
\text { Nodes }\end{array}$ & $\begin{array}{c}\text { O-grid first node } \\
\text { height }(\mathbf{m m})\end{array}$ & $\begin{array}{c}\text { O-grid expansion } \\
\text { ratio }\end{array}$ & $\begin{array}{c}\text { Nodes / } \\
\text { 2D plane }\end{array}$ & $\begin{array}{c}\text { Nodes / } \\
\mathbf{m m}^{2}\end{array}$ \\
\hline \hline G01 & 5 & 10 & $2.0 \times 10^{-5}$ & 2.4 & 3262 & 0.15 \\
G02 & 10 & 19 & $1.0 \times 10^{-5}$ & 1.3 & 11989 & 0.53 \\
G03 & 8 & 23 & $5.0 \times 10^{-6}$ & 1.3 & 25604 & 0.85 \\
G04 & 10 & 23 & $5.0 \times 10^{-6}$ & 1.3 & 30976 & 1.02 \\
G05 & 13 & 28 & $5.0 \times 10^{-6}$ & 1.1 & 38283 & 1.27 \\
G06 & 18 & 28 & $5.0 \times 10^{-6}$ & 1.1 & 52142 & 1.72 \\
\hline \hline
\end{tabular}


Grid G01 was the initial mesh and represents the starting point for the grid independence study. It is also the coarsest grid, as shown in Table 4.4. The other grids, G02 through G06, then represent systematic refinements of the parameters (a) through (f).

The number of trailing edge nodes, parameter (a), dictates the grid density within the downstream wake region. An inadequate number of trailing edge nodes will affect the smoothness and accuracy of the downstream wake. Pitchwise distributions of the total pressure coefficient $\left(C_{P 0}\right)$, dynamic pressure coefficient $\left(C_{q}\right)$ and the outlet flow angle $(\beta)$ are shown in Figure 4.16. Sub-figures (a) through (c) represent data from the $1.20 C_{x}$ location while (d) through (f) are from the $1.40 C_{x}$ location. In Figure 4.16, the distributions corresponding to G01 are inconsistent with those from the other grids. At both axial locations, G01 reports lower total and dynamic pressure coefficients. The outlet flow angle $(\beta)$ is also consistently higher, by about $0.5^{\circ}$, over the entire pitch. The distributions from G02 through G06 show only small localized differences.

In Figure 4.16 (a), there is a region of particular interest labelled "overshoot". Here, the $C_{P 0}$ value is greater than 0 indicating that the local total pressure is higher than the inlet freestream value. This is not physically possible and appears to be an artifact caused by inadequate spatial discretization around the blade trailing edge. Figure 4.17 shows a zoomed view of the trailing edge region. The colour flood contours represent the $C_{P 0}$ values and the grid is overlaid to show the spatial discretization. The positive $C_{P 0}$ values appear to originate from very high total pressures that are just downstream of the blade trailing edge, within the pressure side separated shear layer. Further downstream, at the $1.20 C_{x}$ and $1.40 C_{x}$ positions, the total pressure overshoots decrease slightly through viscous mixing but still yield positive $C_{P 0}$ values. These overshoots are undesirable and should be minimized to reduce the effects on the integrated profile losses.

Referring to Table 4.4 and Figure 4.16 (a) and (d), it appears that increasing the number of trailing edge nodes reduces these overshoots. Grid G06 has the highest number of trailing edge 

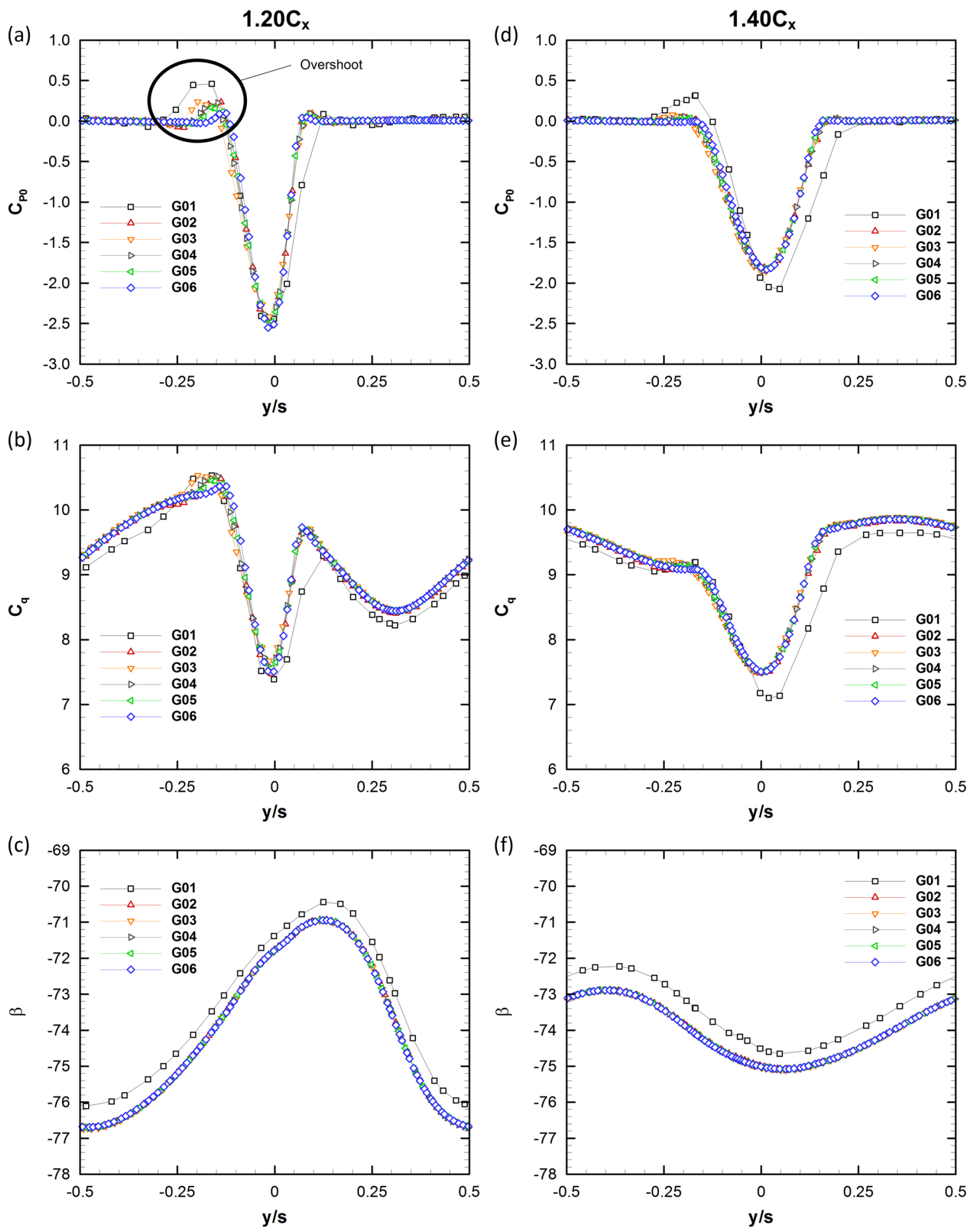

Figure 4.16: Two-dimensional distributions of $C_{P \theta}, C_{q}$ and $\beta$ at $1.20 C_{x}(\mathrm{a}-\mathrm{c})$ and $1.40 C_{x}(\mathrm{~d}-\mathrm{f})$ 


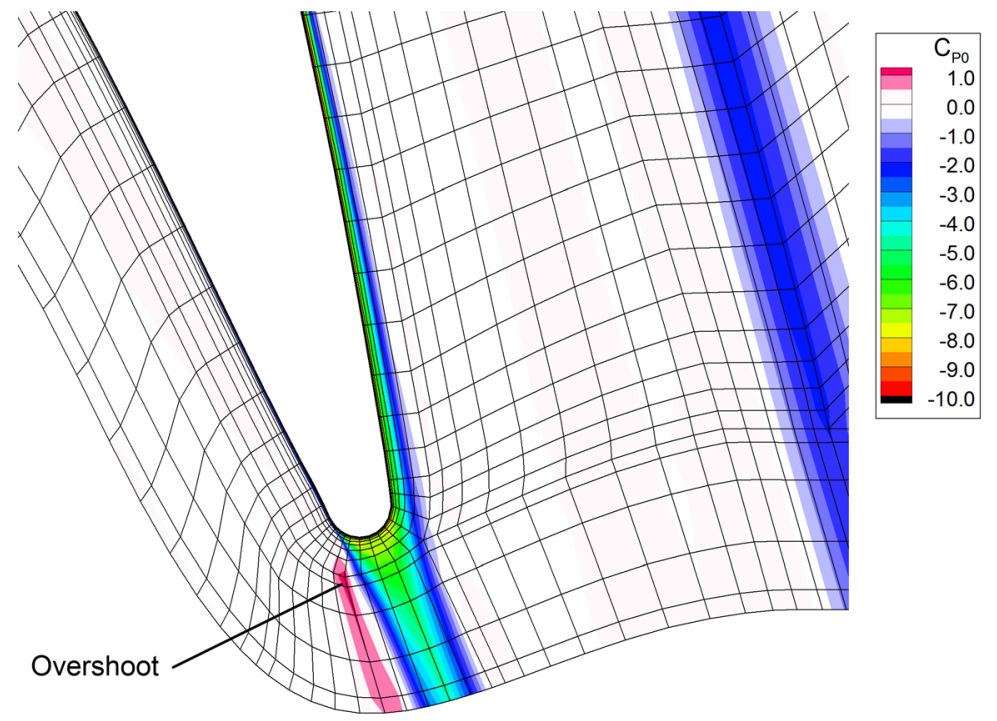

Figure 4.17: Total pressure coefficient $\left(C_{P 0}\right)$ at the blade trailing edge

nodes (18) and the smallest overshoot. In fact, at the $1.40 C_{x}$ location the G06 overshoot has completely disappeared. From this perspective G06 is the preferred grid. However, when also considering the overall node count it appears that 10 trailing edge nodes is a reasonable trade-off. Thus, G02, G04, G05 and G06 are regarded as having satisfactory downstream total pressure distributions.

Figures 4.18 (a) and (b) show the integrated loss coefficients and pitchwise area-averaged flow angles at the $1.40 C_{x}$ location. In terms of the profile losses, grid G01, followed by G03, shows the largest difference relative to the experimental value. This is not surprising, given the differences observed in Figure 4.16 (a) and (b). At the $1.20 C_{x}$ location, the minimum $C_{P 0}$ value of the G01 and G03 distributions are slightly higher than for the other grids; however, the wakes are also slightly wider. This widening appears to be a consequence of the low number of trailing edge nodes (5 and 8 ) and is largely the reason for the higher profile losses.

The integrated losses of Figure 4.18 (a) also show that with increasing node density, for grids G03 through G06, the profile losses approach the experimental value. The exception is grid G02, which produces the same profile losses as G06 $\left(Y_{p}=0.031\right)$. As shown in Table 4.4, Grids 
G02 and G06 both have 10 or more trailing edge nodes but have different first node heights (c) and expansion ratios (d). In general, parameters (c) and (d) seem to affect the location of transition and the predicted state of the near-surface boundary layer. Inaccurate prediction of the blade surface boundary layer will lead to inaccuracies in the profile losses. The similar profile losses of G02 and G06 suggest that values of parameters (c) and (d) are satisfactory for this cascade.
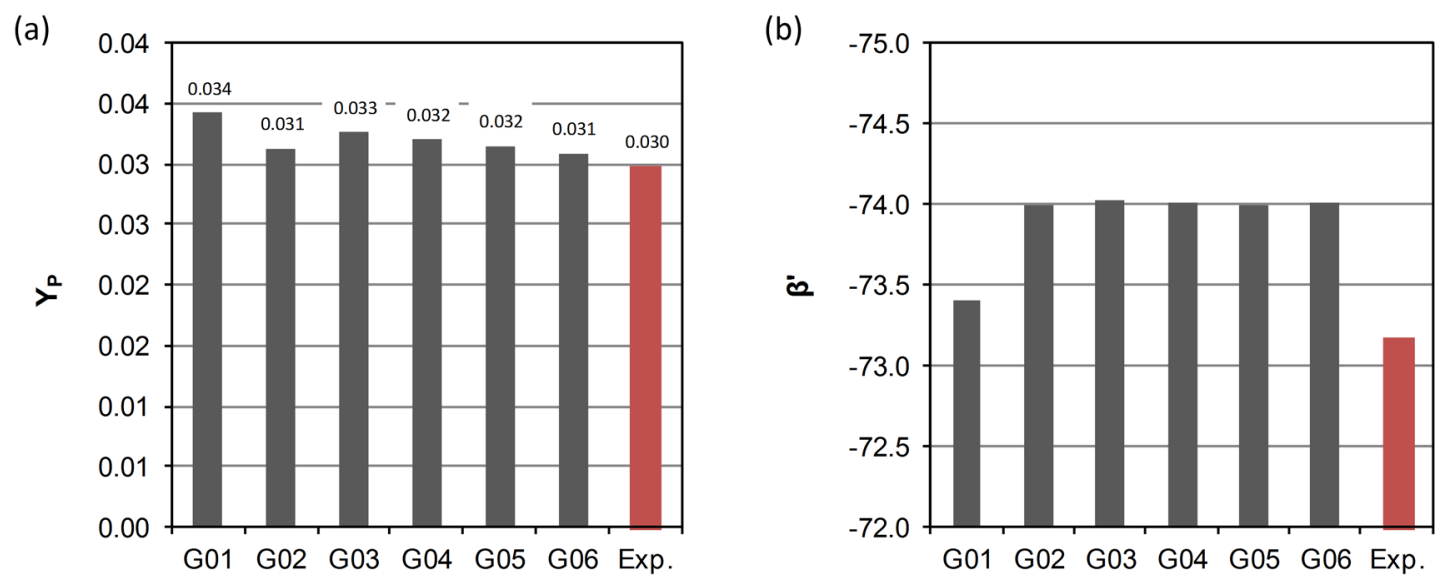

Figure 4.18: (a) Profile loss coefficient and (b) pitchwise area averaged flow angle (b) at $1.40 C_{x}$

Although grids G04 and G05 also have 10 or more trailing edge nodes, they still yield slightly larger profile losses. This suggests that it is not only the number of trailing edge nodes, but perhaps also the distribution of these nodes that influences the profiles losses for this cascade. Grids G02 and G06 produce the smallest overshoots and the smoothest distributions in Figure 4.16 (a) and (d). As a result, G02 and G06 provide the best and most grid independent predictions of the profile losses.

Figure 4.18 (b) shows the integrated flow angles. Grid G01, with lowest grid density, actually has the closest value to the experiment. However, all the cases produced similar integrated flow angles that are close to the experimental uncertainty $\left( \pm 0.6^{\circ}\right)$. Grids G02 through G06 produced nearly identical values $\left(-74.0^{\circ}\right)$. 
The downstream results have shown that grids G02 and G06 produce the most grid independent results. The near surface flow is examined next in terms of the blade loadings and suction surface skin friction coefficients.

Figure 4.19 shows the blade surface static pressure coefficients $\left(C_{P S, i}\right)$, or blade loadings, for select grids. The measured results are overlaid. As shown, the predicted loadings show negligible differences and there is also very good agreement with the experimentally measured loadings. All cases produced grid independent blade loadings.

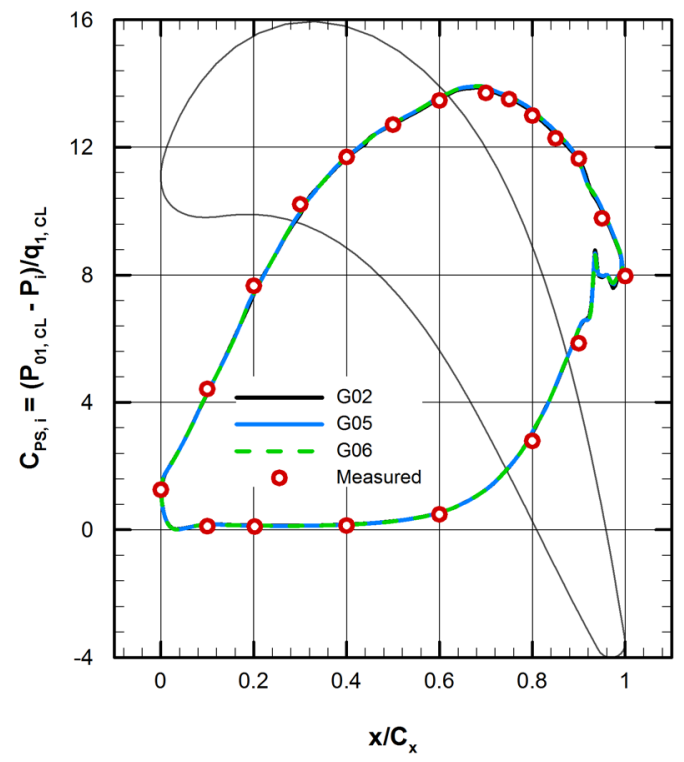

Figure 4.19: Blade surface static pressure coefficient

The suction surface skin friction coefficients $\left(C_{f}=\tau_{w} / q_{1, C L}\right)$ for each grid are shown in Figure 4.20 (a). This parameter provides an indication of the state of the boundary layer along the suction surface. In general, good agreement exists between all cases. However, the G01 distribution shows the most noticeable differences relative to G02 and G06 distributions. The largest differences occur near the leading edge from $0.1 C_{x}$ to $0.40 C_{x}$. All three grids also show differences towards the trailing edge from $0.8 C_{x}$ to $1.0 C_{x}$. Grid G06 produces the smoothest $C_{f}$ distribution which is likely the combined result of the chosen first node height and expansion 
ratio as well as the high density of streamwise nodes. These results again reinforce that the near endwall parameters of G01 are not acceptable; however, those of grids G02 through G06 do appear to be acceptable.

(a)

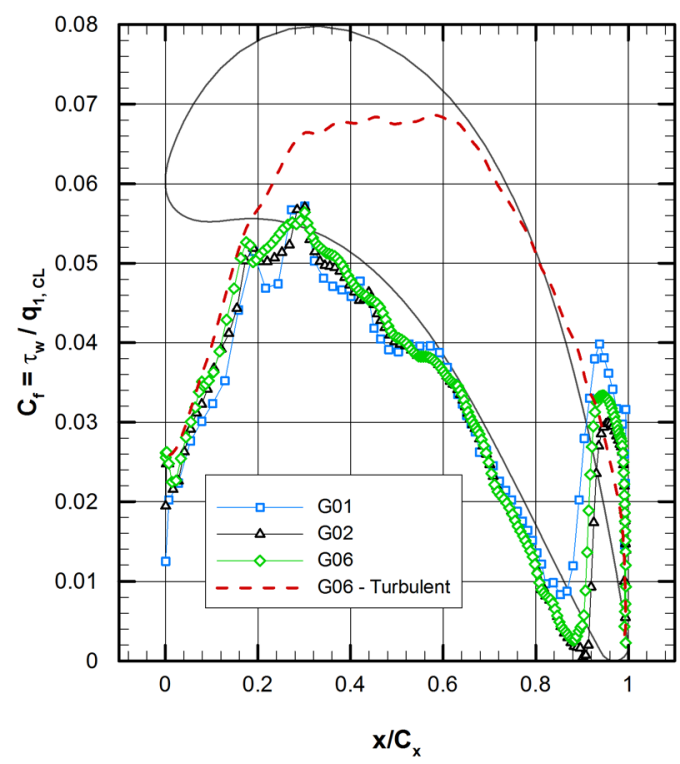

(b)

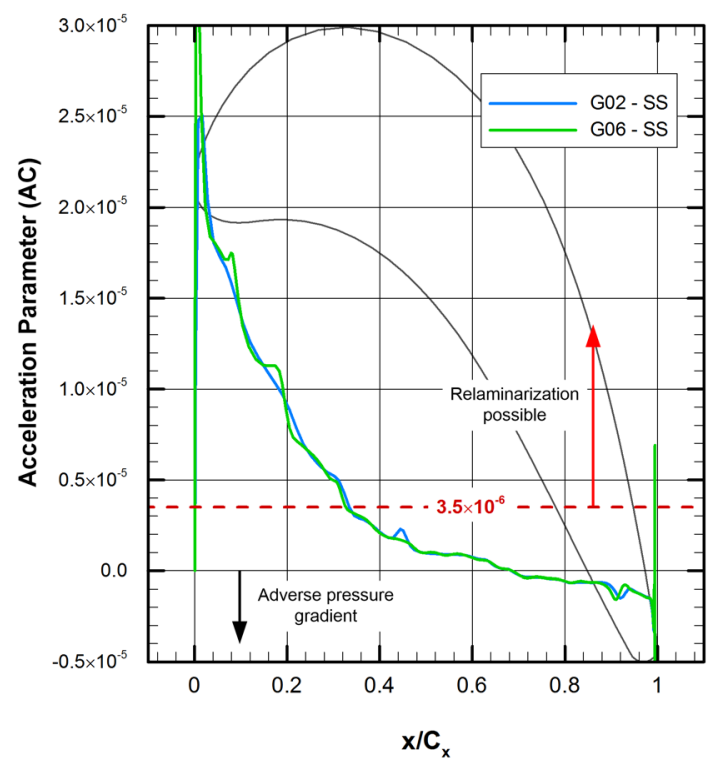

Figure 4.20: (a) Blade suction surface skin friction coefficient $\left(C_{f}\right)$ and (b) the suction surface pressure gradient acceleration parameter (AC)

As mentioned in section 4.3.3, the CFX transition model has recommended blade surface $\mathrm{y}+$ values of about 1 and wall normal expansion ratios of about 1.1. The first node height of grid G02 and G06 yield maximum suction surface $y^{+}$values of approximately 2 and 1, respectively. The respective expansion ratios are 1.3 and 1.1. As such, grid G02 does not conform to the recommendations of the transition model. However, the previously shown agreement between G02 and G06 suggests that for this cascade, and operating point, grid G02 is still acceptable.

The results from a fully turbulent simulation using grid G06 are also shown 4.20 (a). As expected, there are significant differences between the $C_{f}$ distributions from the transitional and fully turbulent simulations. For all of the transitional cases, it appears that transition first occurs 
quickly near the leading edge and a turbulent boundary layer exist downstream up to about $0.20 C_{x}$. After this point the suction surface boundary layer shows signs of relaminarization.

Mayle (1991) suggests that in positive pressure gradients, where the local acceleration is above an empirical threshold, relaminarization of the boundary layer is possible. The threshold of $3.5 \times 10^{-6}$ is applicable for a the non-dimensional acceleration parameter defined as

$$
A C=\frac{v}{V_{e}^{2}} \frac{d V_{e}}{d s}
$$

where $v$ is the laminar viscosity, $V_{e}$ is the boundary layer edge velocity and $s$ is the streamwise distance along the blade surface. The acceleration parameter then represents the streamwise pressure gradient along the blade surface.

Figure 4.20 (b), shows the suction surface acceleration parameter as function of the axial chord for the G02 and G06 grids. Both grids have very similar distributions and show that relaminarization is possible $\left(A C>3.5 \times 10^{-6}\right)$ up to about $0.35 C_{x}$. The transitional $C_{f}$ distributions of Figure 4.20 (a) are significantly different from the fully turbulent case from about $0.18 C_{x}$ up to about $0.92 C_{x}$. The lower $C_{f}$ values over this range are indicative of a boundary layer that is predicted to have relaminarized. Downstream of the suction peak, $0.65 C_{x}$, an adverse pressure gradient is present as indicated in Figure 4.20 (b). For grids G02 and G06, transition begins again at about $0.88 C_{x}$ and the boundary layer is fully turbulent at the trailing edge separation. For the coarser G01 grid, the transition location moves forward, which is consistent with the benchmarking results of the CFX transition model published by Menter et al. (2006).

For this cascade it is evident that the transition model is necessary. The results from grids G02 and G06 are largely grid independent. While G06 produces slightly smoother results, G02 has an overall node density that is about 70\% less than G06. Therefore, G02 was chosen along with G06 to be extruded for the fully three-dimensional simulations. 


\subsubsection{Three-dimensional Grid Sensitivity Study for the Flat Endwall Case}

Table 4.5 shows the mesh sensitivity study parameters for the three-dimensional grids. Each grid represents a spanwise extrusion of either the G02 or G06 grids. As shown, only the spanwise node count (a) was varied. The endwall first node height (b) was kept constant to avoid the convergence problems discussed in Section 4.5. Also, the wall normal expansion ratio (c) was set to 1.1 - the value recommended for the CFX transition model (Langtry et al., 2006; Menter et al., 2006).

The results are compared using downstream data from the $1.40 C_{x}$ plane. Figure 4.21 (a) through $(d)$, show the predicted floods of the steamwise vorticity coefficient $\left(C_{\omega s}\right)$ overlaid with line contours of the total pressure coefficient $\left(C_{P 0}\right)$. Figure $4.21(\mathrm{e})$ shows the corresponding experimental results.

Table 4.5: Summary of the 3D grid sensitivity parameters

\begin{tabular}{cccccc} 
Grid & $\begin{array}{c}\text { (a) } \\
\text { Spanwise } \\
\text { Nodes }\end{array}$ & $\begin{array}{c}\text { Endwall first node } \\
\text { height }(\mathbf{m m})\end{array}$ & $\begin{array}{c}\text { (d) } \\
\text { Endwall } \\
\text { expansion ratio }\end{array}$ & $\begin{array}{c}\text { Max. } \\
\text { endwall } \\
\mathbf{y}+\end{array}$ & $\begin{array}{c}\text { Total } \\
\text { Nodes }\end{array}$ \\
\hline \hline G02-I & 76 & $1.0 \times 10^{-5}$ & 1.1 & 2.55 & $6.95 \times 10^{5}$ \\
G06-I & 88 & $1.0 \times 10^{-5}$ & 1.1 & 2.42 & $3.28 \times 10^{6}$ \\
G06-II & 117 & $1.0 \times 10^{-5}$ & 1.1 & 2.42 & $4.37 \times 10^{6}$ \\
G06-III & 178 & $1.0 \times 10^{-5}$ & 1.1 & 2.42 & $6.81 \times 10^{6}$ \\
\hline \hline
\end{tabular}

The streamwise vorticity coefficient represents the local rotation of the fluid about the streamwise direction. The large negative regions represent the passage vortex, while the positive values show the counter and corner vortices, as labelled in sub-figure (a). Overall, the total pressure predictions are similar between the CFD cases. Each sub-figure includes labels that indicate the minimum total pressure contour as well as the local values within the passage vortex region. The magnitude and position of these local peaks are similar for each case. Also, the size and position of vortical features, as indicated by the floods of streamwise vorticity, are similar. 


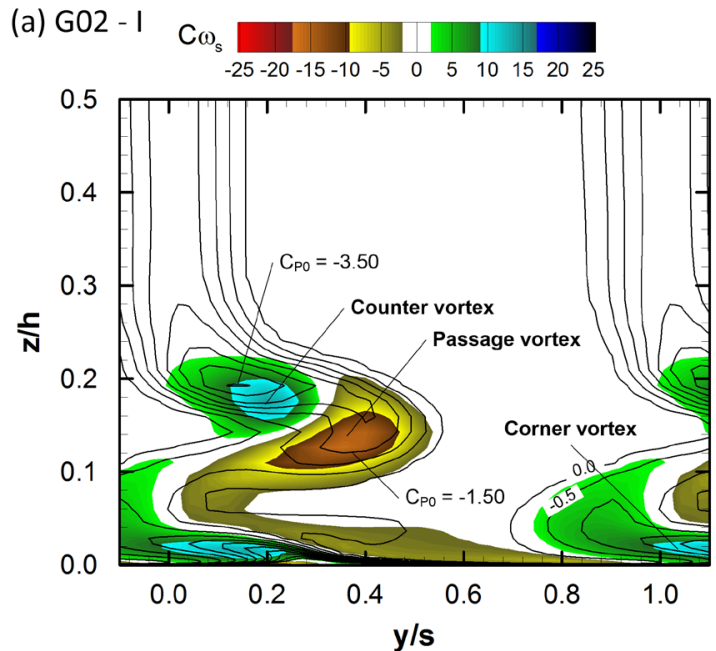

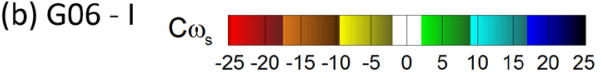

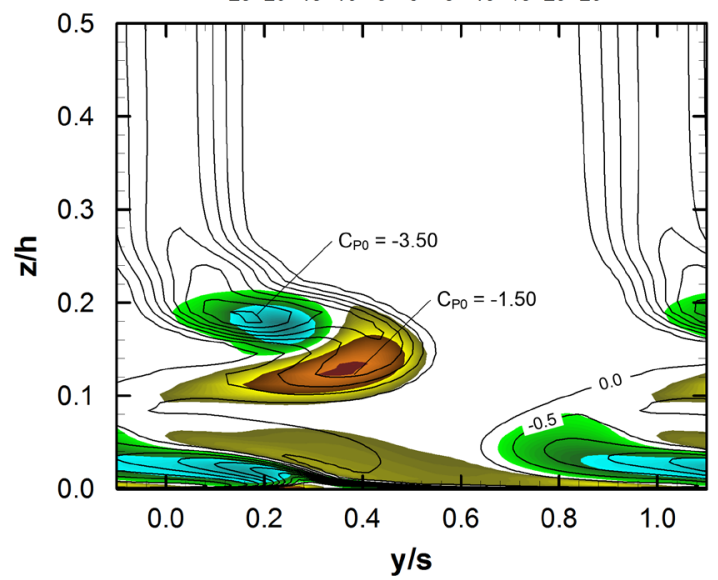

(c) G06 - II $\mathrm{C} \omega_{\mathrm{s}}$

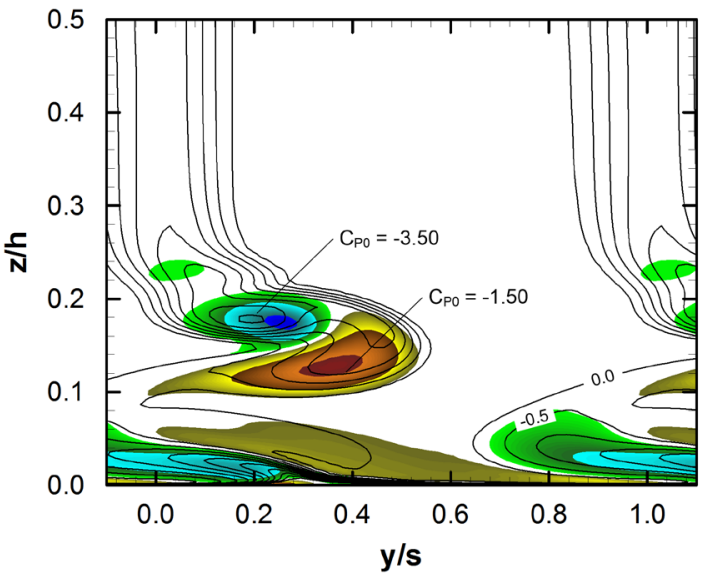

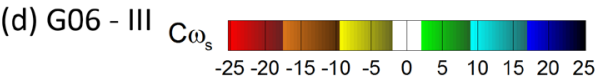

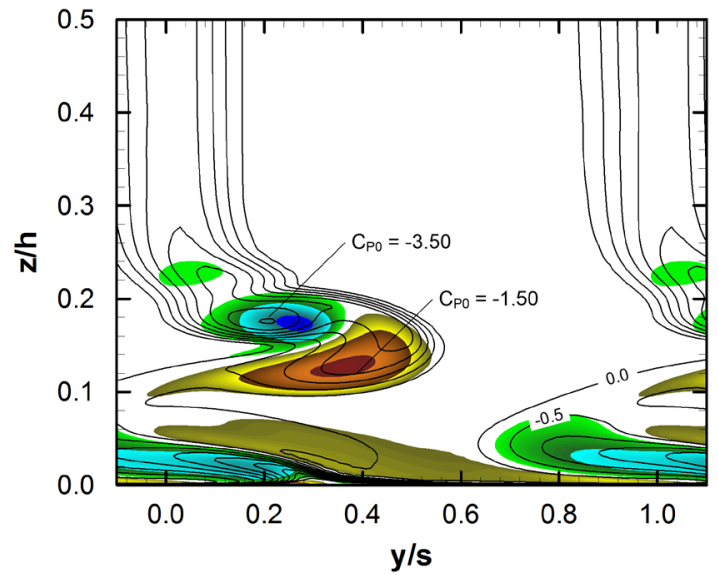

(e) Exp.

$\mathrm{C}_{\mathrm{s}}$

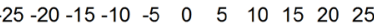

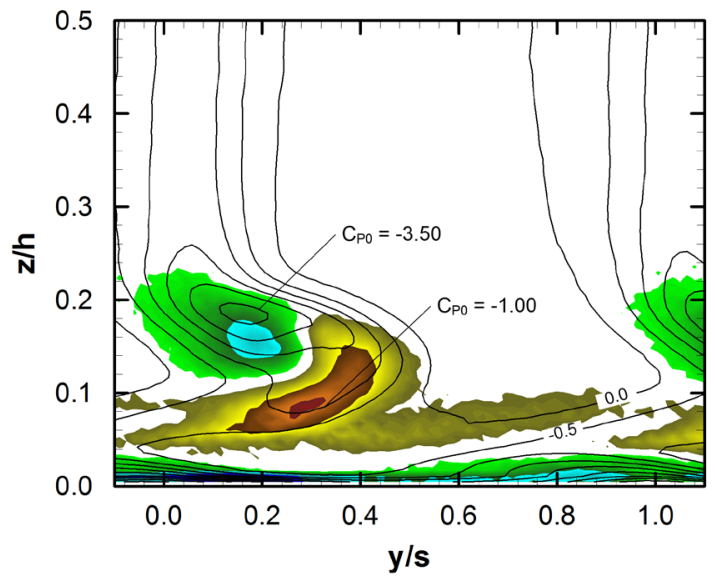

Figure 4.21: Colour floods of the streamwise vorticity coefficient $\left(C_{\omega s}\right)$ overlaid with line contours of the total pressure coefficient $\left(C_{P 0}\right)$ at $1.40 C_{x}$ 
Grids G06-II and G06-III have the largest spanwise node counts and produce the most grid independent results. They have the smoothest loss distributions and the vortical structures have the largest values of streamwise vorticity. Also, there is a region of positive streamwise vorticity that is located above the counter vortex, labelled "vortex A" in Figure 4.21 (c) and (d). This structure is not present in the G02-I, G06-I or the experimental results suggesting that perhaps this feature has dissipated more rapidly in these cases.

Grid G02-I has the lowest node count and produces results that are the most diffuse. In Figure 4.21 (a) the total pressure contour lines are spread over a larger area and the vortical structures have lower peak values of streamwise vorticity. Although grid G02-I produces results that are slightly inconsistent with the others grids, interestingly these results are also the closest match to the experimental measurements.

The pitchwise mass-averaged loss coefficients are shown in Figure 4.22 (a). Consistent with the in-plane data, G06-II and G06-III are the most grid independent results with only minor differences in their distributions. Grid G06-I is also very similar and only shows significant differences at the peak loss location at about $z / h=0.18$. Grid G02-I differs the most relative to the other grids in this peak loss region. At the minimum loss location, at about $z / h=0.10$, grid G02-I also shows significant differences. In general, grid G02-I has a pitchwise loss distribution with smaller peaks and shallower troughs, both of which are wider and more diffuse than for the other grids. Although different from the other grids, the shape of the G02-I distribution is again closest to the experimental results.

Figure 4.23 (a) shows the overall loss coefficients. The secondary losses are shown separately in Figure 4.23 (b). The local differences observed in the pitchwise distributions are not borne out in the overall integrated values. Grid G02-I, G06-II and G06-III have equal loss coefficients of 0.047 , while G06-I has the lowest value at 0.046 . The experimentally measured value is 0.048 . In general, the overall loss values are well predicted by all grids. The secondary losses, in Figure 4.23 (b), follow a similar trend since the predicted profile losses are all equal. 

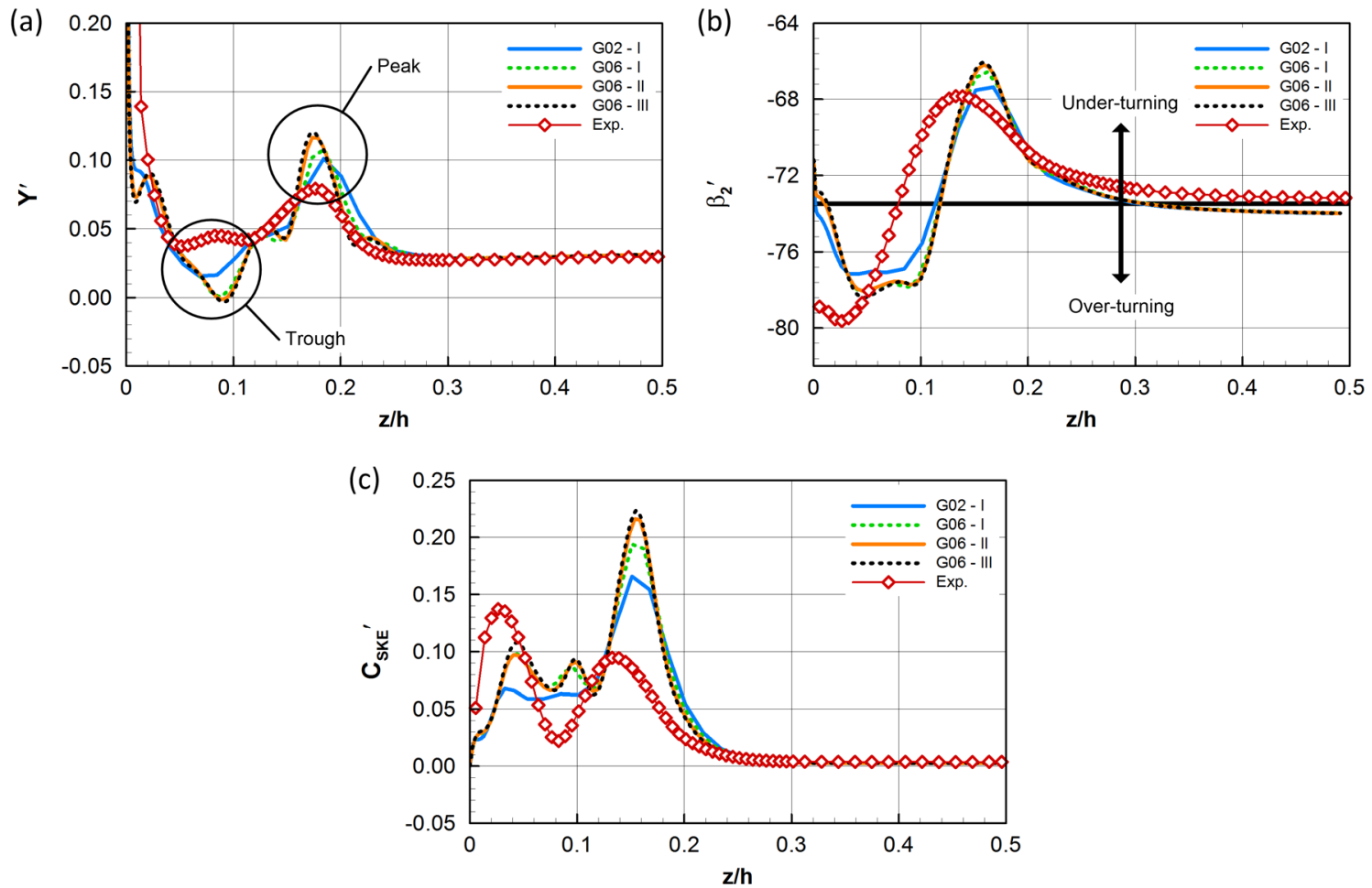

Figure 4.22: Pitchwise averaged loss coefficient (a), outlet pitchwise flow angle (b) and the secondary kinetic energy coefficient at $1.40 C_{x}$
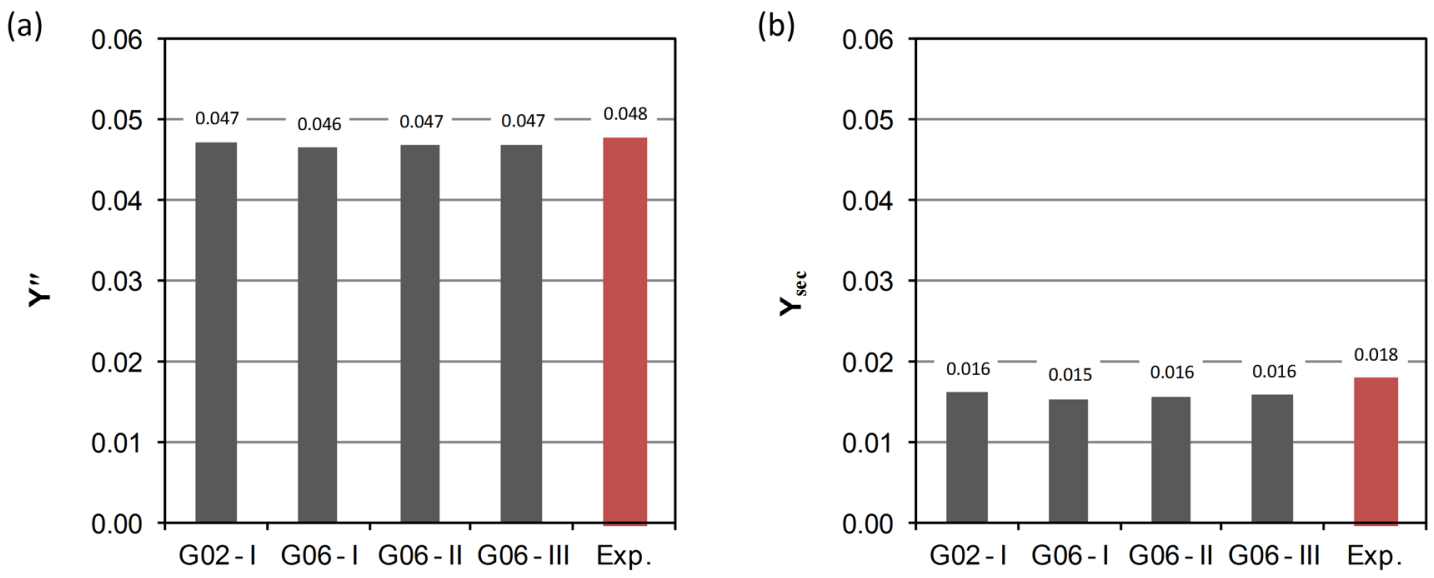

Figure 4.23: (a) Overall loss coefficient and (b) Secondary loss coefficient at $1.40 C_{x}$ 
Figure 4.22 (b) shows the pitchwise averaged outlet flow angle $\left(\beta^{\prime}\right)$. As indicated in the secondary flow region, the spanwise distributions exhibit the classic over-turning and underturning relative to the midspan flow angle. Grids G06-I through G06-III produce results that are largely grid independent with only minor differences at the minimum over-turning and peak under-turning locations. G02-I predicts about $1^{\circ}$ more over-turning and $1^{\circ}$ less under-turning than the other cases.

The pitchwise mass-averaged secondary kinetic energy coefficients $\left(C_{S K E}\right)$ are show in Figure 4.22 (c). As discussed in Section 3.11.5, the secondary kinetic energy represents the kinetic energy associated with the velocity vectors that are perpendicular to the streamwise direction. Thus, towards midspan the coefficients have near zero values and within the secondary flow there are significant levels of $C_{S K E}$. Here, G06-I through G06-III are the most grid independent results. Grid G02-I is the outlier with smaller peaks and shallower troughs. Again, this is consistent with a more diffuse flow that is closer to the experimental distribution.

To summarize the three-dimensional study, grids G06-II and G06-III produced the most grid independent results. Grid G02-I produced some inconsistent results with respect to other grids, but also produced the best prediction of the experimental flow. From an engineering perspective, Grid G02-I produces excellent results given that the node count is approximately $16 \%$ and $10 \%$ of that of grids G06-II and G06-III, respectively. Minimizing the node count for the flat endwall case was not necessarily a priority. However, for cases with purge flow the added flow paths increased the node count by approximately $2.0 \times 10^{6}$. For grid G06-III the node count would then approach $10.0 \times 10^{6}$. For these flows, this is impractical and not representative of the grids used for turbine design purposes. Therefore, G02-I was chosen for the design level simulations of Chapters 6 and 7.

\subsubsection{Three-dimensional Grid Sensitivity Study for the Endwall Cavity Case}

The endwall cavity geometry requires a significantly modified grid from that used for the 3D flat endwall case. For this grid study, the blocking from G02-I was adapted for the endwall 
cavity geometry as shown in Figure 4.5 in Section 4.3.3. In the region labelled "near endwall blocks", the G02-I mesh was mapped to the cavity-shaped endwall. Table 4.6 shows the meshing parameters for this grid study. There are three-grids based on the G02-I, namely G02C-I through G02C-III. The addition of the near endwall blocking layer introduces a new meshing parameter: the spanwise number of spanwise nodes within the endwall cavity region (b). In this study, the number of spanwise nodes (a) represents the number of spanwise nodes from these near-endwall blocks to midspan. The other mesh parameters were kept constant to maintain consistency with the flat endwall grid.

Table 4.6: Summary of the 3D grid sensitivity parameters for the endwall cavity configuration

\begin{tabular}{|c|c|c|c|c|c|c|}
\hline Grid & $\begin{array}{c}\text { (a) } \\
\text { Spanwise } \\
\text { nodes } \\
\text { Interior blocks }\end{array}$ & $\begin{array}{c}\text { (b) } \\
\text { Spanwise } \\
\text { nodes } \\
\text { Endwall cavity }\end{array}$ & $\begin{array}{c}\text { (c) } \\
\text { First node } \\
\text { height } \\
\text { (mm) }\end{array}$ & $\begin{array}{l}\text { (d) } \\
\text { Expansion } \\
\text { ratio }\end{array}$ & $\begin{array}{c}\text { Max. } \\
\text { endwall } \\
\mathbf{y}^{+}\end{array}$ & $\begin{array}{c}\text { Total } \\
\text { Nodes }\end{array}$ \\
\hline G02C-I & 30 & 30 & $1.0 \times 10^{-5}$ & 1.1 & 1.47 & $7.14 \times 10^{5}$ \\
\hline G02C-II & 45 & 40 & $1.0 \times 10^{-5}$ & 1.1 & 1.96 & $1.04 \times 10^{6}$ \\
\hline G02C-III & 65 & 50 & $1.0 \times 10^{-5}$ & 1.1 & 1.95 & $1.42 \times 10^{6}$ \\
\hline
\end{tabular}

The following study is presented in a similar manner to that of the previous section. Again, the data is extracted at the $1.40 C_{x}$ plane for comparison purposes. Colour floods of streamwise vorticity coefficient $\left(\mathrm{C}_{\omega \mathrm{S}}\right)$ with line contours of the total pressure coefficient $\left(C_{P 0}\right)$ are shown in Figure 4.24. The pitchwise-averaged parameters are shown in Figure 4.25 and the overall integrated loss coefficients are given in Figure 4.26.

Figure 4.25 shows that the results for grids G02C-I through G02C-III are in good agreement. The vortical structures are similar in size and have near equal values of peak streamwise vorticity. The $C_{P 0}$ contours are also all in relatively good agreement; however, grids G02C-II and G02-III produce the most grid independent results. The experimental measurements are shown in Figure 4.25 (d) and show significant differences relative to the CFD predictions. The peak values of streamwise vorticity are significantly larger than for the CFD and the total pressures are more diffuse with lower peak values. 
Figure 4.25 (a) shows that the pitchwise mass-averaged loss coefficients from the CFD are in good agreement. Again, grids G02C-II and III show negligible differences, while grid G02C-I shows small differences in the peak and trough regions, as labelled. The experimental results show clear differences in the passage vortex region $(0.4<z / h<0.12)$. These differences are consistent with the pitchwise-averaged flow angle $\left(\beta^{\prime}\right)$ and secondary kinetic energy $\left(C_{S K E}\right)$ distributions of Figure 4.25 (b) and (c). Here, the CFD results are consistent but show higher over-turning as well as higher levels of $C_{S K E}$ than the experiment. 
(a) G02C-I

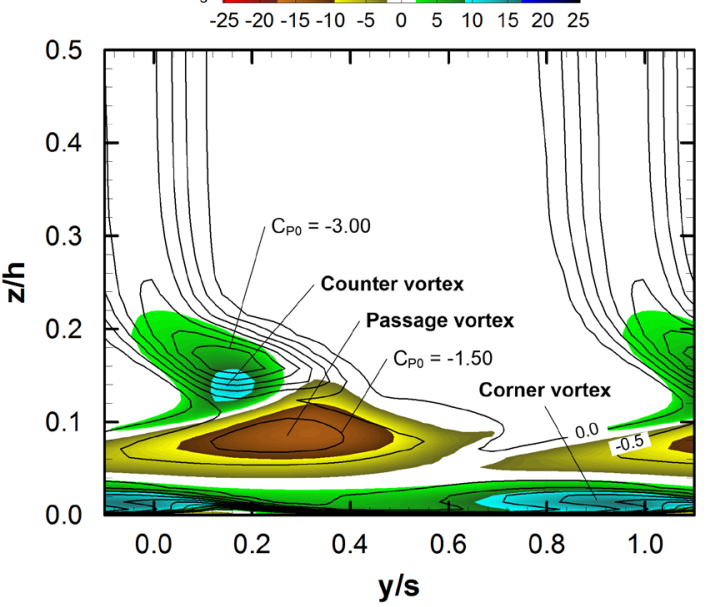

(b) G02C-II

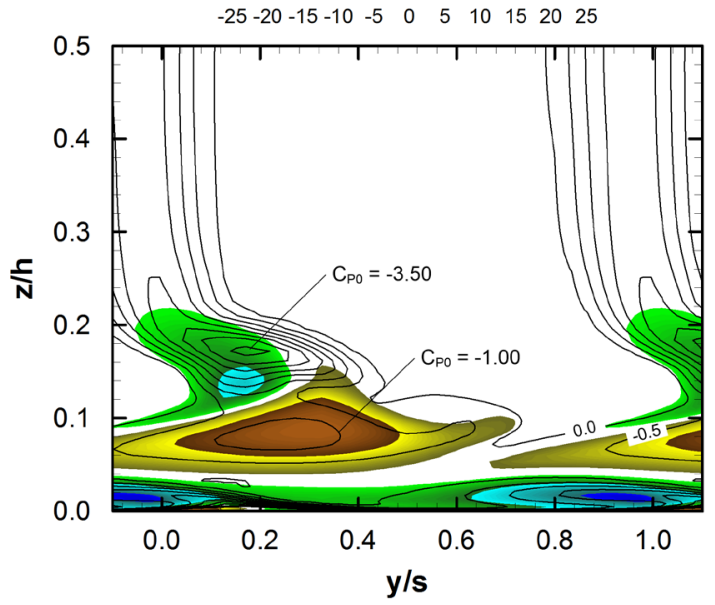

(d) Exp.

$\mathrm{C} \omega_{\mathrm{s}}$

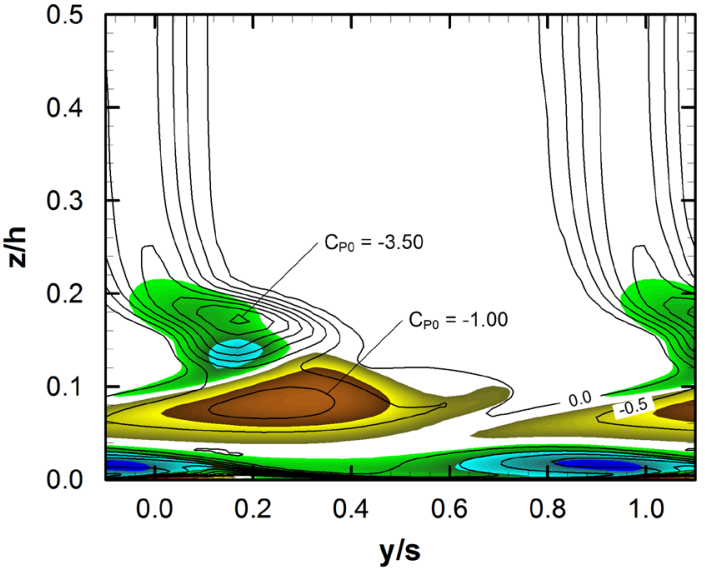

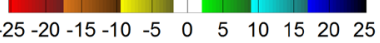

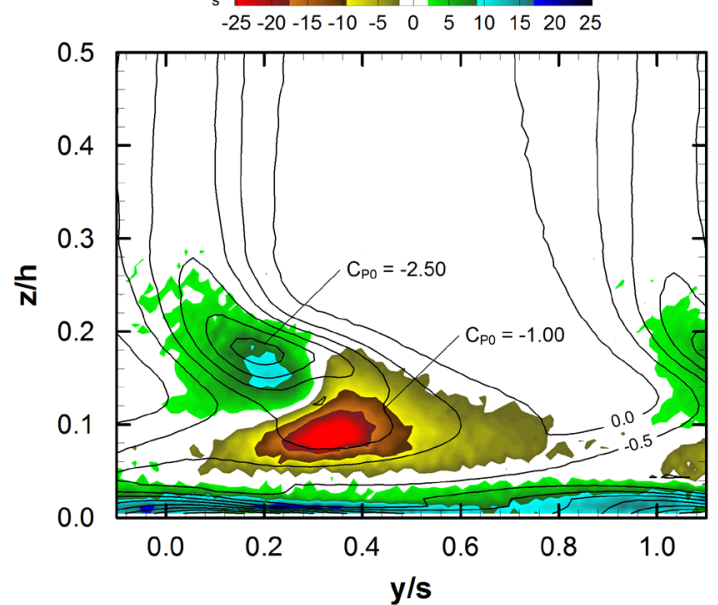

Figure 4.24: Colour floods of the streamwise vorticity coefficient $\left(C_{\omega s}\right)$ overlaid with line contours of the total pressure coefficient $\left(C_{P 0}\right)$ at $1.40 C_{x}$

In terms of the overall losses, all three grids predict near equal values at the $1.40 C_{x}$ plane, as shown in Figure 4.26. Grids G02C-II and G02C-III are essentially grid independent solutions. Since, the node counts for all three grids were manageable and also representative of design levels, the G02C-II grid was selected for all cases involving the endwall cavity geometry. The blocking of G02C-II was then altered slightly for the endwall contouring and elliptical platform overlap geometries. 
In summary, the studies presented in this chapter document the approach taken to arrive at grid independent CFD solutions. The final grids for Chapters 6 and 7 are based on the G02-II and G02C-II three-dimensional grids. As shown, these grids represent trade-offs between the level of grid independence, the solution accuracy with respect to the experiment and the computational resources required. The latter point was a driving factor in reducing the overall node count to levels that are representative for design applications. Interestingly, these grids also tended to provide the best match to the experimental results.
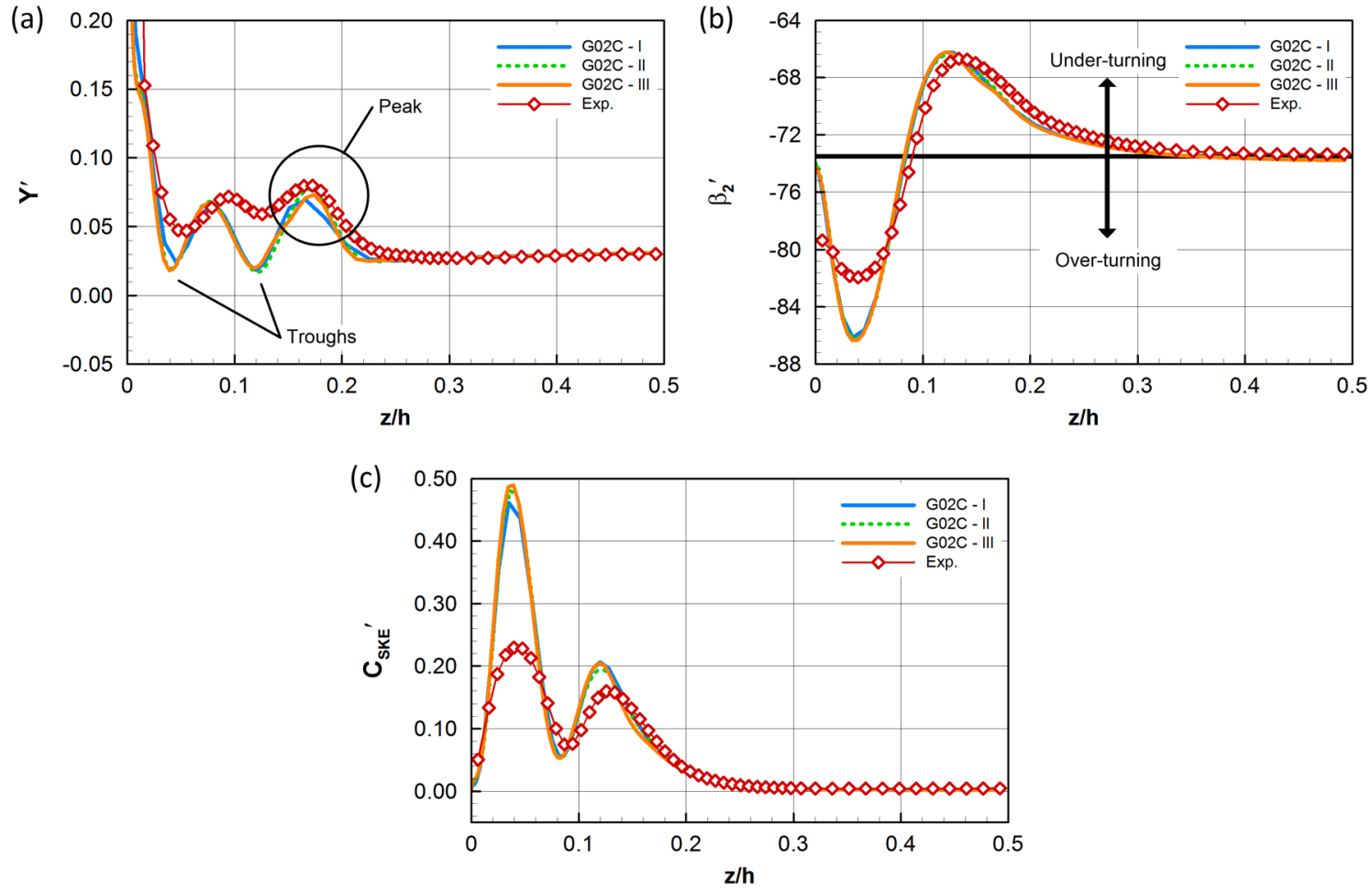

Figure 4.25: Pitchwise averaged loss coefficient (a), outlet pitchwise flow angle (b) and the secondary kinetic energy coefficient at $1.40 C_{x}$ 
(a)

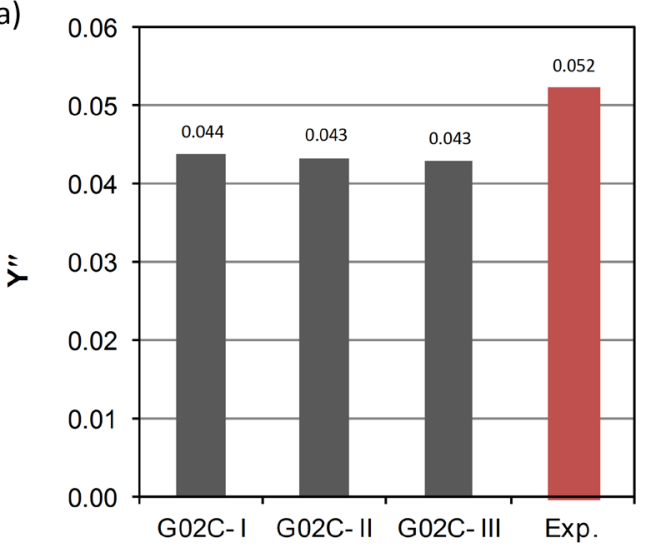

(b)

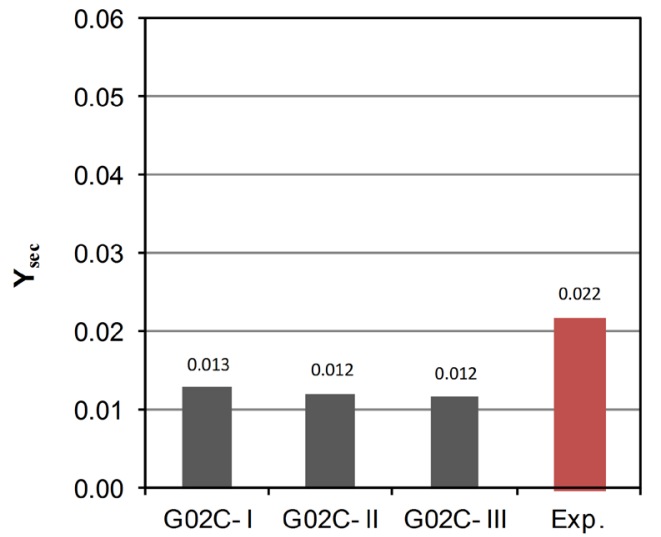

Figure 4.26: (a) Overall loss coefficient and (b) Secondary loss coefficient at $1.40 C_{x}$ 


\section{Chapter 5}

\section{Reynolds Stresses and Loss Production within the Secondary Flow Downstream of a Turbine Cascade}

\subsection{Introduction}

The present chapter investigates how the turbulence field of the secondary flow affects the total pressure loss production downstream of a linear turbine cascade. Measurements were made for the Baseline configuration at an inlet Reynolds number of 50,000 using the seven-hole and $x$-type hotwire probes. The secondary losses are examined in relation to the production of turbulence. In particular, the effects of the Reynolds stresses are examined. The chapter concludes with a comparison to the corresponding CFD results. Comments are made regarding the applicability of two-equation turbulence models for turbine secondary flows. 


\subsection{Motivation}

As described in Section 2.6, turbine secondary flows have been well studied; however, the sources of endwall losses are still not fully understood (Praisner et al., 2007). More specifically, a precise connection between the mean and turbulent flow fields, specific to secondary flows, has yet to be established, as noted in several studies: Moore et al., 1987; Gregory-Smith et al., 1988b; Perdichizzi et al., 1992; Vicharelli and Eaton, 2006; Gustafson et al., 2007; Porreca et al., 2007. The final losses within a turbulent flow are only realized when turbulent eddies completely dissipate to internal energy through viscous interactions. Thus, accurate predictions of secondary losses require turbulence models that model accurately the true physics.

For turbine design computations, it is typical to use a RANS solver with a two-equation turbulence model, such as the shear stress transport (SST) model. This model is based on the assumption that the eddy viscosity field is isotropic. The Reynolds stresses are then proportional to the mean velocity gradient. In swirling flows, like the secondary flow through the turbine bladerow, the validity of this assumption is known to be inaccurate. Although more elaborate computational schemes exist, for the purposes of design, two-equation eddy-viscosity-based models are typically considered a satisfactory compromise between computational accuracy and cost.

In attempts to validate the inaccuracies introduced by the isotropic eddy viscosity assumption, different conclusions based on the measured eddy viscosity fields have been reported. Gregory-Smith and Cleak (1992) showed that the eddy viscosity is fairly isotropic downstream of the cascade in the secondary flow region. However, Perdichizzi et al. (1992) concluded that at higher outlet velocities (greater than about $\mathrm{Ma}=0.3$ ) the eddy viscosity of the secondary flow is in fact highly anisotropic downstream of the cascade. Only at axial locations farther downstream, after significant mixing has taken place, were isotropic conditions approached. Porreca et al. (2007) concluded the highest anisotropic regions were a result of large streamwise fluctuations in velocity, whereas Ristic et al. (1999) reported only fluctuations in the radial components as being significant. These examples show that there is no consistent 
understanding of the Reynolds stress field of turbine bladerows. There are also contradictions regarding the role of the turbulence field in the production of the secondary losses. Thus, one of the objectives here was to obtain detailed experimental results that would improve the physical understanding of the loss production mechanisms. A second objective was to examine the differences between the measured and the predicted flows with the goal of better understanding the consequences of the isotropic eddy viscosity assumption.

\subsection{Approach}

The experimental measurements from the PAKH cascade are presented first. For this data set the cascade inlet Reynolds number was set to 50,000. Downstream measurements were made using the seven-hole and $x$-type hotwire probes described in Sections 3.8.5 and 3.8.7 respectively. The cascade inlet flow field and cascade blade loadings are discussed first, followed by the downstream mean and turbulent flow fields. An examination of the mean flow kinetic energy budget and the secondary loss production mechanisms conclude the experimental measurements section.

The corresponding CFD predictions follow in the subsequent sections. The predictions were made following the procedures described in Chapter 4. Briefly, the steady-state RANS solutions were obtained using the ANSYS CFX v12.0 solver. The two-equation SST turbulence model with the $\gamma-\operatorname{Re}_{\theta}$ transition model was used. The discussion includes many comparisons to the experimental measurements where the typical deficiencies of the CFD predictions are shown. Finally, the validity of the isotropic eddy viscosity assumption, as applied in two-equation turbulence models, is examined. 


\subsection{Inlet Flow Field and Cascade Blade Loadings}

The inlet endwall boundary layer profile was measured using a Pitot probe at three different pitchwise positions $(y / s=0,0.25$ and 0.75$)$ over the centre passage. As shown in Figure 5.1, there is minimal pitchwise variation of the inlet boundary layer profile; the lower velocities towards midspan are caused by the inlet turbulence generating grid. The observed spanwise variations are consistent with the previous work of Knezevici (2011) and are accounted for by mass-averaging the inlet total pressures (using three-hole probe measurements over one pitch and one half-span) to give $C_{P 0_{1}}{ }^{\prime \prime}$. The boundary layer parameters are presented in Table 5.1.

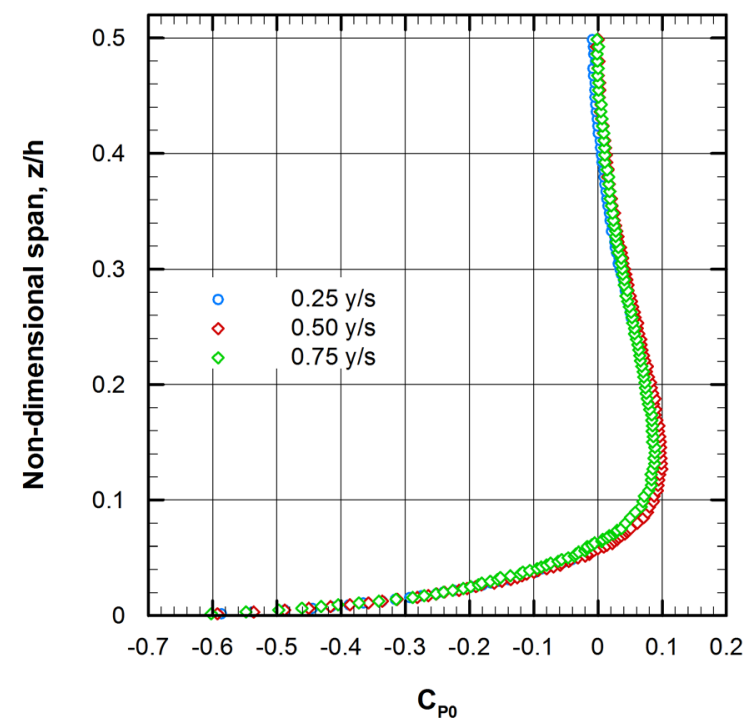

Figure 5.1: Inlet boundary layer total pressure distribution $\left(\operatorname{Re}_{1}=\mathbf{5 0 k}\right)$

Table 5.1: Boundary layer parameters

\begin{tabular}{lcccc}
\hline \hline Boundary layer thickness & $\delta / h$ & & 0.092 \\
Displacement thickness (mm) & & $\delta^{*}$ & 2.13 \\
Momentum thickness (mm) & $\theta$ & & 1.62 \\
Boundary layer shape factor & & $H_{S F}$ & 1.32 \\
Free Stream turbulence intensity & & $T u_{l o c}$ & $3.3 \%$ \\
Free stream length scale & $\Lambda / C_{x}$ & 0.25 \\
\hline \hline
\end{tabular}


As discussed in Chapter 4, the measured inlet boundary layer was used as the boundary condition for the CFD simulations. The inlet turbulence distribution was computed using a predomain as described in Chapter 4.

Figure 5.2 shows the midspan pitchwise variation of the inlet $\left(-1.90 C_{x}\right)$ turbulence quantities obtained using the $x$-type hotwire probe described in Section 3.8.7. The turbulence intensity,

$$
T u=\frac{\sqrt{\frac{1}{3}\left(u^{\prime 2}+v^{\prime 2}+w^{\prime 2}\right)}}{V_{l o c}} \times 100
$$

is non-dimensionalized by the local mean velocity, $V_{l o c}$, and is approximately $3.3 \%$ at the centre passage. This value is consistent with previous studies (Mahallati, 2003; Knezevici, 2011) and approximates engine levels. The inlet turbulence intensity decreases with increasing pitch position, apparently linearly from a maximum of $3.4 \%$ to about $3.15 \%$. This is caused by the inclination of the cascade leading edge relative to the turbulence grid (see Figure 3.2 of Section 3.3).

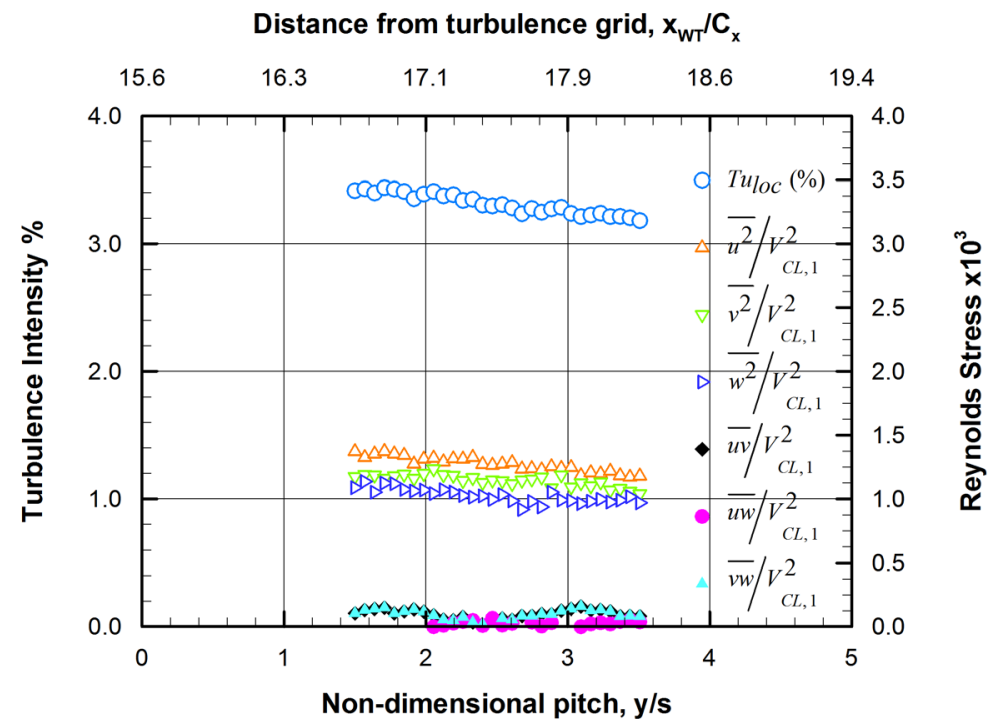

Figure 5.2: Midspan turbulence intensity (left) and Reynolds stresses (right) 
The midspan non-dimensional Reynolds stresses (normal and shear) are also shown in Figure 5.2. The turbulence field appears to be nearly isotropic: the normal stresses in the three coordinate directions have very similar magnitudes, varying from about 1.0 to $1.5 \times 10^{-3}$, and the shear stresses have very low (nearly zero) magnitudes.

Figure 5.3 shows the measured and predicted blade surface static-pressure distributions at midspan. The local static pressures are shown as a static pressure coefficient,

$$
C_{P S_{i}}=\frac{P_{01, C L}-P_{S_{i}}}{q_{1, C L}}
$$

where $i$ is the static tap number. Measurements were made using the specially designed blade described in Section 3.10.4. The loadings shown here are similar to the predictions, indicating that cascade was setup as intended. The suction surface peak is located at approximately $x / C_{x}=0.68$ and there appears to be a small separation bubble that extends from $x / C_{x}=0.85$ to 0.95 in the measurements. The computational loadings support this observation and the near surface velocity vectors show a small region of reversed flow. This apparent bubble is a region of very low shear stress and also shows up in the surface flow visualizations.

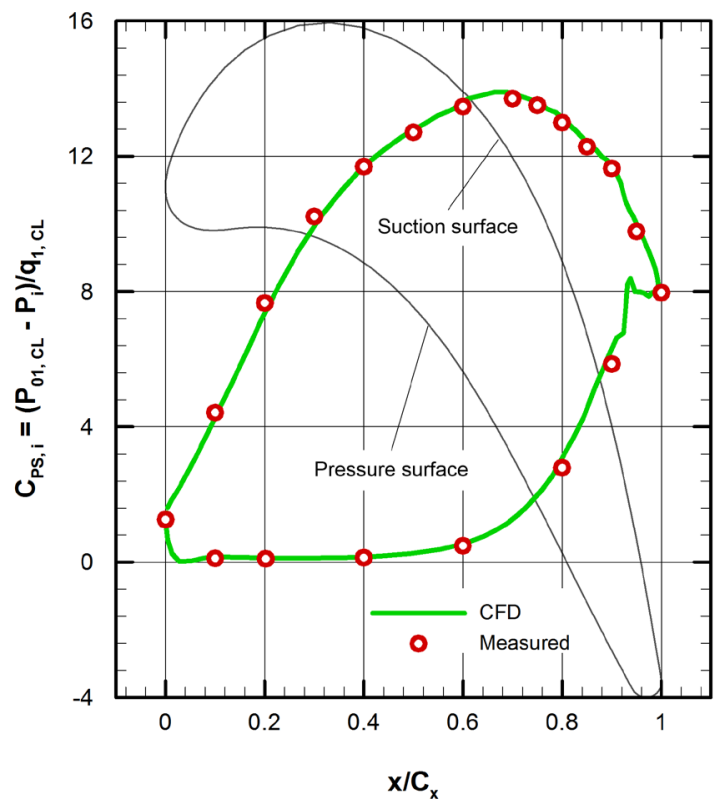

Figure 5.3: Blade surface static pressure distribution measured at midspan $(\mathrm{z} / \mathrm{h}=\mathbf{0 . 5})$ 


\subsection{Experimental Downstream Flow Field}

\subsubsection{Introduction}

The following sub-sections present the experimentally measured downstream flow field. Pressure probe and turbulence measurements were made at three axial locations, 1.00, 1.20 and $1.40 C_{x}$. The flow development with downstream distance is discussed first followed by an analysis of the integrated flow parameters and then the turbulence field. The last sub-section discusses the mechanisms of turbulence production from the mean flow.

\subsubsection{Development of the Secondary Flow with Downstream Distance}

The development of the total pressure losses are discussed in terms of the streamwise vorticity and the secondary velocity vectors. Reference is also made to the typical secondary flow structures described Section 2.5.2, namely the counter, corner and passage vortices. Figure 5.4 presents colour flood contour plots of the total pressure coefficient, $C_{P 0}$, at the three axial locations. The high loss regions are represented by red, lower loss regions by blue and no loss regions by white. Figure 5.5 presents the streamwise vorticity coefficient, $C_{\omega_{s}}$, at the same axial locations. The total pressure coefficients from Figure 5.4 are overlaid as equal increment contour lines. The streamwise vorticity coefficient represents the local rotation of the fluid relative to the streamwise direction. Thus, the yellow and red regions indicate a negative (clockwise) rotation about the streamwise axis while the green and blue regions represent a positive rotation (counter-clockwise). Note that at the $1.00 C_{x}$ plane only about $85 \%$ of the full pitch was traversed due to interference between blade surface and the probe stem. Also, the scale of streamwise vorticity at the 1.20 and $1.40 C_{x}$ planes, Figures $5.5(\mathrm{~b})$ and (c), is smaller than at the $1.00 C_{x}$ plane to make the vortical structures visible. The secondary velocity vectors are also shown in Figure 5.5. 
(a) $1.00 \mathrm{C}_{\mathrm{x}}$

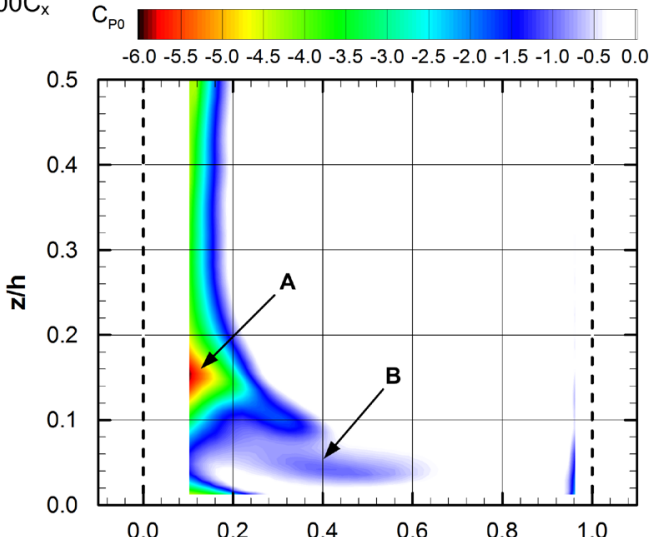

(b) $1.20 \mathrm{C}_{\mathrm{x}}$

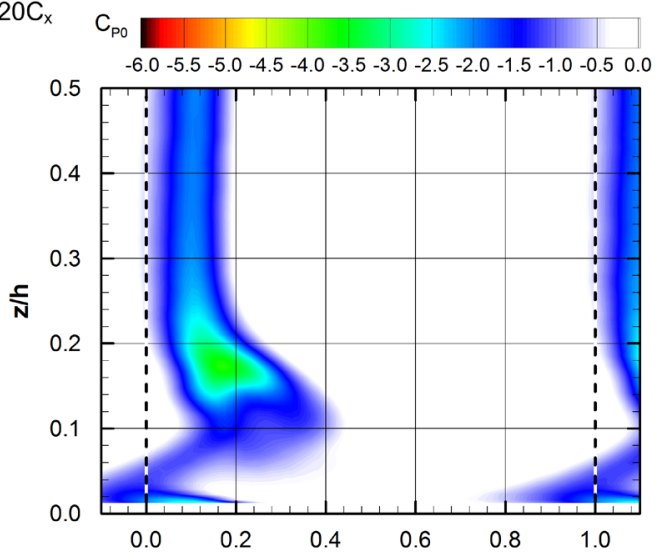

(c) $1.40 \mathrm{C}_{\mathrm{x}}$

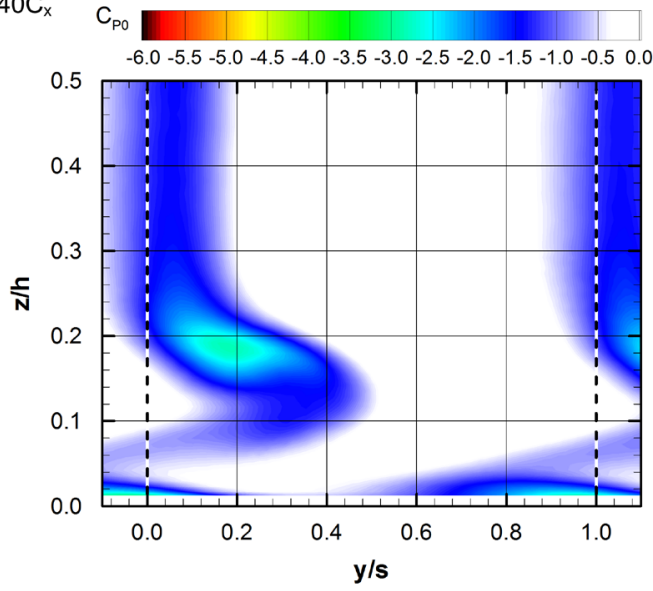

Figure 5.4: Total pressure coefficient $\left(C_{P 0}\right)$

floods at (a) $1.00 C_{x}$, (b) $1.20 C_{x}$ and (c) $1.40 C_{x}$ (a) $1.00 \mathrm{C}$

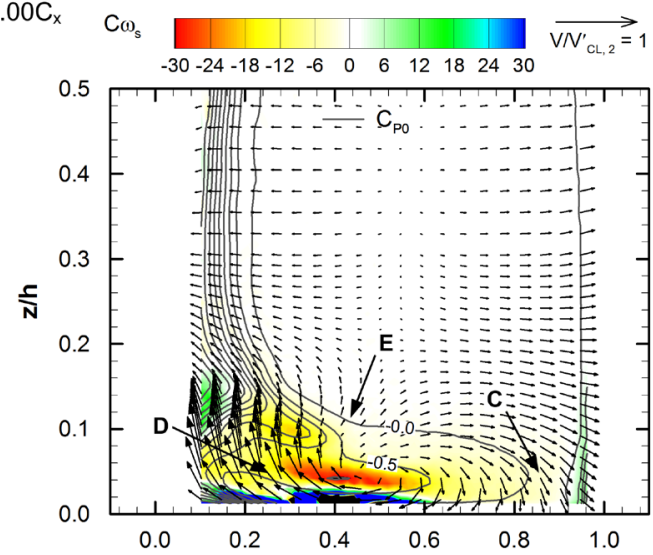

(b) $1.20 C_{x}$

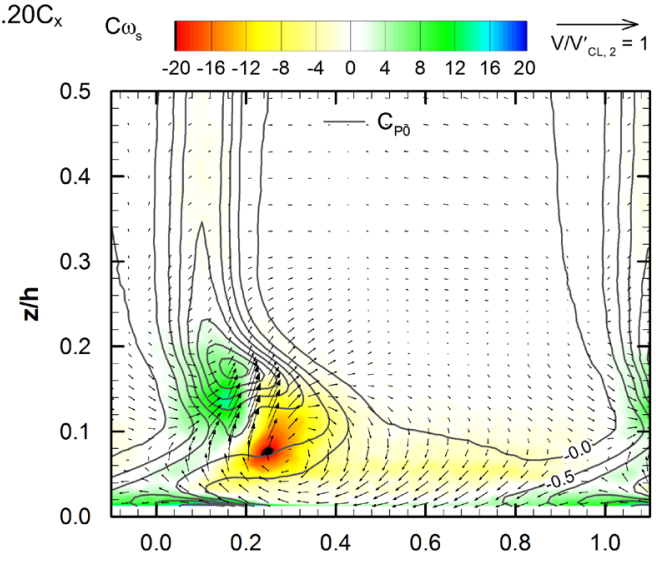

(c) $1.40 C_{x}$

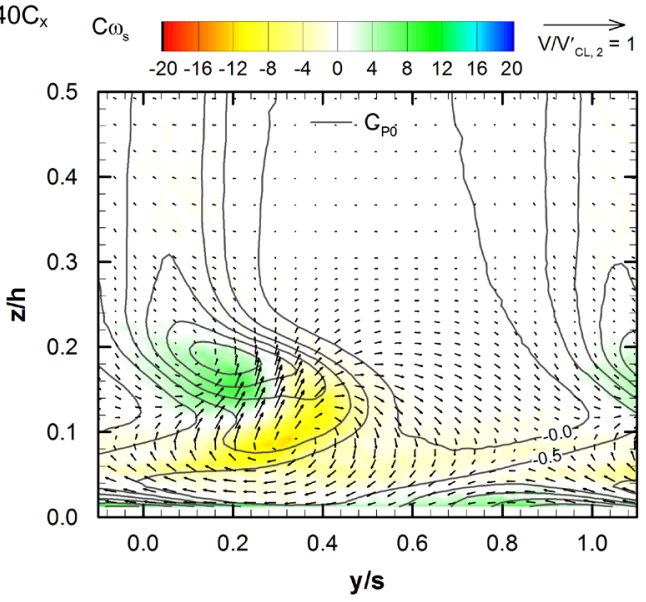

Figure 5.5: Streamwise vorticity coefficient $\left(C \omega_{s}\right)$ floods with contour lines of total pressure coefficient $\left(C_{P 0}\right)$ and secondary velocity vectors at (a) $1.00 C_{x}$, (b) $1.20 C_{x}$ and (c) $1.40 C_{x}$ 
At the $1.00 C_{x}$ plane, shown in Figure 5.4 (a), the high loss fluid is concentrated near the suction surface of the blade with the peak, labelled A, located at approximately $y / s=0.15$ and $z / h=0.16$. The fluid in this region is likely a combination of the low momentum fluid that originated from the suction surface and endwall boundary layers. This accumulation is caused by the rotation of passage and counter vortices. As discussed in Section 2.2, the interaction of the passage vortex with the blade suction surface tends to drive the suction surface boundary layer away from the endwall towards midspan. The counter vortex, rotating in the opposite direction, pushes this fluid away from the blade surface towards the centre of the passage. The shearing and mixing between the passage and counter vortices also contributes to the peak losses in region A. Figure 5.4 (a) also shows another region of elevated loss, labelled B, which is much lower in magnitude. This fluid is likely the remains of the endwall boundary layer that is swept cross passage by the blade-to-blade cross passage pressure gradient.

The floods of streamwise vorticity show evidence of the vortical motion described above. In Figure 5.5 (a), there is a region of positive vorticity (the counter vortex) that corresponds to the peak loss location (A). The larger negative vorticity region, corresponding to the loss core $\mathrm{B}$, is associated with the passage vortex. The corner vortex is indistinguishable on this measurement plane. The colour flood of total pressure coefficient shows that fluid is pulled from the edge of the peak loss core (A) towards the endwall and centre of the passage where the other elevated loss region (B) resides. This spreading is caused by the motion of the counter vortex which acts to convect high loss fluid from the blade surface towards the boundary of the inviscid fluid. At this interface, the inviscid fluid mixes with the impinging higher loss fluid and as a result, the peak loss core (A) appears to stretch towards the lower loss core (B).

As expected, the secondary velocity vectors of Figure 5.5 (a) show a strong clockwise rotation about the passage vortex region. At the point labelled $\mathrm{C}$, the low loss fluid from the inviscid core is clearly drawn from the centre of the passage and pushed towards the endwall. The fluid is turned and driven across the passage as indicated by the strong pitchwise velocities in the very near endwall region; evidence of the high level of shearing experienced by the endwall 
boundary layer. Near the suction surface and endwall junction, labelled D, large spanwise velocities develop as the blade potential pushes the fluid away from the endwall and up the blade suction surface. The local losses are high here as the fluid has origins from the both the blade suction surface and endwall boundary layers. The counter-clockwise rotation of the counter vortex appears to push this fluid into the passage where it mixes with the higher momentum inviscid core. At point $\mathrm{E}$, the secondary velocity vectors also show the initiation of a funneling pattern caused by the opposite rotation of the passage and counter vortex. This region becomes more apparent on the $1.20 C_{x}$ and $1.40 C_{x}$ planes.

Figures 5.4 (b) and (c) show the results of the described mixing processes on the downstream total pressure distributions. At the $1.20 C_{x}$ plane, the loss core previously associated with the passage vortex is no longer distinguishable. The passage vortex, shown in Figure 5.5 (b), that was previously compressed against the endwall has moved away from the endwall and towards the suction surface side of the passage. On the other hand, the counter vortex has moved away from the blade suction surface and towards the endwall. The translation of both vortical structures has resulted in a combined loss core that is larger and has lower loss levels than at the $1.00 C_{x}$ plane. The secondary velocity vectors, in Figure 5.5 (b), show a distinct funnelling pattern between the main vortical structures. Again the direction of these vectors will tend to transport the high loss fluid into the inviscid regions of the flow.

At the $1.40 C_{x}$ plane, the spreading of the secondary flow due to the downstream mixing process reduces the magnitude of the vorticity of the vortical structures and lowers the magnitude of the secondary velocity vectors. Both the passage vortex and counter vortex lose angular momentum by mixing with the inviscid core. As the rotation is slowed, the fluid will tend to spread over a larger area as shown in Figures 5.4 (b) and (c). The direction of the secondary velocity vectors will also cause the loss cores to migrate towards the centre of the passage. With downstream distance, the dissipation of secondary kinetic energy is observed through the reduction in the secondary velocities. The resulting loss production is observed through more diffuse floods of the total pressure coefficient. 


\subsubsection{Integrated Losses and Other Flow Quantities}

Often in parametric CFD simulations, such as those used for the design of endwall contouring, the integrated losses are used to assess the blade row performance. In general, the losses predicted from CFD are unreliable as shown by Yan et al. (1999), Hartland et al. (2000), and Harvey et al. (2000). An additional parameter, the secondary kinetic energy (SKE) is sometimes used as a design metric to assess the benefits from proposed geometric modifications. The merits of this approach are unclear from the open literature and therefore the contribution of secondary kinetic energy to the overall measured and mixed-out losses is investigated here. Averaging was performed using the procedures described in Section 3.11.2.

Table 5.2 presents the integrated total pressure, secondary kinetic energy and turbulent kinetic energy (TKE) coefficients. The integrated data at each plane are plotted in Figure 5.6. The dashed lines between the $1.00 C_{x}$ and the $1.20 C_{x}$ data points are intended to indicate the underestimation of the $1.00 C_{x}$ integrations caused by the shortened pitchwise traverse plane. Thus, the discussion will focus on the data from the $1.20 C_{x}$ and $1.40 C_{x}$ planes.

The magnitude of the total pressure coefficient increases by approximately $10 \%$ from the 1.20 to the $1.40 C_{x}$ plane, indicating that there is an increase in the loss production. The secondary kinetic energy decreases by approximately $32 \%$ indicating significant dissipation from the mean flow. It is commonly thought that this reduction in secondary kinetic energy accounts for much of the loss production through and downstream of a bladerow passage (Yan et al., 1999; Hartland et al., 2000; Harvey et al., 2000; Praisner et al., 2007). However, if this were true then the summation of the secondary kinetic energy and the total pressure losses, $\left|C_{P 0}{ }^{\prime \prime}\right|+C_{S K E}{ }^{\prime \prime}$, at each axial plane should equal to a consistent value. This is not the case here, as shown in Figure 5.6.

The value of $\left|C_{P 0}{ }^{\prime \prime}\right|+C_{S K E}{ }^{\prime \prime}$ increases by about $6 \%$ from the 1.20 to $1.40 C_{x}$ plane, indicating that the increase in downstream losses is not solely a result of secondary kinetic energy dissipation. It is possible that the growth of the endwall boundary layer and its interaction with the passage vortex causes this increase. 
Table 5.2: Integrated flow quantities

\begin{tabular}{|c|c|c|c|}
\hline Parameter & $1.00 C_{x}$ & $1.20 C_{x}$ & $1.40 C_{x}$ \\
\hline$\left|C_{P 0}{ }^{\prime \prime}\right|$ & 0.138 & 0.422 & 0.464 \\
\hline$\left|C_{P 0, \text { mixed }}\right|$ & 0.415 & 0.500 & 0.544 \\
\hline$C_{S K E}{ }^{\prime \prime}$ & 0.134 & 0.046 & 0.031 \\
\hline$C_{T K E}{ }^{\prime \prime}$ & 0.023 & 0.022 & 0.022 \\
\hline$\left|C_{P 0}^{\prime \prime}\right|+C_{S K E}^{\prime \prime}$ & 0.272 & 0.468 & 0.496 \\
\hline
\end{tabular}

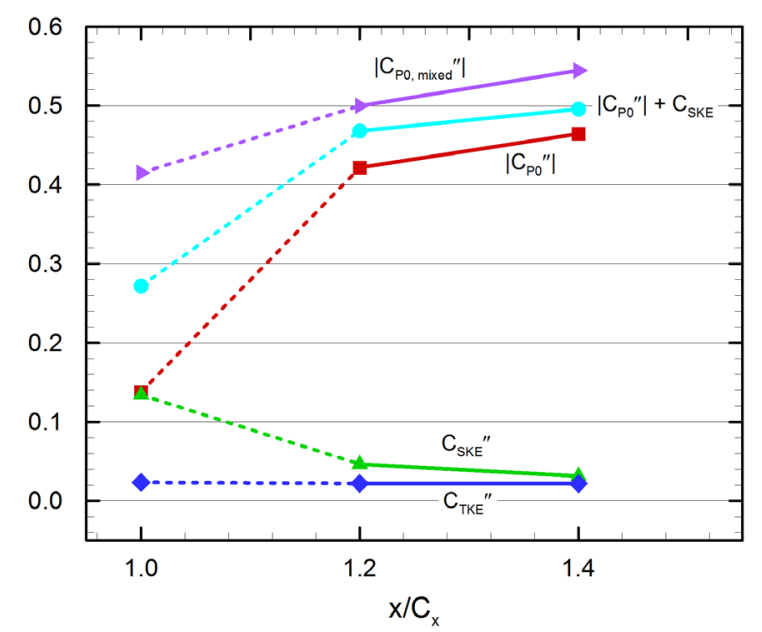

Figure 5.6: Integrated flow quantities at $1.00 C_{x}, 1.20 C_{x}$ and $1.40 C_{x}$

The mixed-out flow quantities are examined next to assess the contribution of the SKE to the mixing losses. The term mixing losses is used here to indicate the difference between the mixed-out and measured total pressure coefficients $\left(C_{P 0, \text { mixed }}{ }^{\prime}-C_{P 0}{ }^{\prime \prime}\right)$. The mixing analysis described in section 3.11.4 was applied to each of the measurement planes. The mixed-out total pressure coefficients are shown in Table 5.2 and plotted in Figure 5.6. At the mixed-out plane the secondary kinetic energy is completely dissipated, appearing as total pressure loss. As such, the secondary kinetic energy is often an indicator of the losses yet to come with further downstream 
mixing. However, additional mixing losses are also realised through the recovery of static pressure and the mixing-out of non-uniformities in the primary kinetic energy (PKE). Therefore, if the secondary kinetic energy is to be used as an alternative design metric then it should represent a significant portion of the mixing losses.

As shown in Figure 5.7 this is not the case for the present cascade. At the 1.20 and $1.40 C_{x}$ plane the secondary kinetic energy accounts for approximately $59 \%$ and $39 \%$ of the mixing losses respectively. The corresponding values are given in Table 5.3.

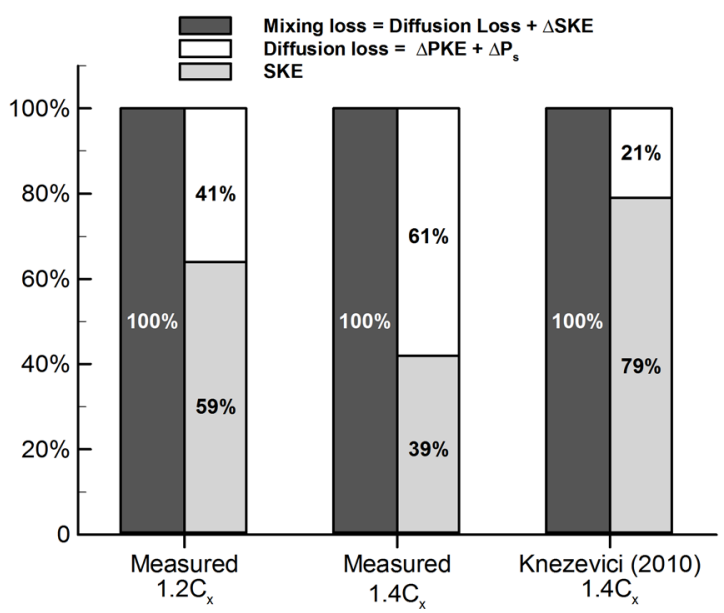

Figure 5.7: Mixing loss breakdown at $1.20 C_{x}$ and $1.40 C_{x}$

For this cascade the recovery of static pressure and the non-uniformities in primary kinetic energy contribute substantially to the mixed-out losses. Thus, in this case it appears that the dissipation of secondary kinetic energy does not account for the majority of the downstream mixing losses as it is sometimes assumed and was largely the case in Knezevici et al. (2010) experiments. It is thought that the relatively high convergence ratio, $C R$, which results in high acceleration through the passage, suppresses the development of the secondary kinetic energy, but also gives rise to streamwise velocity gradients. Consequently, the static pressure recovery and primary kinetic energy contribute significantly to the mixing losses. 
Studies of the secondary losses in different turbine cascades by Benner et al. (2004) and Knezevici et al. (2009; 2010), showed that the SKE contribution to the total mixing losses was at the lowest $62 \%$ and the highest $79 \%$ for different cascades. The apparent variation of the secondary kinetic energy contribution to the overall mixing losses raises questions as to the usefulness of the secondary kinetic energy as a metric for evaluating the benefits of geometric changes during design.

The turbulent kinetic energy (TKE) coefficient, defined as

$$
C_{T K E}=\frac{1}{2} \frac{\left(\overline{u^{\prime 2}}+\overline{v^{\prime 2}}+\overline{w^{\prime 2}}\right)}{V_{C L, 1}^{2}}
$$

is also shown in Figure 5.6. The TKE is relatively constant with axial distance, indicating that the production of turbulence and its dissipation to internal energy appear to be occurring at similar rates. The additional loss production that occurs with increasing downstream distance is not accounted for by the turbulence kinetic energy. The production of turbulence (increasing TKE) is associated with a loss of kinetic energy from the mean flow and thus results in total pressure losses. Although the turbulent kinetic energy is quantified by the Reynolds normal stresses and not the shear components, as shown in Equation 5.3, it will be shown that the shear stresses interaction with the velocity gradients plays an important role in production rate of TKE (see Section 5.5.5) and thus losses from the mean flow.

Table 5.3: Summary of mixing loss breakdown

\begin{tabular}{|c|c|c|c|}
\hline Parameter & $1.20 C_{x}$ & $1.40 C_{x}$ & $\begin{array}{c}\text { Knezevici (2010) } \\
1.40 C_{x} \\
\end{array}$ \\
\hline Measured loss, $C_{P 0}{ }^{\prime \prime}$ & -0.422 & -0.464 & -0.098 \\
\hline$\Delta \mathrm{SKE}=C_{S K E, \text { mixed }}^{\prime \prime}-C_{S K E}^{\prime \prime}$ & -0.046 & -0.031 & -0.015 \\
\hline$\Delta \mathrm{PKE}=C_{P K E, \text { mixed }}^{\prime \prime}-C_{P K E}^{\prime \prime}$ & -0.124 & -0.109 & -0.030 \\
\hline$\Delta \mathrm{P}_{\mathrm{S}}=C_{P S, \text { mixed }}^{\prime \prime}-C_{P S}^{\prime \prime}$ & 0.092 & 0.060 & 0.026 \\
\hline Diffusion loss $=\Delta \mathrm{PKE}+\Delta \mathrm{P}_{\mathrm{S}}$ & -0.032 & -0.049 & -0.004 \\
\hline Mixing loss $=$ Diffusion loss $+\Delta \mathrm{SKE}$ & -0.078 & -0.080 & -0.019 \\
\hline Mixed-out losses, $C_{P 0, \text { mixed }}$ & -0.500 & -0.544 & -0.117 \\
\hline
\end{tabular}




\subsubsection{Downstream Turbulence Intensity}

Figure 5.8 shows floods of turbulence intensity with contour lines of total pressure coefficient for the $1.00,1.20$ and $1.40 C_{x}$ planes. The turbulence intensity shown here $\left(T u_{C L, 2}\right)$ is non-dimensionalized by the midspan mass-averaged velocity of the current measurement plane $\left(V_{C L, 2}^{\prime \prime}\right)$. This is different than the traditional method of non-dimensionalizing by the local velocity $\left(V_{l o c}\right)$, as shown in Equation 5.1, which would tend to amplify the turbulence intensity in regions of low velocity.

The turbulence intensity distribution at each downstream location has a general shape that is consistent with the shape of the total pressure contours. The peak intensities do not coincide with the peak loss cores; however, the regions of highest intensity reside within regions of high total pressure loss. At the $1.00 C_{x}$ plane, Figure $5.8(\mathrm{a})$, there exist two distinct peaks of turbulence intensity, labelled A and B, much like the two peaks of total pressure coefficient discussed previously. The largest of the two (A) has an intensity of about $11 \%$ and is located at $y / s=0.44$ and $z / h=0.04$. The other core of high intensity fluid (B), of approximately $9 \%$ intensity, is located at $y / s=0.15$ and $z / h=0.16$. These regions of elevated turbulence intensity are associated with the counter and passage vortices, but do not coincide with their respective positions. Elevated turbulence is also observed near the blade pressure surface extending from the endwall to midspan at $y / s=0.95$. The maximum value, labelled $\mathrm{C}$, is about $13 \%$ at a spanwise location of $z / h=0.05$. It is thought this region is associated with a pressure side corner vortex, but the close proximity to the blade makes it difficult to identify a coherent structure. This region is not examined further.

At the $1.20 C_{x}$ plane the turbulence intensity associated with passage and counter vortices begin to blend into one region of elevated turbulence, labelled D. The peak intensity (D) is approximately $10.5 \%$ and is located at $y / s=0.15$ and $z / h=0.10$. The motion of the passage and counter vortices has moved the high intensity fluid from positions $\mathrm{A}$ and $\mathrm{B}$ on the $1.00 C_{x}$ plane towards the centre of the passage, much like the translation of the peak total pressure deficit discussed previously. The secondary velocity vectors shown in Figure 5.5 (b) indicate that the 
peak turbulence intensity resides in a region of significant shear, suggesting that this shearing is responsible for the production of turbulence in this region. This is further examined in Section 5.5.5. The turbulence intensity associated with the passage and counter vortices is $9.5 \%$ and $9 \%$ respectively.

At the $1.40 C_{x}$ plane the peak turbulence intensity, labelled E, is approximately $9.5 \%$ and is associated with the passage vortex, as indicated by the floods of streamwise vorticity shown in Figure 5.5 (c). Relative to the $1.20 C_{x}$ plane the peak intensity associated with passage vortex shows negligible dissipation. However, the turbulence intensity associated with the counter vortex is approximately $7.5 \%$, a reduction of $1.5 \%$ from the previous plane. It appears that the counter vortex dissipates turbulent energy more quickly than the passage vortex. Figure 5.5 (c) indicates that the counter vortex is smaller in size and strength and generally has less secondary kinetic energy than the passage vortex. As noted above, the highest rates of turbulence production appear to be within the high shear region between the counter and passage vortex. The larger passage vortex seems to entrain more of this fluid and thus has higher turbulence intensities than the counter vortex.

The mixing process is evident from the three axial planes in Figure 5.8. The turbulence intensity appears to diffuse much like the total pressure coefficient with downstream distance. In the blade wake region the turbulence intensity decays more quickly than in the secondary flow region, from $8 \%$ to $4 \%$ over $0.4 C_{x}$. In the secondary flow region the peak turbulence intensities decay from $11 \%$ to $9.5 \%$. A similar trend is noted for the total pressure distribution. As will be shown in Section 5.5.5 the rate of turbulence production is nearly four times lower in the blade wake than within the secondary flow. This is the likely why the turbulence intensities within the wake region appear to decay more rapidly than in the secondary flow.

An examination of the mean kinetic energy budget for turbulent incompressible flow will provide further insights into how turbulence and losses are produced from the mean flow. 
(a) $1.00 C_{x}$

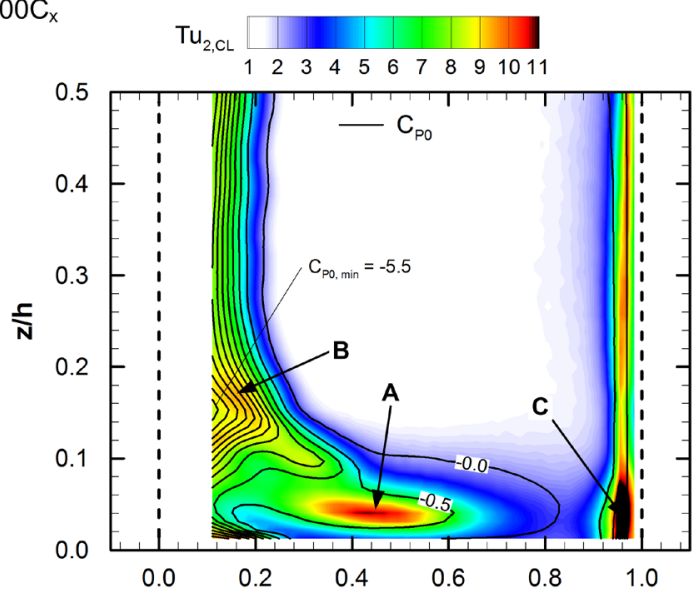

(b) $1.20 C_{x}$

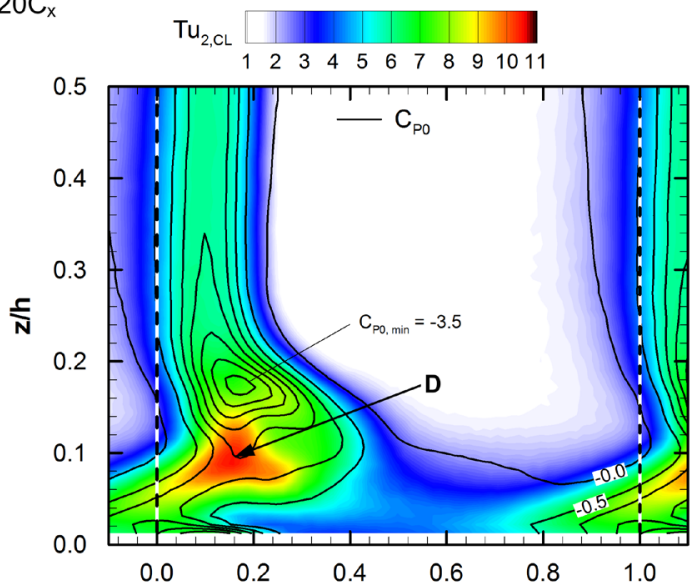

(c) $1.40 \mathrm{C}_{\mathrm{x}}$

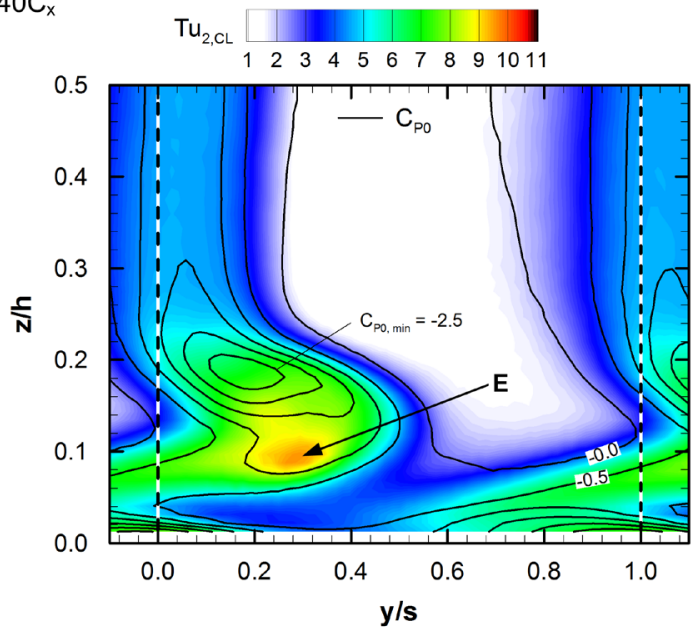

Figure 5.8: Floods of local turbulence intensity $\left(T u_{l o c}\right)$ with contour lines of total pressure coefficient $\left(C_{P 0}\right)$ at (a) $1.00 C_{x}$, (b) $1.20 C_{x}$ and (c) $1.40 C_{x}$. 


\subsubsection{Turbulence and Loss Production from the Mean Flow}

The production of turbulence is the first step to the production of losses through the dissipation of turbulent kinetic energy (Gregory-Smith et al., 1988b). The equation of mean kinetic energy (MKE) for a turbulent flow is shown in Equation 5.4. It is derived from the equations of motion for an incompressible turbulent flow, as shown by Moore et al. (1987). The

mean velocity vector is designated by $\bar{u}_{i}$ and the Reynolds averaged stresses by $\overline{u_{i}^{\prime} u_{j}^{\prime}}$. Expressions for the mean axial velocity gradients were derived using the method described in Section 3.11.5.

$$
\begin{aligned}
& \frac{1}{2} \frac{\partial}{\partial t}\left(\rho \bar{u}_{i}^{2}\right)+\frac{\partial}{\partial x_{j}} \bar{u}_{j}\left(P_{S}+\frac{1}{2} \rho \bar{u}_{i} \bar{u}_{i}\right)+\frac{\partial}{\partial x_{j}} \rho \overline{u_{i}^{\prime} u_{j}^{\prime}} \bar{u}_{i} \ldots \\
& \text { I } \\
& \ldots-\frac{\partial}{\partial x_{j}} \mu \bar{u}_{i}\left(\frac{\partial \bar{u}_{i}}{\partial x_{j}}+\frac{\partial \bar{u}_{j}}{\partial x_{i}}\right)=\rho \overline{u_{i}^{\prime} u_{j}^{\prime}} \frac{\partial \bar{u}_{i}}{\partial x_{j}}-\mu\left(\frac{\partial \bar{u}_{i}}{\partial x_{j}}+\frac{\partial \bar{u}_{j}}{\partial x_{i}}\right) \frac{\partial \bar{u}_{i}}{\partial x_{j}}
\end{aligned}
$$

IV $\quad \mathrm{V}$

The terms in the equation have the following physical interpretation (Moore et al., 1987):

(I) the rate of change of mean kinetic energy

(II) the rate of convective transport of total pressure

(III) the rate of mean kinetic energy transport by turbulent fluctuations

(IV) the rate of mean kinetic energy transport by mean viscous stresses

(V) the deformation work of the mean flow by turbulence stresses. The rate at which turbulence is produced from the mean flow as turbulent kinetic energy

(VI) the direct viscous dissipation of mean kinetic energy

In the following section, Equation 5.4 is decomposed into the primary and secondary flow directions $\left(x_{\text {prim }}, y_{\text {sec }}, w_{\text {sec }}\right)$, rather than the Cartesian coordinate directions $(x, y, z)$, as defined in Figure 3.7. Thus, $i, j=1,2$ or 3 correspond to the $x_{p r i m}, y_{s e c}$, and $w_{s e c}$ directions respectively. In this coordinate system, the energy transfer through the production of turbulence, is observed as a loss from the primary mean flow. Term $(\mathrm{V})$, the deformation work, is used to visualize the rate of 
turbulence production from the mean flow and is an indication of the rate of total pressure loss production.

Figures 5.9 (a) and (b) show floods of the total deformation work term, $\Psi_{i, j}$, with contour lines of the total pressure coefficient, $C_{P 0}$, and the secondary velocity vectors for the 1.20 and $1.40 C_{x}$ planes. The non-dimensional total deformation work coefficient, defined by:

$$
\Psi_{i, j}=\frac{\overline{u_{i}^{\prime} u_{j}^{\prime}}}{V_{C L, 1}^{2}} \cdot \frac{\partial\left(\bar{u}_{i} / V_{C L, 1}\right)}{\partial\left(x_{j} / C_{x}\right)}
$$

is the summation of all nine terms. Negative values represent the rate at which mean kinetic energy is lost to the production of turbulence through the Reynolds stresses, and thus the rate of total pressure loss production. Positive values represent a gain of kinetic energy from the action of the Reynolds stress field. It is difficult to visualize how the mean flow can receive energy from Reynolds stress field; however, this effect has been observed in a number of other studies (Moore et al., 1987; Gregory-Smith et al., 1988b; Perdichizzi et al., 1992; Gustafson et al., 2007). Such positive values are discussed later with reference to Figure 5.9.

Term (VI) in Equation 5.4, $-\mu\left(\frac{\partial \bar{u}_{i}}{\partial x_{j}}+\frac{\partial \bar{u}_{j}}{\partial x_{i}}\right) \frac{\partial \bar{u}_{i}}{\partial x_{j}}$, represents the viscous dissipation of mean kinetic energy and therefore contributes to the generation of total pressure loss. At all points in the flow term (VI) is a loss. The following derivation demonstrates that the sign of term (VI) is always negative.

If the mean strain rate tensor is given by $S_{i j}=\frac{1}{2}\left(\frac{\partial \bar{u}_{i}}{\partial x_{j}}+\frac{\partial \bar{u}_{j}}{\partial x_{i}}\right)$ then term (VI) can be simplified as follows:

$$
-2 \mu S_{i j} \frac{\partial \bar{u}_{i}}{\partial x_{j}}
$$


The mean strain rate tensor, $S_{i j}$, by definition is the symmetric part of the mean velocity gradient, $\frac{\partial \bar{u}_{i}}{\partial x_{j}}$. It can be shown that the product of a symmetric tensor (i.e. $S_{i j}$ ) with another tensor (i.e. $\frac{\partial \bar{u}_{i}}{\partial x_{j}}$ ) is equal to the symmetric tensor times the symmetric part of the other tensor. A full proof is given by Kundu and Cohen (2004a), such that:

$$
S_{i j} \frac{\partial \bar{u}_{i}}{\partial x_{j}}=S_{i j}\left(\frac{1}{2}\left(\frac{\partial \bar{u}_{i}}{\partial x_{j}}+\frac{\partial \bar{u}_{j}}{\partial x_{i}}\right)\right)=S_{i j} S_{i j}
$$

It then follows that term (VI) is simplified as:

$$
-2 \mu S_{i j} \frac{\partial \bar{u}_{i}}{\partial x_{j}}=-2 \mu S_{i j}\left(\frac{1}{2}\left(\frac{\partial \bar{u}_{i}}{\partial x_{j}}+\frac{\partial \bar{u}_{j}}{\partial x_{i}}\right)\right)=-2 \mu S_{i j} S_{i j}
$$

As shown, the product of the mean strain rate, $S_{i j} S_{i j}$, is positive for all points in the flow. Thus, term (VI) is always negative and represents the viscous dissipation of mean kinetic energy to heat (Kundu and Cohen, 2004b).

In the present work term (VI) was evaluated from the measured mean velocity gradient. The values of term (VI) were several orders of magnitude less than the deformation work, term $(\mathrm{V})$. This is not a unique result as it is well accepted that term VI, the mean kinetic energy lost to viscous dissipation, is small (Kundu and Cohen, 2004b). Therefore, the deformation work (the production of turbulence) is the main contribution to the production of loss from the mean flow, which appears as total pressure loss in the mean flow pressure measurements.

The deformation work term also appears in the equation of turbulent kinetic energy (not shown here) with the sign reversed. The physical implication is that a loss of mean kinetic energy from the mean flow (negative deformation work) results in a gain of turbulent kinetic energy. Once the turbulence is produced, the loss from the mean flow has been realized. However, the turbulent energy must then dissipate by viscous action to constitute a final loss to internal energy. 
In the equation of turbulent kinetic energy there exists a term analogous to the (VI) term of Equation 5.4, as follows:

$$
\mu \overline{\left(\frac{\partial u_{i}^{\prime}}{\partial x_{j}}+\frac{\partial u_{j}^{\prime}}{\partial x_{i}}\right) \frac{\partial u_{i}^{\prime}}{\partial x_{j}}}
$$

It represents the viscous dissipation of the turbulent kinetic energy. It is also well known that the gradients of the velocity fluctuations result in a term that is comparable in magnitude to the deformation work term (Kundu and Cohen, 2004b). The implication is that there is significant dissipation caused by the gradients of the turbulent fluctuations. As previously shown in Figure 5.6, the overall integrated values of the turbulent kinetic energy show little change with axial distance. The significant turbulence production, shown by the high levels of negative deformation work in Figures 5.9 (a) and (b), is therefore balanced by the viscous dissipation of the mean and turbulent kinetic energy.

In Figure 5.9 (a), the highest rate of turbulence production is in a region of high shear at $y / s=0.08$ and $z / h=0.07$. As shown by the secondary velocity vectors, this is the location where the counter and passage vortices interact. The two vortices have different magnitudes of streamwise vorticity, resulting in turbulence production as they begin to interact and mix. Other regions of elevated negative deformation work exist where the secondary flow interacts with the inviscid core. As previously described, in the discussion of the total pressure losses in Section 5.5.2, the high momentum inviscid core will lose energy to the rotating vortical structures along this boundary. In Figure 5.9, the yellow and red regions indicate that mean energy is lost to the production of turbulence. The measurements indicate that the Reynolds shear stresses, as opposed to the normal stresses, and the corresponding mean velocity gradients are the main contributors to this loss of mean kinetic energy. It is therefore evident that the Reynolds shear stresses are fundamentally important to the production of total pressure losses downstream of the cascade. Further evidence, discussed later, is presented in Figures 5.10 and 5.11 (a) to (c). 

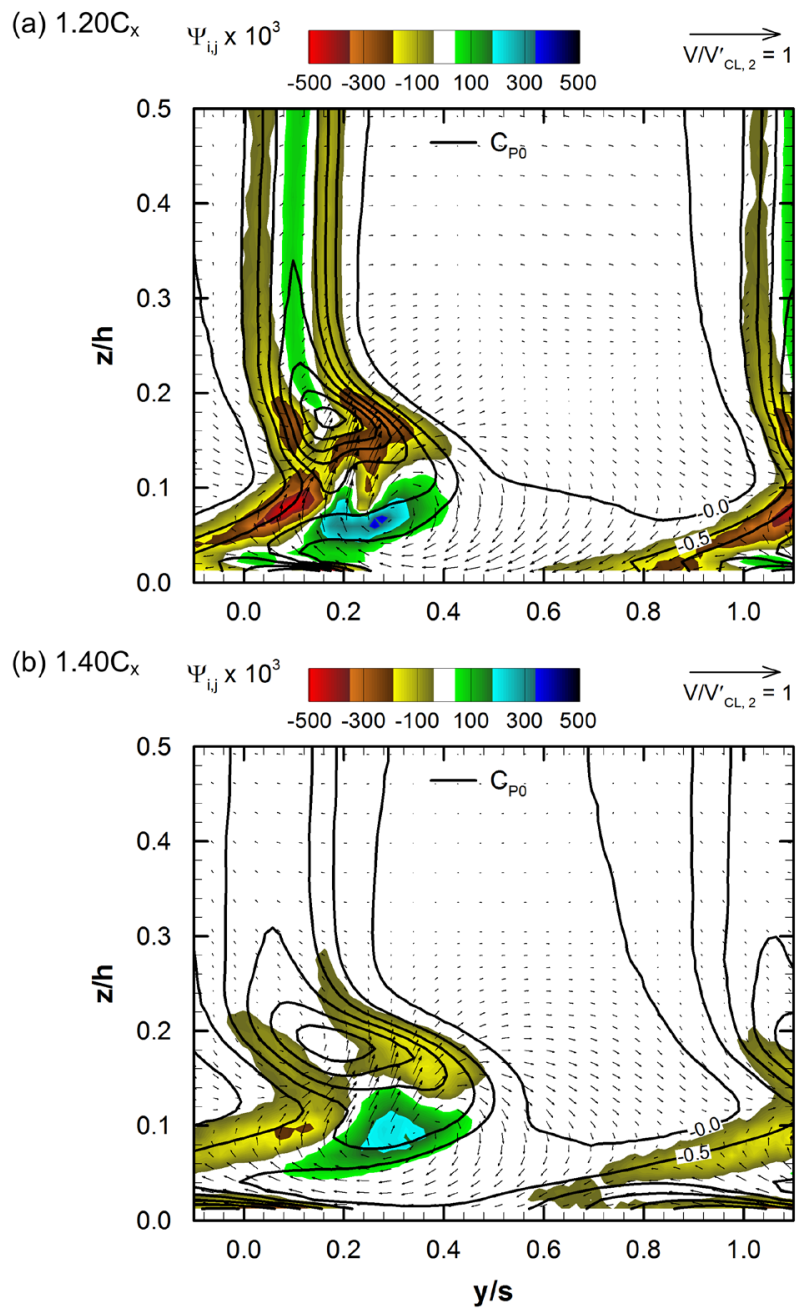

Figure 5.9: Floods of the deformation work term, $\Psi_{\mathrm{i}, \mathrm{j}}$, with contour lines of total pressure coefficient and the secondary velocity vectors at (a) $1.20 C_{x}$ and (b) $1.40 C_{x}$

There are significant areas of positive deformation work, as indicated by the green regions of Figure 5.9. These occur mainly within the region of the passage vortex and also in the twodimensional wake region. As will be shown later in Figures 5.10 and 5.11, the Reynolds normal stresses are the dominant components in these positive regions. The velocity gradients are small, but the normal stresses are large and consequently the deformation work term has a relatively large positive magnitude. This suggests that in regions where the flow is locally accelerated the mean flow can gain primary kinetic energy from the turbulent normal stresses. In a similar 
cascade experiment, Perdichizzi et al. (1992) also reported regions of positive deformation work within the secondary flow. Actually, their sign convention was reversed. Thus, these regions were referred to as negative deformation work but similarly represent a return of kinetic energy to the mean flow. These regions appeared on the downstream plane (at $1.30 C_{x}$ ) and were adjacent to the passage vortex. Much like the present work, Perdichizzi et al. (1992) concluded that a return of energy to the mean flow is caused by the action of the normal stresses in locally accelerating regions (positive velocity gradient). However, they also note that the $\overline{u^{\prime} w^{\prime}}$ shear stress also contributed to the positive regions (reported as negative) since the corresponding velocity gradient $(\partial \bar{u} / \partial z)$ had the same sign. In the present work, the shear components appear to have a negligible contribution. Also, the positive regions tend to weaken with downstream distance, while Perdichizzi et al.'s (1992) results show an increase in positive deformation work (reported as negative) with downstream distance.

The results from the $1.40 C_{x}$ plane are shown in Figure 5.9 (b). Here, both positive and negative regions of deformation work are again evident. As before, the negative regions are located within the high shear regions of the flow field. The action of the deformation work from the $1.20 C_{x}$ plane was to spread the regions of total pressure deficit into the inviscid core through mixing. This mixing is evident in Figure 5.9 from the broadening of the secondary flow region. The peak rate of turbulence production (negative deformation work) at the $1.40 C_{x}$ plane has been reduced by approximately $50 \%$ as the flow mixes to more uniform conditions with downstream distance. The positive region of deformation work is local to the passage vortex and its magnitude has been reduced by approximately $40 \%$ relative to the $1.20 C_{x}$ plane.

From Figures 5.9 (a) and (b) it is not evident which components of the deformation work are most important to the turbulence production. Figures 5.10 and 5.11 show pitchwise distributions of the nine components at the $1.20 C_{x}$ and $1.40 C_{x}$ planes. The data are extracted at three spanwise locations: midspan, the location of the peak total pressure loss and the location of the passage vortex core. As shown, the sub-plots are categorized into rows such that figures (a) through (c) correspond to the midspan, (d) through (f) the peak loss core and (g) through (i) the 
passage vortex core. The components corresponding to the shear and normal Reynolds stresses are plotted separately, as indicated. At the bottom of the figure there are two additional sub-plots showing the location of the pitchwise distributions relative to colour floods of the streamwise vorticity coefficient and the total deformation work. Contour lines of the total pressure coefficient are also overlaid.

At midspan of the $1.20 C_{x}$ measurement plane, Figures 5.10 (a) to (c), the $\Psi_{1,2}$ component has the largest values of negative deformation work within the wake and is therefore the most significant contribution to the local turbulence production. By definition, the $\Psi_{1,2}$ component corresponds to the $\overline{u^{\prime} v^{\prime}}$ Reynolds stress and the pitchwise gradient of primary velocity $(\partial \bar{u} / \partial y)$. Since, the flow at mid-span is primarily two-dimensional it is expected that the $\overline{u^{\prime} v^{\prime}}$ Reynolds stress would be dominant in this area. Also, this Reynolds stress and velocity gradient are opposite in sign through the wake which results in the regions of negative deformation work. As shown, there are two troughs of the $\Psi_{1,2}$ component that straddle the location of minimum total pressure deficient (at $y / s=0.10$ ). The trough on the right is larger than the one on the left. This bias is likely caused by the larger blade normal velocity gradients present within the suction surface boundary layer. The larger gradients result in more turbulence production over the suction surface as well as downstream of the blade trailing edge.

In Figure 5.10 (a), the $\Psi_{2,1}$ component has a similar distribution to the dominant $\Psi_{1,2}$, albeit with smaller troughs through the wake region. Since both components are computed using the same Reynolds stress $\left(\overline{u^{\prime} v^{\prime}}\right)$ the observed differences are a result of the smaller velocity gradient $(\partial \bar{v} / \partial x)$. The consistent shape of the two distributions does however indicate that it is in fact $\overline{u^{\prime} v^{\prime}}$ Reynolds stress that is significant to the production of the turbulence, and thus loss, within the blade wake. As expected for the midspan location, the $\overline{u^{\prime} w^{\prime}}$ component is insignificant. 

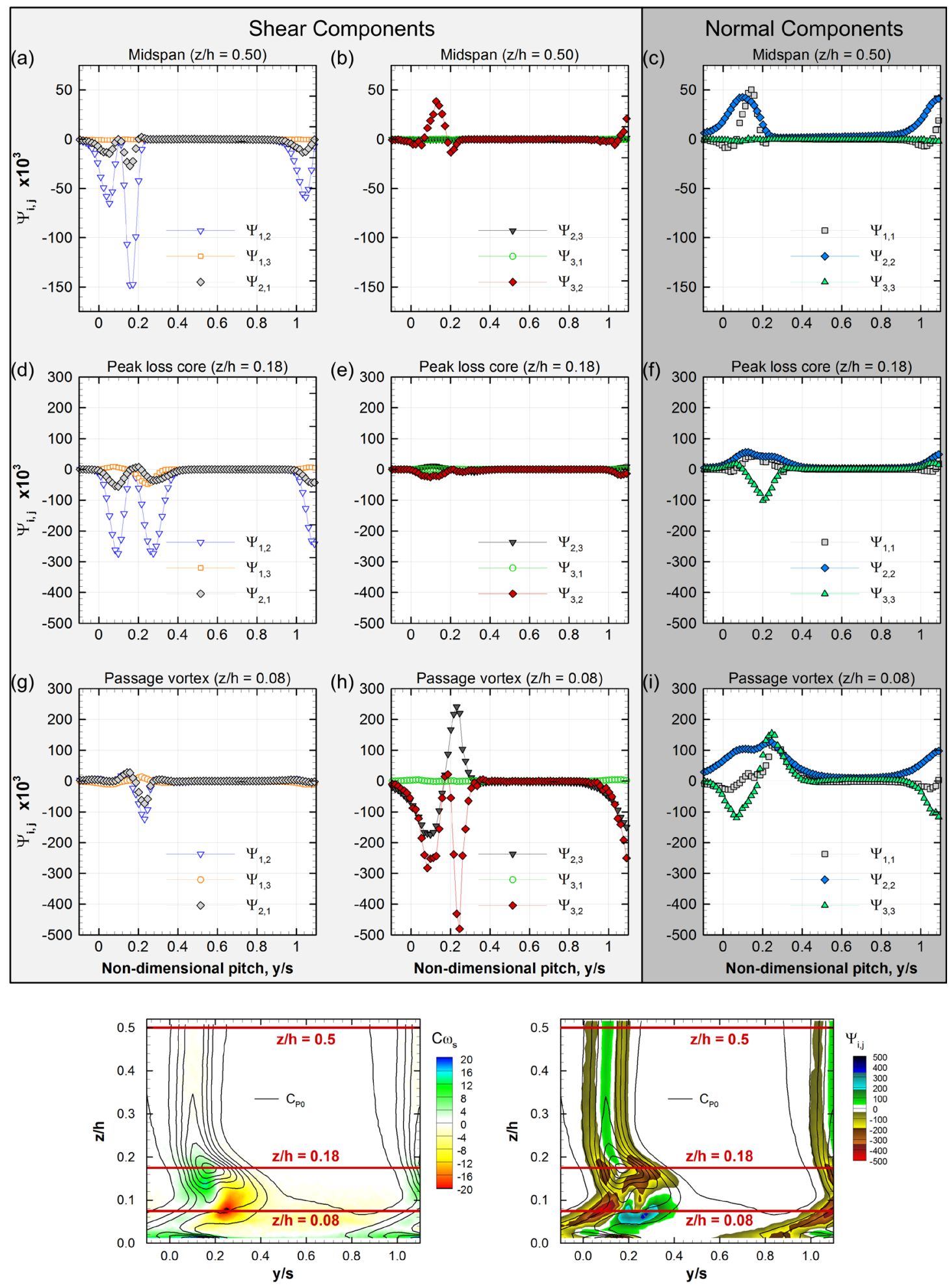

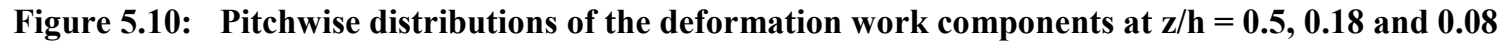
from the $1.20 C_{x}$ plane 
Figure 5.10 (c) shows that positive deformation work observed within the wake region is primarily a result of the $\Psi_{1,1}$ and $\Psi_{2,2}$ components. The corresponding normal Reynolds stresses, $\overline{u^{\prime 2}}$ and $\overline{v^{\prime 2}}$, have large positive values and the gradients, $\partial \bar{u} / \partial x$ and $\partial \bar{v} / \partial y$, are small but also positive. As shown in Figure 5.10 (b), the $\Psi_{3,2}$ component also makes a positive contribution. In this case the $\overline{v^{\prime} w^{\prime}}$ stress and the corresponding velocity gradient $(\partial \bar{w} / \partial y)$ are both negative. This result is not a general one. As will be shown for the other pitchwise distributions, the regions of positive deformation work tend to be dominated by the action of the normal stresses while the shear components tend to dominate the turbulence production (negative deformation work).

Figures 5.10 (d) and (f) show the pitchwise distributions through the peak loss location at $z / h=0.18$ of the $1.20 C_{x}$ plane. Note that the vertical axis scale is larger than that midspan to accommodate the larger values of deformation work. In general, the overall shape of the distributions is very similar to those at midspan. The largest turbulence production comes from the same $\Psi_{1,2}$ component which similarly shows two troughs on either side of the minimum total pressure deficient $(y / s=0.18)$. Since the flow here is only slightly three-dimensional with small spanwise mean velocities, the similarities are not surprising. A notable difference, however, is the turbulence production associated with the $\Psi_{3,3}$ component, as shown in Figure 5.10 (f). Previously at midspan, the normal components were shown to transfer only kinetic energy back to the mean flow (positive deformation work). Here, within the peak loss core, positive values of the $\overline{w^{\prime 2}}$ normal Reynolds stress occur where the corresponding velocity gradient $(\partial \bar{w} / \partial z)$ is negative, thereby resulting in the turbulence production. Thus, it appears that in this slightly three-dimensional region the normal Reynolds stresses may act to generate turbulence or return kinetic energy to the mean flow depending on the sign of the local gradient. However, it should be noted that the turbulence production here is dominated by the shear component $\left(\Psi_{1,2}\right)$ which is nearly three times larger than the normal component $\left(\Psi_{3,3}\right)$. 
Similar conclusions regarding the roles of the normal and shear Reynolds stresses can be drawn from the distributions extending through the passage vortex core, shown in Figures 5.10 (g) through (i). Again, a shear component is the most dominant in the turbulence production. However, here it is the $\Psi_{3,2}$ component and the corresponding $\overline{v^{\prime} w^{\prime}}$ shear Reynolds stress. The magnitude of the shear stress alone is moderate, but when combined with the corresponding velocity gradient $(\partial \bar{w} / \partial y)$ the rate of loss production is observed to be relatively high. This indicates again that the Reynolds shear stresses, large or small in magnitude, are important to the production of total pressure losses.

In Figure $5.10(\mathrm{~h})$ the minimum value of the $\Psi_{3,2}$ component occurs at $y / s=0.24$. This corresponds with the minimum value of streamwise vorticity through the passage vortex as shown by the colour flood. Although significant turbulence production occurs here, it is offset by the action of the normal stresses and their associated positive deformation work shown in Figure 5.10 (i). Consequently, the minimum total deformation work (summation of the nine components) is adjacent to the passage vortex core. As shown by the two-dimensional flood of streamwise vorticity, this is a high shear region where the passage and counter vortex interact.

The data from the $1.40 C_{x}$ plane are shown in Figure 5.11. Note that the range of the vertical axis is smaller than that used for the $1.20 C_{x}$ planes. Overall, the trends at the $1.40 C_{x}$ plane are very similar to those at $1.20 C_{x}$. At mid-span, Figures 5.11 (a) through (c), the $\Psi_{1,2}$ is the dominant component. Again, the two troughs straddle the location of the peak total pressure loss with turbulence production biased to the suction side of the blade wake. The magnitude of the $\Psi_{1,2}$ component is about $1 / 3$ of that from the previous plane. Through the peak loss core, Figures 5.11 (d) through (e), the $\Psi_{1,2}$ is again dominant and has a similar distribution to that at the $1.20 C_{x}$ plane. The magnitude of the troughs is reduced here by about $1 / 3$. Differences, relative to the $1.20 C_{x}$ plane, are noted in the passage vortex region at $z / h=0.09$, Figure $5.11(\mathrm{~g})$ through (i). Unlike at the $1.20 C_{x}$ plane, the $\Psi_{3,2}$ component in Figure 5.11 (h) does not show a 

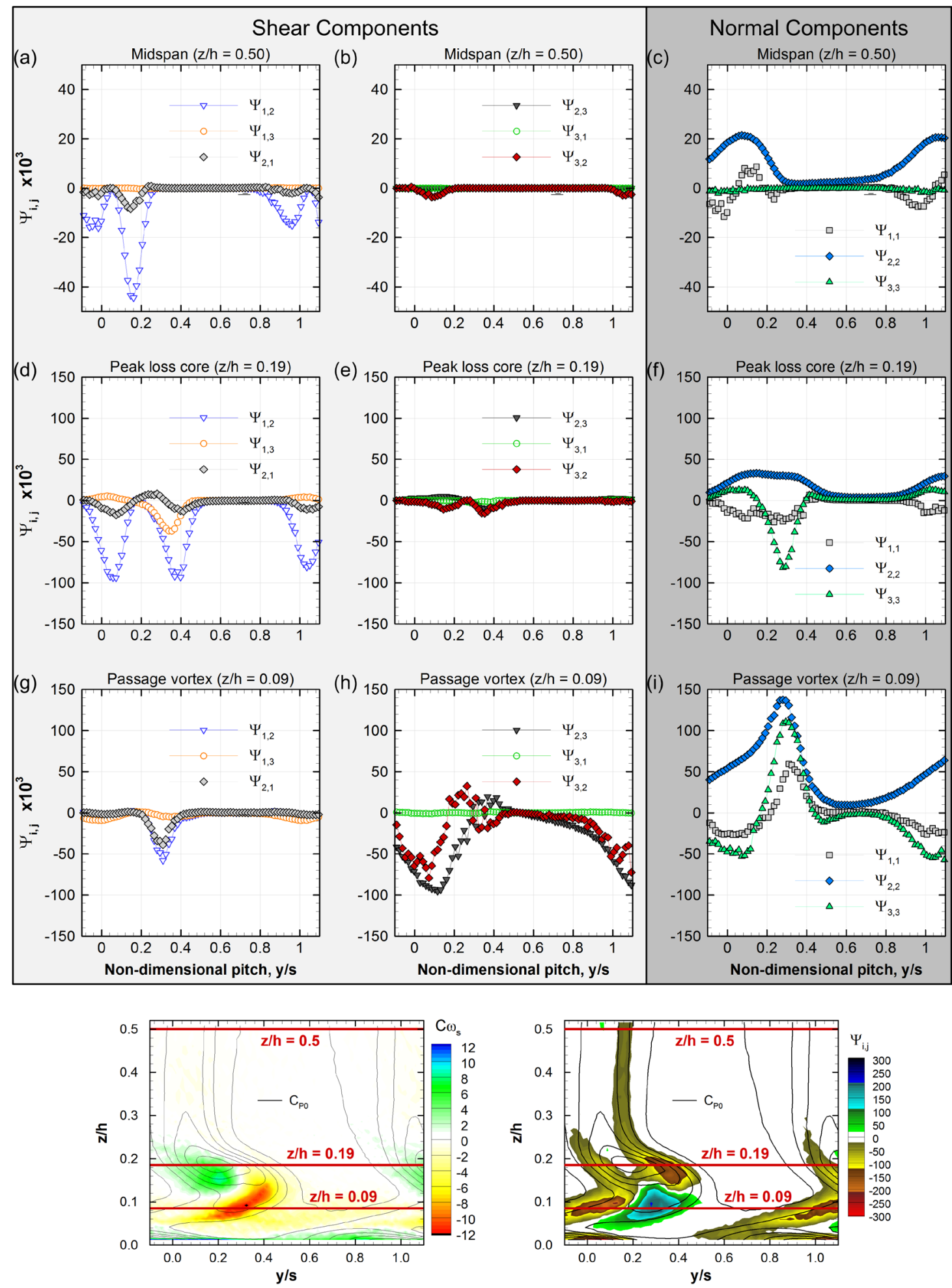

Figure 5.11: Pitchwise distributions of the deformation work components at $\mathrm{z} / \mathrm{h}=\mathbf{0 . 5 , 0 . 1 8}$ and 0.08 from the $1.40 C_{x}$ plane 
distinct peak that corresponds to the location of minimum streamwise vorticity through the passage vortex $(y / s=0.24)$. In this case, it is the $\Psi_{1,2}$ and $\Psi_{2,1}$ components, shown in Figure $5.11(\mathrm{~g})$, that are responsible for the turbulence production within the vortex core. However, like at the $1.20 C_{x}$ plane, the overall production at this location is offset by the action of the normal stresses. Here, the $\Psi_{2,2}$ component shows similar magnitudes to those at the $1.20 C_{x}$ plane, while the $\Psi_{1,1}$ and $\Psi_{3,3}$ components are reduced by about $50 \%$ and $30 \%$ respectively.

In the region adjacent to the passage vortex at $y / s=0.14$, the $\Psi_{2,3}$ and $\Psi_{3,2}$ components are still the primary source of loss production (negative deformation work), as shown in Figure $5.11(\mathrm{~h})$. As previously mentioned, this is the region of high shear where the passage and counter vortex interact. The large secondary velocity gradients, coupled with moderate levels of Reynolds shear stress result in significant rates of turbulence production. Similar to the $1.20 C_{x}$ plane, the $\Psi_{3,3}$ normal component also contributes to the losses in this region as shown in Figure 5.11 (i). The magnitude of this component is about $50 \%$ less than at the $1.20 C_{x}$ plane.

In summary, similar trends are observed at the two measurement planes. The physics are consistent and the mixing process is evident by the reduction of the local peaks of the deformation work. In general, the Reynolds shear stresses and corresponding gradients are largely responsible for much of the turbulence, and thus loss, production. On the other hand, the Reynolds normal stresses generally act to return some turbulent energy back to the mean flow within the passage vortex region (positive deformation work). This is the action of positive normal stresses in locally accelerated (positive velocity gradient) regions of the flow. 


\subsection{Computational Results for the Downstream Flow Field}

\subsubsection{Introduction}

The following sections present the predicted flows corresponding to the measured results of Section 5.5. The computational procedures were described in Chapter 4. The mean flow fields are compared first followed by a discussion of the turbulence quantities. This section repeats a number of experimental results for comparison purposes.

Often the differences between predicted and measured results are attributed to short comings of two-equation turbulence models. In these models the eddy viscosity is isotropic, as defined by Boussinesq's approximation, which is unrealistic for many practical situations including the secondary flows in turbomachinery. However, two-equation models such as these are still largely used for turbomachinery design applications due to their robustness and low computational cost. Thus, this section will investigate the anisotropy of the implied eddy viscosity tensor, calculated from the measured Reynolds stresses and the mean velocity gradient. The implied eddy viscosity is not a physical quantity. It is only presented in the following subsections for comparison purposes with the isotropic eddy viscosities of the predicted flow field.

\subsubsection{Development of the Secondary Flow with Downstream Distance}

The CFD results are examined for the $1.20 C_{x}$ and $1.40 C_{x}$ planes. Figure 5.12 shows floods of the measured and predicted total pressure coefficients. Figure 5.13 shows corresponding floods of the streamwise vorticity coefficient and the secondary velocity vectors.

Figures 5.12 (a) and (b) are for the $1.20 C_{x}$ axial plane. In the measured results, Figures 5.12 (a), there is one distinct loss core, labelled A. As previously mentioned, this region of high loss does not coincide with any of the major vortical structures, as also seen from Figure 5.13 (a). The peak loss is concentrated in the region of high shear between the passage vortex and the counter vortex. The corresponding flood of the predicted total pressure coefficient 
(a) $1.20 C_{x}$ Measured

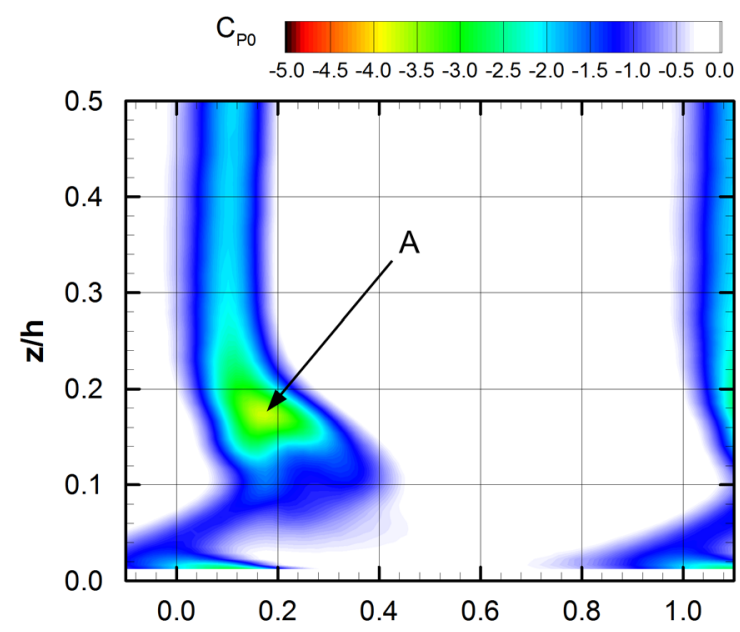

(c) $1.40 \mathrm{C}_{\mathrm{x}}$ Measured

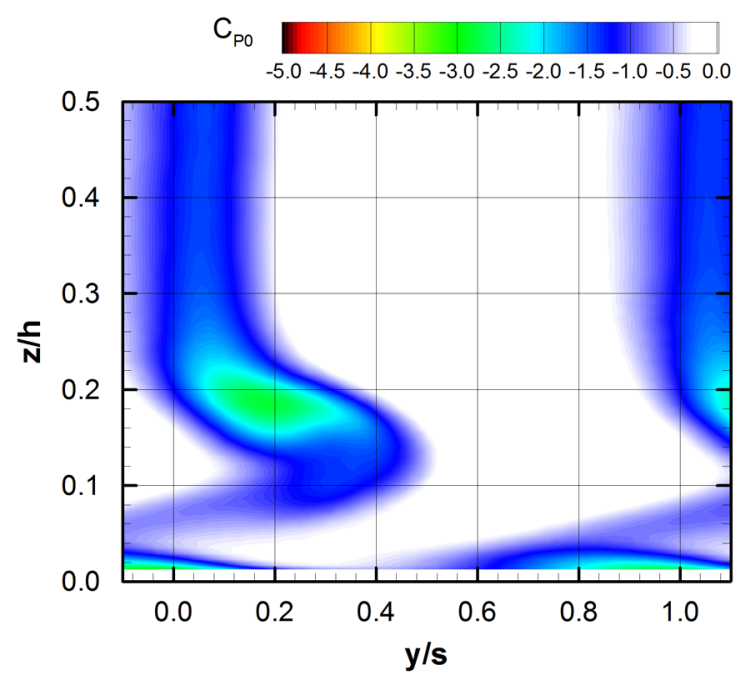

(b) $1.20 C_{x}$ CFD

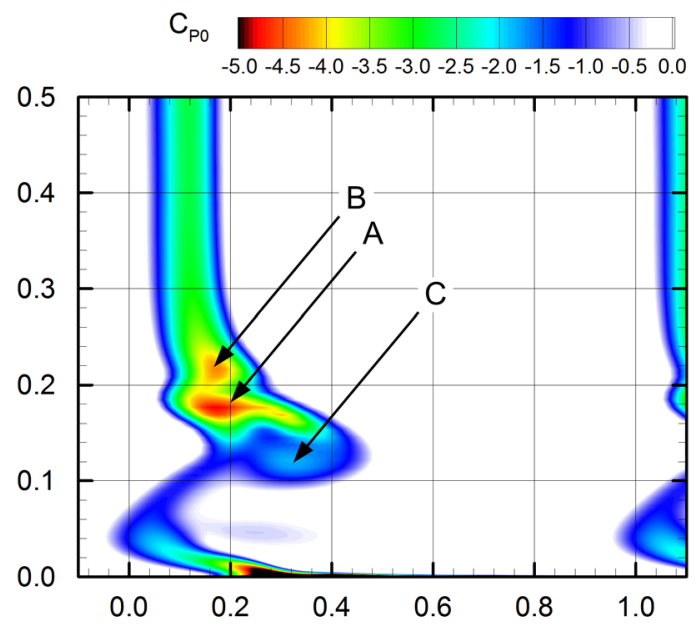

(d) $1.40 C_{x}$ CFD

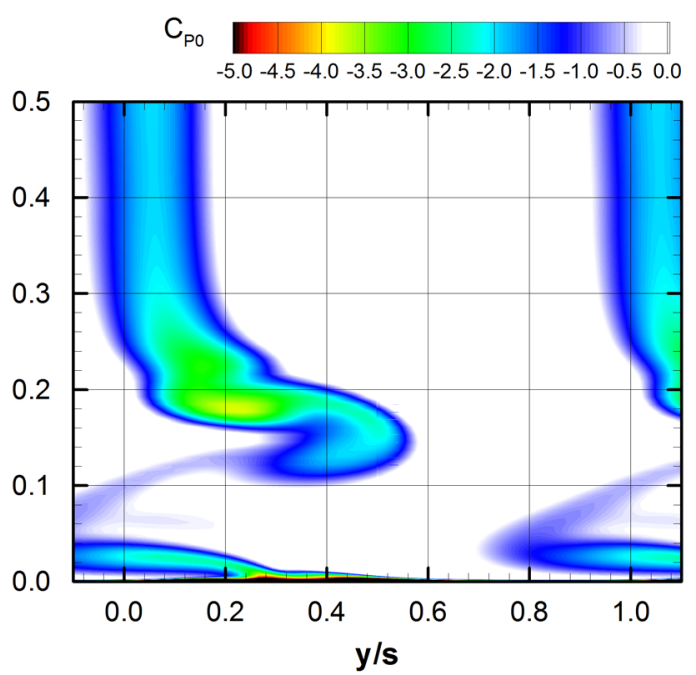

Figure 5.12: Measured and computed total pressure coefficient $\left(C_{P 0}\right)$ floods at $1.20 C_{x}$ and $1.40 C_{x}$

is shown in Figure 5.12 (b). The highest losses are located very close to the endwall, at about $y / s=0.26$ and $z / h=0.01$. This region was too close to the endwall to be measured and therefore the following discussion will concentrate on the large region of high losses away from the endwall.

Compared to the measurements, the predicted losses here are locally much greater in magnitude and are more highly concentrated. The peak loss, labelled A, is about $22 \%$ higher than the measured and is located slightly more towards midspan, at $y / s=0.18$ and $z / h=0.18$. Unlike 
the measured results, the predicted peak loss is nearly coincident with the location of the counter vortex, at $y / s=0.17$ and $z / h=0.18$, suggesting that significant loss production occurs within that vortex. The computations also show two additional distinct loss cores, labelled B and C. Loss core $\mathrm{B}$ is located above the counter vortex and coincides with a region of lower negative streamwise vorticity, as shown in Figure 5.13 (b). This structure appears as a weak coherent vortex core in the computations but is not observed experimentally. In the experimental flow such a structure may already have been dissipated by viscous action or have become entrained by the counter-vortex. Loss core $\mathrm{C}$ is considerably weaker than the other two and is local to the passage vortex, as shown from Figure 5.13 (b). The measurements also show a total pressure deficit in the passage vortex region, although the magnitude is again lower than in the computations.

In general, it appears that the vortical structures are larger in the measurements than the computations. The high loss fluid is spread over a larger area and the peak losses of the measurements are reduced. At the same time, a region of measured high loss production exists in the highly sheared flow between the passage and counter vortices. High loss production in this inter-vortex region is also likely present in the computations, and may be the origin of the tail of high losses associated with loss core A in Figure 5.13 (b). However, the highly concentrated vortices and the corresponding high losses in the predictions seem to mask this inter-vortex loss production.

The results for $1.40 C_{x}$ plane show significant spreading of the fluid associated with the secondary vortices. Also, there are reductions in the peak losses compared with the $1.20 C_{x}$ plane, for both the measured and computed results. For example, the measured streamwise vorticity associated with the passage vortex, is reduced by approximately $40 \%$, while the predictions show a reduction of about $17 \%$. Similarly, the peak streamwise vorticity for the counter vortex is reduced by $38 \%$ for the measurements and $28 \%$ for the computed results. Thus, the spreading of the vortical fluid, which implies a reduction in the strength of the vortices, occurs consistently more quickly in the experiment than in the computations. 
(a) $1.20 C_{x}$ Measured

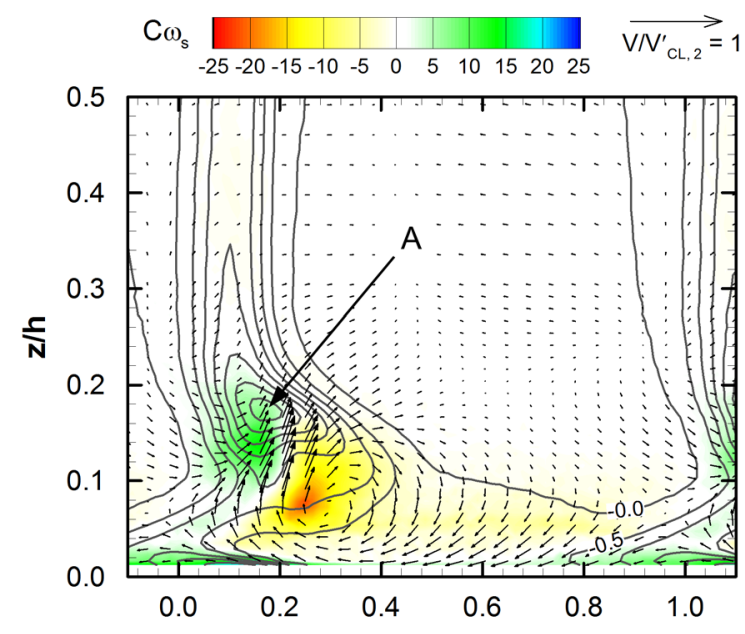

(c) $1.40 \mathrm{C}_{\mathrm{x}}$ Measured

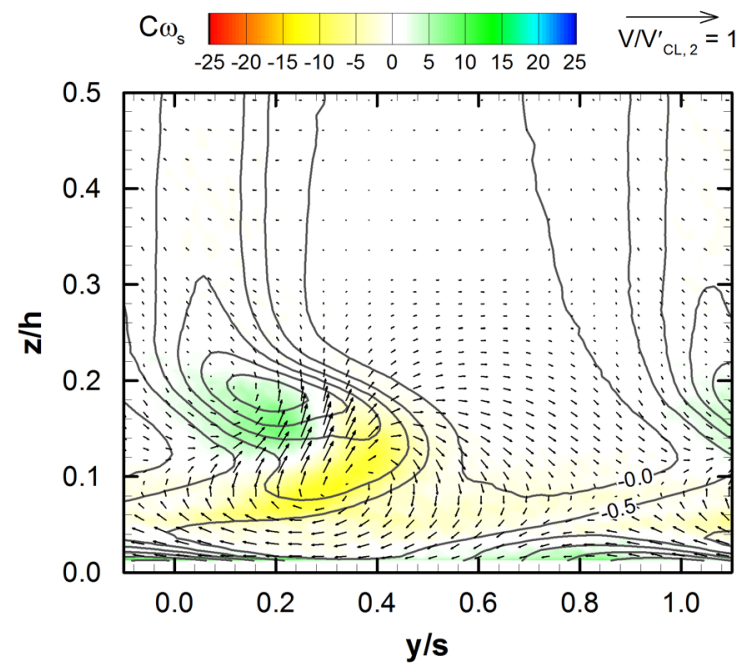

(b) $1.20 C_{x}$ CFD

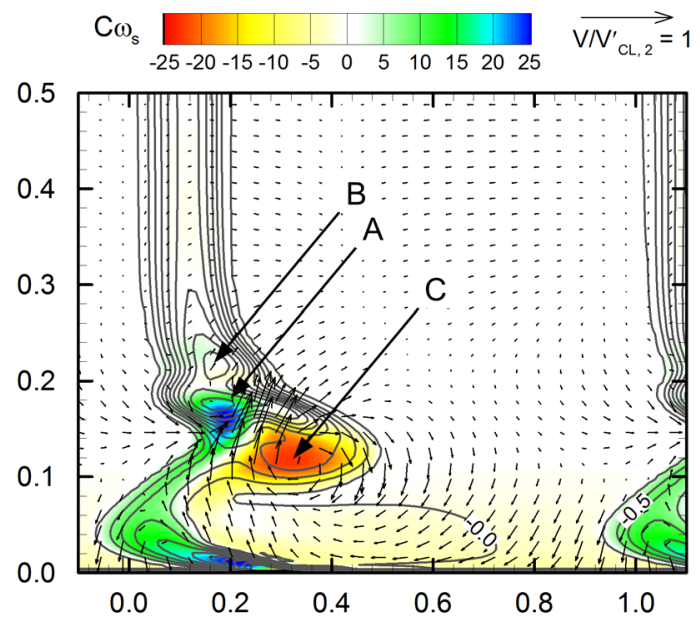

(d) $1.40 \mathrm{C}_{\mathrm{x}} \mathrm{CFD}$

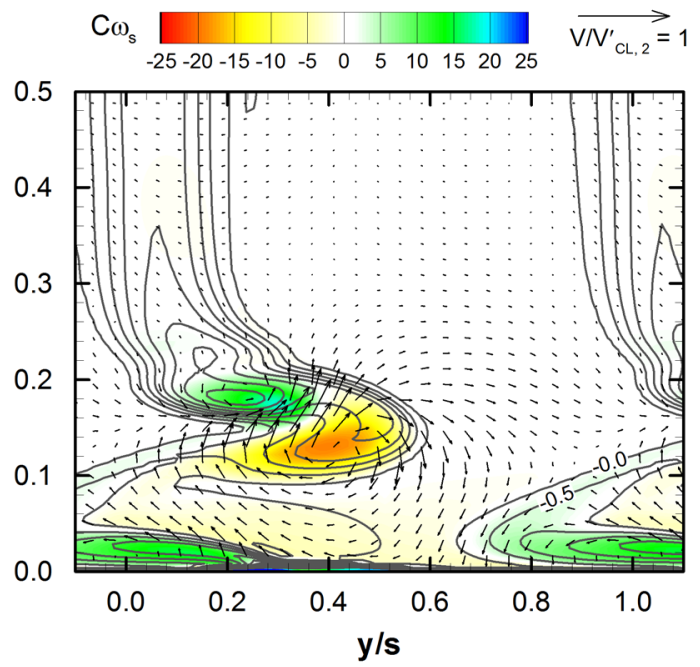

Figure 5.13: Measured and computed streamwise vorticity coefficient $\left(C_{\omega s}\right)$ floods and secondary velocity vectors at $1.20 C_{x}$ and $1.40 C_{x}$

\subsubsection{Integrated Losses and Other Flow Quantities}

The integrated results from the two axial planes are summarized in Table 5.4. Figure 5.14 shows the relative magnitudes of the flow quantities, normalized by the measured mass-averaged total pressure coefficient from the respective planes. At $1.20 C_{x}$ and $1.40 C_{x}$ the measured massaveraged total pressure loss is $5.7 \%$ and $7.5 \%$ greater than the computed values respectively. 
This result is surprising given that the computed peak losses are much higher than the measured values (for example, $22 \%$ higher for the $1.20 C_{x}$ plane). This seems to be the result of two effects. Firstly, the measured losses are somewhat more widely distributed than for the computed flow. Secondly, the axial velocities, and thus the local mass flow rates, are noticeably lower in the high loss regions for the computed results. The net effect is that the mass-averaged losses are actually slightly higher for the measured results.

Table 5.4: Summary of the integrated flow quantities

\begin{tabular}{|c|c|c|c|c|c|c|}
\hline \multirow{2}{*}{ Parameter } & \multicolumn{2}{|c|}{ Measured } & \multicolumn{2}{|c|}{ CFD } & \multicolumn{2}{|c|}{$\begin{array}{c}\text { Measured - CFD)/ } \\
\text { Measured x100 } \\
\end{array}$} \\
\hline & $1.20 C_{x}$ & $1.40 C_{x}$ & $1.20 C_{x}$ & $1.40 C_{x}$ & $1.20 C_{x}$ & $1.40 C_{x}$ \\
\hline$C_{P 0}^{\prime \prime}$ & -0.422 & -0.464 & -0.403 & -0.434 & $-4.6 \%$ & $-6.5 \%$ \\
\hline$C_{P 0, \text { mixed }}$ & -0.500 & -0.544 & -0.495 & -0.515 & $-0.9 \%$ & $-5.3 \%$ \\
\hline$C_{S K E}{ }^{\prime \prime}$ & 0.046 & 0.031 & 0.063 & 0.046 & $37 \%$ & $48 \%$ \\
\hline$C_{T K E}{ }^{\prime \prime}$ & 0.022 & 0.022 & 0.014 & 0.012 & $-36 \%$ & $-45 \%$ \\
\hline
\end{tabular}

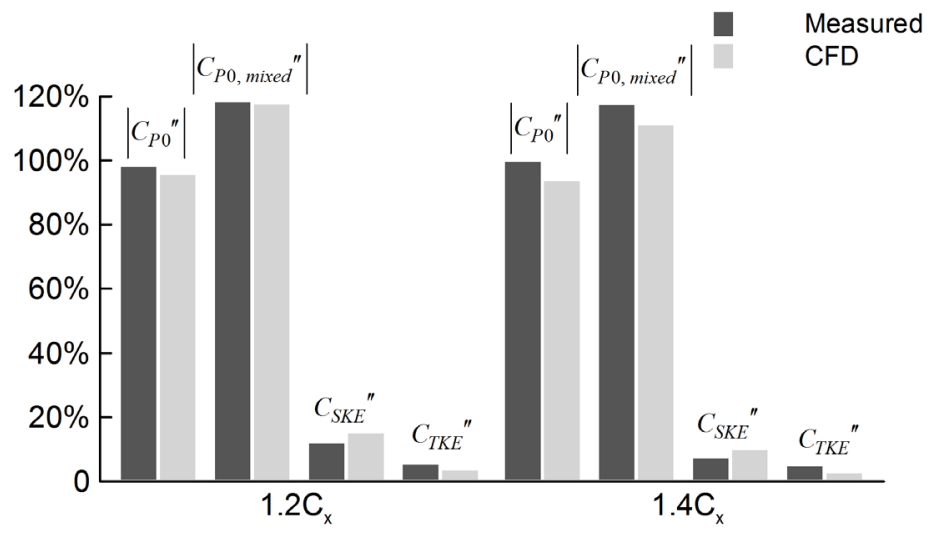

Figure 5.14: Comparison between the measured and CFD integrated flow parameters

The results from the $1.20 C_{x}$ plane show good agreement between the mixed-out values of total pressure loss for the measurements and computations. The dissipation of the secondary kinetic energy contributes about $68 \%$ of the mixing losses. The higher secondary kinetic energy for the computed flow thus partly offsets the lower measured losses, giving similar final mixed- 
out losses. At the $1.40 C_{x}$ plane the predicted mixed-out losses agree less well with those from the measurements. It is also evident that mixing out of the secondary kinetic energy, though significant, only accounts for only about $57 \%$ of the additional mixing losses.

Table 5.4 and Figure 5.14 also include values of the area-averaged turbulent kinetic energy coefficient, $C_{T K E}$. This represents energy extracted from the mean flow and not yet dissipated to internal energy. The measured $C_{T K E}$ shows negligible change between the two measurement planes, indicating that the turbulence is being produced from the mean flow at a similar rate to that at which it is being dissipated by viscous action. This is not the case in the computations. The predicted turbulent kinetic energy is reduced by about $15 \%$ from the $1.20 C_{x}$ to the $1.40 C_{x}$ plane. This is evidently one of the reasons that the computations predict the development of the losses with downstream distance somewhat inaccurately.

\subsubsection{Downstream Turbulence Field}

As shown in the previous section, the measured results show higher levels of turbulent kinetic energy, greater rates of kinetic energy dissipation, higher integrated losses, and larger secondary loss distributions than the computations. Losses are realized first through the production of turbulence from the mean flow and then ultimately by the dissipation of the resulting turbulence (Gregory-Smith et al., 1988b). In computational simulations, typical of those used in design, eddy viscosity-based turbulence models are commonly used. Such models apply the Boussinesq approximation to relate the Reynolds stress tensor in the RANS equations to the mean strain rate tensor, $S_{i j}$, using a scalar value of the eddy viscosity, $v_{T}$, according to

$$
-\overline{u_{i} u_{j}}=2 v_{T} S_{i j}-\frac{2}{3} k \delta_{i j}
$$

where $\delta_{i j}$ is the Kronecker delta (Wilcox, 1998). Thus, the eddy viscosity is assumed to be isotropic. Schmitt (2007) discusses the validity of this assumption in general turbulent flows. He suggests that the wide range of turbulence length scales observed in turbulent flows invalidates the computation of stress based on a single mean scale. An eddy viscosity tensor might provide 
some directional sensitivity, but would still be an oversimplification of the complexity of the turbulence. In Chapter 6 of Wilcox (1998), he discusses the deficiencies of Boussinesq approximation for many practical flows. He suggests that rapid changes in the mean strain rates, also referred to as "extra rates of strain" by Bradshaw (1973), locally invalidate the Boussinesq approximation. Wilcox (1998) then summarizes the flow conditions under which the Boussinesq approximation should fail:

1. flows with sudden changes in mean strain rate;

2. flow over curved surfaces;

3. flow in ducts with secondary motions;

4. flow in rotating and stratified fluids ;

5. three-dimensional flows;

6. flows with boundary-layer separation.

In three-dimensional turbomachinery flows, like those examined here, items 1 through 6 all apply. In general, it is recognized that the assumption of isotropic eddy viscosity is a weakness of the turbulence models commonly used in engineering design. The application of these models continues because of their stable convergence and low computational cost. Here, the experimentally measured Reynolds stresses and mean velocity gradient are used to calculate an implied eddy-viscosity tensor. It should be noted that this tensor is not a physical quantity. It is only calculated here to compare with the predicted isotropic eddy viscosity.

The computed scalar eddy viscosity for the SST turbulence model used in the present computations is non-dimensionalized by the laminar viscosity, $v_{L}$, and is given by:

$$
\frac{v_{T}}{v_{L}}=\frac{a_{1} k}{\max \left(a_{1} \omega, S F_{2}\right)} \cdot \frac{1}{v_{L}}
$$


where $k$ is the turbulence kinetic energy, $\omega$ specific turbulence dissipation rate, $a_{1}$ is a constant, $F_{2}$ is a blending function that restricts the production limiter to the near-wall boundary layer and $S$ is the magnitude of the strain rate,

$$
S_{i j}=\frac{1}{2}\left(\frac{\partial \bar{u}_{i}}{\partial x_{j}}+\frac{\partial \bar{u}_{j}}{\partial x_{i}}\right)
$$

The predicted Reynolds stress tensor, $\overline{u_{i}^{\prime} u_{j}^{\prime}}$, is computed by applying the Boussinesq approximation, as defined in Equation 5.7, using the predicted values of the eddy viscosity $\left(v_{T}\right)$, mean strain rate $\left(S_{i j}\right)$ and the turbulence kinetic energy $(k)$. In the experiment the Reynolds stresses are measured directly. For each Reynolds stress there is a corresponding implied eddy viscosity component. Thus, the non-dimensional "effective" eddy viscosity tensor for the experimental results is given by:

$$
\frac{v_{i j}}{v_{L}}=\frac{-\overline{u_{i}^{\prime} u_{j}^{\prime}}}{2 S_{i j}} \cdot \frac{1}{v_{L}}
$$

where $i$ and $j=1,2$ or 3 represent the $x_{\text {prim }}, y_{\text {sec }}$, and $w_{\text {sec }}$ coordinate directions as defined in Figure 3.7.

The predicted eddy viscosities at the $1.20 C_{x}$ plane are shown in Figure 5.15 as a colourflood contour plot overlaid with line contours of the total pressure coefficient. Comparisons with the floods of streamwise vorticity in Figure 5.13 (b) indicate that the peak values of eddy viscosity occur within the vortical structures. The maximum value is located within the passage vortex, as labelled, and is approximately 150 times the laminar viscosity. As shown, this region is not coincident with the peak losses. Also, the eddy viscosity field appears continuous over the entire plane, even in regions where the mean strain rates are changing rapidly. In the measurements, it will be shown that the eddy viscosity assumption breaks down in these regions. In fact, extremely high values of the eddy viscosity with sharp discontinuities through the secondary flow are observed. In the computations, the under prediction of the eddy viscosity, 
particularly in regions where the strain rates are changing rapidly, is a likely cause for the lower overall losses relative to the experiment.

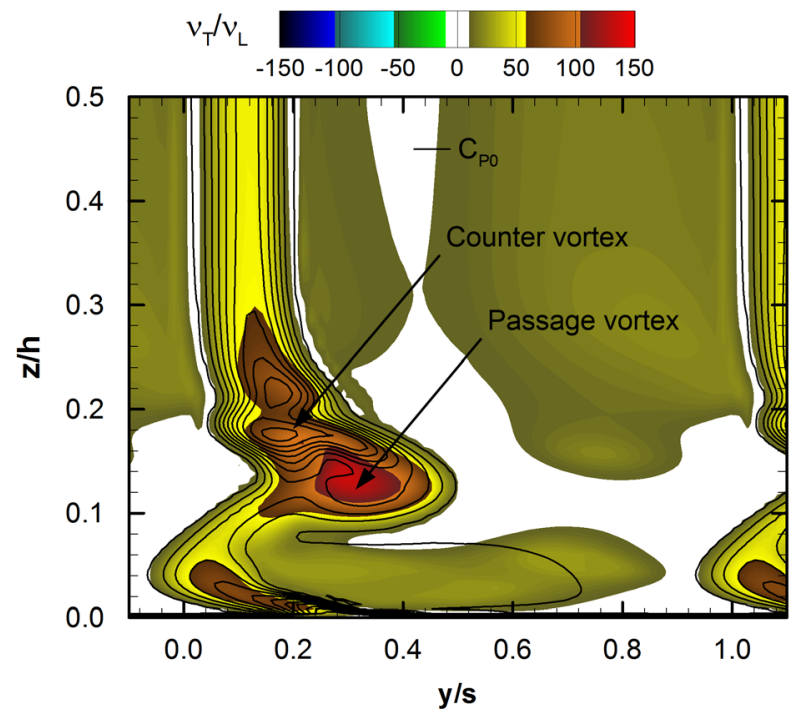

Figure 5.15: Predicted non-dimensional eddy viscosity at $1.20 \mathrm{Cx}$

In the turbulence model (see Equation 5.8), a high value of eddy viscosity would also tend to be associated with elevated turbulence kinetic energy. The turbulence kinetic energy defined as,

$$
k=\frac{1}{2}\left(\overline{u^{\prime 2}}+\overline{v^{\prime 2}}+\overline{w^{\prime 2}}\right)
$$

is a function of the Reynolds normal stresses only, not the shear components. As was previously shown in the experimental results, it was the shear stresses that were dominant in the production of turbulence, and therefore loss, from the mean flow. Therefore, it appears that the importance of the shear stresses could be undervalued by the turbulence model.

For the experimental results, it is not possible to generate a contour plot of a single scalar eddy viscosity for comparison of the computational results of Figure 5.15. For each of the measured Reynolds stresses there is a corresponding eddy viscosity component that is computed 


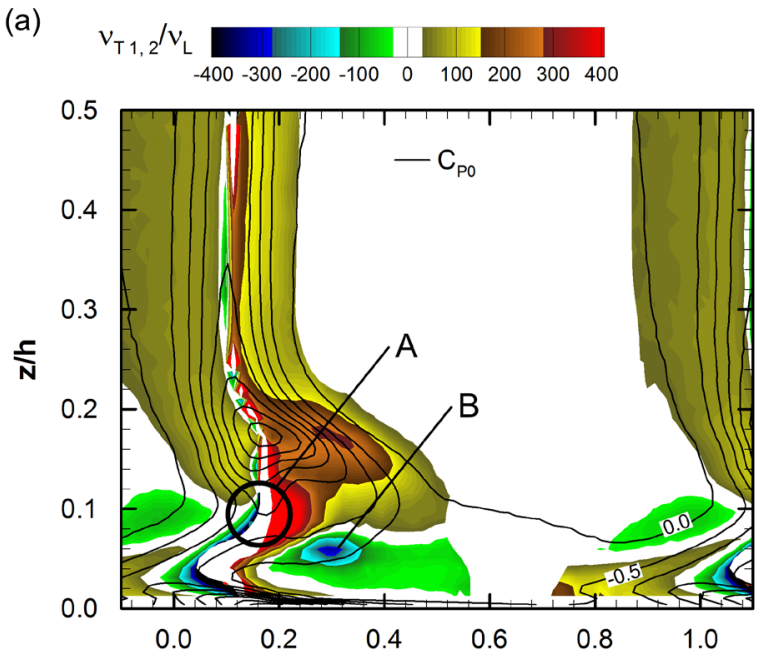

(b)

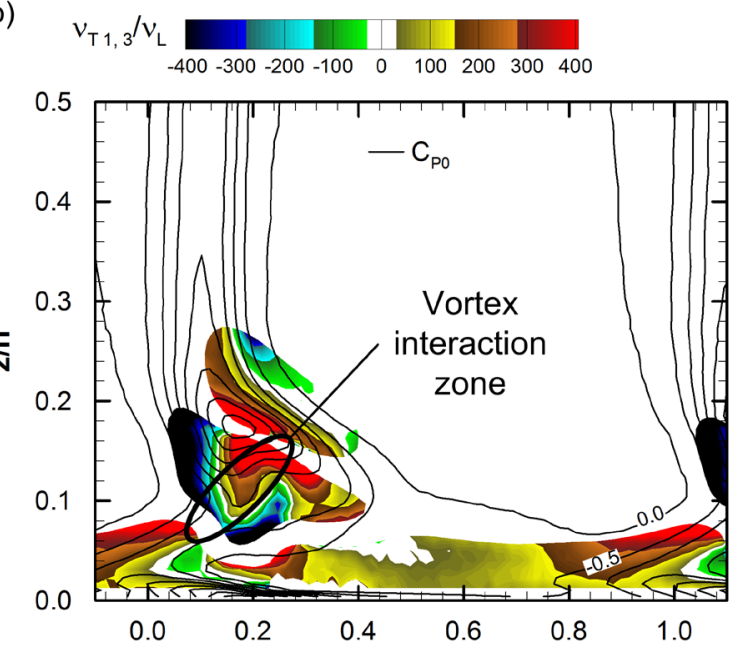

(c)
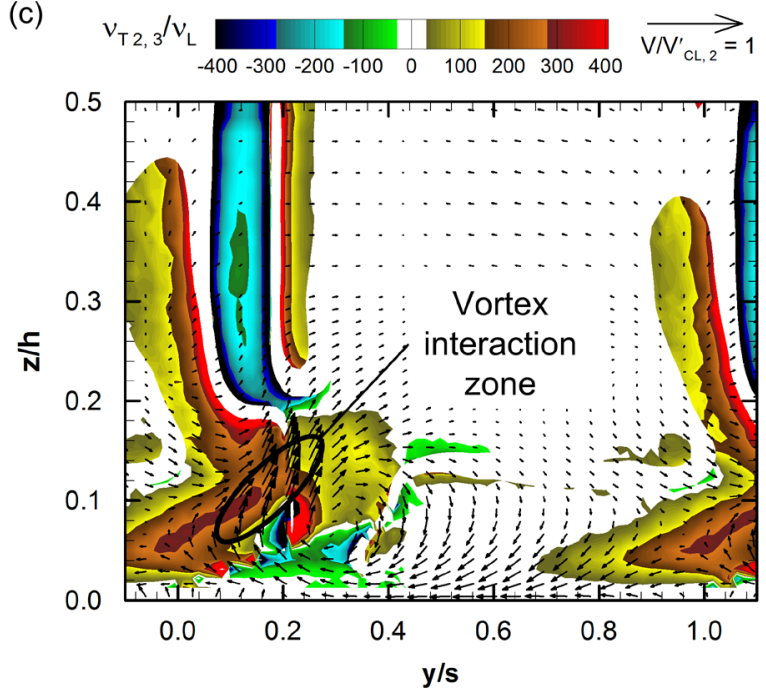

Figure 5.16: Floods of the measured non-dimensional shear eddy viscosity components at $1.20 C_{x}$ 
according to Equation 5.10. The measured eddy viscosity components corresponding to the $\overline{u^{\prime} v^{\prime}}$, $\overline{u^{\prime} w^{\prime}}$ and $\overline{v^{\prime} w^{\prime}}$ Reynolds shear stresses are shown in Figure 5.16 (a) through (c) respectively. Note that the scale for the measured eddy viscosities is more than twice than that used for the predicted values in Figure 5.15. As shown in Equation 5.10, the data reduction procedure for the measured eddy viscosity involves the division of the Reynolds stress by the mean strain rate $\left(S_{i, j}\right)$. In regions where the shear strain rates tend to zero the corresponding eddy viscosities approach extremely high values. Therefore, to visualize the measured fields, the data are only shown for the following range:

$$
\left|S_{i j} \cdot \frac{C_{x}}{V_{C L}}\right| \geq 0.3
$$

where $S_{i j} \cdot \frac{C_{x}}{V_{C L}}$ is the non-dimensionalzed mean strain rate and the limit $(0.3)$ is approximately $2.5 \%$ of range of the non-dimensional mean strain rates at the axial measurement plane.

To validate the clipping procedure applied to the contour floods, pitchwise distributions of the non-dimensional eddy viscosity, the corresponding mean strain rate and the corresponding Reynolds stress where taken at many different spanwise locations. Figure 5.17, shows an example of a distribution taken at $z / h=0.10$ of the $v_{1,2}$ flood of Figure 5.16 (a). Figure 5.17 (a) shows all of the data points in the distribution, while Figure 5.17 (b) shows the results with the data set clipped according to Equation 5.12. The excluded data points are shown as filled black symbols.

In Figure 5.17 (a) there is an extremely large negative value of eddy viscosity, labelled A, at $y / s=0.18$. This value is the product of a negative Reynolds stress and a very small positive mean strain rate. As shown, the eddy viscosity field is locally discontinuous here and the extreme local magnitudes make the rest of the eddy viscosities difficult to visualize. Figure 5.17 (b) shows the clipped data and thus the variation of the effective eddy viscosity over the entire pitch is no longer masked by extreme local values. The discontinuity at $y / s=0.18$ is still visible and large positive and negative eddy viscosity values are to the left and right of the 
discontinuity. As suggested by Wilcox (1998), it appears that the eddy viscosity assumption is not satisfactory in this localized region of rapidly changing mean strain rate.

In Figure 5.17 (b), another region of elevated eddy viscosity, labelled B, was removed by the clipping method. Here, the large local eddy viscosities were the result of a small Reynolds stress and a small corresponding mean strain rate. Thus, these elevated eddy viscosities have no physical significance and were discarded from the visualizations.

As mentioned, several other cut planes and different limits were tested to assess the effects of the clipping method on the interpretation of Figure 5.16 (a) to (c). The chosen levels where selected to best represent the data without masking the details of the distributions. In doing so, the unphysical nature of the eddy viscosity quantity became more apparent. The large discontinuities and widely ranging values suggest the eddy viscosity assumption is inadequate in many regions of this flow.

(a) For all $\left|S_{1,2}\right|$

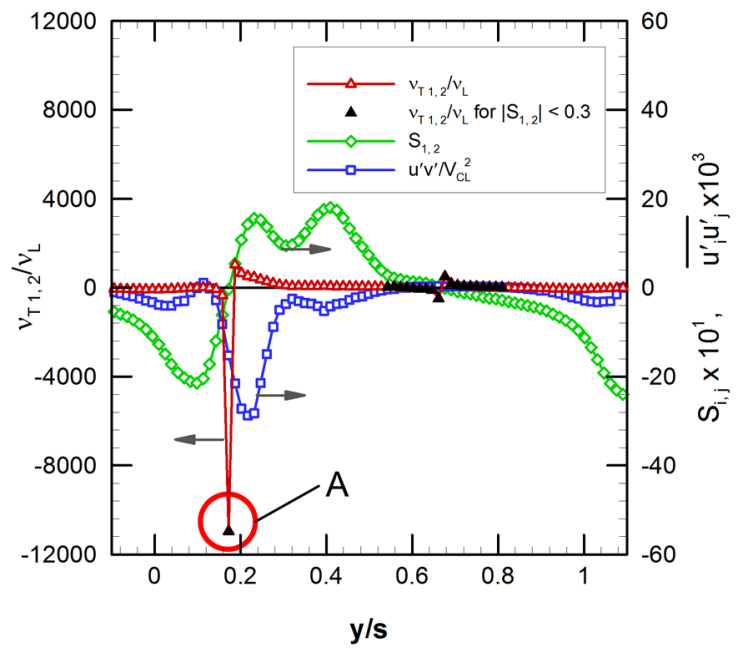

(b) For $\left|S_{1,2}\right| \geq 0.3$

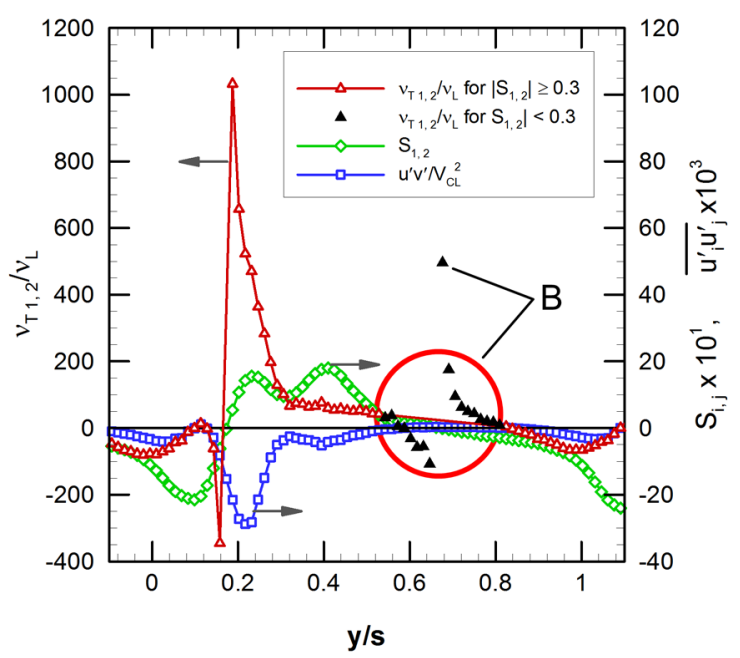

Figure 5.17: Pitchwise distributions of the non-dimensional eddy viscosity $\left(v_{t 1,2} / v_{L}\right)$, mean strain rate $\left(S_{1,2}\right)$ and Reynolds stress $\left(\overline{u^{\prime} v^{\prime}}\right)$ at $\mathrm{z} / \mathrm{h}=\mathbf{0 . 1 0}$ of the $1.20 C_{x}$ plane 
Figure 5.16 (a) shows the colour flood of the shear eddy viscosity component $\left(v_{1,2}\right)$ corresponding to the $\overline{u^{\prime} v^{\prime}}$ Reynolds stress. Within the secondary flow region, at a pitchwise location of approximately $y / s=0.2$, there exists a thin region of high positive eddy viscosity with an adjacent region of apparently negative eddy viscosity, labelled A. Negative eddy viscosity values are physically counter-intuitive, at least in a two-dimensional shear flow, since it implies momentum transport against the mean velocity gradient. Here, in the three-dimensional secondary flow, negative eddy viscosities occur where the corresponding shear stress and mean strain rate are positively correlated. Away from the secondary flow, within the blade wake region, the flow is essentially two-dimensional $(w \approx 0)$ and only positive values of the $v_{1,2}$ eddy viscosity component are measured.

Negative values in the eddy viscosity field of turbine flows have been inferred from measurements by previous researchers. For example, Perdichizzi et al. (1992) showed experimental eddy viscosities within the secondary flow downstream of a linear turbine cascade. They reported only positive values for the $v_{1,2}$ component; however, they did find negative values for the $v_{1,3}$ component which is associated with the $\overline{u^{\prime} w^{\prime}}$ Reynolds stress. They suggest this eddy viscosity distribution is "unrealistic" and that it invalidates the eddy viscosity assumption for turbine secondary flows. The presence of apparently negative values of eddy viscosity has been identified in other types of flows. Liberzon et al. (2007) investigated negative eddy viscosities using particle tracking velocimetry in a cubic Rayleigh-Bénard convection cell. They measured negative eddy viscosity values and acknowledge that this implies momentum transport against the mean gradients. In the open literature, for non-turbomachinery flows, there are many other investigations that have reported negative eddy viscosities and share similar interpretations (Rudiger, 1980; Gama et al., 1994a, b; Wirth et al., 1995; Chaves and Gama, 2000; Cummins and Holloway, 2010). Thus, as it pertains to this work, the presence of negative eddy viscosities is in fact possible in turbine secondary flows. This then violates the Boussinesq assumption of most two-equation eddy-viscosity based turbulence models. 
Negative eddy viscosities however are only one indication of anisotropy. In any given isotropic tensor the shear components should be zero and the diagonal terms equal, yielding a single scalar value. The eddy viscosity components shown in Figures 5.16 (a) through (c) are all very different. This clearly demonstrates that the use of a single scalar value for predicting these flows is over simplified. To this end, the discontinuities of the eddy viscosity field that are local to regions of rapidly changing mean strain rate become impossible to model using a common two-equation turbulence model.

Figure 5.16 (b) shows the eddy viscosity corresponding to the $\overline{u^{\prime} w^{\prime}}$ Reynolds shear stress. The maximum eddy viscosity is approximately 400 times the laminar viscosity and is located in the high shear region between the passage and the counter vortex, labelled as the vortex interaction zone. In the predicted results, the analogous vortex interaction zone is located at about $y / s=0.15$ and $z / h=0.22$, as indicated in Figure 5.13 (b) by secondary velocity vectors. Here, the eddy viscosities are about 100 times the laminar viscosity. This under-prediction is a likely cause for the lower dissipation and overall lower loss production observed in the computations.

Figure 5.16 (b) also shows significant regions of high negative eddy viscosity. They appear to surround the region of positive eddy viscosity in the vortex interaction zone. To validate the integrity of this eddy viscosity field, similar pitchwsie distributions to those in Figure 5.17, but at $z / h=0.14$, are shown in Figure 5.18. Again, the clipped data points are shown as black filled symbols and the clipping procedure has removed the extreme data points of Figure 5.18 (a). The remaining distribution in Figure 5.18 (b) shows that both the negative and positive eddy viscosities result from Reynolds stresses and mean strain rates of significant magnitude. The clipping procedure appears effective here. It is also worth pointing out that where negative eddy viscosities were obtained, in all cases presented here, they were obtained consistently from the measurements at a significant number of adjacent measurement points in both the spanwise and pitchwise directions. Again, the results of Figure 5.16 (b) indicate the high degree of anisotropy of the eddy viscosity tensor. 
Figure 5.16 (c) shows the eddy viscosities corresponding to the $\overline{v^{\prime} w^{\prime}}$ component of the measured Reynolds shear stress. Large positive values are found within the vortex interaction zone, as labelled. This is a region of high shear, as indicated by the secondary velocity vectors, and is also local to the largest values of negative deformation work (turbulence production) as shown in Figure 5.9 of Section 5.5.5. Figures 5.19 (a) and (b) show the pitchwise distributions through the vortex interaction zone at $z / h=0.08$. At about $y / s=0.10$, the large $\overline{v^{\prime} w^{\prime}}$ Reynolds stress and corresponding mean strain rate clearly result in the large positive eddy viscosity. At $\mathrm{y} / \mathrm{s}=0.20$, the mean strain rate changes rapidly and the eddy viscosity distribution shows a sharp discontinuity with extreme positive and negative values. This is another example of a flow region that would be impossible to predict accurately using an isotropic eddy viscosity turbulence model.

(a) For all $\left|S_{1,3}\right|$

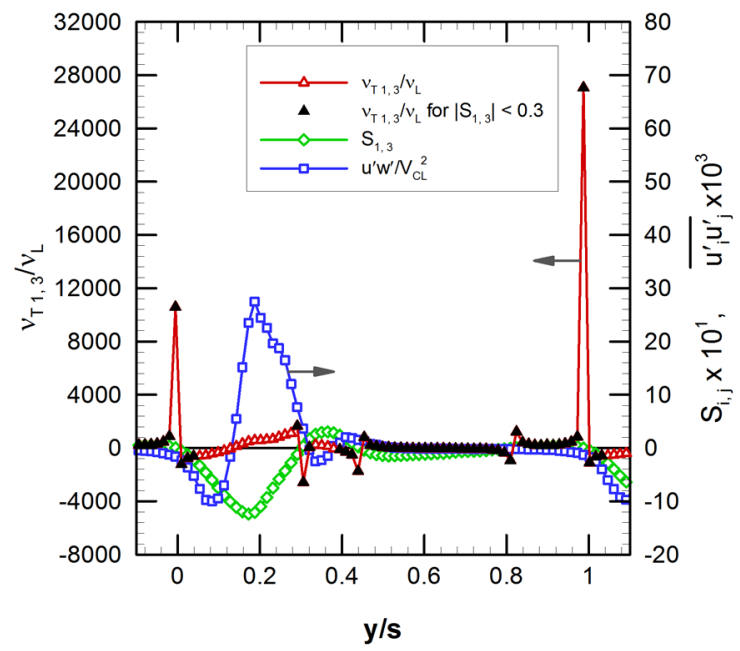

(b) $\operatorname{For}\left|S_{1,3}\right| \geq 0.3$

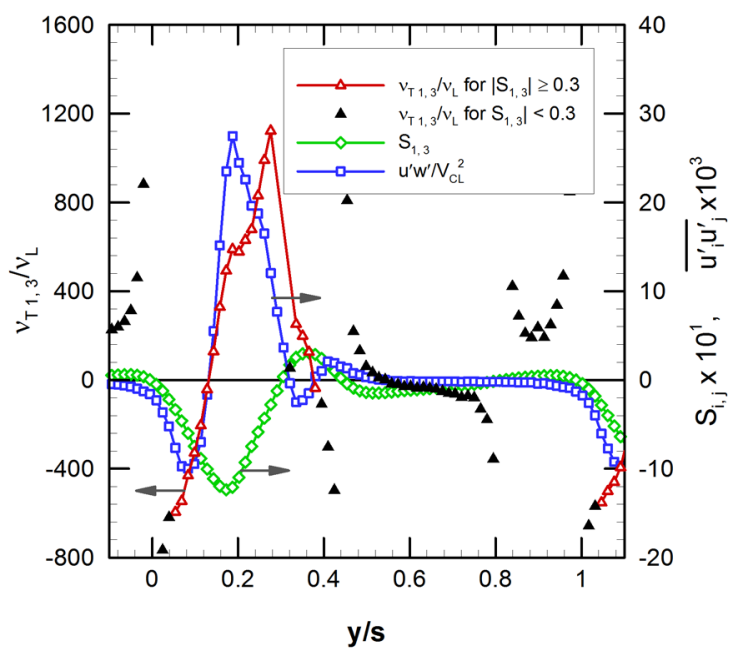

Figure 5.18: Pitchwise distribution of the non-dimensional eddy viscosity $\left(v_{t 1,3} / v_{L}\right)$, mean strain rate $\left(S_{1,3}\right)$ and Reynolds stress $\left(\overline{u^{\prime} w^{\prime}}\right)$ at $\mathbf{z} / \mathbf{h}=\mathbf{0 . 1 4}$ of the $1.20 C_{x}$ plane.

Significant negative eddy viscosities are also shown away from the secondary flow within wake region of Figure 5.16 (c). In this two-dimensional region, negative values are unexpected. As shown in the pitchwise distributions of Figures 5.19 (c) and (d), the negative eddy viscosities are difficult to dismiss as they arise from local mean strain rates and Reynolds stresses of 
significant magnitude. In a perfectly two-dimensional flow one would expect the $S_{23}$ mean strain rate to be zero. Here, this is only the case away from the wake, towards the centre of the passage at $y / s=0.5$.

To further investigate, Figure 5.20 (a) shows the corresponding pitchwise distributions of the secondary velocities ( $v$ and $w$ ) non-dimensionalized by the local velocity vector $\left(V_{l o c}\right)$. Figure 5.20 (b) shows the velocity gradients that correspond to the $S_{23}$ mean strain rate. As

(a) For all $\left|S_{2,3}\right|$ at $z / h=0.08$

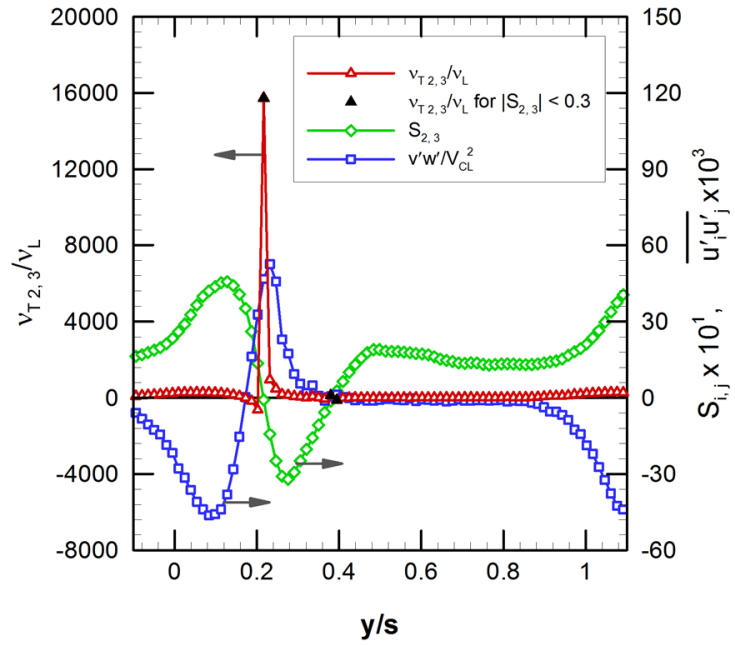

(c) For all $\left|\mathrm{S}_{2,3}\right|$ at $\mathrm{z} / \mathrm{h}=0.40$

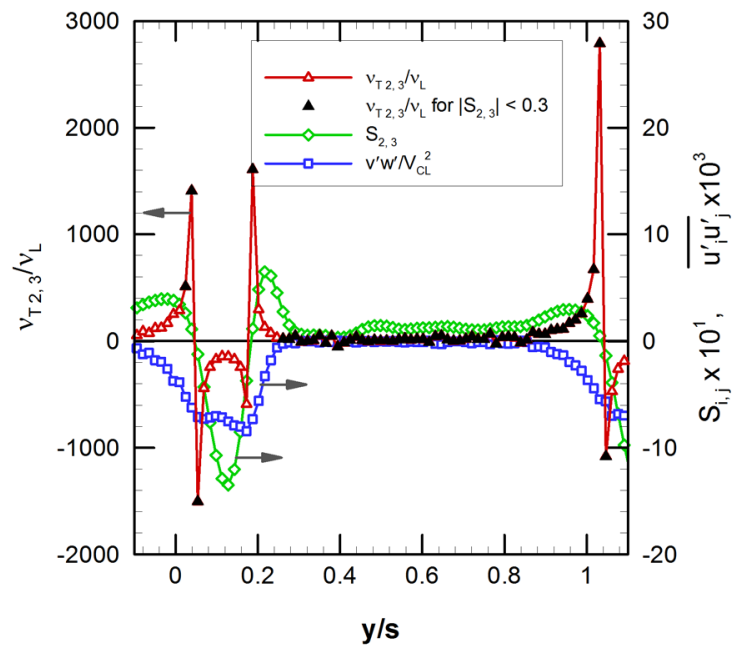

(b) For $\left|S_{2,3}\right| \geq 0.3$ at $z / h=0.08$

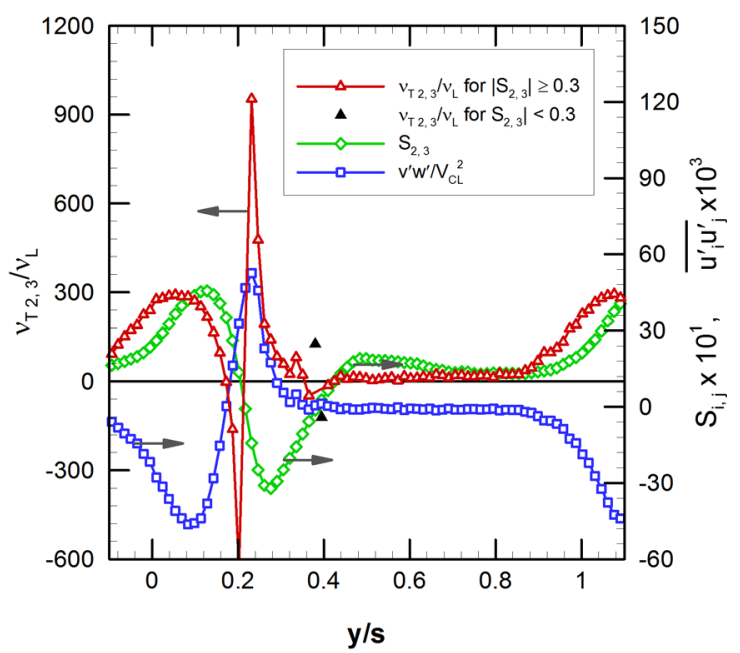

(d) For $\left|S_{2,3}\right| \geq 0.3$ at $z / h=0.40$

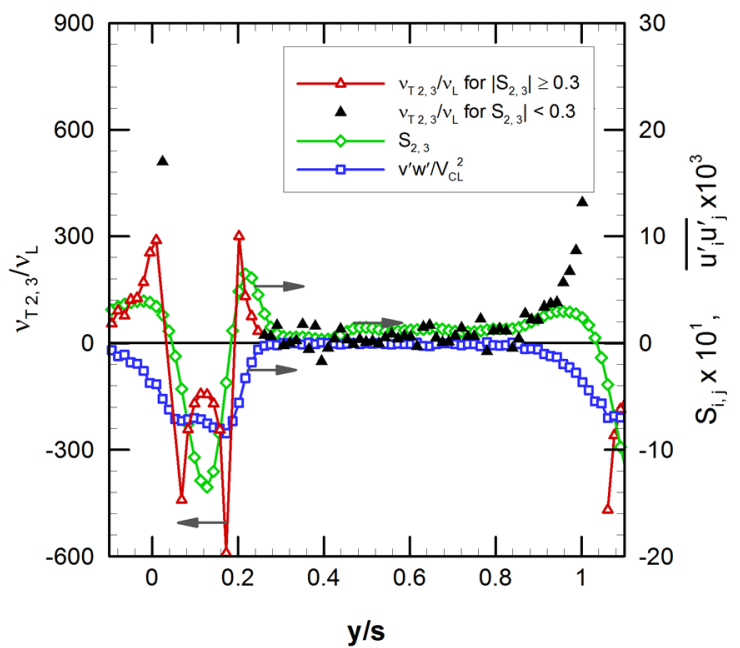

Figure 5.19: Pitchwise distributions of the non-dimensional eddy viscosity $\left(v_{T 2,3} / v_{L}\right)$, mean strain rate $\left(S_{2,3}\right)$ and Reynolds stress $\left(\overline{v^{\prime} w^{\prime}}\right)$ at $\mathbf{z} / \mathbf{h}=\mathbf{0 . 0 8}(\mathbf{a}-\mathbf{b})$ and $\mathrm{z} / \mathrm{h}=\mathbf{0 . 4 0}$ (c-d) of the $1.20 C_{x}$ plane 
shown, the secondary velocities ( $v$ and $w$ ) have small values through the wake which indicate a slight level of three-dimensionality. As a result, the $\partial \bar{w} / \partial y$ gradient is measurable, the $S_{23}$ is of significant magnitude and the negative eddy viscosities appear valid. However, practical significance of this region of negative eddy viscosity is not great. Any energy transfer of the $\overline{v^{\prime} w^{\prime}}$ Reynolds stresses with the mean flow would be insignificant relative to the other Reynolds stresses that have much larger corresponding mean velocities. Perhaps a more important point is that even within the wake, where the flow is essentially two-dimensional, there appears to be a level of anisotropy in the eddy viscosity tensor.

Overall, Figures 5.16 (a) to (c) show very significant anisotropy in the measured eddy viscosity tensor. The peak measured values are in many regions more than three times larger than those obtained with the turbulence model. Although it appears that these measurements invalidate the isotropic eddy viscosity assumption employed in the computations, it is unclear how, and the extent to which, this aspect of the turbulence model alters the resulting predicted flow physics. This issue is examined next.

(a)

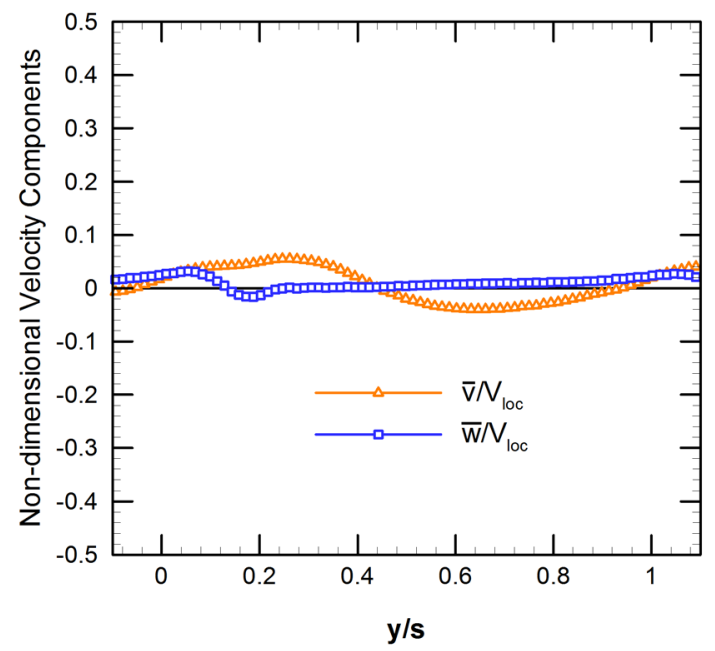

(b)

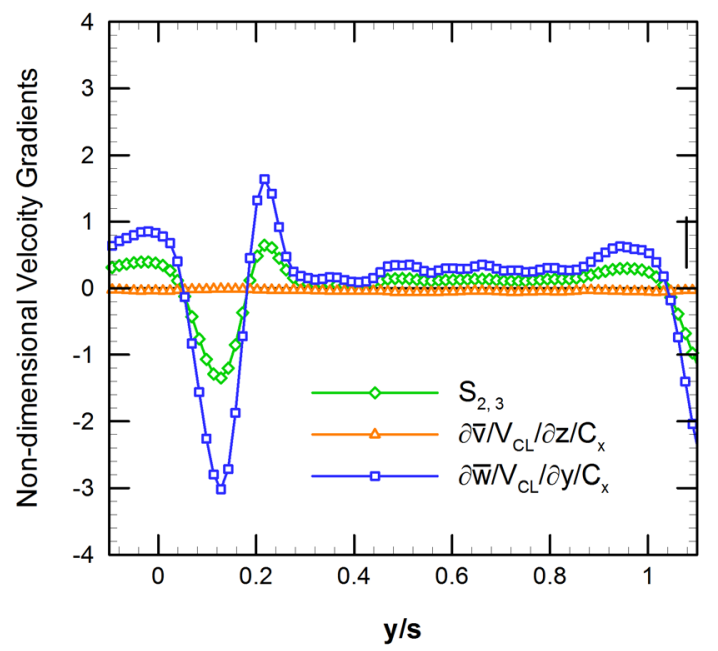

Figure 5.20: Pitchwise distributions of (a) the non-dimensional secondary velocity components and (b) the velocity gradients at $\mathrm{z} / \mathrm{h}=\mathbf{0 . 4 0}$ 
Figures 5.21 and 5.22 show the Reynolds normal and shear stresses from the $1.20 C_{x}$ plane respectively. Line contours of the streamwise vorticity coefficient $C_{\omega_{S}}$ are overlaid to show the relative position of the main vortical features. The solid lines indicate positive values and show the counter and corner vortices, while the dashed lines are negative and show the passage vortex. As previously described, the predicted flows are less diffuse, with smaller vortical structures that are more concentrated and positioned farther from the endwall. Thus, the predicted stress distributions also follow a similar pattern as they are less diffuse with the peak values positioned farther from the endwall. Overall the predicted fields appear significantly different from the measured. However, there are some similarities within the main vortical features despite the large differences in the eddy viscosity fields. For example, the measured $\overline{u^{\prime 2}}$ stress field, shown in Figures 5.21 (a), has local stresses within both the counter and passage vortex that differ by a maximum of $10 \%$ with respect to the predictions shown in Figure 5.21 (b). The other stresses show similar agreement with the largest differences occurring for the $\overline{v^{\prime 2}}$ stress of Figures 5.21 (e) and (f). Here, the largest difference occurs within the passage vortex and is about $20 \%$ of the measured value.

In general, it appears that the values of the predicted Reynolds normal stresses are only locally well predicted within the secondary vortices. However, the differences in the overall distributions point to differences in the origins of turbulence production. The predicted stresses show local peak values that generally coincide with the centre of the vortical features while the measured results show the local peaks between the passage and counter vortices. Presumably, this indicates a fundamental difference in the flow development as well as the mean kinetic energy dissipation. The production of turbulence, which is fundamental to the dissipation of energy from the mean flow, appears to be inaccurately predicted by the CFD.

The deformation work parameter, described in Section 5.5.5, indicates the rates of turbulence production, and therefore losses, from the mean flow. Figure 5.23 compares the predicted deformation work to that measured for the $1.20 C_{x}$ and $1.40 C_{x}$ planes. Again, the line 
(a) Measured

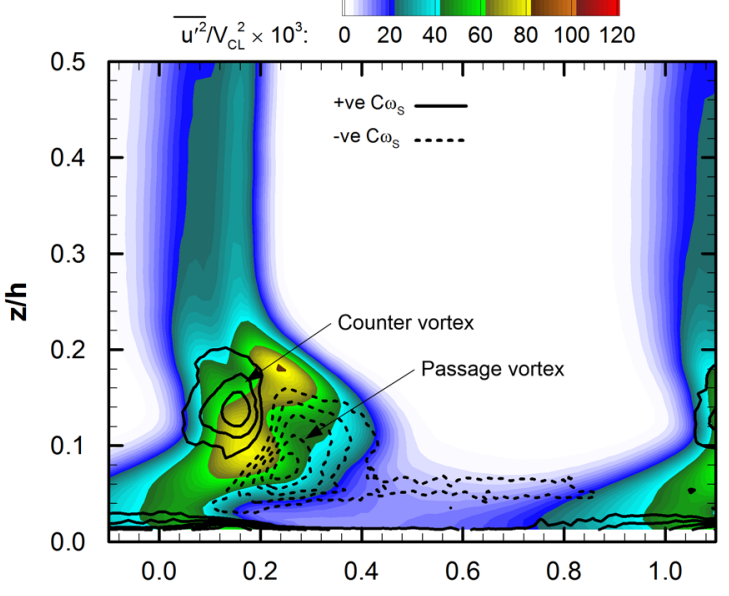

(c) Measured

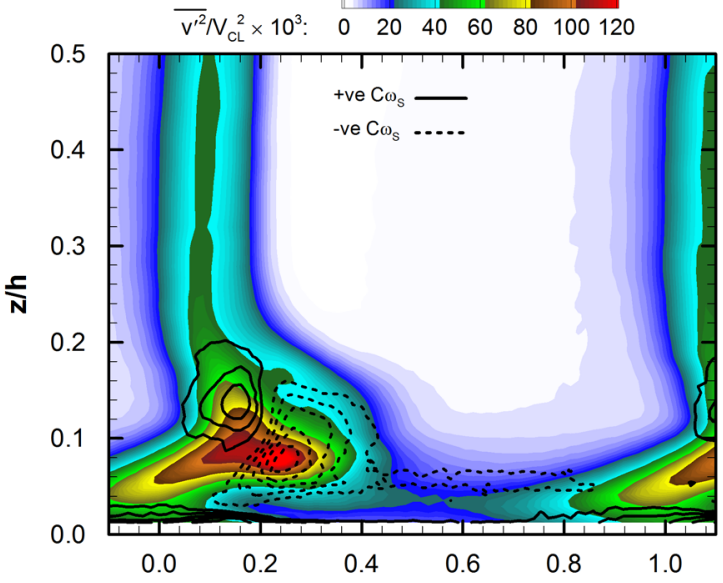

(e) Measured

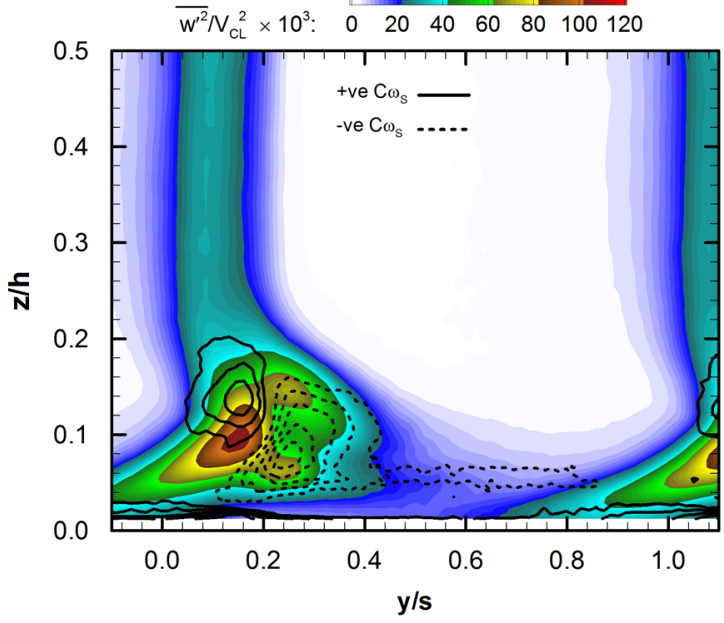

(b) CFD

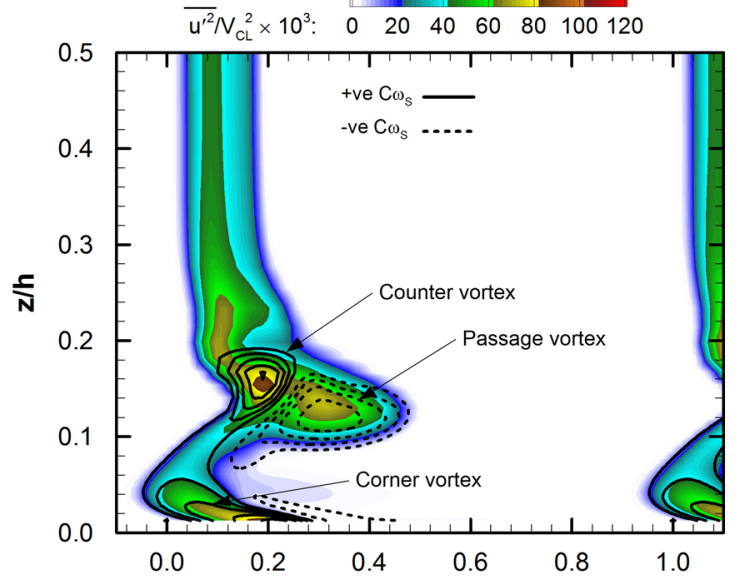

(d) CFD

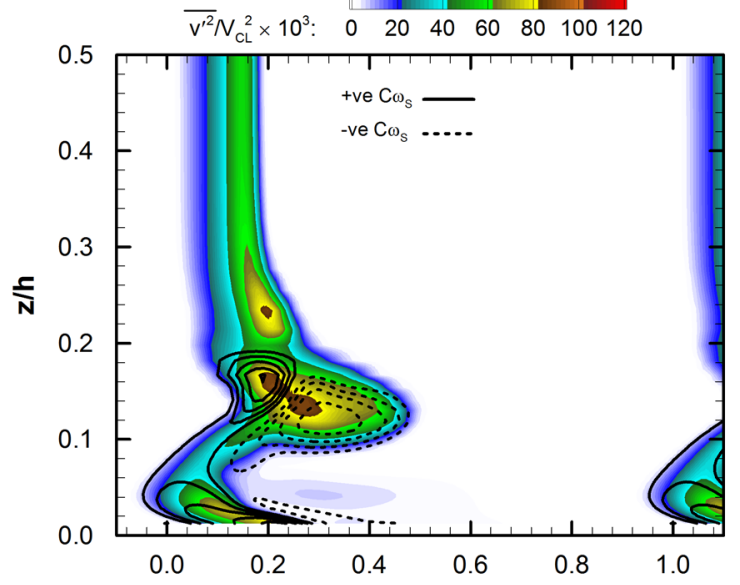

(f) CFD

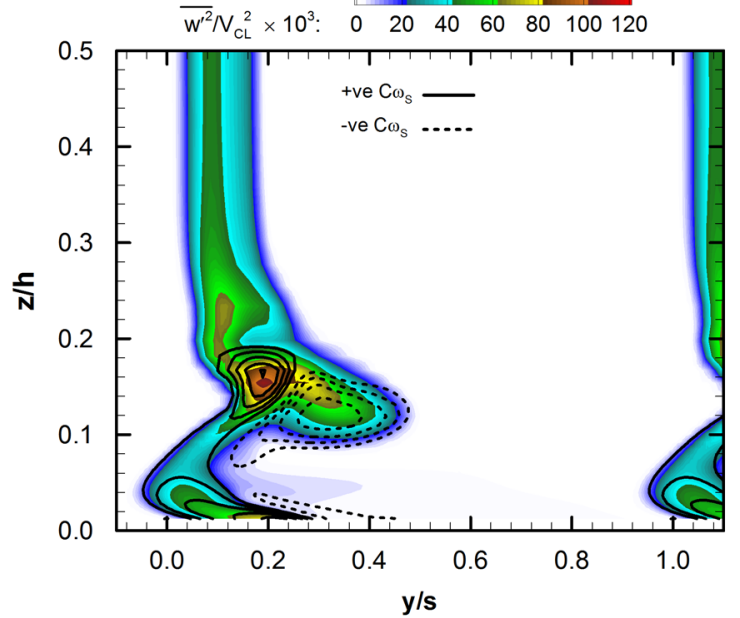

Figure 5.21: Floods of the measured and predicted Reynolds normal stresses with line contours of the streamwise vorticity coefficient at $1.20 C_{x}$ 
(a) Measured

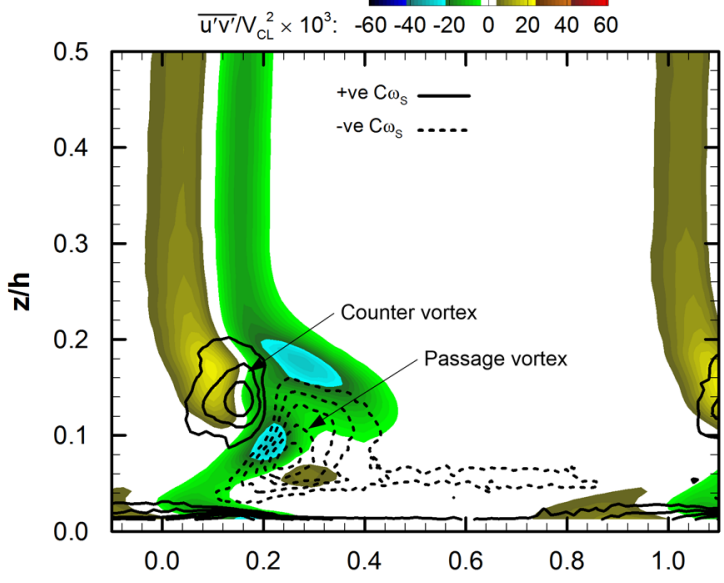

(c) Measured

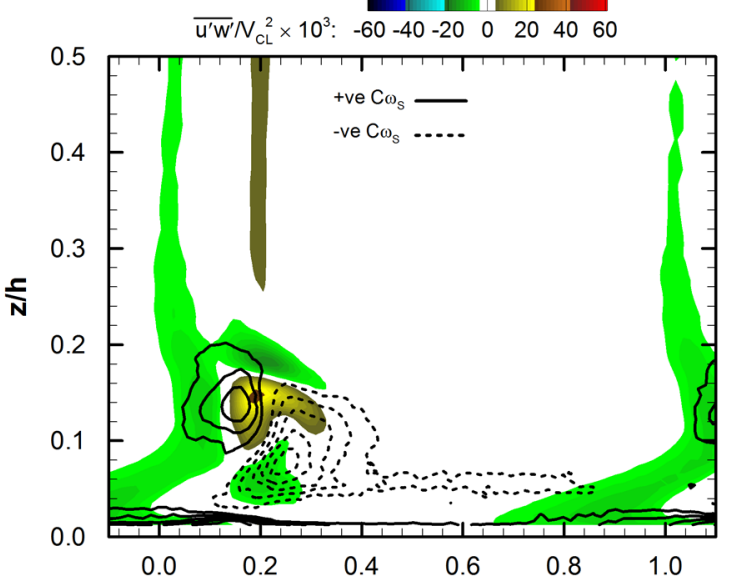

(e) Measured

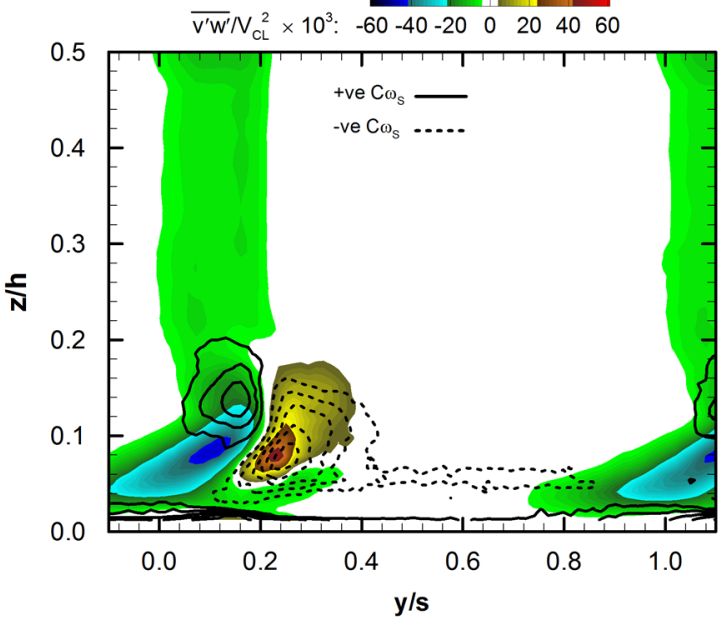

(b) CFD

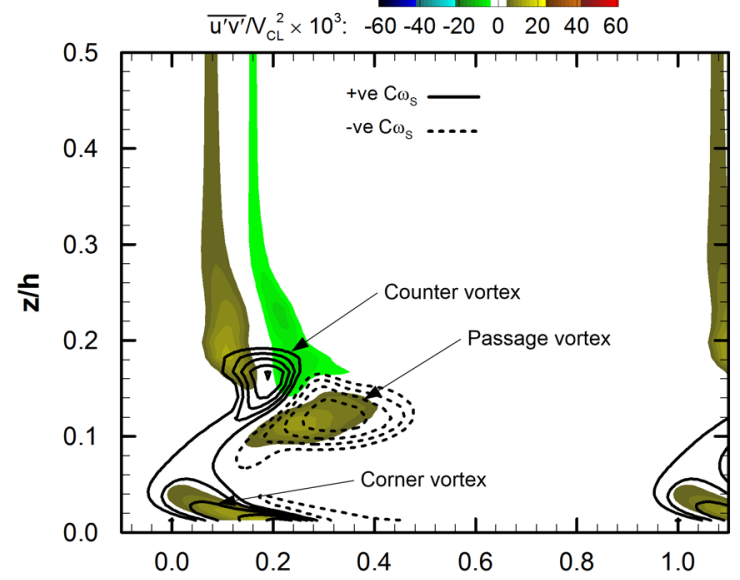

(d) CFD

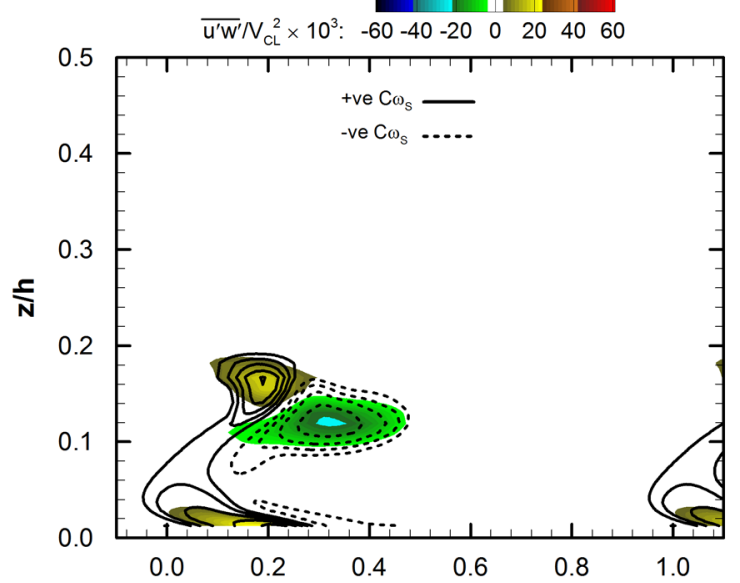

(f) CFD

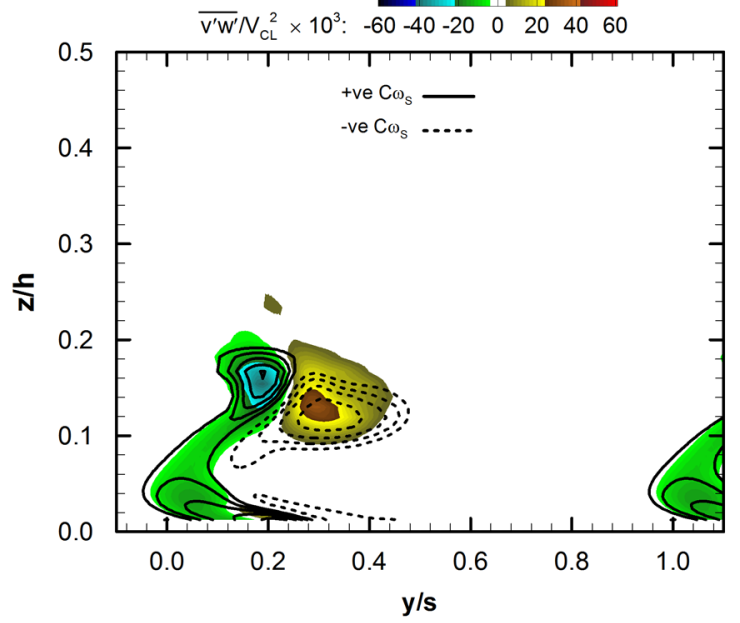

Figure 5.22: Floods of the measured and predicted Reynolds shear stresses with line contours of the streamwise vorticity coefficient at $1.20 C_{x}$ 
contours of the streamwise vorticity show the positions of the vortical features. In Figures 5.23 (a) and (b), the computations show higher (more negative) levels of turbulence production. These regions are located near the center of the passage and counter vortices, with the highest levels occurring within the counter vortex. The measured results have the highest levels of production between these vortices in the region previously referred to as the vortex interaction zone, as labelled in Figures 5.23 (a). The predicted deformation work distributions are consistent with the Reynolds stress distributions of Figures 5.21 and 5.22 which showed elevated stress values within the vortical structures, rather than within the vortex interaction zone. For example: in Figures 5.22 (e) and (f), the predicted $\overline{v^{\prime} w^{\prime}}$ Reynolds shear stress is approximately one third measured value within the vortex interaction zone while nearly twice as large within the counter vortex.

The measured fields of Figures 5.23 (a) also show a large region of positive deformation work within the passage vortex, indicating a gain in momentum from the turbulence field. As discussed previously, the high values of the Reynolds normal stresses and corresponding gradients were responsible for this positive region. In the computations, Figures 5.23 (b), positive regions are small and nearly indiscernible and the passage vortex is associated with large negative deformation work (production of turbulence). This appears to be caused by the significantly lower values of predicted normal Reynolds stresses associated with the passage vortex, as shown in Figures 5.21 (a) through (f). Also, in the computations the shear Reynolds stresses must be opposite in sign to the corresponding velocity gradients, as defined by the Boussinesq approximation given in Equation 5.7. Thus, the predicted deformation work terms associated with the shear stress components must be negative by definition. In the experiment, this was not necessarily the case as the measured eddy viscosity tensor was shown to be highly anisotropic. Thus, in the experiment it was possible to obtain positive deformation work from the shear stresses components. This occurred for the $\Psi_{2,3}$ component through the passage vortex, as shown in Figure $5.10(\mathrm{~h})$. 
(a) $1.20 C_{x}$ Measured

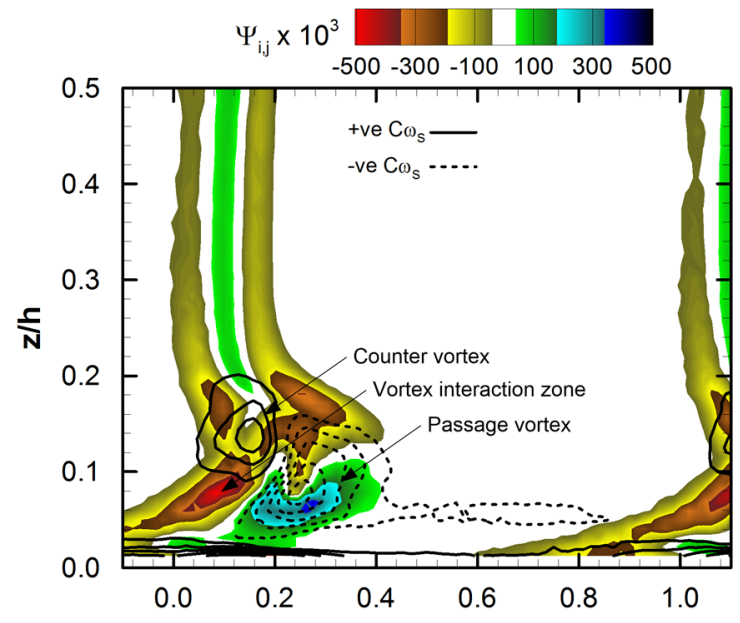

(c) $1.40 \mathrm{C}_{x}$ Measured

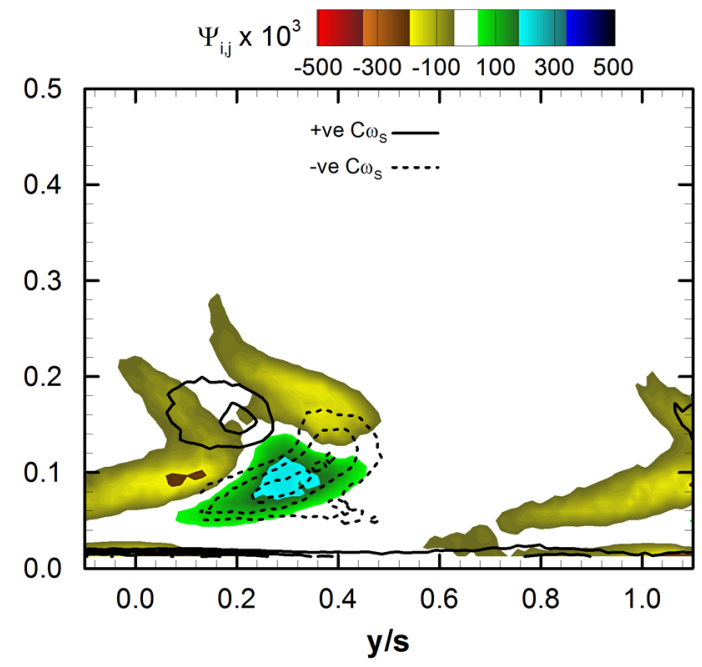

(b) $1.20 \mathrm{C}_{\mathrm{x}} \mathrm{CFD}$

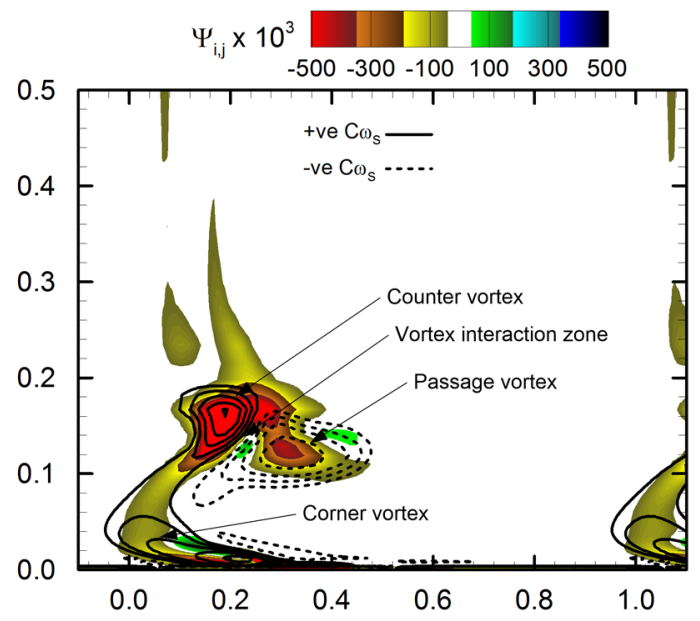

(d) $1.40 C_{x}$ CFD

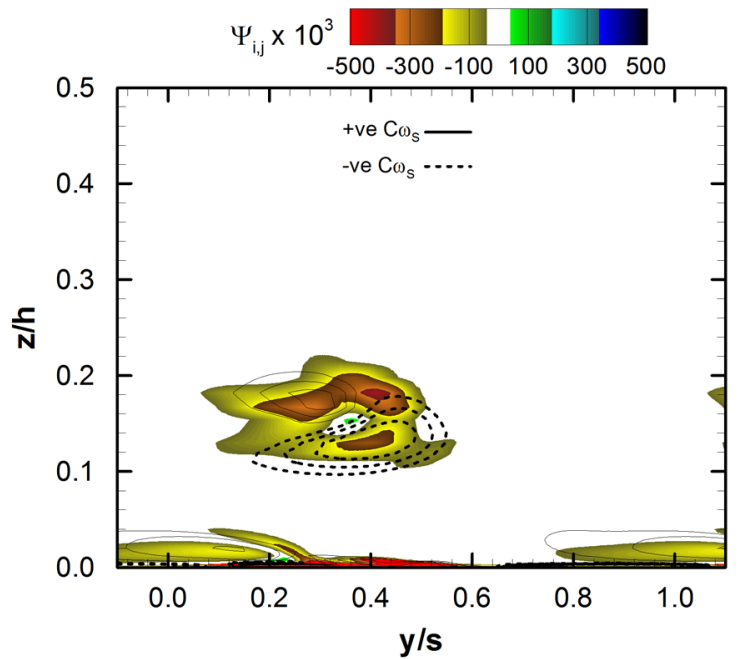

Figure 5.23: Floods of the measured and predicted deformation work, $\Psi$, with line contours of the streamwise vorticity coefficient at $1.20 C_{x}$ and $1.40 C_{x}$

At the $1.40 C_{x}$ plane, Figures $5.23(\mathrm{c})$ and (d), there is a significant reduction in the turbulence production rates for both the measurements and the computations. From the colour floods, the reduction in the deformation work parameter appears similar between the predicted and measured flows. The CFD results still show larger peak values of negative deformation work which remain associated with the passage and counter vortex; however, the largest peak is no longer associated with the counter vortex. Instead, it is away from the vortex cores towards the 
centre of the passage at the boundary between the secondary flow and inviscid core. The measurements in Figure 5.23 (c) show similar regions to the $1.20 C_{x}$ plane, albeit lower in magnitude.

Overall, it appears the turbulence, and thus loss production, within the secondary flow is not well predicted by the RANS solver with the SST turbulence model. Specifically, the CFD shows excessive turbulence production within the counter vortex while it does not show elevated levels within the passage and counter vortex interaction zone. Also, the CFD does not predict the positive deformation work that is clearly observed in the measurements.

To further investigate why the computations show peak levels of production within the counter vortex, as opposed to within the vortex interaction zone like in the measurements, the development of the counter vortex through the blade row is examined using the CFD results. A well established weakness of two-equation turbulence models is the over prediction of the turbulence production within stagnated regions (Menter, 1994). Of course, the core of the counter vortex is not stagnated but this fluid does have origins from a trailing edge recirculation zone with very low velocities.

Figure 5.24, is a visualization of the counter vortex development. Three-dimensional streamlines, coloured by the local $C_{T K E}$, emanate from the core of the counter vortex. The downstream plane, at $1.20 C_{x}$, shows line contours of the streamwise vorticity coefficient, $C_{\omega_{S}}$. As depicted, the counter vortex is a combination of the pressure side, suction side and endwall boundary layer fluid. The suction side flow at the trailing edge contains significant endwall fluid. It has significant spanwise velocity, caused by the passage vortex rotation, which initiates the rollup of the counter vortex prior to the trailing edge. A similar mechanism is discussed by Benner et al. (2004) and Knezevici (2011) where the counter vortex is referred to as the wallinduced vortex. Hodson and Dominy (1987b) alternatively suggest the counter vortex is formed solely from the trailing edge shed vorticity. For this airfoil, it appears both mechanisms are present. Just downstream of the trailing edge, the low momentum pressure side fluid is entrained into the counter vortex resulting in significant three-dimensional mixing and $C_{T K E}$ production. 
The zoomed portion of Figure 5.24 shows very high levels of $C_{T K E}$ within the trailing edge recirculation zone. It is here that the two-equation model appears to over predict the turbulence levels. As the fluid from the recirculation zone moves downstream it is entrained by the counter vortex. By the $1.20 C_{x}$ plane the high turbulence levels, combined with large velocity gradients within the vortex, result in the excessive production rates shown Figures 5.23 (b).

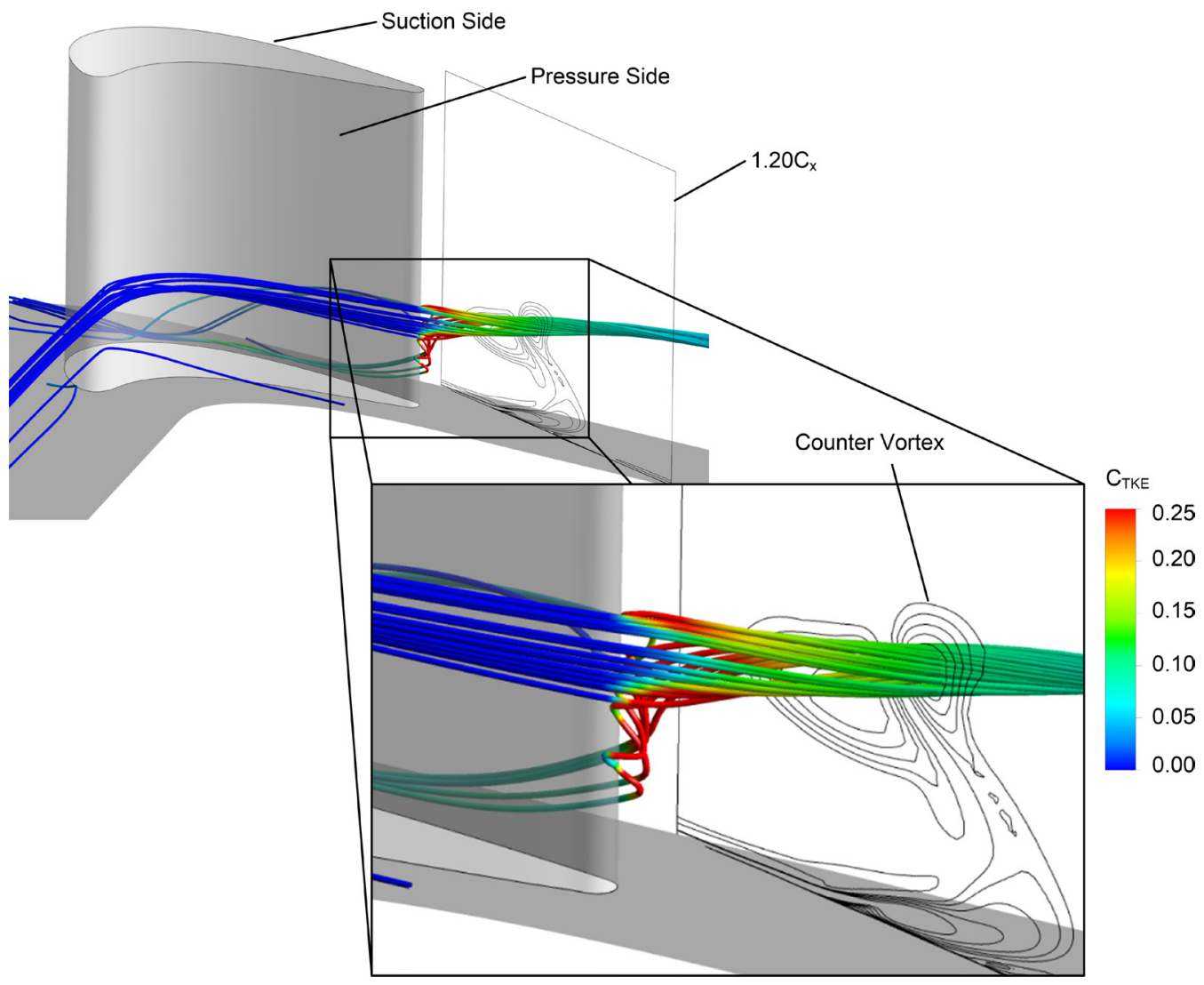

Figure 5.24: Streamlines through the counter vortex core coloured with the local turbulence kinetic energy coefficient, $C_{T K E}$

By contrast, the measurements do not show the same turbulence levels within the counter vortex. Instead, peak values in Figure 5.23 (a) occur at the intersection between the counter and passage vortices. Since the measurements show more diffuse flow with overall higher losses, it is quite possible that the counter vortex develops in a similar manner to the CFD but with increased kinetic energy dissipation. For example, the turbulence associated with the counter vortex core 
may have largely dissipated upstream of the $1.20 C_{x}$ plane and thus appears a total pressure loss. As was previously mentioned the much larger values of the eddy viscosity observed in the measurements is one factor that may contribute to the lower dissipation observed in the computations.

The over production of TKE shown here is only one example that contributes to the differences observed between the measured and predicted results. There could be several areas within and upstream of the passage where similar differences occur. The upstream saddle point, recirculation zones and the low momentum regions along the pressure-side surface are all plausible examples. The numerical and grid discretization as well as the boundary condition approximation (turbulence quantities at inlet boundary) are also significant factors. Although there are other ways to tune the CFD solutions for potentially more accurate results, such as altering the turbulence production limiters (this was not explored here), it is inconceivable that tuning could be generalized for the wide range of possible turbine geometries and the various operating conditions. For practical engineering applications, tuning would inevitably rely on some form of experimental benchmark. To this end, it is unlikely that tuning would eliminate the many inaccuracies present in the CFD solutions.

The comparisons made here have shown detailed differences between the predictions and the measurements. The purpose was to highlight these differences to better understand the limitations of RANS solver employing two-equation turbulence models. From an engineering design perspective, the overall accuracy of the CFD solutions is quite satisfactory and the results are still very useful. The main flow features, as well as the overall integrated values, are reasonably well predicted. Insights into the flow physics can also be gained in regions were measurements are difficult or inaccessible. CFD also allows multiple design iterations to be performed quickly, which allows component optimization and reduces development costs. In the remainder of the thesis, the CFD is used mainly as a tool to support the interpretation of the measured results. 


\subsection{Concluding Discussion}

Experimental measurements of the mean and turbulent flow field were completed downstream of a linear turbine cascade on two-dimensional planes located at three different axial positions. The total pressure distributions from each axial location provide a number of insights into the development of the downstream losses. The peak loss core originates from the blade suction surface and appears to be associated with the counter vortex and not the passage vortex. The passage and counter vortices are responsible for the diffusion of the peak loss core into the inviscid and low loss regions.

The overall mass-averaged total pressure losses were shown to increase with downstream distance. Although the secondary kinetic energy is commonly used as a design correlation parameter, its dissipation with downstream distance does not fully account for the increasing downstream mixing losses. For this cascade, the static pressure recovery and mixing-out of the primary flow non-uniformities contribute significantly to the losses. This result raises questions about the merit of using the level of secondary kinetic energy as a design correlation parameter.

The integrated turbulent kinetic energy from the measurements shows negligible differences between the two downstream planes; which is explained by the fact that the turbulence is being produced from the mean flow and dissipated to heat at similar rates. The production of turbulence constitutes a loss of mean kinetic energy and is the first step to the generation of total pressure losses through viscous dissipation of the turbulence.

The generation of downstream loss was examined by investigating the kinetic energy budget for turbulent flow. Regions of negative deformation work indicate the production of turbulence, and therefore a loss of mean kinetic energy. Negative areas were found within high shear regions of the flow, such as the boundary between the passage and counter vortices as well as the boundary between the secondary flow and inviscid core. On the other hand, positive regions of deformation work were observed within the passage vortex, indicating a return of 
kinetic energy from the Reynolds stresses acting against the mean velocity gradient. The normal stresses were found to dominate these positive regions.

The Reynolds shear stresses and associated velocity gradients dominate the rate of turbulence production from the mean flow. Therefore, the shear stresses are important to the development of total pressure losses. This study supports the concept that the accurate computational prediction of absolute losses requires the turbulence to be accurately modeled.

The computational results show high peak losses that are localized within the secondary vortices. The measured results show significantly lower peak losses that do not coincide with either the passage or counter vortices. The computational results showed at least one weak vortical structure that was not observed experimentally. Overall, the measured results appear more diffuse in both the wake and three-dimensional regions.

The eddy viscosity implied by the measurements downstream of the cascade was found to be highly anisotropic, particularly within the secondary flow region. The peak magnitudes of the eddy viscosities associated with the shear stresses were also found to be more than three times larger than those generated by the turbulence model. Significant regions of negative eddy viscosity were also obtained, which served as an indication that the two equation turbulence model, based on the Boussinesq assumption, is simplified for these flows. The lower values of the predicted eddy viscosity, particularly in the regions of rapidly changing mean strain, is one likely reason for the lower overall losses observed computationally.

The use of two-equation models for design purposes will likely continue for many years. The work here does not invalidate the use of these models. However, it does emphasize the possible limitations the Boussinesq assumption imposes on the accuracy of turbine secondary flow predictions.

The predicted and measured Reynolds stresses fields are overall different. However, the local values within the main vortical features showed maximum differences of only $20 \%$. The predictions show peak stresses within the vortex cores, which demonstrates a fundamental 
difference to production mechanisms relative to the experiment. It appears the two-equation model over predicts the turbulence production in low momentum regions of the flow. This may account for the high levels of turbulence production within the counter vortex that are not observed in the experimental measurements. 


\section{Chapter 6}

\section{Secondary Flows of Turbine Cascades with Simplified Upstream Platform Overlaps and Endwall Cavities}

\subsection{Introduction}

The present chapter investigates the secondary flows of three different rim-seal configurations, two of which are geometric simplifications. The purpose of this chapter is to demonstrate that flows for cases with simplified geometric rim-seals may appropriately approximate the flows of a typical rim-seal with zero net purge flow. Measurements were made with the seven-hole probe downstream of the Baseline, Ramp, Step and Cavity configurations described in Section 3.5.4. The secondary flows are examined in terms of total pressure losses, streamwise vorticity and secondary kinetic energy. The pitchwise and overall integrated quantities are also included. The chapter concludes with interpretations of the flow field using CFD and surface flow visualizations. The physical interpretations then set the basis for the following chapters that examine the interaction of the purge and mainstream flows. 


\subsection{Motivation}

Turbine cascade studies for the investigation of secondary flows have traditionally been performed under conditions that are idealized relative to a real engine environment. From a design perspective, this raises questions about how our current understanding of secondary flows is affected by real engine features. For instance, in a real turbine the rim-seal geometry creates endwall surfaces that are discontinuous. The endwall is separated to provide mechanical clearance between the stator ring and rotor disk-wheel. As shown schematically in Figure 1.2, the platform overlap and endwall cavity, form a relatively large step at the wheel-space and main gaspath interface. This step introduces a large disruption to the endwall boundary layer and thus significantly alters the secondary flow relative to that of a traditional flat endwall cascade. In addition to the step, the secondary flows are further complicated by the injection of purge flow which is required to cool the blade disk and prevent ingress of the hot mainstream gas.

As mentioned in Section 2.8, a primary design objective of the rim-seal is to protect the disk wheel-space while also limiting the amount of purge mass flow injected into the mainstream. In general, the literature discussed in Section 2.8 shows that turbine bladerows with a leading edge rim-seals have higher losses and stronger vortical structures than the corresponding flat endwall cases. Thus, it would appear desirable to incorporate rim-seal geometry into the early stages of the turbine CFD analyses; however, this can be problematic as such geometric details may only be finalized after much of the turbine design is complete, adding additional iterations to the design process. In cases where the endwall geometry is to be optimized for secondary loss mitigation (e.g. endwall contouring), the inclusion of the upstream geometry becomes even more important.

To simplify the design process, the rim-seal geometry may initially be approximated as gradual ramp, as shown in Figure 6.1 (a). The purpose of the ramp is to reduce the complexity of the CFD mesh within the endwall cavity region while not excluding the major geometric features of the rim-seal geometry (step height and distance from the blade leading edge). This has 
obvious simplifying benefits for shape optimized endwalls, which typically involve automatic optimization schemes that are computationally intensive.

The present chapter will examine the endwall flows and secondary losses of a linear turbine cascade with three different platform overlap geometries, two of which incorporate geometric simplifications. The objective of the chapter is to demonstrate that the endwall flow is relatively insensitive to minor simplifications of the platform overlap geometry. Thus, simplifying the rim-seal may be a viable approximation during the early stages of turbine CFD analyses. The results shown here are from test cases with zero net purge flow and thus form the basis for results of Chapter 7 which includes complimentary measurements for cases with simulated purge flow.

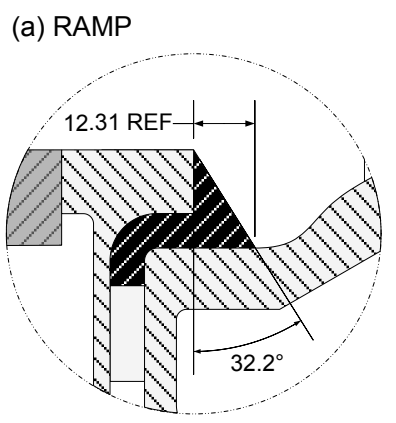

Detail A-A (b) STEP

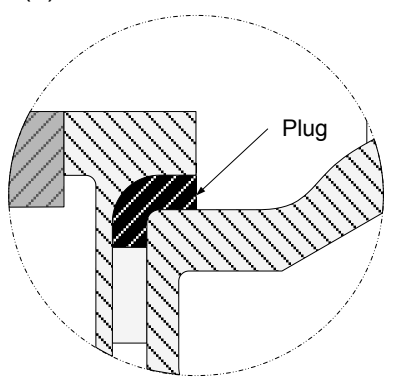

Detail A-A (c) CAVITY

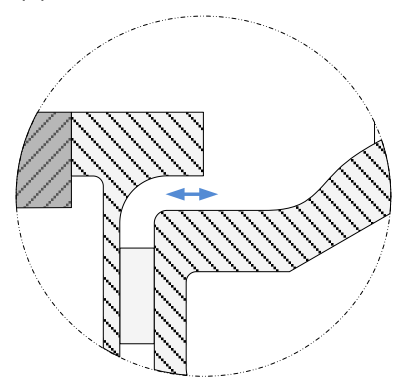

Detail A-A

Plenum sealed at supply plenum inlet

Figure 6.1: Geometric simplifications of the platform overlap (dimensions in $\mathbf{m m}$ )

\subsection{Approach}

Experimental measurements from the PAKH cascade were made for the design inlet Reynolds number of 75,000. There are four geometric cases considered: the Baseline flat endwall, the Ramp, Step and Cavity cases. The latter three are shown schematically in Figure 6.1. Downstream measurements were made using the seven-hole probe described in Section 3.8.5. The CFD presented in this chapter is largely used to facilitate the interpretation of 
the intra- passage flow field. However, the CFD results are also compared to the experiment to validate its usefulness for flow interpretation. The CFD predictions were completed following the procedures described in Chapter 4.

\subsection{Inlet Flow Field and Blade Loadings}

As previously mentioned, the inlet Reynolds number for the cases presented in the current and remaining chapters is 75,000 . This is different from the previous results shown in Chapter 5 which were measured at an inlet Reynolds number of 50,000. The lower operating point of Chapter 5 ensured the hot-wire measurements were within the range of the velocity calibrations. Figure 6.2 shows the experimentally measured boundary layer for the inlet Reynolds number of 75,000. For reference, the corresponding results measured at $\operatorname{Re}_{1}=50,000$ were previously presented in Figure 5.1. The two boundary layers show negligible differences and are essentially self similar.

The measured inlet boundary layer is also applied as the inlet condition for the CFD simulations. Details of the CFD procedures are given in Section 4.4.

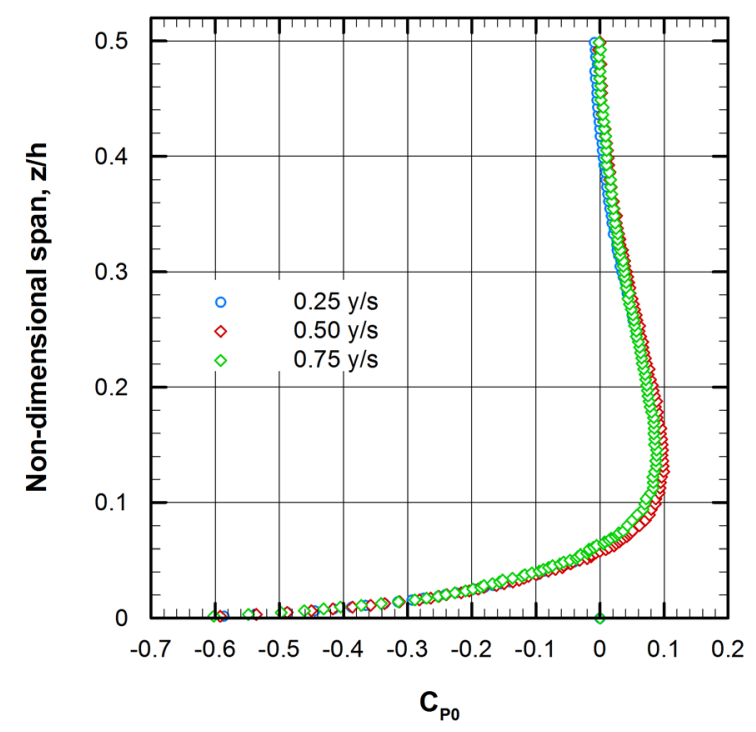

Figure 6.2: Inlet boundary layer total pressure distribution $\left(\operatorname{Re}_{1}=\mathbf{7 5 k}\right)$ 
The experimentally measured blade loadings corresponding to the $\operatorname{Re}_{1}=75,000$ operating condition are shown in Figure 6.3. Sub-figures (a) and (b) show the loadings corresponding to the blade midspan $(z / h=0.5)$ and near the endwall $(z / h=0.05)$ locations, respectively.

In Figure 6.3 (a), at the blade midspan, the loadings from the three modified endwall configurations are consistent with the baseline flat endwall. There are minor differences at the blade leading edge $\left(x / C_{x}=0\right)$ and towards the trailing edge along the pressure surface at about $x / C_{x}=0.8$ and 0.9. The three cavity endwall configurations are consistent in this area. Overall, the midspan results demonstrate that profile flow for this airfoil is independent of the endwall flows and the airfoil aspect ratio is sufficient for half span downstream measurements.

(a) Measured

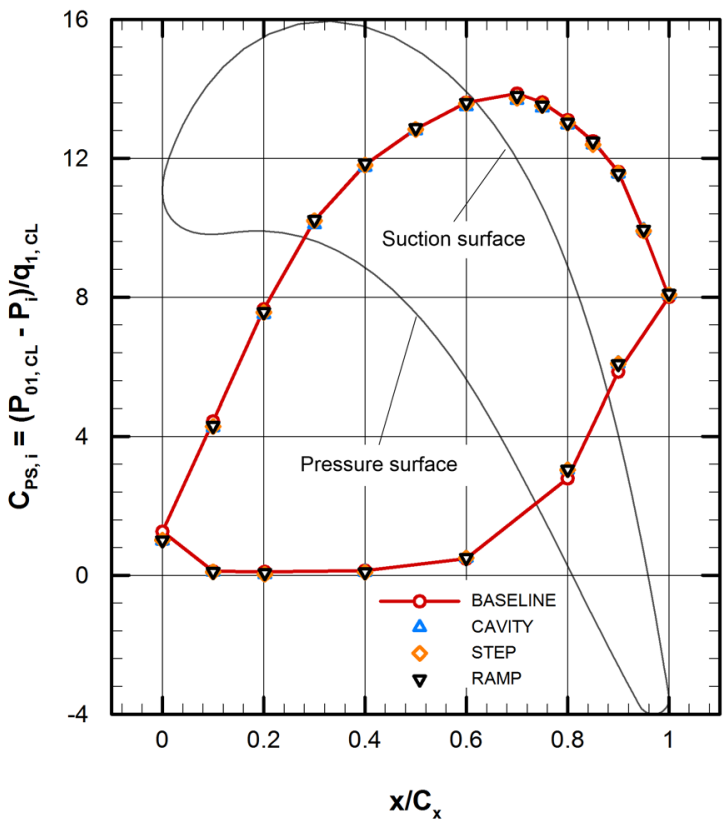

(b) Measured Near Endwall $(\mathrm{z} / \mathrm{h}=0.05)$

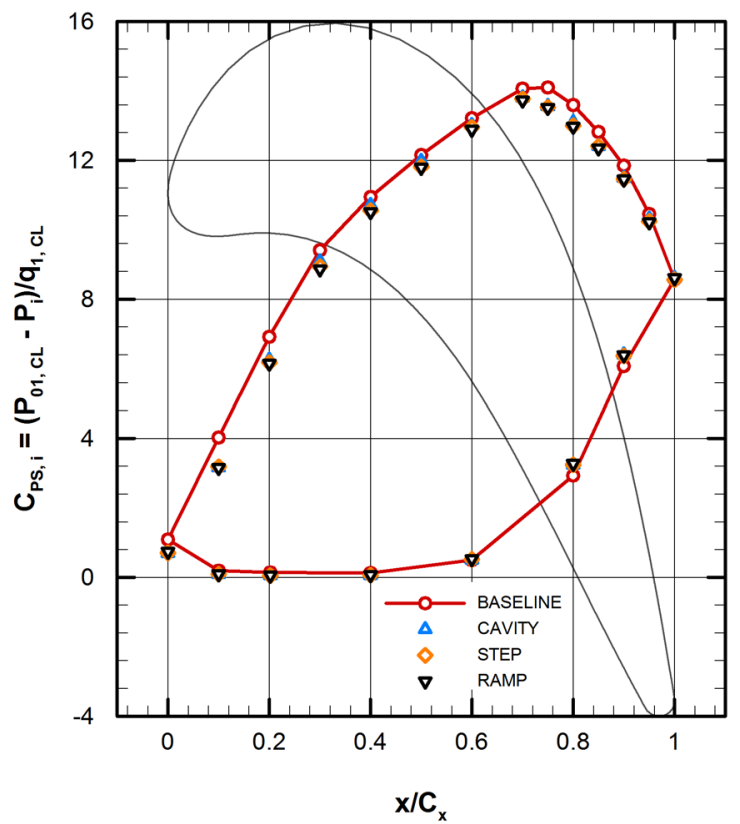

Figure 6.3: Measured blade surface static pressure distribution at $\mathrm{z} / \mathrm{h}=0.5$ and $\mathrm{z} / \mathrm{h}=\mathbf{0 . 0 5}$

At the near endwall location, in Figure 6.3 (b), the loadings of the three modified endwalls are essentially equal. However, along the suction surface there is a distinct unloading of the airfoil relative to the baseline configuration. The blade loading in this region is affected by the non-uniformities of the static pressure field introduced by the secondary flow. As will be shown 
in the following sections, the secondary flows of these cases are significantly altered from the flat endwall. It is therefore not surprising that the loadings in this region are different. Perhaps the most important finding here is that despite the simplifications of the Ramp and Step configurations relative to the Cavity case the near endwall blade loadings are essentially unaffected.

The corresponding loadings from the CFD predictions are shown in Figure 6.4. At the midspan location, in Figure 6.4 (a), the predictions are well matched to the experimental measurements. As previously mentioned in Chapter 4, this is a good indication of consistency between the experimental and simulated conditions.

(a) CFD

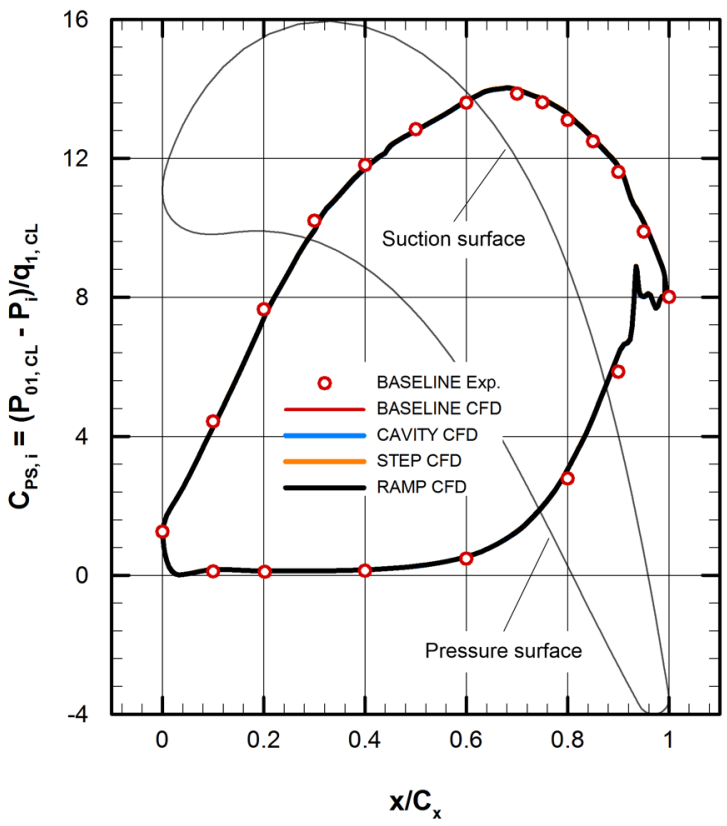

(b) CFD

Near Endwall $(z / \mathrm{h}=0.05)$

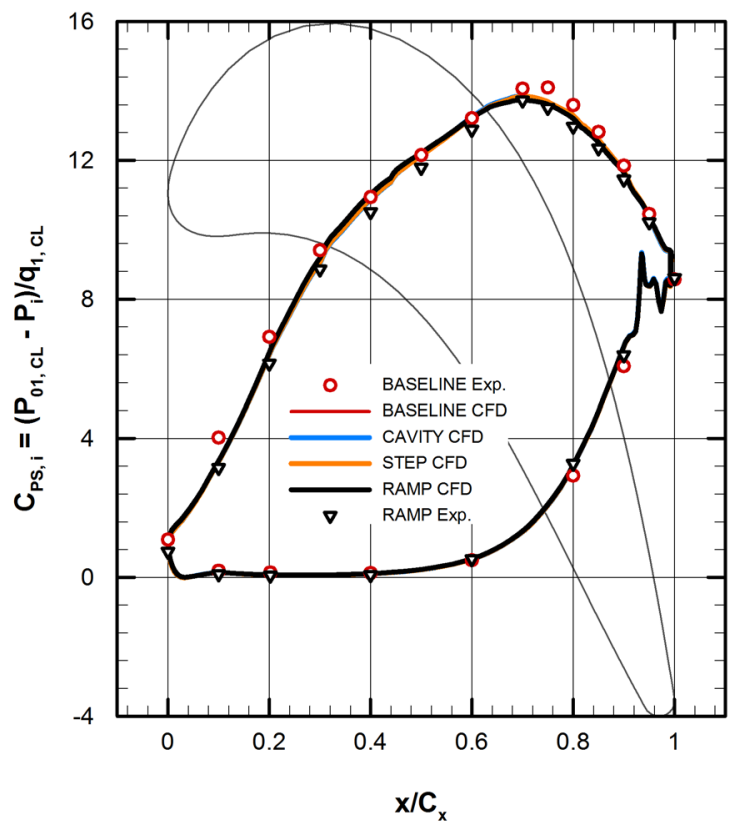

Figure 6.4: Predicted blade surface static pressure distribution at $\mathrm{z} / \mathrm{h}=0.5$ and $\mathrm{z} / \mathrm{h}=\mathbf{0 . 0 5}$

In Figure 6.4 (b), the near endwall predictions for three cavity cases are again essentially equal. For reference, the experimental measurements from the Baseline and Ramp case are overlaid. As shown, the baseline predictions show larger differences relative to the experimental measurements than the three cavity cases. For the cavity configurations, only minor differences 
(relative to the experiment) are noted along the SS between $x / C_{x}=0.2$ and 0.7. Overall, the similarities between the Ramp, Step and Cavity predictions indicate consistency between their respective secondary flows.

\subsection{Downstream Experimental Results}

Experimental flow field measurements were made at the $1.40 C_{x}$ axial plane for the Baseline flat endwall case and the three rim-seal configurations. Figure 6.5 shows colour-flood contour plots of the streamwise vorticity overlaid with line contours of the total pressure coefficient for the four cases. The passage vortex can be identified by negative vorticity (red and yellow), while the counter and corner vortices are indicated by positive vorticity (blue and green).

Of the four cases shown in Figure 6.5, the secondary flow of the baseline (flat endwall) case, Figure 6.5 (a), is notably different from that of each rim-seal configuration. In general, the presence of the upstream geometry appears to intensify the passage vortex, resulting in local regions with higher total pressure losses and larger values of streamwise vorticity.

In Figure 6 (a), there are three vortical features distinguishable from the streamwise vorticity flood. These are identified by the schematic arrows. The passage vortex is the largest structure and has the highest values of streamwise vorticity. The counter-rotating vortex is smaller and has a peak magnitude of streamwise vorticity that is $40 \%$ lower than the passage vortex. The corner vortex is small (nearly indistinguishable) and is located close to the endwall and the blade trailing edge projection. Each vortical feature is associated with low momentum, high loss fluid, as indicated by the line contours of total pressure coefficient. The highest losses, $C_{P 0}=-2.50$, are adjacent to the location of the counter-rotating vortex, as shown in Figure 6 (a), and are not coincident with the position of any of the secondary flow vortices. Knezevici et al. (2010) suggest this high loss fluid likely originates within the blade passage and inlet endwall boundary layer and is swept to the current location by the action of the passage and counter vortices. Since similar local loss peaks are observed near the counter vortex for each of 
(a) BASELINE (measured)

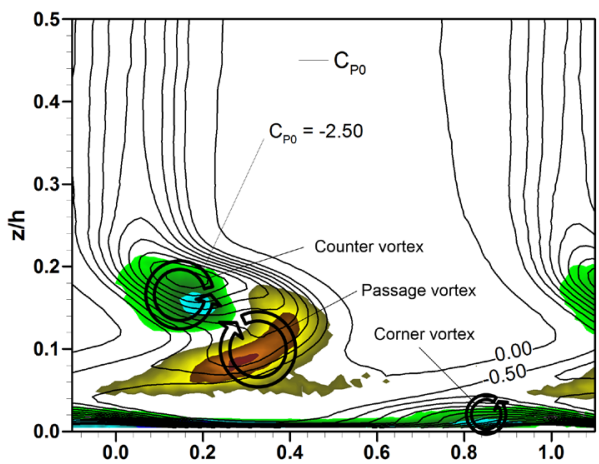

(c) STEP (measured)

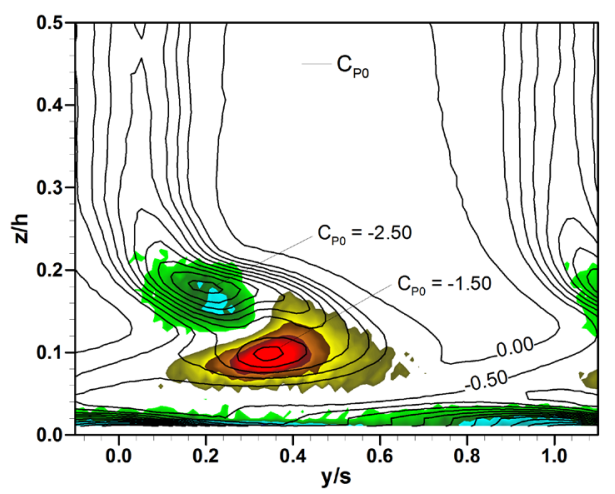

(b) CAVITY (measured)

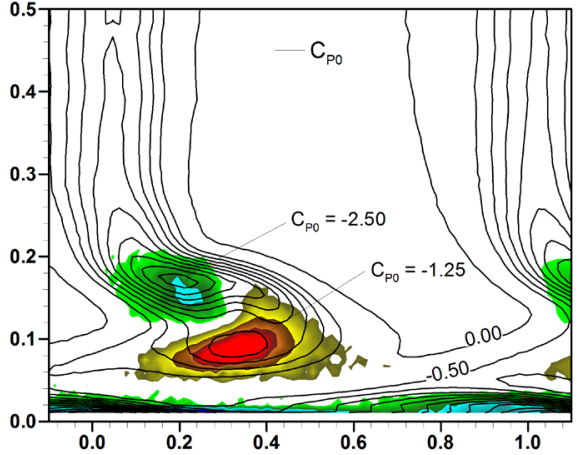

(d) RAMP (measured)

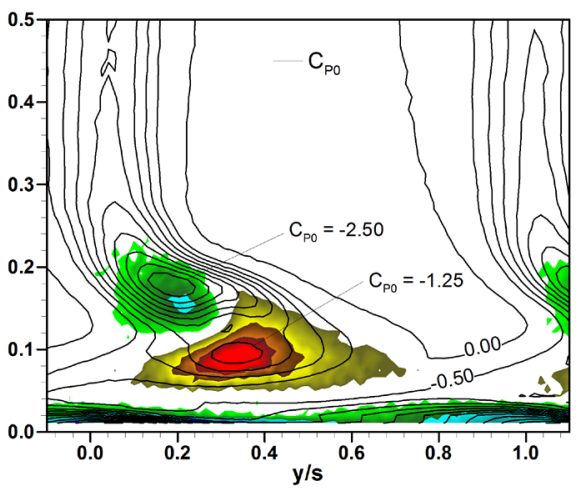

$\mathrm{C} \omega_{\mathrm{s}}$

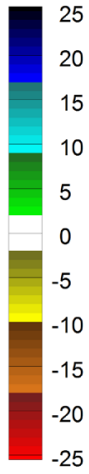

Figure 6.5. Floods of the experimentally measured $C \omega_{S}$ overlaid with the line contours of $C_{P 0}$ for the (a) Baseline, (b) Cavity, (c) Step and (d) Ramp configurations

the rim-seal configurations, Figures 6.5 (b) through (d), it appears that the formation of this loss region is relatively insensitive to the upstream rim-seal geometry.

As will be shown in Section 6.7, the presence of the upstream rim-seal geometry increases the overall integrated in-plane losses by about $10 \%$ relative to the baseline case. The majority of this increase can be attributed to elevated losses within the region of the passage vortex. Distinct peak loss cores are shown in Figures 6.5 (b) through (d) that are coincident with peak values of negative vorticity (the passage vortex region). Table 6.1 shows a summary of these peak values for each case. The Cavity, Step and Ramp configurations show losses within the passage vortex that are 25,50 and $25 \%$ higher relative to the baseline case and the values of streamwise vorticity 
that are 88,94 and $72 \%$ larger. The link between a stronger passage vortex and higher losses is clearly evident.

A stronger passage vortex not only has the effect of increasing the peak losses, but also of increasing the overall size of the secondary flow. The rim-seal cases shown in Figure 6.5 (b) through (d) have larger secondary flows than the baseline case shown in Figure 6.5 (a). Consequently, the region composed of zero loss fluid, commonly referred to as the inviscid core, is smaller for cases with an endwall cavity. Table 6.1 shows the percentage of the exit plane area (one full pitch and one half span) that consists of the inviscid fluid. For flows with the same profile region (ie. the same wake thickness), large values will indicate a smaller secondary flow. As shown, the Step case has the strongest passage vortex, the highest passage vortex losses and the largest secondary flow. As will be shown in the next section, the Step case also has the highest overall integrated losses.

Table 6.1: Summary of peak losses and streamwise vorticity within the passage vortex (PV) and the relative in-plane area of the inviscid core

\begin{tabular}{|l|c|c|c|c|}
\hline & Baseline & Cavity & Step & Ramp \\
\hline Peak $C_{P 0}$ in PV core & -1.00 & -1.25 & -1.50 & -1.25 \\
\hline Peak $C_{\omega s}$ in PV core & -18 & -34 & -35 & -31 \\
\hline$\%$ area of inviscid core & $36.2 \%$ & $32.0 \%$ & $29.8 \%$ & $31.4 \%$ \\
\hline
\end{tabular}

Figure 6.6 shows colour floods of the secondary kinetic energy (SKE) coefficient. As previously mentioned, this represents the mean kinetic energy of the fluid associated with the components of velocity (the secondary velocities) in the plane that is perpendicular to the primary flow direction. The secondary velocities are represented by vectors in Figure 6.6. The vortex centers can be identified by regions (within the secondary flow) of low secondary velocity and low secondary kinetic energy. Away from the vortex cores, the vector field shows the rotation of the fluid about the streamwise axis thereby making the counter vortex, passage vortex and the near endwall cross flow clearly visible. High levels of $C_{S K E}$ are local to high secondary velocities and there seems to be two distinct regions for each case. 
(a) BASELINE (measured)

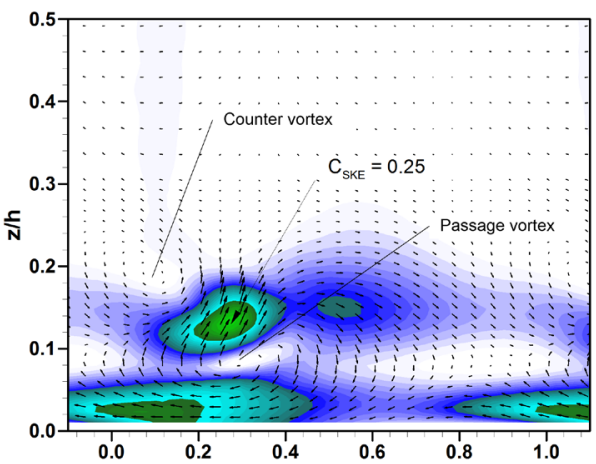

(c) STEP (measured)

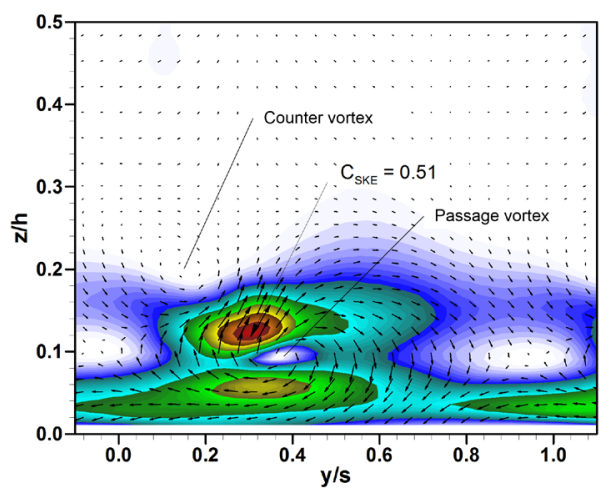

(b) CAVITY (measured)

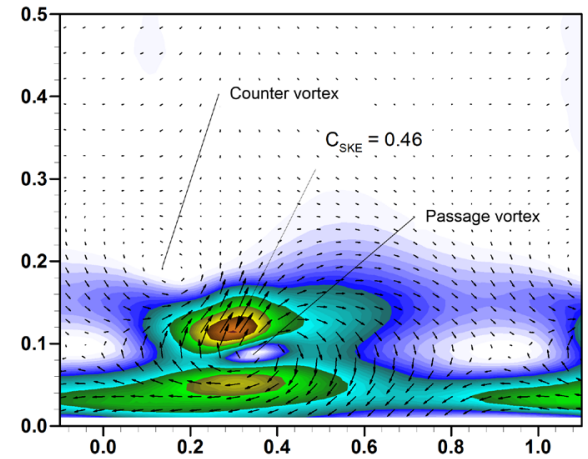

(d) RAMP (measured)

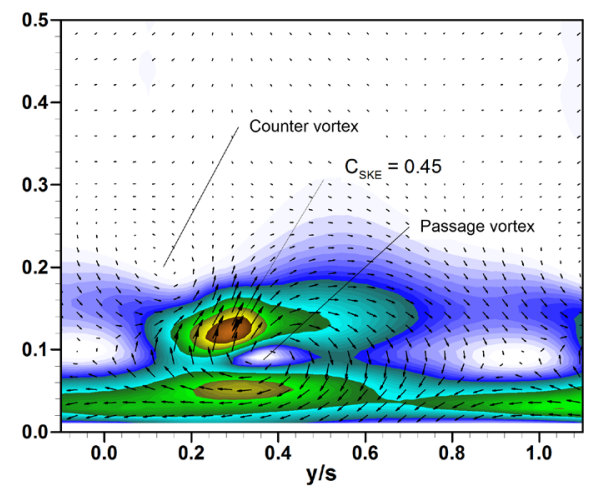

$\overrightarrow{\mathrm{VN}^{\prime} \mathrm{CL}_{2}}=1$

$\mathrm{C}_{\text {SKE }}$

0.550

0.500

0.450

0.400

0.350

0.300

0.250

0.200

0.150

0.100

0.050

0.000

Figure 6.6. Floods of the experimentally measured $C_{S K E}$ overlaid with the secondary velocity vectors for the (a) Baseline, (b) Cavity, (c) Step and (d) Ramp configurations

The first region is local to the maximum $C_{S K E}$ and is positioned where the passage and counter vortices begin to interact; for the baseline case shown in Figure 6.6 (a) the peak value of $C_{S K E}$ is 0.25 and is positioned at $y / s=0.30$ and $z / h=0.14$. Figures 6.6 (b) through (d) show that the peak value of $C_{S K E}$ is 84,104 and $80 \%$ larger than the baseline case for the Cavity, Step and Ramp configurations, respectively. Of the three rim-seal cases, the Step configuration generates a slightly stronger passage vortex (see Figure 6.5) which results in higher peak and overall integrated values of $C_{S K E}$. The secondary kinetic energy, once generated, will eventually dissipate with downstream distance through viscous mixing with the primary flow. Thus, the $C_{S K E}$ is also an indication of the additional losses that are realized at fully mixed-out conditions. As shown in the next section, the Step case also has the highest overall mixed-out losses. 
The second region of elevated $C_{S K E}$ is located below the passage vortex towards the suction side of the passage. Here, the secondary velocities are relatively large and oriented in the crosspassage direction. Knezevici et al. (2010) suggest that elevated $C_{S K E}$ in the cross-flow region is evidence of the commonly observed flow angle over-turning that occurs in the near endwall region of turbine bladerows. Figures 6.6 (b) through (d) show that the rim-seal cases have about $50 \%$ higher $C_{S K E}$ in this cross flow region than the baseline case. As a result, the pitchwise averaged flow angles, shown in Figure 6.9 (c), have approximately $2^{\circ}$ more over-turning. Again, this is evidence that the presence of the upstream cavity strengthens the passage vortex and increases both the in-plane and mixed-out losses.

\subsection{Downstream Computational Results}

The purpose of this sub-section is to demonstrate that CFD predicts the flow physics reasonably well for these cases. Of course, the typical limitations of CFD simulations outlined in Chapters 4 and 5 still apply. The general agreement between the experiment and CFD will then add merit to CFD results that are used later to further examine the intra-passage flow field.

Figure 6.7 shows colour floods of the streamwise vorticity with line contours of the total pressure coefficient overlaid. The baseline case is shown in sub-figure (a) and the Cavity, Step and Ramp cases are shown in sub-figures (b) through (d) respectively. In each case, the passage and counter vortices are clearly visible. The comer vortex is also visible and more pronounced in the CFD when compared to the experiments. For the three cavity cases, the secondary flow appears more compressed against the endwall than in the baseline case, which is consistent with the experimental measurements. The peak values of streamwise vorticity associated with the passage vortex are significantly lower than the experimental values for the three cavity cases, while the baseline case appears to be relatively well predicted.

The fact that the baseline passage vortex is well predicted but the cavity cases are less so suggests that the passage vortex development is attenuated in the CFD for the three cavity cases. 
(a) BASELINE (CFD)

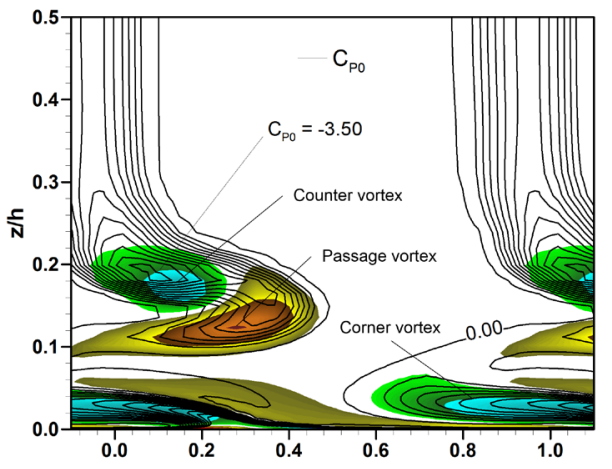

(c) STEP (CFD)

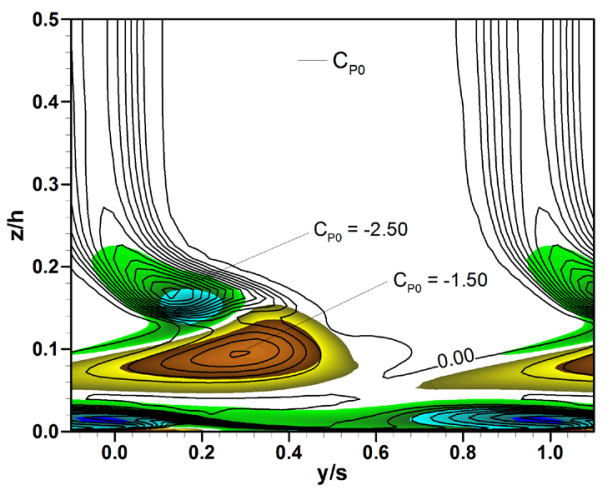

(b) CAVITY (CFD)

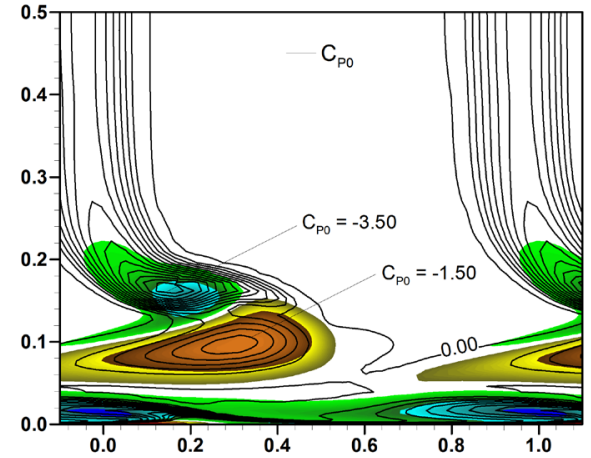

(d) RAMP (CFD)

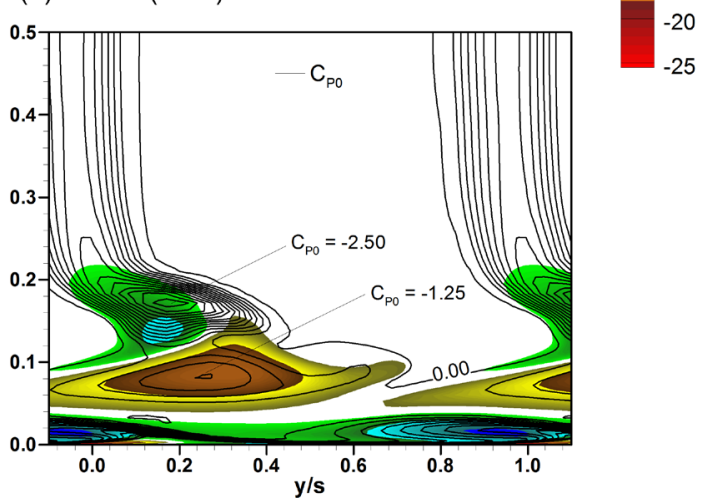

Figure 6.7: Floods of the predicted (CFD) $C_{\omega S}$ overlaid with the line contours of $C_{P 0}$ for the (a)

Baseline, (b) Cavity, (c) Step and (d) Ramp configurations

As will be shown in Section 6.8, the passage vortex development is closely linked to the formation of a recirculation zone downstream of the platform overlap. Therefore, it is possible that these differences relative to the baseline case likely originate within the predicted cavity recirculation regions.

The overlaid total pressure contours of Figure 6.7 follow similar trends to the experiment. As discussed in Chapters 4 and 5, it is typical for the CFD predictions to appear less diffuse with higher peak loss values than the experiment. The peak losses, although larger than the experiment are similarly adjacent to the counter vortex. Also, in each case there is an elevated loss region that is local to the passage vortex and is nearly coincident with the local peak value of 
(a) BASELINE (CFD)

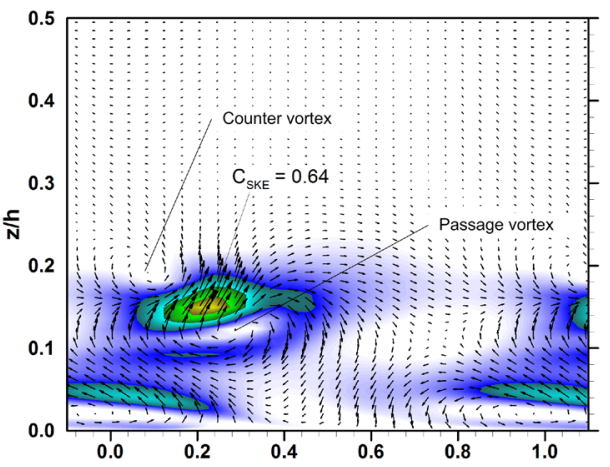

(c) STEP (CFD)

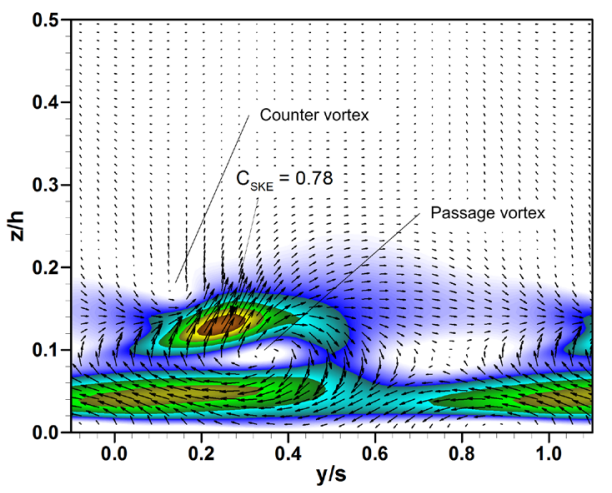

(b) CAVITY (CFD)

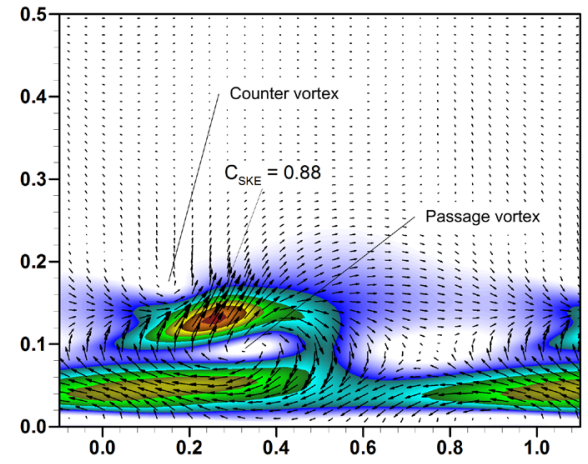

(d) RAMP (CFD)

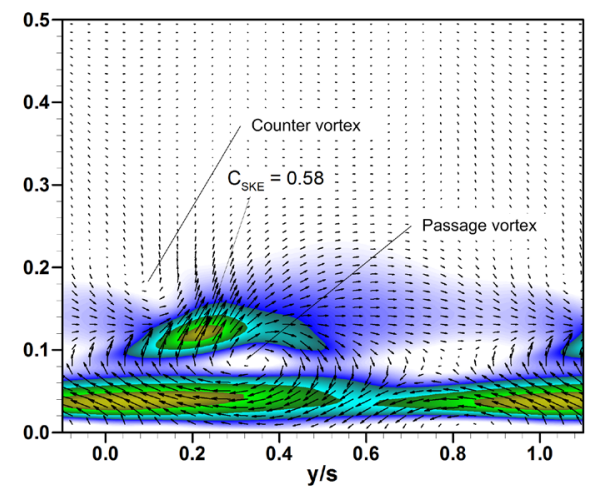

$\overrightarrow{\mathrm{V} / \mathrm{CL}_{\mathrm{CL}}}=1$

$\mathrm{C}_{\text {SKE }}$

1.000
0.900

0.800

0.700

0.600

0.500

0.400

0.300

0.200

0.100

0.000

Figure 6.8: Floods of the predicted $C_{S K E}$ overlaid with the secondary velocity vectors for the (a)

\section{Baseline, (b) Cavity, (c) Step and (d) Ramp configurations}

negative streamwise vorticity. The flows from the three cavity cases are very similar, with the Ramp case showing the most diffuse passage vortex with the lowest peak losses.

Overall, the total pressure contours and floods of streamwise vorticity show that the CFD predicts similar trends to the experiment regarding the loss distribution and position of the main vortical features. However, the magnitude of the flow quantities is largely different from the experiment which is an expected outcome for typical CFD predictions.

Figure 6.8 shows the predicted floods of the secondary kinetic energy (SKE) coefficient with the secondary velocity vectors overlaid. Note the scale for the $C_{S K E}$ is nearly double that of experimental floods shown in Figure 6.5. Here, the CFD predicts the highest levels of SKE within the vortex interaction zone, the region between the passage and counter vortices. This was 
also true of the experimental results. The secondary motion of the passage and counter vortices pushes fluid towards the inviscid core leading to high secondary velocities and large values of secondary kinetic energy. As shown in the three cavity cases, there is another region of elevated secondary kinetic energy located closer to the endwall below the passage vortex. As indicated by the secondary vectors, there is significant cross-passage flow here which leads to the locally high levels of secondary kinetic energy.

In general, the distributions of $C_{S K E}$ of Figure 6.8 are similar to the experiment; however, the peak values are significantly higher. This is an indication of less viscous dissipation since secondary kinetic energy dissipates through viscous mixing with downstream distance. As discussed in Chapter 4 and 5, these results exhibit the typical shortcoming of CFD simulations. Overall, the interaction of the secondary structures and the distributions of total pressure loss, streamwise vorticity and secondary kinetic energy appear to be well predicted.

\subsection{Experimental and Computational Integrated Results}

This section presents pitchwise and overall integrated flow field quantities for the measured and CFD results at the $1.40 C_{x}$ axial plane. Area- and mass-averages were computed based on data cell centres as mentioned in Section 3.11.2. A summary of the overall integrated values including the fully mixed-out flow quantities is shown in shown in Table 6.2. Table 6.3 presents the same data, but normalized by the corresponding baseline experimental values. The pitchwise averaged results are shown in Figure 6.9. Sub-figure (a) shows spanwise distributions of pitchwise mass-averaged loss coefficients, normalized by the baseline experimental mid-span value, for each of the cavity cases. Figures 6.9 (b) and (c) show the corresponding pitchwise mass-averaged $C_{S K E}$ and area-averaged streamwise error angle, $\Delta \beta_{2}$, respectively.

In Figure 6.9 (a), the spanwise loss distributions have two local peaks which correspond to the position of the passage and counter vortices. For each cavity case, the higher losses relative to the baseline are clearly associated with the stronger passage vortex whereas the losses 
associated with the counter vortex appear unaffected relative to the baseline case. In general, the CFD predictions are qualitatively well correlated with the experimental measurements; however, the predictions show evidence of lower mixing rates and consequently less diffusion of the low momentum fluid. The predicted loss distributions have larger spanwise gradients and local loss peaks that are smaller and located closer to the endwall.

The predicted distributions of $C_{S K E}$, shown in Figure 6.9 (b), have peaks that are larger than the experiment which indicates less viscous dissipation and higher cross-flow in the near endwall region. Again, this is consistent with the discussions of Chapters 4 and 5.

In Figure 6.9 (c), the predictions show larger values of flow over-turning in the near endwall region, also an indication of a stronger blade-to-blade cross-flow, while there are only slight differences in flow under-turning away from the endwall. Although the predicted results demonstrate some of the common short comings of CFD simulations, the results shown here appear satisfactory for design level computations. Overall, the interpretation of the flow physics would not be skewed if one only had access to the predicted results.

Table 6.2 and Table 6.3 show that the predicted in-plane losses for the three rim-seal configurations are similar, with differences that are less than 1\%. The Ramp case has the lowest value of secondary kinetic energy and consequently has the lowest mixed-out losses, a normalized value of $110 \%$ relative to the baseline experiment. The Cavity and Step cases have normalized mixed-out loss values of 115 and $113 \%$ respectively. In general, the predicted overall losses appear to be relatively insensitive to simplifications of the rim-seal geometry.

The experimental overall losses show subtle differences between the flows for the three rim-seal geometries. Similar to the predictions, the Step produces the highest in-plane losses, a normalized value of $113 \%$, whereas the Cavity and Ramp configurations have values that are 6\% and $4 \%$ lower respectively. The corresponding normalized mixed-out loss values are 140, 132 and $135 \%$ for the Step, Cavity and Ramp cases respectively. Figure 6.9 (a) shows that the differences in the loss values for the three rim-seal cases are localized within the passage vortex region; only small differences are noted in the vicinity of the counter vortex. This suggests that 
the rim-seal cavity, even without a net purge flow, has the effect of reducing the strength of the passage vortex relative to the other two rim-seal cases (Ramp and Step), thereby lowering the losses, the cross-flow and near wall over-turning. A physical explanation for this phenomenon is suggested by de la Rosa Blanco et al. (2009) and is further examined in Section 6.8.3 using 3D CFD visualizations.

Table 6.2: $\quad$ Summary of the measured and predicted overall integrated flow quantities

\begin{tabular}{|c|c|c|c|c|c|c|c|c|}
\hline \multirow[b]{2}{*}{ Flow Quantity } & \multicolumn{2}{|c|}{ Baseline } & \multicolumn{2}{|c|}{ Cavity } & \multicolumn{2}{|c|}{ Step } & \multicolumn{2}{|c|}{ Ramp } \\
\hline & Exp. & $C F D$ & Exp. & $C F D$ & Exp. & $C F D$ & Exp. & $C F D$ \\
\hline $\mathrm{Y}$ & 0.048 & 0.047 & 0.051 & 0.044 & 0.054 & 0.044 & 0.052 & 0.043 \\
\hline$Y_{p}$ & 0.030 & 0.031 & 0.031 & 0.031 & 0.031 & 0.031 & 0.030 & 0.031 \\
\hline $\mathrm{Y}_{\mathrm{sec}}$ & 0.018 & 0.015 & 0.020 & 0.013 & 0.023 & 0.012 & 0.022 & 0.012 \\
\hline$\beta_{2}^{\prime \prime}$ & -72.7 & -73.4 & -73.0 & -73.5 & -73.1 & -73.5 & -73.0 & -73.5 \\
\hline$\beta_{2, \text { midspan }}$ & -73.2 & -74.0 & -73.4 & -73.8 & -73.4 & -73.7 & -73.3 & -73.8 \\
\hline $\mathrm{C}_{\mathrm{SKE}}{ }^{\prime \prime}$ & 0.032 & 0.043 & 0.051 & 0.081 & 0.057 & 0.079 & 0.056 & 0.070 \\
\hline$Y_{\text {mixed-out }}$ & 0.057 & 0.054 & 0.063 & 0.055 & 0.067 & 0.054 & 0.064 & 0.052 \\
\hline$Y_{p, \text { mixed-out }}$ & 0.031 & 0.033 & 0.032 & 0.032 & 0.032 & 0.032 & 0.031 & 0.032 \\
\hline$Y_{\text {sec, mixed-out }} / Y_{\text {baseline (Exp.) }}$ & 0.026 & 0.022 & 0.031 & 0.023 & 0.035 & 0.022 & 0.033 & 0.020 \\
\hline
\end{tabular}

Table 6.3: Summary of the measured and predicted overall integrated flow quantities relative to the baseline measurements

\begin{tabular}{|c|c|c|c|c|c|c|c|c|}
\hline \multirow[b]{2}{*}{ Flow Quantity } & \multicolumn{2}{|c|}{ Baseline } & \multicolumn{2}{|c|}{ Cavity } & \multicolumn{2}{|c|}{ Step } & \multicolumn{2}{|c|}{ Ramp } \\
\hline & Exp. & $C F D$ & Exp. & $C F D$ & Exp. & $C F D$ & Exp. & $C F D$ \\
\hline $\mathrm{Y} / \mathrm{Y}_{\text {baseline (Exp.) }}$ & $100 \%$ & $97 \%$ & $107 \%$ & $92 \%$ & $113 \%$ & $91 \%$ & $109 \%$ & $91 \%$ \\
\hline$Y_{p} / Y_{\text {baseline (Exp.) }}$ & $62 \%$ & $66 \%$ & $65 \%$ & $65 \%$ & $66 \%$ & $65 \%$ & $63 \%$ & $65 \%$ \\
\hline$Y_{\text {sec }} / Y_{\text {baseline (Exp.) }}$ & $38 \%$ & $32 \%$ & $42 \%$ & $27 \%$ & $48 \%$ & $26 \%$ & $46 \%$ & $25 \%$ \\
\hline$\beta_{2}^{\prime \prime}$ & -72.7 & -73.4 & -73.0 & -73.5 & -73.1 & -73.5 & -73.0 & -73.5 \\
\hline$\beta_{2, \text { midspan }}$ & -73.2 & -74.0 & -73.4 & -73.8 & -73.4 & -73.7 & -73.3 & -73.8 \\
\hline $\mathrm{C}_{\mathrm{SKE}}{ }^{\prime \prime}$ & 0.032 & 0.043 & 0.051 & 0.081 & 0.057 & 0.079 & 0.056 & 0.070 \\
\hline$Y_{\text {mixed-out }} / Y_{\text {baseline (Exp.) }}$ & $119 \%$ & $114 \%$ & $132 \%$ & $115 \%$ & $140 \%$ & $113 \%$ & $135 \%$ & $110 \%$ \\
\hline$Y_{p, \text { mixed-out }} / Y_{\text {baseline (Exp.) }}$ & $65 \%$ & $68 \%$ & $67 \%$ & $68 \%$ & $67 \%$ & $68 \%$ & $65 \%$ & $68 \%$ \\
\hline$Y_{\text {sec, mixed-out }} / Y_{\text {baseline (Exp.) }}$ & $54 \%$ & $46 \%$ & $65 \%$ & $47 \%$ & $72 \%$ & $46 \%$ & $69 \%$ & $42 \%$ \\
\hline
\end{tabular}


(a) Pitchwise Mass-averaged Normalized Loss Coefficient
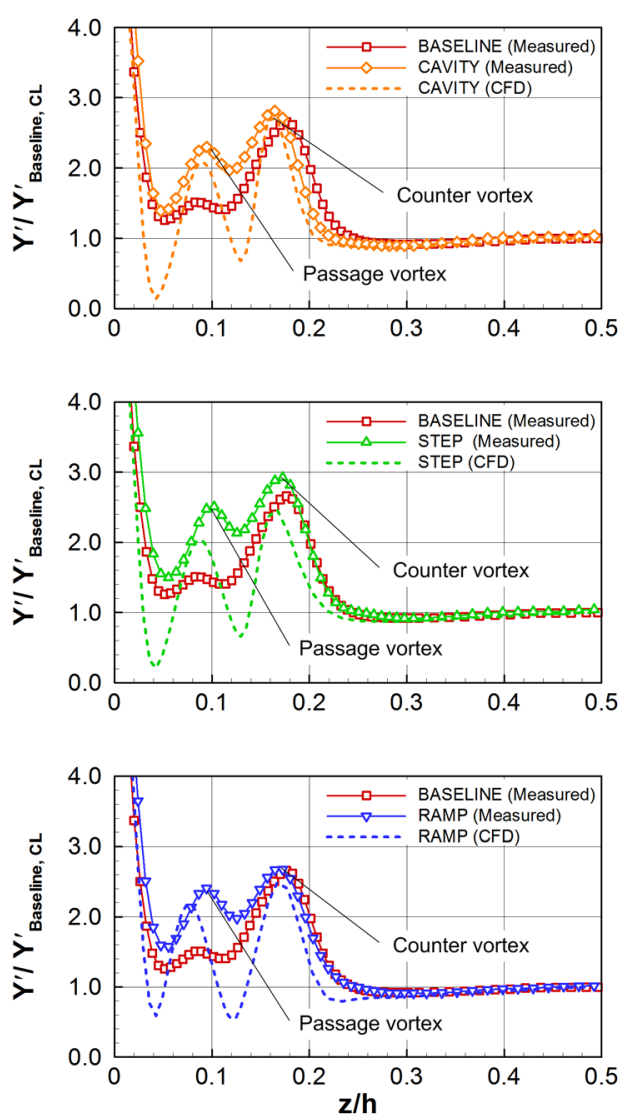

(b) Pitchwise Mass-averaged Secondary Kinetic Energy Coefficient

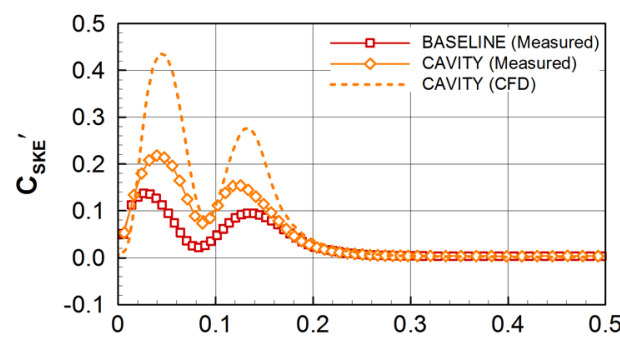

(c) Pitchwise Area-averaged Flow Angle
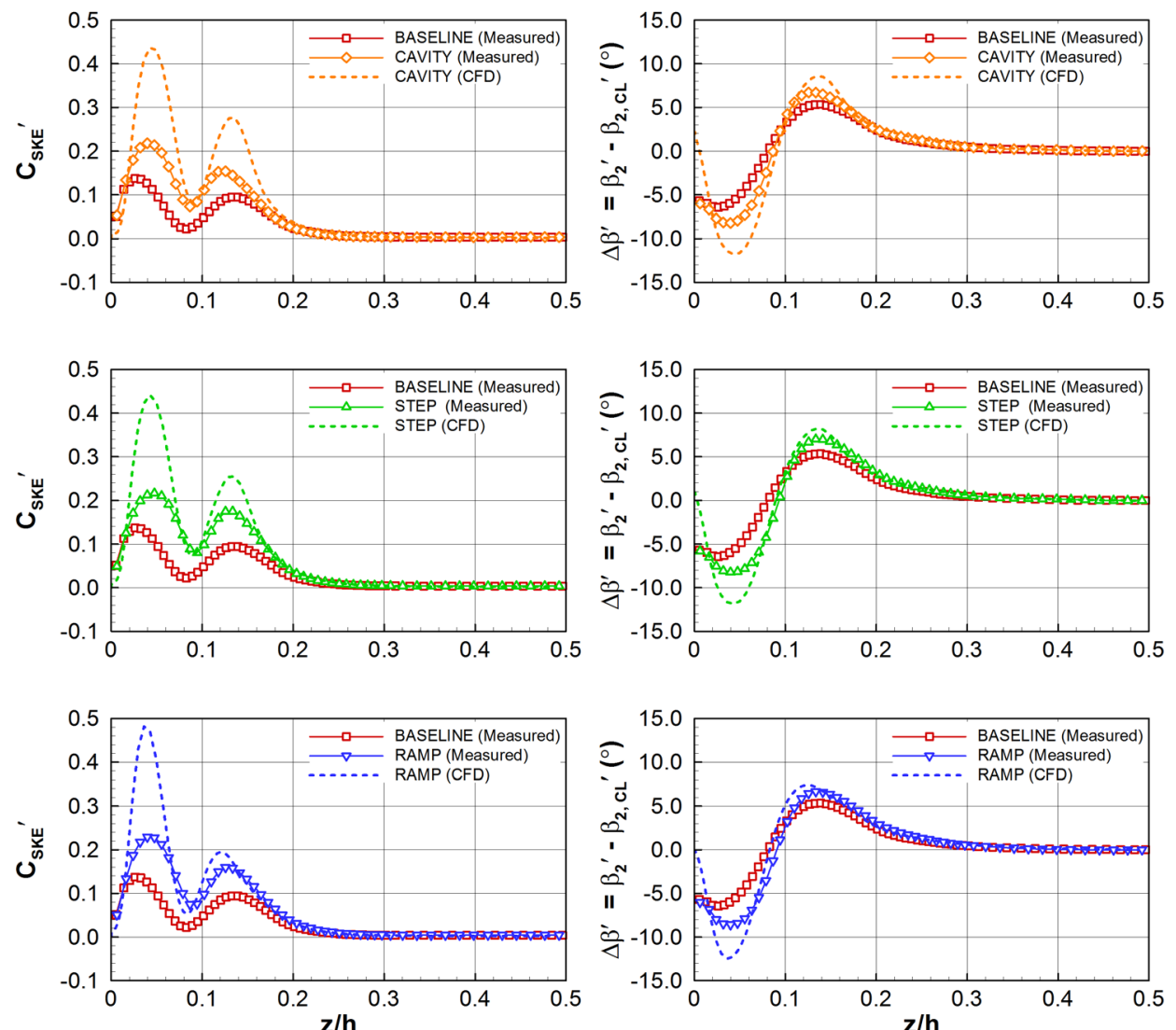

Figure 6.9: Experimental and computational pitchwise-averaged results 


\subsection{Visualization of the Endwall Cavity Flow Field}

\subsubsection{Introduction}

The purpose of this section is to visualize the effects of the upstream platform overlap on the secondary flow development relative to that of the Baseline flat-endwall case. The downstream measurements have shown that the flow fields of three cavity cases are very similar. Also, the CFD simulations predict the experimental physics reasonably well and within the typical limitations of CFD. Since experimental intra-passage measurements were not performed, the CFD is used in the following sub-sections to aid the interpretation of the flow physics. Subsection 6.8.2 presents computational 3D visualizations and endwall surface flow visualizations of the Baseline and Cavity flow fields. Experimental visualizations of the Cavity case are also presented. Sub-section 6.8.3 further examines why the Cavity case has the lowest overall losses and a slightly attenuated passage vortex relative to the Step and Ramp cases.

\subsubsection{Interpretation of the Flow Fields for the Flat and Cavity Endwall Configurations}

The experimental results have shown that the presence of an upstream rim-seal strengthens the passage vortex and increases the secondary losses relative to the same bladerow with a flat endwall. As discussed in Chapter 2, this is largely the case in many similar investigations. The work of Abo El Ella et al. (2012) details a loss mechanism, described below, by which the endwall cavity strengthens the passage vortex. They present downstream measurements and surface flow visualizations from two transonic cascades, one with a flat endwall and the other with a ramp-type endwall cavity similar to that of Figure 6.1 (a). The experimental measurements of their study are complimentary to this work and are part of a larger research program at Carleton University with PWA. The CFD results shown here are used to further understand the cavity flow field and passage vortex interactions. Introductory $3 \mathrm{D}$ visualizations 
are presented first followed by endwall surface flow visualizations. Lastly, the predicted Cavity surface visualizations are compared to the experimental surface flow visualizations.

Figures 6.10 (a) and (b) show the 3D visualizations of the predicted secondary flow for the Baseline and Cavity cases respectively. ISO-surfaces, set to a constant value of turbulence kinetic energy (TKE), are overlaid with surface streamlines to show the passage vortex development through the bladerow. The flat endwall exhibits a typical secondary flow as shown in Figure 6.10 (a). Blue velocity vectors show the inlet boundary layer while red stream-ribbons, emanating from within the upstream endwall boundary layer, represent the flow as it approaches the bladerow. As shown, the endwall boundary layer separates at the saddle point; the red stream-ribbons are split, developing into the pressure and suction side of the horseshoe vortex. The passage vortex is formed as the blade-to-blade pressure field drives the pressure-side leg of the horseshoe vortex across the passage causing it to entrain more inlet boundary layer fluid. As the passage vortex meets the suction side of the adjacent blade, the suction-side leg of the horseshoe vortex is entrained. Strong interactions between the passage vortex and the blade surface boundary layers give rise to the counter and corner vortices (not show here).

The corresponding visualization for the Cavity rim-seal configuration is shown in Figures 6.10 (b). As previously noted, Abo El Ella et al. (2012) provide a description of a similar flow field using experimental surface flow visualizations and downstream measurements. They showed that a three-dimensional recirculation zone exists within the cavity and is oriented with its axis perpendicular to the mainstream flow. In Figure $6.10(\mathrm{~b})$, the inlet boundary fluid (represented by red stream-ribbons) is shown to separate from the platform overlap and forms a similar three-dimensional recirculation zone. Much like the flat endwall case, the red streamribbons are split forming two legs of a much larger horseshoe vortex. However, the split appears to initiate away from the endwall and above the recirculation zone, rather than at a distinct saddle point as shown for the flat endwall case. 
(a) BASELINE

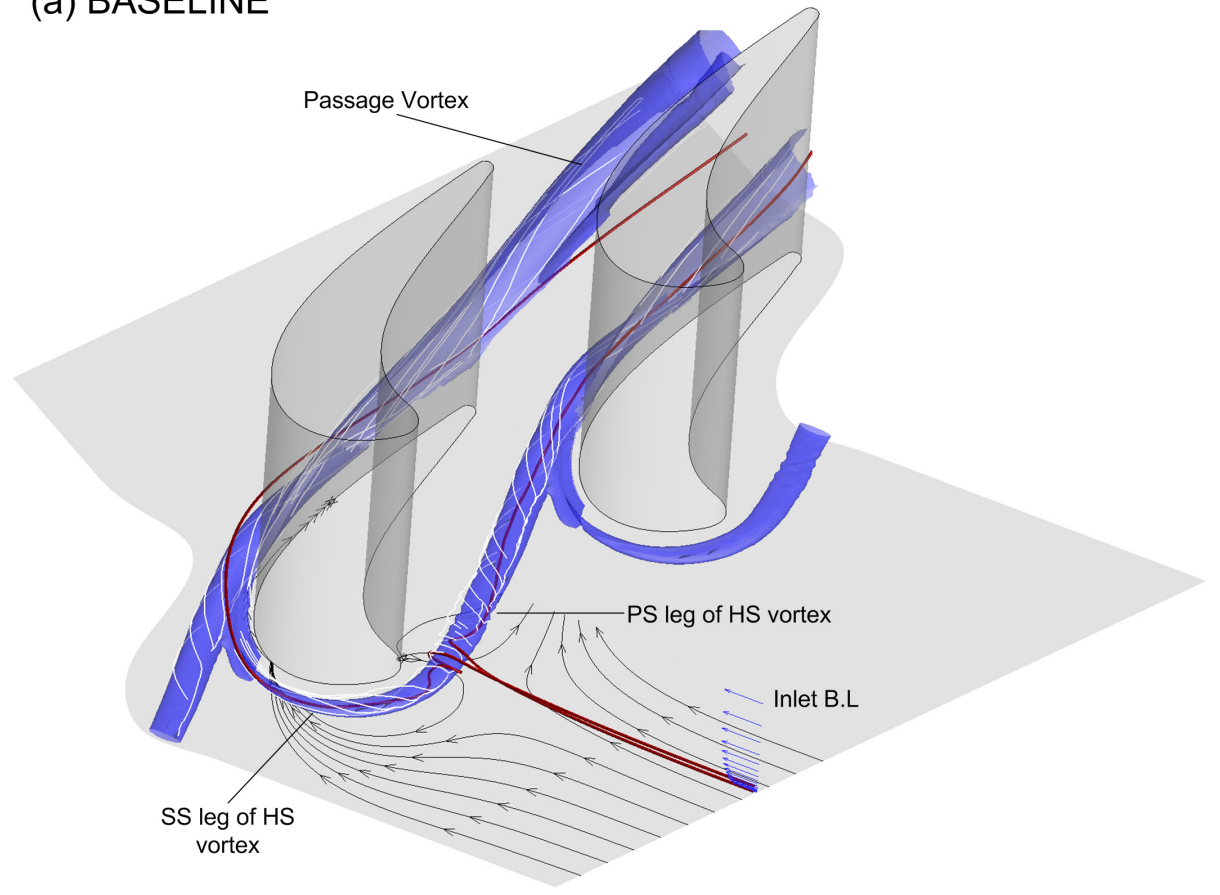

(b) CAVITY

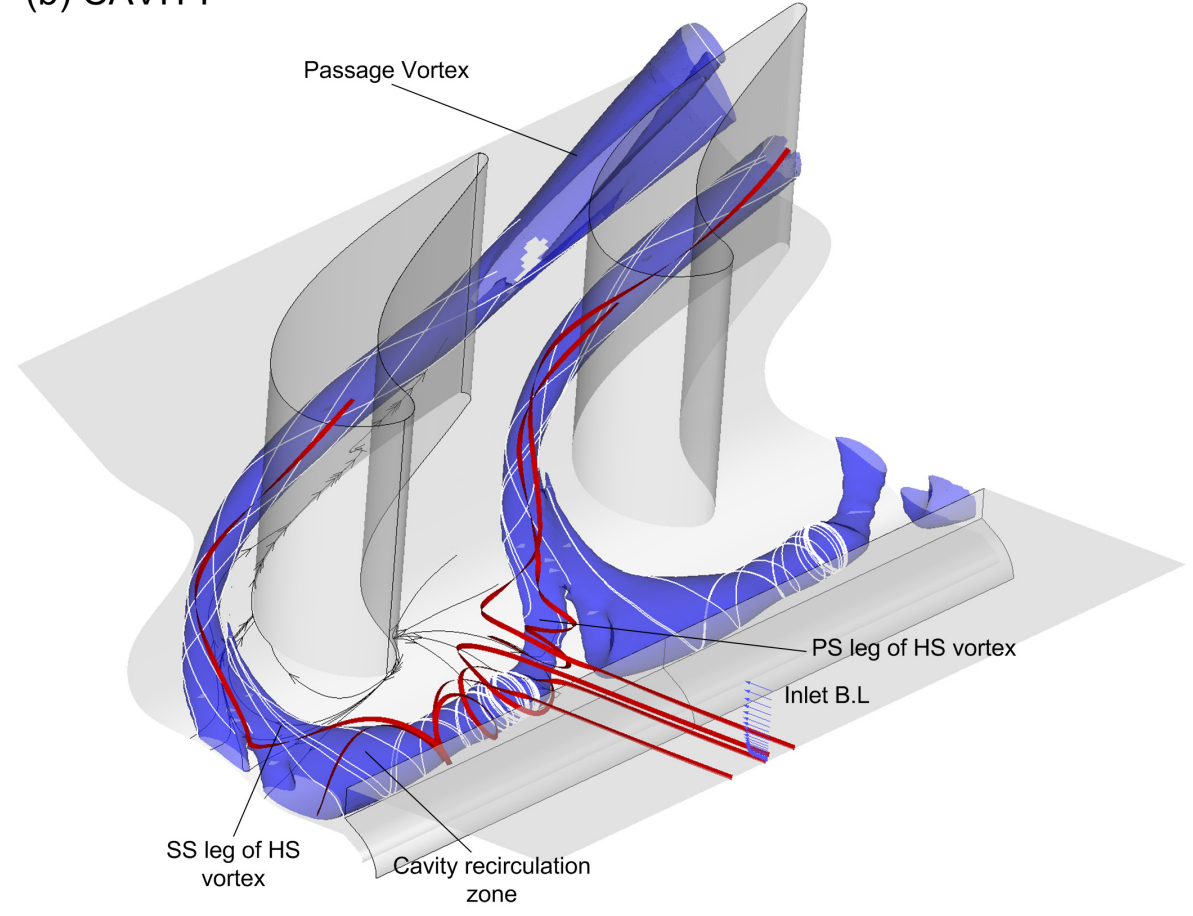

Figure 6.10: ISO-surface of constant TKE and surface streamlines showing the secondary flow of the (a) baseline and (b) cavity case 
Abo El Ella et al. (2012) suggest that the recirculation zone is about one pitch in length and that it terminates at distinct points (periodically spaced) upstream of the bladerow. At these points the authors suggest that small vortices may be shed from the recirculation zone, energizing the pressure-side leg of the horseshoe vortex, and consequently strengthening the passage vortex.

The predicted flow shown in Figure 6.10 (b) is slightly different. Within the endwall cavity, at the mid-pitch $(y / s=0.5)$ location, it appears that the blade-to-blade pressure gradient causes the recirculation zone to turn downstream and move towards the blade suction surface, rather than terminating as suggested by Abo El Ella et al. (2012). As the recirculation zone is turned downstream it resembles the pressure-side leg of the classic horseshoe vortex; however, it is clearly strengthened by the cavity recirculation zone. At the opposite side of the endwall cavity, towards the suction side of the blade, the cavity recirculation appears to be larger, indicating a reduction in the vortex strength. This suction-side leg interacts with the pressure-side and becomes entrained by the passage vortex further downstream, similar to the classic flat endwall secondary flow. The net result for the cavity case, as observed in the experimental results, is a stronger and larger passage vortex with higher losses as well as elevated levels of secondary kinetic energy and streamwise vorticity.

Computational endwall surface visualizations showing the endwall limiting streamlines are discussed next with reference to Figure 6.11. Sub-figure (a) and (b) identify the Baseline and Cavity endwalls respectively. As shown, the flow enters from the right at the design inlet flow angle. The colour flood contour represents the non-dimensional shear stress, $C_{f}$, and the surface shear stress streamlines are overlaid. The limiting streamlines are marked by red lines. Wherever possible the notation of Sieverding (1985) is applied.

For the Baseline case, Figure 6.11 (a), the upstream saddle point, marked $\mathrm{A}_{1}$, is a region of very low shear stress. The primary separation, $\mathrm{S}_{1}$, marks the separation of the inlet boundary layer as it encounters the blade pressure field. The pressure and suction side of the primary separation are labelled $\mathrm{S}_{1 \mathrm{~S}}$ and $\mathrm{S}_{1 \mathrm{P}}$ respectively. The secondary separation, $\mathrm{S}_{2}$, corresponds to the "lift-off" line of the horseshoe (HS) vortex. As shown, the pressure-side inlet boundary layer 
separation $\left(\mathrm{S}_{1 \mathrm{P}}\right)$ and the pressure-side leg of the HS vortex $\left(\mathrm{S}_{2 \mathrm{P}}\right)$ are driven across the blade passage by the cross-passage pressure gradient. The passage vortex evolves from the pressureside leg as it moves downstream and across the passage. The endwall cross-flow is clearly indicated by intra-passage shear stress streamlines. Towards the suction side of the blade, the horseshoe vortex and the inlet boundary layer separations wrap around the blade. The intersection of these two limiting streamlines marks a strong interaction with blade surface. Further downstream, along the suction surface, the passage vortex interacts with the blade surface flow giving rise to the corner vortex, $\mathrm{S}_{3}$. Similarly, the counter vortex formation is induced by the passage vortex and the trailing edge separation (not shown).

In Figure 6.11 (b), the limiting surface streamlines for the Cavity case are notably different from those of the Baseline case. In this figure, the edge of the platform overlap is labelled for reference. As previously described, the cavity recirculation zone forms downstream of the platform overlap as the inlet boundary separates from the platform overlap. Visual indicators of the cavity recirculation zone are the reattachment line, $\mathrm{R}_{\mathrm{C}}$, just upstream of the blade leading edge and the separation further upstream within the cavity, labelled $\mathrm{S}_{\mathrm{C}}$. The inlet boundary layer fluid that separated from the platform overlap reattaches at $\mathrm{R}_{\mathrm{C}}$. Within the cavity, the surface streamlines show the endwall fluid is pushed upstream into the cavity away from the reattachment $\left(R_{C}\right)$. As the flow moves deeper into the cavity, it separates along the separation line, $\mathrm{S}_{\mathrm{C}}$, as it approaches the platform overlap and purge slot. The fluid is then pushed in the spanwise direction into the main passage thereby completing the cavity recirculation.

Analogous to the Baseline HS vortex, the cavity recirculation is divided into pressure-side and suction-side regions, or legs. Perhaps the most complex region of this cavity flow is the interaction between the two legs at about mid-pitch. Here there are very low shear stresses and a saddle point is marked $A_{1}$. There may also be a second saddle point as the streamlines appear to diverge at other points in the vicinity of $A_{1}$. Sieverding (1985) mentions that it is possible for two saddle points to form upstream of the turbine passage, however, this has not been observed 
(a) BASELINE CFD

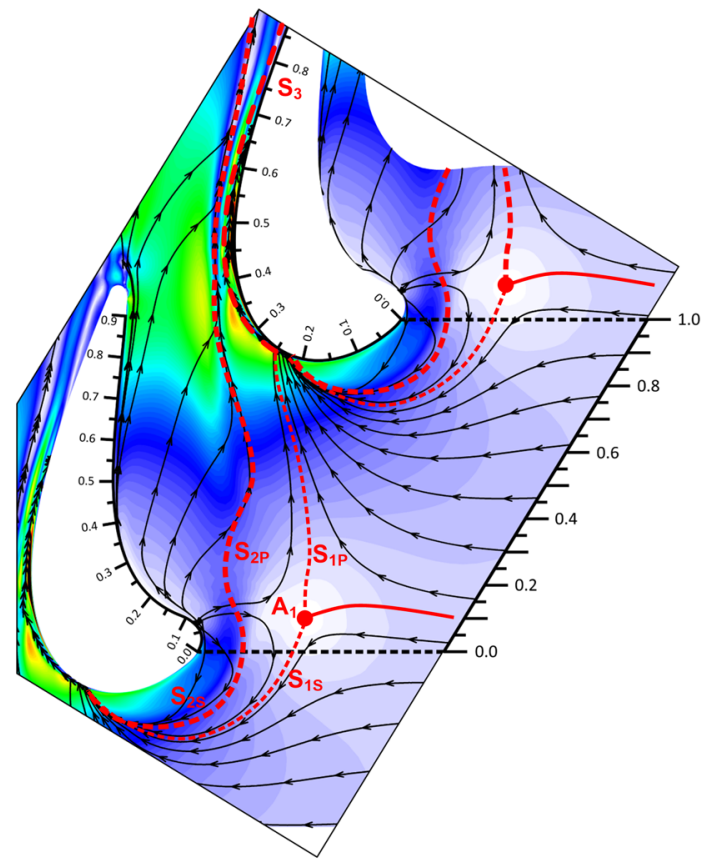

(b) CAVITY CFD

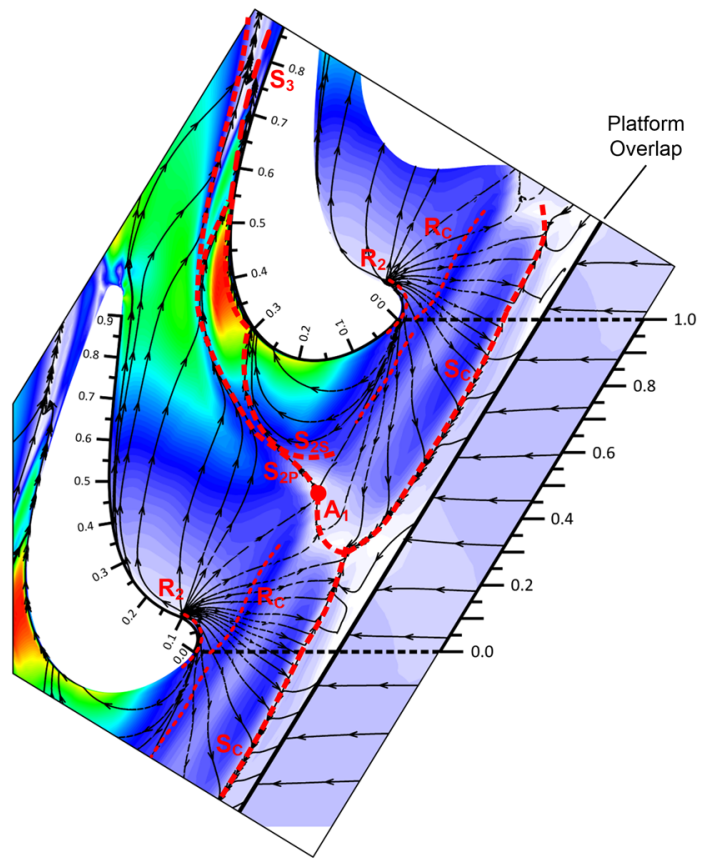

$\mathrm{C}_{\mathrm{f}} \times 10^{-3}: \quad 0 \quad 5 \quad 5 \quad 101520 \quad 25 \quad 30 \quad 3540 \quad 45 \quad 50 \quad 55 \quad 60 \quad 65 \quad 70 \quad 75 \quad 80$

(c) CAVITY Exp.

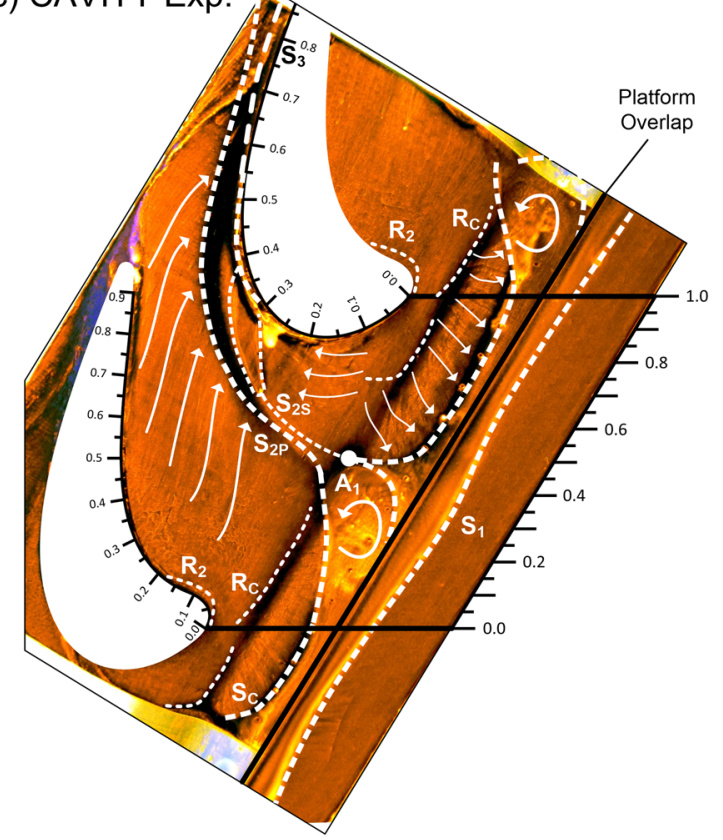

Figure 6.11: Endwall surface visualization and interpretation 
consistently in other studies and is perhaps cascade specific. The complexity of flow in the cavity region makes it difficult to decipher further detail than shown in the present interpretation.

The secondary separation, $\mathrm{S}_{2 \mathrm{P}}$, also appears to emanate downstream from the saddle point $\left(A_{1}\right)$. Similar to the Baseline case, the $\mathrm{S}_{2 \mathrm{P}}$ line is the "lift-off" line of the horseshoe vortex. For the cavity case, the $S_{2 P}$ line is clearly displaced towards the blade suction surface relative to the flat endwall visualization. The opposite is true for the suction-side separation, $\mathrm{S}_{2 \mathrm{~S}}$, as it is displaced by the cavity reattachment $\left(\mathrm{R}_{\mathrm{C}}\right)$ towards the $\mathrm{S}_{2 \mathrm{P}}$ separation. As shown, it appears there is an interaction as $S_{2 P}$ and $S_{2 S}$ intersect within the passage. Significant endwall shear stress is observed just downstream of the interaction and appears to be on of the primary causes for the stronger secondary flows associated with the cavity cases. Further downstream from this interaction, the strengthened passage vortex leads to high endwall shearing at the endwall and blade suction surface junction. The corner vortex is formed similarly to the baseline case as it emanates from the interaction of the passage vortex and blade suction surface. As previously the described, the corner vortex is strengthened relative to the baseline case; likely a consequence of the stronger passage vortex.

To this point, the above CFD visualizations were useful for visualizing the passage flow field. However, to ensure the above interpretations are representative of the true physics the following paragraphs will examine the experimental endwall visualization of the Cavity case. The experimental flat endwall visualizations were not completed and are not discussed further.

Figure 6.11 (c) shows the endwall surface flow visualization for the experimental Cavity case. The visualization was prepared following the techniques described in Section 3.10.5. The limiting streamlines are overlaid as white dashed lines. Shear stress lines are shown as solid white lines with arrows. The experimental visualization is very similar to the corresponding predictions shown in Figure 6.11 (b). Evidence of the cavity recirculation zone is again visible. The reattachment line, $\mathrm{R}_{\mathrm{C}}$, is identified by the region of low residual pigment upstream of the blade leading edge that extends in the pitchwise direction. Abo El Ella et al. (2012) identifies a similar region and indicates this is likely the reattachment line of the separated inlet boundary 
layer. Upstream of the reattachment, the shear levels are reduced as the fluid appears to disperse into the cavity. The low shear levels occur near the base of the endwall cavity in a region of locally high curvature and may indicate an endwall separation. The CFD shows similar low shear regions within the cavity; however, an inspection of the near endwall velocity vectors shows no evidence of a separated boundary layer. Further upstream in Figure 6.11 (c), the $\mathrm{S}_{\mathrm{C}}$ separation is clearly defined. Similar to the CFD, $\mathrm{S}_{\mathrm{C}}$ forms as the cavity recirculation fluid is forced up into the mainstream flow by the platform overlap.

One difference between the experimental and CFD endwall visualizations is the distinct separation, $\mathrm{S}_{1}$, observed upstream of the platform edge in Figure 6.11 (c). It appears that the experimental inlet endwall boundary layer separates prior to the platform termination. The CFD shows no evidence of this separation as the inlet boundary layer is fully attached up to the platform trailing edge. The observed separation $\left(\mathrm{S}_{1}\right)$ does not appear to significantly alter the flow physics relative to the CFD. The abrupt rectangular platform geometry causes the formation of a recirculation zone that is not strongly influenced by the position of $\mathrm{S}_{1}$. However, as will be discussed in Chapter 8, the location of the $S_{1}$ separation becomes important when the shape of the platform overlap is altered to reduce strength of the cavity recirculation.

Within the endwall cavity region of Figure 6.11 (c), the interaction of the suction- and pressure-side legs of the cavity recirculation is slightly different than the CFD. The $\mathrm{S}_{2 \mathrm{P}}$ leg appears to turn downstream more closely to the blade pressure side rather than emanating from the saddle point $A_{1}$. It is possible that the $S_{2 P}$ line actually forms from a second saddle point as suggested by Sieverding (1985), but the visualizations could not confirm this. The $\mathrm{S}_{2 \mathrm{~S}}$ line is positioned more closely to the suction-side of the passage than in the CFD. The opposing pitchwise displacement of $S_{2 S}$ and $S_{2 P}$ creates a large region of low shear stress between the $S_{2 S}$ and $\mathrm{S}_{2 \mathrm{P}}$ within the endwall cavity. A counter-clockwise rotation is indicated here by a solid line. The formation of this region was observed visually during the experimental testing. The counterclockwise motion of the endwall pigment was clearly observed which suggests there is a large 
three-dimensional vortex oriented with its axis perpendicular to the endwall surface positioned within the interaction region.

As mentioned, Abo El Ella et al. (2012) also examined this interaction region. They suggest the possibility of small vortices being shed from the termination of the cavity recirculation at a similar location. Their interpretation gives the impression that these vortices are small structures oriented similarly with the rotation axis perpendicular to the endwall surface. In the present work, the vortex structure within the interaction region appears to be relatively large. Perhaps the unobstructed purge slot of the present Cavity configuration allows the cavity flow to re-circulate upstream into the purge slot thereby facilitating the formation of a larger structure within this interaction region.

The differing observations between the present work and that of Abo El Ella et al. (2012) could also stem from the different flow and geometric conditions. Abo El Ella et al. (2012) performed their tests at transonic conditions with the platform overlap geometry approximated as gradual ramp without a purge slot cavity. In their case, the interaction region would then be restricted to within the endwall cavity and perhaps reduced the size of the interaction. Nevertheless, the present work is still very similar to the results Abo El Ella et al. (2012). The most distinct characteristics of these flows are the large endwall cavity recirculation, the strong interaction between the suction and pressure-side legs, the enhanced strength of the passage and corner vortices and the increase in secondary losses.

\subsubsection{The Effect of Restricting the Bladerow Potential Field with a Simplified Step}

The experimental results presented in Sections 6.5 and 6.7 showed that the Cavity case has a weaker passage vortex than the Step case and therefore has lower measured and mixed-out losses. The Step case showed the highest integrated losses and SKE as well as the highest peak values of streamwise vorticity within the passage vortex. This experimental trend was not borne out in the CFD predictions as the results of all three cases were very similar. The integrated losses were all within $1 \%$. However, as discussed in the previous section the CFD does appear to 
predict the general physics of the secondary flow with reasonable accuracy. Therefore, this section will use the CFD to will visualize the cavity flow and examine a possible explanation for the attenuated losses in the experimental Cavity case.

De la Rosa Blanco et al. (2009) performed measurements downstream of a linear cascade with an upstream cavity and platform overlap geometry with and without net purge flow. In their study they suggest that the presence of the rim-seal cavity, without any net mass flow, can reduce the intensity of the passage vortex. They propose that by allowing the blade potential to push fluid into the cavity purge slot, rather than restricting it as in the Step case, the strength of the horseshoe vortex can be reduced. The benefits are then realized through a reduction in the passage vortex strength and lower secondary losses. As shown previously in this chapter, the downstream losses of the Cavity case are lowest amongst the three cavity geometries, thereby suggesting the mechanisms proposed by de la Rosa Blanco et al. (2009) also apply here. Although the Step case is hypothetical and such geometry would not be present in a real engine, the relative loss reduction of the Cavity case may have identified a potential area of improvement for real turbine design. Thus, the cavity flows of the Step and Cavity cases are further examined here.

Figures 6.12 (a) and (b) show four cut-planes, parallel to the design inlet flow angle, with superimposed streamlines for the Step and Cavity cases, respectively. The streamlines show the size and intensity of the cavity recirculation zone at different pitchwise locations. In Figures 6.12 (a), the cavity recirculation zone is visible in the planes at $y / s=+0.25,0.00$ and -0.25 . The overall size and shape of the recirculation zone shows little variation in the pitchwise direction. At mid-pitch $(y / s=-0.50)$, the cavity recirculation is no longer visible as it has turned downstream and formed the pressure-side leg of the horseshoe vortex. Figure 6.13 (a) shows the static pressure coefficient $\left(C_{P S}\right)$ through the cavity recirculation at a spanwise location of one half the purge slot height, $z / h=-0.048$. The lowest static pressures appear to follow the axis of the 
(a) STEP

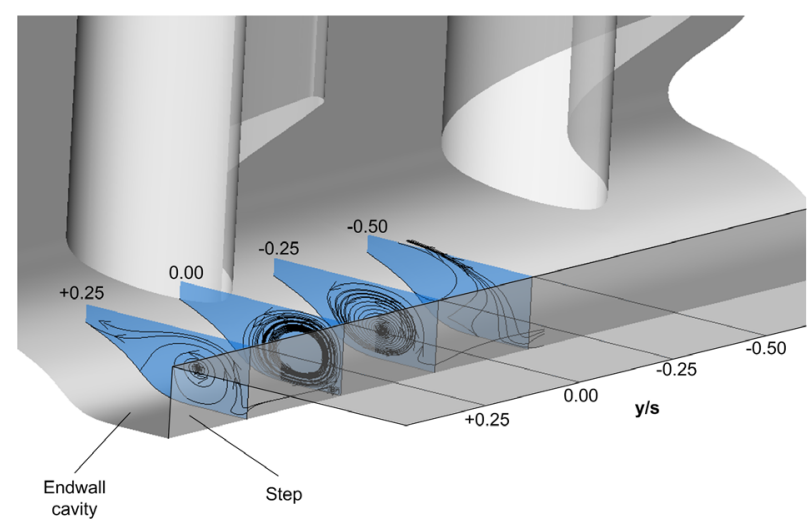

(b) CAVITY

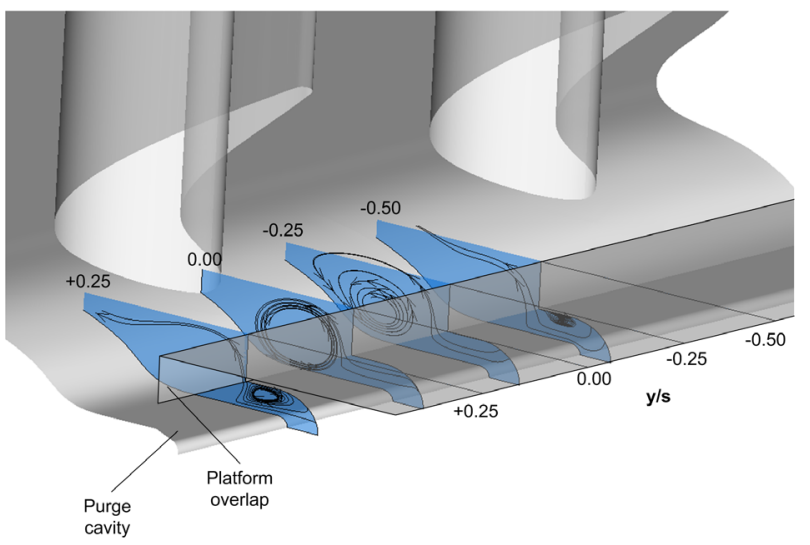

Figure 6.12: Cut-planes oriented with the design inlet flow angle showing the endwall cavity streamlines for the (a) Step and (b) cavity case

(a) STEP

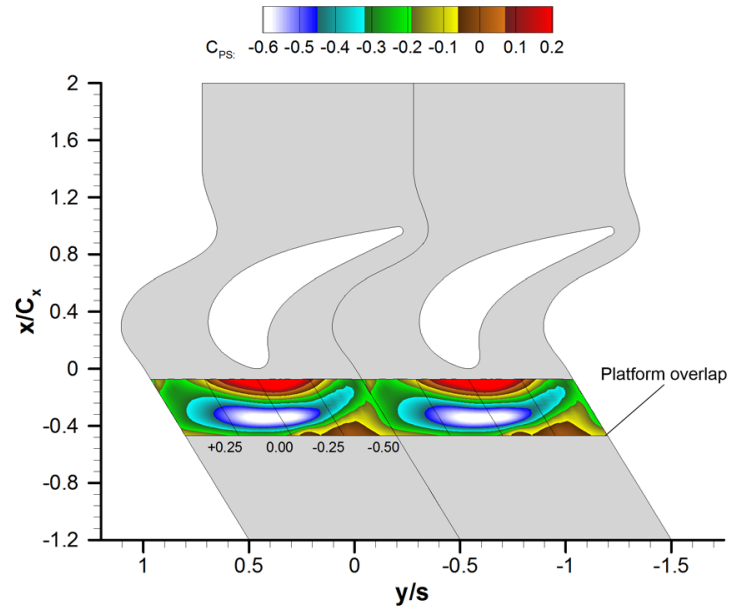

(b) CAVITY

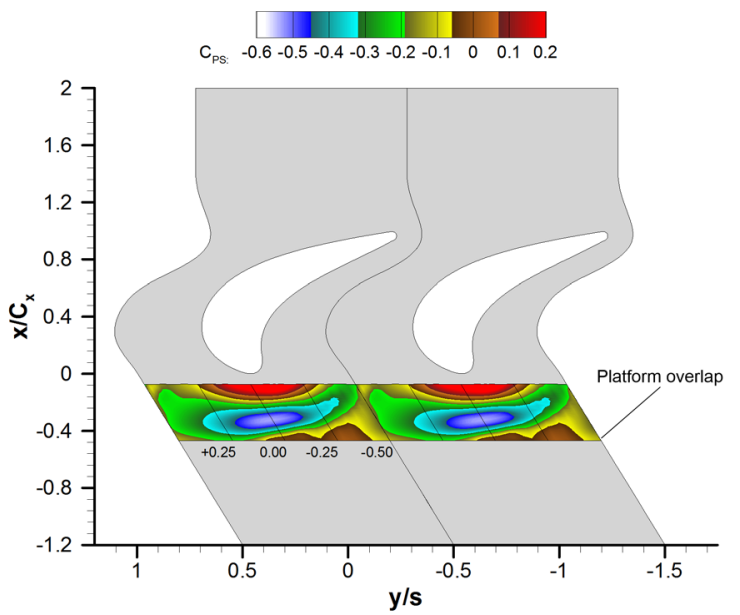

Figure 6.13: Cavity static pressure at $\mathrm{z} / \mathrm{h}=\mathbf{- 0 . 0 4 8}$ (one half the step height) 
cavity recirculation zone. In the pitchwise direction, away from the blade leading edge projection $(y / s=0.00)$, the static pressure increases up to a local maximum at $y / s=-0.50$. Here, the pressure and suction-side legs of the cavity recirculation zone intersect.

The Cavity case shown in Figures 6.12 (b) and 6.13 (b) is different than the Step case. The cavity recirculation zone is reduced in its pitchwise extent and there are other structures visible within the purge slot. In fact, it appears that the cavity recirculation zone, in regions of high static pressure $(y / s=+0.25$ and -0.50$)$ is pushed into the purge slot and in regions of low static pressure $(y / s=-0.25)$ is circulated back into the mainstream. Figure 6.14 shows a colour flood of the axial velocity at the purge slot exit plane. As shown, there is significant ingestion corresponding to the higher pressure region at the $y / s=+0.25$ location. A smaller injection region, designated by positive axial velocities, is positioned within the lower pressure region between the $y / s=0.00$ and $y / s=-0.25$. The ingestion and injection across the purge slot exit affects the development of the secondary flow and reduces the strength of the passage vortex relative to the Step case. This mechanism is described in the following paragraphs.

Both de la Rosa Blanco et al. (2009) and Popovic and Hodson (2013a) describe the effects of the purge slot recirculation on the mainstream flow. In the higher pressure regions, the suction-side of the cavity recirculation is pushed into the purge slot, as observed for the Cavity case. The favourable pressure gradient increases the near endwall velocities, thereby energizing the suction-side leg of the cavity recirculation. Conversely, in the lower pressure regions the injected fluid with positive axial velocities opposes the near-endwall motion of the pressure-side leg. Attenuating the pressure-side leg has the most prominent effect of reducing the strength of the passage vortex. The strengthened suction-side leg caused by the ingestion does not offset the benefits of the injection. As a result, the lower losses are obtained for the Cavity case relative to the Step case.

The lower overall losses obtained in the Cavity case was an unexpected result. Prior to testing, it was thought that the enhanced mixing within the purge slot would result in higher overall losses. As shown here, the recirculation within the cavity slot can have added benefits by 
reducing the strength of the passage vortex. Perhaps this idea may be exploited for secondary loss mitigation. Although not explored in the present thesis, adding periodic contours into the purge cavity could potentially be used to achieve further benefits.

As mentioned, the results of this chapter do not include the effects of purge flow. The following chapter will show that purge flow is important to the secondary flow development. Therefore, the benefits discussed here regarding the cavity shape may not be realizable for engine conditions. However, it is also possible that the purge flow could be directed using the platform shaping to reduce the secondary losses relative to an unshaped purge slot. This present thesis will not examine these ideas further, but were mentioned here as potential future work.

The following chapter (7) will focus on the effects of purge flow for the Cavity geometry. Chapter 8 will then examine the effects of two secondary loss mitigation techniques: intrapassage endwall contouring and an elliptically shaped platform.

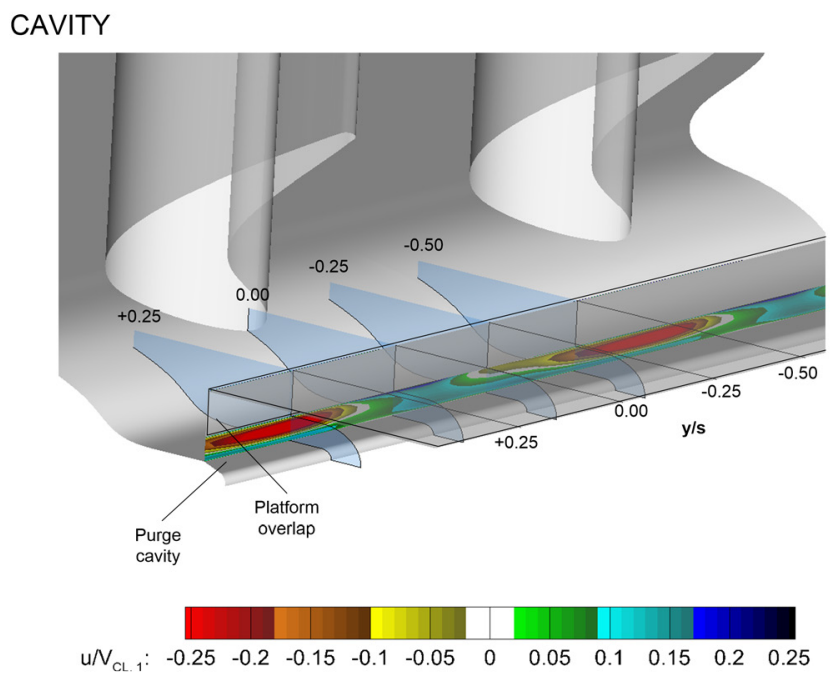

Figure 6.14: Purge slot axial velocity 


\subsection{Concluding Discussion}

The present chapter investigated the effects of upstream platform overlap and endwall cavity geometry on the secondary flow of a low-speed linear turbine cascade. Measurements and computations were performed for a baseline flat endwall case and three rim-seal configurations, namely the Cavity, Step and Ramp. The Cavity case is representative of engine-type hardware (with zero net purge flow), while the Step and Ramp cases have geometric simplifications that can be used to simplify design-oriented computations.

Downstream measurements show that the rim-seal geometry increases the streamwise vorticity and peak losses within the passage vortex region relative to the baseline case. Increased levels of secondary kinetic energy are observed in the near endwall region which are a consequence of more blade-to-blade cross flow and higher values of flow overturning. The counter vortex region appears to be relatively insensitive to the upstream rim-seal as the peak losses and vorticity show only small differences relative to the baseline.

In general, upstream geometry has the local effect of strengthening the passage vortex which results in larger values (relative to the baseline case) of integrated in-plane and mixed-out losses, as much as 13 and $40 \%$ respectively. Differences of this magnitude are significant and therefore the present author recommends including rim-seal representative geometry in the design computations as early as possible.

When the three rim-seal geometries are directly compared, the experimental results show subtle differences. Overall, the Step case produced the highest overall losses while the Cavity and Ramp cases had in-plane losses that were 6 and 4\% lower. The predicted flow fields largely capture the experimentally observed flow physics and were consequently used to visualize the development of the passage vortex within the endwall cavity region. The relatively good agreement between the experiment and predictions suggests that the ramp-type simplification of the rim-seal is appropriate for design-level computations. The Ramp configuration becomes 
particularly attractive for use in applications that require iterative optimizations schemes such as endwall contouring.

As mentioned throughout the chapter, the purge flow will be shown to have a prominent effect on the cascade secondary flows. The benefits of rim-seal simplification shown in this chapter are likely limited to the early design-level computations. It appears that for the most accurate predictions of turbine secondary flows, simulated purge injection should be included. 


\section{Chapter 7}

\section{Secondary Flow of a Turbine Cascade with Simulated Purge Flow}

\subsection{Introduction}

The present chapter investigates the effects of simulated purge flow on the secondary flow of a linear cascade. The purpose is to examine the effects of varying the purge mass flow rate on the downstream secondary flows. As mentioned in Chapter 2, the purge flow of a real engine is required to cool the blade disk and prevent ingress of the hot mainstream gas into the disk cavity. Although the mass flow rate of the purge stream is relatively small compared to the main gas path, the interaction of the two streams occurs upstream of the blade and therefore significantly alters the development of the secondary flow through the blade passage. The measurements presented herein were made with the seven-hole probe downstream of the PAKH cascade with the rectangular platform overlap and flat intra-passage endwalls (RF). The secondary flows are examined in terms of total pressure losses, streamwise vorticity and secondary kinetic energy. The pitchwise and overall integrated quantities are also included. Similar to the previous chapter, CFD is used in combination with surface flow visualizations to interpret the cavity and intrapassage flow field. Also, the prediction capabilities of the commercial solver are evaluated. 


\subsection{Motivation}

As discussed in Chapter 2 the careful design of the rim seal geometry can lead to reductions in the required purge flow (Bohn et al. (1999)). Improvements to the amount of cooling flow directly benefit the engine fuel consumption. McLean et al. (2001) acknowledges the importance of limiting the purge flow rate but also urges designers to consider the effects of the purge flow on the endwall boundary layer, mainstream pressure field and the vortex development. Thus, two important design objectives of the rim-seal are: (1) limiting the required purge mass flow and (2) reducing the interaction with the main gas path to limit additional loss generation.

Chapter 2 shows that several researchers have investigated the effects of different purge flow aspects on the secondary losses. The purge mass flow rate and the relative tangential velocity are two characteristics of the rim-seal that are linked to increased secondary losses. Popovic and Hodson (2013a) investigated both characteristics using linear cascade experiments and corresponding CFD simulations. One key finding was that there was a non-linear relationship between the leakage losses and the purge flow rate. In fact, there were ranges of the purge flow ratio for which the interaction between the purge flow and main gas path were different.

As discussed in Chapter 2 there are many other interpretations of the purge and main stream interaction, some of which are different than that of Popovic and Hodson (2013a). Likely, the varying interpretations are a consequence of the flow complexity and the flow sensitivity to the specific conditions studied. An objective of the present study is to put forth another interpretation of the purge and mainstream interaction, thereby adding to the evolving general interpretation in open literature.

The cascade investigated in the current chapter has a rectangular platform overlap and a flat intra-passage endwall. Chapter 8 will investigate the effects of an elliptical overlap and the 
effects of intra-passage endwall contouring. The results of this chapter serve as the baseline for the loss mitigation technologies discussed in Chapter 8.

Lastly, CFD is applied in this chapter to visualize the flow within the endwall cavity region. Since CFD is also widely used for turbine design the predictions are compared to the experimental results to assess the prediction capabilities of the commercial solver (CFX).

\subsection{Approach}

Experimental measurements from the PAKH cascade were made for the design inlet Reynolds number of 75,000. A single geometric case, designated RF, is considered in the present chapter. The cascade has a rectangular (R) overlap and flat (F) intra-passage endwalls. The purge flow mass flow rate was independently varied for each test case. As described in Section 3.5.4, the purge flow ratio, $\phi$, is the ratio between the purge flow rate and the main gas path mass flow for a single blade passage, expressed as a percentage. Each case is then also designated by 3 digits representing the $\phi$ value of the respective case. For example, RF 075 refers to the rectangular flat endwall with a purge flow level of $0.75 \%$.

Downstream measurements were made using the seven-hole probe described in Section 3.8.5. The CFD results presented in this chapter are largely used to facilitate the interpretation of the intra- passage flow field. The prediction methods followed the procedures described in Chapter 4.

\subsection{Inlet Flow Field and Purge Flow Velocity Triangles}

For the present chapter the inlet conditions of the cascade are the same as described in Section 6.4. The inlet boundary layer for the current chapter was indistinguishable from that shown in Figure 6.2. 
The inlet velocity triangles for the purge flow and mainstream are shown in Figures 7.1 (a) and (b) for low and high purge mass flow rates, respectively. Since the actual purge flow velocities are small relative to the mainstream the purge flow triangles are scaled by $5 \mathrm{X}$ in Figure 7.1. As summarized in Section 2.8.3, Popovic and Hodson (2013a) describe the effects of two characteristics of the purge flow: (1) the leakage fraction which is analogous to the purge flow ratio $(\phi)$ used in the present work and (2) the magnitude of the purge flow tangential velocity. In a real engine the purge flow tangential velocity is dependent on the blade speed, $U$, and the injected purge mass flow rate $\left(\dot{m}_{p}\right)$. In the present work the injection angle of the purge flow $(\gamma)$ is fixed by the turning vanes in the purge injection channel, see Figure 3.4 (a). Thus, an increase to the purge mass flow rate results in a proportional increase in the tangential component of the purge flow velocity, as shown in Figures 7.1 (a) and (b). By contrast, Popovic and Hodson (2013a) were able to independently varying the leakage fraction and the purge flow tangential velocity.

(a) Low Purge Mass Flow Ratio

$(\phi=0.75 \%)$

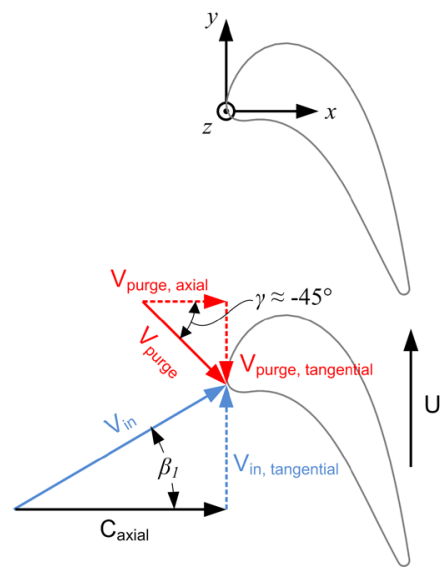

(b) High Purge Mass Flow Ratio

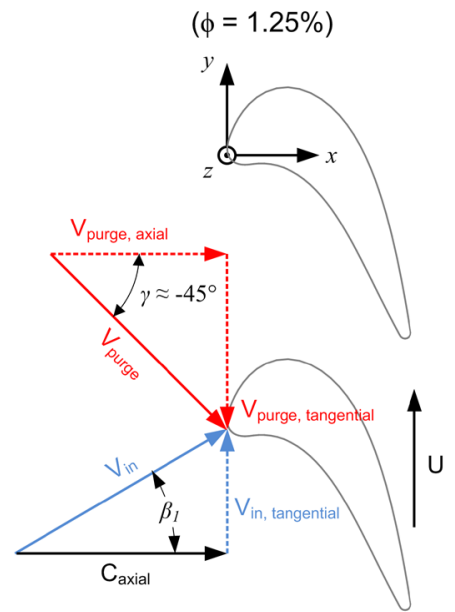

Figure 7.1: Purge flow velocity triangles ( $5 x$ the mainstream scale) with a fixed injection angle for (a) low and (b) high purge mass flow ratios 


\subsection{Blade Loadings with Purge Flow Injection}

The measured blade loadings corresponding to the mid-span $(z / h=0.50)$ and the near endwall location $(z / h=0.05)$ are shown in Figure 7.2 (a) and (b), respectively. At mid-span there is good agreement between the cases with purge flow (RF 075 to 125); however over the suction surface there are slight differences relative to the case with zero net purge flow (RF 000). The slight increase in loading over the suction surface is indicative of higher free stream velocities within the passage. The higher midspan velocities may be caused by flow convergence at midspan. The axial velocity ratio (AVR) parameter is often used to infer the level of flow convergence at midspan. It is defined as:

$$
A V R=\frac{u_{2 C L}^{\prime}}{u_{1, C L}^{\prime}}
$$

where $u_{1, C L}^{\prime}$ and $u_{2, C L}^{\prime}$ are the pitchwise mass-averaged inlet and outlet axial velocities measured at the blade midspan. AVR values greater than one $(A V R>1)$ indicate a converging flow path since the axial velocity increases through the blade passage. Table 7.1 shows the AVR corresponding to each case shown in Figure 7.2. As shown, with increasing purge mass flow the convergence (AVR) increases.

Table 7.1: Influence of purge flow on the measured AVR

\begin{tabular}{|c|c|c|c|c|c|}
\hline \multirow{2}{*}{ AVR } & \multicolumn{5}{|c|}{ Purge mass flow ratio, $\phi \mathbf{( \% )}$} \\
\cline { 2 - 6 } & $\mathbf{0 . 0 0}$ & $\mathbf{0 . 7 5}$ & $\mathbf{0 . 9 0}$ & $\mathbf{1 . 1 0}$ & $\mathbf{1 . 2 5}$ \\
\hline & 0.999 & 1.017 & 1.021 & 1.023 & 1.026 \\
\hline
\end{tabular}

Initially, it was thought that the flow convergence at midspan was an anomaly. One possible explanation was the midspan flow was not entirely two-dimensional. Perhaps, the twodimensional flow that is typically measured at mid-span had shifted due to the asymmetry of the test-section endwalls. CFD was used to test this hypothesis. Simulations were performed with a domain modelled as one half-span with a symmetry condition applied at midspan. The symmetry 
(a) Measured Midspan $(\mathrm{z} / \mathrm{h}=0.5)$

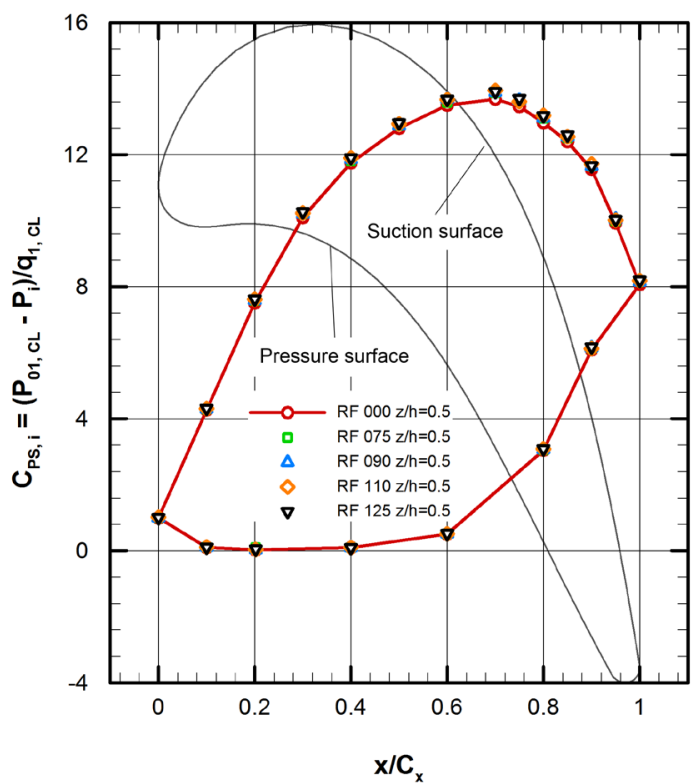

(b) Measured Near Endwall $(\mathrm{z} / \mathrm{h}=0.05)$

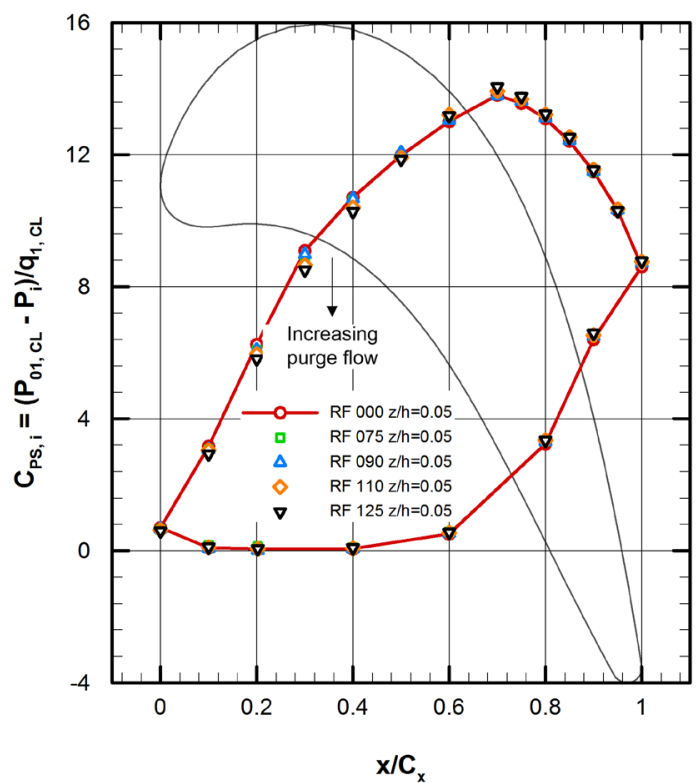

Figure 7.2: Measured blade surface static pressure distribution at $\mathrm{z} / \mathrm{h}=0.5$ and $\mathrm{z} / \mathrm{h}=0.05$ with varying levels of purge flow

(a) CFD

Midspan $(\mathrm{z} / \mathrm{h}=0.5)$

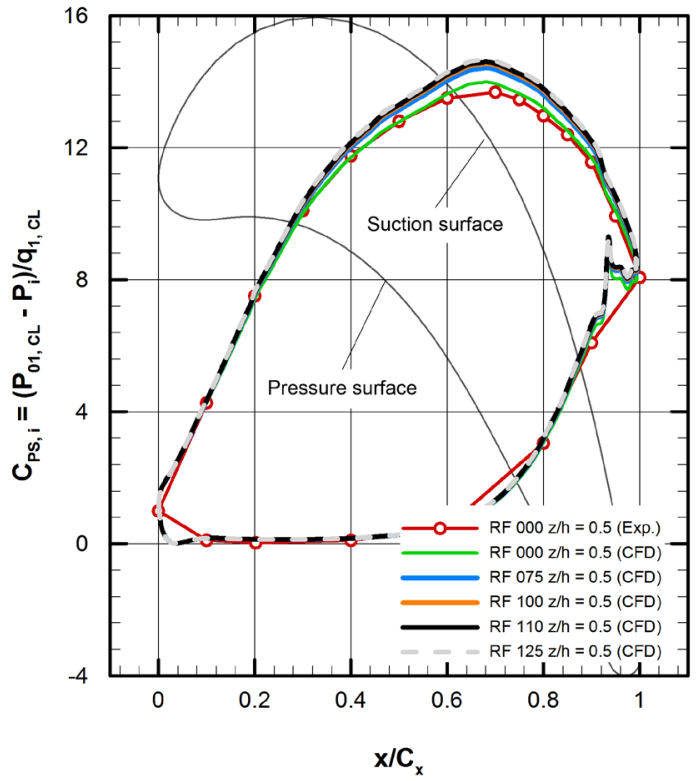

(b) CFD Near Endwall $(z / \mathrm{h}=0.05)$

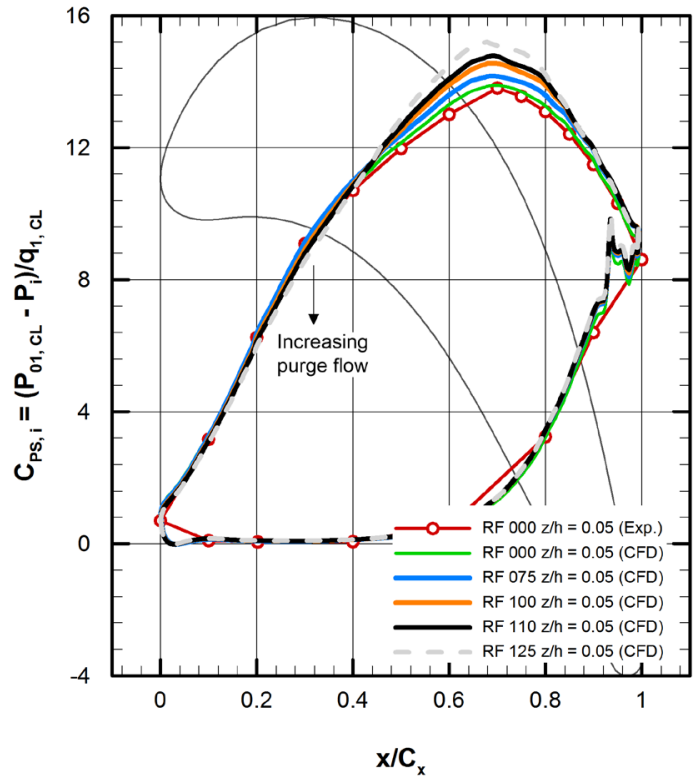

Figure 7.3: Predicted blade surface static pressure distribution at $\mathrm{z} / \mathrm{h}=0.5$ and $\mathrm{z} / \mathrm{h}=0.05$ with varying levels of purge flow 
condition would ensure that the endwall flows were identical and the midspan flow was truly two-dimensional. Therefore, if the predicted midspan loadings were found to be similar to the experiment then the apparent convergence was not caused by the asymmetric endewall geometry.

The CFD loadings are shown in Figures 7.3 (a) and (b). For the midspan results of Figure 7.3 (a) the suction surface loadings demonstrate a similar increasing pattern to the experiment with increasing purge mass flow. The predicted AVR values are shown in Table 7.2 and also increase with increasing purge flow. Figure 7.4 compares the measured and predicted AVR values as a function of the purge mass flow ratio. The magnitudes of the experimental and predicted AVR values are different for all levels of positive purge flow. However, with increasing purge flow the AVR increases monotonically for both the experiments and predictions. This agreement indicates that the experimentally observed convergence is in fact true and is not a result of the endwall asymmetry.

To the present author's knowledge there are no other studies in the open literature that have documented the effect of purge flow on the midspan loading. Paniagua et al. (2004), however, shows rotor blade static pressures from a HP turbine stage at $15 \%$ span for three different purge flow rates (i.e. one suction and two injection rates). Their results show that increasing the purge flow ratio decreases the blade loading, an opposite trend to midspan results shown here. They explain the increase in blade static pressures with increasing purge flow as being caused by a reduced exit Mach number. In their case, the decrease in loading appears near the blade leading edge where incidence effects are most prominent. In the present results, incidence is not likely the cause for the observed increase in loading since the loading distribution is altered in the vicinity of the suction peak and not the blade leading edge.

Table 7.2: Influence of purge flow on the predicted AVR

\begin{tabular}{|c|c|c|c|c|c|}
\hline \multirow{2}{*}{} & \multicolumn{5}{|c|}{ Purge mass flow ratio, $\phi(\%)$} \\
\cline { 2 - 6 } & $\mathbf{0 . 0 0}$ & $\mathbf{0 . 7 5}$ & $\mathbf{1 . 0 0}$ & $\mathbf{1 . 1 0}$ & $\mathbf{1 . 2 5}$ \\
\hline AVR & 1.000 & 1.021 & 1.029 & 1.032 & 1.038 \\
\hline
\end{tabular}




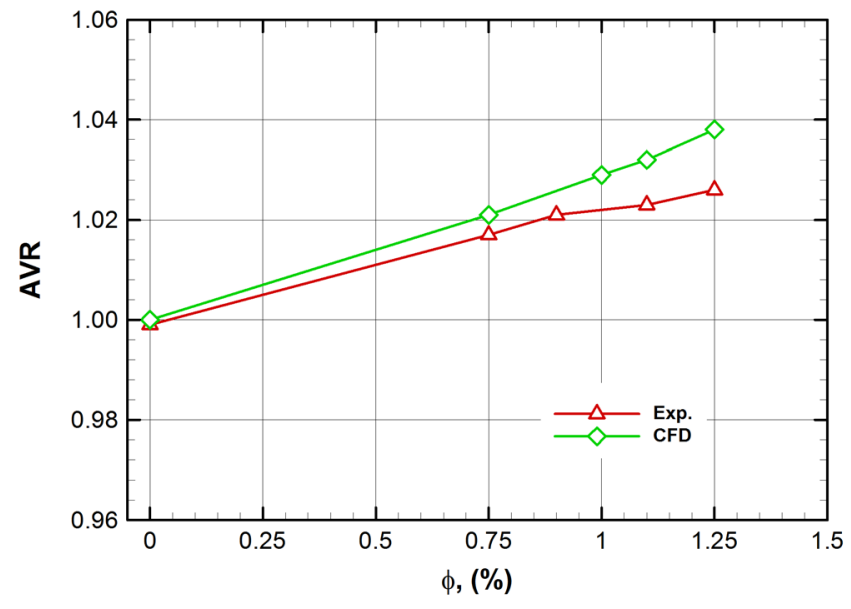

Figure 7.4: Measured and predicted axial velocity ratio (AVR) with increasing purge mass flow ratio $(\phi)$

The loading results of Paniagua et al. (2004) are likely more comparable to the near endwall $(z / h=0.05)$ measurements and predictions presented shown here in Figures $7.2(\mathrm{~b})$ and 7.3 (b), respectively. In the present experimental results, the airfoil appears to unload over the forward part of the blade suction surface with increasing purge flow, as indicated. Since the loadings here are influenced by the three-dimensionality of secondary flow, the apparent unloading is likely caused by incidence resulting from the secondary velocities. Further downstream of the suction peak $\left(x / C_{x}=0.5\right)$ the loadings increase slightly with increasing purge flow.

The corresponding CFD results are shown in Figure 7.3 (b) and show notable differences compared with the experimental results. For example, the reduced loading over the forward part of the suction surface is less evident in the CFD than the experiment. Also, over the suction peak the increase in loading with increasing purge flow is significantly larger than in the experiment. Since the loadings can only be compared at a single near-endwall location the differences shown here are likely caused by differences in the secondary flows of the experiment and computations. As observed in Chapters 4 and 5, the CFD predicts the secondary flow physics reasonably well; however, the size and position of the structures are not necessarily coincident with the experiment 
throughout the passage. Thus, at a single spanwise location it is quite possible for the loadings to show significant differences.

In general, the CFD predictions appear to capture similar trends within increasing purge flow, albeit with different loading levels than the experiment. Section 7.7 will further examine the computational results. It will be shown that for cases with purge flow injection the CFD predictions are consistent with the experimental trends and are therefore still useful for the interpretation of the intra-passage flow physics.

\subsection{Downstream Experimental Results}

\subsubsection{Introduction}

The following sub-sections investigate the effects of purge flow on the cascade secondary flows. Experimentally measured downstream flow fields are shown at the $1.40 C_{x}$ plane for cases with the rectangular platform overlap and the flat intra-passage endwall. Flow field measurements were made using the seven-hole pressure probe described in Section 3.8.5. Prior to making downstream measurements on the centre passage, the periodicity of the secondary flow over multiple passages was measured. These results are presented first in the following subsection. The subsequent sub-sections then discuss the effects of purge flow on the downstream flow field using distributions of the total pressure, streamwise vorticity, secondary kinetic energy and secondary velocity vectors.

\subsubsection{Periodicity of the Secondary Flow with Purge Flow Injection}

In Section 3.4, it was mentioned that the purge flow uniformity was monitored using static taps within the endwall cavity. Acceptable uniformity was observed within the endwall cavity over the range of operating points (see Figure 3.6 in Section 3.4); however, the periodicity of the downstream secondary flow was unknown. More specifically, it was unknown whether the purge 
injection was evenly distributed through the passages, thereby creating near equal secondary flows for the centre and adjacent passages.

Measurements were made over three blade passages using the seven-hole probe positioned at the downstream measurement plane $\left(1.40 C_{x}\right)$. Figures 7.5 (a) and (b) show two traverses through the secondary flow from different spanwise locations, $z / h=0.12$ and $z / h=0.20$. The losses differ by a maximum of $6 \%$ between peaks for both spanwise positions. The pitchwise flow angle shows a slight gradient equal to about $+2^{\circ}$ and $-1^{\circ}$ per pitch spacing as shown in Figure 7.5 (a) and (b) respectively.

Full planar measurements were also made for two adjacent passages, as shown in Figure 7.6. The data shown was not collected in a single run. Measurements were first made over the centre passage and several months later data was collected over the adjacent passage. In fact, the data for the lower passage was actually collected in an effort to trouble-shoot the jagged appearance of the contours. As will be discussed later in the present Chapter, the "rough" data were attributed to unsteadiness arising at purge flow ratios with values near 1. As shown, the second passage agrees reasonably well with the centre passage. There are minor differences in the size and position of loss core associate with the passage vortex region. The difference between the overall integrated losses is about $7 \%$, which can largely be attributed to higher losses associated with the corner vortex of the lower passage.

The higher losses associated with the passage on the right-hand side are thought to be caused by the finite length of the purge slot. The purge slot terminates upstream of the blade row between the bottom two blades, as shown in Figure 7.7. As a result, the incoming flow of the fifth blade (as labelled) would likely be disrupted by the end-effects of the purge slot. It is therefore not a surprise that the corner vortex generated between the endwall and suction surface of the fifth blade is considerably stronger than that of the centre passage.

Overall the periodicity of the secondary flow is satisfactory. It is recommended for future work that the cascade have more blades to improve the periodicity of the centre passages. 
(a) Outlet Flow $\left(1.40 C_{x}, z / h=0.12\right)$
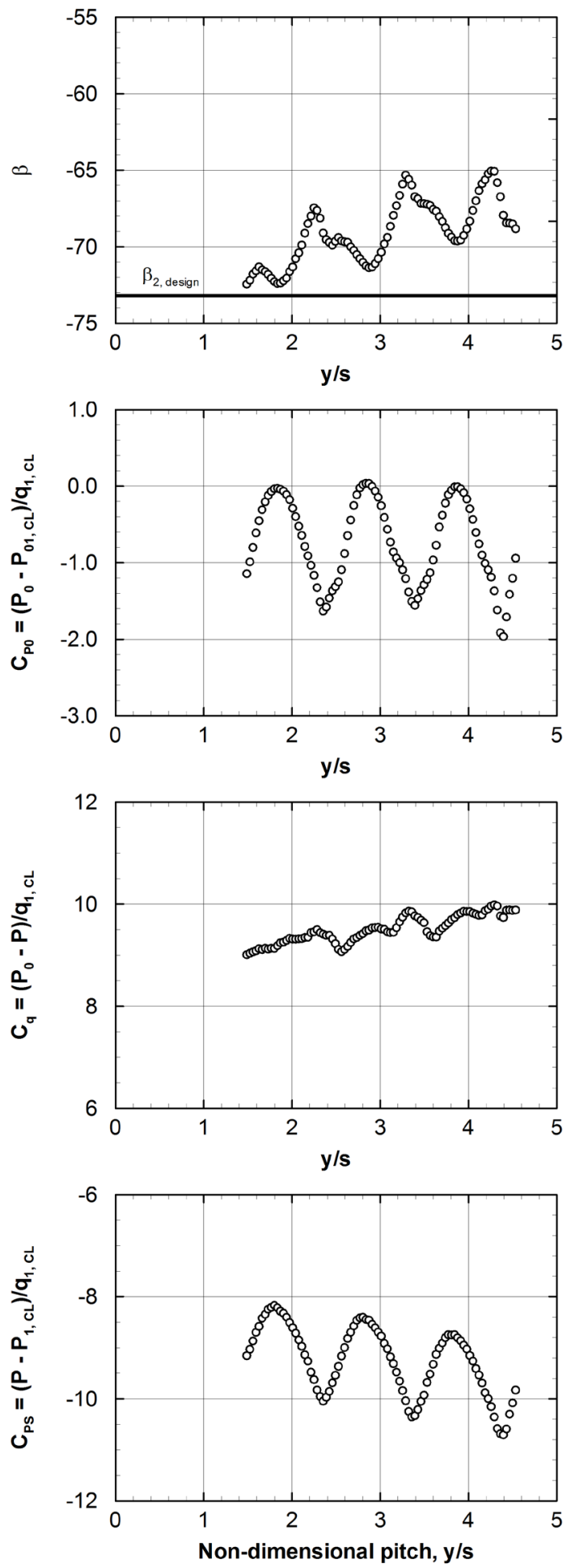

(b) Outlet Flow $\left(1.40 \mathrm{C}_{\mathrm{x}}, \mathrm{z} / \mathrm{h}=0.20\right)$
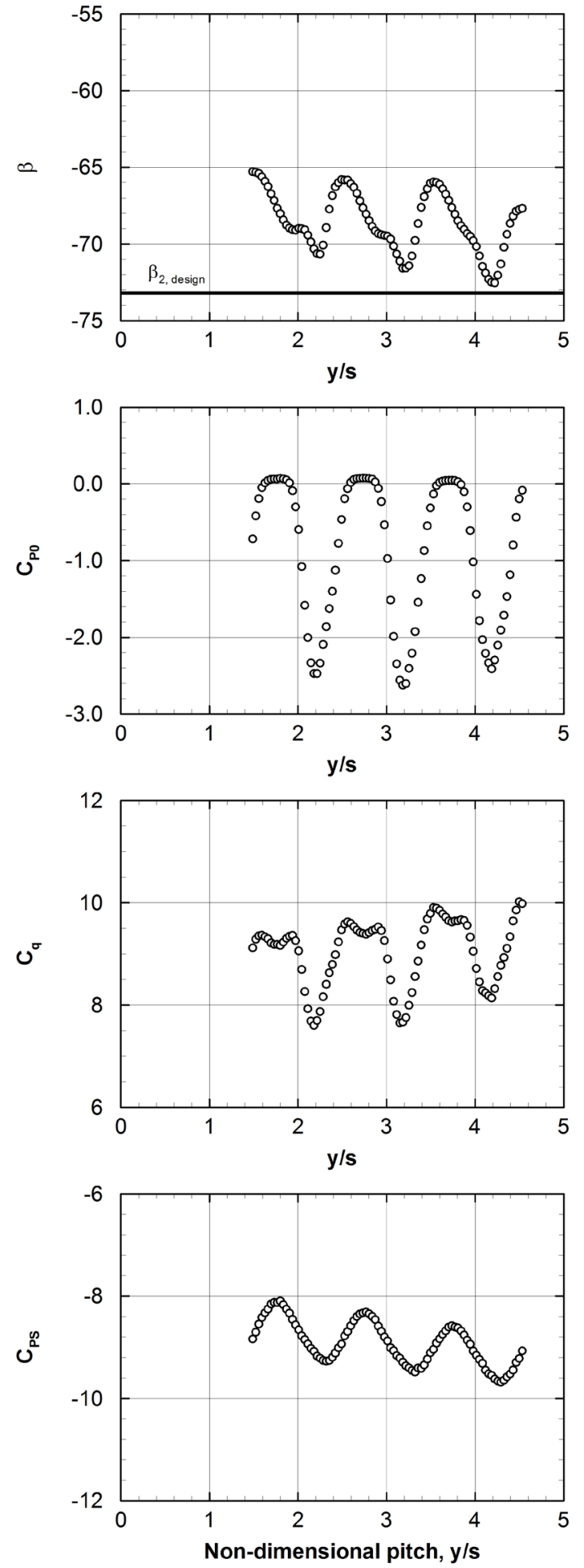

Figure 7.5: Secondary flow periodicity measurements at different spanwise locations for $\phi=0.90$ 


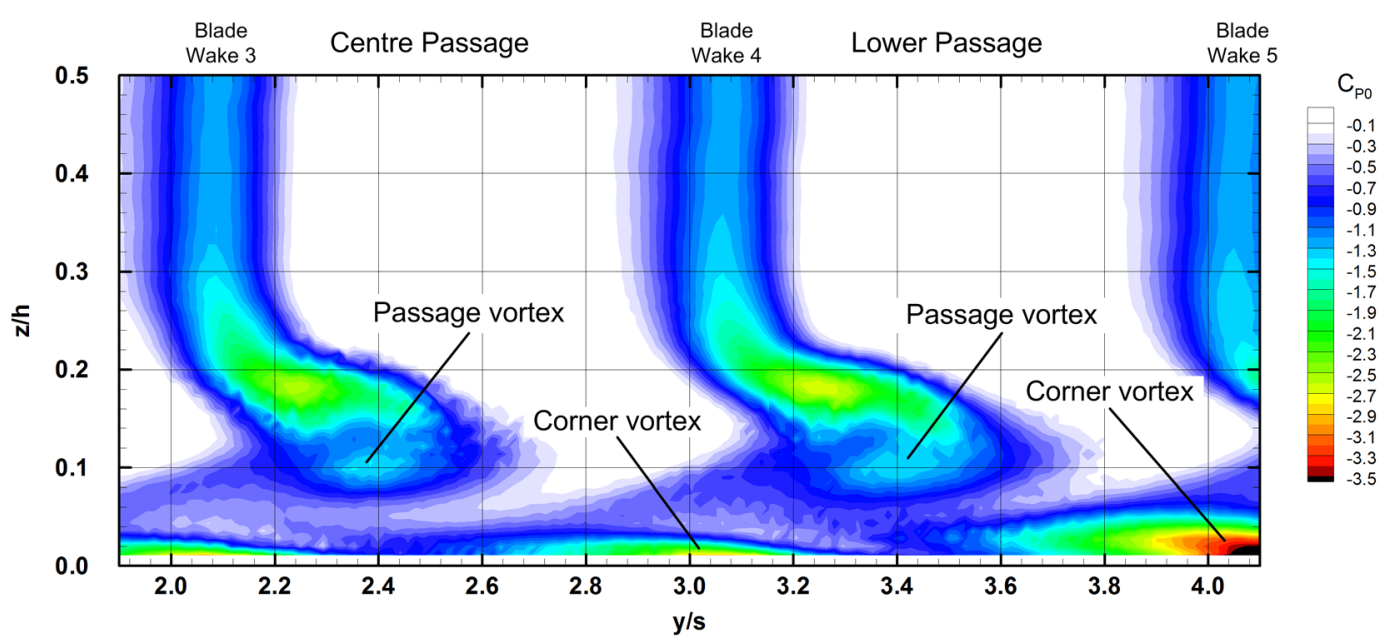

Figure 7.6: Total pressure coefficient measured over two adjacent passages for $\phi=0.9$ at $1.40 C_{x}$

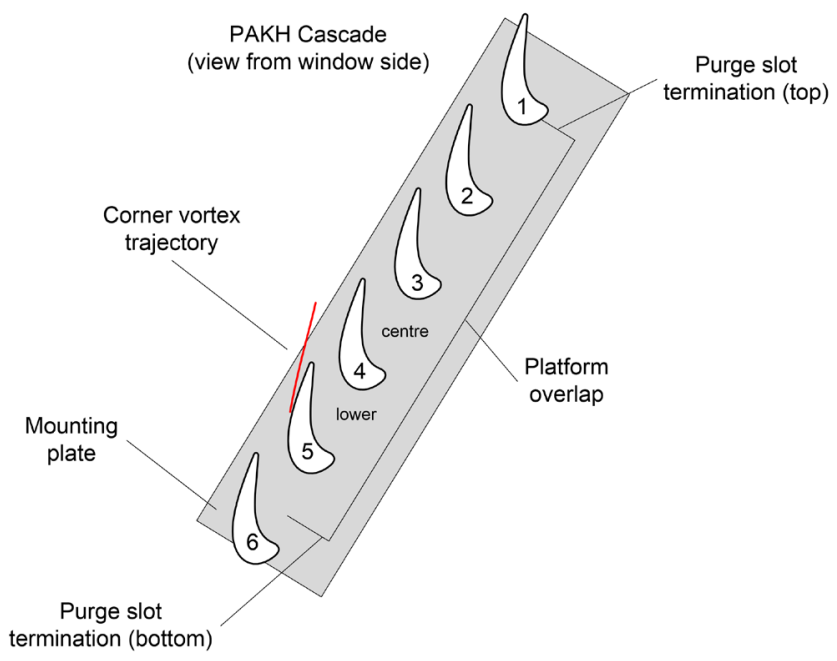

Figure 7.7: Schematic of the cascade numbering and purge slot termination points

\subsubsection{The Effects of Purge Flow on the Downstream Flow field}

The following section presents the experimentally measured downstream flow field. Planar distributions of the total pressure, streamwise vorticity and secondary kinetic energy are shown for the $1.40 C_{x}$ plane in Figures 7.8 through 7.11. Corresponding measurements were also 
made for the $1.20 C_{x}$ plane, but for brevity are only shown in Appendix F. Pitchwise and the overall integrated flow quantities are discussed in Section 7.8.

Figure 7.8 (b) through (f) show colour flood contour plots of the total pressure coefficient for increasing levels of purge flow measured at the $1.40 C_{x}$ plane. For reference, the baseline flat endwall case is shown in sub-figure (a). Sub-figure (b) corresponds to the zero net purge flow case (RF 000) discussed in Chapter 6.

For cases RF 075 through RF 110 shown in sub-figures (c) through (e) the pressure gradients appear discontinuous which is somewhat different from the smooth contours generated in the other cases. It was speculated that the discontinuous data were caused by unsteadiness, or resonance, developing within the purge flow system at certain purge flow operating points. The purge flow ratio of about $\phi=1.0 \%$ was avoided for this reason. These effects were also observed at different measurement planes for the same operating conditions as shown in Appendix $\mathrm{F}$ for the $1.20 C_{x}$ plane. A number of troubleshooting efforts were attempted to smooth the data but none were successful. Some examples of the troubleshooting include: repeating the measurements, stiffening the pressure probe support, installing baffles in the purge flow plenum, altering the pressure sampling rates and examining the frequency spectra of the measured signals. With reference to the latter, there were no obvious frequencies that could be interpreted in the pressure measurements. To the author's knowledge similar effects have not been published in other studies. In particular, Lynch et al. (2013) performed aerodynamic and heat transfer measurements on a larger scale version of the present cascade, albeit at lower purge flow levels and in a different wind-tunnel facility. They did not observe these effects.

In Chapter 8 , measurements of the same bladerow with an elliptically shaped platform overlap geometry are presented. For the same range of purge flows investigated the scattered data are not observed. It is quite possible that altering the overlap geometry from the rectangular shape shown here changes the resonance frequency of the purge flow system. Thus, for the current flow conditions the unsteadiness is attenuated. With the current set of data it is not 
(a)

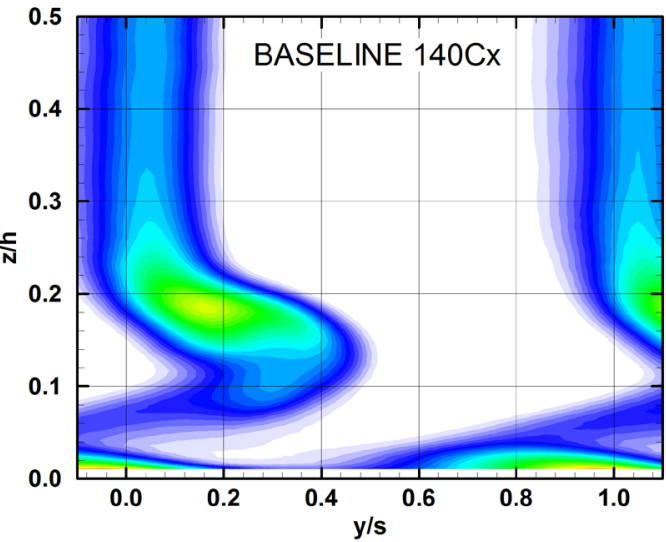

(c)

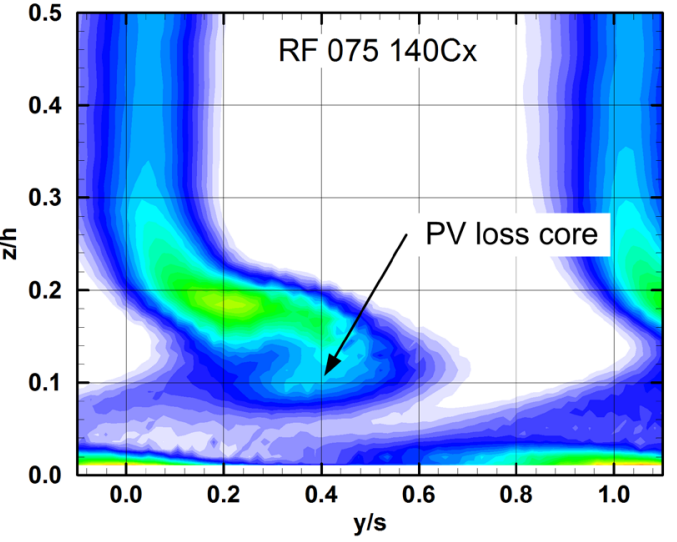

(e)

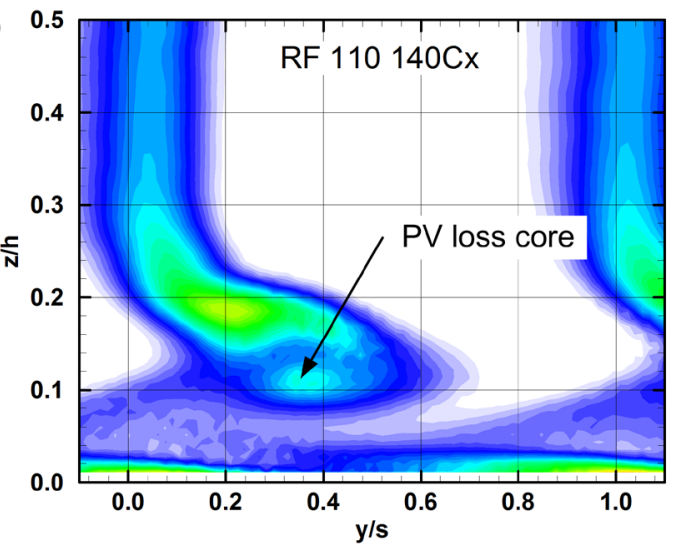

(b)
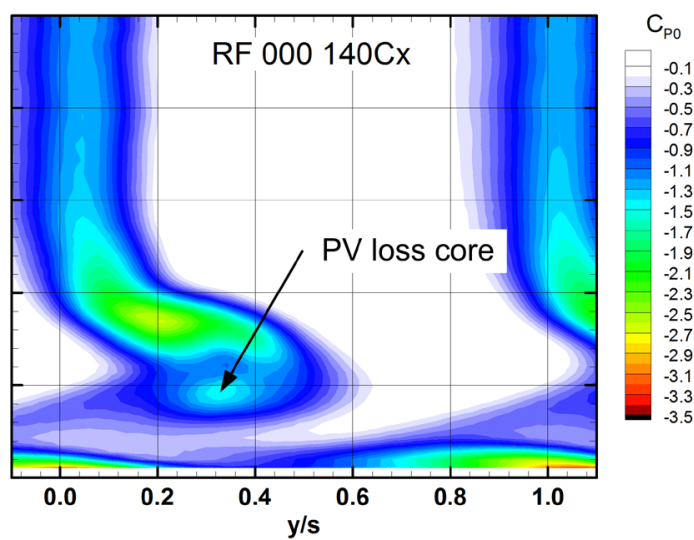

(d)

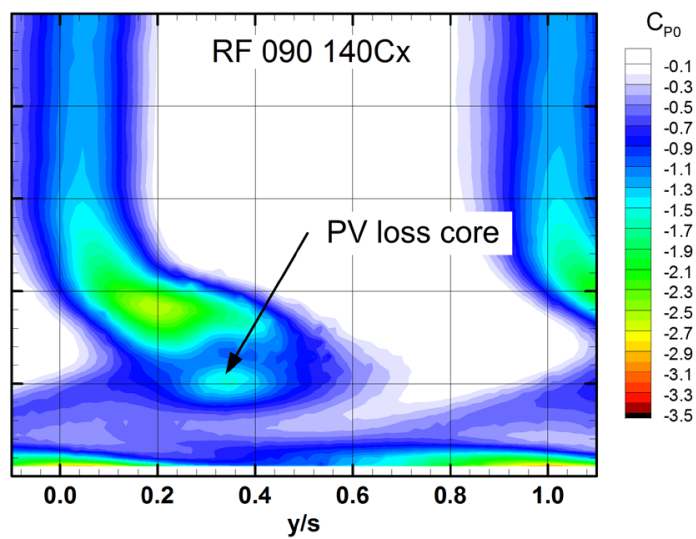

(f)

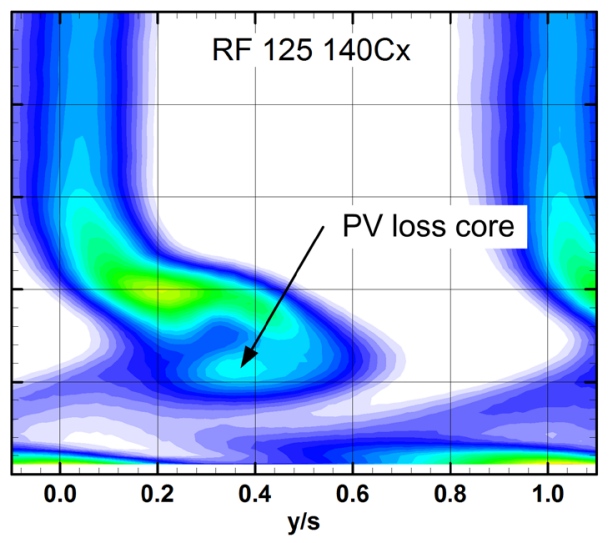

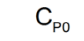

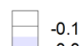

Figure 7.8: Floods of the total pressure coefficient $\left(C_{P 0}\right)$ for varying levels of purge flow measured at $1.40 C_{x}$ 
(a)

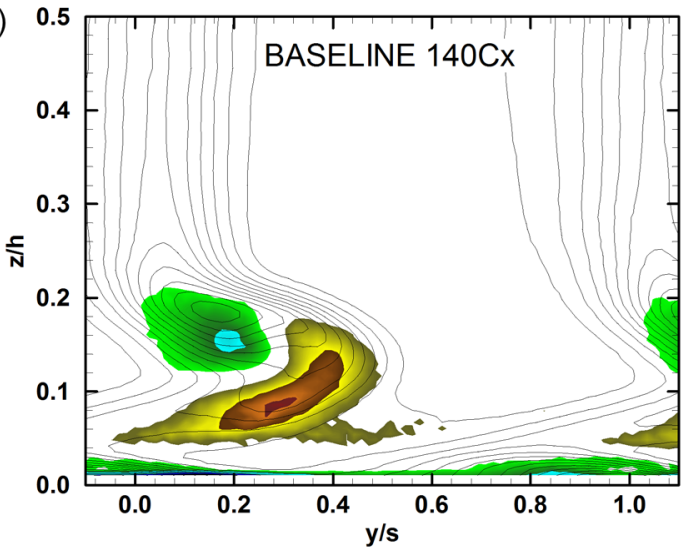

(c)

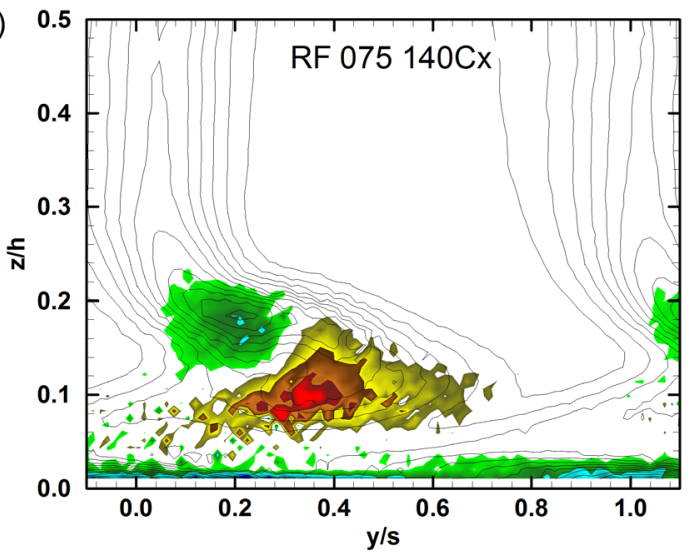

(e)

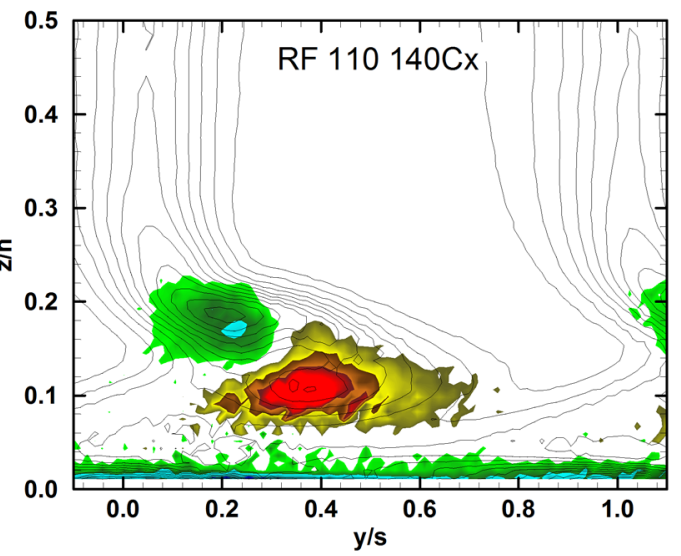

(b)

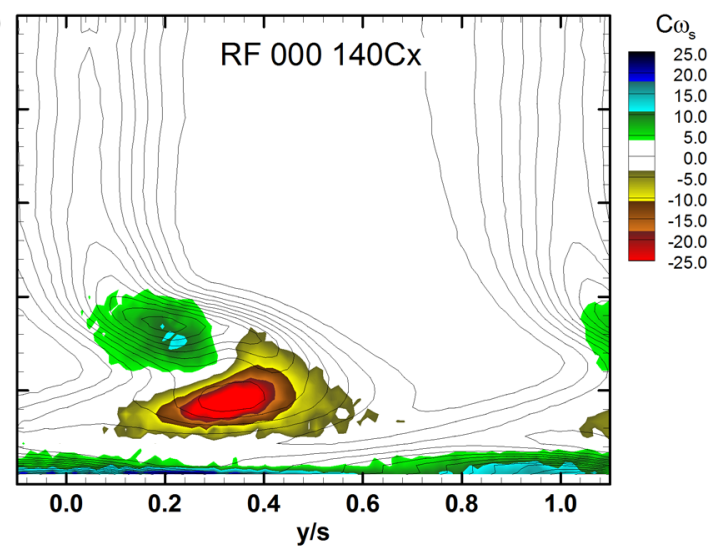

(d)

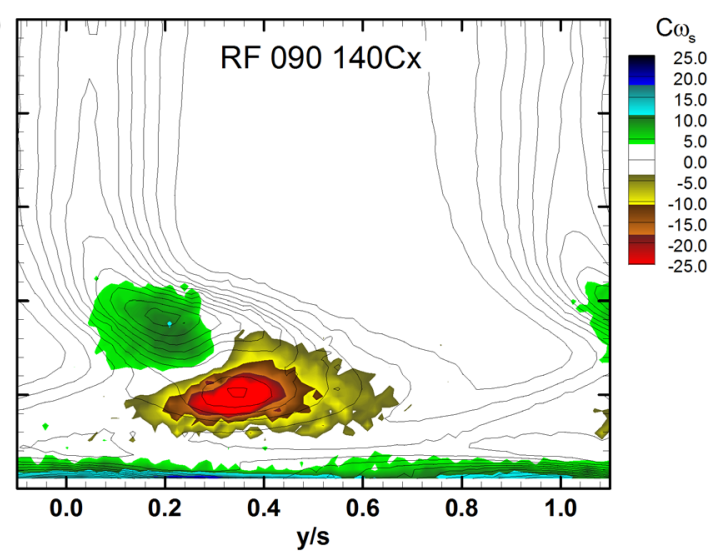

(f)

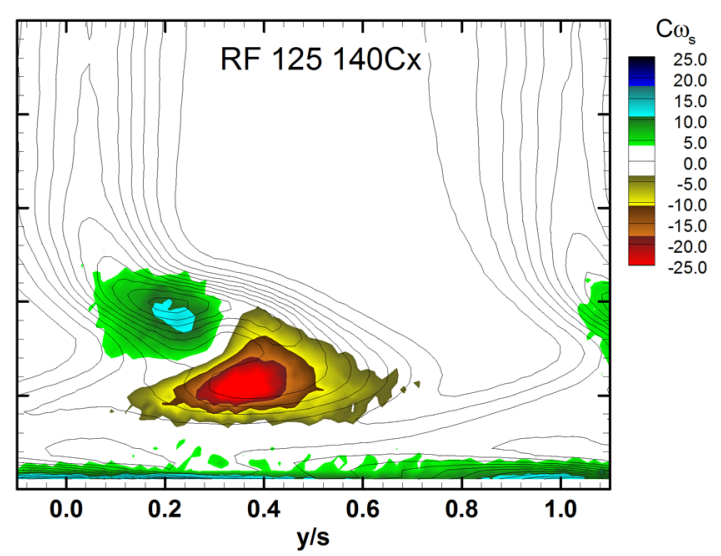

Figure 7.9: Floods of the streamwise vorticity coefficient $\left(C_{\omega S}\right)$ with contour lines of the total pressure coefficient $\left(C_{P 0}\right)$ for varying levels of purge flow measured at $1.40 C_{x}$ 
possible to distinguish if the scattered data are caused by unsteadiness related to these flows in general or if these results are specific to the current test-section setup. Nevertheless, the flow structures are still distinguishable and useful for the interpretation of these flows.

The total pressure and streamwise vorticity coefficients shown in Figures 7.8 (b) and 7.9 (b) show the effects of the stepped geometry on the downstream secondary flow, as previously discussed in Chapter 6 . When compared to the Baseline case, the stepped case (RF 000) has a distinct loss core associated with the location of the passage vortex, as labelled. Also, the passage vortex is clearly larger and has higher local losses than the Baseline case. For the cases with positive net purge flow, Figures 7.8 and 7.9 (c) through (f), the addition of purge flow alters the location of the passage vortex, the overall size of the secondary flow, the peak loss and streamwise vorticity within the passage vortex. More quantitatively, Table 7.3 and Figure 7.10 summarize these flow quantities for each purge flow level.

Table 7.3: Summary of peak losses and streamwise vorticity within the passage vortex (PV), the passage vortex location and the relative in-plane area of the inviscid core

\begin{tabular}{|l|c|c|c|c|c|}
\hline & RF 000 & RF 075 & RF 090 & RF 110 & RF 125 \\
\hline Peak $C_{P 0}$ in PV core & -1.47 & -1.30 & -1.52 & -1.52 & -1.48 \\
\hline Peak $C_{\omega s}$ in PV core & -39 & -32 & -36 & -39 & -32 \\
\hline PV pitchwise location, y/s & 0.35 & 0.37 & 0.37 & 0.39 & 0.39 \\
\hline PV spanwise location, z/h & 0.088 & 0.099 & 0.099 & 0.103 & 0.110 \\
\hline Inviscid core \% area of pitch & $39.7 \%$ & $35.6 \%$ & $32.5 \%$ & $32.8 \%$ & $32.5 \%$ \\
\hline
\end{tabular}

Figure 7.10 (a) shows the position of the passage vortex centre relative to that of the zero net purge flow case (RF 000). With increasing flow rate the passage vortex migrates away from the endwall (increasing span) and towards the centre of the passage (increasing pitch). The pitchwise migration of the passage vortex is nearly linear with increasing purge flow as shown by the best-fit line. The spanwise migration is perhaps non-linear, particularly at the highest flow rate of $\phi=1.25 \%$. A second order polynomial or exponential appears to be a better fit.

Figure 7.10 (b) shows that with increasing purge flow the size of the secondary flow increases. Here, the size of the secondary flow was inferred by integrating the area of the inviscid 
core: the white regions of Figure 7.8. By comparing the size of the streamwise vorticity regions associated with the passage vortices shown in Figure 7.9, it appears that the increasing size of secondary flow is largely a result of a larger passage vortex.

Figure 7.10 (c) and (d) show the peak losses and streamwise vorticity within the passage vortex, respectively. The values of the RF 075 case were omitted as possible outliers. For the remaining cases, the peak losses show small differences relative to the zero flow case. However, there is a clear trend of increasing peak vorticity within the PV. It appears the addition of purge flow tends to increase the passage vortex rotation about the streamwise axis which in turn leads to

(a)

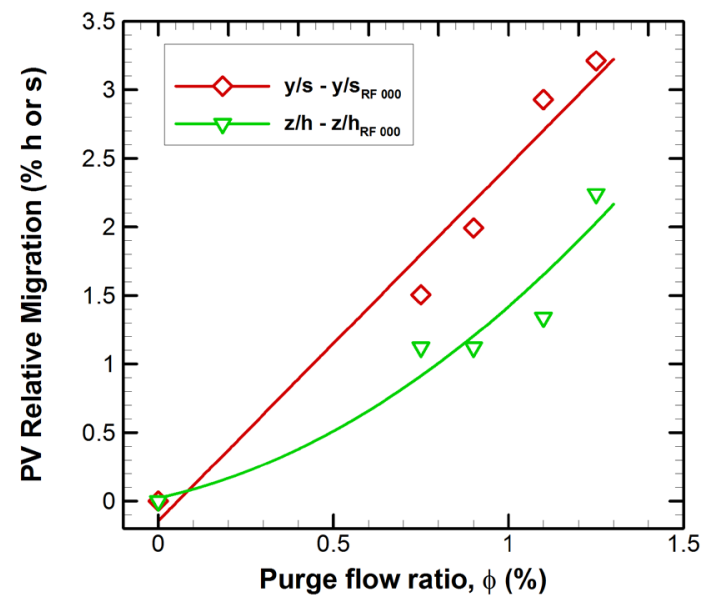

(c)

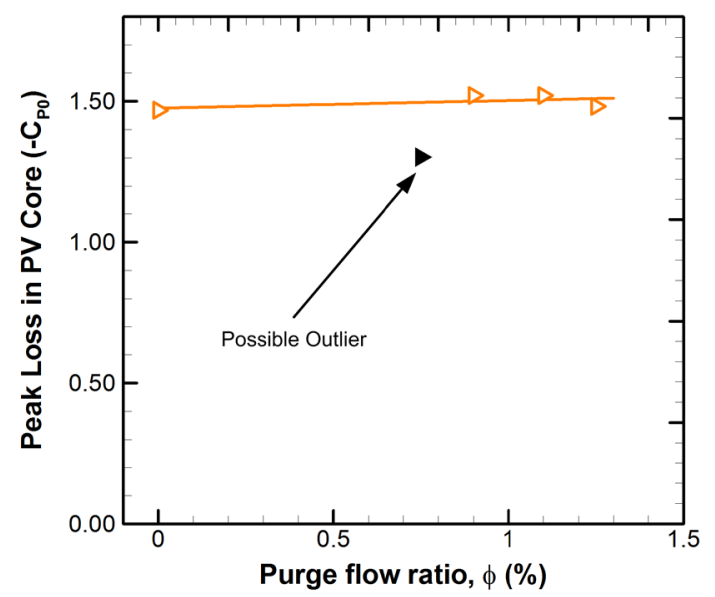

(b)

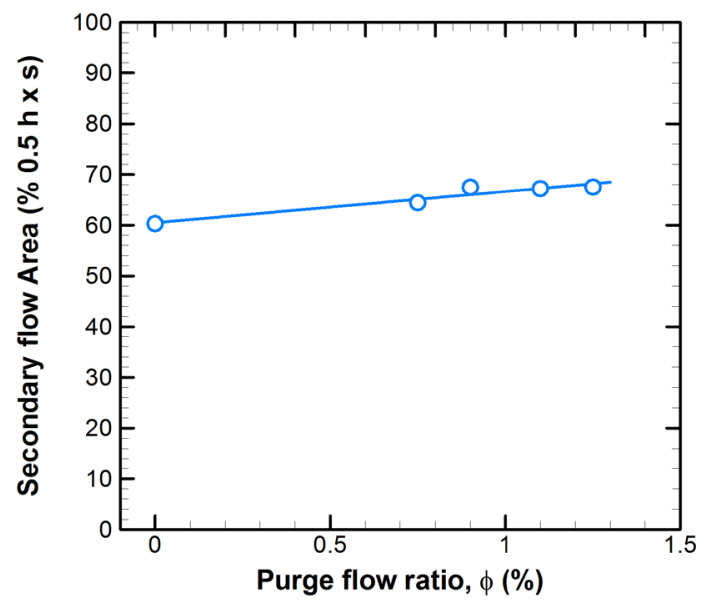

(d)

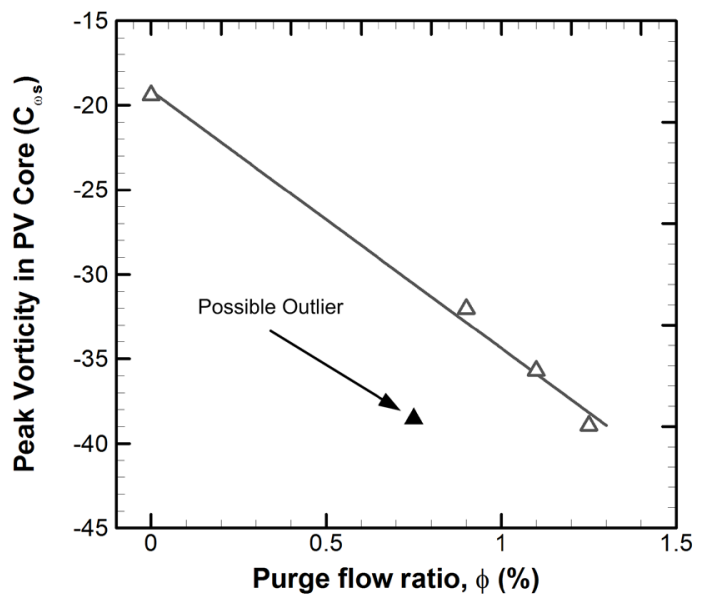

Figure 7.10: Measured passage vortex flow quantities with increasing flow purge flow 
higher mixing levels with the inviscid fluid. The mixing causes the high loss fluid to diffuse into lower loss regions causing the observed increase in size of secondary flow. As will be shown in the following sub-section the overall integrated losses also increase with additional purge flow.

Figure 7.11 shows the corresponding floods of the secondary kinetic energy coefficient with the secondary velocity vectors overlaid for each of the cases. In Figures 7.11 (c) through (f), the addition of purge flow appears to enhance the interaction between the passage and counter vortices. The region labelled the "vortex interaction zone" is local to high secondary kinetic energy as a result of the high secondary velocities. Here, the passage and counter vortices funnel fluid away from the endwall and towards the centre of the passage. As discussed in Chapter 5 the shearing between the vortices leads to high rates of loss production. As shown, the secondary kinetic energy and secondary velocities in the vortex interaction zone increase with increasing purge flow. As will be shown, the higher levels of secondary kinetic energy contribute to the higher mixed-out losses observed for the high purge flow cases.

In Figures 7.11 (c) through (f), there is another peak of secondary kinetic energy located near the endwall within the vicinity of the passage vortex, labelled the secondary lobe. The size and magnitude of this lobe appears to slightly increase with the increasing purge flow level and reaches a maximum for the RF 110 case. At the highest purge level (RF 125) the lobe is reduced in size and magnitude. The RF 075 case appears to be an outlier with significantly lower levels of SKE over the entire distribution; a likely consequence of the scattered data observed for this purge level.

A second observation shown in Figures 7.11 is the spanwise shift of the near endwall lobe with increasing purge flow. This migration of the SKE is consistent with the spanwise shift of the passage vortex observed in Figure 7.9 and shown quantitatively in Figure 7.10 (a). The spanwise displacement is most significant for the RF 125 case. Figure 7.11 (f) also shows a region of very low SKE (white) below the displaced passage vortex, as labelled. Following the work of Popovic and Hodson (2013a), it is likely the fluid here was energized by the purge injection. Section 2.8.3 
(a)

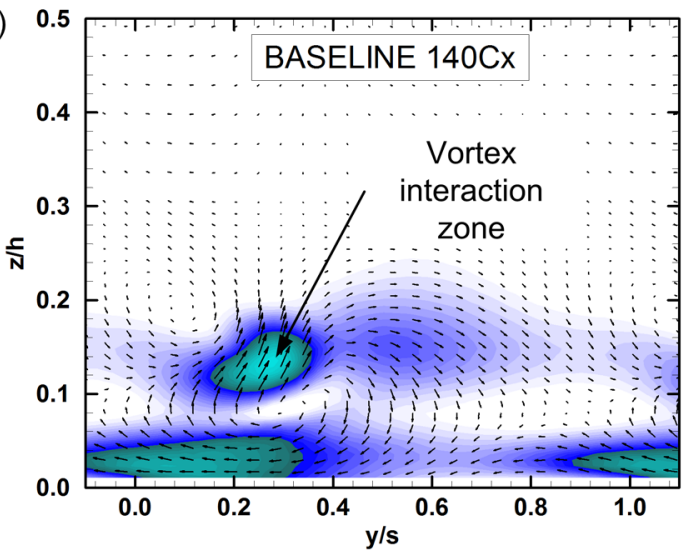

(c)

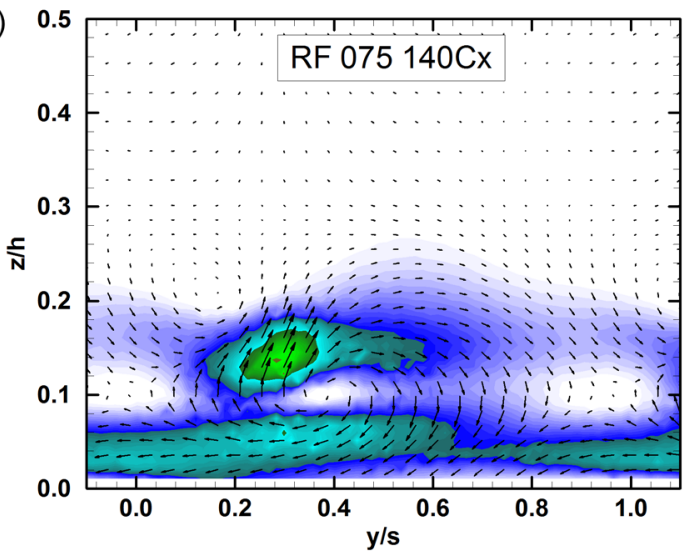

(e)

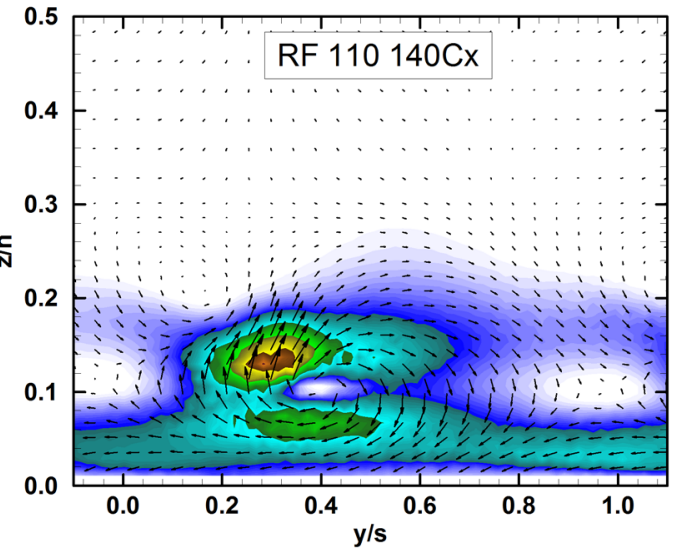

(b)
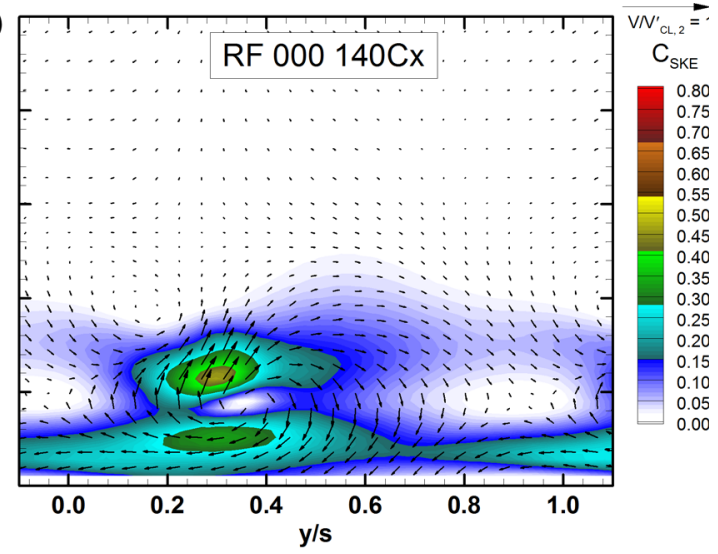

(d)

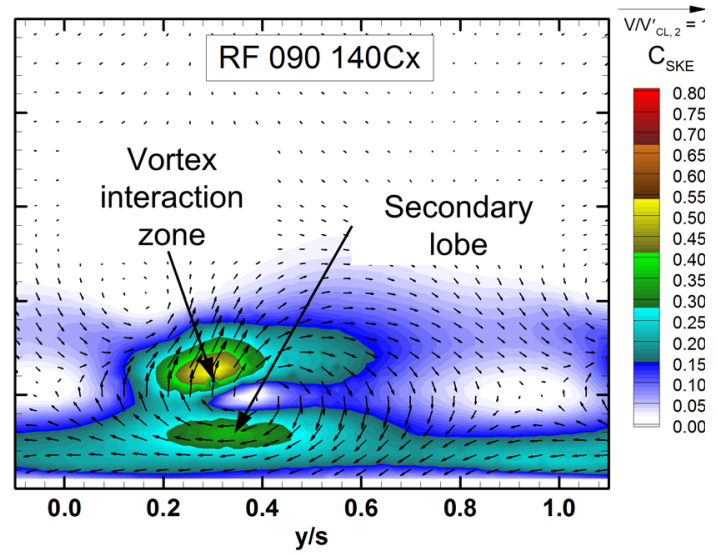

(f)

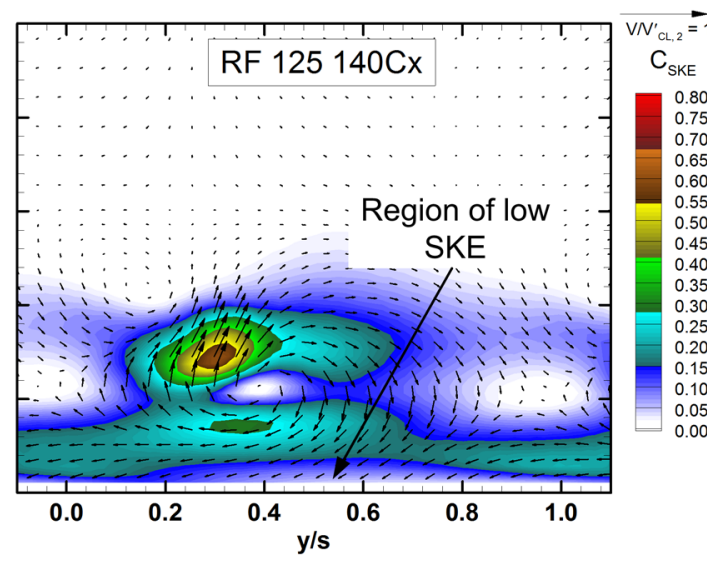

Figure 7.11: Floods of the secondary kinetic energy coefficient $\left(C_{S K E}\right)$ with the secondary velocity vectors overlaid for varying levels of purge flow measured at $1.40 C_{x}$ 
summarizes the work of Popovic and Hodson (2013a). They showed that the leakage losses that occur from purge injection increase with increasing purge flow in a non-linear manner. They described three regimes. At low purge levels, the momentum of the purge flow is insufficient to prevent ingress of the mainstream flow into the purge cavity which thereby thins the mainstream boundary layer and attenuates the development of the secondary flow. For the middle regime with moderate purge levels the leakage losses increase at the highest rate (steepest slope). The purge level is then sufficient to prevent ingress and tends to increase the thickness of the mainstream boundary layer resulting in a stronger secondary flow. In the third regime, at the highest leakage fractions, the losses tend to level off because the momentum of the purge fluid is high enough to energize the mainstream boundary layer. The energized boundary layer then acts to reduce the endwall cross-flow within the passage and thus attenuates any additional secondary losses.

The RF 125 case shows evidence of the third regime described by Popovic and Hodson (2013a). The near endwall lobe is smaller and has lower values of secondary kinetic energy. The pocket of fluid below the lobe, with low secondary kinetic energy, is likely the remnants of the energized endwall boundary layer. As described by Popovic and Hodson (2013a) it is quite possible that this fluid attenuates the endwall shearing leading to the observed lower levels of SKE in this region. Section 7.9 will further examine the endwall flow for different purge levels using the endwall surface visualizations and the computational passage flow visualizations. Section 7.8 presents the integrated losses and will show three regimes similar to that described by Popovic and Hodson (2013a).

\subsection{Downstream Computational Results}

The current section presents the corresponding CFD results from the $1.40 C_{x}$ plane. The

objective of this section is to determine whether the predicted flows with purge injection are in 
reasonable agreement with measured results. The CFD simulations were performed following the procedures outlined in Chapter 4.

Figure 7.12 shows floods of the streamwise vorticity coefficient overlaid with contour lines of the total pressure coefficient for cases with purge flow ratios $(\phi)$ equal to $0.00,0.75,1.00$ and $1.25 \%$. The CFD cases follow the same naming convention used for the experiments. There is one difference in the simulation test matrix relative to the experiment. The RF 090 case of the experiment is replaced by the RF 100 case from the simulations. As mentioned previously, the scattered data effect of the experiment was most prominent at purge flow ratios below the $0.75 \%$ and around $1.00 \%$. The RF 100 case was simulated to test the steadiness of the solution. As shown in Figure 7.12 the flow field is smooth and the scattered effects of the experiment are not present.

Figure 7.12 (a) shows streamwise vorticity and total pressure coefficients of the RF 000 case which was previously referred to as the Cavity case in Chapter 6 . As described previously, when compared to the experimental measurements the CFD results for the RF 000 (Cavity case) show different magnitudes of the total pressure, streamwise vorticity and SKE. However, the CFD did show similar distributions of these quantities relative to the position of the main vortical structures. It was concluded that the predictions are representative of the experimental flow field and any differences were considered typical shortcomings of CFD.

Figures 7.12 (b) through (d) show the corresponding figures for the cases with increasing positive net purge flow. When compared with the experimental measurements shown in Figure 7.9, the predictions show significantly lower levels of streamwise vorticity within the passage vortex. Also, the region of negative vorticity associated with the passage vortex is spread over a larger area. The predicted total pressure contours within this region are less diffuse than the experiment and more concentrated around the centre of the passage vortex. It is thought that the lower strength of the predicted passage vortex results in a smaller associated loss region than the experiment. Of course, as mentioned in Chapter 4, the differences in dissipation rates 
between the predictions and measurements contribute to the concentrated loss contours shown in the predictions.

Similar to the experiments, increasing the purge flow tends to affect the position of the passage vortex core, the overall size of the secondary flow and the peak associated losses and streamwise vorticity. In Figures 7.12 (b) through (d) the increasing purge flow pushes the passage vortex away from the endwall. At the highest purge level the spanwise migration is the most significant. The peak losses within the passage vortex also increase with positive purge flow.

(a) RF 000 (CFD)

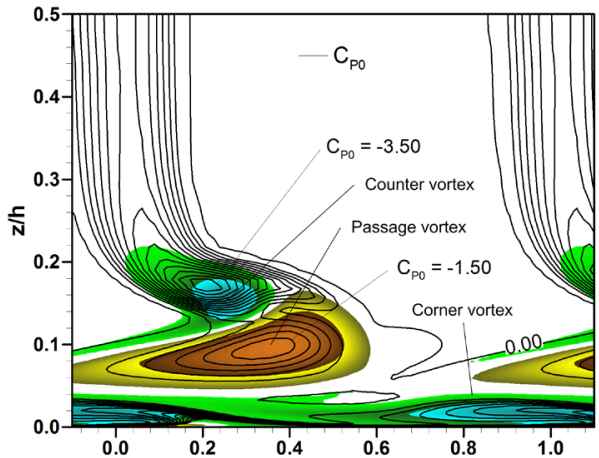

(c) RF 100 (CFD)

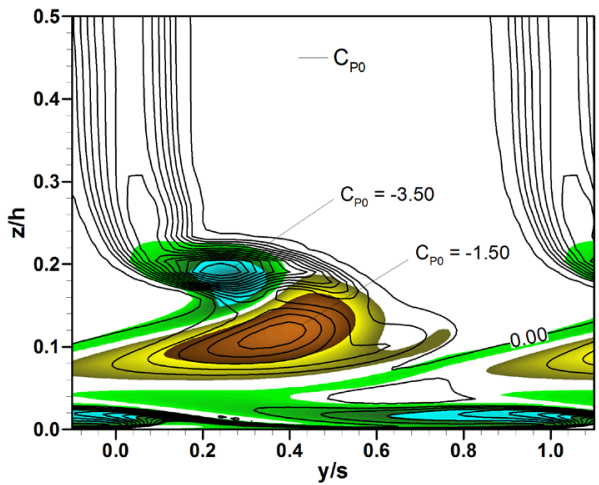

(b) RF 075 (CFD)

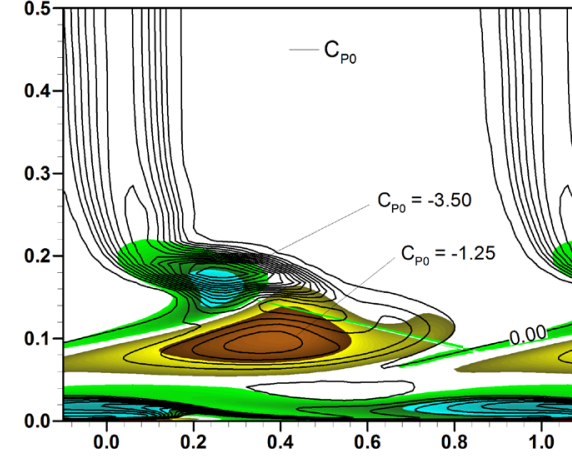

(d) RF 125 (CFD)

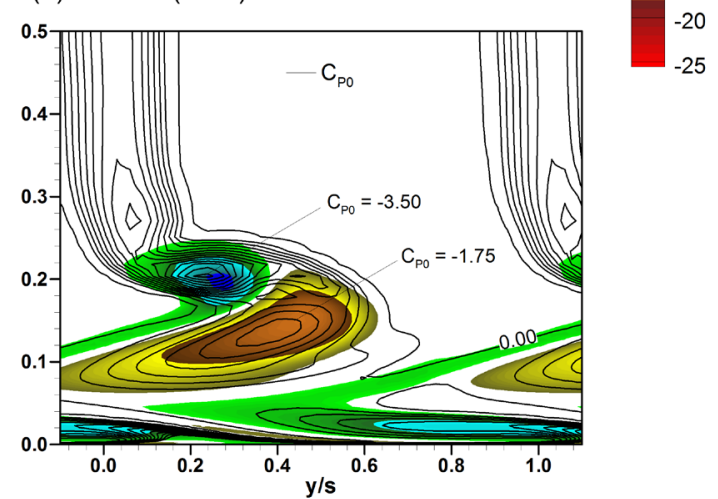

Figure 7.12: Floods of the predicted $C_{\omega S}$ overlaid with the line contours of $C_{P 0}$ for $\phi=$ (a) 0.00, (b) 0.75 , (c) 1.00, and (d) 1.25

Figure 7.13 shows the corresponding distributions of the secondary kinetic energy coefficient with the secondary velocity vectors overlaid. Relative to the experiment the 
predictions show higher peaks values of secondary kinetic energy, evidence of the lower dissipation rates relative to the experiment.
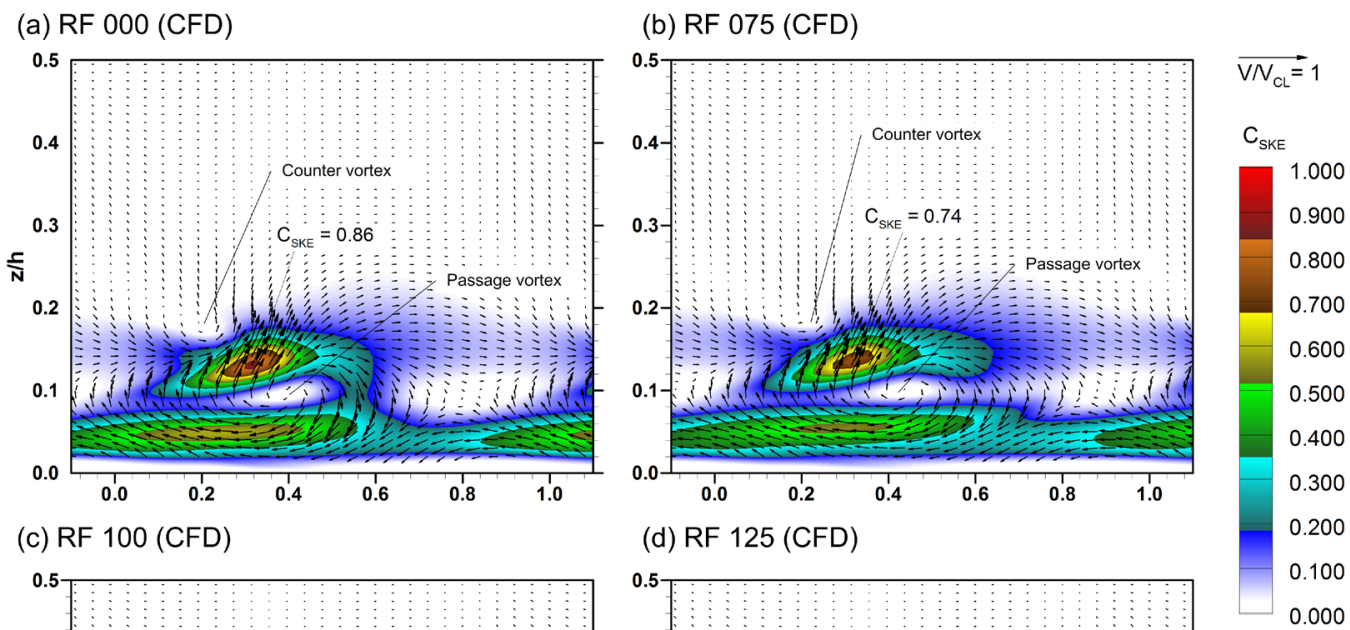

(c) RF 100 (CFD)
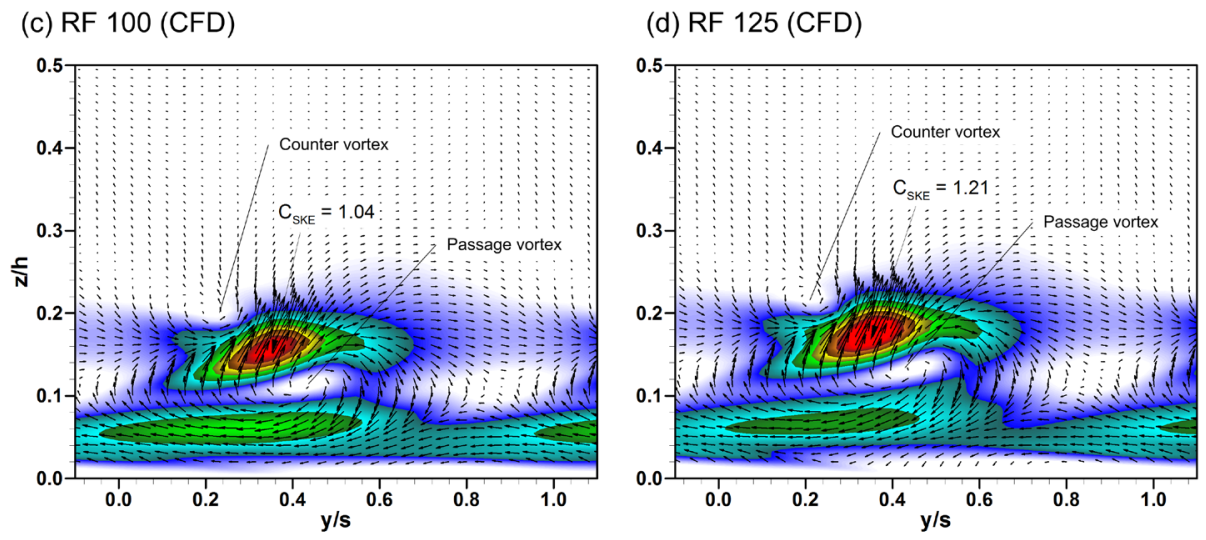

0.000

Figure 7.13: Floods of the predicted $C_{S K E}$ overlaid with the secondary velocity vectors for

$$
\phi=\text { (a) } 0.00 \text {, (b) 0.75, (c) 1.00, and (d) } 1.25
$$

Overall the predicted distributions of secondary kinetic energy are similar to those in the experiment. The highest levels of secondary kinetic energy occur within the vortex interaction zone and these peaks increase monotonically with increasing purge flow. Also, similar to the measurements, there is a distinct second lobe of secondary kinetic energy near the endwall below the passage vortex. The peak value of secondary kinetic energy within the lobe is attenuated at the highest purge injection level (RF 125), similar to the measurements. Also, at the highest purge level there is a pocket of low secondary kinetic energy fluid very close to the endwall. As mentioned in Section 7.6.3, the low secondary kinetic energy is likely remnants of the purge 
injection. Section 7.9 will further examine the near-endwall flow of the computations and measurements.

Figure 7.14 summarizes the effects of purge injection on the predicted passage vortex. In sub-figure (a), the migration of the passage vortex is shown. The experimental results are included for reference. As shown, the CFD predicts more spanwise migration than the experiments for purge ratios higher than $1.0 \%$. In the pitchwise direction, the migration is also higher than in the experiments, but is less sensitive to the level of purge injection at purge ratios greater than $0.75 \%$ (lower slope).

(a)

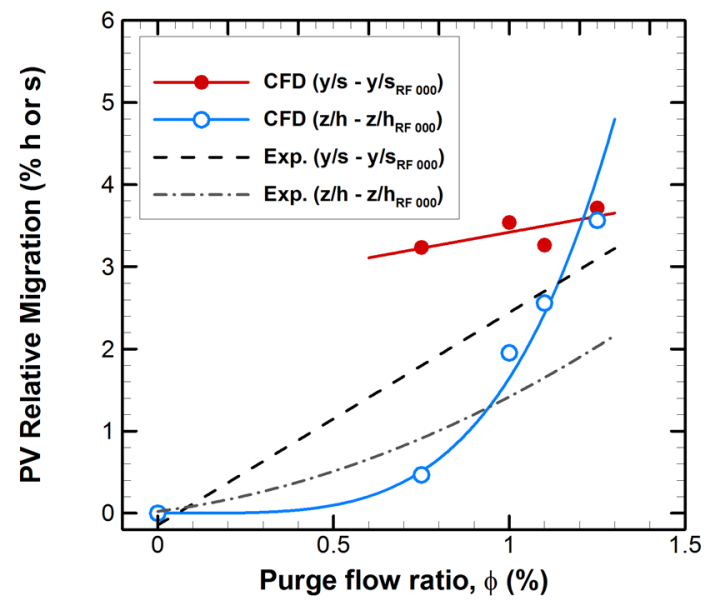

(c)

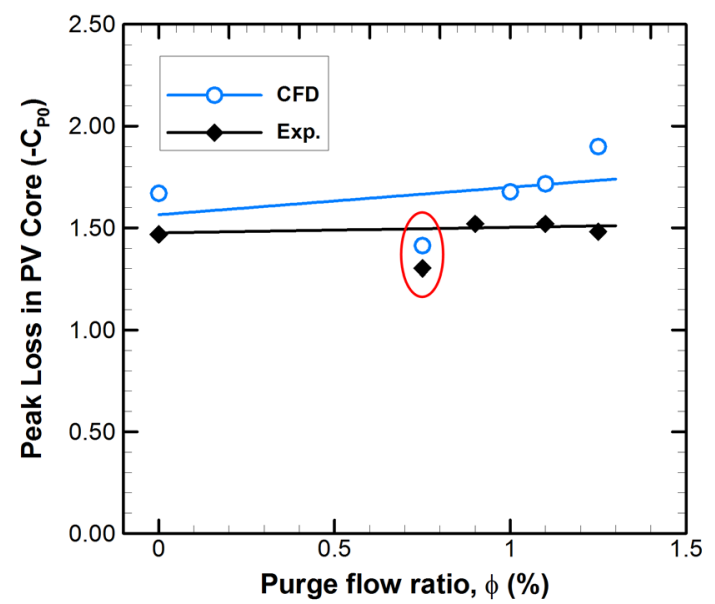

(b)

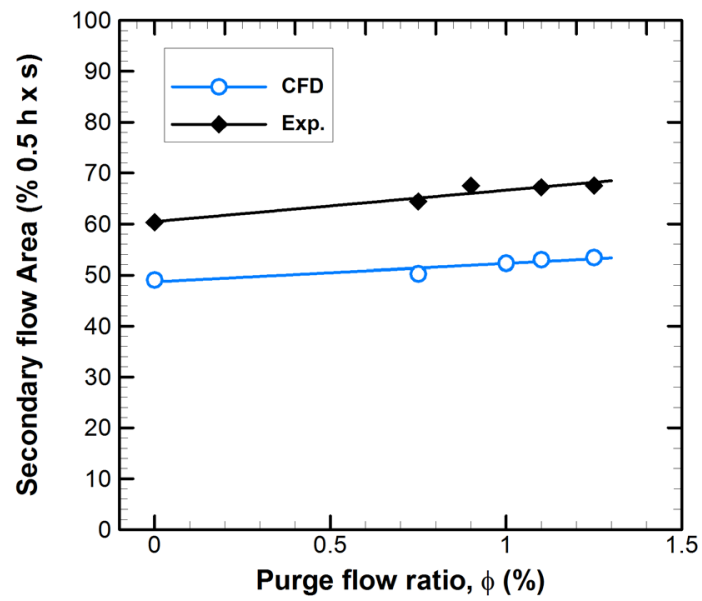

(d)

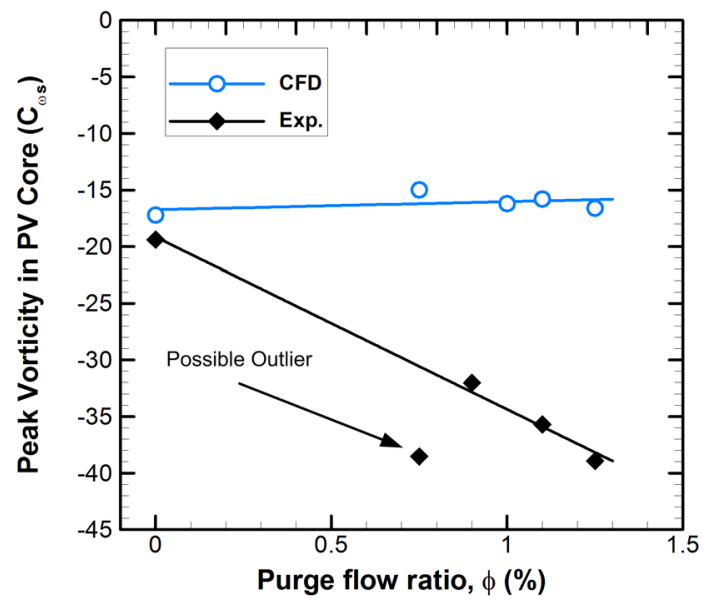

Figure 7.14: Predicted and measured passage vortex flow quantities with increasing flow purge flow 
In Figure 7.14 (b), the size of the secondary flow is expressed as a percentage of the planar area for increasing purge flow ratios. Relative to the experiment, the predicted secondary flows are approximately $10 \%$ smaller in area over the range of purge ratios shown. The smaller predicted secondary flows are caused by the lower dissipation rates observed in the predictions, as described previously. The rate at which the area changes with increasing purge ratios (slope) can be expressed as: $\Delta$ Area / $\Delta \phi$. For the predictions and measurements the slopes are 3.5 and 6 , respectively.

Figure 7.14 (c) shows the peak losses within the passage vortex for increasing purge flow ratio. As previously shown in Figures 7.9 and 7.12, the predicted peak losses are higher than in the experiment. As shown here, the CFD peak loss results tend to increase slightly with increasing purge flow, whereas the experimental peak losses are relatively insensitive to the purge injection level. The exception is the RF 075 case as highlighted in Figure 7.14 (c). In the experiment, the RF 075 case was deemed an outlier due to the scattering of the data. Interestingly, the CFD similarly predicts a lower loss value that does not conform to the linear trend. Thus, it is quite possible that the linear trend shown is not the best fit. However, without more data, especially at lower purge levels, it is difficult to suggest a more appropriate trend. The similarity between the two values at the RF 075 case does however suggest the experimental value may not be as misleading as thought, despite the apparently questionable data.

Figure 7.14 (d), demonstrates a distinct difference between the predictions and measurements. As shown for the predictions, the peak streamwise vorticity within the passage vortex remains relatively constant with increasing purge flow. The measurements show that the streamwise vorticity decreases with increasing purge flow. If the streamwise vorticity is used as a proxy for the strength of the passage vortex, the CFD results shown here under-predict the change of the passage vortex strength with increasing purge injection. Considering that passage vortex attenuation is largely the focus of many endwall-loss mitigation techniques, the inability to properly capture the effect of purge injection on the vortex strength is unfortunate. The results here do not imply that CFD is unusable for studying these flows, since the physical structures and 
loss distributions are fairly consistent with the measurements. In fact, the results simply indicate a particular shortcoming that likely needs further evaluation in future work.

\subsection{Experimental and Computational Integrated Losses and Other Flow Quantities}

The current section presents the measured and predicted integrated results from the $1.40 C_{x}$ plane. Both pitchwise and overall integrations are presented.

The measured and predicted pitchwise mass-averaged loss coefficients $\left(Y^{\prime}\right)$ are shown in Figures 7.15 (a) and (b), respectively. In both the measurements and predictions the local peaks represent the passage and counter vortices, as labelled. The predictions show narrow peaks with higher values for the both the passage and counter vortices. The local troughs between the vortical structures are also more pronounced in the predictions. The higher peaks and lower troughs are believed to be a result of the lower dissipation rates of the CFD predictions.

The effects of purge flow show some differences between the measurements and CFD predictions. In Figure 7.15 (a), the losses associated with the passage vortex increase gradually from the zero to highest purge flow cases (RF 000 to RF 125). The peak also migrates slightly in the spanwise direction. Recall that Figure 7.10 (a) showed these same trends, albeit for local peak values as opposed to the pitchwise integrations shown here. The CFD results of Figure 7.15 (b) show only small differences in the peak losses associated with the passage vortex with increasing purge flow. The addition of purge flow also tends to widen the loss peak associated with the passage vortex and push it towards midspan. The spanwise shift is more evident in the predictions than the experiment. This is consistent with the spanwise migrations shown in Figure 7.10 (a).

Figures 7.15 (c) and (d) show the outlet flow angles for the measurements and CFD respectively. In both the measurements and predictions the classic over-turning (trough) under- 
turning behaviors (hump) are shown. In the measured results, sub-figure (a), there are small differences $\left(<1^{\circ}\right)$ between the maximum overturning and under turning with increasing purge flow rate. In fact, the most notable difference is the spanwise shift of the under-turning hump with increasing purge flow rate. Relative to the lowest flow rate case, the under-turning hump shifts from about $z / h=0.12$ to $z / h=0.16$, about $4 \%$ of span. The shift of the overturning trough is small, less than $1 \%$ span.

The amount of flow angle variation at the downstream plane is an important consideration for multi-row turbine designs. Variations with span can introduce incidence on the downstream bladerow which can adversely affect the local blade loading and the subsequent losses. Here, the results suggest that the addition of purge flow, within the levels shown, does not significantly affect the magnitude of the downstream flow angles. However, designers should consider the spanwise shift of the under-turning hump.

The predicted flow angle distributions are shown in Figures 7.15 (d). The effects of the purge flow are more evident in the predictions. The zero flow case shows about $4^{\circ}$ more overturning than the measurements and $2^{\circ}$ more under-turning. At the highest purge flow rates the over-turning decreases by $2^{\circ}$ and the under-turning increases by $2^{\circ}$. The over-turning trough shifts by about $3 \%$ span while the under-turning hump shifts by about $5 \%$ span. Compared to the measurements the predicted outlet flow angle distributions are more sensitive to purge flow.

As described in Chapter 4, the CFD results shown here are true predictions and are not calibrated post-dictions. From a turbine design perspective, one would likely try to calibrate the simulation model using post-dictions that artificially increase the dissipation rates of the CFD to more closely match the experiment. Agreement then between the outlet flow angle distributions would likely be one metric to evaluate the calibrations. Improving the dissipation rates of the CFD may lead to better agreement of the flow angle sensitivity to the purge flow rate.

Figures 7.15 (e) and (f) show the pitchwise distributions of the secondary kinetic energy coefficient for the measurements and predictions, respectively. Both sets of distributions show two local humps corresponding to the near endwall lobe and the vortex interaction zone, as 
labelled. As described previously, the predictions show notably higher levels of SKE than the experiments. For the zero purge flow cases, the near endwall lobe has higher levels of SKE than the vortex interaction zone. With the addition of purge flow, the SKE within the vortex interaction zone increases while the near endwall levels decrease. Near the endwall, the lower levels of SKE are likely caused by the purge stream altering the endwall boundary layer. As mentioned, the next section will examine the endwall flows in more detail.

Measured $1.40 \mathrm{C}_{\mathrm{x}}$

(a)

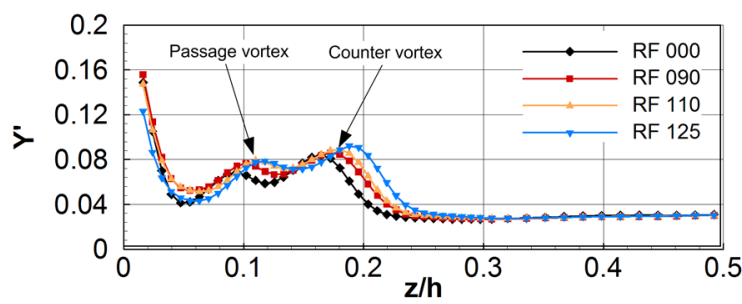

(c)

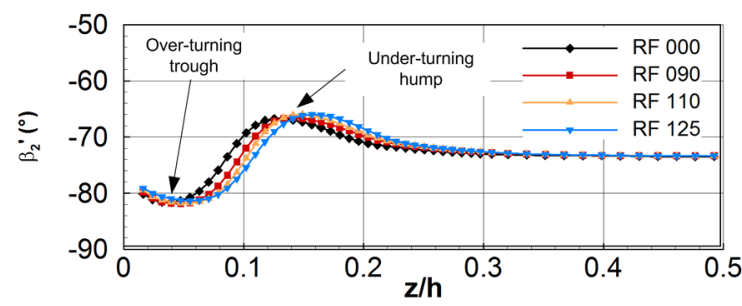

(e)

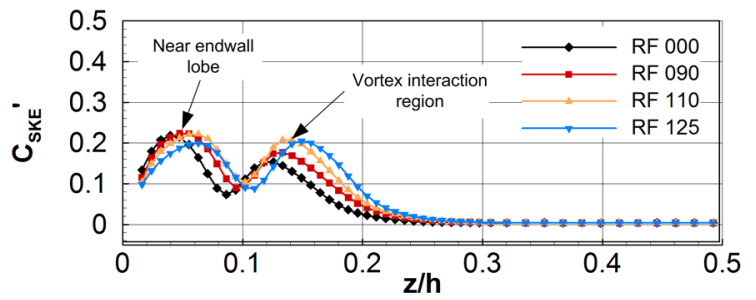

(b)

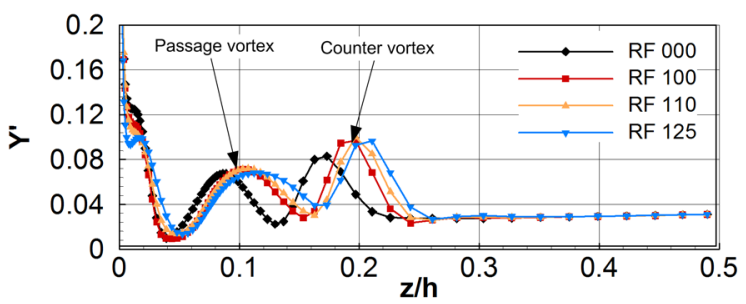

(d)

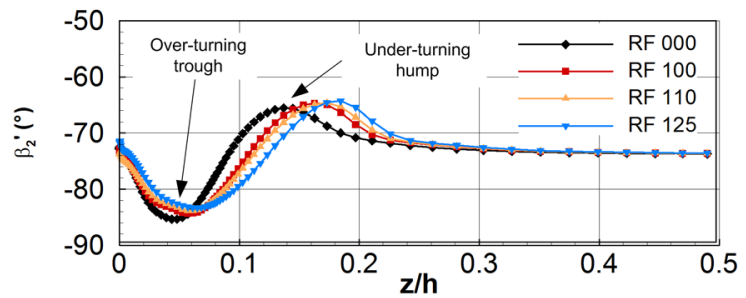

(f)

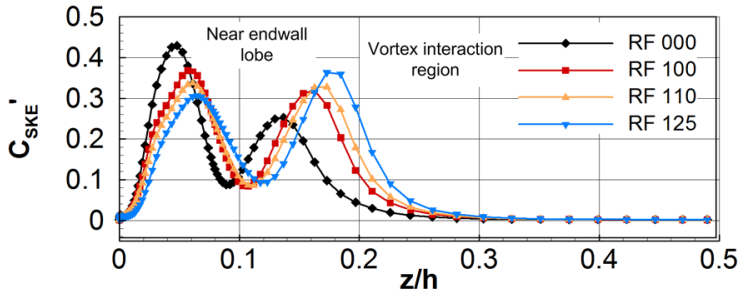

Figure 7.15: Pitchwise integrated flow quantities for different purge flow levels at the $1.40 C_{x}$ axial plane 
For the measurements and predictions, the $C_{S K E}$ of the vortex interaction zone increases by about $33 \%$ and $38 \%$ at the highest purge rates relative to the zero purge flow, respectively. As discussed in Chapter 5 the vortex interaction region is local to the highest rates of loss production. At the current plane, the generation of SKE in the vortex interaction zone represents a loss to the primary flow. However, the true losses are only realized as the SKE dissipates with further downstream mixing. The results shown here indicate the addition of purge flow not only increases the in-plane losses but also increases the losses realized at fully mixed-out conditions.

The overall integrated in-plane and mixed-out losses are shown in Figures 7.16 (a) and (b) for increasing purge flow ratios. All the loss values are normalized by the measured in-plane loss coefficient of the RF 000 case. For reference, the true and normalized losses are also tabulated in Tables 7.4 and 7.5 , respectively.

In Figure 7.16 (a), the measured in-plane losses increase with increasing purge flow ratio. As discussed previously, the three regimes described by Popovic and Hodson (2013a) are shown here. The first regime (I) corresponds to purge flow ratios less than $0.90 \%$. The change in slope with increasing purge flow is moderate for regime (I). As described previously, the purge flow level here is insufficient to prevent ingress, thus the inlet boundary layer is thinned resulting in moderate changes to the loss. The middle regime (II) corresponds to purge flow ratios between 0.90 and $1.10 \%$. The slope here is the steepest of the three regimes and represents the highest loss change for a given increase in purge flow rate. At these purge levels, the ingress is reduced and the mainstream boundary layer thickens resulting in higher secondary losses. The third regime corresponds to purge ratios greater than $1.10 \%$ and has the lowest sensitivity to increases in purge ratio. At this level, the purge flow is sufficiently high and energizes the boundary layer and pushes the passage vortex away from the endwall thereby lowering the near-endwall losses but enhancing the losses within the passage.

The measured mixed-out losses shown in Figure 7.16 (b) similarly show the same three regimes. The slopes within each regime are higher for the mixed-out losses. For the middle flow rates (II), the loss change rate is nearly three times that of the in-plane measurements. 
(a)

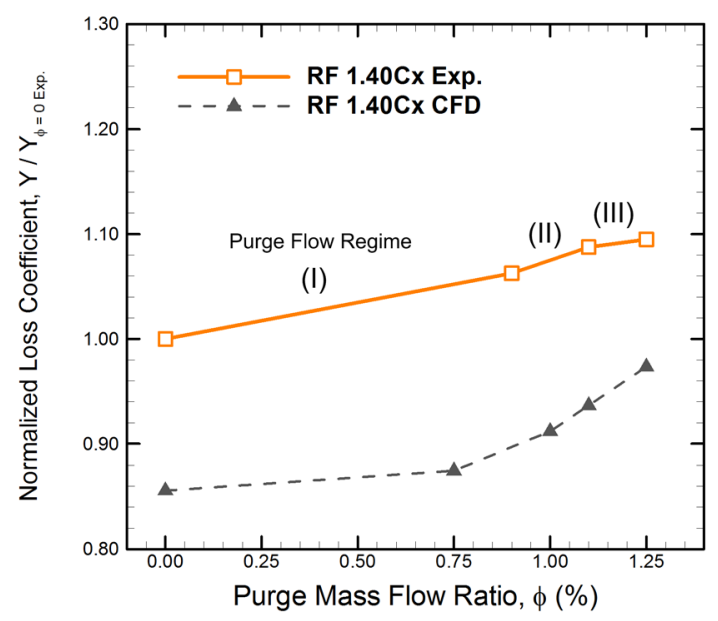

(b)

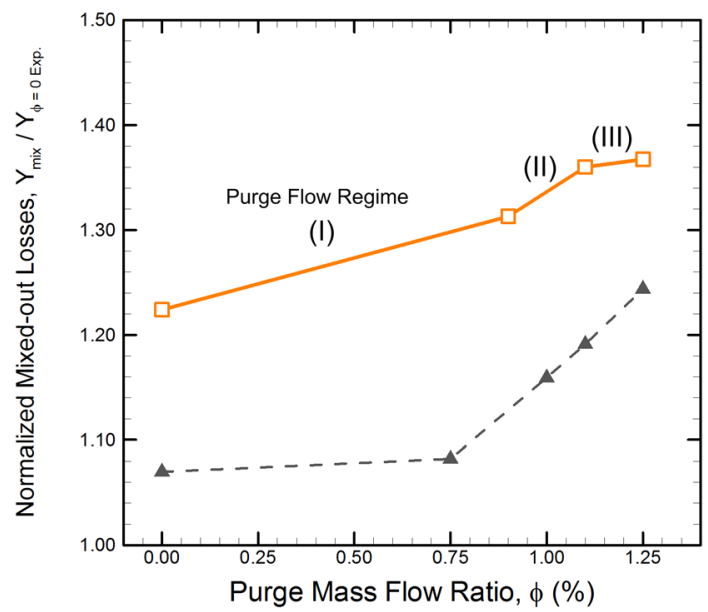

Figure 7.16: Overall in-plane $(Y)$ and mixed-out $\left(Y_{\text {mixed }}\right)$ loss coefficients for different levels of purge flow at $1.40 C_{x}$

As described above the purge injection notably increases the levels of secondary kinetic energy. Tables 7.4 and 7.5 also include the overall integrated secondary kinetic energy coefficients $\left(C_{S K E}\right)$. From the zero flow case to the highest flow rate the $C_{S K E}$ " increases from 0.051 to 0.065 , an increase of approximately a $27 \%$. The corresponding in-plane losses differ by $9 \%$ while the mixed-out losses increase by $15 \%$ over the same interval. At the highest purge flow rate, the mixed-out losses are $37 \%$ higher than the in-plane zero purge flow case.

It is clear that increasing the purge flow ratio causes significant increases to in-plane losses and the secondary kinetic energy. From the pitchwise distributions shown in Figure 7.15 it is evident that the loss increases arise from a strengthening of the passage vortex and increased interaction with the counter vortex. Figure 7.15 (a) shows that with increasing purge flow the losses associated with the passage vortex, the vortex interaction zone and the counter vortex all increase. Also, increasing the purge flow rate tends to attenuate the production of near-endwall losses and secondary kinetic energy, as shown in Figure 7.15 (a) and (e). The near endwall attenuation is small compared to the enhanced interaction observed between the passage and 
counter vortices. The latter ultimately leads to increases in the in-plane losses and secondary kinetic energy.

Table 7.4: Summary of the measured and predicted overall integrated flow quantities

\begin{tabular}{|c|c|c|c|c|c|c|c|c|}
\hline \multirow[b]{2}{*}{ Flow Quantity } & \multicolumn{2}{|c|}{ RF 000} & \multicolumn{2}{|c|}{ RF 090} & \multicolumn{2}{|c|}{ RF 110} & \multicolumn{2}{|c|}{ RF 125} \\
\hline & Exp. & $C F D$ & Exp. & $C F D^{*}$ & Exp. & $C F D$ & Exp. & $C F D$ \\
\hline $\mathrm{Y}$ & 0.051 & 0.044 & 0.055 & 0.047 & 0.056 & 0.048 & 0.056 & 0.050 \\
\hline $\mathrm{Y}_{\mathrm{p}}$ & 0.031 & 0.031 & 0.030 & 0.031 & 0.030 & 0.031 & 0.030 & 0.031 \\
\hline $\mathrm{Y}_{\mathrm{sec}}$ & 0.020 & 0.013 & 0.024 & 0.016 & 0.025 & 0.017 & 0.026 & 0.019 \\
\hline$\beta_{2}^{\prime \prime}$ & -73.0 & -73.5 & -73.1 & -73.4 & -73.1 & -73.4 & -73.1 & -73.4 \\
\hline$\beta_{2, \text { midspan }}$ & -73.4 & -73.8 & -73.3 & -73.6 & -73.3 & -73.6 & -73.3 & -73.6 \\
\hline $\mathrm{C}_{\mathrm{SKE}} "$ & 0.051 & 0.081 & 0.060 & 0.092 & 0.066 & 0.092 & 0.065 & 0.096 \\
\hline $\mathrm{Y}_{\text {mixed-out }}$ & 0.063 & 0.055 & 0.067 & 0.060 & 0.070 & 0.061 & 0.070 & 0.064 \\
\hline$Y_{p, \text { mixed-out }}$ & 0.032 & 0.032 & 0.031 & 0.032 & 0.031 & 0.032 & 0.031 & 0.032 \\
\hline $\mathrm{Y}_{\text {sec, mixed-out }}$ & 0.031 & 0.023 & 0.036 & 0.027 & 0.753 & 0.565 & 0.758 & 0.617 \\
\hline
\end{tabular}

* Simulation preformed for $\phi=1.0$, not $\phi=0.9$

Table 7.5: Summary of the measured and predicted overall integrated flow quantities relative to the zero net purge flow case (RF 000)

\begin{tabular}{|c|c|c|c|c|c|c|c|c|}
\hline \multirow[b]{2}{*}{ Flow Quantity } & \multicolumn{2}{|c|}{ RF 000} & \multicolumn{2}{|c|}{ RF 090} & \multicolumn{2}{|c|}{ RF 110} & \multicolumn{2}{|c|}{ RF 125} \\
\hline & Exp. & $C F D$ & Exp. & $C F D^{*}$ & Exp. & $C F D$ & Exp. & $C F D$ \\
\hline $\mathrm{Y} / \mathrm{Y}_{\mathrm{RF}} 000$ (Exp.) & $100 \%$ & $86 \%$ & $106 \%$ & $91 \%$ & $109 \%$ & $94 \%$ & $109 \%$ & $97 \%$ \\
\hline$Y_{p} / Y_{R F 000 \text { (Exp.) }}$ & $61 \%$ & $61 \%$ & $59 \%$ & $61 \%$ & $59 \%$ & $61 \%$ & $59 \%$ & $61 \%$ \\
\hline $\mathrm{Y}_{\mathrm{sec}} / \mathrm{Y}_{\mathrm{RF} 000 \text { (Exp.) }}$ & $39 \%$ & $25 \%$ & $47 \%$ & $31 \%$ & $50 \%$ & $33 \%$ & $50 \%$ & $37 \%$ \\
\hline$\beta_{2}^{\prime \prime}$ & -73.0 & -73.5 & -73.1 & -73.4 & -73.1 & -73.4 & -73.1 & -73.4 \\
\hline$\beta_{2, \text { midspan' }}$ & -73.4 & -73.8 & -73.3 & -73.6 & -73.3 & -73.6 & -73.3 & -73.6 \\
\hline $\mathrm{C}_{\mathrm{SKE}}{ }^{\prime \prime}$ & 0.051 & 0.081 & 0.060 & 0.092 & 0.066 & 0.092 & 0.065 & 0.096 \\
\hline$Y_{\text {mixed-out }} / Y_{R F} 000$ (Exp.) & $122 \%$ & $107 \%$ & $131 \%$ & $116 \%$ & $136 \%$ & $119 \%$ & $137 \%$ & $124 \%$ \\
\hline$Y_{p, \text { mixed-out }} / Y_{R F} 000$ (Exp.) & $62 \%$ & $63 \%$ & $61 \%$ & $63 \%$ & $61 \%$ & $63 \%$ & $61 \%$ & $63 \%$ \\
\hline$Y_{\text {sec, mixed-out }} / Y_{\mathrm{RF} 000 \text { (Exp.) }}$ & $60 \%$ & $44 \%$ & $70 \%$ & $53 \%$ & $75 \%$ & $57 \%$ & $76 \%$ & $62 \%$ \\
\hline
\end{tabular}


The increase of secondary kinetic energy at the measurement plane significantly increases

the mixed-out losses. Since the mixed-out losses account for the dissipation of secondary kinetic energy and the recovery of static pressure with downsteam distance, the mixed-out losses represent the final losses that would be generated at the fully mixed-out conditions without considering any frictional losses generated along the endwall. Thus, mixed-out losses are therefore the recommended metric to assess the losses for bladerows with purge injection.

In Figure 7.16 (a) and (b) the corresponding CFD losses are shown. The CFD shows two distinct loss growth regimes, opposed to the three shown in the experimental results. From the $\phi=0.00$ to the $\phi=0.75$ the loss growth rate is gradual and corresponds to regime (I) of the experimental results. At higher purge levels the losses increase linearly within increasing purge flow. The losses do not level off as they do for regime (III) of the experiment. It appears the predicted mixing and loss generation within endwall cavity is not entirely representative of the experiment. Considering the complexity of these flows, it is not surprise that the predictions of the loss growth rate with increasing purge flow is not entirely representative of the experiment.

\subsection{Flow Visualization of the Endwall Flow for Different Levels of Purge Flow Injection}

The purpose of this section is to visualize the effects of purge flow injection on the secondary flow of the current cascade. Endwall surface flow visualizations are compared for the zero and highest purge flow cases, namely RF 000 and RF 125. Both predictions and experimentally obtained visualization are discussed. The CFD visualizations show colour floods of the endwall shear stress coefficient with shear stress limiting streamlines overlaid. The intermediate purge flow cases were not visualized.

In general, the previous sections showed that the predicted flow fields with purge flow are similar to the measurements. The secondary flow structures, their relative positions and the associated loss distributions are generally well predicted. One of the primary shortcomings of the 
CFD was the incorrect prediction of the increasing passage vortex strength with increasing purge flow rate. However, the size of the passage vortex, as indicated by the streamwise vorticity floods, was observed to increase with increasing purge flow. Despite some of the previously mentioned shortcomings, the following section will show good agreement between the predicted and experimentally obtained endwall visualizations. The predicted endwall visualization are described first followed a discussion of the experimental visualization and results.

The predicted endwall visualizations are shown in Figures 7.17 (a) and (b) for the RF 000 and RF 125 cases, respectively. The endwalls are coloured by the non-dimensional wall shear stress coefficient, $C_{f}$. The corresponding experimental visualizations are shown in Figures 7.17 (c) and (d) and were obtained following the procedures described in Section 3.10.5. The zero purge injection cases of sub-figures (a) and (c) correspond to the Cavity case of Chapter 6 and were previously discussed in Section 6.8.2. The figures are repeated here for reference. The purge flow cases of sub-figures (b) and (d) are oriented and sized to match subfigures (a) and (c). The inlet flow enters from the right and the nominal purge injection angle is shown for reference. The nomenclature follows that of Sieverding (1985) where possible and is consistent with the previous results.

In Figures 7.17 (a) and (b), the predicted flows similarly show the inlet flow entering from the right and separating after it passes over the platform overlap. The elevated shear region within the endwall cavity just upstream of the blade leading edge marks the reattachment, $\mathrm{R}_{\mathrm{C}}$, of the separated inlet boundary layer. The reattached fluid is driven upstream into the cavity by the blade pressure field and is pushed towards the platform overlap. As the flow encounters the adverse pressure gradient of the platform the endwall flow separates along the cavity separation line, $\mathrm{S}_{\mathrm{C}}$. A similar endwall cavity recirculation is formed in both cases with it axis aligned parallel with the platform overlap. 
(a) RF 000 CFD

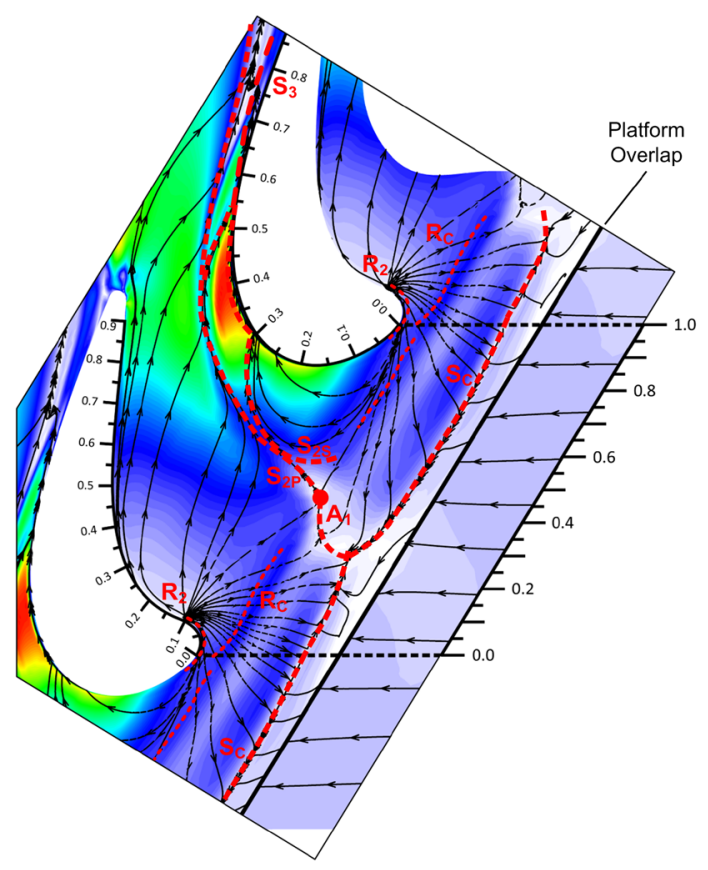

(b) RF 125 CFD

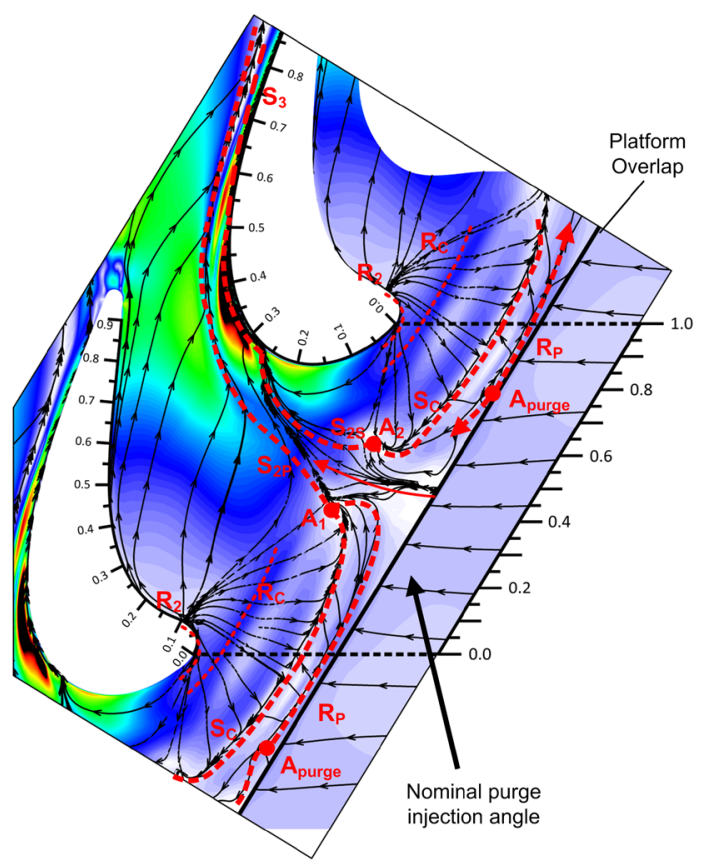

$C_{f} \times 10^{-3}: 0 \quad 5 \quad 101520253035404550556065707580$

(c) RF 000

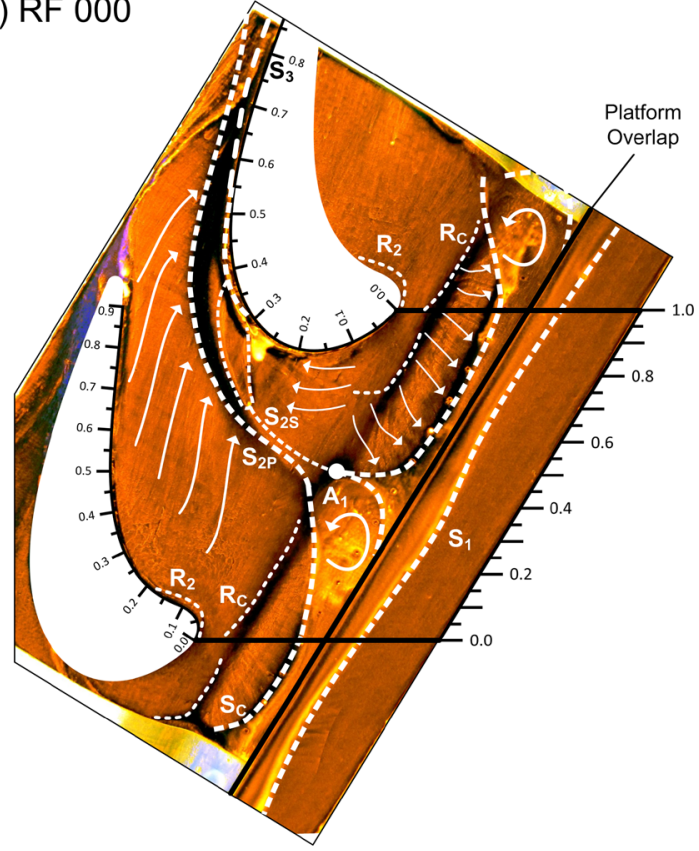

(d) RF 125

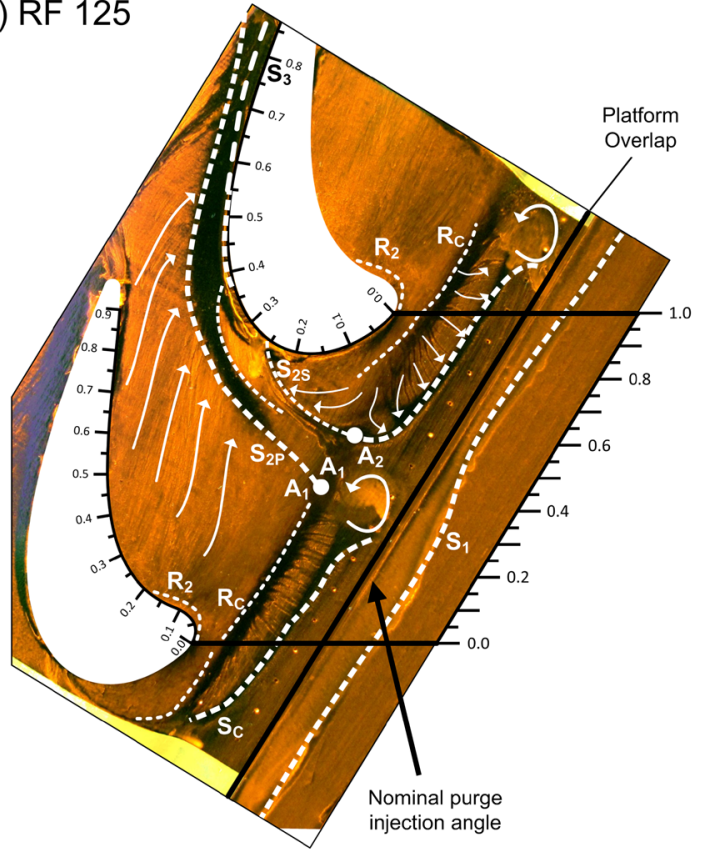

Figure 7.17: Endwall surface visualization and interpretation 
One purpose of the purge injection is to prevent ingress of the mainstream fluid into the purge cavity. As shown in sub-figure (b), the addition of purge injection is effective in shifting the cavity separation slightly downstream towards the blade leading edge. Additional evidence of the ingress barrier is the formation of a second vortex within the endwall cavity, namely the purge flow counter vortex. A reattachment line associated with the purge flow vortex is marked $R_{P}$ in Figure 7.17 (b). The formation of purge flow counter vortex is best described using a cut-plane through the endwall cavity region. Figure 7.18 (a) shows the endwall cavity of the cascade with cut-planes oriented parallel to the mainstream inlet flow angle. Figure 7.18 (b) and (c) show views of the cavity along the pitchwise direction. The cut-planes correspond to the $y / s=-0.25$ position for the RF 000 and RF 125 cases, respectively. For brevity, only one cut-plane is visualized for each configuration. The velocity streamlines identify the key features within the endwall cavity. In both sub-figures, the cavity recirculation zone is identified as the large vortex, as labelled. In Figure 7.18 (b), the net purge injection is zero which allows the mainstream fluid to be either pushed into or ejected from the cavity as dictated by the local pressure field. For the pitchwise location shown, there is local flow ejection from the purge slot. This should not be confused with purge flow injection. As described in Chapter 6, a pitchwise recirculation of mainstream fluid is established within the purge cavity in the absence of purge injection (see Figure 6.12). The pitchwise recirculation leads to local regions of ingress and ejection.

In Figure 7.18 (c), the purge injection is easily identified within the purge slot as it exits into the endwall cavity. As shown, the purge fluid is forced up into the passage by the cavity recirculation and is curled upstream as it encounters the mainstream flow. The reattachment line, $\mathrm{R}_{\mathrm{P}}$, previously identified in the endwall visualization is also labelled here. As shown, it is associated with a small separation that forms below the cavity recirculation and purge flow counter vortex. This separation is used to identify the purge flow counter vortex. With purge injection the endwall flow becomes significantly more complex. The additional vortex interaction within the endwall cavity region, relative to the zero flow case, is a likely source of the additional losses observed for the purge injection cases. 
The axes of the vortices shown in Figure 7.18 (b) and (c) are primarily aligned along the pitchwise direction - parallel to the platform overlap. In Figure 7.17 (b), the pitchwise extent of the cavity recirculation zone and the purge flow counter vortex are shown by the respective separation and reattachment lines. As shown, the purge flow counter vortex, traced by the reattachment $R_{P}$, follows the trajectory of the cavity recirculation. Similar to the cavity recirculation, the purge flow counter vortex is also divided into two legs by a saddle point, labelled $A_{\text {Purge }}$. Each leg follows $R_{P}$ in opposite directions and is entrained at the mid-pitch location where the pressure and suction side legs of the endwall cavity recirculation are funnelled together.

(a) Cut-plane position

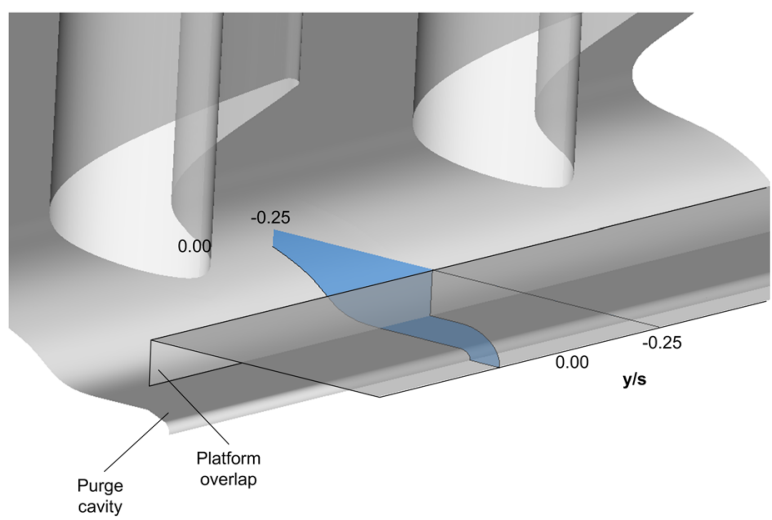

(b) RF 000 at $y / s=-0.25$

(c) RF 125 at $y / s=-0.25$
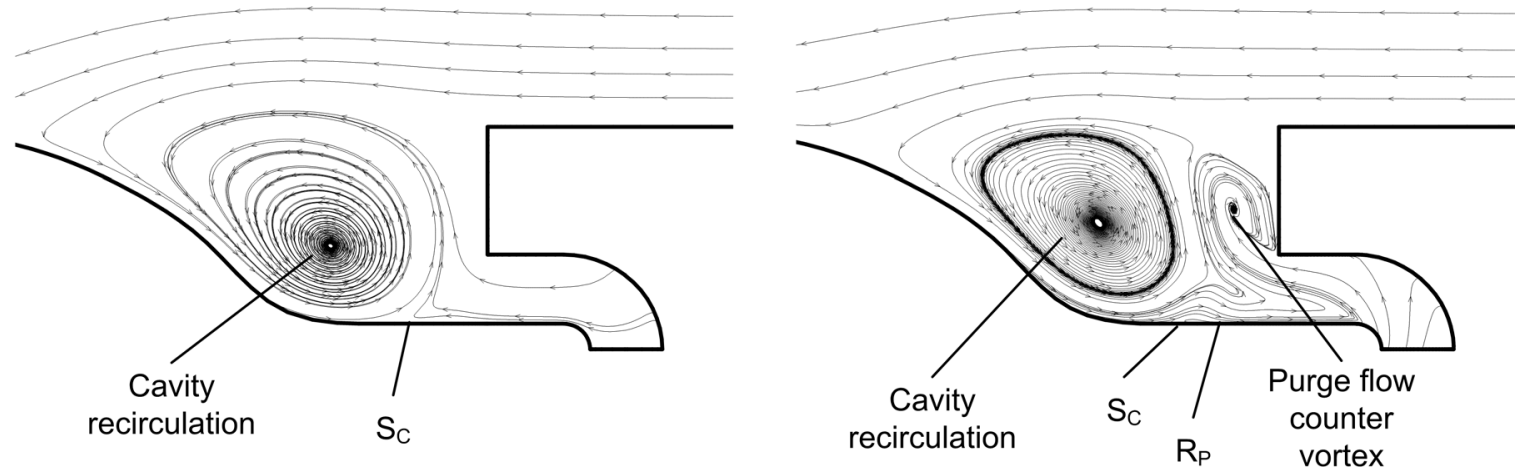

Figure 7.18: Velocity streamlines within the endwall cavity for the (b) RF 000 and (c) RF 125 cases 
Purge injection alters the flow field significantly at the mid-pitch location within the endwall cavity. For the zero purge flow case, Figure 7.17 (a), there is a single distinct saddle point, $A_{1}$, marked by the low values of shear stress and diverging streamlines. For the purge flow case, Figure 7.17 (b), the addition of purge injection appears to split the pressure and suction side legs creating a funnelled region between two saddle points, $A_{1}$ and $A_{2}$. The flow here is complex and highly three-dimensional. Emanating from the $\mathrm{A}_{1}$ and $\mathrm{A}_{2}$ saddle points are the lift-off lines of the pressure and suction side legs of the endwall cavity recirculation, labelled $S_{2 P}$ and $S_{2 S}$. The legs of the endwall cavity recirculation are analogous to those of the horseshoe (HS) vortex of a typical flat endwall secondary flow. From here on, HS vortex refers to the endwall cavity recirculation.

Further downstream from the saddle points the cross-passage pressure gradient pushes the pressure side of the HS vortex, $\mathrm{S}_{2 \mathrm{P}}$, towards the suction side of the passage thereby initiating an interaction between the pressure and suction-side legs. Similar to the zero purge flow case, the pressure side leg is fed by the cross-passage pressure gradient and separates from the endwall surface along, $\mathrm{S}_{2 \mathrm{P}}$. The suction side leg is forced to curl towards the blade suction surface by the blade-to-blade pressure gradient. As shown, the $\mathrm{S}_{2 \mathrm{~S}}$ line follows a trajectory into the blade suction surface. Just downstream of this intersection is a region of elevated endwall shearing. For the purge flow case, the shearing level here is significantly higher than for the zero purge flow case.

The origin of this high shear region was examined using visualizations of the CFD intrapassage flow field for the RF 125 case. Figure 7.19 shows the local flow velocity vectors projected on an intra-passage plane. The plane is perpendicular to the blade suction surface and intersects the blade surface at a non-dimensional suction surface length $\left(\mathrm{SS} / \mathrm{SS}_{\max }\right)$ of about 0.35 . The passage vortex is clearly visualized by the secondary velocities, as labelled. As shown, its close proximity to the blade surface induces high local velocities near the endwall and blade surface intersection. Some fluid is forced up onto the blade surface, while some curls over forming a suction side endwall corner vortex, as labelled. The increased viscous action along the 
endwall surface is marked by the high shear stresses beneath the corner vortex. The higher shear levels observed for the purge flow case, relative to the zero flow case, is a distinct difference that leads to the observed higher overall losses.

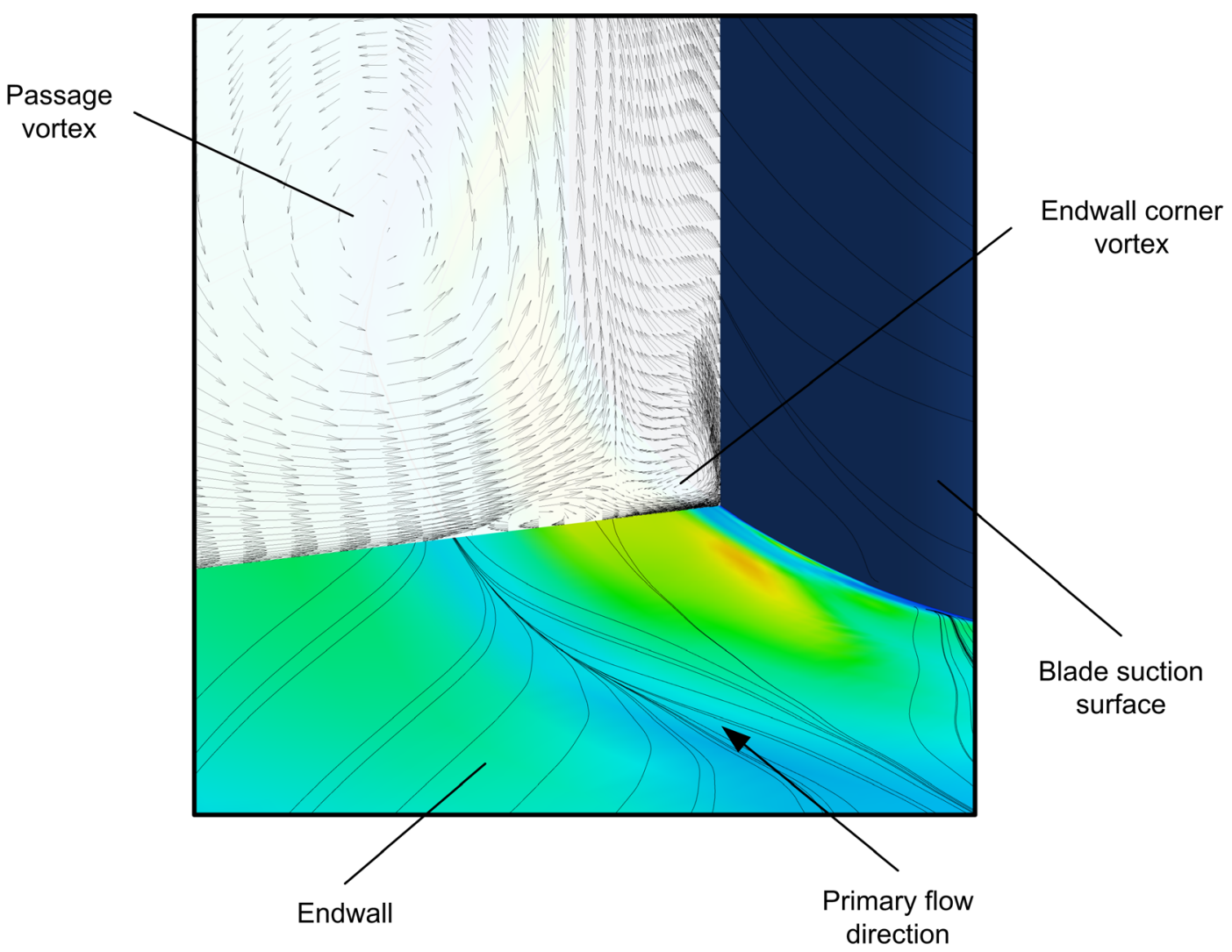

Figure 7.19: Visualization of the intra-passage flow field

The intra-passage flow was further examined. The suction side corner vortex mentioned above completely dissipates with downstream distance prior to the blade trailing edge. Similar to the suction side leg of the HS vortex, marked by $S_{2 S}$, the suction side corner vortex is also pushed against the blade suction surface by the cross-passage pressure gradient. In Figures 7.17 (a) and (b), the tail of the high shear region at about $\mathrm{SS} / \mathrm{SS}_{\max }=0.5$ marks the termination of the suction side corner vortex. Thus, this vortex should not be confused with the corner vortex identified previously on the downstream measurement planes. The downstream corner vortex has an opposite sense of rotation. It is formed as the endwall flow of adjacent passages intersect 
downstream of the blade trailing edge. The $S_{3}$ line shown in Figures 7.17 (a) and (b) marks the trace of a small vortex that is entrained at the trailing edge during the formation of the downstream corner vortex. In both cases, the $\mathrm{S}_{3}$ line follows a similar path. As shown in Figure 7.12 (a) and (d), the downstream corner vortices are also similar. Therefore, it appears that purge injection has a small effect on the downstream corner vortices. The largest downstream effects are associated with the passage vortex.

In Section 7.6.3, it was observed that with purge flow the passage vortex migrates away from the endwall and towards the centre of the blade passage. The size of the passage vortex also increases. In Figure 7.17 (a) and (b) the trace of the passage vortex follows the $\mathrm{S}_{2 \mathrm{P}}$ separation. For the purge flow case, sub-figure (b), the $\mathrm{S}_{2 \mathrm{P}}$ is closer to the blade suction surface. The suctionside leg is similarly displaced and intersects the blade suction surface earlier than in the zero purge flow case. Noting the nominal direction of the purge flow, it follows that the cross-passage pressure gradient upstream of the blade row is enhanced by purge flow. The suction side leg appears weaker than in the zero purge flow case. Conversely, the pressure side leg is strengthened by the elevated cross-flow leading to a stronger passage vortex. The term stronger here refers to the larger size, based on the streamwise vorticity floods, and the higher secondary kinetic energy observed within the vortex interaction region between the passage and counter vortices. A stronger passage vortex was only observed in the downstream measurements.

The effects of purge flow on the cross-passage pressure gradient at the blade passage entrance are evident. By altering the early development of the passage vortex the effects of purge flow then propagate through the passage. It will be shown in the following section that only a small portion of the additional losses realized from purge injection are generated in the early portions of the passage. More significant are the changes to the passage vortex that led to significant loss generation within and downstream of the passage.

Prior to discussing the loss generation through the passage, the experimental endwall visualizations shown in Figures 7.17 (c) and (d) are discussed briefly. Sub-figure (c) was previously discussed in Chapter 6. Sub-figure (d) corresponds to the purge flow case RF 125 . In 
general, the limiting streamlines of the experiment follow the interpretation given by the CFD results. Similar to the zero flow case, the flow separates from the platform overlap. Unlike, in the CFD, a clear separation line, $\mathrm{S}_{1}$, is observed upstream of the platform overlap. The platform overlap separation was discussed previously in Chapter 6. Within the cavity, evidence of the cavity recirculation zone is marked by the reattachment $R_{C}$ and separation line $S_{C}$. The experimental visualizations do not show the reattachment line $\mathrm{R}_{\mathrm{P}}$ that identified the purge flow recirculation in the CFD results. Thus, the experimental visualization cannot confirm the formation of the purge flow counter vortex; however, the CFD interpretation discussed above is logical and quite probable. Obtaining detailed visualizations of the endwall cavity flow was difficult because of the relatively low shear stresses compared to those within the passage. At one point, different viscosity oil mixtures with different pigments were applied separately to the passage and cavity regions. Unfortunately, the visualizations within the cavity were not significantly enhanced.

In summary, the following lists the similarities between the experimental visualization and the CFD results:

- For the purge flow case shown in Figure 7.17 (b) and (d) the endwall cavity recirculation is split by two saddle points, $A_{1}$ and $A_{2}$

- The high shear levels distinguish the saddle points within the endwall cavity

- The suction and pressure side legs of the HS vortex, marked by $\mathrm{S}_{2 \mathrm{~S}}$ and $\mathrm{S}_{2 \mathrm{P}}$, diverge from the respective saddle points

- With purge flow, the suction side leg, $\mathrm{S}_{2 \mathrm{~S}}$, intersects the blade suction surface earlier than in the zero purge flow case and a high shear region is observed downstream of the intersection

- The trace of the passage vortex, marked by $S_{2 \mathrm{P}}$, is closer to the blade suction surface with purge flow suggesting the cross-passage pressure gradient is enhanced by purge injection 
- The addition of purge flow also significantly strengthens the passage vortex as observed in the downstream results of Section 7.6.3.

The experimental visualizations largely support the interpretations derived from the predictions. The predictions show details of the flow that could not be identified experimentally and therefore the overall interpretation of these flows is significantly improved using CFD.

\subsection{Interpretation of the Loss Growth through the Blade Passage}

In Section 7.8, it was found that with increasing purge flow the downstream losses increased at three different rates. Based on the present interpretation of the flow, the previously suggested reasoning behind the three regimes is perhaps simplistic. For each regime, it was suggested that the mainstream boundary layer is either thinned by ingress into the purge slot or thickened by the purge stream. Both affect the development of the secondary flow. The visualizations shown in the previous section suggest that purge injection significantly alters the endwall cavity flow causing changes that propagate through the entire passage. The endwall visualizations identified three regions of enhanced loss generation relative to the zero flow case:

(1) the formation of the purge flow cavity vortex is a source of additional viscous mixing

(2) the endwall and blade suction surface shearing caused by the suction side corner vortex is also a source of additional loss

(3) finally, the strengthened passage vortex caused by the stronger upstream cross-passage pressure gradient ultimately results in higher losses through additional mixing

The sum of these additional losses leads to the observed increase in losses with purge injection. The visualizations of the previous section are qualitative and do not indicate the relative magnitude of each contribution to the overall losses.

Figure 7.20 shows the relative loss growth through the passage for the predicted purge flow cases. For reference the platform overlap and blade cross section are shown. The relative losses 
at a given axial location are normalized by the cascade exit dynamic pressure $\left(q_{2}^{\prime \prime}\right)$ and represent the difference relative to the zero purge case, as defined by:

$$
\left(Y_{l o c}-Y_{l o c, \phi=0.00}\right) \frac{q_{l o c}{ }^{\prime \prime}}{q_{2}^{\prime \prime}}
$$

where the local loss coefficient at a given axial location is defined as:

$$
Y_{l o c}=\left(C_{P 0_{1}}^{\prime \prime}-C_{P 0_{l o c}}{ }^{\prime \prime}\right)\left(\frac{q_{1, C L}}{q_{l o c}}\right)^{\prime \prime}
$$

In Figure 7.20 the flow enters from the left of the figure. As the flow approaches the platform overlap the losses increase relative to the zero flow case. The relative increase appears to be largely independent of the level of purge flow as only small differences are shown between the cases. However, it appears that the purge injection alone alters the frictional losses generated over the latter portion of the platform overlap. The shear stress levels along the platform overlap were investigated for the cases with different purge flow levels (not shown here). For the zero purge flow case the distribution of the endwall shear stress in the pitchwise direction shows regions of both low and high shear stresses. With purge injection, the shear levels are higher and are more uniform along the pitchwise direction. It appears that the purge injection alters the pressure field sensed by inlet boundary layer. Lower static pressures caused by the purge injection result in local acceleration of the inlet boundary layer. Higher losses at the platform overlap trailing edge are thus observed for cases with purge injection.

The effect of the different purge flow rates is first evident within the endwall cavity. At $x / C_{x}=-0.2$, slightly downstream from the purge flow counter vortex, the losses begin to monotonically increase with purge flow rate. Initially, however, the relative differences in the losses are small. Within the endwall cavity region there is only a minor loss penalty with increasing purge flow rate. The small differences here suggest that the viscous mixing within the purge cavity (item 1 above) is low and perhaps largely deferred until further downstream. 
Within the blade passage the effects of purge flow rate are more prominent. At $x / C_{x}=0.5$, or about $\mathrm{SS} / \mathrm{SS}_{\mathrm{MAX}}=0.3$, the relative losses begin to diverge with increasing purge flow rate. As labelled, the onset of the loss divergence corresponds to the location of the high endwall shearing identified in the visualizations (item 2 above). In Figure 7.19, it was shown that the suction surface corner vortex, induced by the passage vortex, is associated with the elevated endwall shear stresses. It was also shown that the level of endwall shearing increased with increasing purge flow, suggesting that the purge flow enhances the interaction between the passage vortex and blade suction surface. Figure 7.20 shows that the losses downstream of this interaction are strongly dependent on purge flow level.

Based on the flow field interpretations and the growth of the intra-passage losses shown here the effects of purge flow can be generally interpreted as follows. Initially, purge flow strengthens the cross-passage pressure gradient within the endwall cavity and early portion of the blade passage. In turn, the passage vortex is strengthened and driven more quickly towards the blade suction surface (item 3 above). The suction side leg of the horseshoe vortex is also more quickly curled towards the blade suction surface. The level of purge flow significantly affects the interaction between the passage vortex and the blade suction surface. Downstream of the interaction point, the majority of the additional losses realized from purge injection are generated.

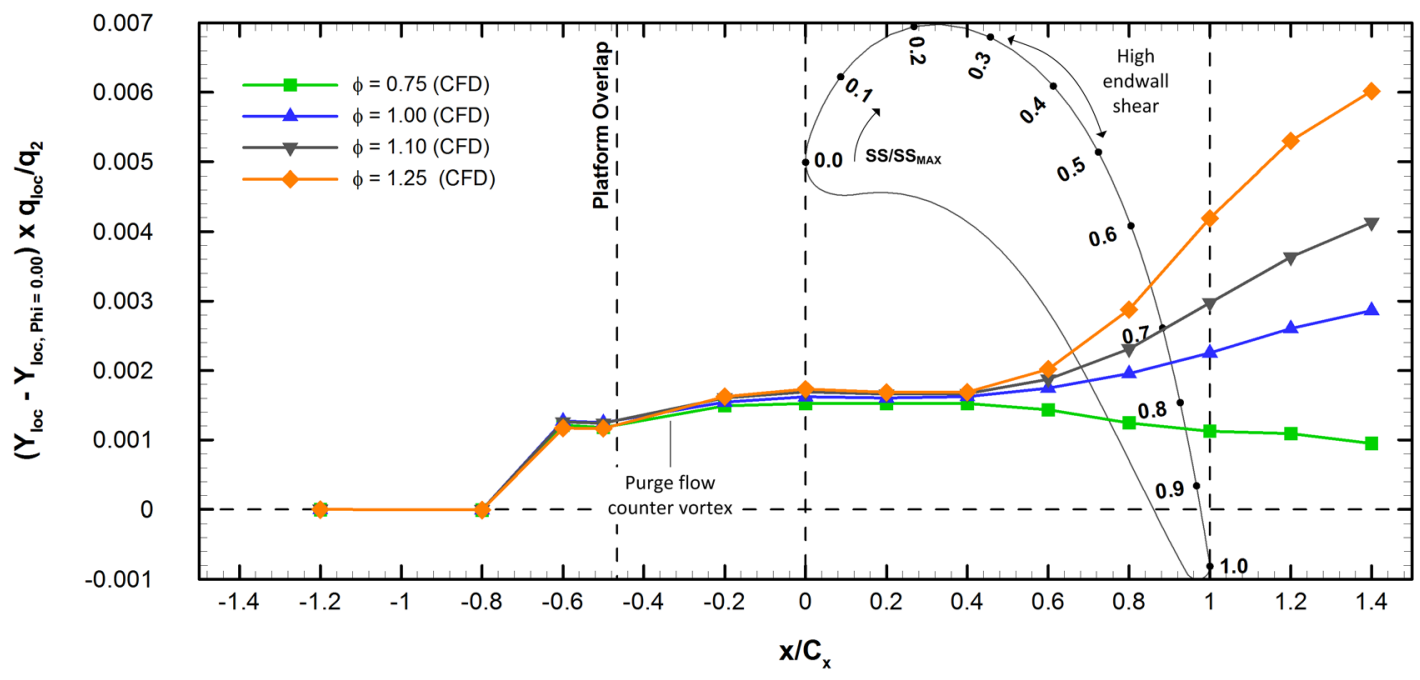

Figure 7.20: Predicted relative loss growth through the passage for varying levels of purge flow 


\subsection{Concluding Discussion}

The present chapter investigated the effects of positive purge flow injection on the secondary flow of a low-speed linear turbine cascade. Measurements were completed on the blade surface and at axial planes downstream of the blade trailing edge. The flow fields were examined in terms of the downstream distributions of the total pressure, streamwise vorticity and secondary kinetic energy coefficients. Pitchwise and overall integrated flow quantities were examined. The flow field interpretation was completed using experimental surface flow visualizations and complimentary intra-passage CFD results.

The blade loading measurements showed that with increasing purge flow the mid-span loading increases over the blade suction surface. Higher free stream velocities are caused by spanwise flow convergence. The axial velocity ratio and CFD predictions confirmed that increasing flow convergence correlates to the increasing purge flow rate.

The downstream measured results show the primary effect of purge injection is to alter the passage vortex. With increasing purge flow rate, the passage vortex is displaced in the spanwise and pitchwise direction. The pitchwise migration increases linearly with purge flow, while the spanwise migration follows an exponential-type trajectory. The associated losses and peak vorticity both increase, indicating that additional purge injection strengthens the passage vortex. The strengthened passage vortex leads to higher levels of secondary kinetic energy within the region between the passage and counter vortices. In a real engine, the increase in secondary kinetic energy is important since it will eventually be realized as additional losses with further downstream mixing.

Purge injection was found to increase the overall losses monotonically with increasing purge flow; however at three different rates. The low, medium and high regimes are consistent with those described by Popovic and Hodson (2013a). The rate of loss growth with purge flow is influenced by how the purge injection alters the mainstream secondary flow development. 
A detailed interpretation of the secondary flow development was discussed using endwall visualisations. The predicted and measured endwall visualizations are in good agreement, indicating that the CFD methods are satisfactory for these flows. The CFD was also used to assess the loss growth throughout the passage. It was shown that the additional losses caused by increasing purge flow are generated in the latter portion of the passage. There is a strong interaction between the passage vortex and the blade suction surface. This interaction is enhanced with purge injection and also marks the onset of the monotonic loss growth with increasing purge levels. 


\section{Chapter 8}

\section{Secondary Loss Mitigation Technology: Non-Axisymmetric Endwall Contouring and an Elliptically Shaped Platform Overlap}

\subsection{Introduction}

The present chapter investigates two different secondary loss mitigation technologies for the current cascade. The two geometric modifications, as described in section 3.5.3, are the nonaxisymmetric contoured endwall and the elliptically shaped platform overlap. These technologies passively manipulate the flow field and were tested at similar purge levels to those examined in the previous chapter.

Non-axisymmetric endwall contouring is a technology that has previously been researched as a secondary loss mitigation technology. Section 2.7 .5 describes much of the relevant literature. The objective of the endwall contour is to reduce the endwall blade-to-blade cross-passage pressure gradient and thereby attenuate the development of the passage vortex. As a relatively 
new technology the robustness of the endwall contouring under more engine representative conditions is not well documented in open literature.

The purpose of the elliptically shaped platform overlap is to provide a more gradual pathway for the inlet endwall boundary layer to pass over the platform overlap into the main passage. As described in the previous chapter, a large separation and recirculation zone forms downstream of the platform overlap which thereby alters the secondary flow. The elliptical overlap was designed to reduce the severity of the separation and attenuate the secondary losses.

The current chapter presents measurements for two geometric configurations. The first is a rectangular platform overlap with non-axisymmetric endwall contouring through the blade passage (RC). The second configuration is an elliptically shaped platform overlap with flat intrapassage endwalls (EF). The original test plan included a third configuration which incorporates both technologies, that is: the elliptical platform overlap with non-axisymmetric endwall contouring (EC). The effects on the flow field for the third configuration are similar to the EF configuration and are therefore only presented in Appendix G.

In the present chapter the results for the $\mathrm{RC}$ case are presented first. The effects of purge flow on the effectiveness of the endwall contouring are discussed. The secondary flows are examined in terms of total pressure losses, streamwise vorticity and secondary kinetic energy. The intra-passage flow physics are interpreted using the endwall flow visualizations.

The remainder of the chapter then focuses on effects of the elliptical platform overlap. More specifically, it will be shown that the elliptical overlap is relatively ineffective as a loss mitigation technology (as applied here). For this reason, the discussion of the elliptical results is brief and focuses on the shortcomings of the application of this technology. 


\subsection{Motivation}

Chapter 1 described the motivation to investigate loss mitigation technologies for gas turbine engines. For aircraft applications, reducing the engine weight while maintaining or improving the current efficiency levels leads to overall improvements for the aircraft fuel consumption. Weight reduction of the turbine section of the engine can be achieved by reducing the blade count. The more widely spaced blades are aerodynamically more highly loaded and tend to have higher secondary losses. Therefore, endwall contouring aims to reduce the loss penalty associated with the more highly loaded blade row.

Chapter 2 described many studies that have shown endwall contouring to be an effective loss migration technology for a variety of flow conditions. However, only a small number of these studies have specifically investigated the effects of the platform overlap geometry and even fewer have investigated the effects of purge flow. The previous chapters have shown that the platform overlap and purge flow significantly affects the secondary flow physics. Thus, it is important to understand how contouring can mitigate the secondary losses when these features are include in the flow path.

Jenny et al. (2011) investigated endwall contouring applied to a cold flow single turbine stage with endwall contouring. The experiments showed that the endwall design effectively reduced the sensitivity of the stage efficiency to the level of purge injection. In another stage test, Schuepbach et al. (2011) showed an opposite trend. The benefit of endwall contouring was reduced with increasing purge flow ratio. In fact, the baseline case (no contouring) was the most resistant to changes in the purge ratio, thereby suggesting a potential vulnerability of endwall contouring in real engine environments.

The current chapter aims to enhance the understanding of the physical interaction between the purge flow and mainstream for turbines with profiled endwalls. Ultimately, this may lead to improvements to the endwall design for turbine blade rows. 
The elliptically shaped platform overlap was also investigated as another loss mitigation technology. The motivation for this technology is to also reduce the secondary losses. Rather than focusing on the development of the passage vortex, the objective was to reduce the severity of the platform overlap separation. Popovic and Hodson (2013c) investigated several engine representative rim-seals. They found that the best designs, which represent a compromise between the increased losses and sealing effectiveness, had the smoothest trajectory from the purge cavity into the main gas-path. In the current thesis, it was hypothesized that the combination of the elliptical overlap and endwall contouring could result in a combined reduction of the secondary losses.

\subsection{Approach}

Experimental measurements of the PAKH cascade were made for the design inlet Reynolds number of 75,000. Downstream measurements were made using the seven-hole probe described in Section 3.8.5. Two geometric cases, designated RC and EF are considered in the present chapter. As mentioned, the results of the EC configuration are presented in Appendix G. The purge flow mass flow rate was independently varied for each test case. Following the convention of the previous chapters, each case is then also designated by 3 digits representing the purge flow ratio, $\phi$, for the respective case.

\subsection{Inlet Flow Field}

The test conditions of the cascade are nearly identical to those described in Sections 6.4 and 7.4. The inlet boundary layer closely matches that shown in Figure 6.2. The designed purge flow inlet velocity triangles are unchanged from those shown in Figure 7.1. 


\subsection{The Effects of Non-axisymmetric Endwall Contouring on the Secondary Flow of the Linear Turbine Cascade}

\subsubsection{Introduction}

The following sub-sections discuss the experimental results for the PAKH cascade with intra-passage endwall contouring. The discussion focuses on the results for the rectangular platform overlap $(\mathrm{RC})$ measured at the $1.40 C_{x}$ plane. The downstream flow fields are described as usual using contour floods of the total pressure, streamwise vorticity and secondary kinetic energy coefficients. A discussion of the overall integrated flow quantities follows. The subsections conclude with an interpretation of the endwall flow using the oil-film surface flow visualizations.

\subsubsection{Downstream Flow Field with Non-axisymmetric Endwall Contouring}

Contour floods of the total pressure coefficient measured at the $1.40 C_{x}$ plane are shown in Figure 8.1. The representative purge flow levels are $\phi=0.00,1.10$ and 1.18. The highest level, $\phi=1.18$, is different than the highest purge level of the RF and EF cases. At the time of testing the purge flow blower could not supply a higher mass flow rate. The results corresponding to the contoured case (RC) are shown on the right-hand side in sub-figures (b, $d$ and $f$ ) while the rectangular overlap cases are shown on the left-hand side in sub-figures (a, c and e). Figures 8.2 and 8.3 show the corresponding floods of the streamwise vorticity and secondary kinetic energy coefficients.

Figure 8.1 shows the effects of the endwall contouring on the downstream total pressure field. In general, the effects of contouring on the downstream flow field are very evident and significant. For the zero purge flow case, sub figure (b), the effect on the passage vortex is clear. The characteristic loss core of the passage vortex is not visible. Instead, the associated losses are lower and less concentrated around the centre of the passage vortex. 
Flat Endwall

(a)

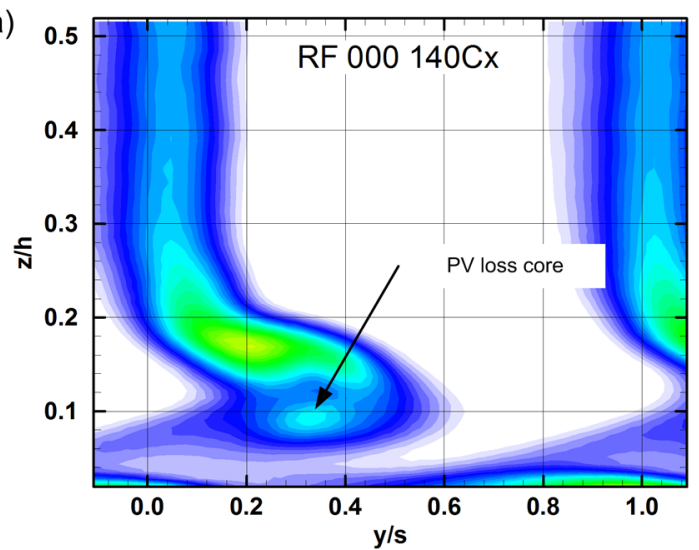

(c)

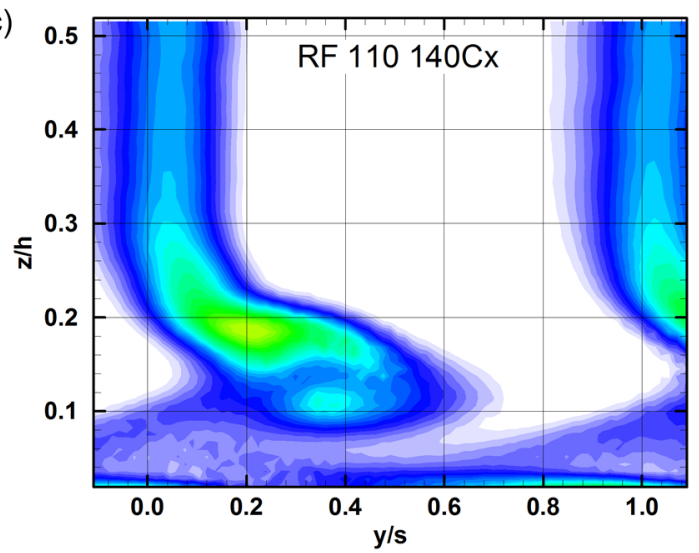

(e)

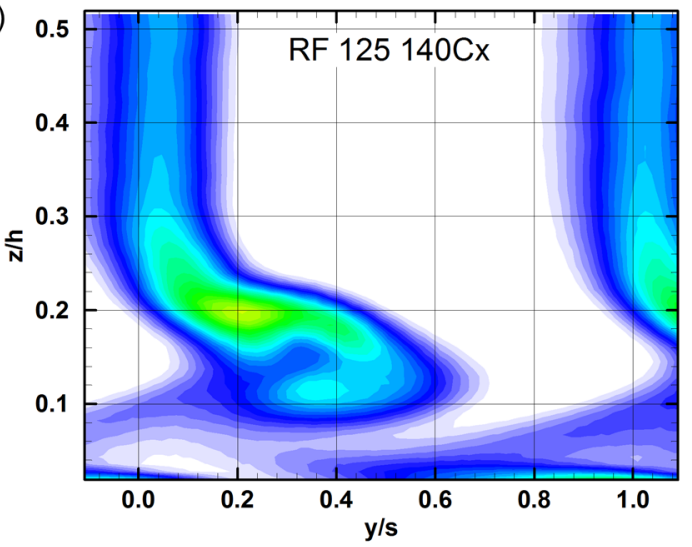

Contoured Endwall

(b)

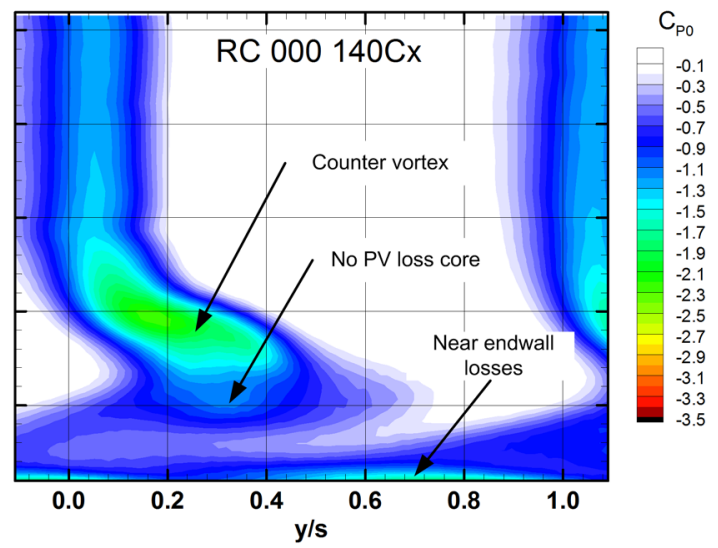

(d)

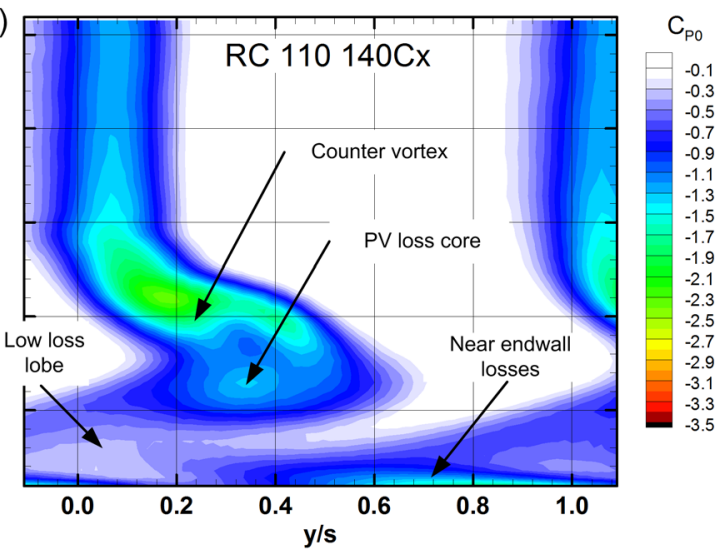

(f)

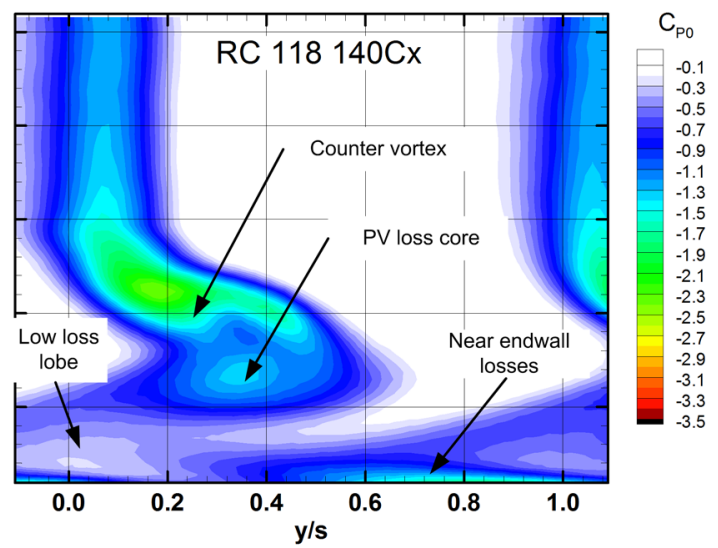

Figure 8.1: Floods of the total pressure coefficient $\left(C_{P 0}\right)$ for varying levels of purge flow measured at $1.40 C_{x}$ for the $\mathrm{RF}$ and $\mathrm{RC}$ configurations 
Two other loss regions are noticeably affected by the endwall contouring: the losses associated with the counter vortex and the near endwall losses adjacent to the corner vortex, as labelled. As shown, these loss regions are reduced relative to the corresponding RF case.

Knezevici et al. $(2009,2010)$ also investigated endwall contouring for low speed cascades, in the same test-section used for the present work, albeit on a traditional cascade without the platform overlap. They showed that contouring reduces the strength of the passage vortex by lowering the pitchwise cross-passage flow as well as the spanwise flow at the endwall and blade suction surface interface. The three loss regions identified in Figure 8.1 (b) are similarly indicative of lower cross flow and less viscous dissipation within the endwall boundary layer.

Figure 8.2 (b) shows the effects of endwall contouring on the streamwise vorticity associated with the passage and counter vortices. The size and shape of the counter vortex is relatively unchanged relative to the RF case. The peak level of streamwise vorticity is slightly lower. However, in the cases with elevated purge flow the peak vorticity is nearly equal to the flat endwall (RF) case. The most significant changes to the streamwise vorticity field occur within the passage vortex. As shown, the peak level of streamwise vorticity within the passage vortex is significantly reduced. The shape and position is also altered. The passage vortex fluid appears more diffuse and is pushed away from the endwall relative to the RF case. Also, the shape of the vortical region appears stretched within the measurement plane. The apparent stretching is likely a visual illusion caused by a change of the relative angle of the vortex axis and the measurement plane. A simple analogy is the slicing of a cylindrical object. At more oblique angles relative to the cylinder axis the slices appear elongated and less circular. Nevertheless, the change in the vortex shape is an indication that the trajectory of the passage vortex has also changed.

In Chapter 2, some of the early endwall contour designs were discussed. The intention of many designs, such as an endwall fence, was to create a physical barrier to reduce the near endwall cross-passage pressure gradient. The contour designs today generally have a mid- 
Flat Endwall

(a)

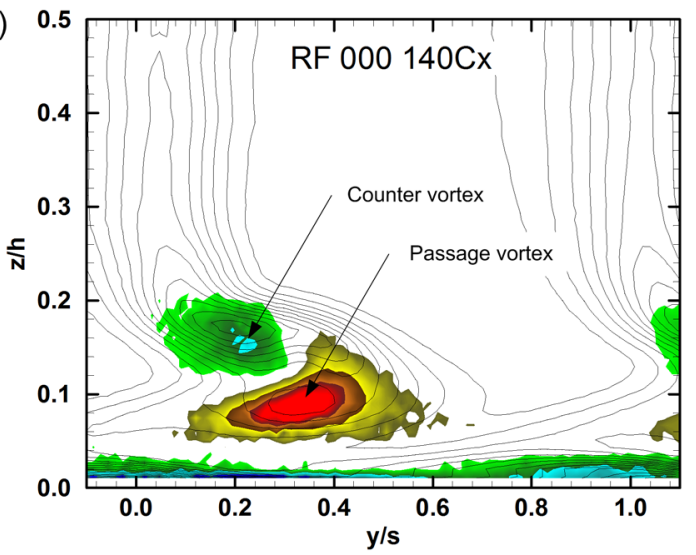

(c)

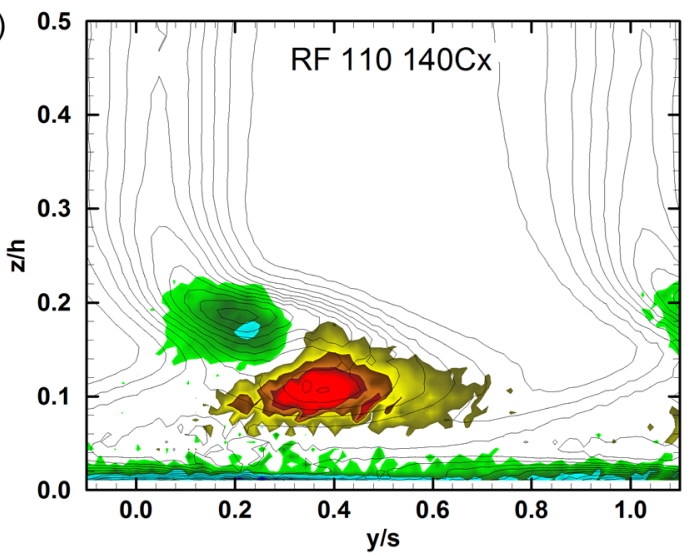

(e)

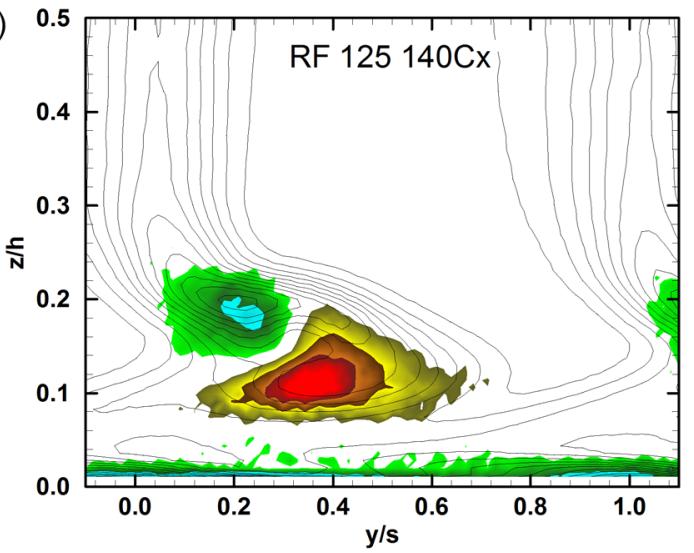

Contoured Endwall

(b)

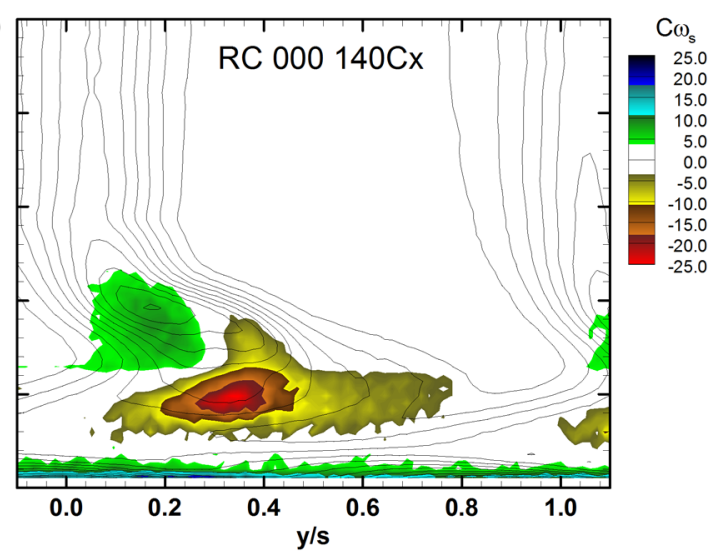

(d)

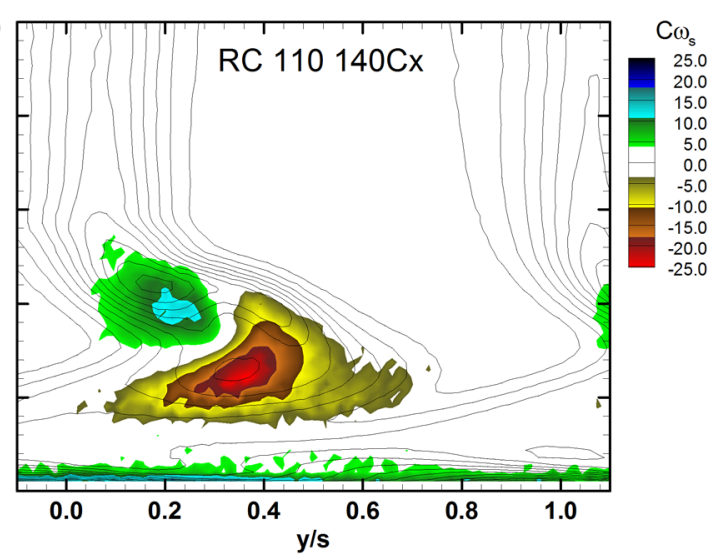

(f)

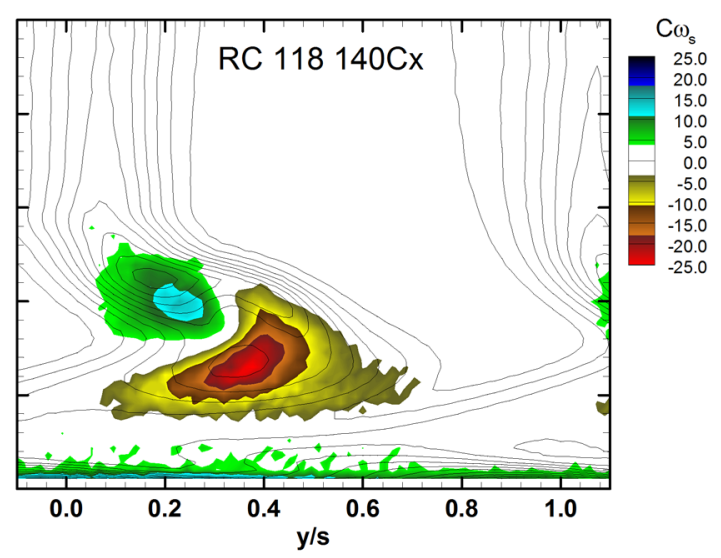

Figure 8.2: Floods of the streamwise vorticity coefficient $\left(C_{\omega S}\right)$ with contour lines of the total pressure coefficient $\left(C_{P 0}\right)$ for varying levels of purge flow measured at $1.40 C_{x}$ for the RF and RC configurations 
passage hump that has similar effects of an endwall fence. The purpose is to gradually alter the blade-to-blade cross-passage pressure gradient and retard the development and pitchwise migration of the passage vortex. The loss contour and streamwise vorticity floods of Figures 8.1 and 8.2 show evidence of a weakened passage vortex and perhaps an altered trajectory.

The corresponding flood of secondary kinetic energy coefficient is shown in Figure 8.3 (b). Similar to the flow fields of the previous chapter, there are two distinct lobes of secondary kinetic energy. The highest level occurs between the passage and counter vortex. As discussed in the previous chapter, the elevated level of secondary kinetic energy is caused by the strong interaction between the passage and counter vortices. The endwall contouring reduces the peak level of the secondary kinetic energy, indicating a reduced interaction between the passage and counter vortices.

The second lobe of secondary kinetic energy is positioned below the passage vortex, as labelled. The elevated secondary kinetic energy in this region is caused by the cross-flow between the endwall and passage vortex. The contouring case shows a lower peak value here because the passage vortex is weakened. Interestingly, although the peak value of secondary kinetic energy within this lobe is lower, higher levels of secondary kinetic energy are spread further across the passage in the pitchwise direction. As a result, the pitchwise integrated levels of secondary kinetic energy in this near endwall region are nearly identical (see the pitchwise averaged results in the following sub-section). Thus, reductions in the overall averaged secondary kinetic energy are generally a result of the lower secondary kinetic energy within the vortex interaction zone.

Perhaps a negative effect of the current contouring design is evident in Figure 8.1 (b) through 8.3 (b). As shown, the secondary flow is displaced away from the endwall and towards the centre of the passage - increasing the penetration depth. As mentioned in previous chapters, as the secondary flow moves away from the endwall more inviscid fluid can be entrained thereby increasing the associated mixing losses. Also, for low aspect ratio blades the displacement of the 
Flat Endwall

(a)

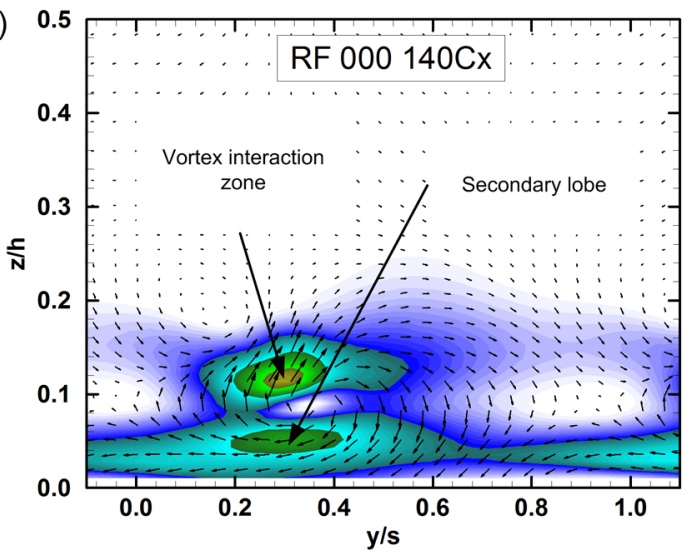

(c)

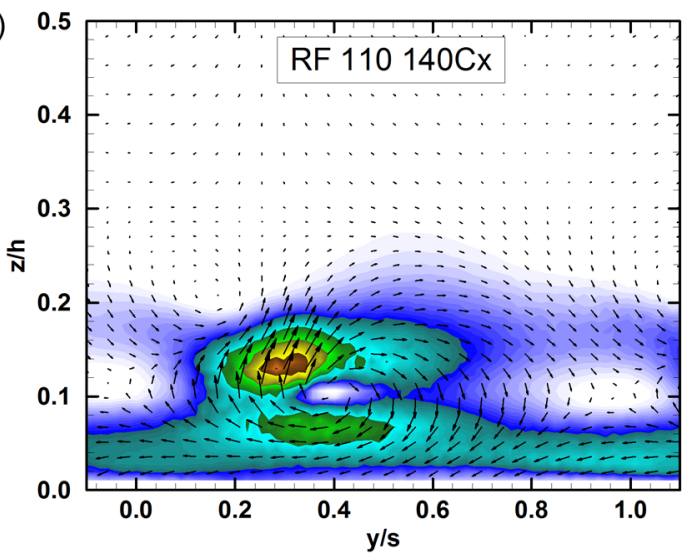

(e)

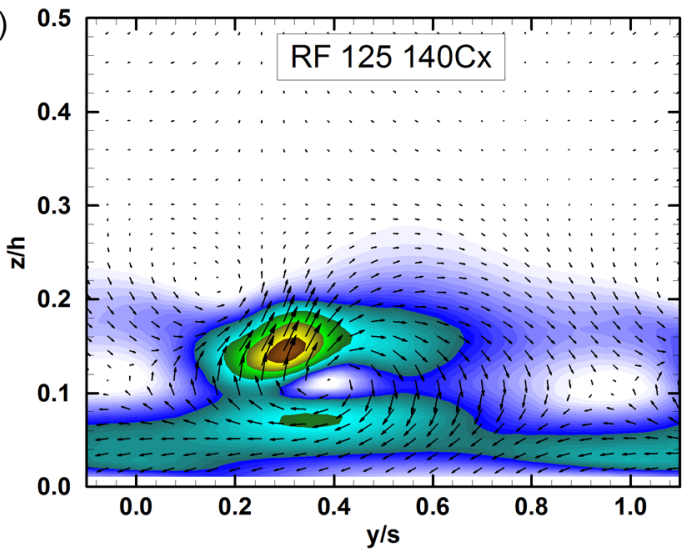

Contoured Endwall

(b)
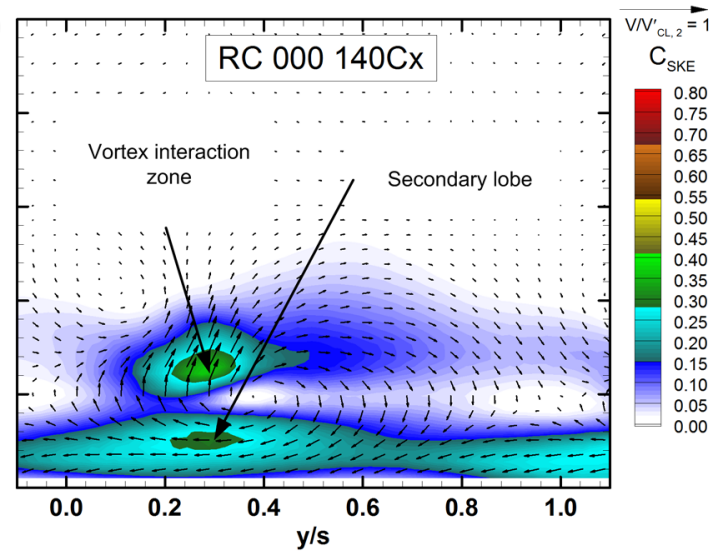

(d)
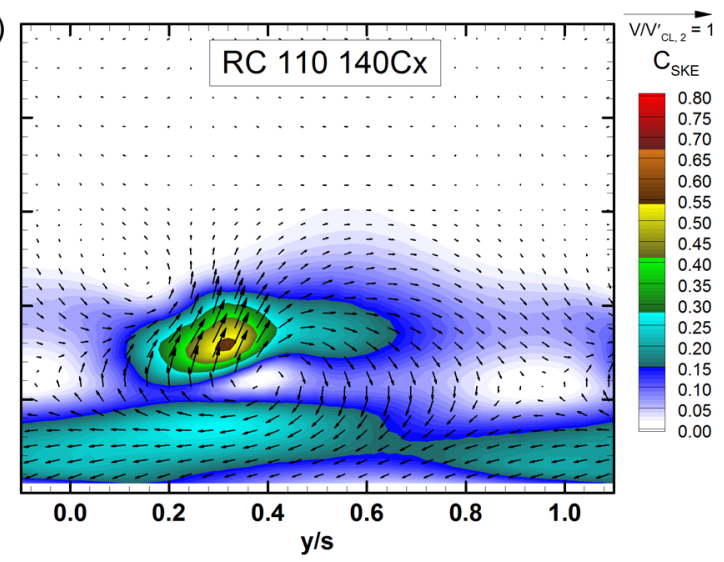

(f)

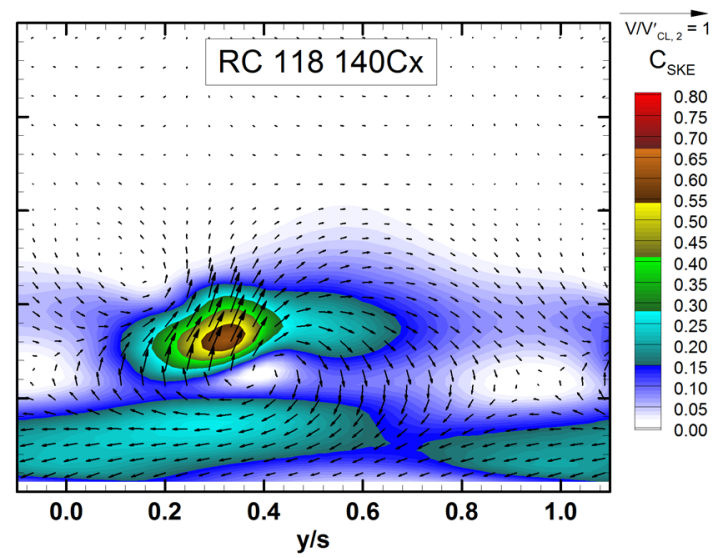

Figure 8.3: Floods of the secondary kinetic energy coefficient $\left(C_{S K E}\right)$ with the secondary velocity vectors overlaid for varying levels of purge flow measured at $1.40 C_{x}$ for the $\mathrm{RF}$ and $\mathrm{RC}$ configurations 
secondary flow towards the centre of the passage can cause a significant interaction with the secondary flow from the opposite endwall.

The effects of purge flow on the contoured flow field are shown in Figures 8.1 through 8.3 in sub-figures (d) and (f). Increasing the purge level for the cases with endwall contouring has similar effects to those shown for the flat endwall cases. The losses and streamwise vorticity associated with the passage vortex region are enhanced with increasing purge flow. In Figures 8.1 (d) and (f), a distinct loss core is apparent for both purge cases with lower peak losses than the corresponding RF case. As labelled, the losses associated with counter vortex increase. The counter vortex streamwise vorticity also increases as shown in Figures 8.2 (d) and (f). The levels are very similar to the RF case.

The near endwall losses, previously identified in Figure 8.1 (b), increase slightly with increasing purge flow, as labelled. The corresponding region for the RF case shows lower losses than the RC case. Also, the purge flow pushes the secondary flow further way from the endwall into the centre of the passage. A distinct low loss lobe is also visible, as labelled. Compared to the corresponding RF cases the low loss lobe has slightly higher loss levels.

The low loss pocket of the higher purge cases is also apparent in the lower purge flow cases, albeit with higher associated losses. For the zero purge flow cases the low loss pocket likely originates from a low loss region of the inlet flow-field. As the flow moves through the passage this low-loss fluid is not well mixed with the higher loss fluid associated with the secondary vortices. The clock-wise rotation of the passage vortex (as shown in Figure 8.2) evidently draws the inviscid fluid towards this low loss pocket. With reference to the purge flow cases, it appears that the addition of purge flow enhances the size of the low loss region. Likely, the injected purge flow has energized this region leading to lower losses over a larger region.

The effects of endwall contouring on the downstream flow field are summarized as follows. In terms of the downstream total pressure losses four regions were significantly altered by endwall contouring. As labelled in Figure 8.1, the four regions are (1) the losses associated with the counter vortex, (2) the losses associated with the passage vortex, (3) the high losses in 
near-endwall region and (4) the low loss near-endwall lobe. The strength of the passage and counter vortex, inferred by the levels of streamwise vorticity, was also changed. Lastly, the endwall countering affected the two peak regions of the secondary kinetic energy.

The counter vortex region for the zero net purge flow case showed lower losses than the RF case. With increasing purge flow the counter vortex losses increase but remain lower than the corresponding RF cases. Although the associated streamwise vorticity was lower than the RF case for the zero net purge flow, the high purge flow cases show near equivalent levels of streamwise vorticity within the counter vortex.

The passage vortex was the flow feature most significantly altered by the endwall contouring. At the zero purge level, the passage vortex did not have an associated loss core. The levels of streamwise vorticity were significantly lower than the RF case. With increasing purge flow the passage vortex develops a distinct loss core similar to that of the RF case; however, the loss levels are lower. The associated streamwise vorticity increases with purge flow, but does not exceed the levels shown in the corresponding RF cases. The passage vortex is clearly weakened by the endwall profiling. The vortex interaction region, between the passage and counter vortices, shows lower secondary kinetic energy which yields benefits at conditions further downstream.

The following sub-section examines the integrated flow quantities. Pitchwise and overall averaged results are discussed.

\subsubsection{Integrated Flow Quantities for the Endwall Contouring Configuration}

The integrated measurements from the $1.40 C_{x}$ plane are presented in the present subsection. The pitchwise and overall averaged flow quantities were averaged following the procedures given in Section 3.11.2. The pitchwise averaged loss coefficient $\left(Y^{\prime}\right)$, pitchwise flow angle $\left(\beta^{\prime}\right)$ and secondary kinetic energy coefficient $\left(C_{\mathrm{SKE}}\right)$ are show in Figure 8.4. Each subfigure shows the results for the contoured (RC) case and the corresponding flat endwall (RF) 
case. The sub-figures are organized in columns by the corresponding purge flow rate, as indicated.

The pitchwise mass-averaged loss coefficients for the zero net purge flow cases are shown in Figure 8.4 (a). The regions corresponding to the passage vortex and counter vortex are labelled. As shown, the RC case shows lower losses than the RF case within the counter vortex region. The corresponding downstream floods of total pressure, shown in Figure 8.1 (a) and (b), similarly show lower losses associated with the counter vortex. The reduction of losses in this region is attributed to the weaker passage vortex, which attenuates the losses generated throughout the passage.

The passage vortex region of Figure 8.4 (a) does not show a loss reduction for the contoured case. Recall that the passage vortex region was visually very different than the RF case at the downstream plane in Figure 8.1. The downstream floods give the impression of lower losses within the passage vortex region. A distinct loss core was not evident within the passage vortex and the loss levels were significantly lower than the corresponding location of the RF case. However, the contoured case also showed higher loss levels adjacent to the passage vortex away from the vortex core. When averaging over the pitch, at a constant spanwise position, the increase in losses adjacent to the passage vortex offset the loss reduction within the passage vortex core. As a result, the pitchwise averaged loss coefficient shows no benefit within the passage vortex region.

Although a loss benefit is not realised for the pitchwise averaged passage vortex region it should be noted that the passage vortex is still attenuated by the profiled endwall. In Figures 8.2 and 8.3, the lower magnitudes of streamwise vorticity and the reduced secondary kinetic energy within the vortex interaction region are evidence of a weaker passage vortex. In fact, the loss reduction associated with the counter vortex is likely caused by the weaker interaction between the passage vortex and blade suction surface flow. 


$$
\phi=0.00
$$

(a)

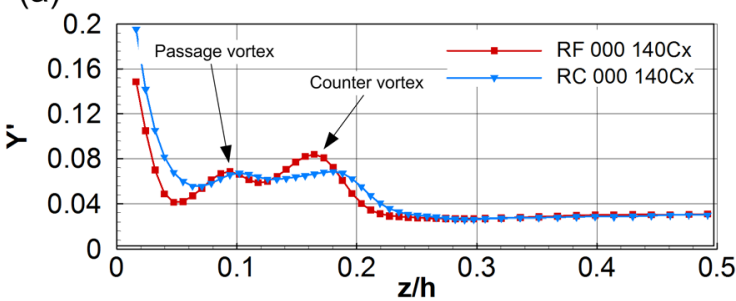

(d)

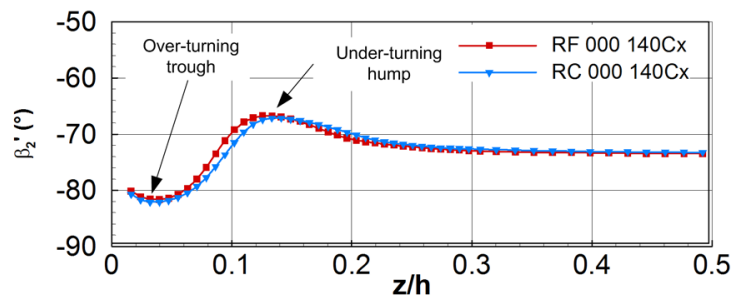

(g)

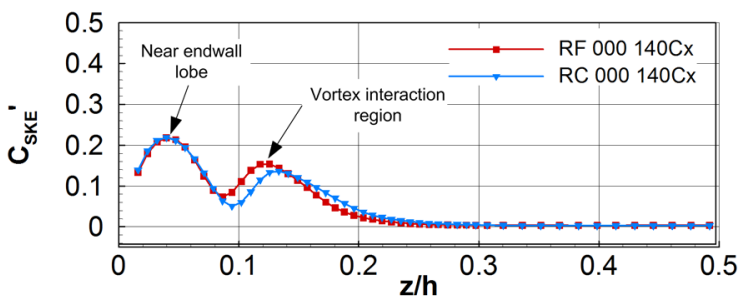

$\phi=1.10$

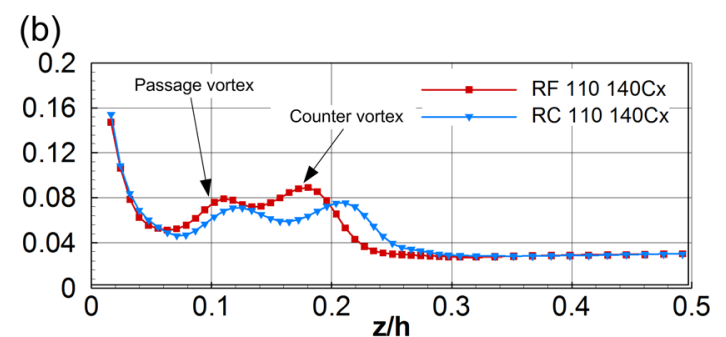

(e)

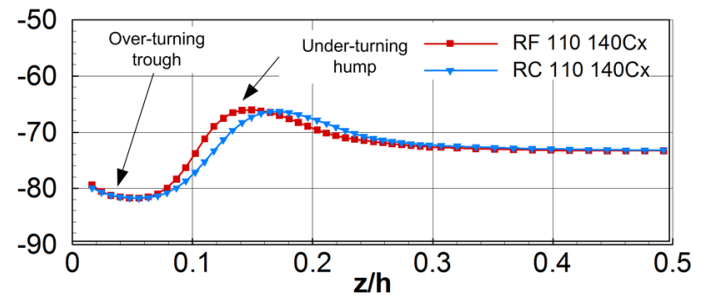

(h)

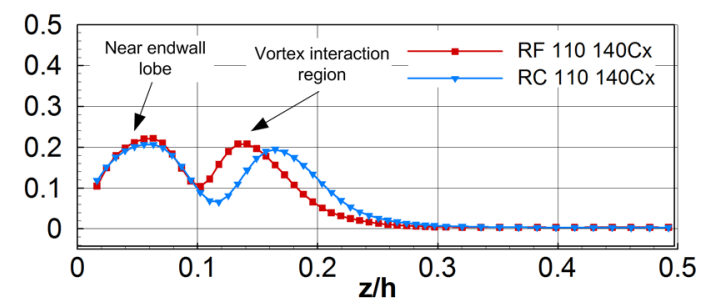

\section{$\phi=1.18$ to 1.25}

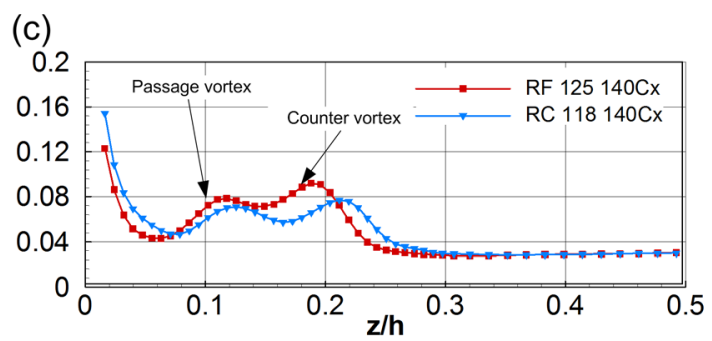

(f)

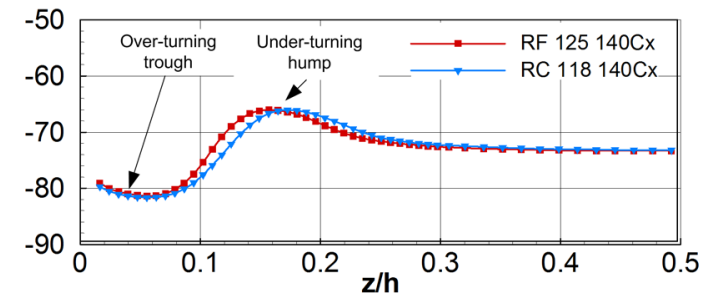

(j)

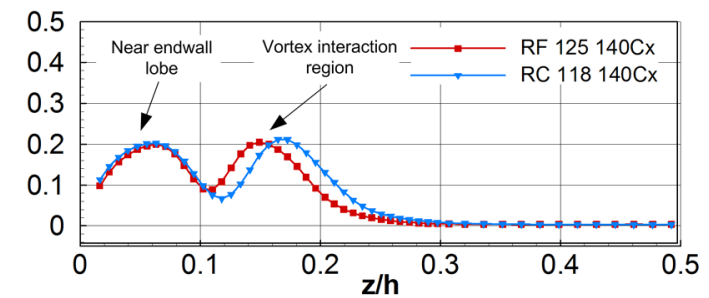

Figure 8.4: Pitchwise-averaged results measured at the $1.40 C_{x}$ plane for the contoured endwall configuration (RC) 
Closer to the endwall in Figure 8.4 (a) the contoured case shows significantly higher losses than the flat endwall case. At the downstream plane, the flood of the total pressure coefficients similarly shows elevated losses at spanwise locations below the passage vortex. In particular, the low loss lobe identified in Figure 8.1 (a) has significantly higher losses for the countered case. The origin of the higher near endwall losses is unclear, but it is suspected the endwall design is not robust at the zero purge level. For the zero net purge flow case, the momentum of the near endwall flow is different than the design intention which may lead to unintended separations along the endwall. The endwall surface visualizations discussed in Section 8.5.4 further investigate the robustness of the endwall design.

At the higher purge levels, Figures 8.4 (b) and (c), the near endwall losses are similar between the RC and RF cases. The effects of contouring at these elevated purge levels are more noticeable near the passage and counter vortex regions. Similar to the zero net purge flow case, the counter vortex region shows the highest benefit. The losses associated with the passage vortex are attenuated, but only slightly. As discussed previously the marginal loss benefit for the passage vortex does not reflect the significant benefits of contouring to the passage vortex structure and surrounding secondary flow.

Figure 8.4 (e) through (f) show the pitchwise averaged flow angle for the three purge levels. As labelled, the over-turning troughs of the RC and RF cases are nearly identical at each purge level. As shown in Chapter 7, with increasing purge flow the over-turning trough becomes slightly deeper and is also wider. A widened trough indicates a larger portion of the span is occupied by flow that is misaligned relative to the design outlet flow angle. The present contouring design therefore shows no effect on the near endwall flow angle (over-turning trough). Others, such as Knezevici et al. $(2009,2010)$, showed that the contouring increases the near endwall secondary kinetic energy thereby causing about a $2^{\circ}$ increase in near endwall overturning. Since increasing the over-turning can have a negative impact on subsequent blade rows, the near equal over-turning shown for the present endwall design should be considered a design success. 
The under-turning that occurs further from endwall is also labelled in Figures 8.4 (d) through (f). As shown, the under-turning is very similar between the RC and RF cases for the contoured cases. The position of the under-turning peak coincides with the region between the passage and counter vortices, namely the vortex interaction region. The high secondary velocities within the vortex interaction region cause the resultant velocity vectors to under-turn relative to the design outlet flow angle. For the present design, the passage vortex is displaced towards midspan and the vortex interaction zone is therefore similarly displaced. The under-turning hump shows a corresponding spanwise shift.

The pitchwise integrated secondary kinetic energy coefficient is shown in Figures 8.4 (g) through (i). The secondary kinetic energy follows a similar pattern to the flow angle. As shown, the near endwall regions of the RC and RF cases have very similar levels of secondary kinetic energy. Recall, that the corresponding downstream plane for the contoured case, shown in Figure 8.3, showed lower peak values of secondary kinetic energy below the passage vortex. However, the elevated levels adjacent to this peak offset the reduction at the peak when pitchwise averaged. Therefore, the resulting pitchwise averaged value is similar to the RF case.

Figures 8.4 (g) through (i) also show that the vortex interaction zone shifts towards midspan for the contoured cases. The shift is well correlated with the shift in the under-turning hump, as discussed above. For this large aspect ratio blade the spanwise shift has a negligible effect on the blade row losses. However, as mentioned previously, for low aspect ratio blades the spanwise shift of the secondary flow may cause an adverse interaction with the secondary flow generated from the opposite endwall.

The overall integrated losses are shown in Figure 8.5 for the different purge levels. The inplane losses are shown in sub-figure (a) and the mixed-out losses are shown in sub-figure (b). For reference the absolute losses and other integrated quantities are given in Table 8.1. The normalized flow quantities relative to the RF 000 case are given in Table 8.2.

In Figure 8.5 (a) the contoured configuration shows lower overall integrated losses for all positive purge flow cases. For the zero net purge flow case, the contoured case shows slightly 
higher losses. As previously mentioned, the contouring design was intended for cases with zero net purge flow. As a result, the contouring has a negative impact on the endwall flow when there is no net purge flow.

(a)

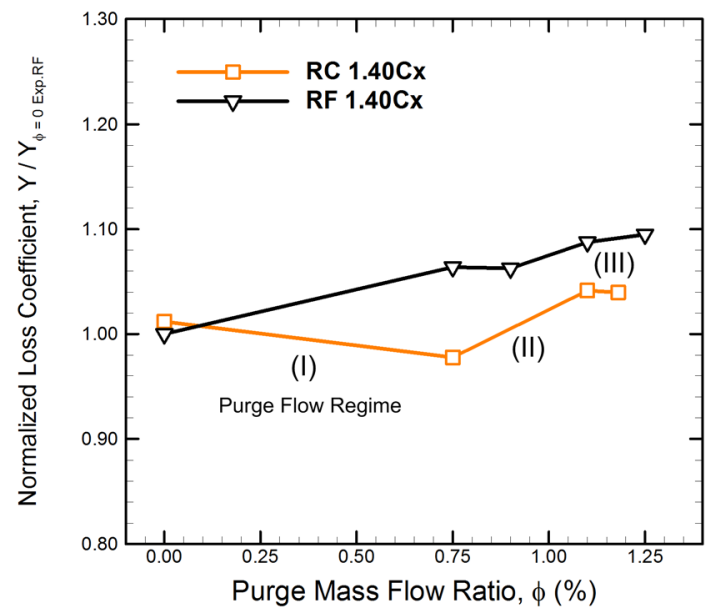

(b)

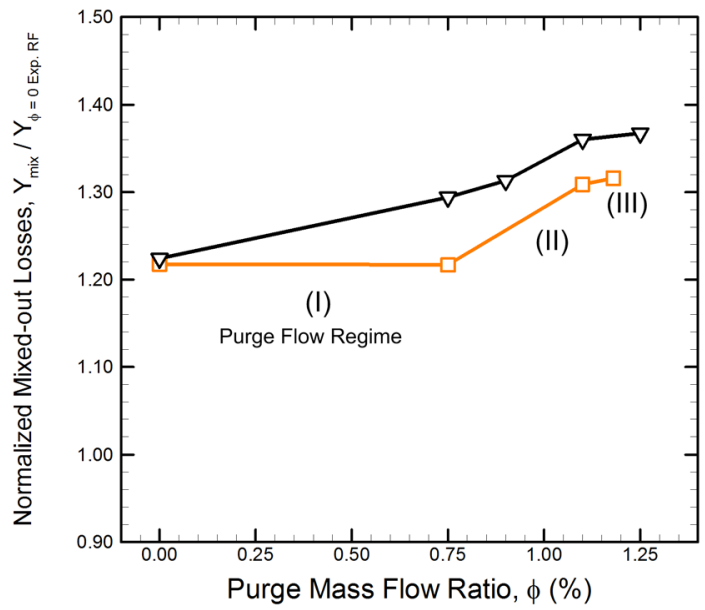

Figure 8.5: Overall $(Y)$ and Mixed-out $\left(Y_{\text {mixed }}\right)$ loss coefficients for different levels of purge flow at $1.40 C_{x}$ for the RF and EF configurations

Table 8.1: $\quad$ Summary of the measured overall integrated flow quantities

\begin{tabular}{|c|c|c|c|c|}
\hline Flow Quantity & RC 000 & RC 075 & RC 110 & RC 118 \\
\hline $\mathrm{Y}$ & 0.052 & 0.050 & 0.053 & 0.053 \\
\hline$Y_{p}$ & 0.030 & 0.030 & 0.030 & 0.030 \\
\hline $\mathrm{Y}_{\mathrm{sec}}$ & 0.022 & 0.020 & 0.023 & 0.023 \\
\hline$\beta_{2}{ }^{\prime \prime}$ & -72.1 & -72.0 & -72.0 & -72.0 \\
\hline$\beta_{2, \text { midspań }}$ & -73.2 & -73.1 & -73.1 & -73.1 \\
\hline $\mathrm{C}_{\mathrm{SKE}}$ & 0.051 & 0.062 & 0.068 & 0.071 \\
\hline$Y_{\text {mixed-out }}$ & 0.063 & 0.062 & 0.067 & 0.068 \\
\hline$Y_{p, \text { mixed-out }}$ & 0.031 & 0.031 & 0.031 & 0.031 \\
\hline$Y_{\text {sec, mixed-out }}$ & 0.032 & 0.032 & 0.036 & 0.037 \\
\hline
\end{tabular}


Table 8.2: Summary of the measured and predicted overall integrated flow quantities relative to the zero net purge flow case (RF 000)

\begin{tabular}{|c|c|c|c|c|}
\hline Flow Quantity & RC 000 & RC 075 & RC 110 & RC 118 \\
\hline $\mathrm{Y} / \mathrm{Y}_{\mathrm{RF}} 000$ (Exp.) & $100 \%$ & $97 \%$ & $103 \%$ & $103 \%$ \\
\hline$Y_{p} / Y_{R F} 000$ (Exp.) & $58 \%$ & $58 \%$ & $58 \%$ & $58 \%$ \\
\hline$Y_{\text {sed }} Y_{\text {RF } 000 \text { (Exp.) }}$ & $42 \%$ & $39 \%$ & $45 \%$ & $45 \%$ \\
\hline$\beta_{2^{\prime \prime}}$ & -72.1 & -72.0 & -72.0 & -72.0 \\
\hline$\beta_{2, \text { midspan' }}$ & -73.2 & -73.1 & -73.1 & -73.1 \\
\hline $\mathrm{C}_{\mathrm{SKE}}{ }^{\prime \prime}$ & 0.051 & 0.062 & 0.068 & 0.071 \\
\hline$Y_{\text {mixed-out }} / Y_{R F} 000$ (Exp.) & $120 \%$ & $120 \%$ & $129 \%$ & $130 \%$ \\
\hline$Y_{p, \text { mixed-out }} / Y_{R F} 000$ (Exp.) & $59 \%$ & $59 \%$ & $59 \%$ & $59 \%$ \\
\hline$Y_{\text {sec, mixed-out }} / Y_{\text {RF }} 000$ (Exp.) & $61 \%$ & $61 \%$ & $70 \%$ & $71 \%$ \\
\hline
\end{tabular}

At the $\phi=0.75$ level, the losses are $9 \%$ lower than the corresponding RF case. Relative to the RF 000 case the losses are reduced by 3\%. It should be noted that the RC 075 case did not exhibit the scattered data shown for the downstream plane of the RF 075 case. The $9 \%$ difference may therefore be exaggerated relative to the uncertain value for the RF 075 case. With increasing purge flow levels the losses increase but remain lower than the corresponding RF case by about $5 \%$ for the $\phi=1.10$. Similar to the RF configuration, at purge flow levels beyond $\phi=1.10$ the losses roughly level off. Overall, the integrated losses shown here indicate the best purge flow rate for the current design is near $\phi=0.75$. Additional data at different purge levels would help define the local minimum.

The losses for the contoured case also exhibit three different regimes as discussed in Section 7.8. As labelled, in the first regime (I) the losses decrease with additional purge flow. It is suspected that the purge flow alters the endwall flow to better suit the endwall contouring design. For example, the purge injection may increase the near endwall momentum thereby reducing unintended separations over the endwall curvature. In the second regime (II), the loss growth rate is the highest (steepest slope). The increased purge flow thickens the endwall boundary layer and therefore enhances the secondary losses. The third regime (III) shows the losses level off and are insensitive to increases in the purge level. As discussed previously, the 
purge level is sufficiently high to push the secondary flow away from the endwall. The displacement of the secondary flow away from the endwall thereby reduces the interaction of the passage vortex and the endwall boundary layer causing the losses to level off with additional purge flow injection.

Figure 8.5 (b) shows the corresponding mixed-out losses. As described in previous chapters, the mixed-out losses include the additional loss contribution from the dissipation of secondary kinetic energy and the static pressure recovery that occurs with downstream mixing. As shown, the mixed-out losses show the similar three-regimes as shown for the in-plane losses of sub-figure (a). For regime (I), the loss growth rate is nearly zero and the local minimum observed for the in-plane losses does not exist. As shown, at $\phi=0.75$, the mixed-out losses are nearly equal to the zero purge flow case. In sub-figure (a) the in-plane losses decrease by about $9 \%$ from the $\phi=0$ to 0.75 . A similar decrease is not realized at the fully mixed-out conditions because the secondary kinetic energy increases significantly. Table 8.1 shows the overall secondary kinetic energy coefficient increases by about $22 \%\left(C_{\mathrm{SKE}}=0.051\right.$ to 0.068$)$ from $\phi=0.00$ to 0.75 . At higher purge levels the increase is less significant. At $\phi=1.10$ and 1.18 , the secondary kinetic energy coefficient are 0.068 and 0.071 respectively. As a result, the mixed-out losses for Regime (II) and (III) increase proportionally relative to the flat endwall cases with increasing purge flow. Through regime (II) and (III) the losses of the contoured case are consistently about $5 \%$ lower than the corresponding RF cases.

In summary, the mixed-out loses of the contoured case show that contouring is effective at mitigating the secondary losses for cascades with positive net purge flow. The downstream floods of total pressure show that contouring reduces the peak losses within the counter vortex region. Since the counter vortex is formed through the interaction of the passage vortex and the blade trailing edge separation, the reduction of losses within the counter vortex region appears to be a consequence of a weaker passage vortex. The passage vortex, although weaker, showed only localized loss benefits. When averaging the results, the loss benefits associated with the passage vortex are offset by increases to the nearby losses. The secondary kinetic energy and pitchwise 
flow angle were only slightly affected by the contouring. The only significant negative effect of contouring was the increased penetration depth of the secondary flow.

The following sub-sections will examine the experimental endwall visualizations. In particular, the effects of contouring on the development of the endwall flow are described.

\subsubsection{Surface flow visualization}

Endwall surface flow visualizations for the contoured endwall configuration were made following the procedures of Section 3.10.5. The visualizations and flow field interpretations are shown in Figure 8.6. The low and high purge levels correspond to sub-figures (b) and (d). The corresponding flat endwall (RF) visualizations are shown for reference in sub-figures (a) and (c).

For reference the endwall contour geometry is shown in Figure 8.7. The colour flood shows the contour amplitudes relative to the baseline RF endwall surface expressed as a percentage of the axial chord $\left(\% C_{x}\right)$. The main features of the endwall design are labelled in

Figure 8.7. Section 3.5.3 describes the purpose of these features in detail. A brief summary is given below.

Near the entrance to the blade passage there are two "humps" labelled $\mathrm{H}_{1}$ and $\mathrm{H}_{2}$. The convex curvature of the humps creates a region of lower static pressure. The concave streamline curvature between the humps forms a trough $(\mathrm{T})$ with locally higher static pressure. Towards the passage exit there is a streamwise "ridge" $(\mathrm{R})$ which is used to guide the trajectory of the passage vortex. Each of the features is identified on the flow visualizations shown in Figure 8.7 (b) and (d). The following paragraphs describe the flow field interpretation and the effects of the contouring features. 
(a) RF 000

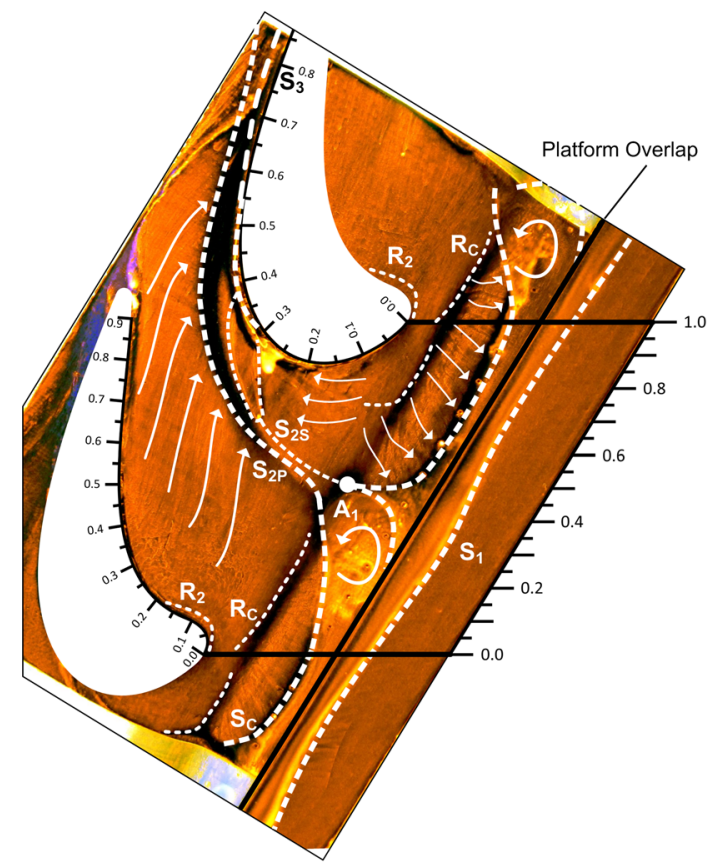

(c) RF 125

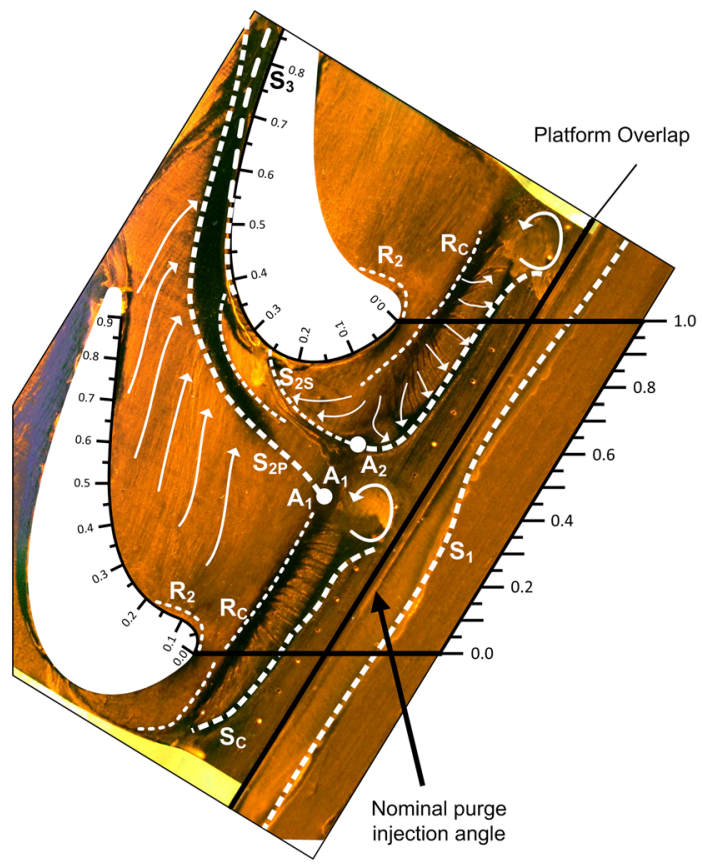

(b) RC 000

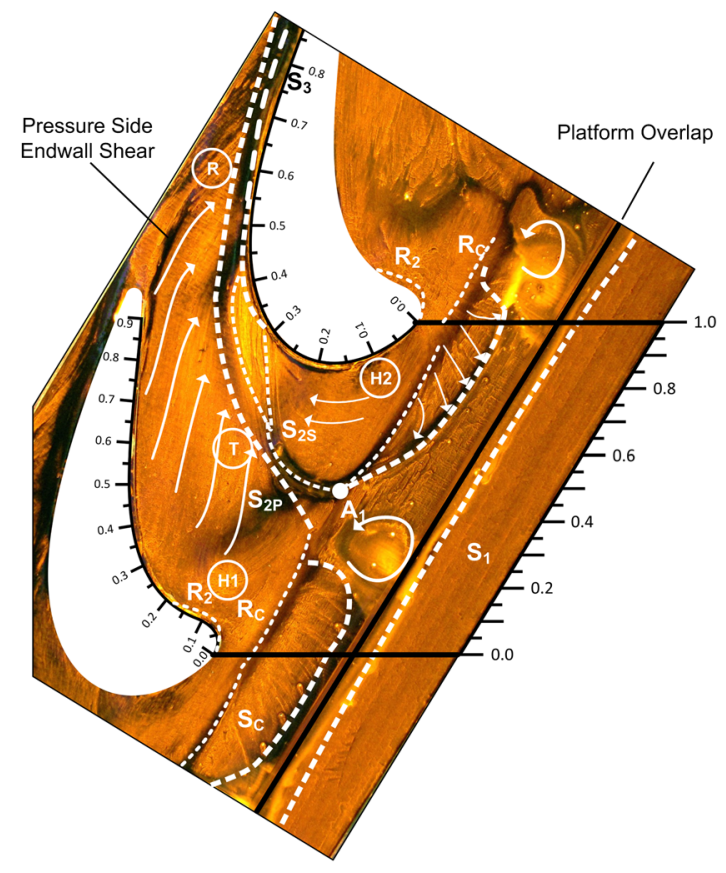

(d) RC 118

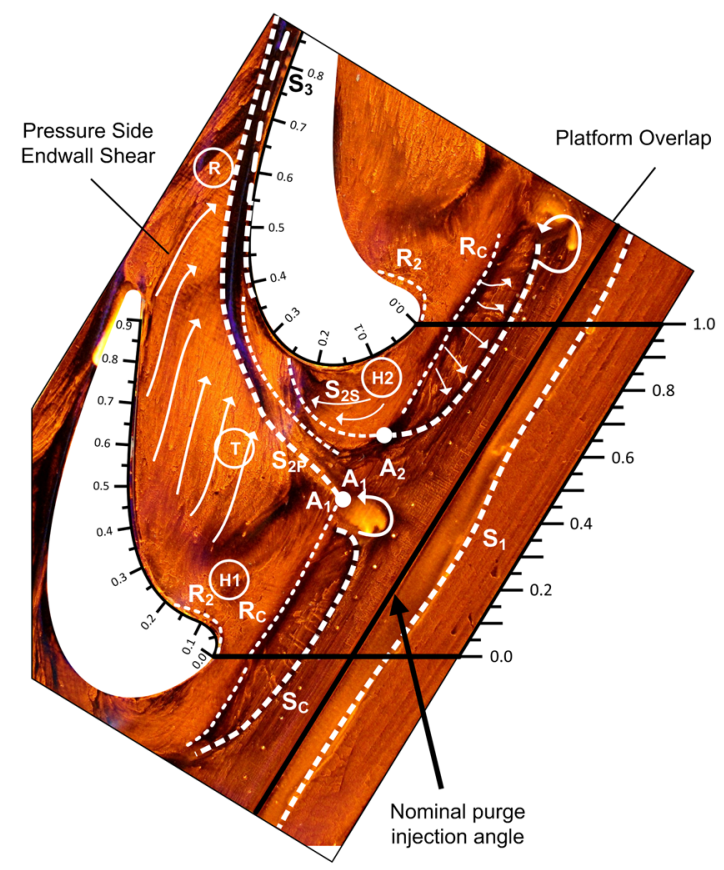

Figure 8.6: Endwall surface visualization and interpretation for the $\mathrm{RC}$ configuration 


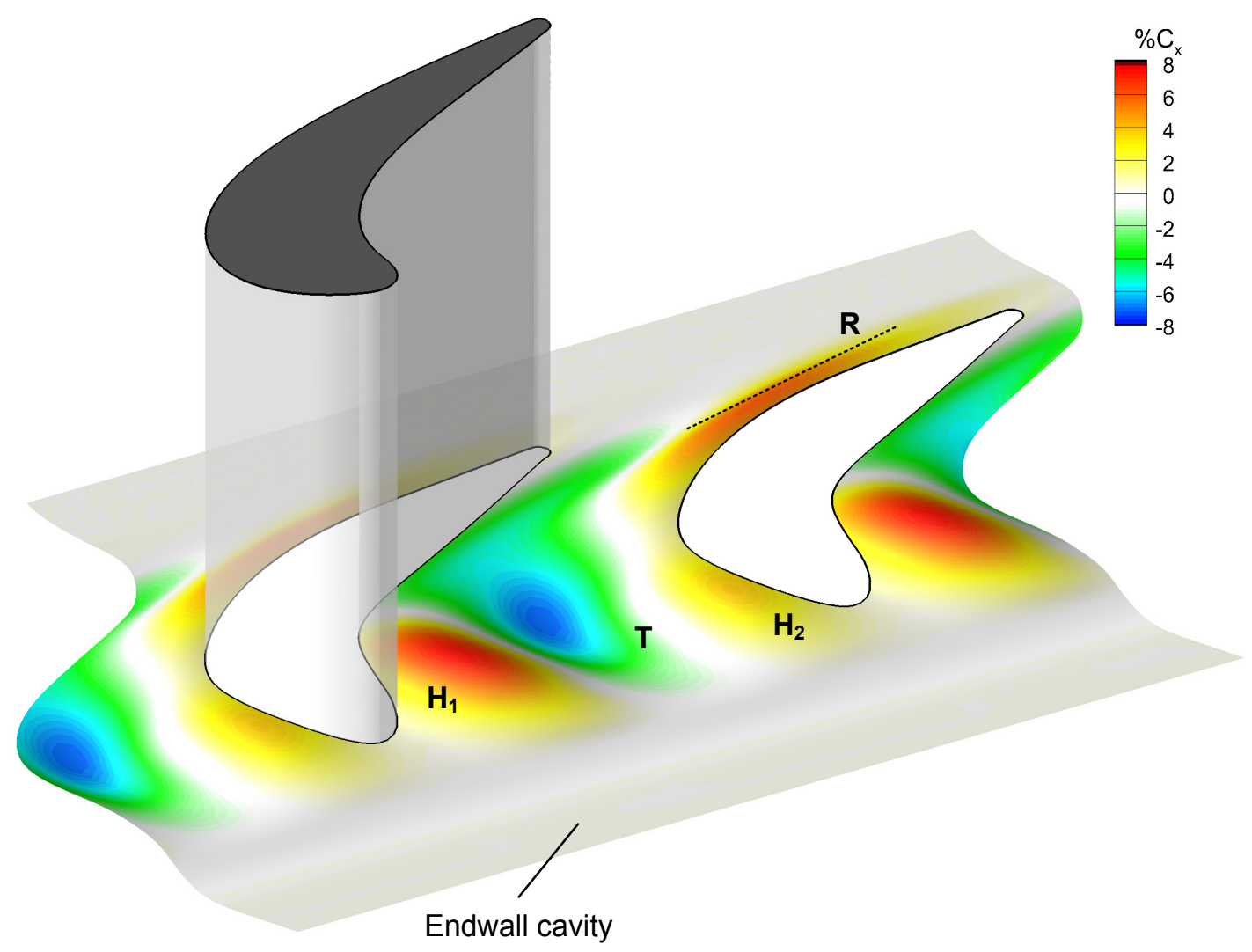

Figure 8.7: Endwall geometry for the RC configuration

Figure 8.6 (b) shows the zero net purge flow visualization and interpretation for the contoured case (RC). As indicated, the flow enters from the right of the figure. Similar to the flat RF case shown in Figure 8.6 (a) the inlet boundary endwall layer separates from the platform overlap, as indicated by the $S_{1}$ separation line. The separation appears to be delayed in the contoured case as the $\mathrm{S}_{1}$ line is positioned more closely to the edge of the platform overlap. A weaker adverse pressure gradient in this region may be the cause for the delayed separation. As will be discussed, the contouring features lead to an altered pressure gradient in the forward portion of the blade - the likely cause for the delayed $S_{1}$ separation.

The axial position of the $S_{1}$ separation also appears to depend on the purge mass flow rate. Consider the low and high purge flow rate cases for both the RF and RC configurations. At the 
high purge flow level the $S_{1}$ separation occurs further upstream. Figure 8.8 shows a schematic interpretation of the endwall cavity flow field. The view is oriented perpendicular to the edge of the platform overlap. Sub-figure (a) represents the zero-net purge flow case and sub-figure (b) the high purge level. The interpretation in Figure 8.8 draws on the discussions of CFD flow field of Figure 7.18. As shown in Figure 8.8 (a), the flow enters from the right and the inlet endwall boundary separates prior to the termination of platform overlap. The cause of the early separation is related to the size and position of the endwall cavity reticulation. The potential field of the recirculation is felt above the platform overlap and causes the endwall boundary layer to separate from the platform. It would be unphysical for the separation to occur prior to the platform edge if the bladerow and flow passage did not exist. It is evident that the adverse pressure gradient of the bladerow alters the cavity flow field such that the recirculation is sensed upstream of the platform overlap edge.

(a) RF 000

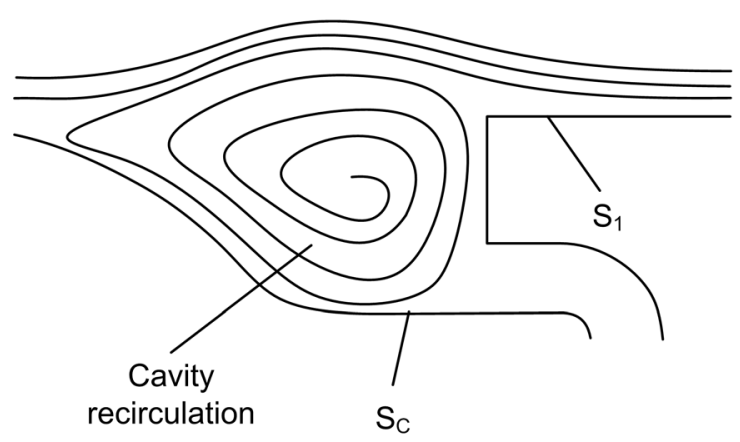

(b) RF 125

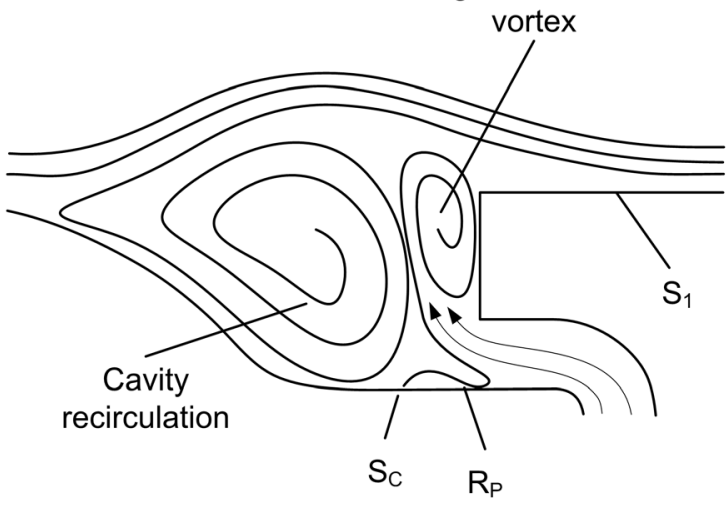

Figure 8.8: Flow field endwall cavity interpretation

In the higher purge flow case of Figure 8.8 (b), the purge injection enhances the adverse barrier for the inlet endwall boundary layer. As shown, the location of the $\mathrm{S}_{1}$ separation is further upstream as the purge flow counter vortex is formed and protrudes into the main passage.

Returning to the zero-purge flow case of Figure 8.6 (b), the separated inlet boundary layer is shown to reattach to the endwall along reattachment line, Rc. The flow is directed into the 
endwall cavity and the endwall cavity recirculation is formed. The position of the Rc reattachment line is further upstream than in the RF case. It appears that the $\mathrm{H}_{1}$ protrusion deflects a portion of the inlet boundary layer into the cavity region, thereby moving the reattachment upstream. Similar to the RF case, the recirculation pushes the endwall fluid further into the endwall cavity and the platform overlap induces the endwall separation along Sc. The cavity fluid is pushed up into the passage at the platform overlap and mixes with the separated inlet-boundary layer.

At the reattachment line Rc, a portion of the separated inlet endwall boundary layer is diverted into the passage rather than into the endwall cavity. The fluid entering the passage must pass over the $\mathrm{H}_{1}$ hump and then into the main passage. The $\mathrm{H}_{1}$ hump lowers the static pressure and locally reduces the cross-passage pressure gradient. As shown, the $\mathrm{S}_{2 \mathrm{P}}$ separation line is positioned closer to the centre of the passage than in the RF case indicating a lower blade-toblade cross-passage pressure gradient in the forward portion of the passage. The reduced pressure gradient reduces the endwall cross flow, which results in a weaker passage vortex. The downstream vorticity floods shown in Figure 8.2 show the passage vortex is attenuated for the contouring cases.

The development of the downstream secondary flow is discussed in the following paragraphs. Compared with the RF case, the $\mathrm{RC}$ case has a similarly positioned saddle point, $\mathrm{A}_{1}$, which marks the interaction of both legs of endwall cavity re-circulation as they turn downstream. The suction side leg of the re-circulation separates along, $\mathrm{S}_{2 \mathrm{~S}}$, which emanates from the saddle point $\mathrm{A}_{1}$. The endwall humps, $\mathrm{H}_{1}$ and $\mathrm{H}_{2}$, appear to funnel the legs of the endwall cavity towards the endwall trough $(\mathrm{T})$. Both humps induce lower static pressures while the trough is a region of locally higher static pressure. The local acceleration of the $\mathrm{H}_{1}$ hump is essential to reducing the cross-passage pressure gradient in the early portion of the passage. The higher momentum fluid is less easily turned and fed into the passage vortex. The $\mathrm{H}_{2}$ hump deflects the suction side leg away from the blade surface and towards the trough $(\mathrm{T})$. The higher static pressure of the trough 
lowers the endwall flow velocities and again reduces flow that feeds the passage vortex growth. As a result, the overall secondary flow is attenuated by the weaker passage vortex.

It is beneficial to have the $S_{2 S}$ separation positioned away from the blade suction surface in order to delay the interaction with the blade suction surface boundary layer. In Section 7.9, it was shown that the downstream losses increase significantly with higher endwall shear levels at the interaction location. As shown here in Figure $8.6(\mathrm{~b})$, the $\mathrm{S}_{2 \mathrm{~S}}$ line appears to intersect the blade suction surface at a suction surface length $\left(\mathrm{SS} / \mathrm{SS}_{\max }\right)$ of about 0.3 . Compared to the RF case in Figure 8.6 (a) the intersection is delayed and is positioned slightly further downstream. Also, for the contoured case, the endwall shear levels appear lower. As discussed in Section 7.9, the lower shear levels are evidence of attenuated secondary flow features. Consistent with the downstream measurements, the lower shear levels of the contoured case here are correlated with the weaker passage vortex shown in the downstream results.

As mentioned above, the pressure-side leg of the endwall cavity re-circulation, marked by the $S_{2 P}$ separation, is positioned closer to the pressure side of the passage. The passage-vortex lift-off line emanates from the $\mathrm{S}_{2 \mathrm{P}}$ line. As shown, the shear levels of the passage vortex track are much lower than in the RC case than the RF case, as indicated by the reduced dark regions along the $S_{2 P}$ line. It appears that the endwall ridge, $R$, reduces the endwall shear in the near endwall region. Again, the lower shear levels are consistent with the reduced strength of the passage vortex shown on the downstream measurement planes.

To this point the endwall visualizations for the zero purge flow cases have shown several benefits of the contouring. However, as shown in Figure 8.5 (a) the overall in-plane losses are actually slightly higher for the zero flow case, RC 000. Although contouring effectively reduces the losses associated with the passage vortex, the near endwall losses increase significantly resulting in no net benefit for the zero purge flow case. More specifically, Figure 8.1 (a) shows the near endwall losses are significantly elevated. As mentioned in Section 8.5.2, the contouring design is perhaps not optimum at the low purge flow level. The endwall visualization of Figure 8.6 (b) shows a potential source of the higher endwall losses. Near the pressure side of the 
passage at the blade trailing edge there is a high shear region, labelled "pressure side endwall shear". As shown, the high shear region appears to emanate from the blade pressure surface near the trailing edge. The elevated shear levels appear to correlate well with the higher near endwall losses shown on the downstream measurement plane of Figure 8.1 (b). Also, a similar trace is not shown for the RF configuration at either the zero or high purge level. The contoured geometry appears to induce unintended loss generation in the vicinity of this high shear region.

The endwall visualizations for the high purge flow cases are shown in Figures 8.6 (c) and (d). The contoured configuration is shown in sub-figure (d) and the RF configuration is shown for reference in sub-figure (c). In general, the endwall contouring with purge flow appears to alter the endwall flow field similarly to the zero purge flow case. The notable similarities and minor differences between the low and high purge flow case are summarized as follows:

- The $\mathrm{S}_{1}$ separation is delayed for the contoured cases compared to corresponding RF cases. The contouring alters the pressure field sensed by the inlet boundary layer. The separation occurs further upstream for the cases with purge flow.

- The reattachment line of the inlet endwall boundary layer, Rc, is positioned further upstream in the contoured cases relative to RF configurations. With purge injection the position of the reattachment line is similar to the corresponding configuration without purge injection.

- The endwall cavity separation, $\mathrm{S}_{\mathrm{C}}$, is positioned further downstream from the purge injection slot for cases with net purge injection. Thus, the endwall cavity recirculation is positioned further downstream.

- The high purge flow cases have similarly positioned saddle points, $A_{1}$ and $A_{2}$. Both RF and $\mathrm{RC}$ cases with purge injection are different from the corresponding zero purge flow cases that show single saddle points.

- The $\mathrm{S}_{\mathrm{P} 2}$ separation of the contoured case (RC 118) emanates from the $\mathrm{A}_{1}$ saddle point similar to the RC 125 case. The lift-off line of the passage vortex follows a similar 
trajectory to RF 125 case. However, the shear levels along the passage vortex trace are significantly reduced in the RC 118 case relative to the RF 125 case.

- The $\mathrm{S}_{2 \mathrm{~S}}$ separation of the contoured case (RC 118) emanates from the $\mathrm{A}_{2}$ saddle point similar to the RC 125 case. The separation line intersects the blade suction surface at nearly the same position as in the RF case. However, the shear levels are notably lower for the contoured case.

- Lastly, near the pressure side of the passage at the blade trailing edge a high shear region is observed. Relative to the zero purge flow case the shear levels for the high purge flow case are considerably lower.

The corresponding surface flow visualizations shown here indicate the contouring geometry generally attenuates the passage vortex while also reducing the $S_{2 S}$ interaction with the blade suction surface. These two actions result in lower losses associated with the passage and counter vortices at the downstream measurement planes.

For the zero net purge injection case, RC 000, the contouring case does not show a loss benefit. Although the passage vortex and peak losses are lower than in the RF 000 case, the endwall losses are significantly higher as shown in pitchwise averaged losses of Figure 8.4 (a). At the higher purge levels, the contouring shows significant loss benefits relative to the corresponding RF cases. By examining the spanwise loss distribution of Figures 8.4 (a) though (c) it is evident that the loss benefit arising from the passage and counter vortex regions is a similar magnitude for all of the cases. The implication is that the contouring affects the losses away from the endwall similarly for all purge levels. The endwall visualizations of the contoured cases support this as the zero and high purge flow cases appear to alter the flow field in a similar manner, as noted by the summary list above. In fact, the endwall visualizations also provide evidence of why the higher purge flow cases have lower near endwall losses. As discussed above, the identified high shear region near the blade passage exit is significantly larger for the zero purge flow case. 
In summary, the endwall contouring geometry for this cascade shows significant benefits at the mid-to-high purge levels. In general, the contouring effectively attenuates the passage vortex. The contoured humps in the forward portion of the passage reduce the rotational motion of the pressure and suction side legs of the endwall cavity recirculation. The trough feature passes through the centre of the passage and locally increases the static pressure. The momentum of the endwall fluid that feeds the passage vortex is reduced. The attenuated passage vortex reduces the loss generation through the passage and lowers the overall losses at the downstream plane.

\subsection{The Effects of an Elliptical Platform Overlap on the Secondary Flow of the Linear Turbine Cascade with Flat Intra-passage Endwalls}

\subsubsection{Introduction}

The following sub-sections present the experimental results from the EF (elliptical flat) case for similar purge flow levels to those of the previous chapter. The downstream results from the $1.40 C_{x}$ measurement plane are presented in the first sub-section. The downstream flow field is described by the contour floods of the total pressure, streamwise vorticity and secondary kinetic energy coefficients. The overall integrated flow quantities are also discussed. The subsections conclude with an interpretation of the endwall flow using the surface flow visualizations. Explanations for the observed differences between the rectangular and platform overlap cases are discussed together with the endwall visualizations.

\subsubsection{The Downstream Flow Field with an Elliptical Platform Overlap}

Contour floods of the total pressure coefficient measured at the $1.40 C_{x}$ plane are shown in Figure 8.9. Three representative purge levels are shown: $\phi=0.00,1.10$ and 1.25 . The results corresponding to the elliptical platform overlap case (EF) are shown on the right-hand side, sub- 
figures ( $b, d$ and $f$ ), while the rectangular overlap cases are shown on the left-hand side, subfigures (a, $\mathrm{c}$ and e). Figures 8.10 and 8.11 show the corresponding floods of the streamwise vorticity and secondary kinetic energy coefficients.

Figure 8.9 shows that the effects of the elliptical overlap are subtle. The differences between floods for the RF and EF configurations are difficult to distinguish. For the zero net purge flow case, EF 000, the peak losses associated with the passage vortex are slightly lower, as labelled. Closer to the endwall however the losses are higher than for the RF 000 case and the overall size of the secondary flow is larger. Thus, when averaging the in-plane losses, the reduction of the peak losses is offset by the higher near endwall losses and larger secondary flow. The next sub-section will discuss the overall integrated losses in more detail.

With the addition of positive net purge flow the effects of the elliptical overlap remain subtle. Similar to the EF 000 case the peak losses within the passage vortex are reduced while the overall size of the secondary flow is larger. Interestingly, all of the RF cases show a pocket of low losses in the near endwall region. This low loss lobe is similar to that observed in the contoured cases (RC) of the previous section. The pocket for the RF 125 and EF 125 cases are labelled in sub-figure (e) and (f). In the RF 125 case this pocket is significantly larger than in the EF 125 case. In general, it appears that the smaller pocket of low losses observed for the EF configuration tends to offset the slight reduction of peak losses within the passage vortex. Thus, the overall integrated losses are generally very similar between the RF and EF configurations. Also, recall from Section 8.5.2 that the low loss lobe of the contouring case is enhanced by purge injection leading to a larger region of lower losses for the higher purge flow cases. Similar behaviour is shown here for the EF configuration.

The other flow quantities are also relatively insensitive to the elliptical overlap. The floods of streamwise vorticity coefficient shown in Figure 8.10 only show subtle differences between the $\mathrm{EF}$ and RF cases. For the zero net purge flow case, the passage vortex is closer to the endwall with slightly lower peak streamwise vorticity. The passage vortex fluid appears stretched over the passage compared to the RF 000 case which is consistent with the larger loss contours 
associated with the secondary flow shown in Figure 8.9 (b). As the purge flow level increases the streamwise vorticity associated with the passage vortex is more similar to the RF 000 case in terms of the size, shape, position and the level of vorticity. At the highest purge level, the floods of streamwise vorticity coefficient are very similar, suggesting the elliptical overlap has little effect on the flow structures at the highest purge levels.

Figure 8.11 shows the corresponding floods of the secondary kinetic energy coefficient with overlaid secondary velocity vectors. Similar to the floods of the streamwise vorticity coefficient the zero net purge flow case shows subtle differences between the EF and RF configurations. Within the vortex interaction zone, the region where the passage and counter vortex interact, the EF 000 case has lower values of secondary kinetic energy. With increasing purge flow, the differences within the vortex interaction zone, between the EF and RF cases, diminish.

Based on the loss contours alone the elliptical overlap does not appear to be an effective secondary loss mitigation technology for this particular cascade. The similarities of the streamwise vorticity and secondary kinetic energy floods show the elliptical overlap has a minor effect on the secondary flow structures. Also, the EF configuration shows no auxiliary benefits that can be realized with downstream mixing. Chapter 5 showed that a reduction of the measured in-plane secondary kinetic energy can translate to loss reductions at the fully mixed-out conditions. As shown here, the secondary kinetic energy for the EF configuration is largely unaffected. In general, for the purge levels shown here the effects of the EF configuration are quite subtle and rather insignificant.

The following sub-section discusses the integrated losses and other flow quantities. Also, an anomaly in the loss behavior at some flow rates is discussed. 
Rectangular Overlap

(a)

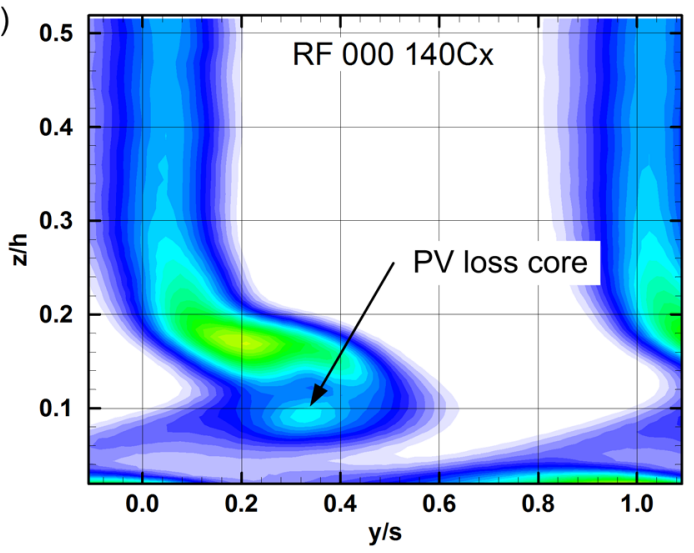

(c)

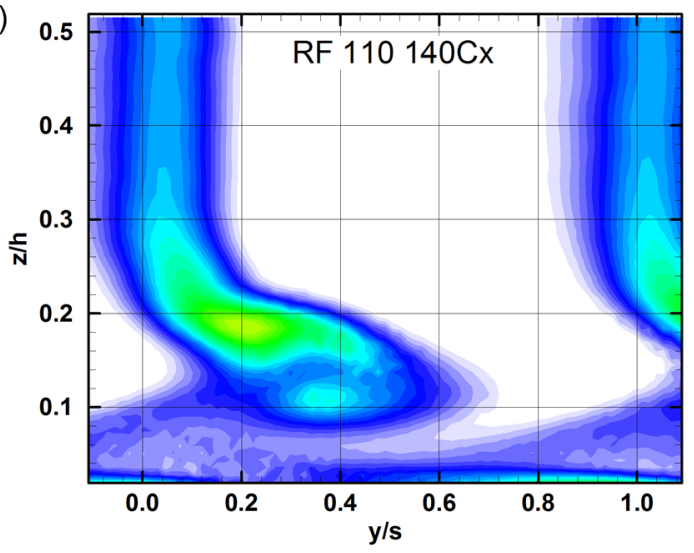

(e)

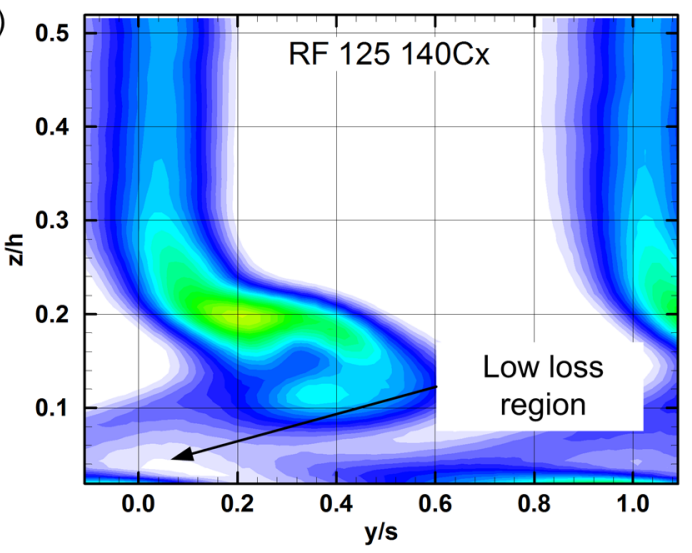

Elliptical Overlap

(b)

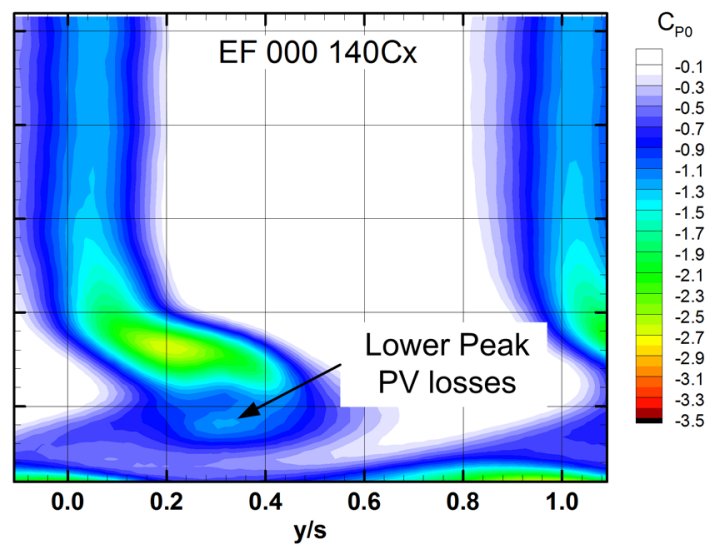

(d)

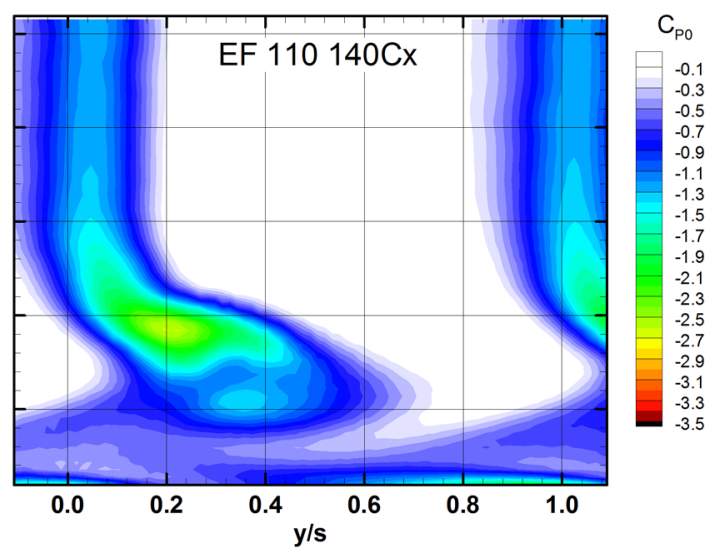

(f)

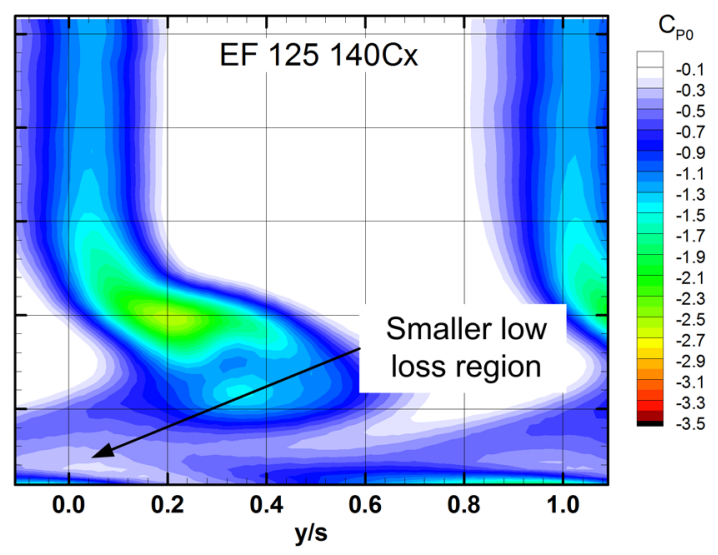

Figure 8.9: Floods of the total pressure coefficient $\left(C_{P 0}\right)$ for varying levels of purge flow measured at $1.40 C_{x}$ for the RF and EF platform overlap configurations 
Rectangular Overlap

(a)

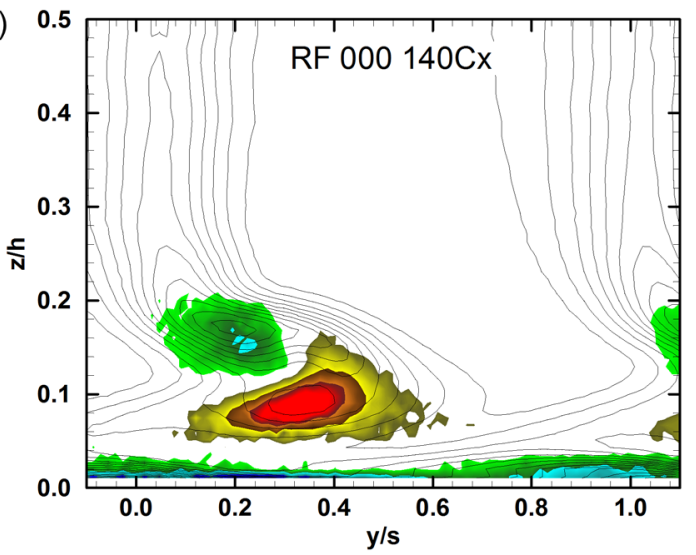

(c)

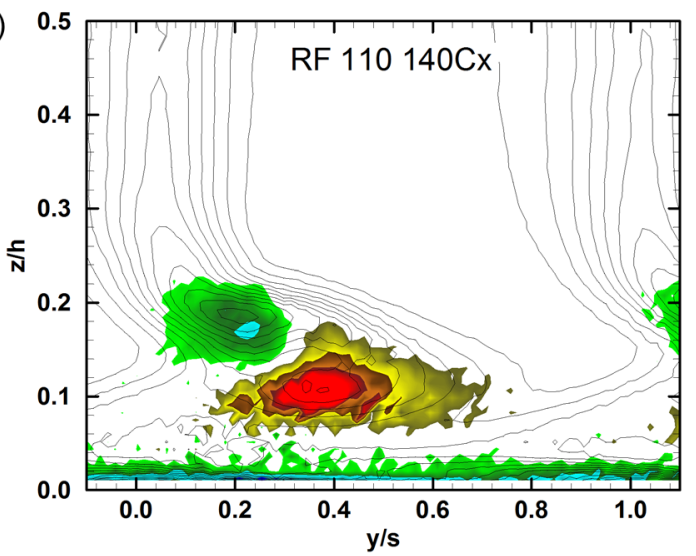

(e)

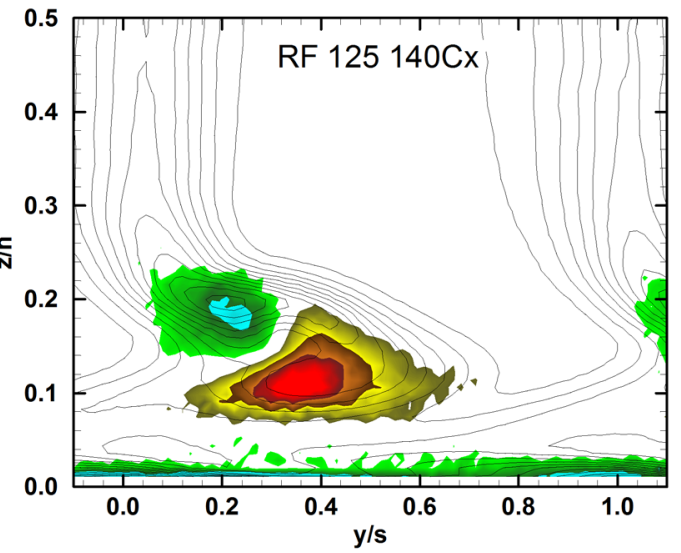

Elliptical Overlap

(b)

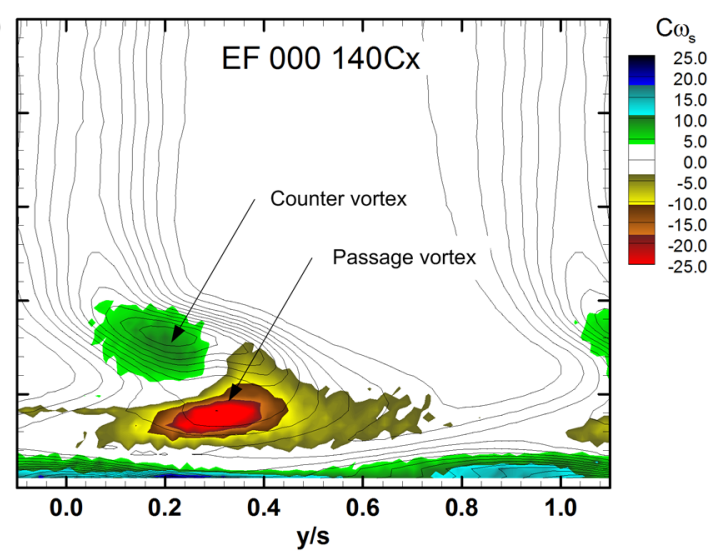

(d)

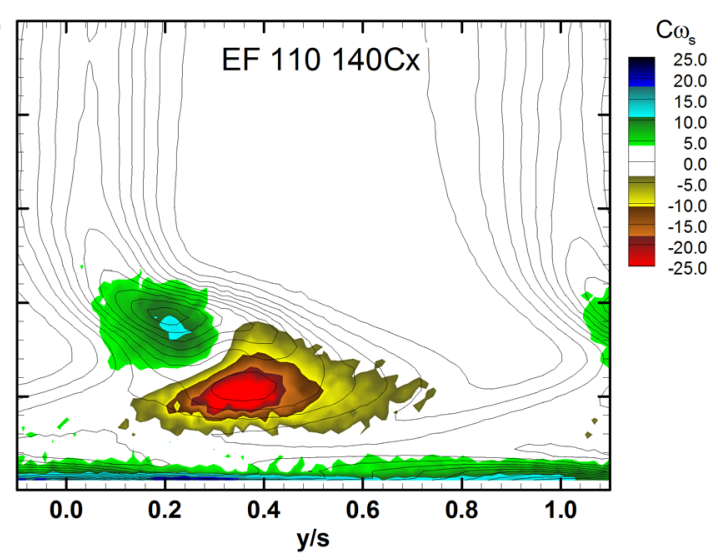

(f)

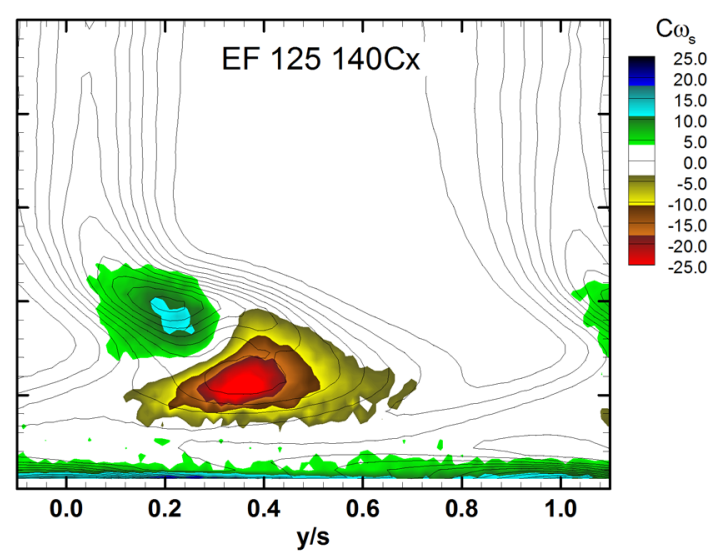

Figure 8.10: Floods of the streamwise vorticity coefficient $\left(C_{\omega S}\right)$ with contour lines of the total pressure coefficient $\left(C_{P 0}\right)$ for varying levels of purge flow measured at $1.40 C_{x}$ for the $\mathrm{RF}$ and $\mathrm{EF}$ platform overlap configurations 
Rectangular Overlap

(a)

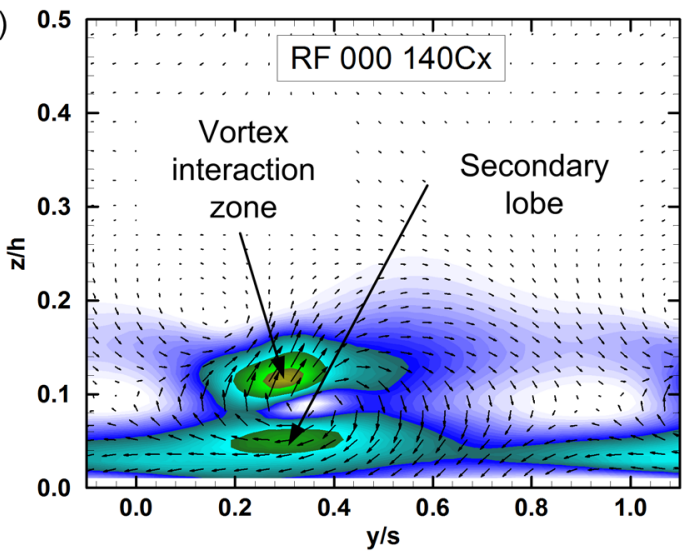

(c)

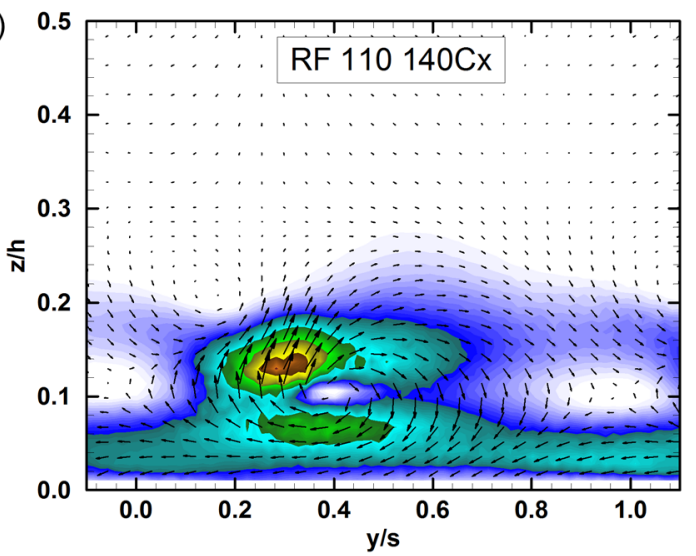

(e)

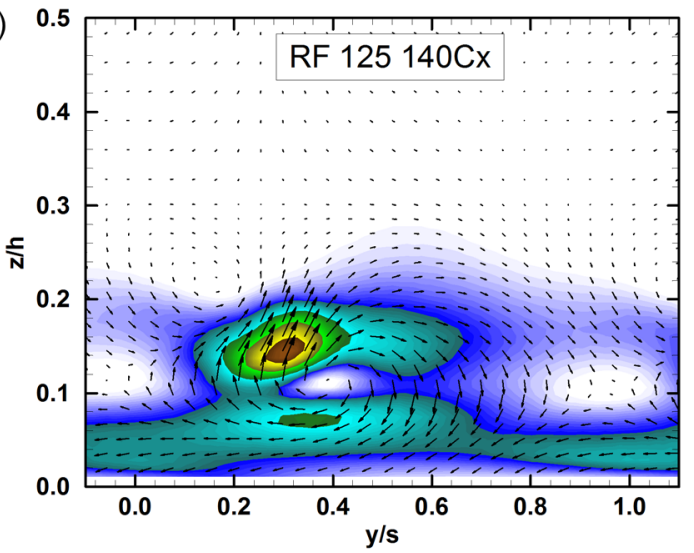

Elliptical Overlap

(b)

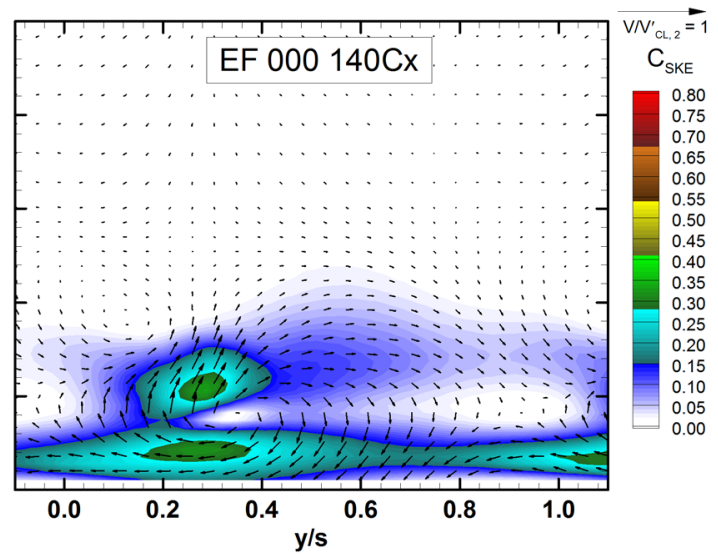

(d)

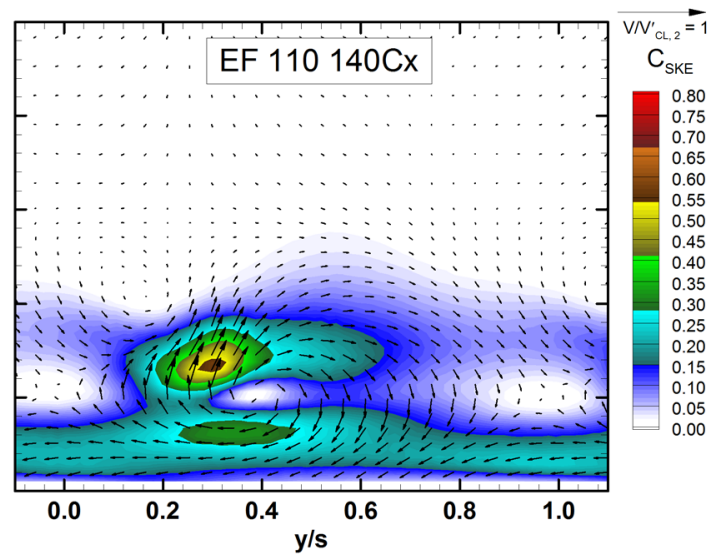

(f)

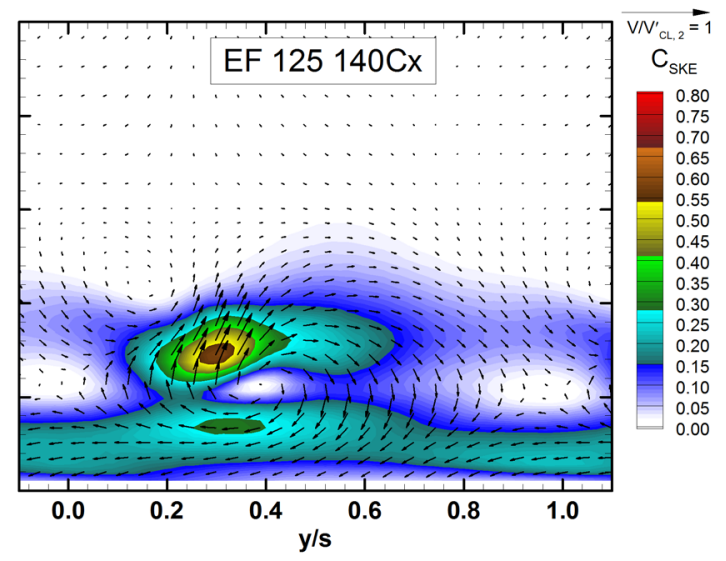

Figure 8.11: Floods of the secondary kinetic energy coefficient $\left(C_{S K E}\right)$ with the secondary velocity vectors overlaid for varying levels of purge flow measured at $1.40 C_{x}$ for the $\mathrm{RF}$ and $\mathrm{EF}$ platform overlap configurations 


\subsubsection{Integrated Flow Quantities for the Elliptical Platform Overlap Configuration}

The normalized overall integrated losses for the EF and RF configurations are shown in Figure 8.12. All of the loss values are normalized by the overall losses of the RF 000 case $\left(Y_{\mathrm{RF} 000}\right)$. In-plane losses are shown in sub-figure (a) and the mixed-out values are shown in subfigure (b). The absolute losses and other flow quantities are given in Table 8.3, while the normalized flow quantities are given in Table 8.4.

In Figure 8.12 (a) the elliptical zero-net purge flow case (EF 000) has in-plane losses that are $1.5 \%$ higher than for the rectangular case. At the next purge level, $\phi=0.75$, the EF losses remain consistently higher than the RF case by about $1.5 \%$. With further increases in the purge level there is an obvious diversion of the losses for the $\phi=0.90$ purge level. For the EF 090 case the losses increase to a peak while the losses for the RF configuration dip forming a local trough. This was unexpected and the data were initially thought to be erroneous. To investigate further two additional cases were measured for the EF geometry for $\phi=0.85$ and 0.95 . As shown, the corresponding losses bracket the peak value shown at $\phi=0.90$. Additionally, corresponding cases were measured for the $1.20 C_{x}$ plane. The normalized losses are also shown in Figure 8.12. The data from the $1.20 C_{x}$ plane show a similar peak that is consistent with the $1.40 C_{x}$ cases. It therefore appears that at this purge level the EF geometry has the effect of increasing the losses.

The observed peak for the EF losses occurs near the $\phi=1.00$ purge level. In Chapter 7, scattered data were observed for the purge levels between $\phi=0.75$ and 1.00 for the RF configuration. Erroneous losses were obtained for cases with significant scatter $(\phi=0.75)$. It was suggested that unsteadiness was generated within the endwall cavity and affected the downstream measurements. For the EF configuration shown here the scattered data were not observed.

To confirm the smoothness of the data at the $\phi=0.90$ level, Figure 8.13 (a) and (b) shows the total pressure coefficient floods for the RF 090 and EF 090 cases. The data is quite smooth for both cases. The EF case shows an enhanced secondary flow with a larger loss region associated with the passage vortex. Thus, for the EF 090 case the enhanced passage vortex 
appears to be the main contribution to the higher overall integrated losses. The total pressure flood for the EF 090 case shows no irregularity and the data appears free of significant error. Furthermore, the additional cases measured at $\phi=0.85$ and 0.95 , not shown here for brevity, also have enhanced losses in the passage vortex region. Therefore, the peak observed in the overall integrated losses at the $\phi=0.90$ purge level appears to be a real phenomenon.

(a)

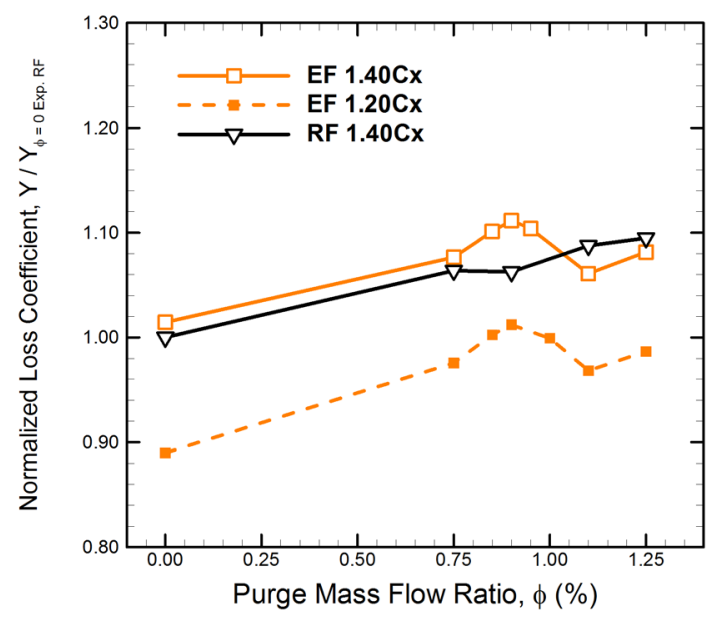

(b)

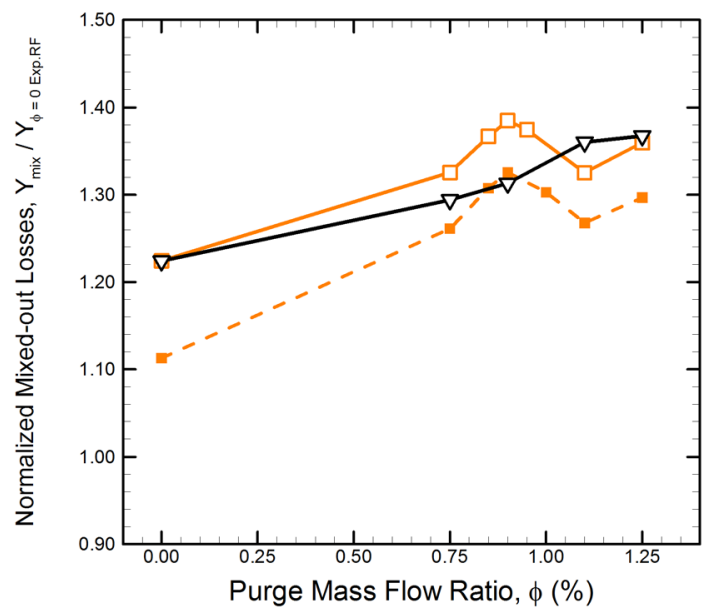

Figure 8.12: Overall $(Y)$ and Mixed-out $\left(Y_{\text {mixed }}\right)$ loss coefficients for different levels of purge flow at $1.40 C_{x}$ for the $R F$ and $E F$ configuations

The corresponding floods of the streamwise vorticity coefficient are shown in sub-figures (c) and (d). The passage vortex of the EF configuration is located further away from the endwall and towards the centre of the passage, an undesirable characteristic for a loss mitigation technology. The displaced passage vortex promotes mixing with the low loss free stream fluid and may lead to overall higher losses. The peak vorticity and the overall size of the passage vortex are similar to the RF case. It appears that the most significant effect of the elliptical overlap is to alter the position of the passage vortex. It is possible the enhanced mixing with the free stream has led to the higher losses observed in passage vortex region.

Overall, the observed peak of the integrated losses around the $\phi=0.90$ purge level (Figure 8.12) is well supported by the in-plane measurements of Figure 8.13. Since, the elliptical 
geometry is designed to alter the endwall cavity recirculation, the significant increase in losses at the $\phi=0.90$ suggests the purge flow significantly enhances the endwall cavity flow field. In Section 8.5.4, the RF and RC cases showed that the inlet boundary layer separates prior to the platform leading edge along the $\mathrm{S}_{1}$ separation line. The axial location of the separation was shown to be sensitive to the purge flow level. It was suggested that the pressure field of the passage and endwall cavity alters the size and position of the endwall cavity recirculation. The inlet boundary layer is then able to sense the potential field of the recirculation and separates prior to the platform edge. Here, at the $\phi=0.90$ purge level, it appears the endwall cavity recirculation is enhanced to a level that causes the losses to reach a local maximum. It is fitting that if the potential field of the bladerow and endwall cavity can affect the inlet boundary layer of a rectangular overlap, the elliptical overlap could quite possibly be more severely affected. Section 8.6.4 will further examine possible explanations for the ineffectiveness of the elliptical overlap with the aid of the endwall flow visualizations.

Table 8.3: $\quad$ Summary of the measured overall integrated flow quantities

\begin{tabular}{|c|c|c|c|c|c|}
\hline Flow Quantity & EF 000 & EF 075 & EF 090 & EF 110 & EF 125 \\
\hline $\mathrm{Y}$ & 0.052 & 0.055 & 0.057 & 0.054 & 0.056 \\
\hline$Y_{p}$ & 0.031 & 0.030 & 0.030 & 0.030 & 0.030 \\
\hline $\mathrm{Y}_{\mathrm{sec}}$ & 0.021 & 0.025 & 0.027 & 0.024 & 0.025 \\
\hline$\beta_{2}^{\prime \prime}$ & -72.3 & -72.3 & -72.2 & -72.1 & -72.2 \\
\hline$\beta_{2, \text { midspan' }}$ & -73.3 & -73.5 & -73.4 & -73.4 & -73.5 \\
\hline $\mathrm{C}_{\mathrm{SKE}}{ }^{\prime \prime}$ & 0.043 & 0.059 & 0.067 & 0.065 & 0.067 \\
\hline$Y_{\text {mixed-out }}$ & 0.063 & 0.068 & 0.071 & 0.068 & 0.070 \\
\hline$Y_{p, \text { mixed-out }}$ & 0.032 & 0.031 & 0.031 & 0.031 & 0.031 \\
\hline$Y_{\text {sec, mixed-out }}$ & 0.031 & 0.037 & 0.040 & 0.037 & 0.039 \\
\hline
\end{tabular}


Table 8.4: Summary of the measured overall integrated flow quantities relative to the zero net purge flow case (RF 000)

\begin{tabular}{|c|c|c|c|c|c|}
\hline Flow Quantity & EF 000 & EF 075 & EF 090 & EF 110 & EF 125 \\
\hline $\mathrm{Y} / \mathrm{Y}_{\mathrm{RF}} 000$ (Exp.) & $100 \%$ & $106 \%$ & $110 \%$ & $105 \%$ & $107 \%$ \\
\hline$Y_{p} / Y_{R F} 000$ (Exp.) & $59 \%$ & $58 \%$ & $58 \%$ & $58 \%$ & $58 \%$ \\
\hline $\mathrm{Y}_{\mathrm{sec}} / \mathrm{Y}_{\mathrm{RF} 000 \text { (Exp.) }}$ & $41 \%$ & $48 \%$ & $51 \%$ & $47 \%$ & $49 \%$ \\
\hline$\beta_{2}^{\prime \prime}$ & -72.3 & -72.3 & -72.2 & -72.1 & -72.2 \\
\hline$\beta_{2, \text { midspan }}{ }^{\prime}$ & -73.3 & -73.5 & -73.4 & -73.4 & -73.5 \\
\hline $\mathrm{C}_{\mathrm{SKE}}{ }^{\prime \prime}$ & 0.043 & 0.059 & 0.067 & 0.065 & 0.067 \\
\hline $\mathrm{Y}_{\text {mixed-out }} / \mathrm{Y}_{\mathrm{RF} 000 \text { (Exp.) }}$ & $121 \%$ & $131 \%$ & $137 \%$ & $131 \%$ & $134 \%$ \\
\hline$Y_{p, \text { mixed-out }} / Y_{R F} 000$ (Exp.) & $61 \%$ & $60 \%$ & $60 \%$ & $60 \%$ & $59 \%$ \\
\hline$Y_{\text {sec, mixed-out }} / Y_{\mathrm{RF}} 000$ (Exp.) & $60 \%$ & $71 \%$ & $77 \%$ & $71 \%$ & $75 \%$ \\
\hline
\end{tabular}

Rectangular Overlap

(a)

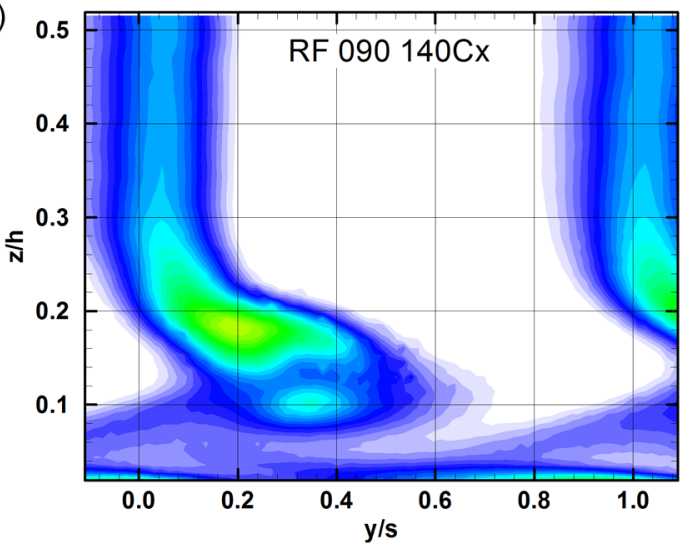

(c)

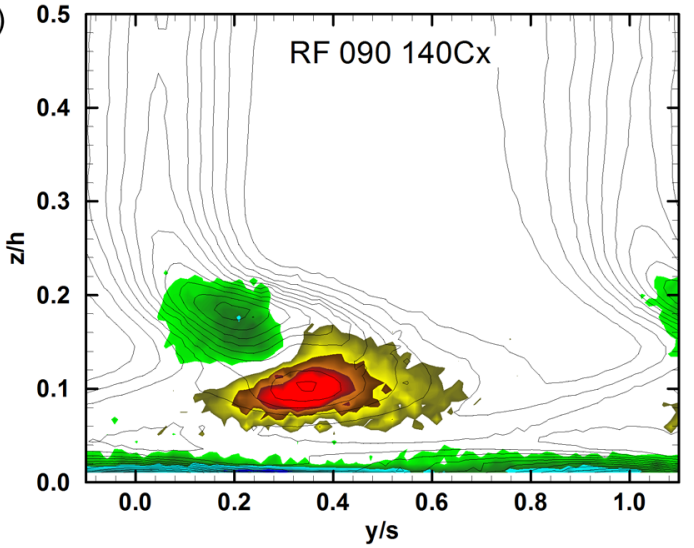

Elliptical Overlap

(b)

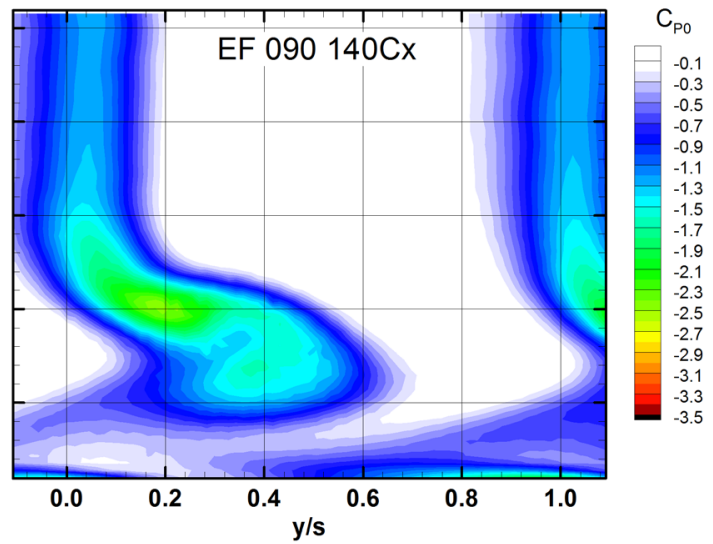

(d)

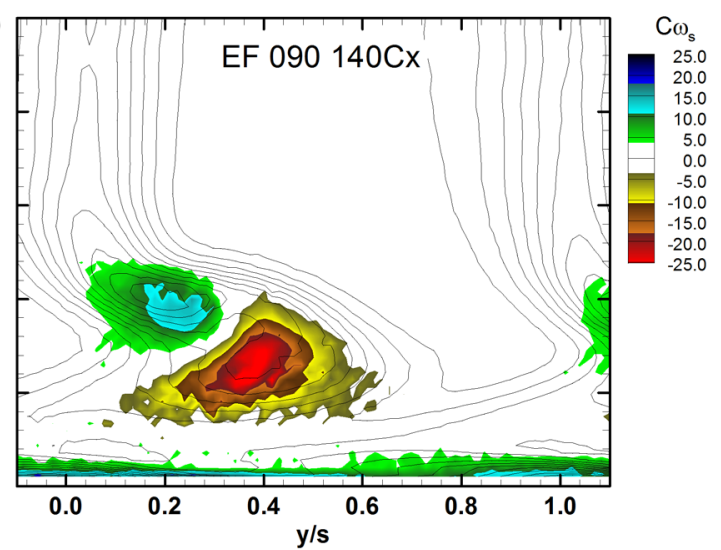

Figure 8.13: Floods of the total pressure coefficient $\left(C_{P 0}\right)$ and the streamwise vorticity coefficient $\left(C_{\omega S}\right)$ for $\phi=0.90$ measured at $1.40 C_{x}$ 
Returning to the integrated losses shown in Figure 8.12, there are two higher purge flow cases corresponding to $\phi=1.10$ and 1.25. The in-plane and mixed-out losses at these purge levels are lower than the observed peak at $\phi=0.90$. Also, the losses are lower than for the RF configuration. The EF in-plane losses are about 3\% and 1.5\% lower than the RF case, respectively. At the fully mixed out conditions the differences are about $3 \%$ and $1 \%$. The results show that EF configuration has potential as secondary loss mitigation technology at these higher purge levels. However, the current design is clearly very sensitive to the purge conditions.

The results shown here demonstrate the importance of considering purge flow in the turbine geometry design as early as possible in the design. For added context, in the current study the elliptical platform overlap was not designed with a rigorous CFD optimization routine. Rather PWA suggested an approximate elliptical profile with the intent of smoothing the transition from the platform overlap into the purge cavity. Varying purge levels were not considered during the design. The current investigation was considered a scoping study to evaluate the potential of such a technology.

The elliptical overlap shows some potential as a loss mitigation technology. However, the downstream floods show only minor changes to the secondary flow structures. Although some benefits were realised for certain purge levels, the elliptical overlap fails to significantly reduce the strength of the passage vortex, as shown in the downsteam floods of streamwise vorticity. Furthermore, the elliptical design does not reduce the levels of secondary kinetic energy, thereby limiting any supplemental benefits that may occur with additional downstream mixing.

\subsubsection{Flow Visualization for the Elliptical Overlap Configuration}

In the present sub-section two possible explanations for the observed ineffectiveness of the elliptical platform overlap are discussed. The first explanation follows the discussion of the loss growth with axial distance shown in Figure 7.20 (Section 7.10). The second investigates the endwall visualizations for high and low purge flow cases. 
In Section 7.10, the CFD simulations and endwall visualizations showed that the most significant loss growth region occurs within the blade passage. In fact, the endwall cavity region upstream of the bladerow showed only moderate loss growth considering the large separation and recirculation zone present in this region. Figure 7.20 and the endwall visualizations of Figure 7.17 showed the highest rate of loss growth occurs within the passage and is related to the losses generated near the highest endwall shear region - near the blade suction surface. This rate of loss growth was well correlated to the level of purge flow. Thus, it appears that the purge level affects the loss growth through the passage more significantly than the loss growth within the endwall cavity. Since the elliptical platform overlap attempts to attenuate the endwall cavity recirculation, it is to be expected that the downstream losses are only mildly affected. The implication is that the level of purge flow is perhaps more significant than the platform overlap geometry to the secondary flow development and associated losses.

In the present chapter the elliptical platform configuration showed subtle effects on the flow structures and loss contours at high purge flow levels. In terms of overall integrated losses, the maximum reduction relative to the RF configuration was about $3 \%$ at the $\phi=1.10$ purge level. At this purge level, the increase in losses caused by purge injection was about $5 \%$ greater than the zero purge case (EF 000). For this particular cascade and overlap design the purge flow has a more significant impact on the downstream losses than the overlap geometry.

As discussed in Chapter 2, others have measured significant benefits when altering the shape of the platform overlap. For example, Popovic and Hodson (2013c) investigated several platform overlap geometries while measuring both the sealing effectiveness and the downstream losses. Their conclusions suggest that the design of the platform overlap is a trade-off between the sealing effectiveness and the "spoiling losses" incurred with increasing purge flow. Thus, they suggest the best design should have a smooth transition from the platform overlap into the rotor passage. In the current work, the small effect of the elliptical overlap suggests that the transition from platform into the passage is perhaps not smooth enough. 
To investigate further, endwall surface oil flow visualizations were generated for the EF configuration with zero net purge flow and for a high purge flow level $(\phi=1.25)$. Figure 8.14 (b) and (d) shows the endwall visualizations for the zero and high purge flow levels for the elliptical overlap. The corresponding RF visualizations are shown for comparison purposes in sub-figures (a) and (c).

In general, the endwall visualizations of the EF cases are very similar to the RF cases. The zero flow case does however show some differences. For instance the endwall cavity separation line, $\mathrm{S}_{\mathrm{C}}$, indicates the endwall cavity recirculation is shorter than for the RF case. The suction side of the endwall cavity recirculation turns downstream near the mid-pitch position, resulting in a shift of the $\mathrm{S}_{2 \mathrm{P}}$ separation towards the centre of the passage. In the corresponding $\mathrm{RF}$ configuration the cavity recirculation turns downstream closer to the suction side of the passage. As discussed in Section 7.10, a shift of the $\mathrm{S}_{2 \mathrm{P}}$ separation towards the centre of the passage indicates a lower cross-passage pressure gradient within the endwall cavity. The lower pressure gradient should cause the pressure side leg to track more slowly across the passage and delay the interaction of the passage vortex with the suction side of the blade. As shown in Figure 8.14 (b), the interaction of the passage vortex and the suction side of the blade, marked by the high endwall shearing, occurs at nearly the same location, approximately $\mathrm{SS} / \mathrm{SS}_{\max }=0.3$. The shear levels also appear similar. Thus, although the endwall cavity recirculation is in fact altered by the elliptical overlap, a region of the intra-passage flow field that is significant to the downstream losses is relatively unchanged. Perhaps the current design of the elliptical overlap is not effective at altering the endwall cavity recirculation to the extent required to attenuate the downstream secondary flow.

Consider the position of the platform overlap separation, $S_{1}$, in Figure 8.14. In the EF cases the separation occurs further upstream than in the rectangular cases. The elliptical shape here appears to be too aggressive. The flow easily separates as it encounters the adverse pressure gradient of the blade row and endwall cavity. In Section 8.5.4, the position of the $S_{1}$ separation 
(a) RF 000

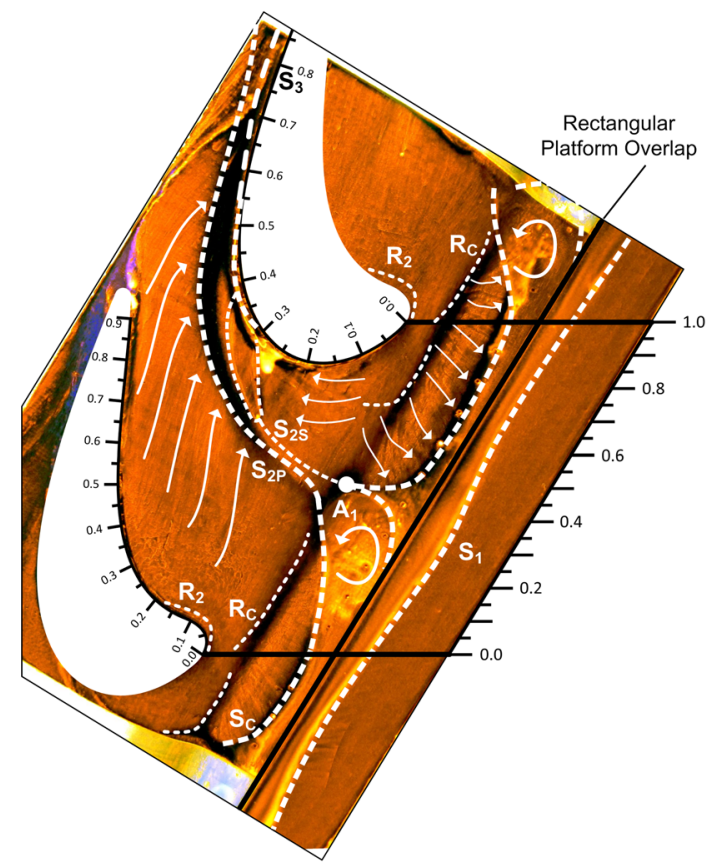

(c) RF 125

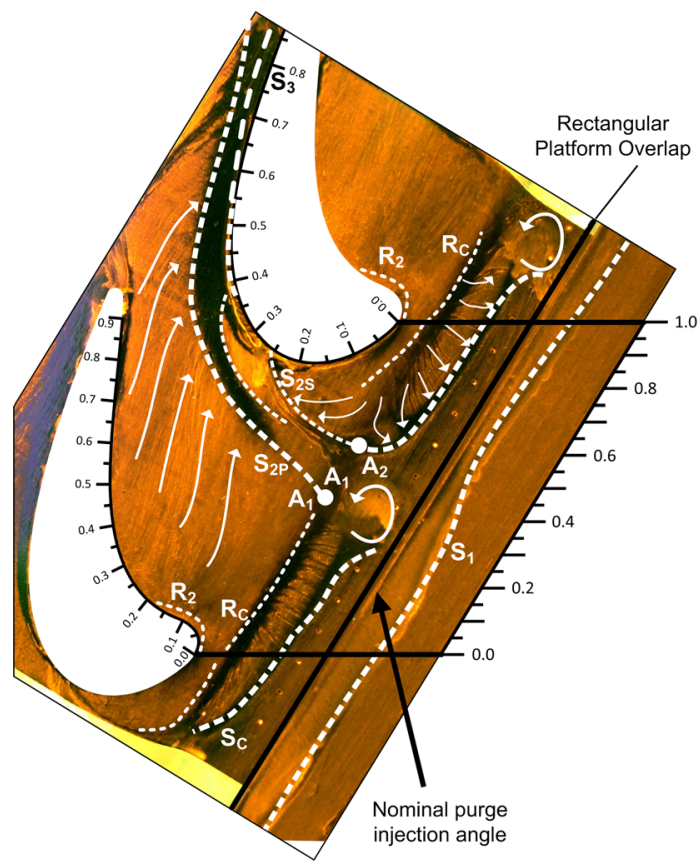

(b) EF 000

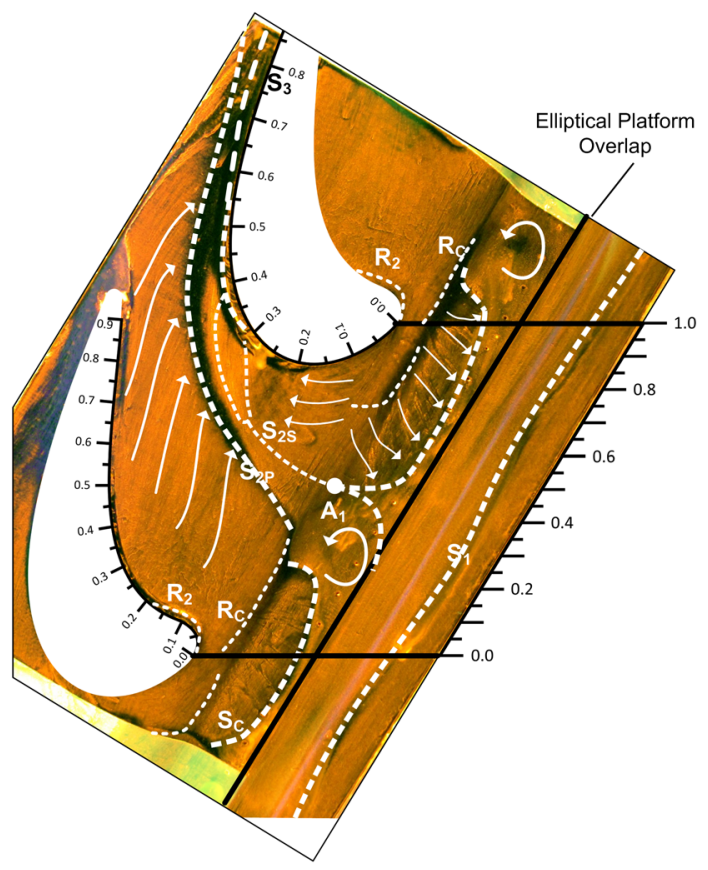

(d) EF 125

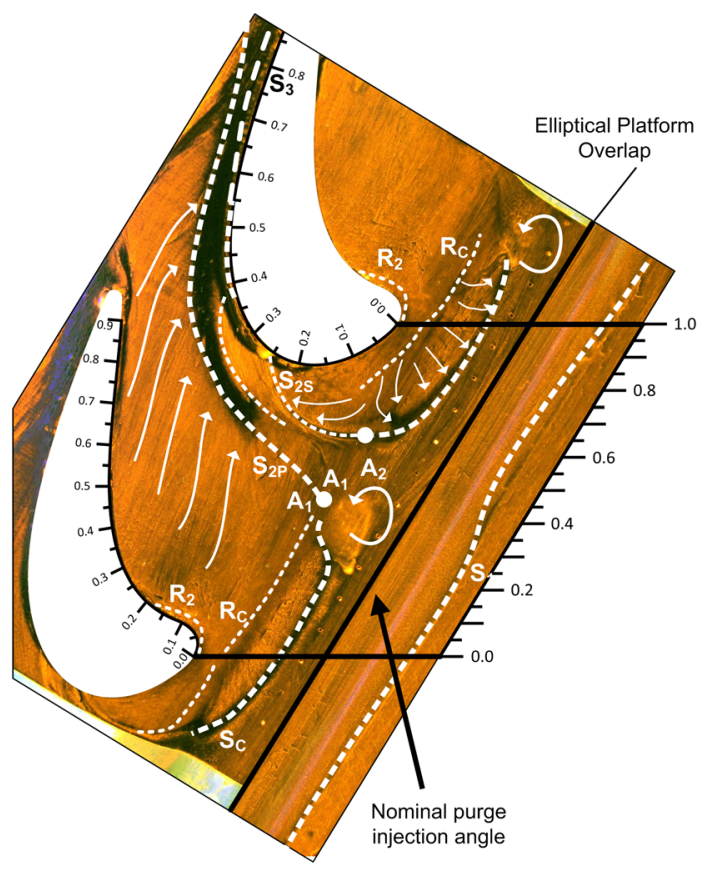

Figure 8.14: Endwall surface visualization and interpretation for the EF configuration 
was shown to be sensitive the purge flow level. In fact, the interpretation shown in Figure 8.8 suggests that the $S_{1}$ separation is sensitive to the size and the spanwise position of the endwall cavity recirculation. Following this interpretation, the adverse pressure field would be sensed further upstream for the elliptical overlap than the rectangular. It is therefore not a surprise that the $S_{1}$ separation occurs further upstream for the elliptical overlap configuration.

At the higher purge level, sub-figures (c) and (d), the results are very similar to the zero flow cases. For the EF configuration, the endwall cavity recirculation is turned downstream further from the blade suction surface (towards the centre of the passage) - evidence of a locally weaker cross-passage pressure gradient. The platform overlap separation, $\mathrm{S}_{1}$, is further upstream in the elliptical case which suggests a stronger endwall cavity recirculation. As a result, the pressure-side of the cavity recirculation tracks across the passage more quickly and interacts with the blade suction surface at a similar position to the RF case. The downstream development of the secondary flow structures is therefore similar, as was shown in Section 8.6.2.

The endwall flow visualizations of the EF configurations provided interesting clues to the infectiveness of the elliptical overlap as a loss mitigation technology. The current platform design was clearly too aggressive and caused an early separation of the inlet endwall boundary layer. However, the visualizations suggest the elliptical overlap does weaken the cross-passage pressure gradient within the endwall cavity. A future design may aim to exploit this benefit by delaying the migration across the passage of the pressure-side leg of the cavity recirculation. Perhaps a more optimized design could delay the appearance of the $S_{1}$ separation - ultimately leading to an elliptical overlap design that is successful in significantly mitigating the secondary loss development. The best design should provide more consistent loss reductions over a wider range of purge levels. 


\subsection{Concluding Discussion}

The present chapter has investigated two loss mitigation technologies, namely the nonaxisymmetric contoured endwall and the elliptical platform overlap. The effects of the each technology on the secondary flow of the PAKH cascade were discussed for a range of purge flow ratios. The results include downstream floods of the total pressure, streamwise vorticity coefficient and secondary kinetic energy coefficients. The interpretation of the secondary flow field combined the downstream flow field measurements, integrated results and the endwall surface visualizations.

The purpose of the elliptical overlap was to create a smooth transition from the mainstream into the endwall cavity. For the present geometry, the elliptical overlap showed minor effects on the secondary flow. The floods of total pressure, streamwise vorticity and secondary kinetic energy coefficients show subtle differences relative to the corresponding rectangular platform overlap case. Small reductions in the losses associated with the passage vortex were observed, while the near endwall losses tended to increase. The integrated losses were reduced for the elliptical geometry at the higher purge levels while at the lower purge levels the integrated losses actually increased relative to the corresponding rectangular overlap cases. Also, the elliptical configuration showed no additional benefits that such as reductions in the secondary kinetic energy.

The endwall visualizations of the elliptical platform overlap configurations provided interesting clues to the ineffectiveness of the elliptical overlap as a loss mitigation technology. In general, the current platform design was not suitable. The inlet boundary was shown to separate prematurely from the platform thereby creating a strong recirculation similar to the rectangular overlap case. The similarities arise because of the adverse pressure gradient the endwall cavity and blade impose on the upstream flow field at the platform overlap. The elliptical overlap has minor effects on the passage vortex. Therefore, the secondary flow develops similarly to the rectangular overlap cases. The overall integrated results suggest that the effects of purge flow on 
the downstream losses are more significant than the maximum loss reductions provided by the elliptical geometry.

A more robust elliptical design should consider the surrounding pressure field and how it will affect the upstream flow field. A successful design that can reduce the strength of the endwall cavity recirculation may provide more consistent loss reductions over a wider range of purge levels. However, in Chapter 7 it was shown that the maximum loss growth through the passage occurs near the blade suction peak and not within the endwall cavity. Therefore, technologies that target the endwall cavity recirculation may not have the same up-side potential as other intra-passage loss mitigation technologies.

The non-axisymmetric endwall contouring was designed to alter the endwall pressure gradient using streamline curvature. The contour consists of two distinct humps at the passage inlet. A trough passes through the centre of the blade passage and a ridge is positioned close to blade suction surface near the passage exit. The design intent of the endwall contour is to reduce the strength of the passage vortex structure. In doing so the other secondary flow features are attenuated reducing the losses generated through the blade passage.

For all levels of purge injection the endwall contouring reduces the strength of the passage vortex, as indicated by the lower levels of streamwise vorticity. The associated loss reductions within the passage vortex are small. In fact, the loss benefits for the contouring cases are attributed to reductions in the peak losses within the region near the counter vortex.

At the lowest purge levels, higher near-endwall losses negate the loss reductions associated with the passage and counter vortices. The increased near endwall losses are attributed to higher shear levels at the passage exit. At higher purge levels, the contouring is more effective. The integrated losses are significantly lower than the corresponding flat endwall cases. The increased loss reductions arise from a weaker passage vortex and reduced interactions with the blade suction surface. A negative effect of the endwall contouring was the increased penetration depth of the secondary flow. At the highest purge levels the secondary flow was significantly displaced away from the endwall compared to the flat endwall case. 
In general, the endwall contouring technology was effective in mitigating the secondary losses for cases with positive net purge flow. For the cases shown here, the endwall contouring reduced the mixed-out losses by about $5 \%$ relative to the flat endwall cases. The current design is robust such that the loss benefits are realised for the range of positive net purge flow investigated. Of course, the apparent robustness shown here does not consider the effectiveness of the endwall contour at off-design incidence of the mainstream inlet flow. 


\section{Chapter 9}

\section{Summary, Conclusions and Recommendations}

\subsection{Summary of the Research Motivation and Approach}

The present thesis investigated aerodynamic topics related to the axial turbine of the commercial aircraft engine. The research was motivated in part by increased pressure on the airtravel industry to reduce the overall aircraft fuel consumption. Reducing the engine weight and improving the fuel efficiency of the axial turbine are important design objectives for the state-ofthe art aircraft engine. These objectives have served as the motivation for the present work.

Weight reductions of the turbine section can be achieved by reducing the number of blades in a given bladerow. Generally, reducing the blade count, while maintaining the same power output, increases the aero-dynamic loading per blade and leads to higher total pressure losses for the bladerow. Loss mitigation technologies can reduce the loss penalties associated with more highly loaded bladerows. The present thesis investigated two different loss mitigation technologies as applied to turbine bladerows. The work examined the mechanisms of total 
pressure loss generation through the turbine bladerow and detailed interpretations of the flow physics were discussed.

The losses that occur in the near-endwall flow of a turbine, termed the secondary flow, are referred to as the secondary losses. Although secondary losses have been thoroughly researched for over 30 years, the sources of endwall losses are still not fully understood (Praisner et al., 2007). Thus, an overall objective of the present research was to improve the physical understanding of the secondary flows of turbine bladerows. A significant contribution of the current thesis was the experimental measurements of the turbulence quantities within the turbine cascade flow field. The mechanisms of energy transfer from the mean to the turbulent flow field were discussed in detail.

A relatively new technology, termed endwall contouring, aims to reduce the secondary losses of an axial bladerow by passively manipulating the flow field. For an axial turbine, the contoured endwall is a specialized endwall shape that alters the blade-to-blade endwall pressure field through streamline curvature. In doing so, the development of the secondary flow can be attenuated and the secondary losses reduced. However, as a new technology, its effectiveness under engine representative conditions is still not well understood. For example, within an actual turbine the inlet flow is complicated by the relatively large step, termed the platform overlap, that bridges the axial gap between the stationary and moving bladerows. Also, a disk cooling flow, termed purge flow, is typically injected into the main gas path from between the blade disks to prevent ingress of the hot mainstream gases. Understanding the effects of different purge flow levels and different platform overlap shapes on the turbine secondary flow was another important objective of the present thesis. In addition, the combined effect of these engine representative conditions on the effectiveness of the endwall contouring technology was investigated.

The turbine flow fields were studied experimentally in a low-speed linear cascade testing facility. A common blade shape, PAKH, was used for the different geometric configurations. Experimental and computational results were presented in Chapters 5 through 8 . Chapter 5 discussed the turbulent flow field for the planar endwall cascade. Chapter 6 examined the 
influence of the upstream platform overlap on the secondary flow without the presence of purge flow. Chapter 7 examined the influence of vary levels of purge flow on the secondary flows for the cascades with a rectangular platform overlap and flat intra-passage endwalls. Lastly, Chapter 8 examined the secondary loss-mitigation technologies. Endwall contouring and an elliptical platform overlap shape were discussed separately.

\subsection{Conclusions and Contributions}

The present section summarizes the significant conclusions of the current thesis. More detailed discussions are given in Chapters 5 through 8 . The present section concludes with a summary of the significant contributions of the present work.

Measurements of the mean and turbulent flow field downstream of the planar endwall cascade were presented in Chapter 5. The objective was to improve the physical understanding of the loss production mechanisms within the secondary flow, downstream of turbine bladerow. The generation of downstream losses were examined by investigating the kinetic energy budget for turbulent flow. The production of turbulence, which represents a loss from the mean flow field, was identified by regions of negative deformation work. The highest rates of turbulence production were found within the high shear regions of the flow, such as the boundary between the passage and counter vortices as well as the boundary between the secondary flow and inviscid mainstream. The Reynolds shear stresses and corresponding velocity gradients were found to be related to the highest rates of turbulence production. The Reynolds shear stresses are therefore important to the generation of total pressure losses within turbine secondary flows.

The turbulence measurements of Chapter 5 and corresponding computational simulations

were also used to investigate the isotropy of the eddy viscosity field within the secondary flow. Computational simulations used for turbine design purposes often use two-equation turbulence models that inherently assume the eddy viscosity field is isotropic, an approximation referred to as the Boussinesq assumption. The implied eddy viscosity tensor calculated from the turbulence 
measurements was shown to be highly anisotropic, particularly within the secondary flow. Also, regions of negative eddy viscosity were also obtained. The results show that the Boussinesq approximation, commonly used in computational predictions of turbine secondary flows, is overly simplified.

The computational predictions also showed peak Reynolds stresses within the vortex cores, opposed to within the high shear regions as shown in the experiments. Consistent with distributions of the Reynolds shear stresses, the distributions of deformation work showed the peak rates of turbulence production, and therefore loss generation, occur within the vortex cores. The experimental measurements were different and showed that the highest rates of turbulence production within the high shear regions between the main vortical structures. Thus, it appears that the mechanisms of loss generation in the computations are different than observed experimentally.

Chapter 6 investigated the effects of upstream platform overlap and endwall cavity geometry on the secondary flow of the PAKH cascade. Measurements and computations were performed for a baseline flat endwall case and three rim-seal configurations, namely the Cavity, Step and Ramp. A common question faced by turbine designs is the extent to which the rim-seal geometry affects the flow field of the downstream bladerow. The downstream measurements showed that the rim-seal geometry increases the streamwise vorticity and peak losses within the passage vortex region relative to the flat-endwall cascade. Increased levels of secondary kinetic energy were observed in the near endwall region which is a consequence of more blade-to-blade cross flow and higher values of flow overturning.

In general, the upstream platform geometry had the local effect of strengthening the passage vortex which increased the mixed-out losses by as much $40 \%$ relative to the flat endwall case. Differences of this magnitude are significant and therefore it is recommended that rim-seal representative geometry be included in the turbine design process as early as possible.

When the three rim-seal geometries were directly compared, the experimental results showed subtle differences. The Ramp configuration is a simplification of typical rim-seal 
geometry and has benefits for design-level computational predictions. The purpose of the ramp is to reduce the complexity of the CFD mesh within the endwall cavity region while not excluding the major geometric features of the rim-seal geometry. The relatively good agreement between the experimental measurements and the predictions suggested that the Ramp-type simplification of the rim-seal may be appropriate for design-level computations.

A detailed interpretation of the secondary flow was developed for the turbine flows with upstream overlap geometry. The differences relative to the secondary flow of traditional flat endwall turbine approximation were marked by the development of the endwall cavity recirculation region downstream of the platform overlap. The endwall cavity recirculation is analogues to the traditional horseshoe vortex and was shown to initiate the development of the passage vortex. A strong interaction between the suction and pressure-side legs of the endwall cavity recirculation leads to stronger passage and corner vortices, ultimately increasing in secondary losses. The upstream rim-seal causes significant changes to the secondary flow, and as stated above, representative geometry should be included in the turbine designs as early as possible.

Chapter 7 investigated the effects of positive net purge flow injection on the secondary flow of the cascade with a rectangular platform overlap (the Cavity configuration) and flat intrapassage endwalls. The downstream measurements showed that the most significant effect of purge injection on the secondary flow was to alter the passage vortex. The associated losses and peak vorticity both increased, indicating that additional purge injection strengthens the passage vortex. The strengthened passage vortex also leads to higher levels of secondary kinetic energy within the region between the passage and counter vortices. The generation of secondary kinetic energy is eventually realised as loss with further downstream mixing as the flow approaches fully-mixed out conditions. Thus, the enhanced secondary kinetic energy realized by purge injection is important to the turbine design.

Purge injection was found to increase the overall losses with increasing purge flow at three different rates. The different rates of loss growth within the blade passage were shown to be 
influenced by the interactions between the purge and mainstream flows at different purge levels. For example, the highest rates of loss growth occurred for regime (II) for purge flow ratios between 0.90 and $1.10 \%$. At these purge levels, ingress of the mainstream flow into the purge slot is reduced and the mainstream boundary layer is thickened by the injected purge flow. The thickened endwall boundary layer results in a stronger secondary flow with higher secondary losses. The different rates of loss growth with increasing purge injection become important for off-design purge injection. For example, at the lowest and highest purge levels, the loss growth is modest for increasing purge flow. Thus, for purge levels within the low (I) and high (III) regimes, the bladerow losses are relatively constant for small changes to the purge flow level. Conversely, in regime (II) the losses are more sensitive to changes in the purge flow level.

A detailed interpretation of the effects of purge injection on the secondary flow was also discussed. The predicted and measured endwall visualizations were in good agreement, indicating that the CFD methods are satisfactory for the general interpretation of these flows. It was shown that the additional losses caused by increasing the purge flow rate were primarily generated in the aft portion of the passage. There is a strong interaction between the passage vortex and the blade suction surface which was shown to be enhanced with purge injection.

In Chapter 8 two secondary loss mitigation technologies were investigated: the nonaxisymmetric contoured endwall and the elliptical platform overlap. Each geometric configuration was tested for a range of purge flow levels.

The non-axisymmetric endwall contouring was designed to attenuate the passage vortex by altering the endwall pressure gradient with streamline curvature. For all levels of purge injection the endwall contouring reduces the strength of the passage vortex, as indicated by the lower levels of streamwise vorticity. The associated loss reductions within the passage vortex are small. However, overall loss benefits are realized through reductions in the peak losses near the counter vortex.

The endwall contouring is most effective at the highest purge levels. The integrated losses are significantly lower than the corresponding flat endwall cases. The increased loss reductions 
arise from a weaker passage vortex and reduced interactions with the blade suction surface. A negative effect of the endwall contouring was the increased penetration depth of the passage vortex.

In general, the endwall contouring technology was effective at mitigating the secondary losses for cases with positive net purge flow. For the range of purge flows investigated, the endwall contouring reduced the mixed-out losses by about $5 \%$ relative to the flat endwall cases. The current design is robust such that the loss benefits are realised for the range of positive net purge flow investigated. At zero net purge injection there was no benefit for the contouring design.

The purpose of the elliptical overlap was to create a smooth transition from the mainstream into the endwall cavity. For the present geometry, the elliptical overlap showed minor effects on the secondary flow. Small reductions in the losses associated with the passage vortex were observed while the near endwall losses tended to increase. At the higher purge levels, the integrated losses were slightly reduced relative to the corresponding rectangular overlap cases. However, at the lower purge levels the integrated losses actually increased.

The endwall visualizations of the elliptical platform overlap configurations showed that the inlet boundary layer separates prematurely from the platform thereby creating a strong endwall cavity recirculation. The overall integrated results suggest that the effects of purge flow on the downstream losses are more significant than the maximum loss reductions provided by the elliptical geometry. Also, the results here indicate that loss mitigation technologies that target the endwall cavity recirculation may not have the same up-side potential as other intra-passage technologies.

In summary, the current research has achieved the primary objective of the thesis by enhancing the understanding of turbine secondary flows. The significant contributions of the work are summarized below: 
- Chapters 6 and 7 contributed to the understanding of secondary flows for turbine cascades with platform overlap geometry and positive net purge flow. The interpretations of these secondary flows are significantly different from the generally accepted interpretations for turbines with flat endwall approximations. As recommended, the effects of the platform overlap and purge injection should be included in turbine designs.

- The results of Chapter 5 have added to the general understanding of loss generation within turbine cascade secondary flows. Since the generation of secondary losses for turbine bladerows is not fully understood in open-literature (Praisner et al., 2007), the relations shown here between the turbulence field and the production of the secondary losses downstream of turbine bladerow are significant. Furthermore, the turbulence measurements have shown that the commonly applied Boussinesq assumption is over simplified for the computational prediction of turbine secondary flows.

- Chapter 8 has contributed to the understanding of the endwall contouring and platform overlap shaping as loss mitigation technologies. The detailed interpretations of the secondary flow physics presented in the current work are significant contributions to the improvement of the state-of-the-art turbine designs. The present endwall contouring design showed loss benefits for a range of positive net purge flows demonstrating endwall contouring, as applied here, is robust for these operating conditions. 


\subsection{Recommendations for Future Work}

In addition to the contributions of the present thesis the current research has identified a number of possible directions for future work.

The turbulence measurements were performed for a single cascade geometry. It is therefore difficult to extrapolate these results and make conclusions regarding the general loss generation mechanisms within turbine blade rows. It is recommended that similar studies be completed for additional cascades. The present cascade is representative of a moderately loaded high pressure turbine bladerow. As turbine engines move towards more highly loaded bladerows, it may be beneficial to explore the turbulence field for a cascade that is more highly loaded. Furthermore, the current cascade is unusual in that it has a high convergence ratio (CR) of 2.95. The streamwise gradients that arise with a high convergence ratio may alter the turbulence field relative to that of a bladerow with a more conventional convergence ratio. Testing a cascade with a lower convergence ratio may help to generalize the results shown here. Also, another logical extension of the current work would be to measure the turbulence field of a corresponding turbine cascade with non-axisymmetric endwall contouring.

Another potential improvement to the current tests would be to measure turbulence quantities that are both temporally and spatially correlated. With the present point measurement techniques temporal correlation is difficult to achieve.

The present work was completed in a low-speed testing facility so that the subtle effects of purge flow and platform overlap geometry could be investigated individually. A compromise of the present work was the inability of the experiment to capture the density and temperature differences between the purge and mainstream gas path of a real engine. It is recommended to pursue a similar study for an equivalent cascade designed for compressible conditions. To this end, cold and hot flow rig-testing are additional future directions.

The current cascade test setup has not considered the periodic unsteady effects caused by the relative motion between the stationary and moving bladerows in a real engine. Since, the 
purge flow in a real engine is injected from within the cavity between the two bladerows, the purge flow is likely affected by the periodically varying pressure field. Future work could investigate this unsteady behaviour using a test section with a stationary and a rotating bladerow.

In the current work, the platform overlap and purge injection was shown to be important to the secondary flow development. An equally important design criterion of the rim-seal is also the sealing effectiveness. The present work has only focused on the downstream effects of the platform overlap and purge injection. Follow-up work, that perhaps considers an optimized rimseal, should investigate the effectiveness of the rim-seal in preventing hot-gas ingestion.

The elliptical overlap showed some benefits as a loss mitigation technology, but was sensitive to the level of purge injection. It is recommended that a future work reconsider the overlap design with the aid of computational optimization.

The endwall contouring investigated in the present work was shown to be robust over a range of positive net purge flows. The loss benefits shown here are modest and there appears to be further room for improvement. The current contouring designs do not extend deep within the within the endwall cavity. It is recommended that future work investigate a loss-mitigation design that together optimizes the overlap geometry, the endwall cavity and the intra-passage endwall.

Lastly, the current work has only considered the effectiveness of the endwall contouring for varying levels of purge injection. In real engine applications the contouring design must be robust for varying engine operating conditions. It is recommended that a future work test the same cascade for different flow incidence angles and perhaps different Reynolds number conditions. 


\section{References}

Abo El Ella, H. M., Sjolander, S. A. and Praisner, T. J. (2012), "Effects of an Upstream Cavity on the Secondary Flow in a Transonic Turbine Cascade," Journal of Turbomachinery, 134, 51009 .

Abraham, S., Panchal, K., Ekkad, S. V., Ng, W., Lohaus, A. S. and Malandra, A. (2012), "Effect of Endwall Contouring on a Transonic Turbine Blade Passage: Part 1 - Aerodynamic Performance,” ASME Paper No. GT2012-68425, Copenhagen, Denmark, June 11-15.

ANSYS ANSYS Inc. (2009), “Ansys CFX-Solver Theory Guide,” ANSYS CFX, Release 12.0.

Bean, H. S. (1971), Fluid Meters: Their Theory and Application, 6th ed., Report of ASME Research Committee on Fluid Meters, New York, NY.

Bearman, P. W. (1971), "Corrections for Ambient Temperature Drift on Hot-Wire Measurements in Incompressible Flow,” DISA Information, 11, pp. 25-30.

Becz, S., Majewski, M. S. and Langsten, L. S. (2004), "An Experimental Investigation of Contoured Leading Edges for Secondary Flow Loss Reduction," 2004 ASME Turbo Expo, June 14, 2004 - June 17, 2004 Paper No. Vienna, Austria,

Becz, S., Majewski, M. S. and Langston, L. S. (2003), "Leading Edge Modification Effects on Turbine Cascade Endwall Loss," 2003 ASME Turbo Expo, June 16, 2003 - June 19, 2003 Paper No. Atlanta, GA, United states,

Benner, M. W. (2003), "The Effects of Leading Edge Geometry on Profile and Secondary Losses in Turbine Cascades," PhD thesis, Carleton University, Ottawa, Canada.

Benner, M. W., Sjolander, S. A. and Moustapha, S. H. (2004a), "The Influence of Leading-Edge Geometry on Secondary Losses in a Turbine Cascade at the Design Incidence," ASME Journal of Turbomachinery, 126, pp. 277-287.

Benner, M. W., Sjolander, S. A. and Moustapha, S. H. (2004b), "Measurements of Secondary Flows Downstream of a Turbine Cascade at Off-Design Incidence," ASME Paper No. GT2004-53786, Vienna, Austria, June 14-17.

Benner, M. W., Sjolander, S. A. and Moustapha, S. H. (2006a), “An Empirical Prediction Method for Secondary Losses in Turbines-Part I: A New Loss Breakdown Scheme and Penetration Depth Correlation," ASME Journal of Turbomachinery, 128, pp. 273-80.

Benner, M. W., Sjolander, S. A. and Moustapha, S. H. (2006b), “An Empirical Prediction Method for Secondary Losses in Turbines - Part II: A New Secondary Loss Correlation,” ASME Journal of Turbomachinery, 128, pp. 281-291. 
Bergh, J., Snedden, G. and Meyer, C. (2012), "Optimization of Non-Axisymmetric End Wall Contours for the Rotor of a Low Speed, $11 \backslash 2$ Stage Research Turbine with Unshrouded Blades," ASME Paper No. GT2012-68569, Copenhagen, Denmark, June 11-15.

Bohn, D. E., Decker, A., Ohlendorf, N. and Jakoby, R. (2006), "Influence of an Axial and Radial Rim Seal Geometry on Hot Gas Ingestion into the Upstream Cavity of a 1.5-Stage Turbine,” ASME Paper No. GT2006-09453, Barcelona, Spain, May 6-11.

Bohn, D. E., Surken, N., Yu, Q. and Kreitmeier, F. (1999), "Influence of Rim Seal Geometry on Hot Gas Ingestion into the Upstream Cavity of an Axial Turbine Stage," ASME Paper No. 99-GT-248, Indianapolis, Indiana, June 7-10.

Boletis, E. (1985), "Effects of Tip Endwall Contouring on the Three-Dimensional Flow Field in an Annular Turbine Nozzle Guide Vane: Part 1 - Experimental Investigation," Journal of Engineering for Gas Turbines and Power, 107, pp. 983-990.

Bradshaw, P. (1973), "Effects of Streamline Curvature on Turbulent Flow," AGARDograph No. 169.

Brear, M. J., Hodson, H. P., Gonzalez, P. and Harvey, N. W. (2002a), "Pressure Surface Separations in Low-Pressure Turbines-Part 2: Interactions with the Secondary Flow," Journal of Turbomachinery, 124, pp. 402-409.

Brear, M. J., Hodson, H. P. and Harvey, N. W. (2002b), "Pressure Surface Separations in LowPressure Turbines-Part 1: Midspan Behavior," Journal of Turbomachinery, 124, pp. 393401.

Brennan, G., Harvey, N. W., Rose, M. G., Fomison, N. and Taylor, M. D. (2003), "Improving the Efficiency of the Trent 500-HP Turbine Using Nonaxisymmetric End Walls-Part I: Turbine Design," ASME Journal of Turbomachinery, 125, pp. 497-504.

Bunker, R. S., Metzger, D. E. and Wittig, S. (1992a), "Local Heat Transfer in Turbine Disk Cavities. Part I. Rotor and Stator Cooling with Hub Injection of Coolant," Journal of Turbomachinery, 114, pp. 211-220.

Bunker, R. S., Metzger, D. E. and Wittig, S. (1992b), "Local Heat Transfer in Turbine Disk Cavities. Part II. Rotor Cooling with Radial Location Injection of Coolant," Journal of Turbomachinery, 114, pp. 221-228.

Burd, S. W. and Simon, T. W. (2000), "Flow Measurements in a Nozzle Guide Vane Passage with a Low Aspect Ratio and Endwall Contouring," Journal of Turbomachinery, 122, pp. 659-666.

Buresti, G. and Di Cocco, N. R. (1987), "Hot-Wire Measurement Procedures and their Appraisal through a Simulation Technique," Journal of Physics E: Scientific Instruments, 20, pp. 87-99. 
Chaves, M. and Gama, S. (2000), "Time Evolution of the Eddy Viscosity in Two-Dimensional Navier-Stokes Flow," Physical Review E (Statistical Physics, Plasmas, Fluids, and Related Interdisciplinary Topics), 61, pp. 2118-20.

Chen, L. D. and Dixon, S. L. (1986), "Growth of Secondary Flow Losses Downstream of a Turbine Blade Cascade," J. Eng. for Gas Turbines and Power, 108, pp. 270-276.

Christiansen, T. and Bradshaw, P. (1981), "Effect of Turbulence on Pressure Probes," Journal of Physics E: Scientific Instruments, 14, pp. 992-997.

Chue, S. H. (1975), "Pressure Probes for Fluid Measurement," Progress in Aerospace Sciences, 16, pp. 147-223.

Chung, J. T. and Simon, T. W. (1993), "Effectiveness of the Gas Turbine Endwall Fences in Secondary Flow Control at Elevated Freestream Turbulence Levels," ASME Paper No. 93-GT-51, Cincinnati, OH, USA, May-24-27.

Chung, J. T., Simon, T. W. and Buddhavarapu, J. (1991), "Three-Dimensional Flow near the Blade/Endwall Junction of a Gas Turbine: Application of a Boundary Layer Fence," ASME Paper No. 91-GT-45, Orlando, FL, USA, June 3-6.

Cleak, J. G. E. and Gregory-Smith, D. G. (1992), "Turbulence Modeling for Secondary Flow Prediction in a Turbine Cascade," Journal of Turbomachinery, 114, pp. 590-598.

Corral, R. and Gisbert, F. (2008), "Profiled End Wall Design Using an Adjoint Navier-Stokes Solver," ASME Journal of Turbomachinery, 130, 021011.

Cummins, P. F. and Holloway, G. (2010), "Reynolds Stress and Eddy Viscosity in Direct Numerical Simulations of Sheared Two-Dimensional Turbulence," Journal of Fluid Mechanics, 657, pp. 394-412.

Cumpsty, N. A. and Horlock, J. H. (2006), “Averaging Nonuniform Flow for a Purpose,” ASME Journal of Turbomachinery, 128, pp. 120-129.

Davidson, L. and Billson, M. (2006), "Hybrid Les-Rans Using Synthesized Turbulent Fluctuations for Forcing in the Interface Region," International Journal of Heat and Fluid Flow, 27, pp. 1028-1042.

de la Rosa Blanco, E., Hodson, H. P. and Vazquez, R. (2009), "Effect of the Leakage Flows and the Upstream Platform Geometry on the Endwall Flows of a Turbine Cascade," ASME Journal of Turbomachinery, 131, 011004.

Deich, M. E., Zaryanskin, A. E., Fillipov, G. A. and Zatsepin, M. (1960), "Method of Increasing the Efficiencey of Turbine Stages Wiht Short Blades," Teploenergetikia, 2, pp. 240-254.

Denton, J. D. (1993), “The 1993 IGTI Scholar Lecture: Loss Mechanisms in Turbomachines," ASME Journal of Turbomachinery, 115, pp. 621-656. 
Döbbeling, K., Lenze, B. and Leuckel, W. (1992), "Four-Sensor Hot-Wire Probe Measurements of the Isothermal Flow in a Model Combustion Chamber at Different Levels of Swirl," Experimental Thermal and Fluid Science, 5, pp. 381-389.

Dossena, V., Perdichizzi, A. and Savini, M. (1999), “The Influence of Endwall Contouring on the Performance of a Turbine Nozzle Guide Vane," Journal of Turbomachinery, 121, pp. 200-208.

Dunn, D., Snedden, G., Von Backstrom, T. and Mdluli, M. P. (2013), "Unsteady Effects of a Generic Non-Axisymmetric Endwall Contour on the Rotor of a 1 1/2 Stage Low Speed Turbine Test Rig," ASME Paper No. San Antonio, Tx, United states, June 3-7.

Förster, F., Sims-Williams, D. and Ingram, G. (2011), "Time Resolved Measurements in the Durham Cascade,” ASME Paper No. GT2011-45836, Vancouver, BC, CA, June 6-11.

Förster, F., Sims-Williams, D. and Ingram, G. (2012), "Reconstruction of the Unsteady Pressure Field in a Low Speed Linear Cascade,” ASME Paper No. GT2012-69156, Copenhagen, Denmark, June 11 - 15.

Gallington, R. W. (1981) "Measurement of Very Large Flow Angles with Non-Nulling SevenHole Probes," Proceedings of the 27th International Instrumentation Symposium, Indianapolis, IN, USA, April 27-30.

Gama, S., Vergassola, M. and Frisch, U. (1994a), "Negative Eddy Viscosity in Isotropically Forced Two-Dimensional Flow: Linear and Nonlinear Dynamics," Journal of Fluid Mechanics, 260, pp. 95-126.

Gama, S., Vergassola, M. and Frisch, U. (1994b) "Two-Dimensional Isotropic Negative Eddy Viscosity: A Common Phenomenon," Proceedings of the 5th European Turbulence Conference, Siena, Italy, Jul 5 - 8.

Germain, T., Nagel, M., Raab, I., Schupbach, P., Abhari, R. S. and Rose, M. (2010), "Improving Efficiency of a High Work Turbine Using Nonaxisymmetric Endwalls - Part I: Endwall Design and Performance,” ASME Journal of Turbomachinery, 132, 021007.

Gerner, A. A., Maurer, C. L. and Gallington, R. W. (1984), "Non-Nulling Seven-Hole Probes for High Angle Flow Measurement," Experiments in Fluids, 2, pp. 95-103.

Gier, J., Stubert, B., Brouillet, B. and De Vito, L. (2003), "Interaction of Shroud Leakage Flow and Main Flow in a Three-Stage LP Turbine," 2003 ASME Turbo Expo, June 16, 2003 June 19, 2003 Paper No. Atlanta, GA, United states,

Gisbert, F., Corral, R., Chia, J. M. and Lopez, C. (2008), "Cavity Effects on the Design of Turbine Profiled End Walls," ASME Paper No. GT2008-51136, Berlin, Germany, June 9-13.

Goobie, S. M. (1989), “A Test Section for Investigating the Off-Design Performance of Planar Cascades," MASc thesis, Carleton University, Ottawa, Canada. 
Gregory-Smith, D., Bagshaw, D., Ingram, G. and Stokes, M. (2008), "Using Profiled Endwalls, Blade Lean and Leading Edge Extensions to Minimise Secondary Flow," ASME Paper No. GT2008-50811, Berlin, Germany, June 9-13.

Gregory-Smith, D. G. and Cleak, J. G. E. (1992), "Secondary Flow Measurements in a Turbine Cascade with High Inlet Turbulence," ASME Journal of Turbomachinery, 114, pp. 173183.

Gregory-Smith, D. G., Graves, C. P. and Walsh, J. A. (1988a), "Growth of Secondary Losses and Vorticity in an Axial Turbine Cascade,” ASME Journal of Turbomachinery, 110, pp. 1-8.

Gregory-Smith, D. G., Walsh, J. A., Graves, C. P. and Fulton, K. P. (1988b), "Turbulence Measurements and Secondary Flows in a Turbine Rotor Cascade," ASME Journal of Turbomachinery, 110, pp. 479-485.

Gustafson, R., Mahmood, G. and Acharya, S. (2007), "Aerodynamic Measurements in a Linear Turbine Blade Passage with Three-Dimensional Endwall Contouring," ASME Paper No. GT2007-28073, Montreal, QC, CA, May 14-17.

Harrison, S. (1990), "Secondary Loss Generation in a Linear Cascade of High-Turning Turbine Blades," ASME Journal of Turbomachinery, 112, pp. 618-624.

Hartland, J. C., Gregory-Smith, D. G., Harvey, N. W. and Rose, M. G. (2000), "Nonaxisymmetric Turbine End Wall Design: Part II- Experimental Validation," ASME Journal of Turbomachinery, 122, pp. 286-293.

Hartland, J. C., Gregory-Smith, D. G. and Rose, M. G. (1998), "Non-Axisymmetric Endwall Profiling in a Turbine Rotor Blade," ASME Paper No. 98-GT-525, Stockholm, Sweden, June 2-June 5.

Harvey, N. W., Brennan, G., Newman, D. A. and Rose, M. G. (2002), "Improving Turbine Efficiency Using Non-Axisymmetric End Walls: Validation in the Multi-Row Environment and with Low Aspect Ratio Blading," ASME Paper No. GT2002-30337, Amsterdam, Netherlands, June 3-6.

Harvey, N. W., Rose, M. G., Taylor, M. D., Shahpar, S., Hartland, J. and Gregory-Smith, D. G. (2000), "Nonaxisymmetric Turbine End Wall Design: Part I- Three-Dimensional Linear Design System," ASME Journal of Turbomachinery, 122, pp. 278-285.

Hilfer, M., Ingram, G. and Hogg, S. (2012), "Endwall Profiling with Tip Clearance Flows," ASME Paper No. GT2012-68488, Copenhagen, Denmark, June 11-15.

Hodson, H. P. and Dominy, R. G. (1987a), “Off-Design Performance of a Low-Pressure Turbine Cascade,” ASME Journal of Turbomachinery, 109, pp. 201-209.

Hodson, H. P. and Dominy, R. G. (1987b), "Three-Dimensional Flow in a Low-Pressure Turbine Cascade at Its Design Condition,” ASME Journal of Turbomachinery, 109, pp. 177-185. 
Houtermans, R., Coton, T. and Arts, T. (2004), “Aerodynamic Performance of a Very High Lift Low Pressure Turbine Blade with Emphasis on Separation Prediction," Journal of Turbomachinery, 126, pp. 406-413.

Ingram, G., Gregory-Smith, D. and Harvey, N. (2005), "Investigation of a Novel Secondary Flow Feature in a Turbine Cascade with End Wall Profiling," ASME Journal of Turbomachinery, 127, pp. 209-214.

Ingram, G., Gregory-Smith, D., Rose, M., Harvey, N. and Brennan, G. (2002), "The Effect of End-Wall Profiling on Secondary Flow and Loss Development in a Turbine Cascade," ASME Paper No. GT2002-30339, Amsterdam, Netherlands, June 3-6.

Jenny, P., Abhari, R. S., Rose, M. G., Brettschneider, M. and Gier, J. (2011), “A Low Pressure Turbine with Profiled End Walls and Purge Flow Operating with a Pressure Side Bubble,” ASME Paper No. GT2011-46309, Vancouver, BC, Canada, June 6-10.

Johnson, G. H. and Reed, L. S. (1985), "Seven Hole Probe in Shear Flow," AIAA 23rd Aerospace Sciences Meeting Paper No. AIAA-85-0076, Reno, NV, USA, Jan. 14-17.

Kacker, S. C. and Okapuu, U. (1982), "Mean Line Prediction Method for Axial Flow Turbine Efficiency," ASME Journal of Engineering for Power, 104, pp. 111-119.

Knezevici, D. C. (2011), "Controlling Secondary Flows in Very Highly-Loaded Low-Pressure Turbine Cascades," PhD thesis, Carleton University, Ottawa, Canada.

Knezevici, D. C., Sjolander, S. A., Praisner, T. J., Allen-Bradley, E. and Grover, E. A. (2009), "Measurements of Secondary Losses in a High-Lift Front-Loaded Turbine Cascade with the Implementation of Non-Axisymmetric Endwall Contouring," ASME Paper No. GT2009-59677, Orlando, FL, USA, June 8-12.

Knezevici, D. C., Sjolander, S. A., Praisner, T. J., Allen-Bradley, E. and Grover, E. A. (2010), "Measurements of Secondary Losses in a Turbine Cascade with the Implementation of Nonaxisymmetric Endwall Contouring," ASME Journal of Turbomachinery, 132, 011013.

Kopper, F. C., Milano, R. and Vanco, M. (1981), "Experimental Investigation of Endwall Profiling in a Turbine Vane Cascade," AIAA Journal, 19, pp. 1033-1040.

Kundu, P. K. and Cohen, I. M. (2004a), Fluid Mechanics, 3rd ed., San Diego, CA: Elsevier Academic Press, pp. 535-540, Chap. 13.

Kundu, P. K. and Cohen, I. M. (2004b), Fluid Mechanics, 3rd ed., San Diego, CA: Elsevier Academic Press, pp. 535-540, Chap. 13.

Langston, L. S. (2001), "Secondary Flows in Axial Turbines-a Review," Annals of the New York Academy of Sciences, 934, pp. 11-26. 
Langston, L. S., Nice, M. L. and Hooper, R. M. (1977), "Three-Dimensional Flow within a Turbine Cascade Passage," ASME Journal of Engineering for Power, 99 Ser A, pp. 2128.

Langtry, R. B., Menter, F. R., Likki, S. R., Suzen, Y. B., Huang, P. G. and Volker, S. (2006), “A Correlation-Based Transition Model Using Local Variables - Part II: Test Cases and Industrial Applications,” ASME Journal of Turbomachinery, 128, pp. 423-434.

Lewis, W. E. (1966), "Fixed-Direction Probes for Aerodynamic Measurements," Proceedings Institution of Mechanical Engineers 1965-66, 180 Part 3J, pp. 141-151.

Liberzon, A., Lüthi, B., Guala, M., Kinzelbach, W. and Tsinober, A. (2007), Progress in Turbulence II, ed., Springer Berlin Heidelberg, pp. 85-88, Chap. 17.

Lipfert, M., Marx, M., Rose, M. G., Staudacher, S., Mahle, I., Freygang, U. and Brettschneider, M. (2014), “A Low Pressure Turbine at Extreme Off-Design Operation,” ASME Journal of Turbomachinery, 136, 031018.

Lott, P. T., Hills, N. J., Chew, J. W., Scanion, T. and Shahpar, S. (2009), "High Pressure Turbine Stage Endwall Profile Optimisation for Performance and Rim Seal Effectiveness," ASME Paper No. GT2009-59923, Orlando, FL, United states, June 8-12.

Luo, J., Liu, F. and McBean, I. (2011), "Optimization of Endwall Contours of a Turbine Blade Row Using an Adjoint Method," ASME Paper No. GT2011-46163, Vancouver, BC, Canada, June 6-10.

Luo, J., Xiong, J., Liu, F. and McBean, I. (2010), "Secondary Flow Reduction by Blade Redesign and Endwall Contouring Using an Adjoint Optimization Method," ASME Paper No. GT2010-22061, Glasgow, UK, June 14-18.

Lyall, M. E., King, P. I., Clark, J. P. and Sondergaard, R. (2013), "Endwall Loss Reduction of High Lift Low Pressure Turbine Airfoils Using Profile Contouring - Part I: Airfoil Design,” ASME Paper No. GT2013-95000, San Antonio, TX, USA, June 3-7.

Lynch, S. P., Thole, K. A., Kohli, A., Lehane, C. and Praisner, T. (2013), “Aerodynamic Loss for a Turbine Blade with Endwall Leakage Features and Contouring," ASME Paper No. GT2013-94943, San Antonio, TX, USA, June 3-7.

MacIsaac, G. D., Taremi, F., Knezevici, D. C., Scribner, C. A. and Sjolander, S. A. (2009), "Challenges in Using Kiel and Seven-Hole Pressure Probes in Highly Three-Dimensional Flows: Application to Turbomachinery Cascade Measurements," $19^{\text {th }}$ ISABE Conference Paper No. ISABE-2009-1209, Montreal, QC, CA, Sept. 11-17.

Mahallati, A. (2003), "Aerodynamic of a Low-Pressure Turbine Airfoil under Steady and Periodically Unsteady Conditions," PhD thesis, Carleton University, Ottawa, Canada. 
Mahallati, A. and Sjolander, S. A. (2007), "Aerodynamics of a Low-Pressure Turbine Airfoil at Low-Reynolds Numbers Part 2: Blade-Wake Interaction," ASME Paper No. GT200727348, Montreal, QC, CA, May 14-17.

Marini, R. and Girgis, S. (2007), "The Effect of Blade Leading Edge Platform Shape on Upstream Disk Cavity to Mainstream Flow Interaction of a High-Pressure Turbine Stage,” ASME Paper No. GT2007-27429, Montreal, QC, CA, May 14-17.

Mayle, R. E. (1991), “The 1991 IGTI Scholar Lecture: The Role of Laminar-Turbulent Transition in Gas Turbine Engines," Journal of Turbomachinery, 113, pp. 509-536.

McLean, C., Camci, C. and Glezer, B. (2001a), "Mainstream Aerodynamic Effects Due to Wheelspace Coolant Injection in a High-Pressure Turbine Stage: Part I - Aerodynamic Measurements in the Stationary Frame," Journal of Turbomachinery, 123, pp. 687-696.

McLean, C., Camci, C. and Glezer, B. (2001b), "Mainstream Aerodynamic Effects Due to Wheelspace Coolant Injection in a High-Pressure Turbine Stage: Part II - Aerodynamic Measurements in the Rotational Frame," Journal of Turbomachinery, 123, pp. 697-703.

Menter, F. R. (1994), “Two-Equation Eddy-Viscosity Turbulence Models for Engineering Applications," AIAA Journal, 32, pp. 1598-1605.

Menter, F. R., Langtry, R. B., Likki, S. R., Suzen, Y. B., Huang, P. G. and Volker, S. (2006), “A Correlation-Based Transition Model Using Local Variables - Part I: Model Formulation," ASME Journal of Turbomachinery, 128, pp. 413-422.

Miyoshi, I., Higuchi, S. i. and Kishibe, T. (2013), "Improving the Performance of a High Pressure Gas Turbine Stage Using a Profiled Endwall," ASME Paper No. GT2013-95148, San Antonio, TX, USA, June 3-7.

Moffat, R. J. (1982), "Contributions to the Theory of Single-Sample Uncertainty Analysis," ASME Journal of Fluids Engineering, 104, pp. 250-258.

Moore, J. and Adhye, R. Y. (1985), "Secondary Flows and Losses Downstream of a Turbine Cascade," ASME Journal of Engineering for Gas Turbines and Power, 107, pp. 961-968.

Moore, J., Shaffer, D. M. and Moore, J. G. (1987), "Reynolds Stresses and Dissipation Mechanisms Downstream of a Turbine Cascade," ASME Journal of Turbomachinery, 109, pp. 258-267.

Morris, A. W. H. and Hoare, R. G. (1975), "Secondary Loss Measurements in a Cascade of Turbine Blades with Meridional Wall Profiling," American Society of Mechanical Engineers (Paper).

Moustapha, S. H. and Williamson, R. G. (1986), "Effect of Two Endwall Contours on the Performance of an Annular Nozzle Cascade," AIAA Journal, 24, pp. 1524-1530.

Niewoehner, J., Poehler, T., Jeschke, P. and Guendogdu, Y. (2015), "Investigation of Nonaxisymmetric Endwall Contouring and Three-Dimensional Airfoil Design in a 1.5 
Stage Axial Turbine-Part II: Experimental Validation,” Journal of Turbomachinery, 137, 081010 .

Owen, J. M. and Wilson, M. (2001), "Some Current Research in Rotating-Disc Systems," Journal of Heat Transfer in Gas Turbine Systems, 934, pp. 206-221.

Panchal, K., Abraham, S., Ekkad, S. V., Ng, W., Brown, B. J. and Malandra, A. (2011), "Investigation of Effect of End Wall Contouring Methods on a Transonic Turbine Blade Passage,” ASME Paper No. GT2011-45192, Vancouver, BC, Canada, June 6-10.

Paniagua, G., Denos, R. and Almeida, S. (2004), "Effect of the Hub Endwall Cavity Flow on the Flow-Field of a Transonic High-Pressure Turbine," ASME Journal of Turbomachinery, 126, pp. 578-586.

Perdichizzi, A. and Dossena, V. (1993), "Incidence Angle and Pitch-Chord Effects on Secondary Flows Downstream of a Turbine Cascade," ASME Journal of Turbomachinery, 115, pp. 383-391.

Perdichizzi, A., Ubaldi, M. and Zunino, P. (1992), "Reynolds Stress Distribution Downstream of a Turbine Cascade," Experimental Thermal and Fluid Science, 5, pp. 338-350.

Piggush, J. D. and Simon, T. W. (2013), "Flow Measurements in a First Stage Nozzle Cascade Having Endwall Contouring, Leakage, and Assembly Features," Journal of Turbomachinery, 135.

Poehler, T., Gier, J. and Jeschke, P. (2010), "Numerical and Experimental Analysis of the Effects of Non-Axisymmetric Contoured Stator Endwalls in an Axial Turbine," ASME Paper No. GT2010-23350, Glasgow, UK, June 14-18.

Poehler, T., Niewoehner, J., Jeschke, P. and Guendogdu, Y. (2015), "Investigation of Nonaxisymmetric Endwall Contouring and Three-Dimensional Airfoil Design in a 1.5Stage Axial Turbine-Part I: Design and Novel Numerical Analysis Method," Journal of Turbomachinery, 137, 081009.

Popovic, I. (2005), "Measured Steady and Unsteady Aerodynamic Performance of a Family of Three Highly-Loaded Low-Pressure Turbine Cascades," MASc thesis, Carleton University, Ottawa, Canada.

Popovic, I. and Hodson, H. P. (2013a), "Aerothermal Impact of the Interaction between Hub Leakage and Mainstream Flows in Highly-Loaded High Pressure Turbine Blades," Journal of Turbomachinery, 135, 061014.

Popovic, I. and Hodson, H. P. (2013b), "The Effects of a Parametric Variation of the Rim Seal Geometry on the Interaction between Hub Leakage and Mainstream Flows in High Pressure Turbines," ASME Journal of Engineering for Gas Turbines and Power, 135, 112501. 
Popovic, I. and Hodson, H. P. (2013c), "Improving Turbine Stage Efficiency and Sealing Effectiveness through Modifications of the Rim Seal Geometry," Journal of Turbomachinery, 135, 061016.

Popovic, I., Hodson, H. P., Janke, E. and Wolf, T. (2013), “The Effects of Unsteadiness and Compressibility on the Interaction between Hub Leakage and Mainstream Flows in HighPressure Turbines," Journal of Turbomachinery, 135, 061015.

Porreca, L., Hollenstein, M., Kalfas, A. I. and Abhari, R. S. (2007), "Turbulence Measurements and Analysis in a Multistage Axial Turbine," Journal of Propulsion and Power, 23, pp. 227-234.

Praisner, T. J., Allen-Bradley, E., Grover, E. A., Knezevici, D. C. and Sjolander, S. A. (2007), "Application of Non-Axisymmetric Endwall Contouring to Conventional and High-Lift Turbine Airfoils," ASME Paper No. GT2007-27579, Montreal, QC, CA, May 14-17.

Praisner, T. J., Allen-Bradley, E., Grover, E. A., Knezevici, D. C. and Sjolander, S. A. (2013), "Application of Nonaxisymmetric Endwall Contouring to Conventional and High-Lift Turbine Airfoils," ASME Journal of Turbomachinery, 135.

Prevost, J. M. (2013), "Controlling Secondary Flows in Highly-Loaded Compressor Cascades," MASc thesis, Carleton University, Ottawa, Canada.

Prumper, H. (1972), "Application of Boundary Layer Fences in Turbomachinery," AGARDograph No. 164, pp. 311-331.

Reid, K., Denton, J., Pullan, G., Curtis, E. and Longley, J. (2006), "The Effect of Stator-Rotor Hub Sealing Flow on the Mainstream Aerodynamics of a Turbine," ASME Paper No. GT2006-90838, Barcelona, Spain, May 6 - 11.

Ristic, D., Lakshminarayana, B. and Chu, S. (1999), “Three-Dimensional Flowfield Downstream of an Axial-Flow Turbine Rotor," Journal of Propulsion and Power, 15, pp. 334-344.

Rodger, P. (1992), "Establishing Tow-Dimensional Flow in a Large-Scale Planar Turbine Cascade," M.Eng. thesis, Carleton University, Ottawa, Canada.

Rose, M. G. (1994), "Non-Axisymmetric Endwall Profiling in the HP NGV's of an Axial Flow Gas Turbine," ASME Paper No. 94-GT-249, Hague, Netherlands, June 13-16.

Rose, M. G., Harvey, N. W., Seaman, P., Newman, D. A. and McManus, D. (2001), "Improving the Efficiency of the Trent 500-HP Turbine Using Nonaxisymmetric End Walls-Part II: Experimental Validation,” ASME Paper No. 2001-GT-0505, New Orleans, Louisianna, USA, June 4-7.

Rudiger, G. (1980), “On Negative Eddy Viscosity in Mhd Turbulence,” Magnetohydrodynamics, 16, pp. 1-10. 
Saha, R., Fridh, J., Fransson, T., Mamaev, B. I. and Annerfeldt, M. (2012), "Experimental Studies of Leading Edge Contouring Influence on Secondary Losses in Transonic Turbines," ASME Paper No. GT2012-68497, Copenhagen, Denmark, June 11-15.

Sangston, K., Little, J., Lyall, M. E. and Sondergaard, R. (2013), "Endwall Loss Reduction of High Lift Low Pressure Turbine Airfoils Using Profile Contouring - Part II: Validation," ASME Paper No. GT2013-95002, San Antonio, TX, USA, June 3-7.

Sauer, H., Muller, R. and Vogeler, K. (2001), "Reduction of Secondary Flow Losses in Turbine Cascades by Leading Edge Modifications at the Endwall," Journal of Turbomachinery, 123, pp. 207-213.

Schmitt, F. G. (2007), “About Boussinesq's Turbulent Viscosity Hypothesis: Historical Remarks and a Direct Evaluation of Its Validity," C. R. Mécanique, 335, pp. 617-627.

Schobeiri, M. T. and Lu, K. (2014), "Endwall Contouring Using Continuous Diffusion: A New Method and Its Application to a Three-Stage High Pressure Turbine," ASME Journal of Turbomachinery, 136, 011006.

Schuepbach, P., Abhari, R. S., Rose, M. G., Germain, T., Raab, I. and Gier, J. (2010), "Effects of Suction and Injection Purge-Flow on the Secondary Flow Structures of a High-Work Turbine," ASME Journal of Turbomachinery, 132, 021021.

Schuepbach, P., Abhari, R. S., Rose, M. G. and Gier, J. (2011), "Influence of Rim Seal Purge Flow on the Performance of an Endwall-Profiled Axial Turbine," ASME Journal of Turbomachinery, 133, 021011.

Schuler, P., Kurz, W., Dullenkopf, K. and Bauer, H. J. (2010), “The Influence of Different Rim Seal Geometries on Hot-Gas Ingestion and Total Pressure Loss in a Low-Pressure Turbine,” ASME Paper No. GT2010-22205, Glasgow, United kingdom, June 14-18.

Schüpbach, P., Abhari, R. S., Rose, M. G., Germain, T., Raab, I. and Gier, J. (2010), “Improving Efficiency of a High Work Turbine Using Nonaxisymmetric Endwalls - Part II: TimeResolved Flow Physics,” ASME Journal of Turbomachinery, 132, 021008.

Scribner, C. A. (2011), "The Effect of Turbulence Intensity and Reynolds Number on the Aerodynamic Behaviour of Kiel, Three-Hole, and Seven-Hole Pressure Probes," MASc thesis, Carleton University, Ottawa, Canada.

Shahpar, S., Lapworth, B., Pablos, T. D. and Taylor, M. (1999), "A Linear Approach to the Multi-Parameter Design of Three-Dimensional Turbomachinery Blades," 37th AIAA Aerospace Sciences Meeting and Exhibit, Paper No. AIAA 99-0363, Reno, NV, January 11-14.

Sharma, O. P. and Butler, T. L. (1987), "Predictions of Endwall Losses and Secondary Flows in Axial Flow Turbine Cascades," ASME Journal of Turbomachinery, 109, pp. 229-236. 
Sieverding, C. H. (1985), "Recent Progress in the Understanding of Basic Aspects of Secondary Flows in Turbine Blade Passages," ASME Journal of Engineering for Gas Turbines and Power, 107, pp. 248-257.

Sjolander, S. A. (1975), "The Endwall Boundary Layer in an Annular Cascade of Turbine Nozzle Guide Vanes," M.Eng. thesis, Carleton University, Ottawa.

Sjolander, S. A. (2007), "Supplementry Course Notes: Fluid Machinery/Turbomachinery" Department of Mechanical and Aerospace Engineering, Carleton University: Ottawa, ON, Canada.

Snedden, G., Dunn, D., Ingram, G. and Gregory-Smith, D. (2009), "The Application of NonAxisymmetric Endwall Contouring in a Single Stage, Rotating Turbine," ASME Paper No. GT2009-59169, Orlando, FL, United states, June 8-12.

Szodruch, J., Grimme, W., Blumrich, F. and Schmid, R. (2011), "Next Generation Single-Aisle Aircraft - Requirements and Technological Solutions," Journal of Air Transport Management, 17, pp. 33-39.

Taremi, F., Sjolander, S. A. and Praisner, T. J. (2013), "Application of Endwall Contouring to Transonic Turbine Cascades: Experimental Measurements at Design Conditions," ASME Journal of Turbomachinery, 135, 011031.

Taylor, B. N. and Kuyatt, C. E. (1994), "Guidelines for Evaluating and Expressing the Uncertainty of Nist Measurement Results," NIST Technical Note, Release - 1297.

Torre, D., Vazquez, R., de la Rosa Blanco, E. and Hodson, H. P. (2011), “A New Alternative for Reduction in Secondary Flows in Low Pressure Turbines," ASME Journal of Turbomachinery, 133, 011029.

Turgut, O. H. and Camci, C. (2012), "Experimental Investigation and Computational Evaluation of Contoured Endwall and Leading Edge Fillet Configurations in a Turbine NGV," ASME Paper No. GT2012-69304, Copenhagen, Denmark, June 11-15.

Turgut, O. H. and Camci, C. (2013), "Influence of Leading Edge Fillet and Nonaxisymmetric Contoured Endwall on Turbine NGV Exit Flow Structure and Interactions with the Rim Seal Flow," ASME Paper No. GT2013-95843, San Antonio, TX, USA, June 3-7.

Vicharelli, A. and Eaton, J. K. (2006), "Turbulence Measurements in a Transonic Two-Passage Turbine Cascade," Experiments in Fluids, 40, pp. 897-917.

Wang, H. P., Olson, S. J., Goldstein, R. J. and Eckert, E. R. G. (1997), "Flow Visualization in a Linear Turbine Cascade of High Performance Turbine Blades," ASME Journal of Turbomachinery, 119, pp. 1-8.

Warner, R. E. and Tran, M. H. (1987), "Recent Developments to Improve High-Pressure and Intermediate-Pressure Turbine Efficiency," Proceedings of the Institution of Mechanical 
Engineers International Conference: Turbomachinery - Efficiency Prediction and Improvement. Paper No. Cambridge, Engl,

Weiss, A. P. and Fottner, L. (1995), "Influence of Load Distribution on Secondary Flow in Straight Turbine Cascades," Journal of Turbomachinery, 117, pp. 133-141.

Wilcox, D. C. (1998), Turbulence Modeling for CFD, 2nd ed., La Canada, Calif:: DCW Industries.

Wirth, A., Gama, S. and Frisch, U. (1995), "Eddy Viscosity of Three-Dimensional Flow," Journal of Fluid Mechanics, 288, pp. 249-264.

Wisler, D. C. (1998), "The Technical and Economic Relevance of Understanding Boundary Layer Transition in Gas Turbine Engines," 1997 Workshop on Boundary Layer Transition in Turbomachines, NASA/CP-1998-206958 in Minnowbrook II, pp. 53-64.

Yan, J., Gregory-Smith, D. and Walker, P. J. (1999), "Secondary Flow Reduction in a Nozzle Guide Vane by Non-Axisymmetric End-Wall Contouring,” ASME Paper No. 99-GT-339, Indianapolis, Indiana, USA, June 7-10.

Yaras, M. (1987), "Flow in the Tip Gap of a Rectilinear Turbine Cascade," MEng thesis, Carleton University, Ottawa, Canada.

Yaras, M. (1990), "Measurement and Prediction of Tip-Clearance Effects in a Linear Turbine Cascade," PhD thesis, Carleton University, Ottawa, Canada.

Yaras, M. I. and Sjolander, S. A. (1992), "Effects of Simulated Rotation on Tip Leakage in a Planar Cascade of Turbine Blades: Part I - Tip Gap Flow," ASME Journal of Turbomachinery, 114, pp. 652-659.

Yaras, M. I., Sjolander, S. A. and Kind, R. J. (1992), "Effects of Simulated Rotation on Tip Leakage in a Planar Cascade of Turbine Blades: Part II - Downstream Flow Field and Blade Loading," ASME Journal of Turbomachinery, 114, pp. 660-667.

Yavuzkurt, S. (1984), "A Guide to Uncertainty Analysis of Hot-Wire Data," Transactions of the ASME. Journal of Fluids Engineering, 106, pp. 181-6.

Zess, G. A. and Thole, K. A. (2002), "Computational Design and Experimental Evaluation of Using a Leading Edge Fillet on a Gas Turbine Vane," Journal of Turbomachinery, 124, pp. 167-175.

Zoric, T. (2006), "Experimental Investigation of Secondary Flows in a Family of Three Highly Loaded Low-Pressure Turbine Cascades," MASc thesis, Carleton University, Ottawa, Canada.

Zoric, T., Popovic, I., Sjolander, S. A., Praisner, T. and Grover, E. (2007a), "Comparative Investigation of Three Highly Loaded LP Turbine Airfoils: Part I - Measured Profile and Secondary Losses at Design Incidence," ASME Paper No. GT2007-27537, Montreal, QC, Canada, May 14-17. 
Zoric, T., Popovic, I., Sjolander, S. A., Praisner, T. and Grover, E. (2007b), "Comparative Investigation of Three Highly Loaded LP Turbine Airfoils: Part II - Measured Profile and Secondary Losses at Off-Design Incidence," ASME Paper No. GT2007-27538, Montreal, QC, CA, May 14-17.

Zweifel, O. (1945), "Spacing of Turbo-Machine Blading, Especially with Large Angular Deflection,” Brown Boveri Review, 32, pp. 436-444. 


\section{Appendix A}

\section{Pressure Transducer Calibration}

The pressure transducers were calibrated using the micromanometer shown in Figure A.1 which was designed and manufactured by Benner (2003) and Mahallati (2003). The full-scale range of the micrometer is $0-30 \mathrm{~cm}$ of $\mathrm{H}_{2} \mathrm{O}$ with a differential measurement uncertainty of $\pm 0.05 \mathrm{~mm}$ of $\mathrm{H}_{2} \mathrm{O}$. The water columns are filled with distilled water so the density can be estimated within $\pm 0.1 \mathrm{~kg} / \mathrm{m}^{3}$. The large diameter water columns reduce the meniscus effect while the pointed needles attached to micrometers reduce the visual ambiguity of water column height.

To calibrate the transducers, the micromanometer output was plumed in parallel to each transducer input. After applying a constant pressure to the water column using a pneumatic syringe, the voltage output $(E)$ was recorded using the data acquisition system. The applied pressure was calculated using $P_{\text {applied }}=\rho g h$, where $h$ is the measured column height difference and the $\rho$ air density. Separate calibrations were performed for the \pm 1 inch and \pm 5 inch nominal range transducers, ASG DRAL501DN and ASG DRAL505DN respectively. The transducer output voltage $(E)$ was related to the applied pressure $(P)$ using the linear relation,

$$
P=C_{1}+C_{2} E
$$

where $C_{1}-C_{2}$ are the calibration coefficients. Prior to making flow field measurements, the value of $C_{1}$ was adjusted by zeroing the transducers at the ambient conditions. This was also routinely done after completing a set of flow field measurements. 


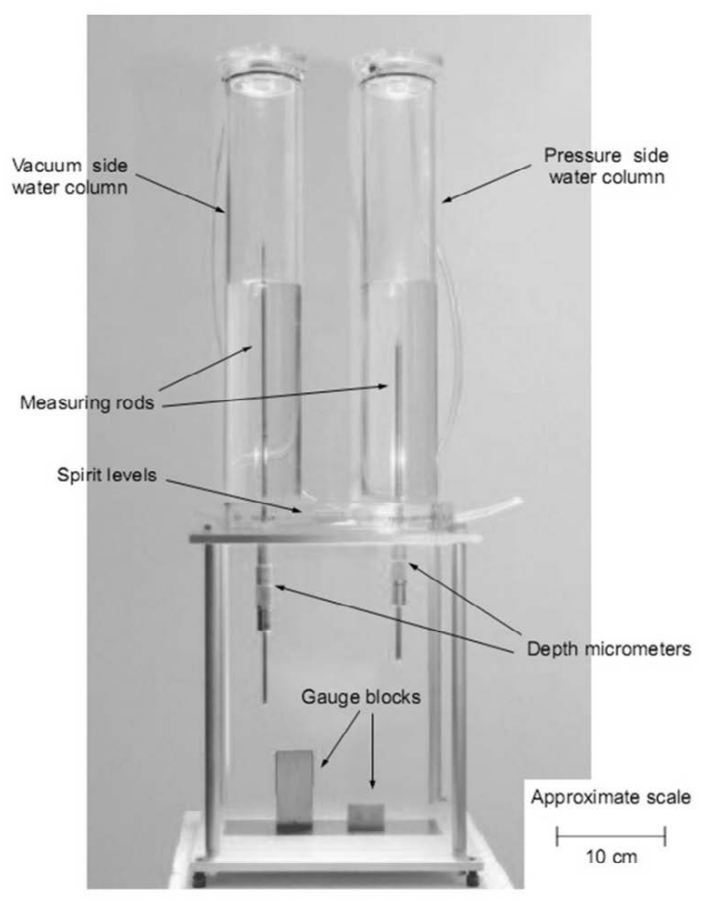

Figure A.1: Water micromanometer, from Benner (2003)

The linear relation of Equation A.1 was a satisfactory fit for the \pm 1 inch transducers; however, several trials showed the \pm 5 inch transducers calibrations were non-linear at higher pressures. Therefore, the relation

$$
P=C_{1}+C_{2} E+C_{3} E^{2}+C_{4} E^{3}
$$

where $C_{1}-C_{4}$ are the calibration coefficients was used for the larger range transducers. Calibration coefficients for the four \pm 1 inch (ASG DRAL501DN) and eight \pm 5 inch (ASG DRAL505DN) transducers are shown in Tables A.1 and A.2 respectively. Sample calibration data with the curve fits are shown in Figures A.2 and A.3.

Table A.1: Calibration coefficients for the ASG DRAL501DN $\left( \pm 1\right.$ inch $\left.\mathrm{H}_{2} \mathrm{O}\right)$ transducers

\begin{tabular}{|c|c|c|}
\hline Transducer & $\mathbf{C}_{\mathbf{1}}$ & $\mathbf{C}_{\mathbf{2}}$ \\
\hline DRAL501DN 1 & -353.1 & 99.2 \\
\hline DRAL501DN 2 & -334.6 & 96.1 \\
\hline DRAL501DN 3 & -330.2 & 96.3 \\
\hline DRAL501DN 4 & -334.4 & 96.1 \\
\hline
\end{tabular}


Table A.2: Calibration coefficients for the ASG DRAL505DN ( \pm 5 inch $\mathrm{H}_{2} \mathrm{O}$ ) transducers

\begin{tabular}{|c|c|c|c|c|}
\hline Transducer & $\mathbf{C}_{\mathbf{1}}$ & $\mathbf{C}_{\mathbf{2}}$ & $\mathbf{C}_{\mathbf{3}}$ & $\mathbf{C}_{\mathbf{4}}$ \\
\hline DRAL505DN 1 & -1710.8 & 494.4 & -0.514 & 0.052 \\
\hline DRAL505DN 2 & -1743.8 & 498.4 & -0.596 & 0.057 \\
\hline DRAL505DN 3 & -1712.6 & 489.4 & -0.559 & 0.054 \\
\hline DRAL505DN 4 & -1754.0 & 521.4 & -8.744 & 0.815 \\
\hline DRAL505DN 5 & -1718.8 & 497.3 & -0.991 & 0.091 \\
\hline DRAL505DN 6 & -1723.3 & 493.8 & -1.002 & 0.094 \\
\hline DRAL505DN 7 & -1736.3 & 509.2 & -5.327 & 0.479 \\
\hline DRAL505DN 8 & -1727.7 & 504.3 & -4.331 & 0.400 \\
\hline
\end{tabular}

(a)

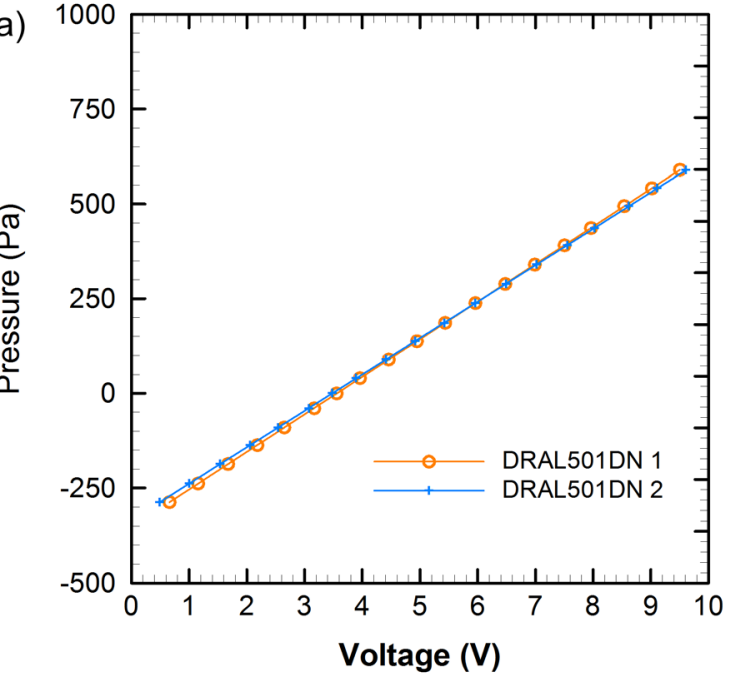

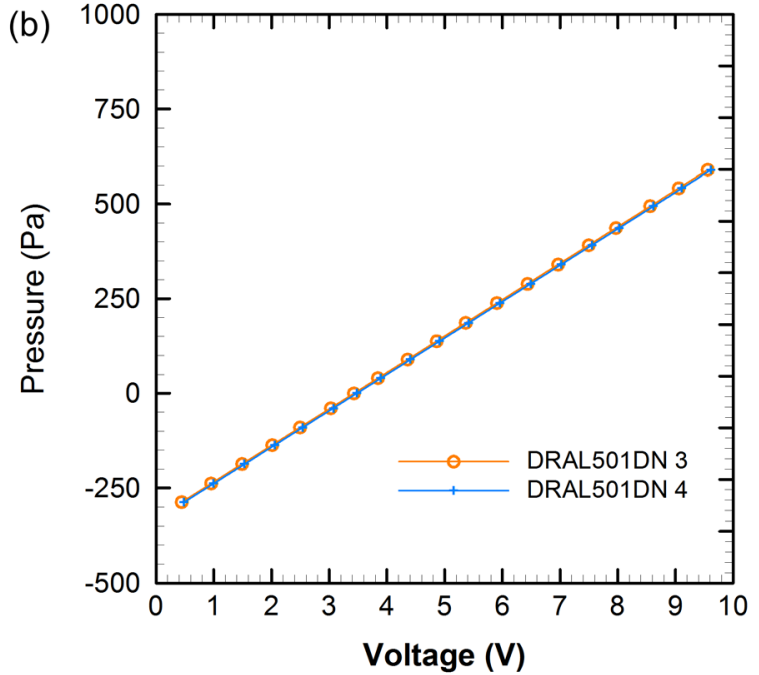

Figure A.2: Calibration data and curve fits for the ASG DRAL501DN $\left( \pm 1\right.$ inch $\left.\mathrm{H}_{2} \mathrm{O}\right)$ transducers 

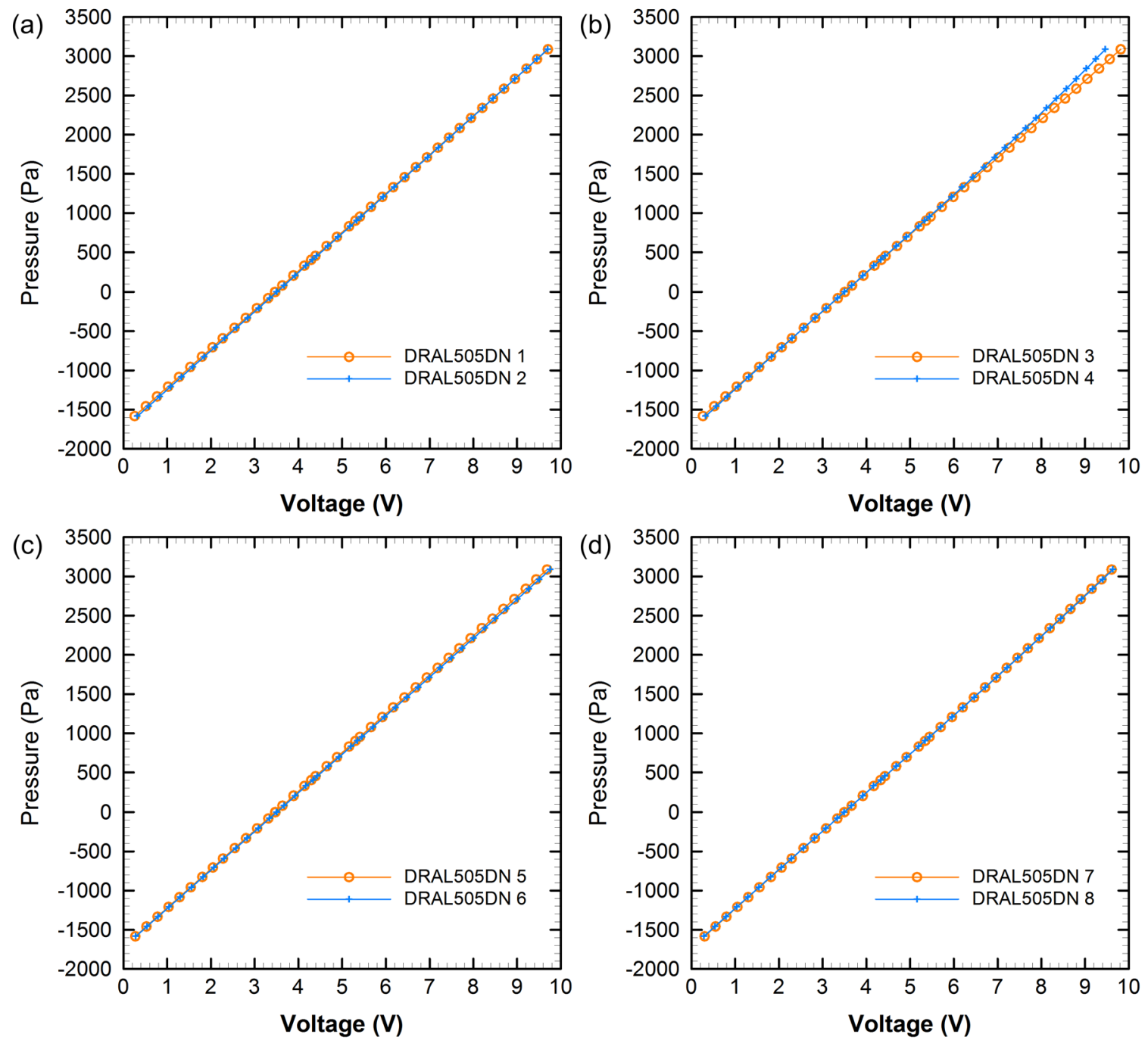

Figure A.3: Calibration data and curve fits for the ASG DRAL505DN $\left( \pm 5\right.$ inch $\left.\mathrm{H}_{2} \mathrm{O}\right)$ transducers 


\section{Appendix B}

\section{Procedure for Determining Experimental Uncertainties}

Experimental measurements are susceptible to errors. Even when the experiments and procedures are carefully designed, the errors, although minimized, cannot be eliminated. For wind tunnel experiments, the $N^{\text {th }}$-order single sample analysis procedure of Moffat (1982) is a well accepted method for uncertainty estimation. In this method, the elemental sources of error are summed using the law of propagation of uncertainty (i.e. the root-sum-square method) which is also documented in a NIST technical note by Taylor and Kuyatt (1994).

Measurement errors may be categorized as bias or random. The bias, or fixed, errors cause a measurement to be consistently different from the true value. In the context of a mature windtunnel experiment, the bias errors are minimized over time through the systematic refinement of the facility procedures. The random, or precision, errors are recognized as scatter from the true value and are introduced by signal discretization, probe positioning, interpolation, repeatability, etc. In many experimental instances it can be difficult to decouple the bias and random components of the error. In the procedure of Moffat (1982) both are accounted for by quantifying the errors introduced through instrument calibration.

For any measured quantity, denoted here by $x$, the uncertainty can be estimated from the calibration data and is given by 


$$
\delta x= \pm 2 \sigma
$$

where $\sigma$ is the standard deviation. If the scatter of the measured quantity follows a Gaussian (normal) distribution then the true value is expected to fall within the uncertainty bandwidth, $\delta x$, with a confidence of $95 \%$.

Many of the reported flow parameters in this thesis are calculated from several individual measurements (e.g the overall loss coefficient, Y). Thus, in this context a calculated parameter, denoted by $X$, is then a function of the elemental errors and is given by

$$
X=X\left(x_{1}, x_{2}, \ldots, x_{N-1}, x_{N}\right)
$$

where $N$ is the total number of errors. The method of Moffat (1982) then shows that the contribution to the uncertainty in $X$ from each independent variable $x_{n}$ is given by

$$
\delta X_{n}=\frac{\partial X}{\partial x_{n}} \delta x_{n}
$$

The partial derivatives are a calculated by independently perturbing the elemental sources of error and observing the effect on overall calculated quantity $(X)$. The overall uncertainty in $X$, with $95 \%$ confidence, is calculated using the root-sum of squares (RSS) method and is then given by

$$
\delta X=\sqrt{\sum_{n=1}^{N}\left(\delta X_{n}\right)^{2}}
$$




\section{Appendix C}

\section{Calibration Procedures and Data Reduction Methods for the Three-hole Pressure Probe}

The following describes the calibration and data reduction procedures for the three-hole probe introduced in Section 3.8.4 and shown schematically in Figure 3.13. Initially, the probe is placed in the calibration jet with the probe tip positioned at the centre of rotation and the body axis aligned with the nozzle. After aerodynamically aligning the probe, the calibration is performed by rotating the probe through the pitchwise angle range of $\phi= \pm 21^{\circ}$ in $0.5^{\circ}$ increments. The measured port pressures from each orientation are corrected to the flow conditions at the probe tip using,

$$
C_{P i}=\frac{P_{i}-P_{S}}{P_{0}-P_{S}}=\left(\frac{P_{i}-P_{C 2}}{P_{C 1}-P_{C 2}}-C_{P S}\right) \cdot \frac{1}{C_{q_{C a l}}} \quad \text { for } i=1,2,3
$$

where $i$ is the probe port number and $C_{q_{C a l}}$ is the dynamic pressure coefficient for the calibration nozzle (see Section 3.9).

The method of Lewis (1966) is then applied to determined the six non-dimensional calibration coefficients,

$$
K_{1}=\frac{P_{1}-P_{S}}{q}=C_{P 1}
$$




$$
\begin{gathered}
K_{12}=\frac{P_{1}-P_{2}}{q}=C_{P 1}-C_{P 2} \\
K_{13}=\frac{P_{1}-P_{3}}{q}=C_{P 1}-C_{P 3} \\
K_{23}=\frac{P_{2}-P_{3}}{q}=C_{P 2}-C_{P 3} \\
K_{\phi 2}=\frac{P_{1}-P_{2}}{P_{1}-P_{3}}=\frac{K_{12}}{K_{13}} \\
K_{\phi 3}=\frac{P_{1}-P_{3}}{P_{1}-P_{2}}=\frac{1}{K_{\phi 2}}
\end{gathered}
$$

Sample calibration data for two different operating points are shown in Figure C.1. The $K_{12}, K_{13}$ and $K_{23}$ coefficients show a slight sensitivity to Reynolds number and it was therefore necessary to calibrate the probe for the expected velocities levels of the cascade inlet and outlet. A total of four calibrations were performed for jet velocities of 10, 15, 30 and $42 \mathrm{~m} / \mathrm{s}$ which correspond to Reynolds numbers (based on the probe tip width) of 1220, 1830, 3660 and 5120 respectively.

To characterize an unknown two-dimensional flow the following procedure is used. First, the port pressures $P_{1}, P_{2}$ and $P_{3}$ are measured and the values of $K_{\phi 2}$ and $K_{\phi 3}$ are calculated according to Equations C.6 and C.7. Theoretically, the flow angle $(\phi)$ can be linearly interpolated from either the $K_{\phi 2}$ or $K_{\phi 3}$ calibration data; however, Figure C.1 (d) shows that the $K_{\phi 2}$ and $K_{\phi 3}$ calibration curves have less scatter for negative and positive flow angles respectively. Therefore, the sign of the flow angle is first determined by comparing the values of $P_{2}$ and $P_{3}$ and then the data reduction continues as follows:

1. If $P_{2}$ reads high, $\phi$ is determined from $K_{\phi 2}$. Otherwise, $\phi$ is found using $K_{\phi 3}$.

2. If $P_{2}$ reads high, $q$ is determined from the value of $K_{12}$ at $\phi$. Otherwise, $q$ is found from $K_{13}$.

3. $P_{S}$ is determined using $q$ and value $K_{l}$ at $\phi$.

4. $P_{0}$ is determined from $P_{0}=P_{S}+q$ 
In practice, the probe is positioned in the wind-tunnel at a fixed orientation (non-nulling mode). In order to reduce errors caused by high levels of flow misalignment, the probe axis is aligned with the expected mean flow direction such that only small flow angles are measured. The angle of the probe axis relative to the cascade $(\varphi)$ was measured using a digital protractor within $\pm 0.1^{\circ}$. The direction of the cascade flow $(\beta)$ was determined by:

$$
\beta=\phi+\varphi
$$

(a)

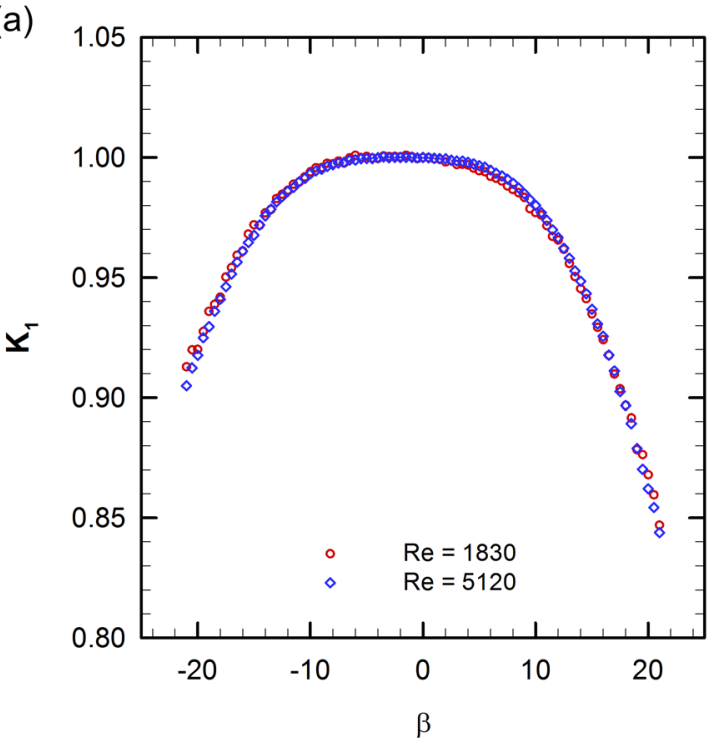

(c)

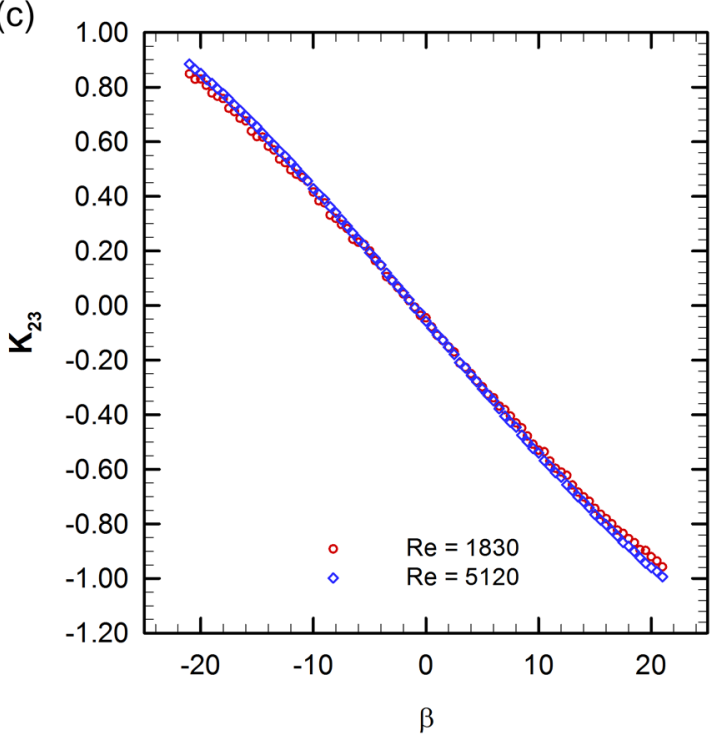

(b)

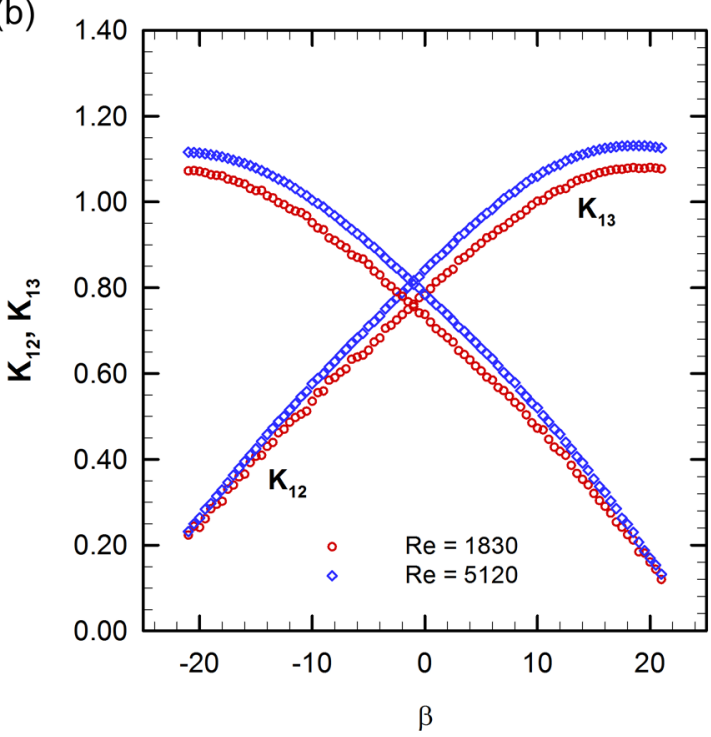

(d)

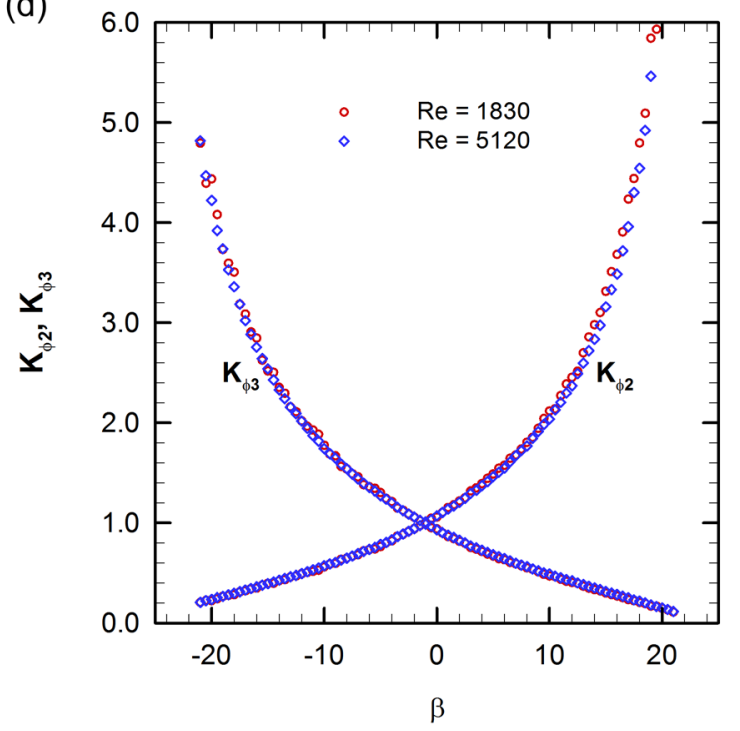

Figure C.1: Sample calibration data for the three-hole probe at two Reynolds numbers 


\section{Appendix D}

\section{Calibration Procedures and Data Reduction Methods for the Seven-hole Pressure Probe}

\section{D.1 Introduction}

The following section describes the calibration and data reduction procedures for the seven-hole probe introduced in Section 3.8.5. Figure D.1 shows the probe port numbering scheme as well as the probe coordinate system. Calibrations were performed for flow misalignment in both the pitch $(\beta)$ and yaw $(\alpha)$ directions following the method of Gerner et al. (1984). Here, the data reduction procedures have been slightly modified to improve the accuracy of the inferred dynamic pressure in low-Reynolds number flows (see Section D.3).

The overall calibration is applicable for an angle range of $\pm 70^{\circ}$. Depending on the level of misalignment, two different flow regimes are possible and each requires different calibration procedures. At low angles ( $\alpha$ or $\beta<25^{\circ}$ ), the flow remains fully attached as it passes over the probe tip and the centre port (7) will read the highest pressure. At high angles $\left(\alpha\right.$ or $\left.\beta>25^{\circ}\right)$, the flow tends to separate from the probe tip surface causing the leeward ports to be insensitive flow misalignment. One of the peripheral ports ( 1 through 6$)$ will read the highest pressure. In this work, the calibrations are performed over for the range of $-50^{\circ}<\alpha, \beta<50^{\circ}$ in $2^{\circ}$ increments for 
several velocity levels. At each data point, the flow is categorized into either the low- or highangle regime and the appropriate calibration procedure is applied. Like the three-hole probe, the measured pressures are first corrected to the measurement plane conditions according to Equation C.1.

Section D.2 will first describe the flow angle nomenclature and sections D.3 and D.4 follow with the data reduction procedures for the low- and high-angle regimes, respectively.
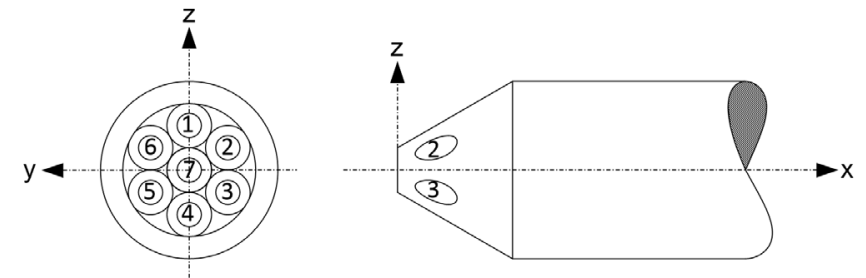

Figure D.1: Probe tip numbering and Cartesian coordinate system

\section{D.2 Flow Angle Nomenclature}

The following provides a brief summary of the subtle, but important, details of the flow angle nomenclature that were highlighted by Scribner (2011). Figures D.2 and D.3 show the two probe coordinate systems required for the seven-hole probe calibration. Figure D.2 shows the tangential coordinate system with the velocity vector, $V$, expressed in terms of the tangential flow angles, $\alpha$ and $\beta$.

$$
\begin{aligned}
& \alpha=\tan ^{-1}\left(\frac{u}{w}\right) \\
& \beta=\tan ^{-1}\left(\frac{u}{v}\right)
\end{aligned}
$$

$\alpha$ is the angle between the velocity component $u$ and the projection of $V$ in the $u w$ plane while $\beta$ is the angle between $u$ and the projection of $V$ in the $u v$ plane. As shown, $\alpha$ is positive when the flow is directed at port 4 causing it to read a higher pressure than port 1. Similarly, $\beta$ is positive 


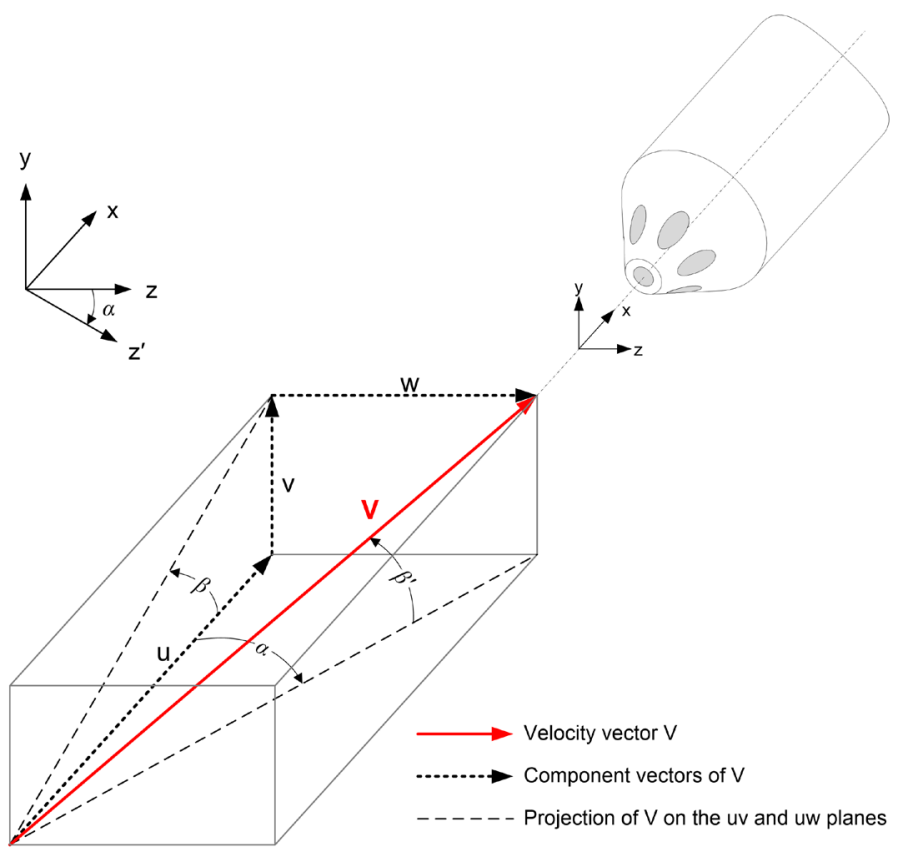

Figure D.2: Tangential probe coordinate system, reproduced from Scribner (2011) and originally adapted from Gerner et al. (1984)

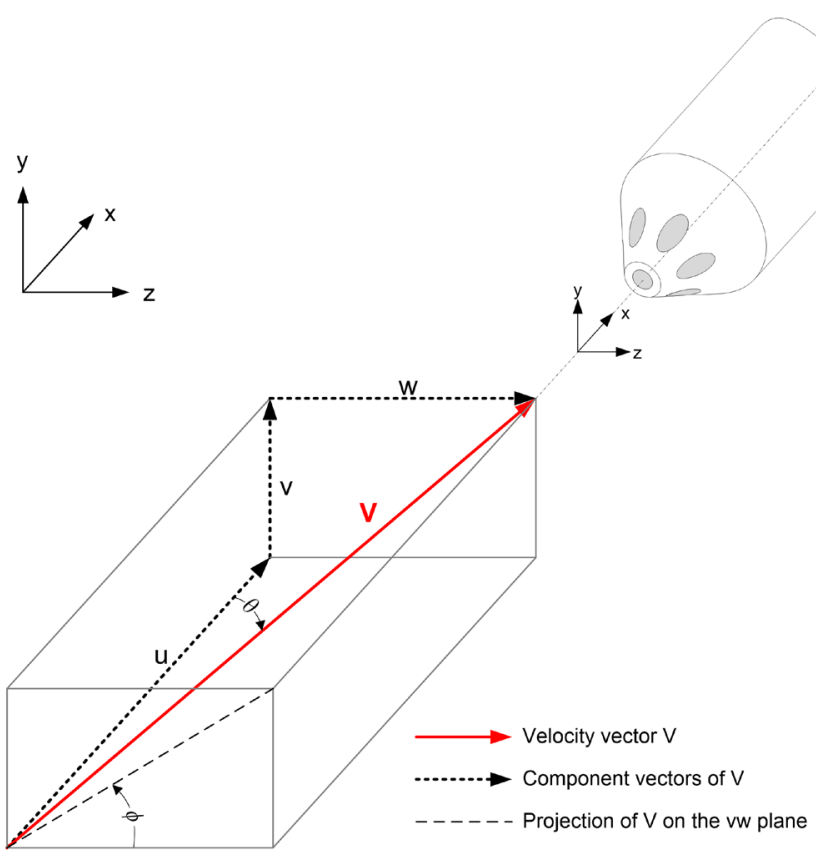

Figure D.3: Polar probe coordinate system, reproduced from Scribner (2011) and originally adapted from Gerner et al. (1984) 
when ports 2 and 3 read higher than ports 5 and 6 . Figure D.3, shows an alternative convention where the velocity vector, $V$, is expressed in terms of polar angles. Here the roll angle, $\phi$, and misalignment angle, $\theta$ are given by

$$
\begin{aligned}
& \phi=\tan ^{-1}\left(\frac{v}{w}\right) \\
& \theta=\cos ^{-1}\left(\frac{u}{V}\right)
\end{aligned}
$$

The calibrations are performed using the two-axis facility described in Section 3.9 and shown schematically in Figure 3.17. In this facility, as noted by Scribner (2011), the probe pitch control is coupled to the probe yaw position. Figure 3.17 shows that yaw turntable is mounted to a fixed surface and therefore independently controls the yaw angle $(\alpha)$ by rotating about the $y$-axis only. The pitch control however is mounted to the yaw turntable and moves with yaw rotations. As a result, pitch movements actually rotate the probe tip about the $z^{\prime}$-axis of Figure D.2 and not the $z$-axis. $\quad \beta^{\prime}$ therefore represents the angular displacement of the pitch control itself, whereas $\beta$ is the rotation of $V$ relative to the fixed z-axis. $\beta$ is then a function the pitch control angle $\left(\beta^{\prime}\right)$ and the tangential angle $(\alpha)$ :

$$
\beta=\tan ^{-1}\left(\frac{\tan \beta^{\prime}}{\cos \alpha}\right)
$$

Finally, the velocity vector components expressed in terms of $\alpha$ and $\beta$ are given by:

$$
\begin{gathered}
u=\frac{1}{\left(1+(\tan \alpha)^{2}+(\tan \beta)^{2}\right)^{1 / 2}} \\
v=u \cdot \tan (\beta) \\
w=u \cdot \tan (\alpha)
\end{gathered}
$$




\section{D.3 Low-Angle Regime}

In the low angle regime the flow is fully attached over the probe tip surface. All of the pressure ports remain sensitive to flow misalignment and together define the non-dimensional calibration coefficients:

$$
\begin{gathered}
C_{1}=\frac{P_{4}-P_{1}}{P_{7}-\bar{P}_{1-6}} \\
C_{2}=\frac{P_{3}-P_{6}}{P_{7}-\bar{P}_{1-6}} \\
C_{3}=\frac{P_{2}-P_{5}}{P_{7}-\bar{P}_{1-6}}
\end{gathered}
$$

where $\bar{P}_{1-6}$ is the average pressure sensed from ports 1 through 6 . The coefficient numerators are formulated using pressures from opposing ports (see Figure D.1). As such, each is most sensitive to flow angularity in one of three equally spaced planes that pass through the probe body axis.

The denominator, $P_{7}-\bar{P}_{1-6}$, is referred to as a "pseudo-dynamic pressure" where $P_{7}$ and $\bar{P}_{1-6}$ approximate the local total and static pressure respectively.

Equations D.9 through D.11 equally divide the angular space (for low angles) about the probe tip. Considering the contribution of each coefficient along the tangential coordinate axes gives the tangential angle coefficients,

$$
\begin{gathered}
C_{\alpha}=\frac{2 C_{1}+C_{2}-C_{3}}{3} \\
C_{\beta}=\frac{C_{2}+C_{3}}{\sqrt{3}}
\end{gathered}
$$

By calibrating in a known flow, the following functions for the yaw angle $(\alpha)$, the pitch angle $(\beta)$, the total pressure coefficient $\left(C_{0}\right)$ and the dynamic pressure coefficient $\left(C_{q}\right)$ are uniquely defined by $C_{\alpha}$ and $C_{\beta}$ : 


$$
\begin{aligned}
& \alpha=f\left(C_{\alpha}, C_{\beta}\right) \\
& \beta=f\left(C_{\alpha}, C_{\beta}\right) \\
& C_{0}=f\left(C_{\alpha}, C_{\beta}\right) \\
& C_{q}=f\left(C_{\alpha}, C_{\beta}\right)
\end{aligned}
$$

where the total and dynamic pressure coefficients $\left(C_{0}\right.$ and $\left.C_{q}\right)$ are defined as

$$
\begin{gathered}
C_{0}=\frac{P_{7}-P_{0}}{P_{7}-\bar{P}_{1-6}} \\
C_{q}=\frac{P_{7}-\bar{P}_{1-6}}{P_{0}-P_{S}}
\end{gathered}
$$

Sample calibration surfaces for $C_{\alpha}, C_{\beta}, C_{0}$ and $C_{q}$ are shown as colour floods in Figures D.4 through D.7, respectively. Additional sub-plots show the coefficient distributions along $\alpha=0^{\circ}$ and $\beta=0^{\circ}$ for three separate calibrations of varying Reynolds number $\left(\operatorname{Re}_{D}=3040,4030\right.$ and 5300). For these levels, the $C_{q}$ coefficient shows significant differences, as large as $2.5 \%$ of local dynamic pressure $\left(P_{0}-P_{S}\right)$, while the other coefficients $\left(C_{\alpha}, C_{\beta}\right.$, and $\left.C_{0}\right)$ appear relatively insensitive to Reynolds number variations.

For an unknown flow of varying velocity levels, the traditional data reduction involves selecting a single calibration with a Reynolds number that best approximates the expected or "average" flow conditions. The sensitivity of the $C_{q}$ coefficient shown here then contributes to a relatively large uncertainty of the inferred dynamic pressures (Benner, 2003). As an improvement, five calibrations were performed over the range of expected in-service Reynolds numbers. The data reduction was then modified and now iteratively solves for, rather than approximating, the local flow velocity using a linear interpolation and table look-up method. 


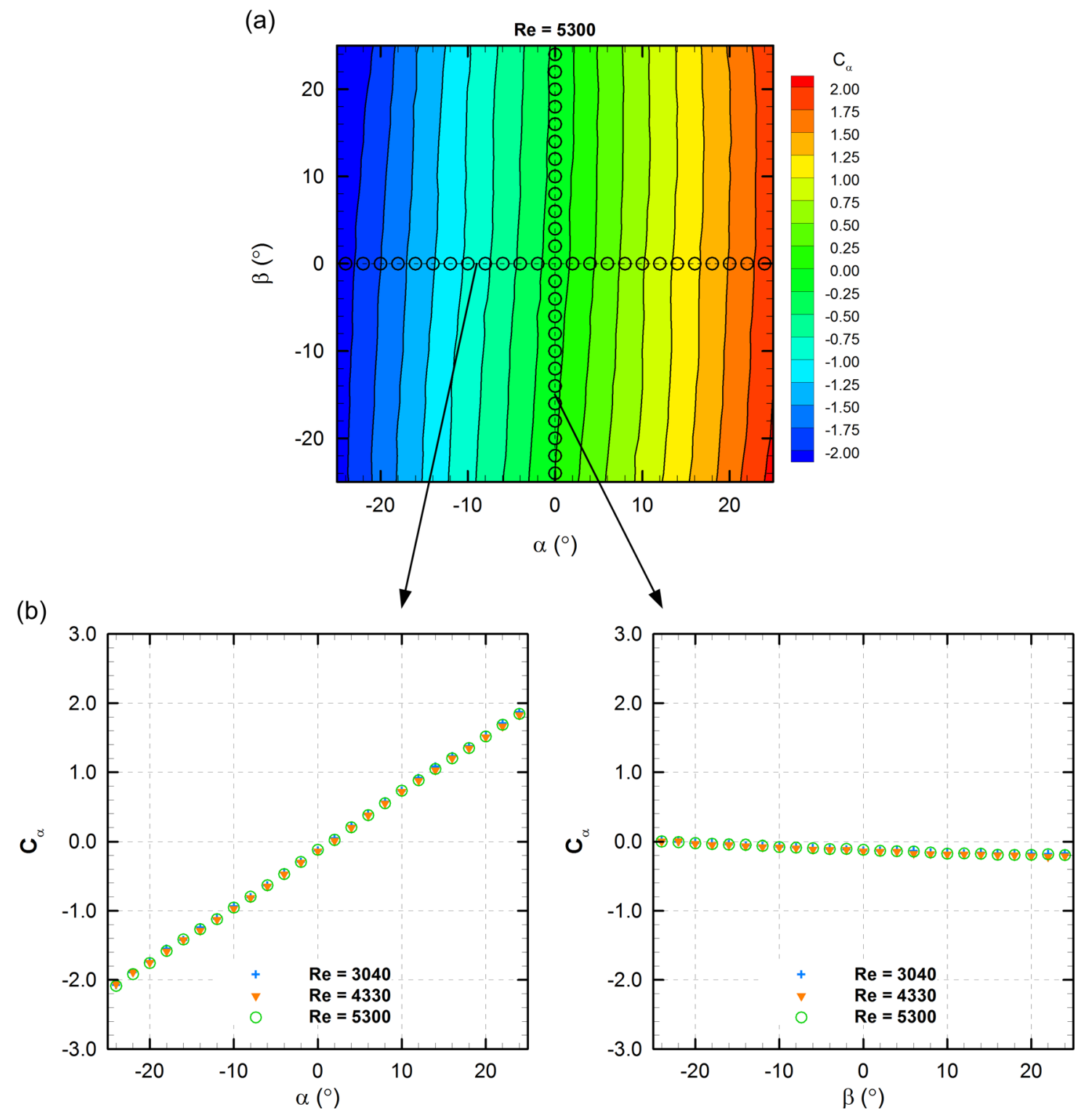

Figure D.4: $\quad C_{\alpha}$ calibration data for sector 7 


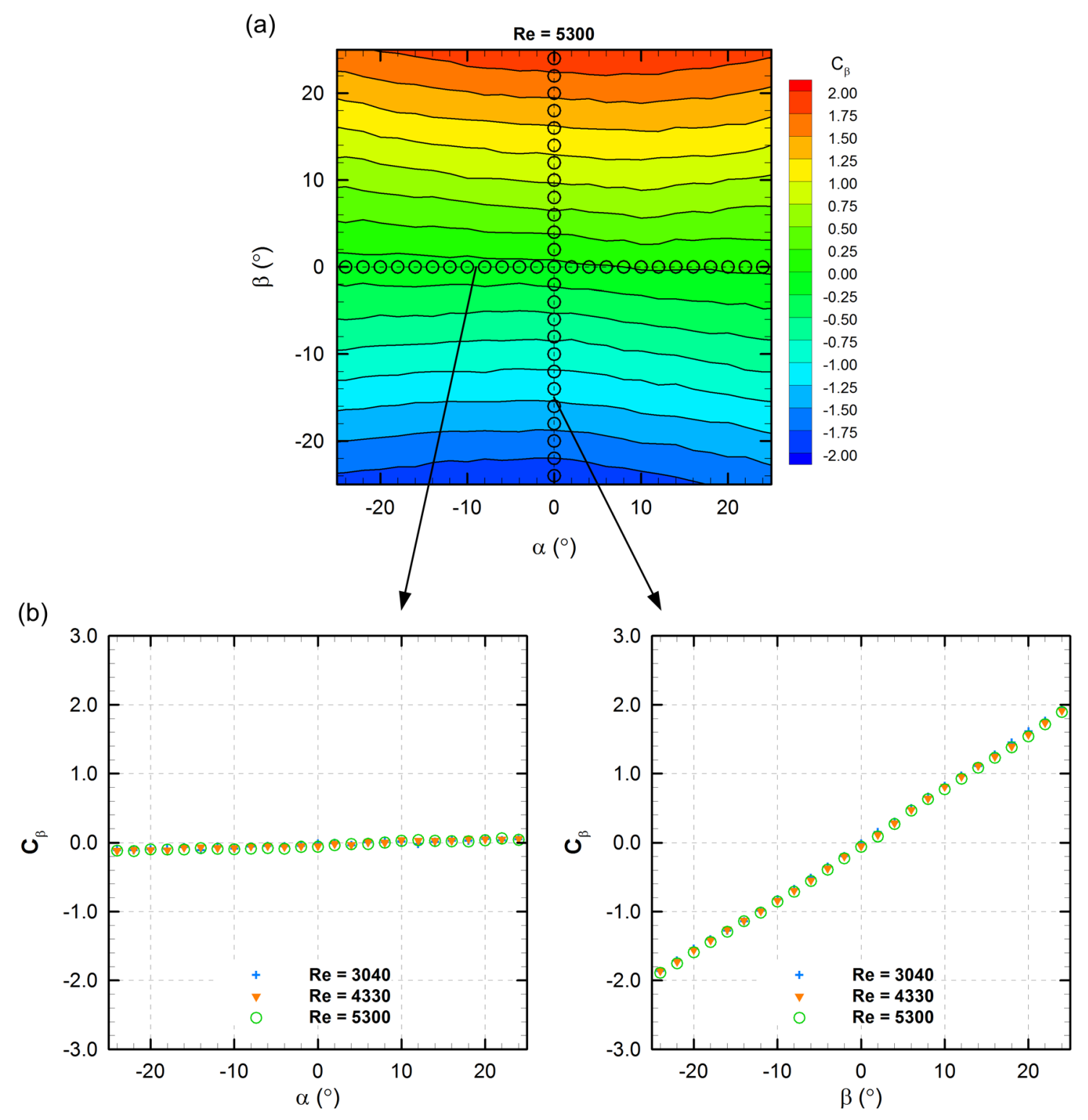

Figure D.5: $\quad C_{\beta}$ calibration data for sector 7 


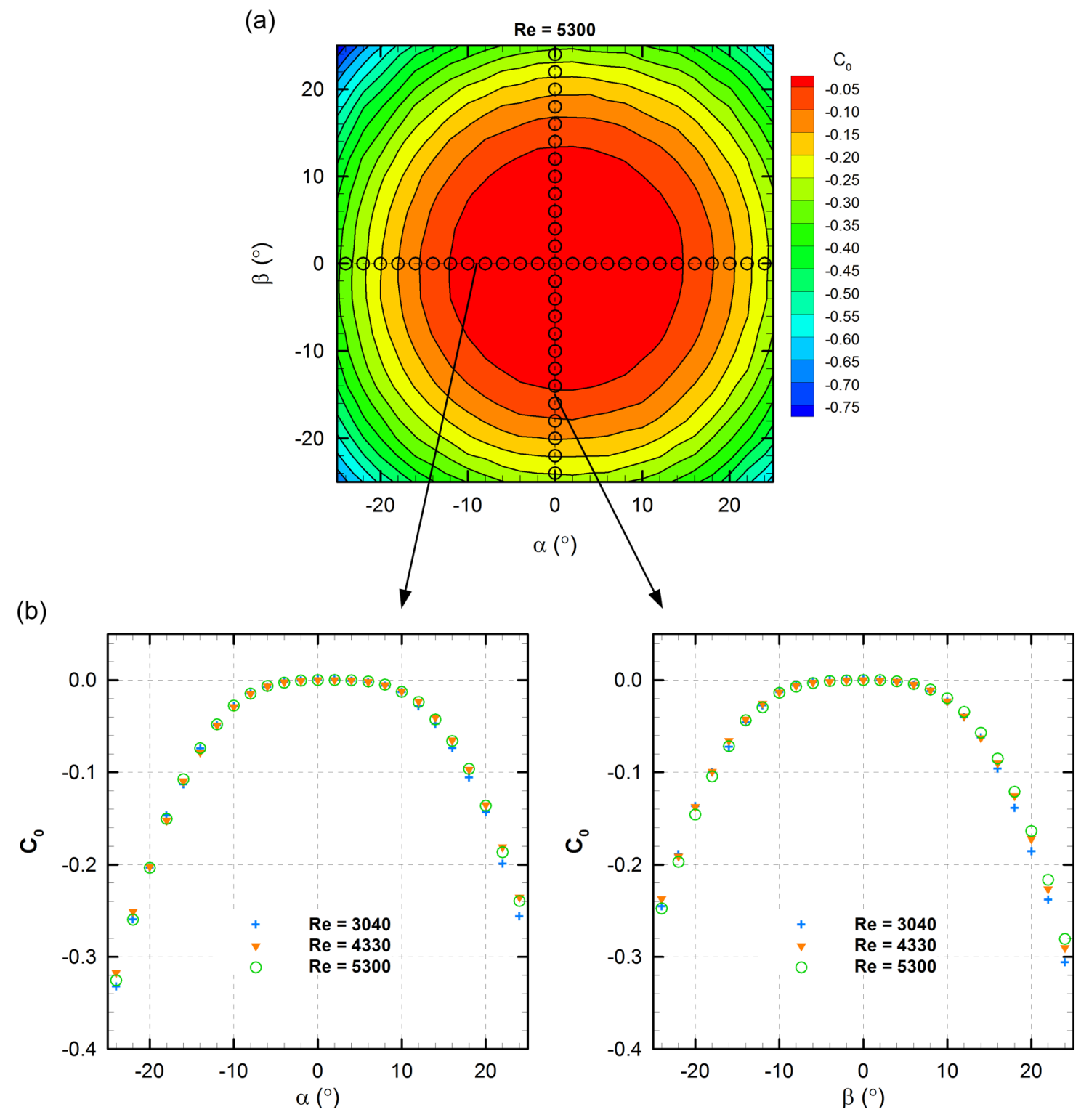

Figure D.6: $\quad C_{0}$ calibration data for sector 7 


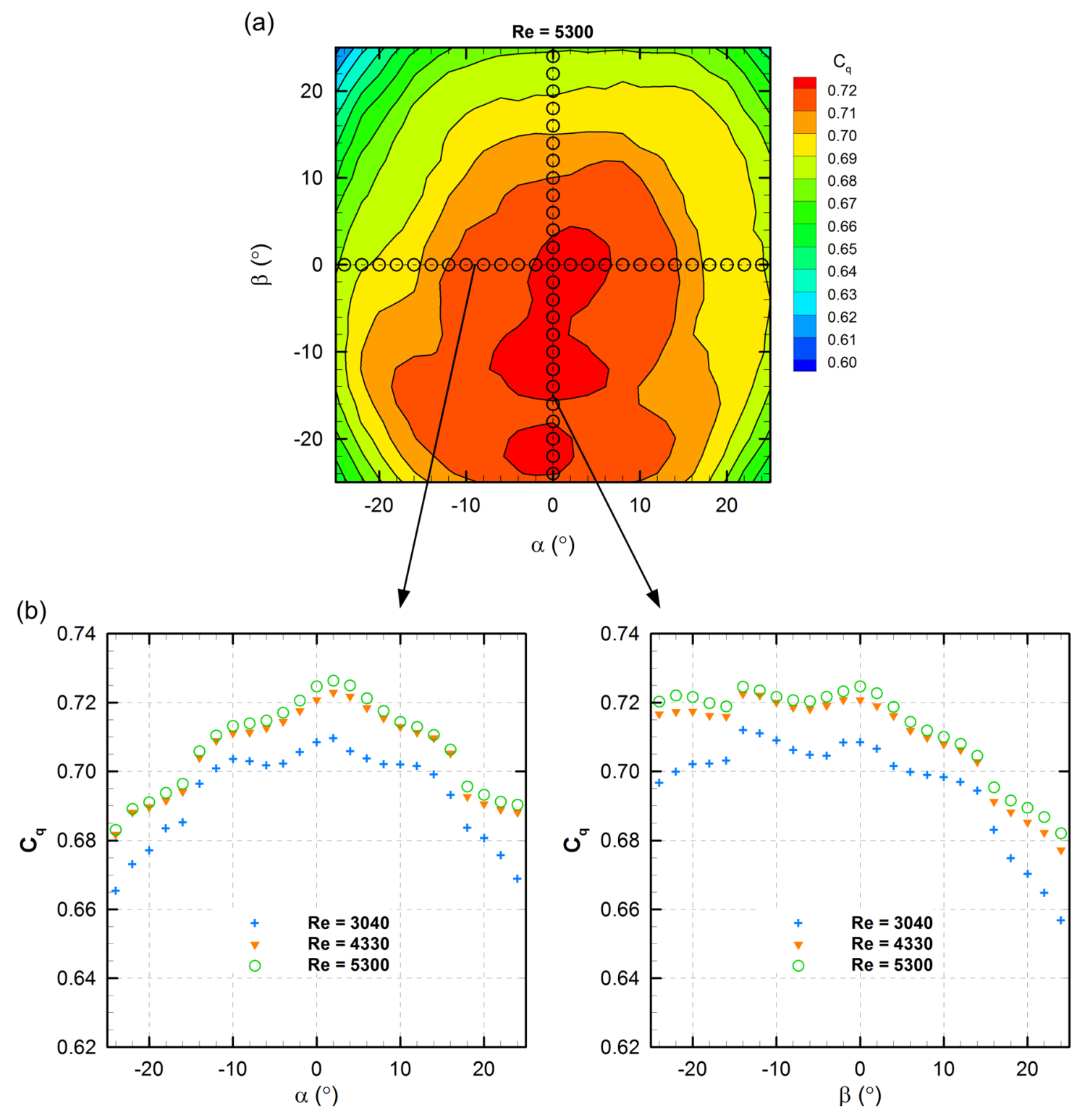

Figure D.7: $\quad C_{q}$ calibration data for sector 7 


\section{D.4 High-Angle Regime}

Flow misalignment larger than about $25^{\circ}\left(\alpha\right.$ or $\left.\beta>25^{\circ}\right)$ increases the pressure gradient on the leeward side of the probe tip and causes the flow to separate from the surface. The leeward ports are rendered insensitive to further misalignment and only the ports within the attached flow are useful. This includes the highest reading peripheral port (1 to 6), the adjacent ports, and the centre port (7). Gallington (1981) suggests the high-angle coefficients should be defined using the polar coordinate system of Figure D.3 and are given by:

$$
\begin{gathered}
C_{\theta n}=\frac{P_{n}-P_{7}}{P_{n}-\left(\frac{P_{n-}+P_{n+}}{2}\right)} \\
C_{\phi n}=\frac{P_{n-}-P_{n+}}{P_{n}-\left(\frac{P_{n-}+P_{n+}}{2}\right)}
\end{gathered}
$$

The subscript $n$ denotes the highest reading pressure port, while $n$ - and $n+$ indicate the adjacent ports in the counter clockwise and clockwise directions, respectively (see Figure D.1). As defined, the coefficients are specific to each of the six angular sectors shown in Figure D.8 (a).

(a)

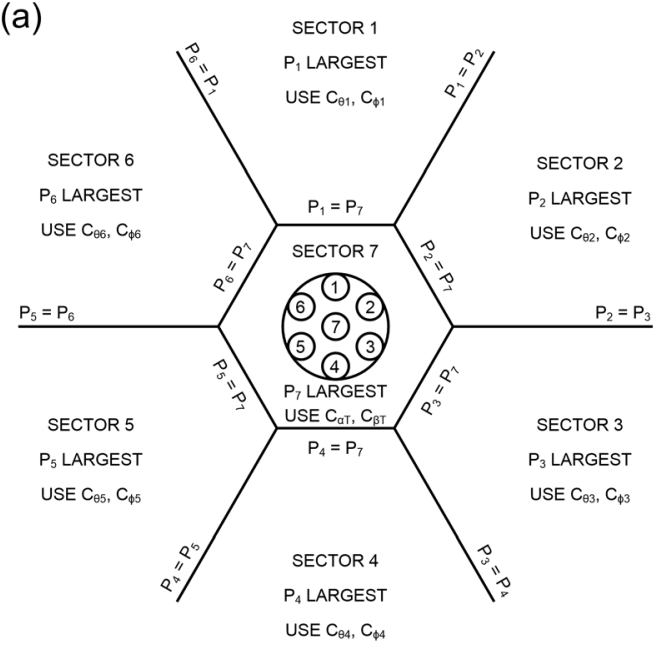

(b)

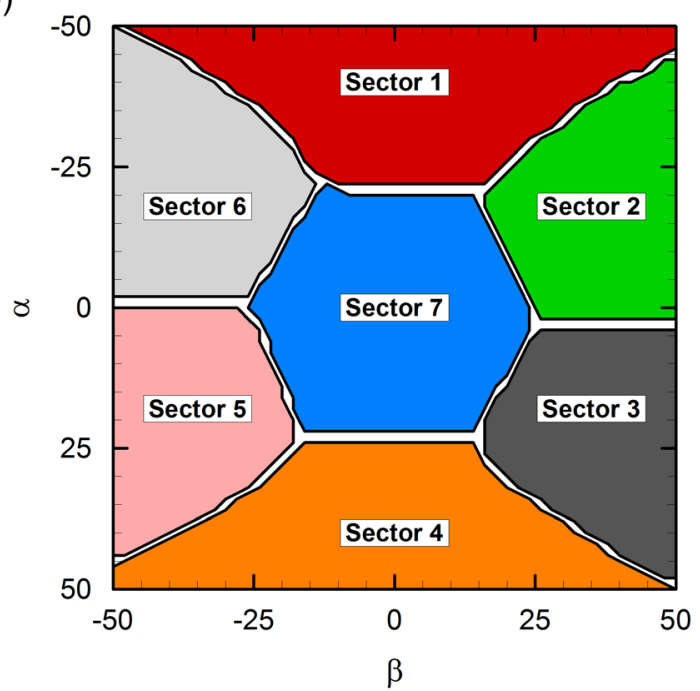

Figure D.8: (a) Division of probe tip angular space, reproduced from Gerner et al. (1984) and (b) the experimental probe tip angular space 
Figure D.8 (b) shows the angular sectors determined from a sample calibration.

Much like the low-angle regime, calibrating in a known flow yields functions that are uniquely defined by Equations D.20 and D.21. The misalignment angle $(\theta)$, the roll angle $(\phi)$, the total pressure coefficient $\left(C_{0}\right)$ and the dynamic pressure coefficient $\left(C_{q}\right)$ are defined by

$$
\begin{gathered}
\theta=f\left(C_{\theta}, C_{\phi}\right) \\
\phi=f\left(C_{\theta}, C_{\phi}\right) \\
C_{0}=f\left(C_{\theta}, C_{\phi}\right) \\
C_{q}=f\left(C_{\theta}, C_{\phi}\right)
\end{gathered}
$$

where the total and dynamic pressure coefficients $\left(C_{0}\right.$ and $\left.C_{q}\right)$ are defined as

$$
\begin{gathered}
C_{0}=\frac{P_{n}-P_{0}}{P_{n}-\left(\frac{P_{n-}+P_{n+}}{2}\right)} \\
C_{q}=\frac{P_{n}-\left(\frac{P_{n-}+P_{n+}}{2}\right)}{P_{0}-P_{S}}
\end{gathered}
$$

The calibration coefficients $\left(C_{\theta}, C_{\phi}, C_{0}\right.$, and $\left.C_{q}\right)$ corresponding to port 4 are shown in Figures D.9 through D.12. Each figure also contains sub-plots showing the coefficients distributions at $\theta=0^{\circ}$ and at $\phi=0^{\circ}$ for five values of $\mathrm{Re}_{D}$. In an unknown flow, the same iterative data reduction procedure described in Section D.3 is applied. 


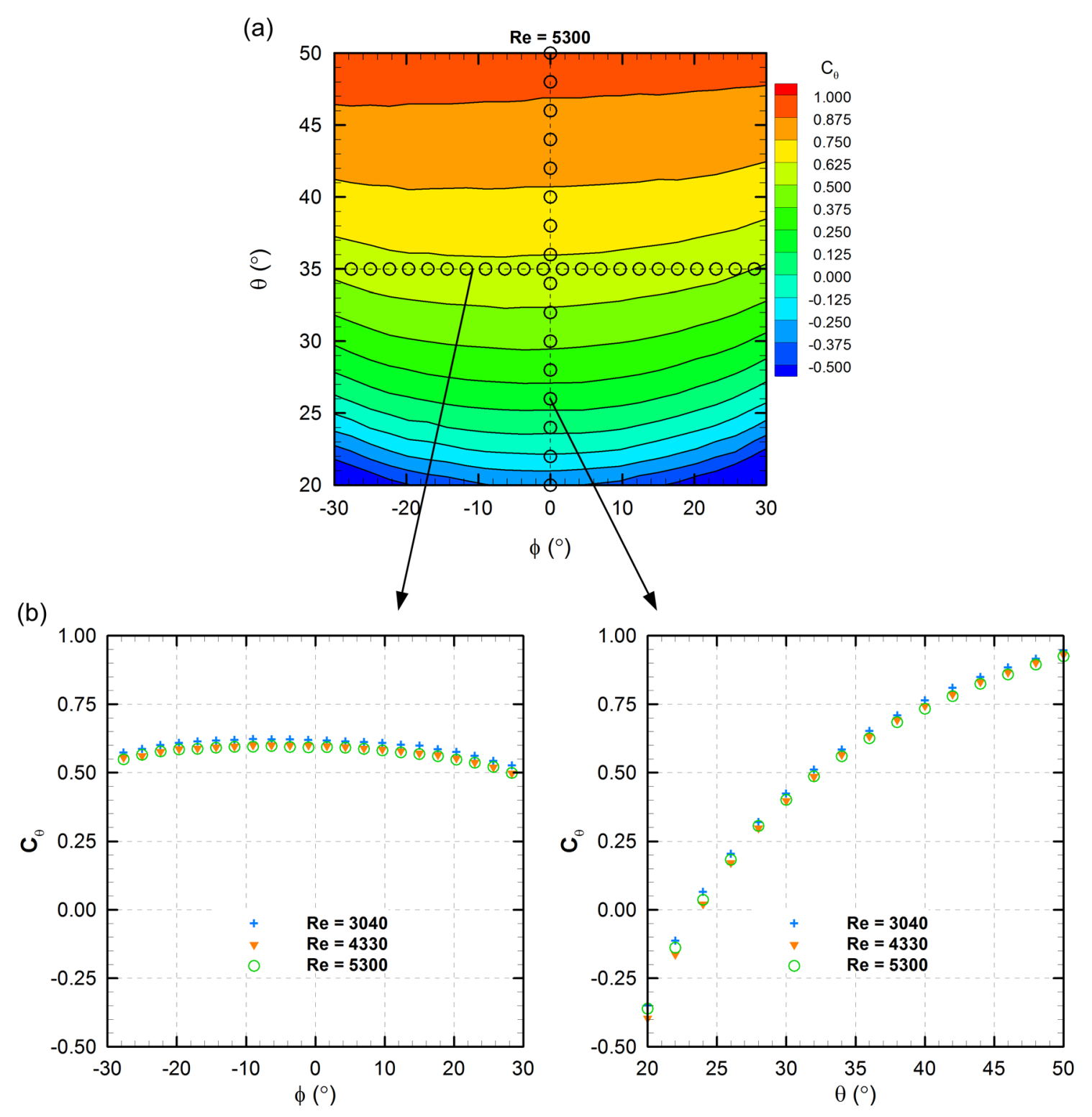

Figure D.9: $\quad C_{\theta}$ calibration data for sector 4 


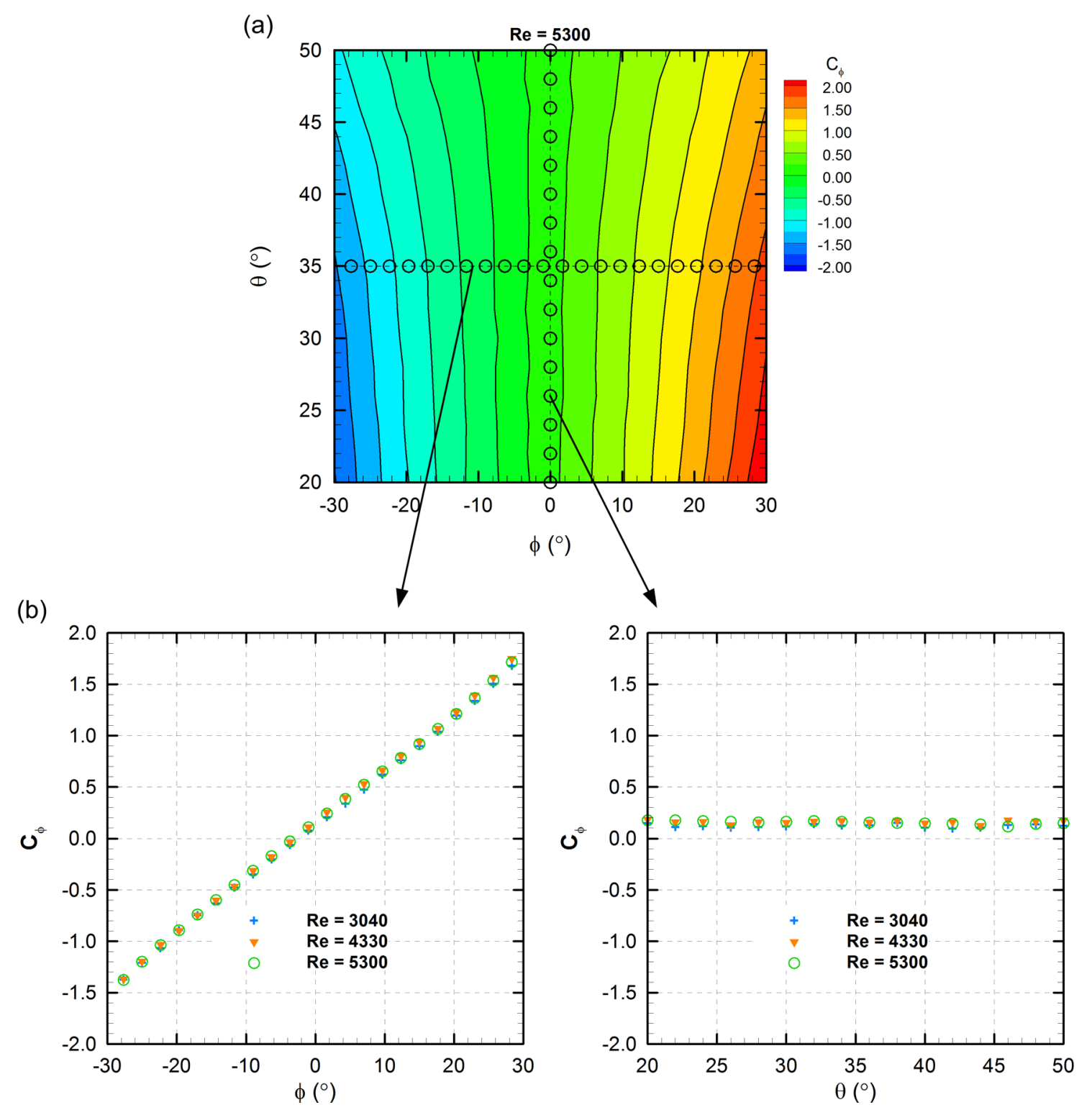

Figure D.10: $C_{\phi}$ calibration data for sector 4 


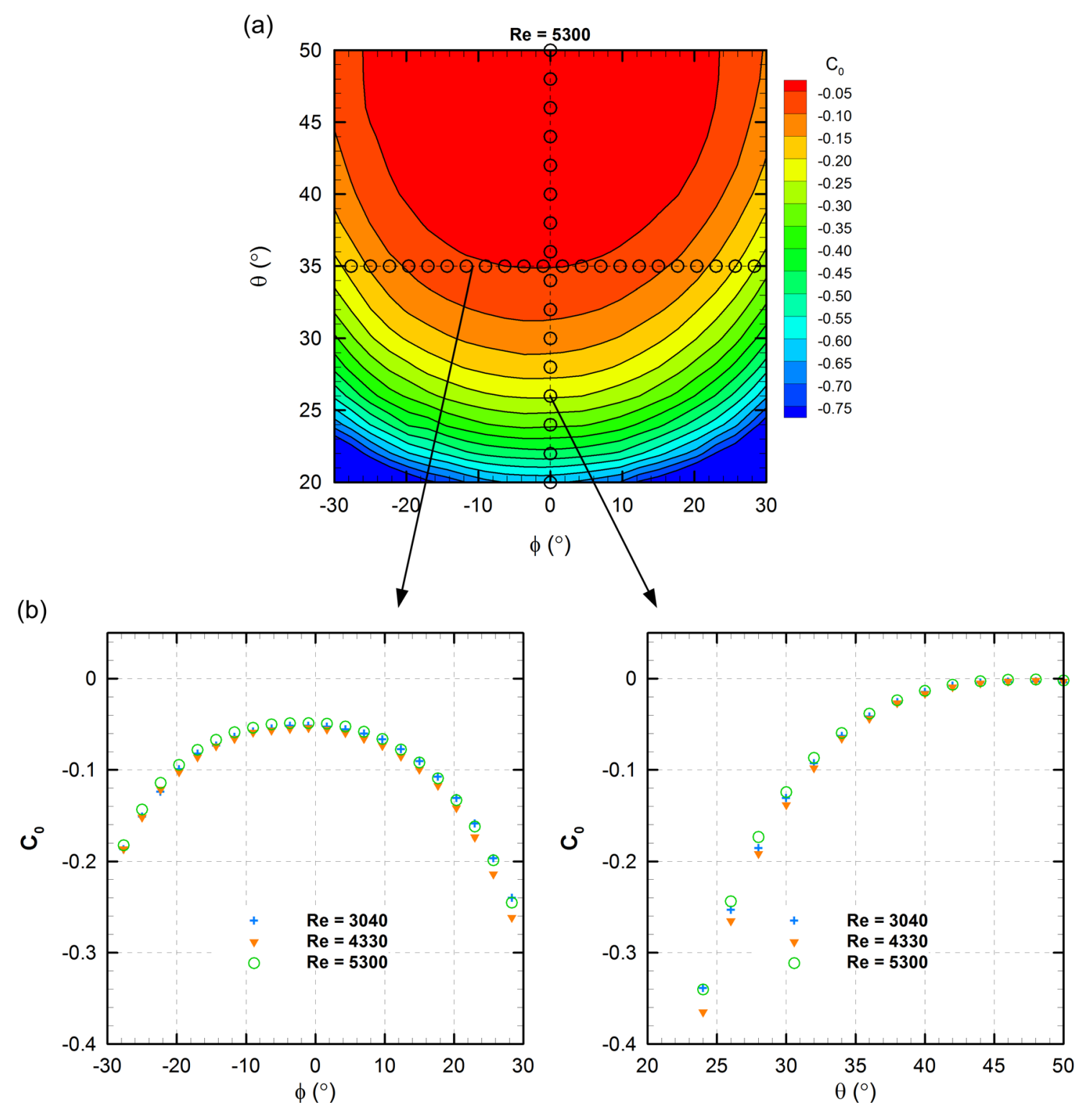

Figure D.11: $C_{0}$ calibration data for sector 4 


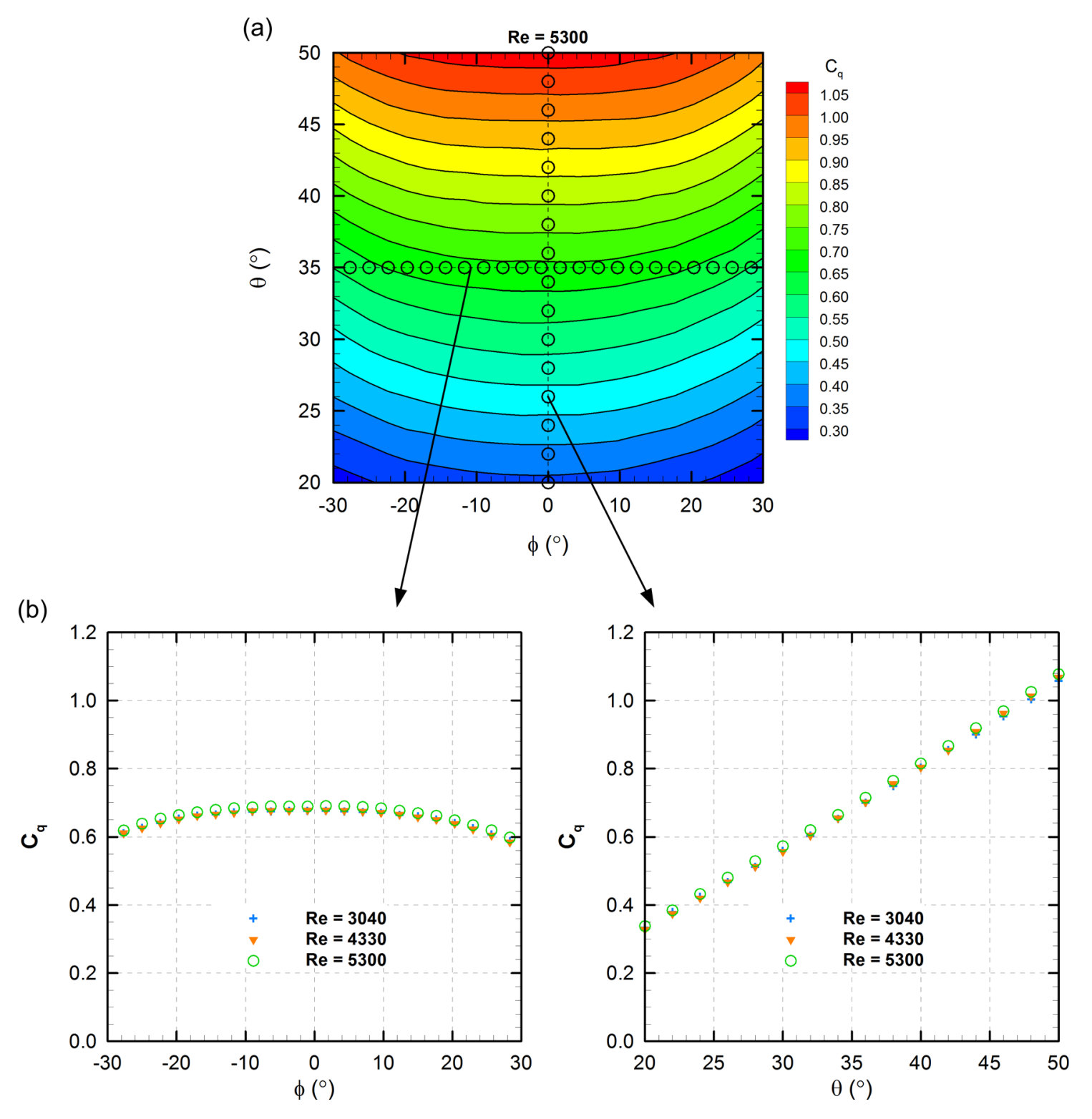

Figure D.12: $C_{q}$ calibration data for sector 4 


\section{D.5 Challenges in Measuring Total Pressure within Turbine Cascade Secondary Flows using Seven-hole Pressure Probes}

During the course of the present work, the same flows were measured using the Kiel and seven-hole pressure probes. Small but repeatable differences in the measured total pressures were observed within the secondary flow. The following section briefly describes these observations and provides insights into why the two probes infer different values under certain conditions. This work led to a subsequent research project by Scribner (2011) which investigated the response of the seven-hole probe over a range of Reynolds numbers and turbulence levels.

In low-speed flows, the pressure sensed by a Pitot-tube can be adversely affected by Reynolds number, flow angularity, shear flow and turbulence levels (Chue, 1975). Each of these factors is present within the secondary flow of a turbine cascade and therefore downstream measurements made with the seven-hole pressure probe are also likely to be affected. Since the seven-hole probe used in this experiment is calibrated in a uniform flow for the effects of flow angularity and Reynolds number, the following section will discuss the effects of shear flow and turbulence.

In an unknown flow, the presence of shear gradients across the face of the probe-tip can lead to an erroneous interpretation of the local flow quantities (Johnson and Reed, 1985). Figure D.13 shows the probe tip in uniform and non-uniform velocity distributions. The local gradient causes a difference in the pressure sensed by oppositely spaced ports which can be misinterpreted as an apparent flow angle. While the present seven-hole probe (7HP-1) was manufactured as small as possible to minimize the shear effects, it was still difficult to assess whether the observed levels of shear were problematic or not. Therefore, comparison measurements of the same cascade flow were made using a different seven-hole probe (7HP-2) with a $31 \%$ larger tip diameter. On the basis of their diameters and the relative spacing of the peripheral pressure ports, one might expect the two probes to respond somewhat differently in the presence of the same shear gradients. The total pressure loss coefficient $\left(C_{P 0}\right)$ showed very good agreement between the two probes. The differences of the area- and mass-averaged values were 
$0.01 \%$ and $0.12 \%$ of the inlet dynamic pressure respectively. The agreement is likely due to the fact that the spatial resolution of the two probes is sufficiently high for the flows investigated. Thus, the shear effects are not expected to adversely affect the results of this work.

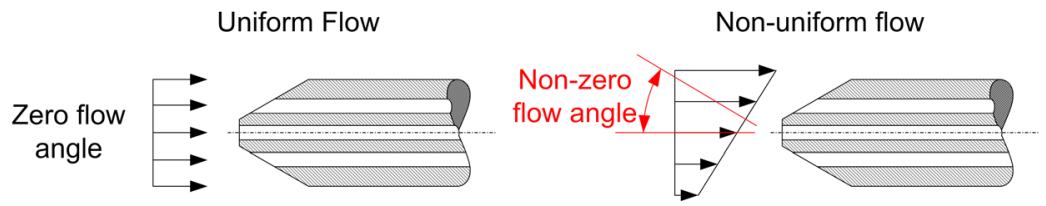

FigureD.13: The effect of shear flow on the inferred flow angle of a seven-hole probe

The secondary flows downstream of turbine cascades have shown that elevated levels of turbulence exist within the passage vortex region (Moore and Adhye, 1985; Moore et al., 1987; Gregory-Smith et al., 1988b). It is known that in flows of high turbulence intensity pressure probes have an inadequate pneumatic response and measure an incorrect value of the timeaveraged pressure (Chue, 1975; Christiansen and Bradshaw, 1981). Pitot-tubes may read higher values than the actual time-averaged pressure while static pressure probes can sense lower pressures or higher values depending on the scales of turbulence (Chue, 1975; Christiansen and Bradshaw, 1981). Therefore, for multi-hole probes, where the individual ports may exhibit characteristics of Pitot or static ports depending on the local flow alignment, it is difficult to assess the impact of elevated turbulence on the seven-hole pressure probe.

It is possible to calibrate the seven-hole probe in elevated turbulence fields but the experimental setup is challenging. Generating uniform flows of different intensity in an accelerating jet while also maintaining appropriate scales is difficult. Recently, an innovative method has been developed by Scribner (2011) for generating uniform elevated turbulent jets in the low-speed calibration facility at Carleton University. Since the Kiel probe is considered the most accurate instrument for making total pressure measurements in misaligned flows (Chue, 1975), it was used as a means of comparison for the seven-hole pressure probe measurements. Although the Kiel probe is also likely affected by the high levels of turbulence intensity, the 
direct measurement of the total pressure is advantageous when compared to the calibration (in a uniform and low intensity flow) that is required by the seven-hole probe.

Figure D.14 (a) shows contour plots of the total pressure coefficient $\left(C_{P 0}\right)$ measured using the Kiel and seven-hole probe at $1.40 C_{x}$ downstream of the leading edge of the cascade, where $C_{x}$ is the blade axial chord. The results are largely in good agreement; however, within the secondary flow region (labelled A and B) there are notable differences. The seven-hole probe reads higher losses or lower total pressures, as shown by the darker red region. Although these differences appear small and locally concentrated, the overall area-averaged values differ by $3.5 \%$. In other cascades measured in the same facility these differences can be as large as $16 \%$. At locations farther downstream, the mixing of the flow reduces the non-uniformities and the dissipation of the turbulence kinetic energy improves the agreement between the Kiel and sevenhole probes.

When comparing the two probes it is useful to examine the total pressure differences pointby-point in the flow domain. Such differences are shown in the colour flood of Figure D.14 (b) and are presented as a percentage of the cascade inlet dynamic pressure:

$$
\% q_{1, C L}=\left|C_{P 0_{\text {Kiel }}}-C_{P 0_{7 H P}}\right| \times 100
$$

(a)

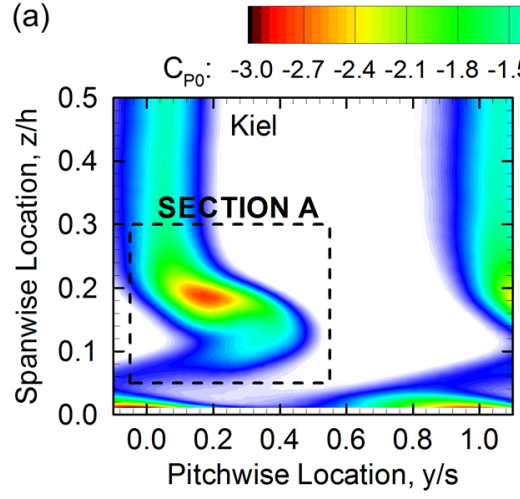

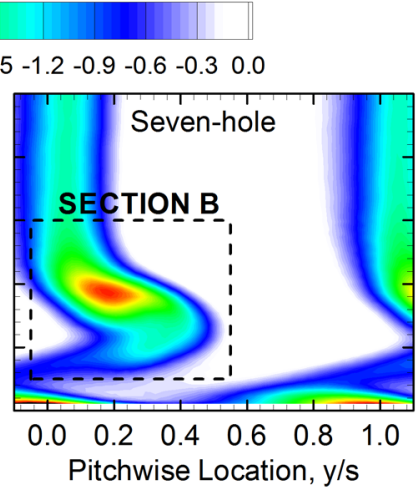

(b) - Tu

$\% q_{C L}: 024681012141618$

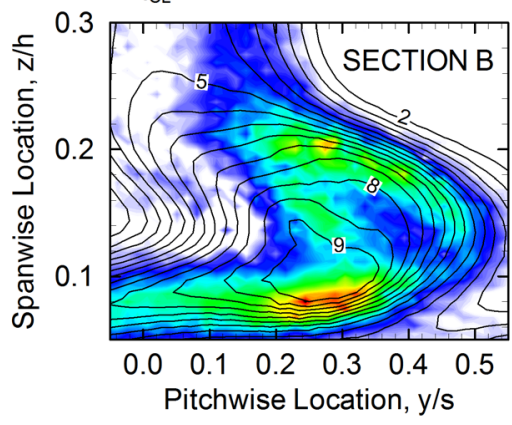

Figure D.14: $C_{P 0}$ measured using the Kiel and seven-hole probe (a) and point by point total pressure difference, $\% q_{1, C L}$, with line contours of $T u_{l o c}$ (b) 
Also shown in Figure D.14 (b) are line contours of the turbulence intensity which were measured using the $x$-type hotwire probe. The largest difference between the two pressure probes is located at $z / h=0.08$ and $y / s=0.3$ and is approximately $18 \%$ of the inlet dynamic pressure. Although this region has elevated levels of turbulence $\left(T u_{l o c} \approx 9 \%\right)$, the peak turbulence intensity is not well correlated with the largest difference. Another region, located at $z / h=0.2$ and $\mathrm{y} / \mathrm{s}=0.3$ where the turbulence intensity is slightly lower $\left(T u_{l o c} \approx 7 \%\right)$, shows differences of about $8 \%$ of the inlet dynamic pressure. It would appear that the largest differences are local to steep gradients of turbulence intensity in regions where the turbulence intensity is also high.

The author has not made turbulence measurements downstream of other cascades and therefore it is somewhat difficult to make general conclusions regarding the response of the Kiel and seven-hole probes in unknown turbulent flows. More details regarding the challenges associated with measuring the total pressure in complex flows is given in by MacIsaac et al. (2009). It was concluded that the response of both probes should be explored in known turbulent flows. As mentioned previously, generating the appropriate turbulent conditions can be difficult and therefore this task was assigned to a separate research project Scribner (2011). At the time, the present author elected to move forward with the intended research using the seven-hole probe as the primary downstream measurement instrument. As mentioned Section 3.8.5, the reported uncertainties associated with the seven-hole probe were updated to reflect the work completed by Scribner (2011). 


\section{Appendix E}

\section{Calibration, Data Reduction Methods and Estimated Uncertainties for the X-type Hot-wire Probe}

\section{E.1 Introduction}

The following appendix details the calibration and data reduction procedure for the $x$-type hotwire probe described in Section 3.8.7. The uncertainty analysis follows in section E.4.

The working principle of thermal anemometry is based on the convective cooling (heat transfer) of a flow over the heated sensor. The resistance of hotwire sensor, given by:

$$
R_{w}=R_{r}\left[1+\alpha_{r}\left(T_{w}-T_{r}\right)\right]
$$

is a function of its average temperature, $T_{w}$, where $R_{r}$ is the sensor resistance at the reference temperature $\left(T_{r}\right)$ and $\alpha_{r}$ is the temperature coefficient of resistance.

In constant temperature anemometry, the hotwire sensor is connected to a Wheatstone Bridge, as shown in Figure E.1, and is heated to a temperature higher $\left(T_{w}\right)$ than the ambient. When the hotwire is exposed to a convective flow the wire is cooled - changing the wire resistance and the voltage input to a servo amplifier. As a result, the amplifier output current is increased and is fed back to the top of the bridge, as shown in Figure E.1. The influx of current 
restores the wire resistance and balances the bridge. The amplifier output voltage, commonly referred to as the top-of-bridge voltage $\left(E_{T O B}\right)$, is then an indirect measure of the wire heat transfer and flow velocity.

In practice, the anemometer output signal $\left(E_{\text {out }}\right)$ is off-set and amplified to reduce noise and improve the digital acquisition. The top-of-bridge voltage is related to the acquired output signal $\left(E_{\text {out }}\right)$ by:

$$
E_{T O B}=\frac{E_{\text {out }}}{G}+E_{\text {offset }}
$$

where $E_{\text {offset }}$ is the voltage off-set and $G$ is the gain.

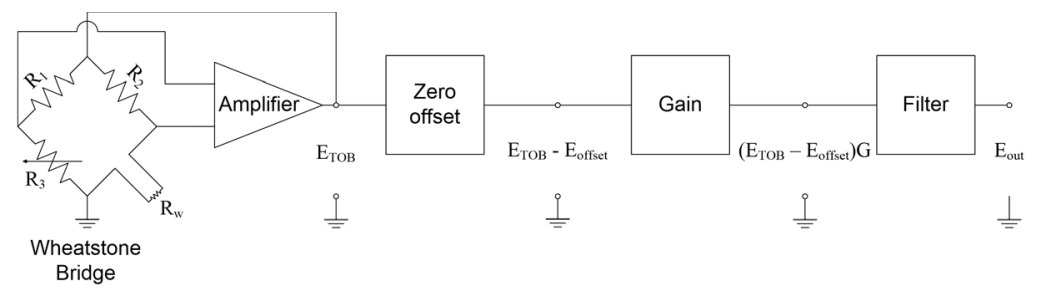

Figure E.1: Thermal Anemometry Circuit

\section{E.2 Calibration Procedures}

The $x$-type probe requires a velocity and directional calibration. For the velocity calibration the measurements are made with the probe axis aligned with the calibration jet for a range of velocities $\left(7<V_{j e t}<42 \mathrm{~m} / \mathrm{s}\right)$. At each velocity level, the effective cooling velocity $\left(V_{\text {eff }}\right)$ of each wire is related to the $E_{T O B}$ using the general form of King's law,

$$
E_{\text {TOB }}^{2}=\left(A+B V_{\text {eff }}^{n}\right)\left(\frac{T_{w}-T_{\text {test }}}{T_{w}-T_{\text {ref }}}\right)
$$

where the $A, B$ and $n$ are the calibration coefficients. The temperature correction by Bearman (1971) was applied if the flow temperature $\left(T_{\text {test }}\right)$ was different from that at which the bridge was balanced $\left(T_{r e f}\right)$. Sample velocity calibrations for the two sensors of the $x$-type probe are shown in shown in Figure E.2. The experimental data are shown as symbols and the 
calculated curves as solid lines. To account for subtle changes to the wire resistance caused by oxidation, the velocity calibrations were performed before each wind-tunnel test.

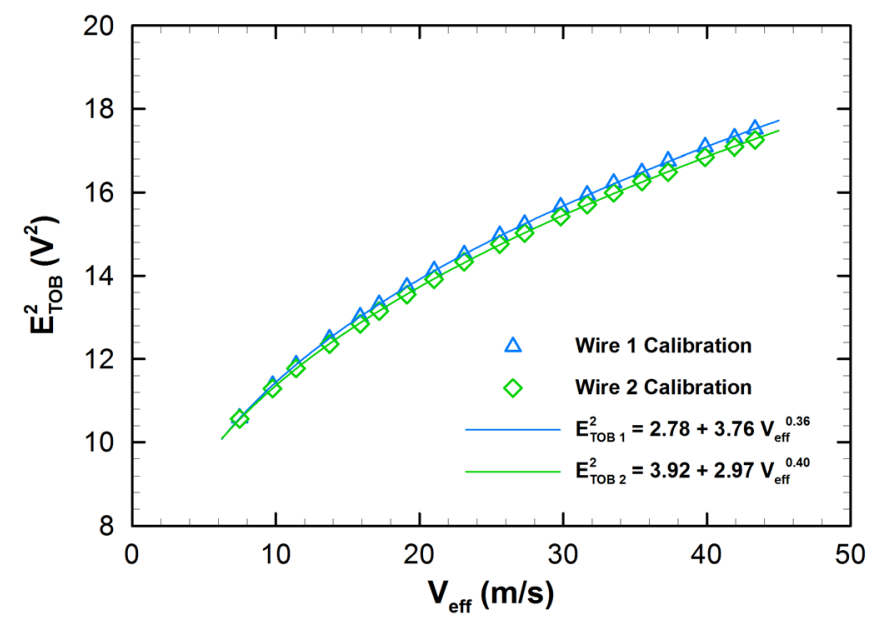

Figure E.2: Sample hotwire velocity calibration

The directional sensitivity of each wire was measured for a range of pitch $(\beta)$ and yaw $(\alpha)$ flow angles $\left(-30^{\circ}<\alpha, \beta<+30^{\circ}\right)$ in $2^{\circ}$ increments. The probe was initially placed with the probe axis aligned with the calibration jet and measurements were made at a constant jet velocity $\left(V_{j e t}\right)$ of $30 \mathrm{~m} / \mathrm{s}$. The sensitivity functions, given by:

$$
g_{i}(\alpha, \beta)=\frac{V_{e f f_{i}}^{2}}{V_{j e t}^{2}}
$$

where $i$ is the wire number were computed for each angular position.

Separate calibrations were performed for three roll orientations: $0^{\circ}, 45^{\circ}$ and $90^{\circ}$. In an unknown flow, only two positions, $0^{\circ}$ and $90^{\circ}$ were required for determining the mean velocities while the third position, $45^{\circ}$, was required to determine Reynolds stresses. Example calibration surfaces for the $0^{\circ}$ position are shown in Figure E.3. The directional sensitivity functions are mainly influenced by the probe tip geometry, and therefore remain relatively constant for a series of wind-tunnel tests. Occasionally, the hotwire sensors were damaged during testing and wire 
repairs were completed in-house. New calibrations were performed prior to any subsequent testing.
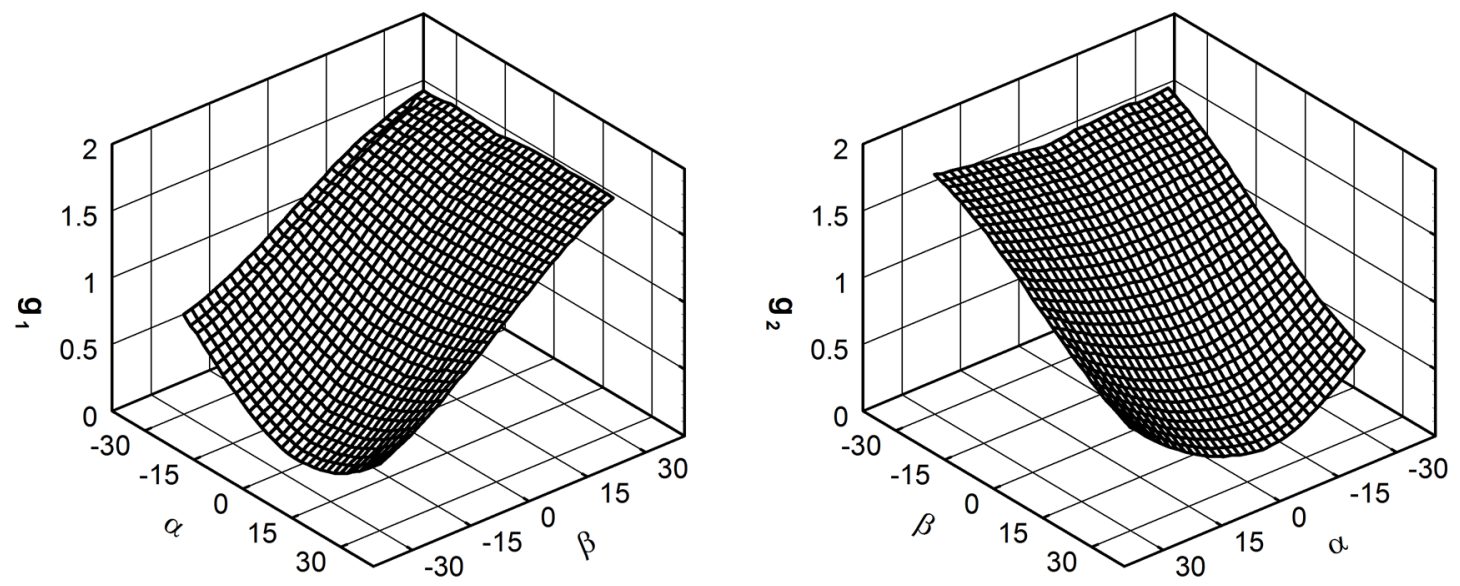

Figure E.3: Directional sensitivity functions for the $x$-type hotwire probe

\section{E.3 Data Reduction Procedures}

As mentioned, a method detailed by Döbbeling et al. (1992), originally derived for quadruple-wire probes, was adapted for the $x$-type probe. Time-averaged velocity measurements were made by collecting data with the $x$-type probe oriented in the two roll positions, $0^{\circ}$ and $90^{\circ}$. Four time-averaged effective cooling velocities, one per wire position, were collected at each data point. The flow angles were determined first by minimizing the error squares sum, $E_{s q}$, given by:

$$
E_{s q}=\sum_{i=1}^{4}\left(\frac{V_{e f f_{i}}^{2}}{\sum_{i=1}^{4} V_{e f f_{i}}^{2}}-\frac{g_{i}(\alpha, \beta)}{\sum_{i=1}^{4} g_{i}(\alpha, \beta)}\right)^{2}
$$

where $g_{i}$ is the value of the sensitivity function for each wire orientation. A look-up table method and an iterative pattern search procedure were required to do so. Upon determining the flow angles and corresponding values of the sensitive functions, the resultant flow velocity is given by: 


$$
V=\frac{1}{4}\left(\sum_{i=1}^{4} \frac{V_{e f f_{i}}^{2}}{g_{i}(\alpha, \beta)}\right)^{1 / 2}
$$

Since the hot-wire signals cannot be recorded simultaneously for the two orientations of the probe, this method cannot provide instantaneous information regarding the complete flow field. Therefore, to extract the turbulence data a separate procedure developed by Buresti and Di Cocco (1987) was applied. This procedure involves solving an over-determined non-linear system of equations derived from the general response equation of the hot-wire sensor:

$$
V_{e f f_{i}}^{2}=A_{1_{i}} u^{2}+A_{2_{i}} v^{2}+A_{3_{i}} w^{2}+A_{4_{i}} v w+A_{5_{i}} u w+A_{6_{i}} v w
$$

where $i$ is the wire number and the coefficients $A_{1}-A_{6}$ are determined using a non-linear least squares fitting procedure applied to the directional sensitivity functions. The system of equations is formed with expressions for the mean effective cooling velocity, $V_{\text {eff }}$, and the mean fluctuating effective cooling velocity, $\overline{v_{e f f}^{\prime 2}}$, in terms of the six Reynolds stresses and the three mean components of velocity. Additional equations, applicable only to an $x$-type probe, for $\overline{\left(v_{e f f_{1}}^{\prime}-v_{e f f_{2}}^{\prime}\right)^{2}}, \overline{\left(v_{e f f_{1}}^{\prime}+v_{e f f_{2}}^{\prime}\right)^{2}}$ and $\overline{v_{e f f_{1}}^{\prime} \cdot v_{e f f_{2}}^{\prime}}$ where the subsrcripts 1 and 2 indicate the wire number, were also included. Using the directional sensitivity functions, $g_{1-4}(\alpha, \beta)$ to determine the mean velocities, the solution for the six Reynolds stresses was obtained using an optimization technique. As mentioned, the solution accuracy was improved by using additional data collected at a third roll orientation of $45^{\circ}$.

\section{E.4 Uncertainties of the X-type Hotwire Probe}

The uncertainties of the hotwire measurement techniques are discussed in the following section. The analysis will first address the uncertainty of the measured mean, $V_{\text {eff }}$, and $r m s$, $\left(\overline{v_{e f f}^{\prime 2}}\right)^{1 / 2}$, effective cooling velocities. These uncertainties are then combined with those introduced by the data reduction methods used to infer the mean velocity vector, flow angles and the Reynolds stresses. 


\section{E.4.1 Uncertainty of the Effective Cooling Velocity}

The uncertainty of the effective cooling velocity was estimated following a procedure given by Yavuzkurt (1984). The procedure combines the uncertainties of the measured jet velocity, $V_{j e t}$, and those introduced through the data reduction and curve fitting procedure. As mentioned in Section 3.9, the jet velocity is calculated from the nozzle contraction pressure difference $\left(\triangle P=P_{C l}-P_{C 2}\right)$ and is given by,

$$
V_{j e t}=\sqrt{\frac{2 \cdot \Delta P \cdot C_{q_{C a l}}}{\rho}}=\sqrt{\frac{2 \cdot \Delta P \cdot C_{q_{C a l}}}{P / R T}}
$$

where $C_{q_{C a l}}$ is the dynamic pressure coefficient and $\rho$ is the air density computed using the ideal gas law $(\rho=P / R T)$. The uncertainty of the jet velocity, $\delta V_{j e t}$, is estimated by applying the method Moffat (1982) to the equation E.8, giving

$$
\delta V_{j e t}=\frac{1}{2} V_{j e t}\left(\left(\frac{\delta \Delta P}{\Delta P}\right)^{2}+\left(\frac{\delta C_{q_{C a l}}}{C_{q_{C a l}}}\right)^{2}+\left(\frac{\delta C_{q_{C a l}}}{C_{q_{C a l}}}\right)^{2}+\left(\frac{\delta P}{P}\right)^{2}+\left(\frac{\delta T}{T}\right)^{2}\right)^{1 / 2}
$$

where $\delta$ is used to denote the uncertainty of a measurement quantity (e.g. $\delta \Delta P$ is the uncertainty of the contraction pressure difference, $\Delta P$ ). The errors introduced by curve fitting and data processing, $\delta V_{C a l}$, were estimated by computing the effective velocity for each calibration point.

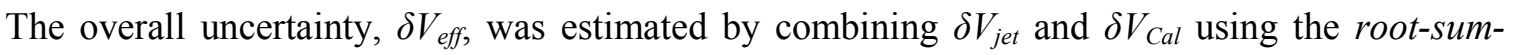
squares (RSS) method. Equation E.9 shows that the overall uncertainty is a function of the contraction pressure difference $(\Delta P)$ and thus larger uncertainties occur at lower velocity levels as shown in Figure E.4. Yavuzkurt also demonstrates that relative uncertainty in the instantaneous effective velocity, $\delta v_{\text {eff }}^{\prime} / v_{\text {eff }}^{\prime}$, is equal to the relative uncertainty of the mean effective velocity, $\delta V_{\text {eff }} / V_{\text {eff }}$, as well as the relative $r m s$ value, $\delta\left(\overline{v_{e f f}^{\prime 2}}\right)^{1 / 2} /\left(\overline{v_{e f f}^{\prime 2}}\right)^{1 / 2}$. Thus, the relative uncertainty distribution shown in Figure E.4 is also applicable to the rms of the effective cooling velocities, and is therefore applicable to the overall uncertainty of the Reynolds stresses. 


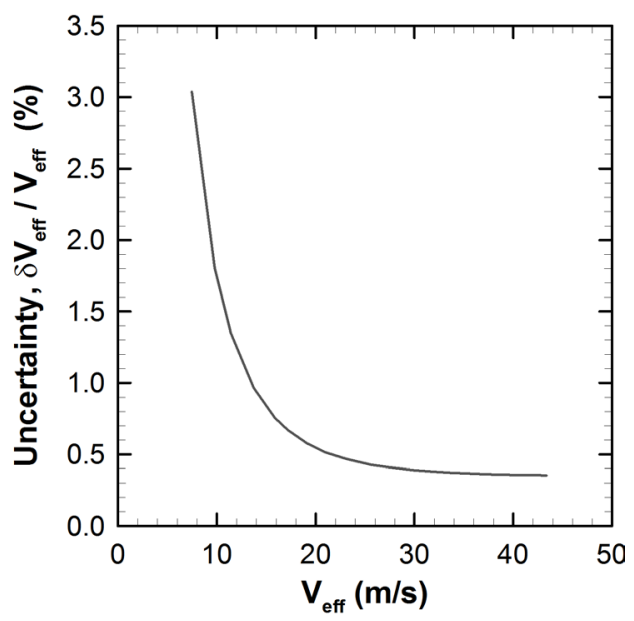

Figure E.4: Estimated Uncertainty of $V_{e f f}$ and $\left(\overline{v_{e f f}^{\prime 2}}\right)^{1 / 2}$

\section{E.4.2 Uncertainty of the Mean Velocity Vector}

As previously described, the mean velocity components in an unknown flow were inferred using the effective velocities from each sensor. Errors introduced by the directional calibration procedure were evaluated by reprocessing the calibration data (from multiple calibrations) as if it were an unknown flow - a procedure similar to that used by Benner (2003) to estimate the uncertainty of a seven-hole probe. The computed velocity and flow angles were compared to the true known values at each measurement location. A total of 1922 points were evaluated to obtain an average value of the uncertainty due to the directional calibration. The uncertainty in the measured mean velocity vector and flow angles, introduced by the directional calibration, was estimated to be $1.29 \%$ of the local velocity and $0.5^{\circ}$ respectively.

Interpolation errors were accounted for separately by removing data points from the directional sensitivity surfaces and re-processing the omitted data. Again, the computed and true values were compared to obtain an average estimate of the uncertainty due to the interpolation procedure. The uncertainty of the mean velocity vector and flow angles, caused by the interpolation procedure, was estimated to be $1.32 \%$ of the local velocity and $0.7^{\circ}$ respectively. 
The method by Moffat (1982) was used to combine the uncertainties introduced by errors in the measured effective cooling velocity, the directional calibration and interpolation. The overall uncertainty is a function of the measured velocity level as shown in Figure E.5. The experimental measurements of the cascade flow (at an inlet $\operatorname{Re}_{1}=50,000$ ) had outlet velocities ranging from 22 to $34 \mathrm{~m} / \mathrm{s}$. At this level the uncertainty is nearly constant at about $2 \%$ of the mean velocity, as shown in Figure E.5. The combined uncertainty of the flow angles of the mean velocity components is $0.9^{\circ}$.

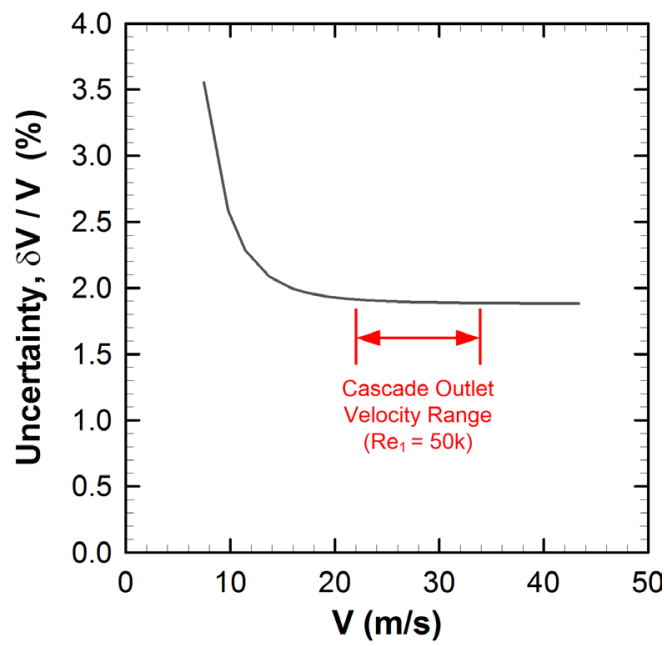

Figure E.5: Overall uncertainty of the measured mean velocity

\section{E.4.3 Uncertainty of the Reynolds Stresses}

It was not possible to experimentally generate flow fields with known and controllable turbulence quantities. Therefore, to estimate the uncertainty of measured Reynolds stresses, computer generated flow fields were used to simulate known, time varying signals with different turbulence statistics. Buresti and Di Cocco (1987) used a similar procedure. These known (or true) flows were then processed using the data reduction procedures for the $x$-type probe. The computed Reynolds stresses were compared to the "true" values from the simulated flows. The evaluated errors then account for: the errors introduced in the hotwire sensitivity coefficients $\left(A_{I}\right.$ to $A_{6}$ ), the errors caused by the truncation of higher order terms in the hotwire response equations 
and the errors introduced by the optimization scheme. The sensitivity of the uncertainty to errors in the mean velocity vector and flow angles was also tested.

A total of five, three-component flows (15 total signals), were artificially generated following a procedure for creating synthesized turbulence by Davidson and Billson (2006) - a common approach for generating inlet boundary conditions for unsteady CFD simulations (LES, URANS, DES). In this analysis the signals were generated for a single point rather than a twodimensional plane. For each of the five flows, three levels of turbulence intensity $(T u=5,10$ and $15 \%)$ and eight velocity combinations were generated, as shown in Table E.1. This totalled 24 cases for each of the five simulated flows.

Table E.1: Summary of velocity cases for each level of turbulence intensity

\begin{tabular}{|c|c|c|c|c|c|c|c|c|}
\hline $\begin{array}{c}\text { Mean velocity } \\
\text { component }\end{array}$ & Case 1 & Case 2 & Case 3 & Case 4 & Case 5 & Case 6 & Case 7 & Case 8 \\
\hline $\mathrm{U}$ & 1 & 1 & 1 & 1 & 1 & 1 & 1 & 1 \\
\hline $\mathrm{V}$ & 0 & 0.1 & 0.2 & 0.1 & 0 & -0.1 & 0 & -0.1 \\
\hline $\mathrm{W}$ & 0 & 0.1 & 0.2 & 0 & 0.1 & 0 & -0.1 & -0.1 \\
\hline
\end{tabular}

Table E.2 summarizes the uncertainty estimates of the Reynolds stresses, normalized by $\overline{u^{\prime 2}}$, for each turbulence level. The relative uncertainty of turbulence intensity is also given. For a given level of turbulence the errors were insensitive to flow angularity and therefore the uncertainties represent an average value from the cases of Table E.1.

The data reduction procedures applied here are only applicable to flows of low to moderate levels of turbulence intensity. As a result, the cases with the highest turbulence intensity $(T u=15 \%)$ produced the highest uncertainty, as shown in Table E.2. In this thesis, the highest measured turbulence intensity does not exceed $10 \%$.

The $\overline{v^{\prime} w^{\prime}}$ Reynolds stress has the highest relative uncertainty of the shear components. Since the $x$-type probe is only rotated about the axis of the probe body, rather than other rotations about the transverse and bi-normal axes, the probe is less sensitive to velocity fluctuations in $y z$-plane of the probe. As shown by Buresti and Di Cocco (1987), the accuracy of this procedure 
is improved by using additional measurements orientations; however these positions are generally not feasible for cascade measurements. A quadruple wire probe may be a suitable alternative; however, these probes are typically larger than the $x$-type and may not provide adequate spatial resolution. For this investigation, the $x$-type hotwire was a suitable trade-off between measurement uncertainty, probe spatial resolution and efficient data collection.

Table E.2: Overall uncertainty of the measured Reynolds stresses and the turbulence intensity (Tu)

\begin{tabular}{|c|c|c|c|}
\hline & $T u=5 \%$ & $T u=10 \%$ & $T u=15 \%$ \\
\hline$\left({\overline{u^{\prime 2}}}_{\text {True }}-\overline{u^{\prime 2}}\right) /{\overline{u^{\prime 2}}}_{\text {True }} \times 100$ & $1.3 \%$ & $1.4 \%$ & $1.6 \%$ \\
\hline$\left({\overline{v^{\prime 2}}}_{\text {True }}-\overline{v^{\prime 2}}\right) /{\overline{u^{\prime 2}}}_{\text {True }} \times 100$ & $2.9 \%$ & $4.2 \%$ & $6.4 \%$ \\
\hline$\left(\overline{w^{\prime 2}}\right.$ True $\left.-\overline{w^{\prime 2}}\right) / \overline{u^{\prime 2}}$ True $\times 100$ & $2.3 \%$ & $3.1 \%$ & $6.8 \%$ \\
\hline$\left({\overline{u^{\prime} v^{\prime}}}_{\text {True }}-\overline{u^{\prime} v^{\prime}}\right) /{\overline{u^{\prime 2}}}_{\text {True }} \times 100$ & $0.2 \%$ & $0.6 \%$ & $1.7 \%$ \\
\hline$\left(\overline{u^{\prime} w^{\prime}}{ }_{\text {True }}-\overline{u^{\prime} w^{\prime}}\right) /{\overline{u^{\prime 2}}}_{\text {True }} \times 100$ & $1.2 \%$ & $1.2 \%$ & $1.3 \%$ \\
\hline$\left(\overline{v^{\prime} w^{\prime}}\right.$ True $\left.^{\prime}-\overline{v^{\prime} w^{\prime}}\right) /{\overline{u^{\prime 2}}}_{\text {True }} \times 100$ & $1.8 \%$ & $1.5 \%$ & $3.2 \%$ \\
\hline$\left(T u_{\text {True }}-T u\right) / T u_{\text {True }} \times 100$ & $1.3 \%$ & $1.4 \%$ & $1.6 \%$ \\
\hline
\end{tabular}




\section{Appendix F}

\section{Additional Experimental Results for the Cascade Configuration with a Rectangular Platform Overlap and Flat Intra-passage Endwalls (RF)}

\section{F.1 Introduction}

For brevity in the discussion chapters some results for the RF configuration were not included. For completeness, additional measurements at different axial planes are included in this appendix.

\section{F.2 Downstream Measurements for the Rectangular Flat (RF) configuration at the $1.20 C_{x}$ measurement plane}

The measurements shown in this section were made for the RF configuration for different levels of purge flow at the $1.20 C_{x}$ axial plane. Floods of the total pressure, streamwise vorticity and the secondary kinetic energy are shown in Figures F.1 through F.3. Corresponding measurements from the $1.40 C_{x}$ plane were presented in Section 7.6.3. 
(a)

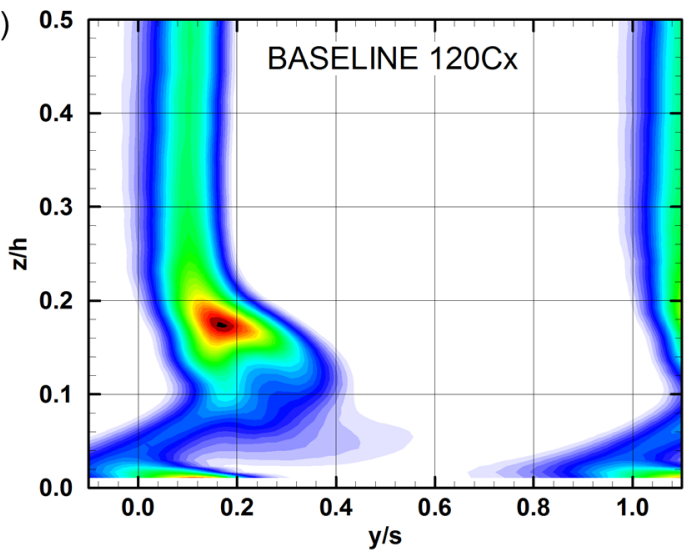

(c)

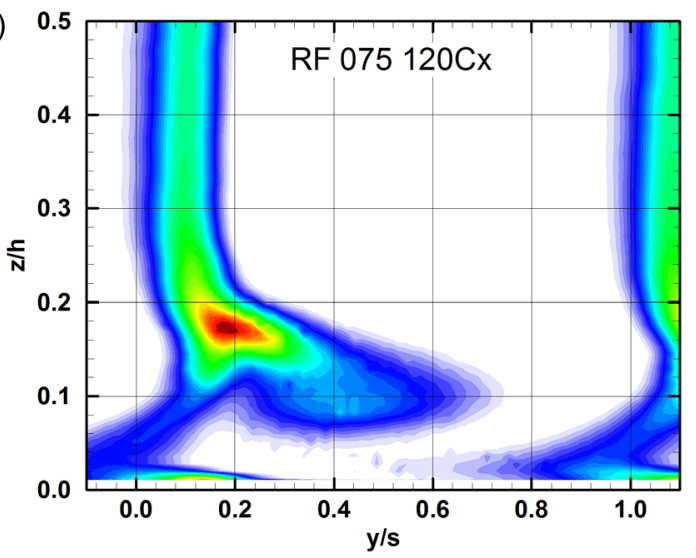

(e)

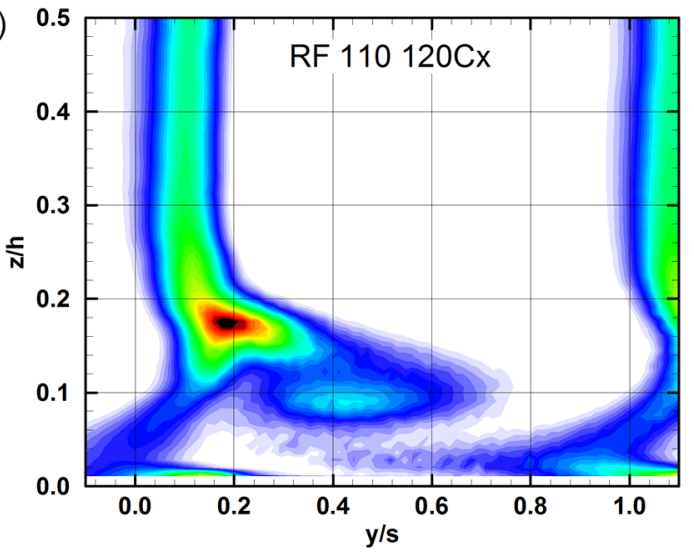

(b)

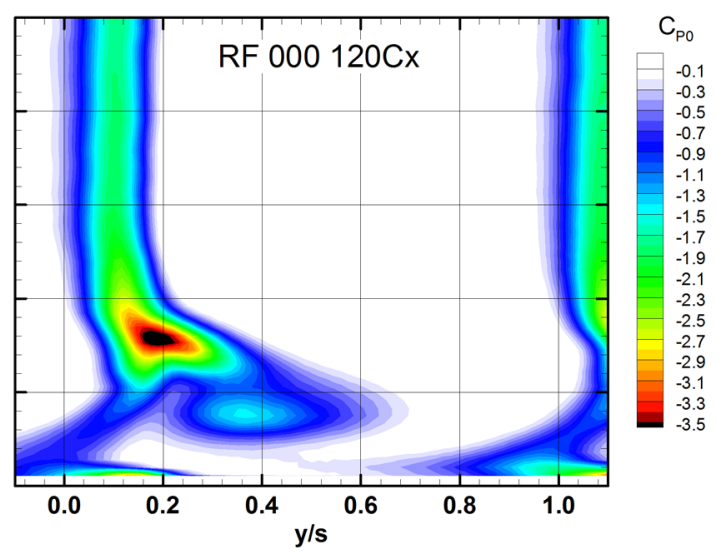

(d)

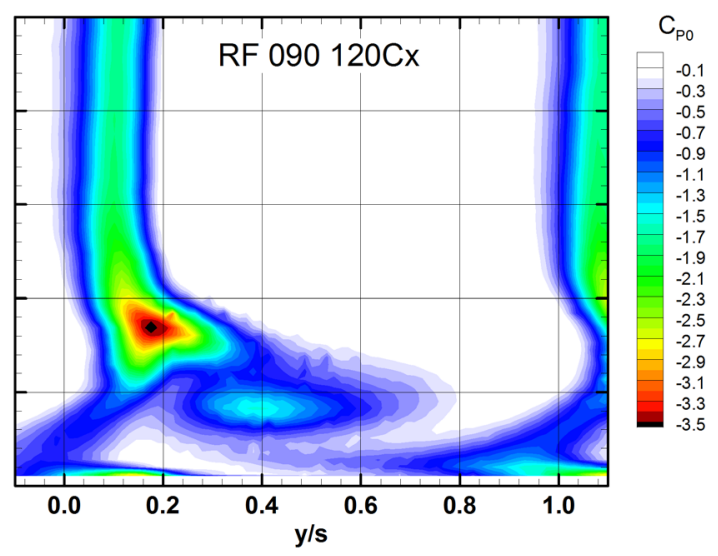

(f)

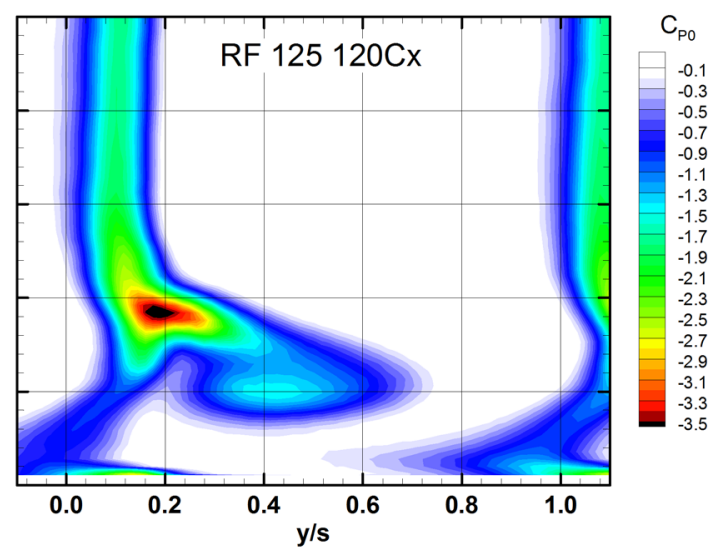

Figure F.1: Floods of the total pressure coefficient $\left(C_{P 0}\right)$ for varying levels of purge flow measured at $1.20 C_{x}$ 
(a)

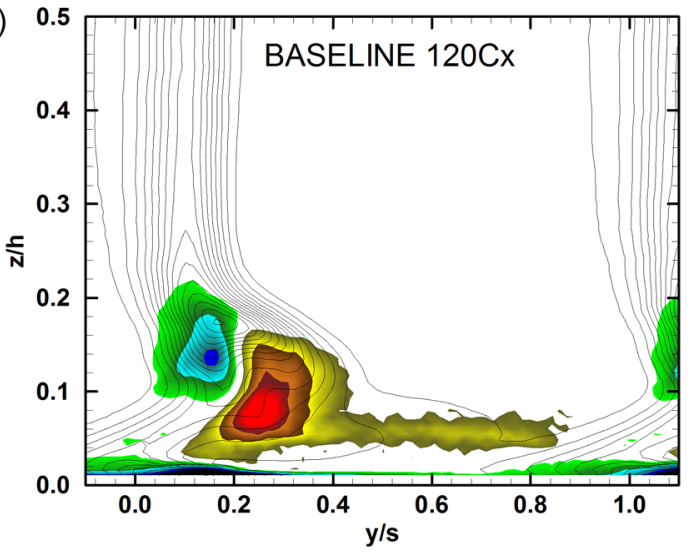

(c)

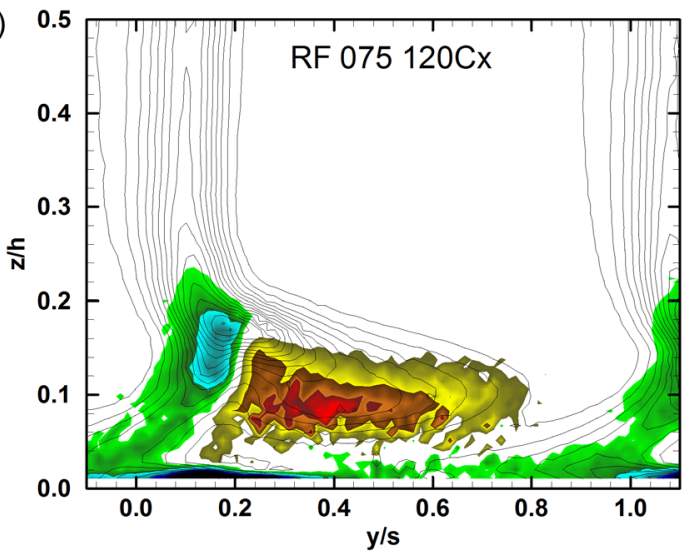

(e)

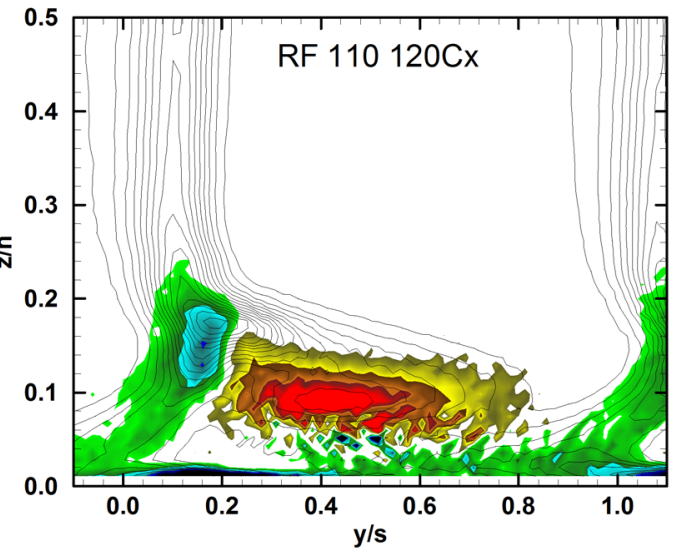

(b)

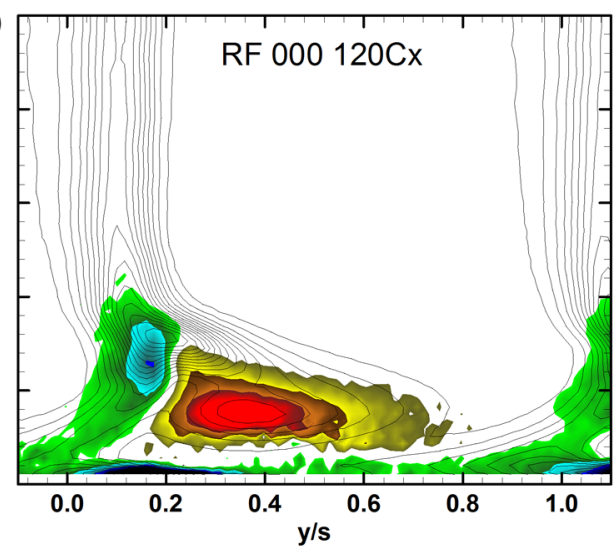

(d)

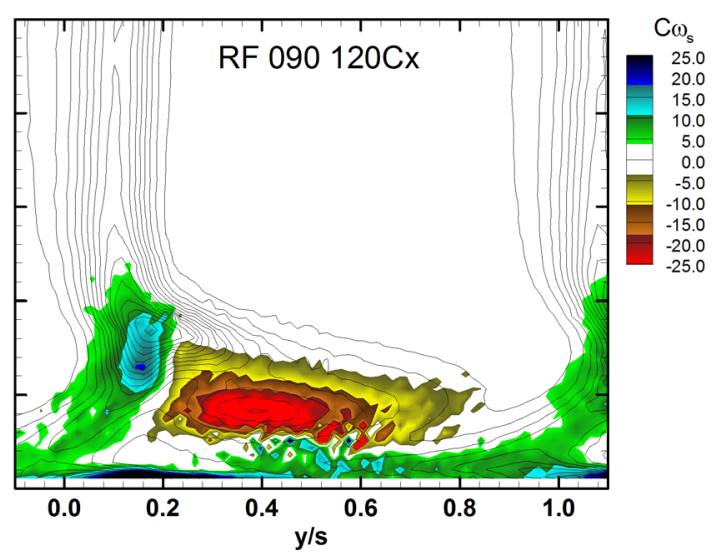

(f)

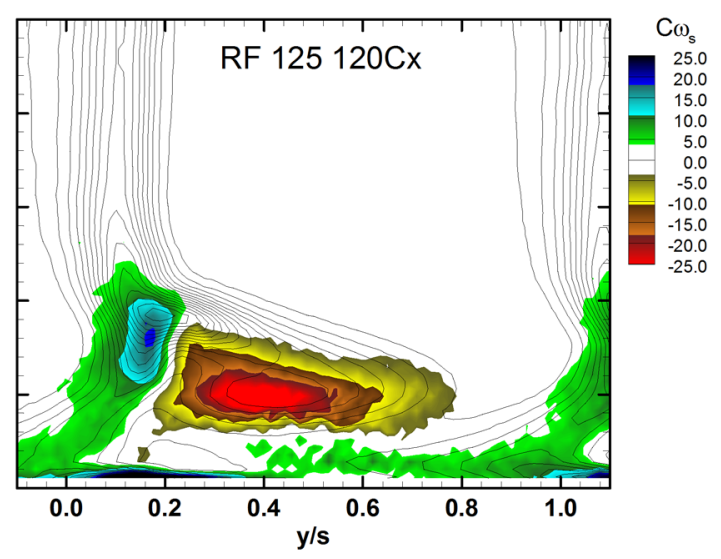

Figure F.2: Floods of the streamwise vorticity coefficient $\left(C_{\omega S}\right)$ with contour lines of the total pressure coefficient $\left(C_{P 0}\right)$ for varying levels of purge flow measured at $1.20 C_{x}$ 
(a)

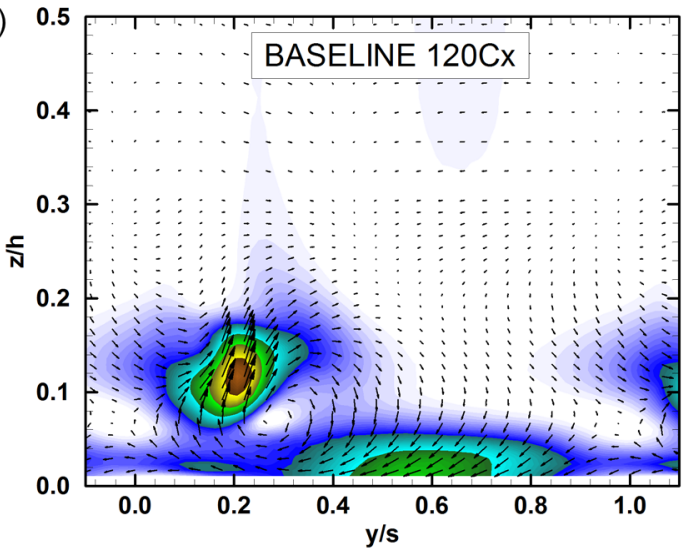

(c)

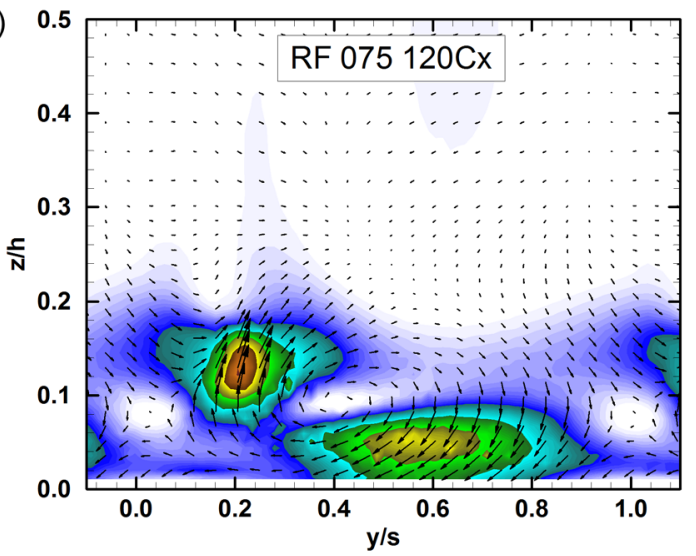

(e)

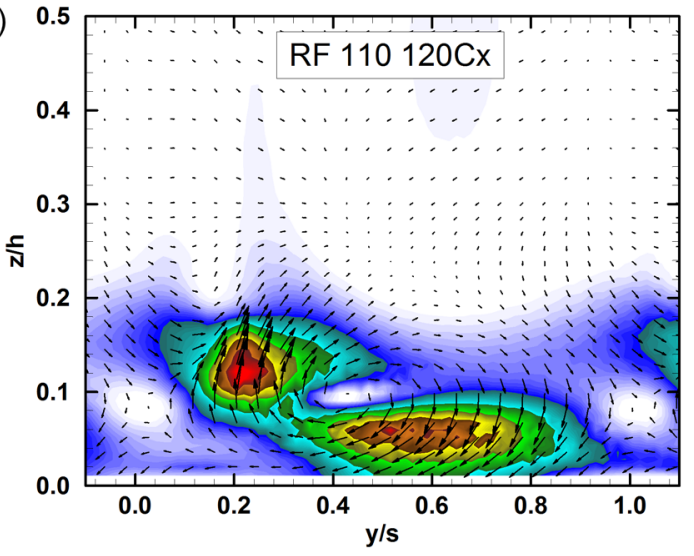

(b)
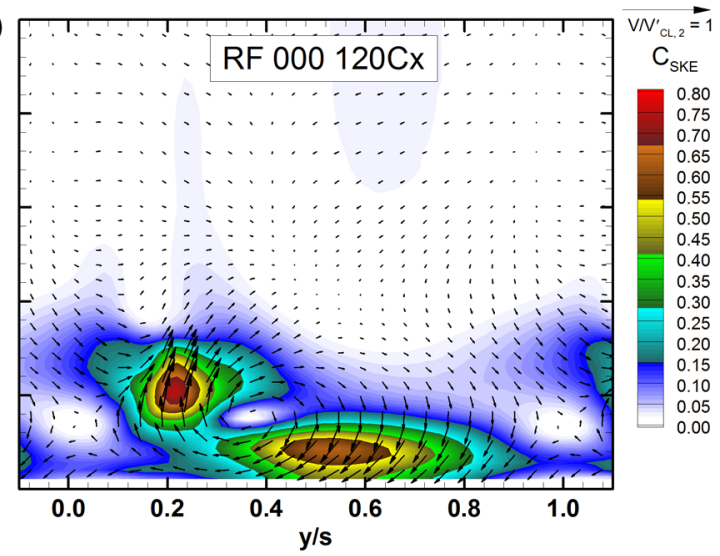

(d)

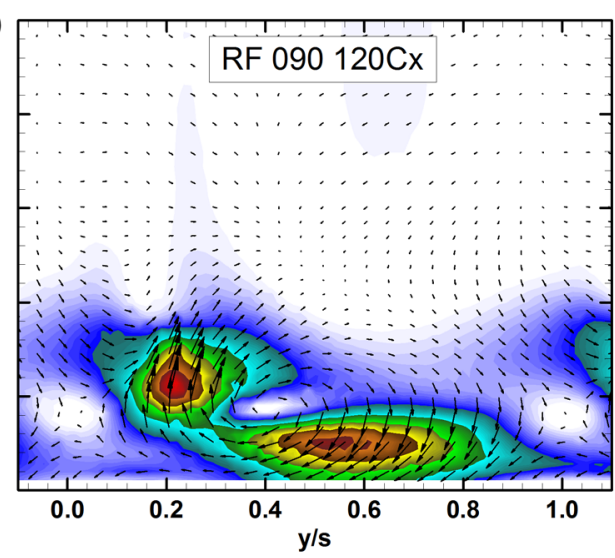

$\mathrm{VN}_{\mathrm{C}-2}=1$

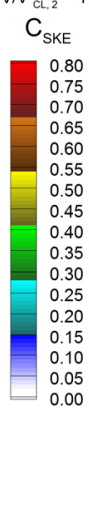

(f)

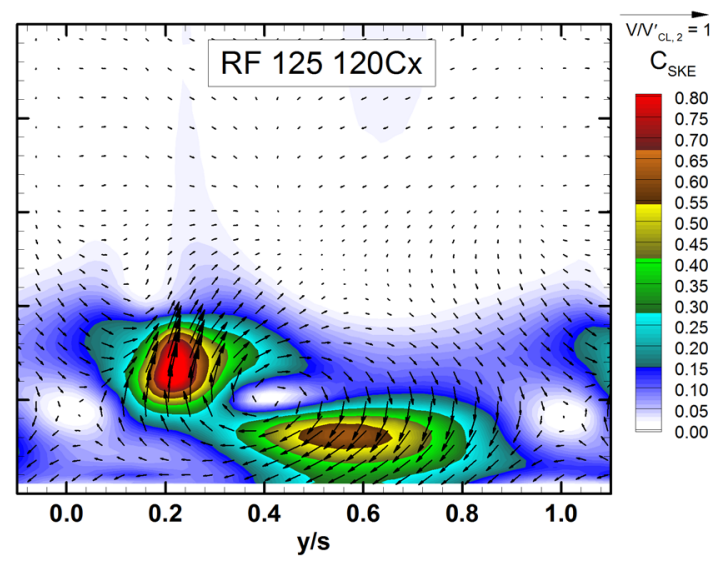

Figure F.3: Floods of the secondary kinetic energy coefficient $\left(C_{S K E}\right)$ with the secondary velocity vectors overlaid for varying levels of purge flow measured at $1.20 C_{x}$ 


\section{Appendix G}

\section{Experimental Results for the Cascade Configuration with an Elliptical Overlap and Non-axisymmetric Endwall Contouring (EC)}

\section{G.1 Introduction}

The present appendix presents the results from the cascade configuration with the elliptical platform overlap and non-axisymmetric endwall contouring applied within the blade passage. This configuration is denoted EC.

\section{G.2 Downstream Measurements and Overall Integrated Flow Quantities for the EF configuration at the 1.40Cx measurement plane}

The measurements shown in this section were made for the EC configuration for different levels of purge flow at the $1.40 C_{x}$ axial plane. Floods of the total pressure, streamwise vorticity and the secondary kinetic energy are shown in Figures G.1through G.3. The in-plane and mixed- 
out losses are shown in Figure G.4. The integrated flow quantities are summarized in Table G.1 and Table G.2

\section{Contoured Endwall}

(a)

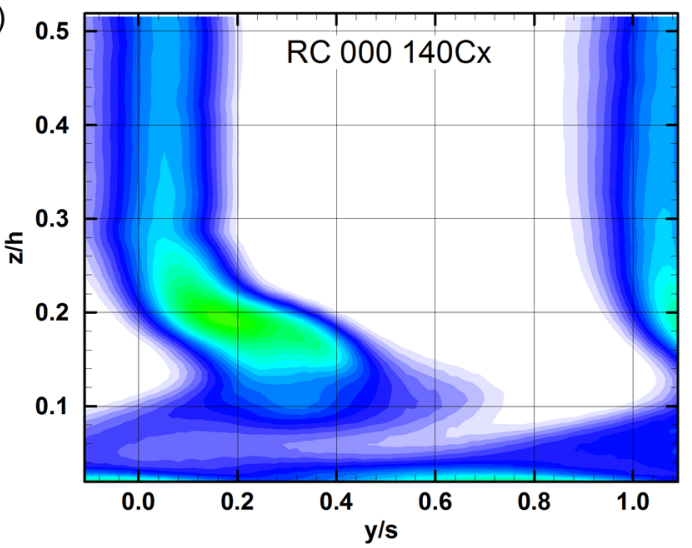

(c)

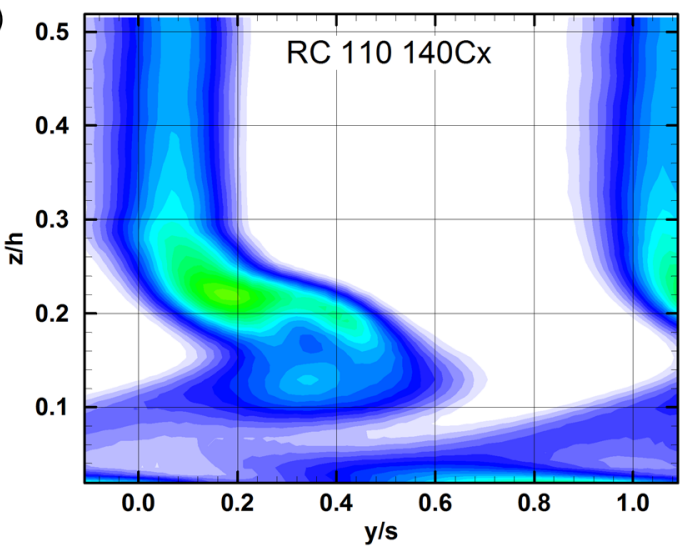

(e)

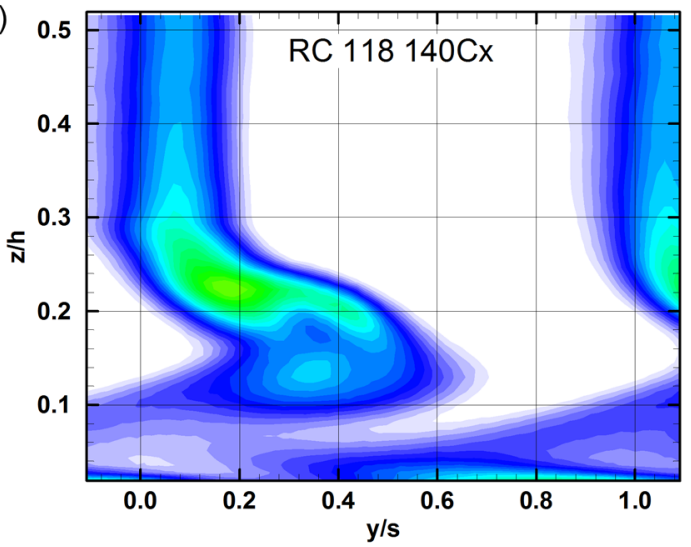

\section{Elliptical Overlap and Contoured Endwall}

(b)

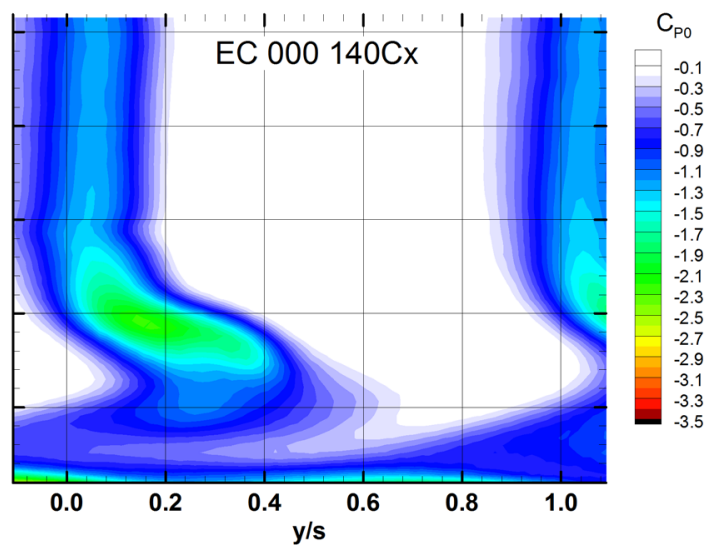

(d)

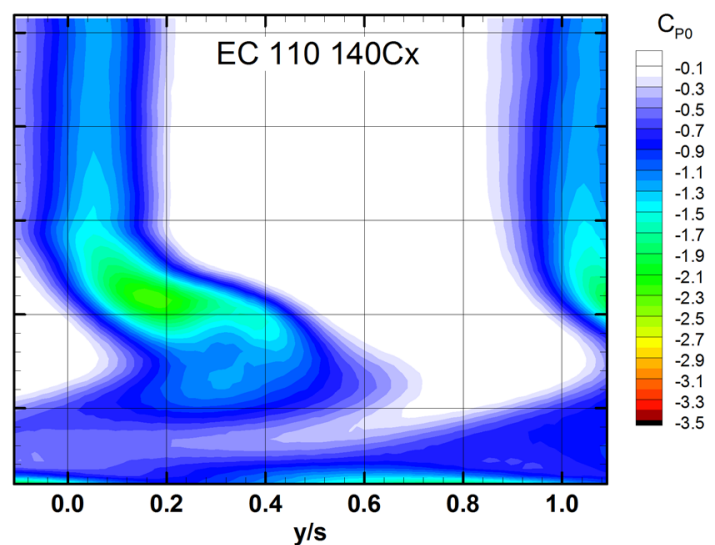

(f)

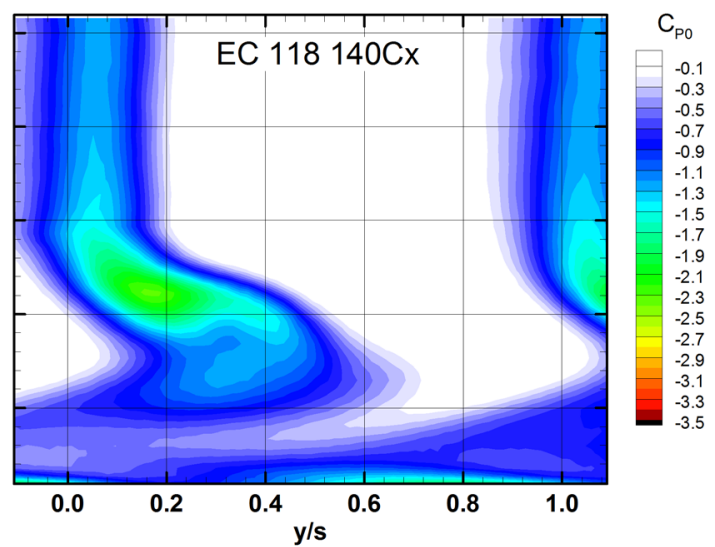

Figure G.1: Floods of the total pressure coefficient $\left(C_{P 0}\right)$ for varying levels of purge flow measured at $1.40 C_{x}$ 
Contoured Endwall

(a)

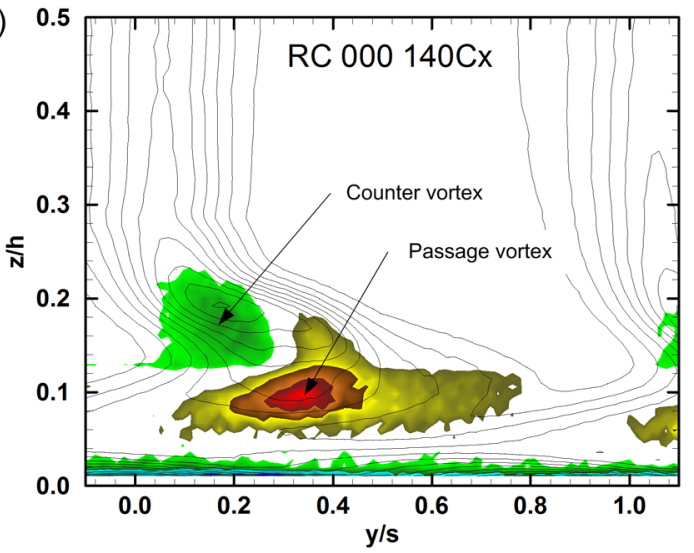

(c)

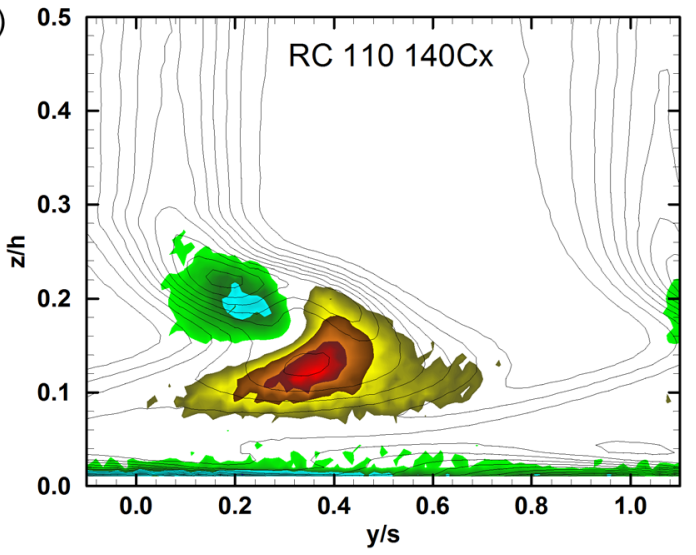

(e)

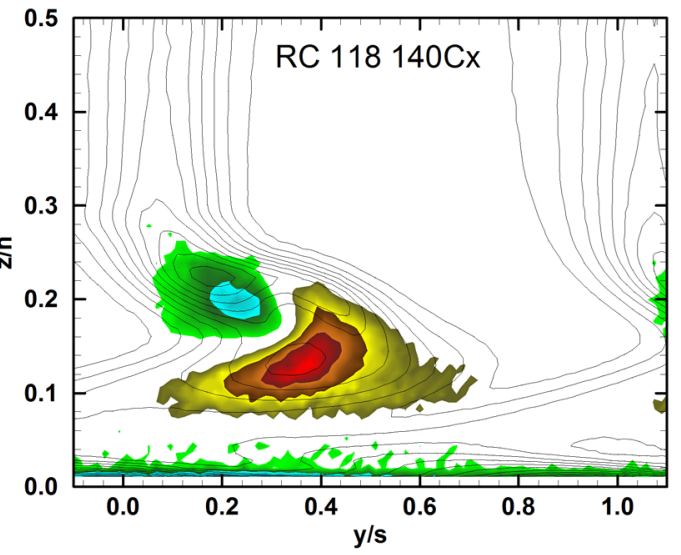

Elliptical Overlap and Contoured Endwall

(b)

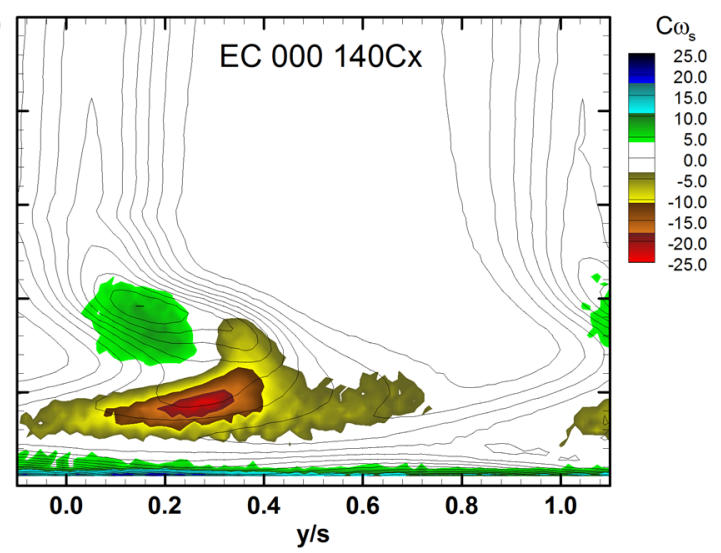

(d)

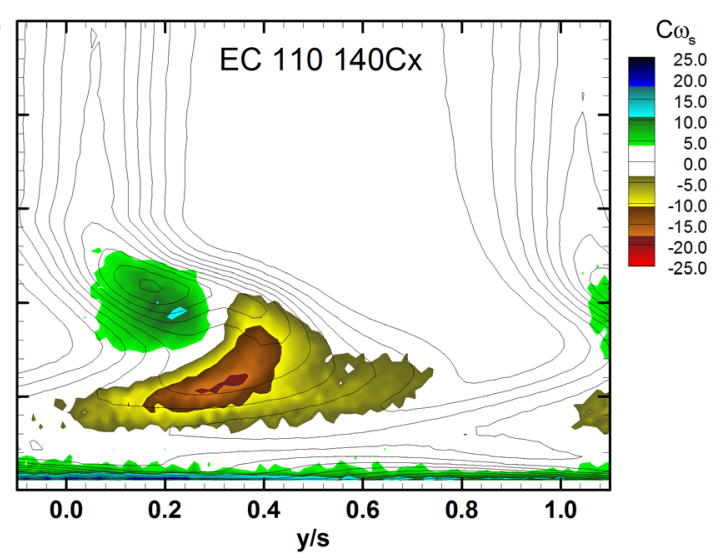

(f)

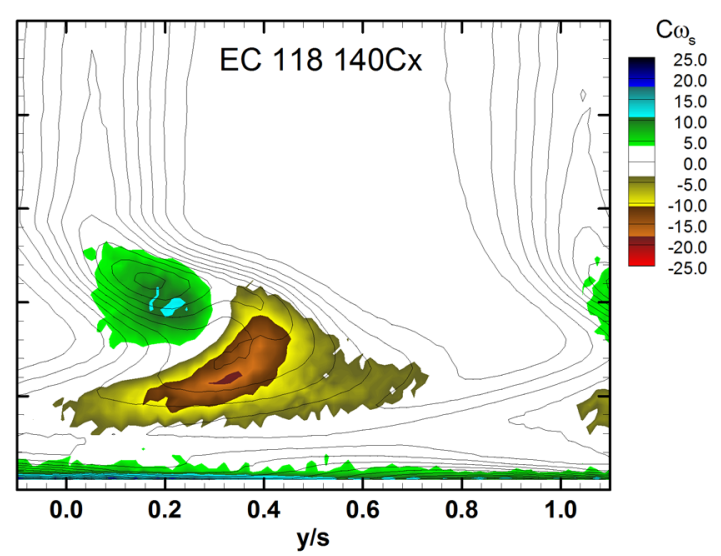

Figure G.2: Floods of the streamwise vorticity coefficient $\left(C_{\omega S}\right)$ with contour lines of the total pressure coefficient $\left(C_{P 0}\right)$ for varying levels of purge flow measured at $1.40 C_{x}$ 
Contoured Endwall

(a)

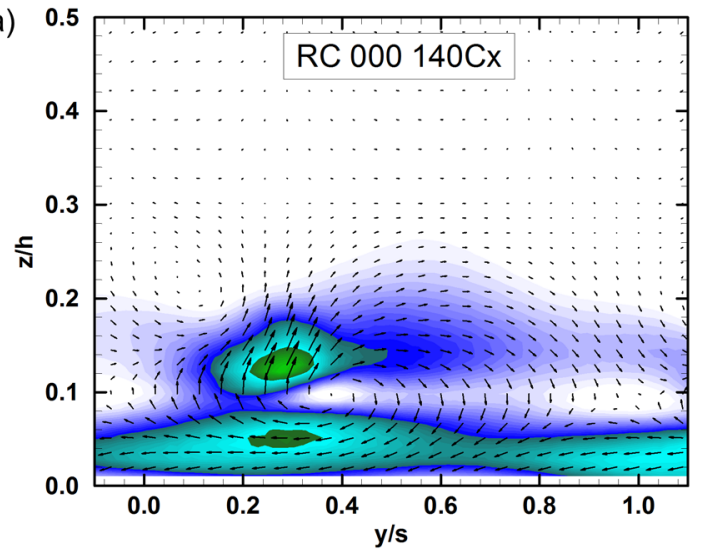

(c)

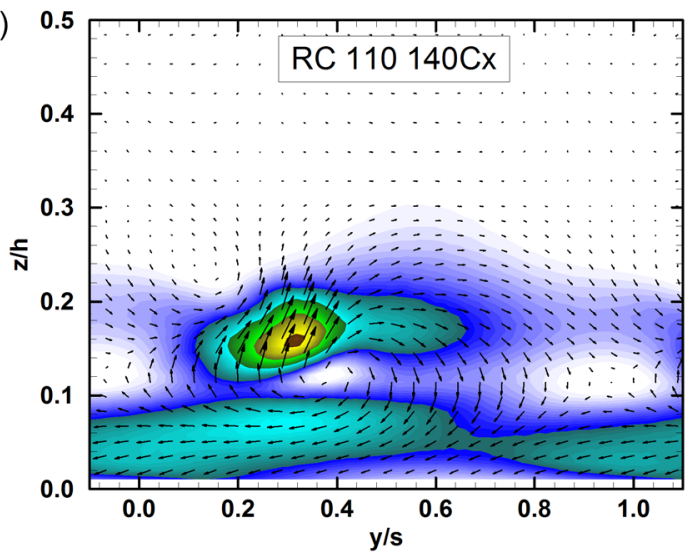

(e)

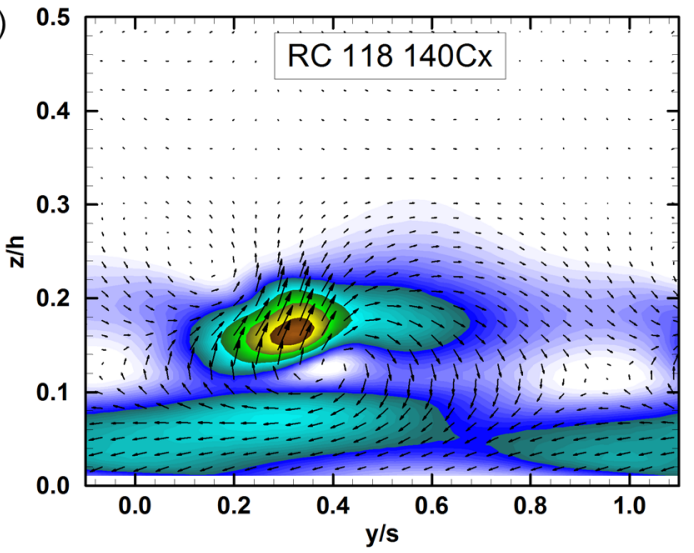

\section{Elliptical Overlap and Contoured Endwall}

(b)

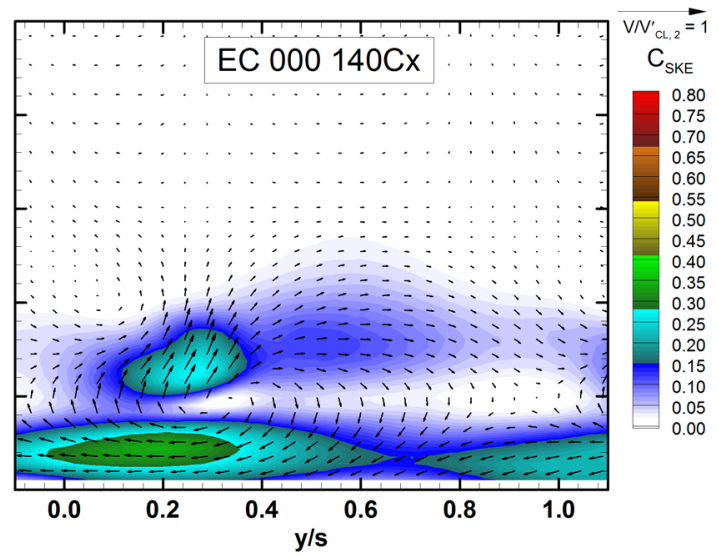

(d)

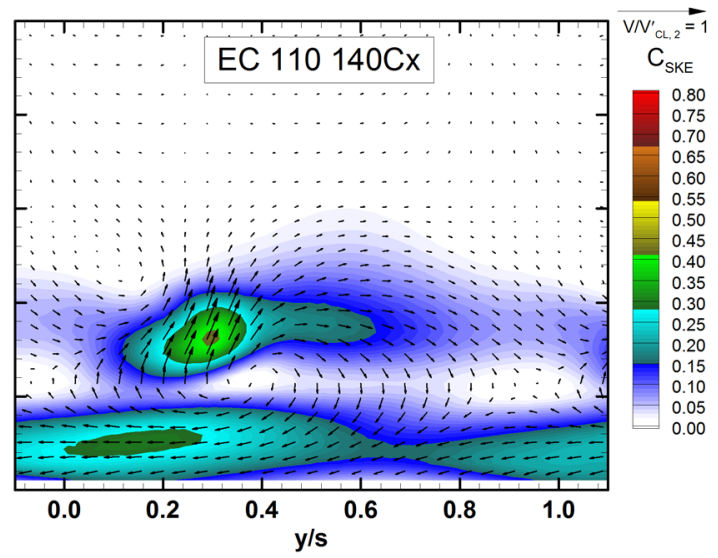

(f)

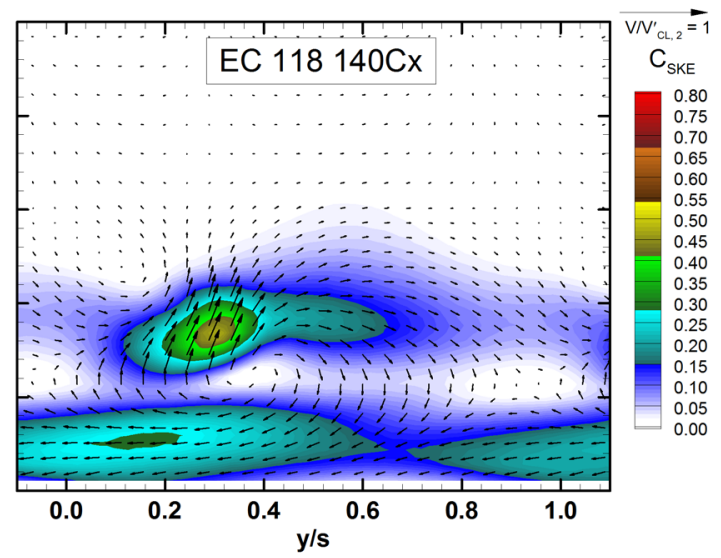

Figure G.3: Floods of the secondary kinetic energy coefficient $\left(C_{S K E}\right)$ with the secondary velocity vectors overlaid for varying levels of purge flow measured at $1.40 C_{x}$ 
(a)

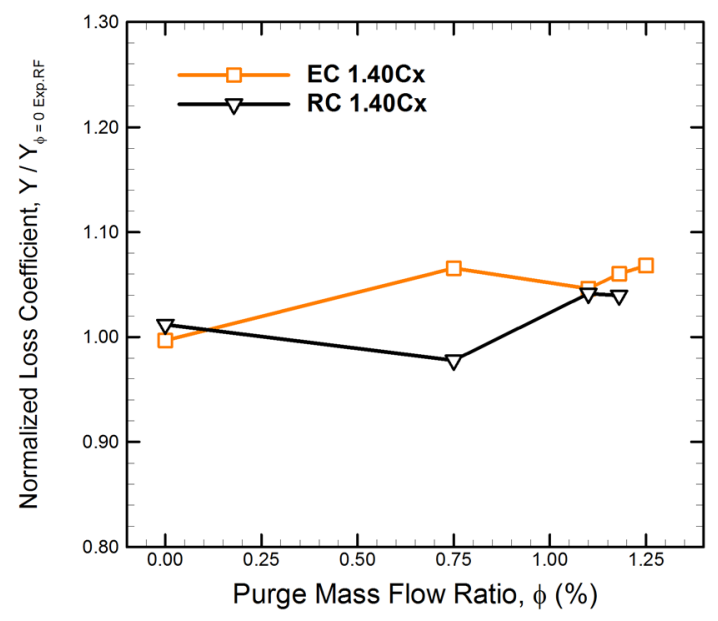

(b)

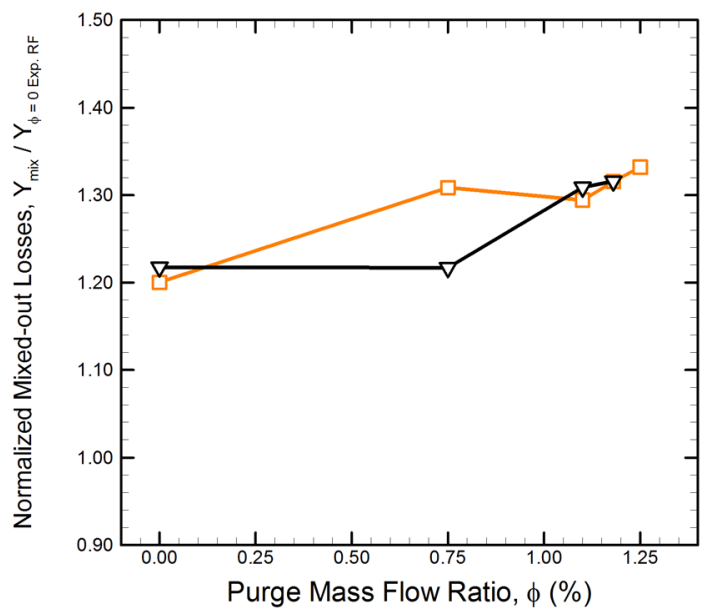

Figure G.4: Overall $(Y)$ and Mixed-out $\left(Y_{\text {mixed }}\right)$ loss coefficients for different levels of purge flow at $\mathbf{1 . 4 0 C}_{\mathrm{x}}$ for the RC and EC configurations

Table G.1: $\quad$ Summary of the measured overall integrated flow quantities

\begin{tabular}{|c|c|c|c|c|}
\hline & EC 000 & EC 075 & EC 110 & EC 118 \\
\hline Flow Quantity & Exp. & Exp. & Exp. & Exp. \\
\hline $\mathrm{Y}$ & 0.051 & 0.055 & 0.054 & 0.054 \\
\hline$Y_{p}$ & 0.030 & 0.030 & 0.030 & 0.030 \\
\hline $\mathrm{Y}_{\mathrm{sec}}$ & 0.021 & 0.025 & 0.024 & 0.024 \\
\hline$\alpha_{2 "}^{\prime \prime}$ & -72.2 & -72.1 & -72.0 & -72.0 \\
\hline$\alpha_{2, \text { midspan' }}$ & -73.3 & -73.2 & -73.2 & -73.2 \\
\hline $\mathrm{C}_{\text {SKE" }}$ & 0.049 & 0.061 & 0.063 & 0.065 \\
\hline$Y_{\text {mixed-out }}$ & 0.062 & 0.067 & 0.066 & 0.068 \\
\hline$Y_{p, \text { mixed-out }}$ & 0.031 & 0.031 & 0.031 & 0.031 \\
\hline$Y_{\text {sec, mixed-out }}$ & 0.031 & 0.036 & 0.035 & 0.037 \\
\hline
\end{tabular}


Table G.2: Summary of the measured and predicted overall integrated flow quantities relative to the zero net purge flow case (RF 000)

\begin{tabular}{|c|c|c|c|c|}
\hline Flow Quantity & $\begin{array}{c}\text { EF } 000 \\
\text { Exp. } \\
\end{array}$ & $\begin{array}{c}\text { EF } 075 \\
\text { Exp. } \\
\end{array}$ & $\begin{array}{c}\text { EF } 110 \\
\text { Exp. } \\
\end{array}$ & $\begin{array}{c}\text { EF } 118 \\
\text { Exp. }\end{array}$ \\
\hline$Y / Y_{R F} 000$ (Exp.) & $100 \%$ & $107 \%$ & $105 \%$ & $106 \%$ \\
\hline$Y_{p} / Y_{R F} 000(E \times p)$. & $59 \%$ & $59 \%$ & $59 \%$ & $59 \%$ \\
\hline$Y_{\text {sed }} Y_{\text {RF } 000 \text { (EXP.) }}$ & $41 \%$ & $48 \%$ & $46 \%$ & $48 \%$ \\
\hline$\alpha_{2 "}$ & -72.2 & -72.1 & -72.0 & -72.0 \\
\hline$\alpha_{2, \text { midspań }}$ & -73.3 & -73.2 & -73.2 & -73.2 \\
\hline $\mathrm{C}_{\mathrm{SKE}}{ }^{\prime \prime}$ & 0.049 & 0.061 & 0.063 & 0.065 \\
\hline$Y_{\text {mixed-out }} / Y_{\text {RF }} 000$ (Exp.) & $120 \%$ & $131 \%$ & $130 \%$ & $132 \%$ \\
\hline$Y_{p, \text { mixed-out }} Y_{R F} 000$ (Exp.) & $61 \%$ & $60 \%$ & $61 \%$ & $60 \%$ \\
\hline$Y_{\text {sec, mixed-out }} Y_{R F} 000$ (Exp.) & $60 \%$ & $71 \%$ & $69 \%$ & $72 \%$ \\
\hline
\end{tabular}

